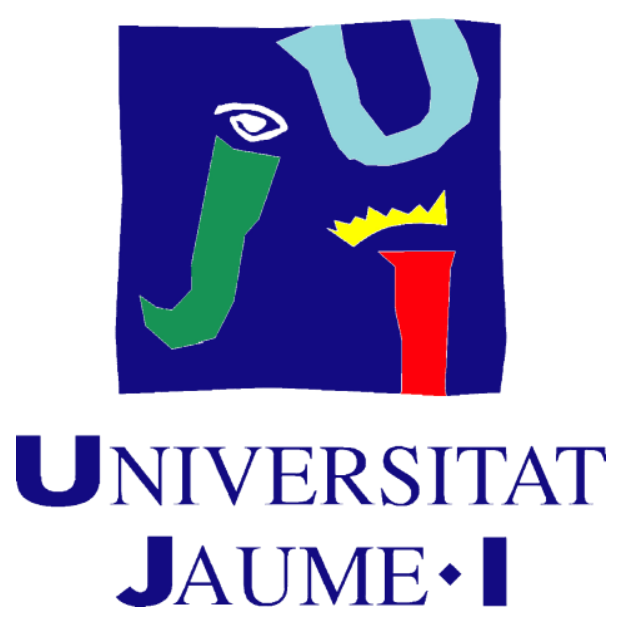

DEPARTAMENTO DE QUIIMICA INORGÁNICA Y ORGÁNICA

\title{
FUNDAMENTAL STUDIES AND
}

\section{APPLICATIONS OF MOLECULAR GELS}

\section{FORMED BY PEPTIDE DERIVATIVES}

Estudios Fundamentales y Aplicaciones de Geles Moleculares Formados por Derivados Peptídicos

PhD Thesis

Cristina Berdugo Gumbau

Supervisors:

Juan F. Miravet Celades and Beatriu Escuder Gil

Castellón, May 2015 


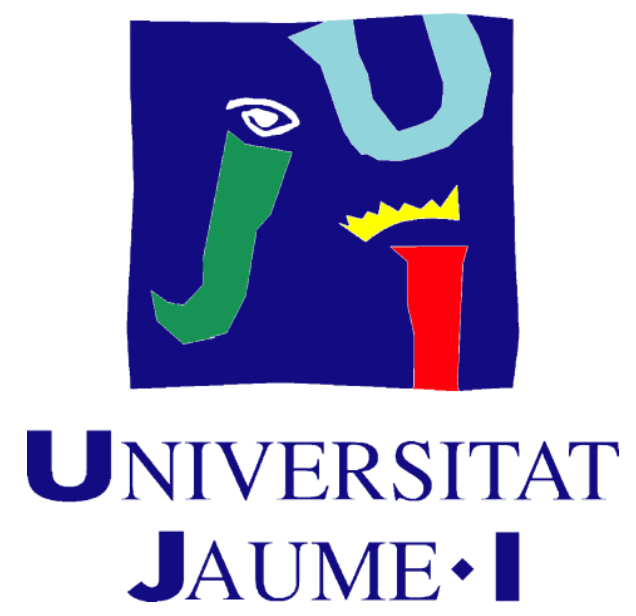

DEPARTAMENTO DE QUIIMICA INORGÁNICA Y ORGÁNICA

Juan Felipe Miravet Celades, Associate Professor at the Department of Inorganic and Organic Chemistry, Universitat Jaume I.

Beatriu Escuder Gil, Associate Professor at the Department of Inorganic and Organic Chemistry, Universitat Jaume I.

CERTIFY that the PhD thesis entitled "Fundamental Studies and Applications of Molecular Gels Formed by Peptide Derivatives" presented by Cristina Berdugo Gumbau, has been developed under their supervision at the Organic Chemistry Section of the Department of Inorganic and Organic Chemistry, Universitat Jaume I.

Castellón, May 2015 



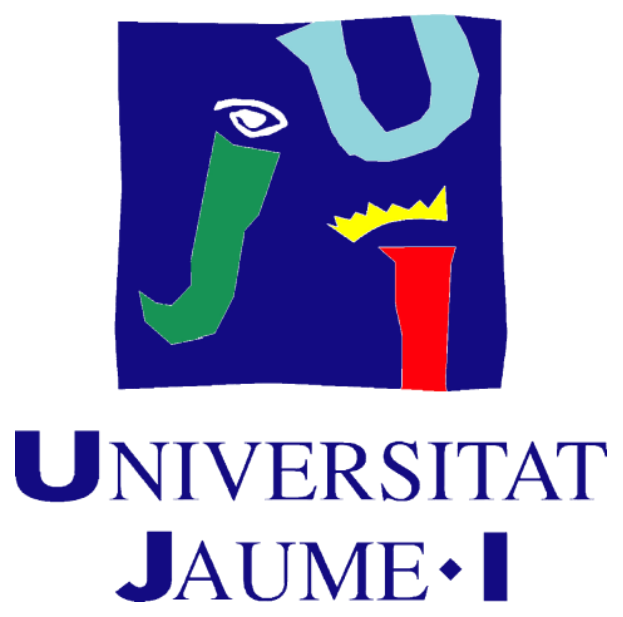

This work has been financed by the Spanish Ministry of Economy and Competitiveness (grants CTQ2009-13961, CTQ2012-37735 and FPI fellowship) and Universitat Jaume I (grant P1.1B2012-25).

Part of this work has been published up to date in the following papers

Substrate Selective Catalytic Molecular Hydrogels: the Role of the Hydrophobic Effect. C. Berdugo; J. F. Miravet; B. Escuder, Chem. Commun., 2013, 49, 10608-10610.

Biocatalytic Self-Assembly of Supramolecular Charge-Transfer Nanostructures Based on n-Type Semiconductor-Appended Peptides. S. K. M. Nalluri; C. Berdugo; N. Javid; P. W. J. M. Frederix; R. V. Ulijn, Angew. Chem. Int. Ed., 2014, 53, 5882 -5887.

Structural Insight into the Aggregation of L-Prolyl Dipeptides and its Effect on Organocatalytic Performance. C. Berdugo; J. F. Miravet; B. Escuder, Org. Biomol. Chem., 2015, 13, 592-600.

Study of the Effect of Polymorphism on the Self-Assembly and Catalytic Performance of an L-Proline Based Molecular Hydrogelator. S. Díaz-Oltra; C. Berdugo; J. F. Miravet; B. Escuder, New J. Chem., 2015, DOI: 10.1039/C5NJ00072F.

\section{A Biocatalytically Driven Dynamic Combinatorial Library Approach for the Discovery} of Aqueous Gel Phase Charge Transfer Nanostructures. C. Berdugo; S. K. M. Nalluri; N. Javid; B. Escuder; J. F. Miravet; R. V. Ulijn (manuscript in preparation). 



\section{Aknowledgements}

Me gustaría dar las gracias en primer lugar a mis directores de tesis, los Doctores Juan Felipe Miravet y Beatriu Escuder, por haberme dado la oportunidad de realizar esta tesis en su grupo de investigación. Además de todo lo aprendido con ellos me llevo conmigo una gran experiencia a nivel personal.

También querría agradecer al Prof. Rein V. Ulijn por haberme dado la oportunidad de realizar una estancia en la Universidad de Strathclyde, Glasgow, así como al Dr. Siva M. Krishna Nalluri por su ayuda en la realización del proyecto. También quisiera agradecer a todas las personas que convivieron conmigo allí durante esos 6 meses y me ayudaron en todo lo posible, gracias a todos ellos por la amistad recibida y por querer compartir todo ello conmigo.

Como no, agradecer a las personas con las que he compartido estos años de duro trabajo en el laboratorio (así como viajes y salidas nocturnas), sin ellos esto no hubiera sido lo mismo. Gracias por los consejos, la paciencia y sobre todo por vuestra amistad, tanto las viejas (Vicent, Marta, Maria Dolores, chicas sois las mejores, gracias de verdad) como a los más nuevos (Juanjo, Carles, Cesar, Nishant, Santi y Marco), Carlos de ti tampoco me olvido. iiiGRACIAS CHICOS!!! Me ha encantado estar con vosotros.

A mis amigos, a los que siempre han estado a mi lado, a los que me he encontrado, y a los que estuvieron, gracias por acompañarme y compartir experiencias durante estos años.

Y por último, a mi familia que siempre me ha apoyado, a mis padres Fernando y Fina, que siempre han confiado en mí, por su afecto y apoyo y sobre todo, gracias a mi hermana Inma por su cariño y ayuda en los momentos más difíciles.

Gracias a todos ellos he conseguido sacar adelante este trabajo. 



\section{Abbreviations}

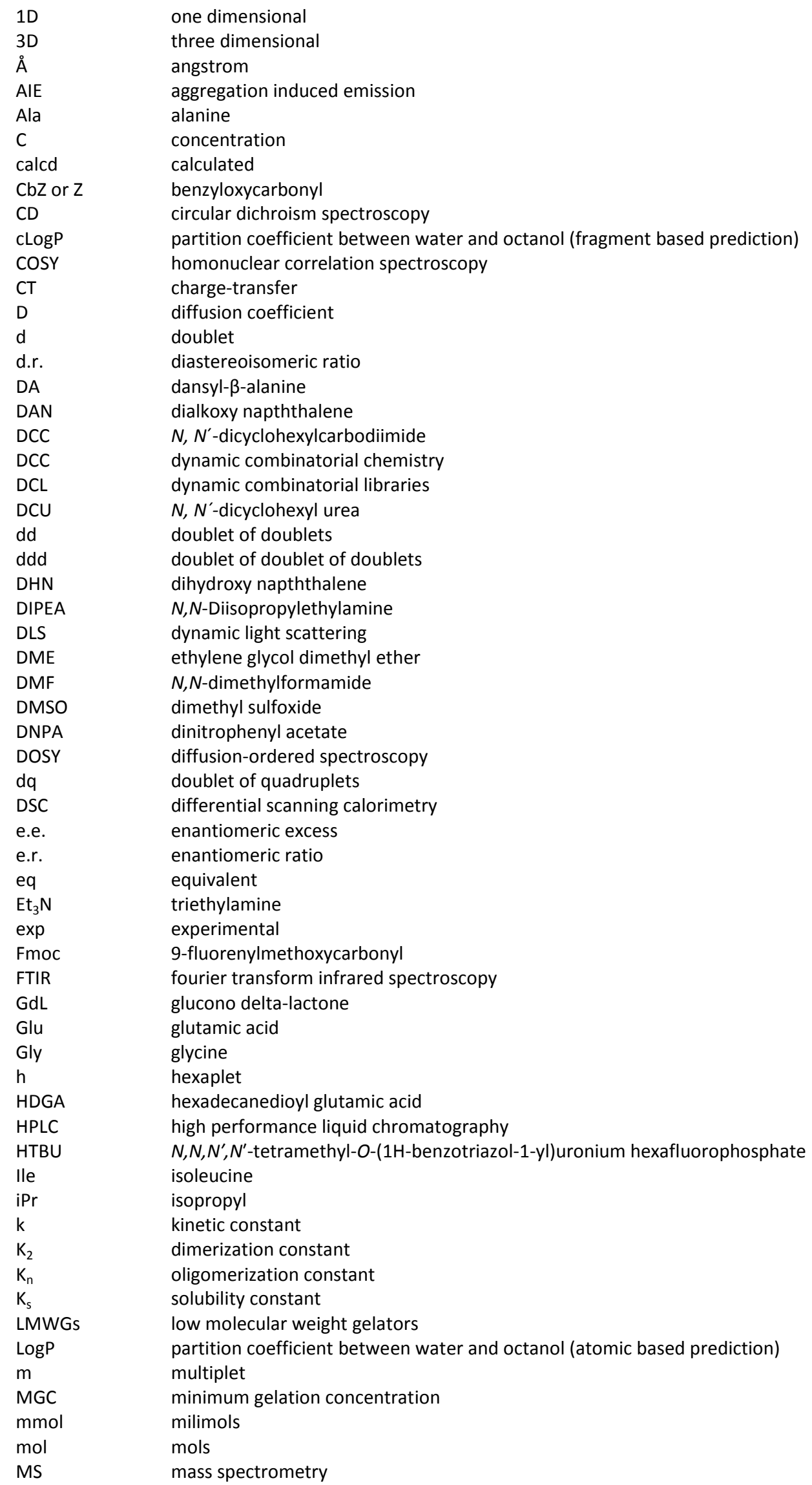




$\begin{array}{ll}\text { MS-CT } & \text { mixed stack charge-transfer } \\ \text { NDA } & 1,4,5,8 \text { - naphthalenetetracarboxylic acid } \\ \text { NDI } & \text { naphthalenediimide } \\ \text { NMR } & \text { nuclear magnetic resonance spectroscopy } \\ \text { NOE } & \text { nuclear overhauser effect } \\ \text { OCSs } & \text { organic solar cells } \\ \text { OFETs } & \text { organic field-effect transistors } \\ \text { PA } & \text { peptide amphiphile } \\ \text { Phe } & \text { phenylalanine } \\ \text { pKa } & \text { acid dissociation constant } \\ \text { Pro } & \text { proline } \\ \text { PrOH } & \text { propanol } \\ \text { q } & \text { quadruplet } \\ \text { quant } & \text { quantitative } \\ \text { rt } & \text { room temperature } \\ \text { S } & \text { singlet } \\ \text { SANS } & \text { small angle neutron scattering spectroscopy } \\ \text { SAXS } & \text { small angle X-ray scattering spectroscopy } \\ \text { SDS } & \text { sodium dodecyl sulfate } \\ \text { SEM } & \text { scanning electron microscopy } \\ \text { t } & \text { triplet } \\ \text { T } & \text { temperature } \\ \text { T2 } & \text { NMR transversal relaxation time } \\ \text { t-Boc } & \text { tert-butoxycarbonyl } \\ \text { TCNQ } & \text { tetracyanoquinodimethane } \\ \text { td } & \text { triplet of doublets } \\ \text { TEM } & \text { transmission electron microscopy } \\ \text { TFA } & \text { trifluoroacetic acid } \\ T_{\text {gel }} & \text { gel-to-sol transition temperature } \\ \text { THF } & \text { tetrahydrofuran } \\ \text { TNF } & \text { trinitrofluorenone } \\ \text { TTF } & \text { tetrathiafulvalene } \\ \text { Tyr } & \text { tyrosine } \\ \text { US } & \text { ultrasound } \\ \text { UV-Vis } & \text { ultraviolet-visible spectroscopy } \\ \text { Val } & \text { valine } \\ \text { WAXD } & \text { wide-angle X-ray diffraction } \\ \text { wt \% } & \\ \text { XRD } & \text { weight percent } \\ \Delta H & \end{array}$




\section{TAble of Contents}

$\begin{array}{lll}\text { Chapter } 1 \text { Introduction } & 1\end{array}$

1.1. Molecular Gels 3

1.1.1. Peptide-Based Molecular Gels: Advances and $\begin{array}{ll}\text { Applications } & 8\end{array}$

1.2. References and Notes 13

$\begin{array}{lll}\text { Chapter } 2 \text { Objectives } & 15\end{array}$

$\begin{array}{ll}\text { 2.1. Objectives } & 17\end{array}$

2.1.2. Specific Objectives 18

2.2. References and Notes 20

Chapter 3 Self-Assembled L-Proline Based Catalysts in Organic Media 21

3.1. Introduction 23

3.1.1. Organocatalysis with L-Proline 23

3.1.2. Supramolecular Catalysis 26

3.1.2.1. Supramolecular Catalytic Gels 27

3.2. L-Proline Dipeptides: Aggregation Study and its Effect in $\begin{array}{ll}\text { Organocatalysis } & 32\end{array}$

3.2.1. Synthesis 33

3.2.2. Conformational Changes Associated to the Aggregation of L-Proline Dipeptides 36

3.2.3. Catalytic Studies $\quad 46$

3.3. L-Proline Derived Bolaamphiphiles: Gel-Phase Study of the Organocatalytic Performance $\quad 49$

3.3.1. Characterisation of Gels in Toluene 49

3.3.2. Proline Organogels as Catalysts for the Michael

Reaction in Toluene 


\section{Chapter 4 A Self-Assembled L-Proline Based Amphiphilic Catalyst:}

4.1. Introduction

4.1.1. Supramolecular Catalysis in Water Mediated by Proline Derivatives

4.2. Study on the Effect of Polymorphism on the Self-Assembly and Catalytic Behaviour of a L-Proline Based Hydrogelator

5.2.1. $\mathrm{pH}-$ Sensitive Hydrogelators: Potentiometric 
Chapter 6 Biocatalytic Gelation-Driven Dynamic Combinatorial Library to Discover Charge-Transfer Peptide Nanostructures

6.1. Introduction

6.1.1. Supramolecular Electronics

6.1.2. Supramolecular Charge-Transfer Nanostructures

6.1.3. Dynamic Combinatorial Chemistry

6.1.4. Enzyme Driven Gelation

6.2. Synthesis

190

6.2.1. Synthesis of NDI-Tyr Acceptor

6.2.2. Synthesis of Dialkoxy Naphthalene Donors

6.3. Biocatalytic Gelation-Driven Dynamic Combinatorial Library as a Tool to Discover New Charge-Transfer Systems

6.4. Study of a Supramolecular Nanostructure Based on NDI-Dipeptide in a Charge-Transfer Complex

6.5. Conclusions

6.6. Supporting Information

216

6.7. References and Notes

Chapter 7 Conclusions

8.1. General Remarks.

8.2. Synthesis and Characterisation of New Compounds

8.3. Methodology of Characterisation Gels

8.4. Catalysis Study

8.5.Polymorphism Study 
8.7.Study of Dynamic Combinatorial Libraries

8.8. References and Notes

Annex I. Summary in Spanish

Annex II. List of Molecules 



\subsection{Molecular Gels}

Nowadays gels are well-known materials commonly used in everyday life (Figure 1.1.1), from cosmetics to cleaning products or food. These gel materials present unique properties (flexibility, malleability, storage capacity, etc.) that make them interesting in fields such as medicine, biology or electronics.

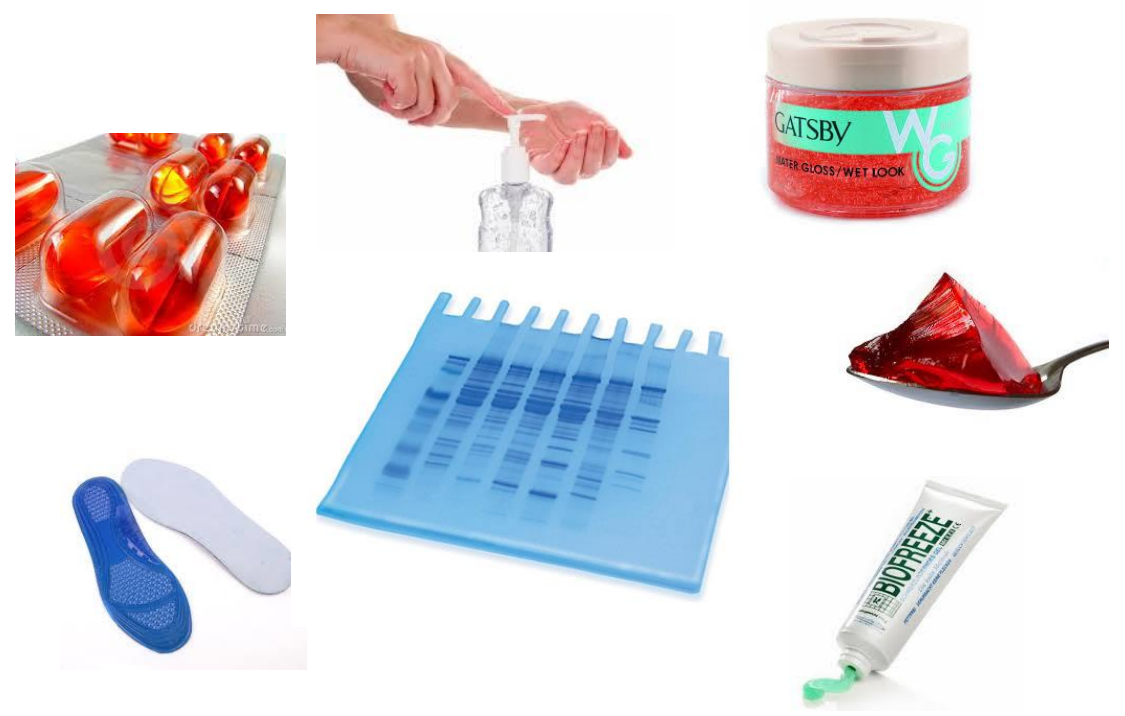

Figure 1.1.1. Examples of common gel materials.

From a scientific perspective an absolute definition of a gel is still elusive, mainly due to its variety and complexity. The first attempt was made by Dr. Dorothy Jordon Lloyd in 1926 who said "...the gel, is easier to recognise than to define". ${ }^{1}$ This and other empirical definitions have been made based on visual observations. Some decades later, in 1974, in an effort to link macroscopic with microscopic properties, Flory defined a gel as a two-component colloidal dispersion with a continuous structure with macroscopic dimensions that is permanent on the time scale of the experiment and as a result, the material is "solid-like" in its rheological behaviour despite being mostly liquid. ${ }^{2}$

Generally, a gel is formed from two components, a small amount of solid (usually 0.1-10 wt \%, minor component) named as gelator that is able to form a continuous three dimensional (3D) network that can immobilise a large volume of solvent (major component), supporting its own weight without collapsing and is the origin of the 
viscoelastic behaviour (tested by inversion, if no flow is observed, the solution is said to have become a gel). ${ }^{3}$

Gels can be classified according to their constitution, type of interaction involved in the formation of the network or the media where they are formed (see Figure 1.1.2). Thus, in accordance with the media, materials termed as organogels refers to those cases where the fluid component is an organic compound and hydrogels when it is water. Often the solvent is removed, in such cases aerogels are formed (the 3D network remains unaltered) or xerogels (collapse of the network upon removing the solvent). Gels can be further divided by their constitution as polymeric gels; those formed by the cross-linking of macromolecules, or those formed by single molecules, molecular gels. In the case of polymeric gels the network formation arises as a result of cross-linked macromolecules via physical interactions (non-covalent) or chemical (covalent) interactions. Reversibility can only be found when non-covalent interactions are involved. Finally, the assembly of small organic compounds by the action of non-covalent (physical) intermolecular interactions (solvophobic, ionic, H-bonding, $\pi-\pi$ stacking, van der Waals, metal coordination, etc.) ${ }^{4}$ leads to supramolecular (molecular) gels. These small organic compounds are called low molecular weight gelators (LMWGs). All work reported herein is based on this last category of gelation.

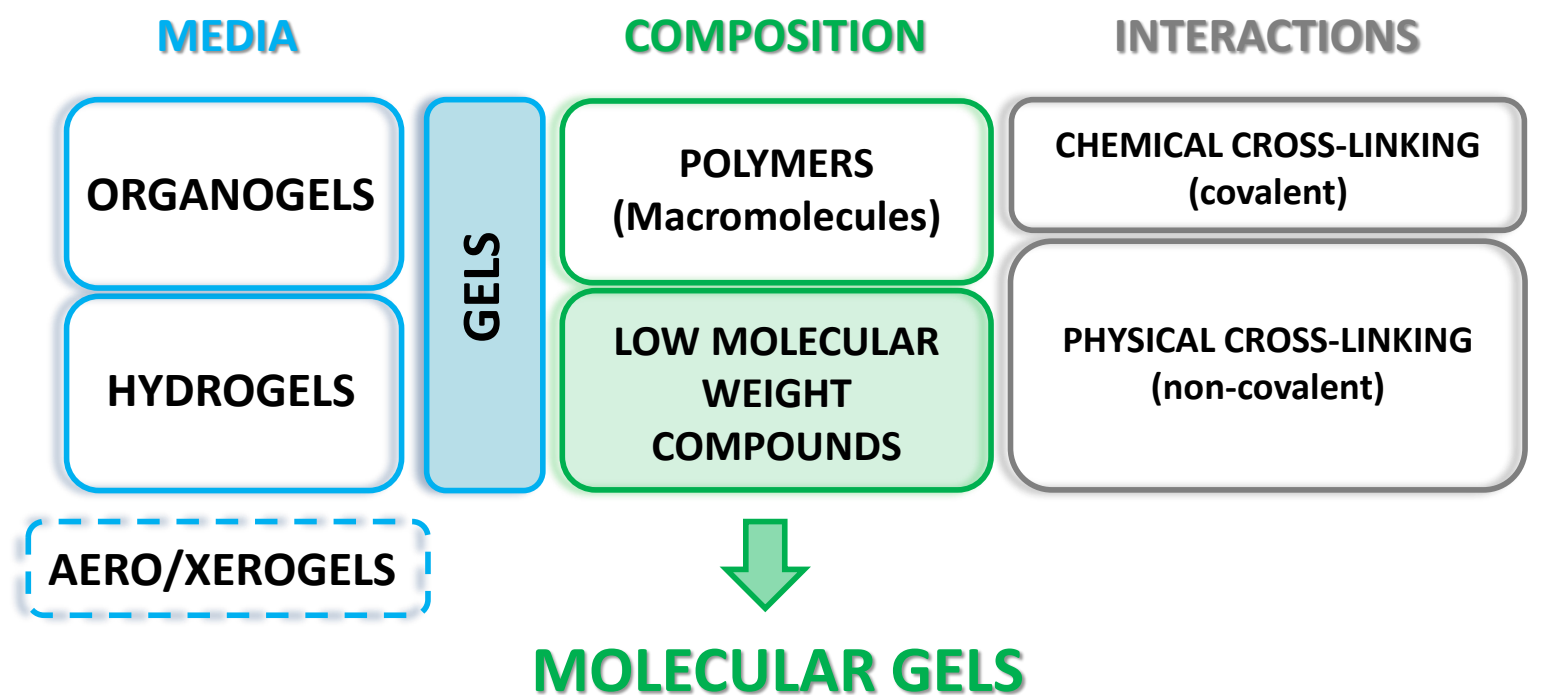

Figure 1.1.2. Classification of gels. 
Some authors define the gelation process as a 'frustrated' attempt of crystallization. ${ }^{5}$ Both mechanisms are based on the arrangement of the molecules in a homogenous solution after being heated and left to cool (annealing) in response to the differences in solubility (see Figure 1.1.3). In crystallisation, the molecules are condensed and arranged in an ordered three-dimensional pattern. Conversely, during the gelation the gelator molecules are arranged in one-dimensional fibres that self-assemble into complex aggregates forming a three-dimensional network that entraps the solvent.
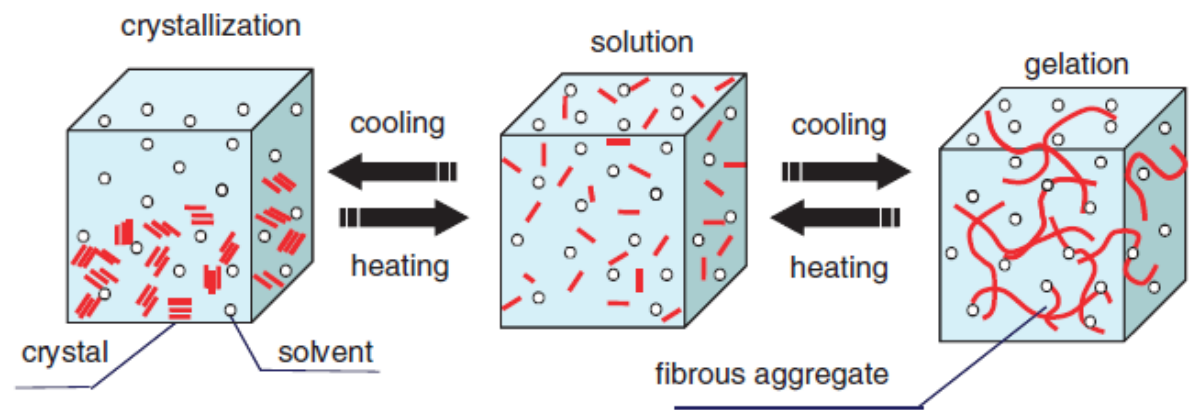

Figure 1.1.3. Comparative scheme between crystallisation and gelation of small molecules. ${ }^{6}$

Molecular gels are most commonly prepared by heating a solution of the gelator and then cooling, leading to a supersaturated solution. Gelation occurs when gelator molecules hierarchically self-assemble in a bottom-up fashion through non-covalent interactions into elongated fibrils of typically 5-100 nm in diameter. These fibrils aggregate further in order to generate a 3D architecture that can grow into large assemblies forming the final fibrillar network, trapping the solvent molecules and thus forming the gel material (see Figure 1.1.4). Most LMWG gels, due to their non-covalent nature are thermally reversible, with the melting temperature of the gel being nearly independent of the concentration of gelator. 


\section{Molecules}

1D Assembly

Fibres

Gel
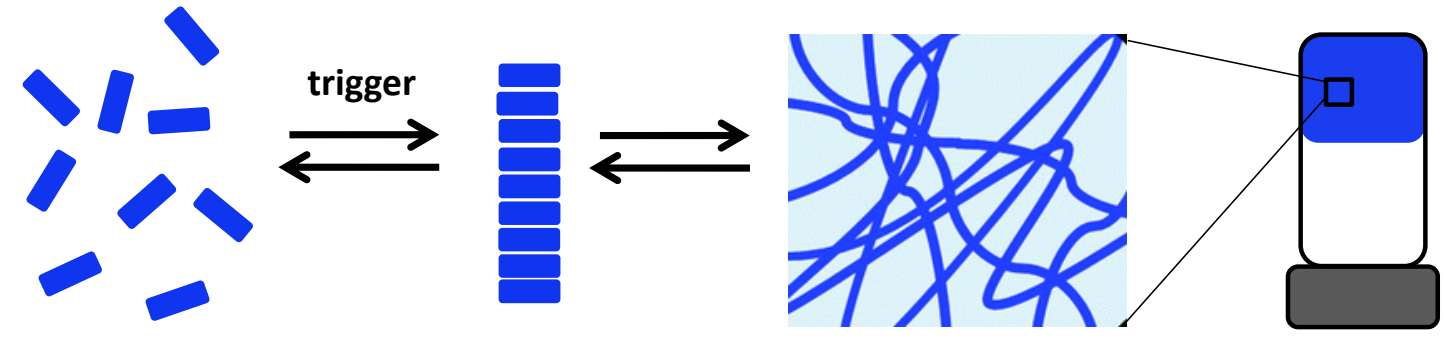

Figure 1.1.4. Representation of the aggregation process that leads to the gel formation.

The first gelator molecule was reported in 1841 employing lithium urate, ${ }^{7}$ but it was not until in 1891 when a description of LMWGs was published using 1,3:2,4-di-Obenzylidene-D-sorbitol. ${ }^{8}$ However, for many years there was almost no research interest in molecular gels until the early 1990s when an exponential growth was observed with significant efforts to exploit these new materials (see Figure 1.1.5). ${ }^{9}$

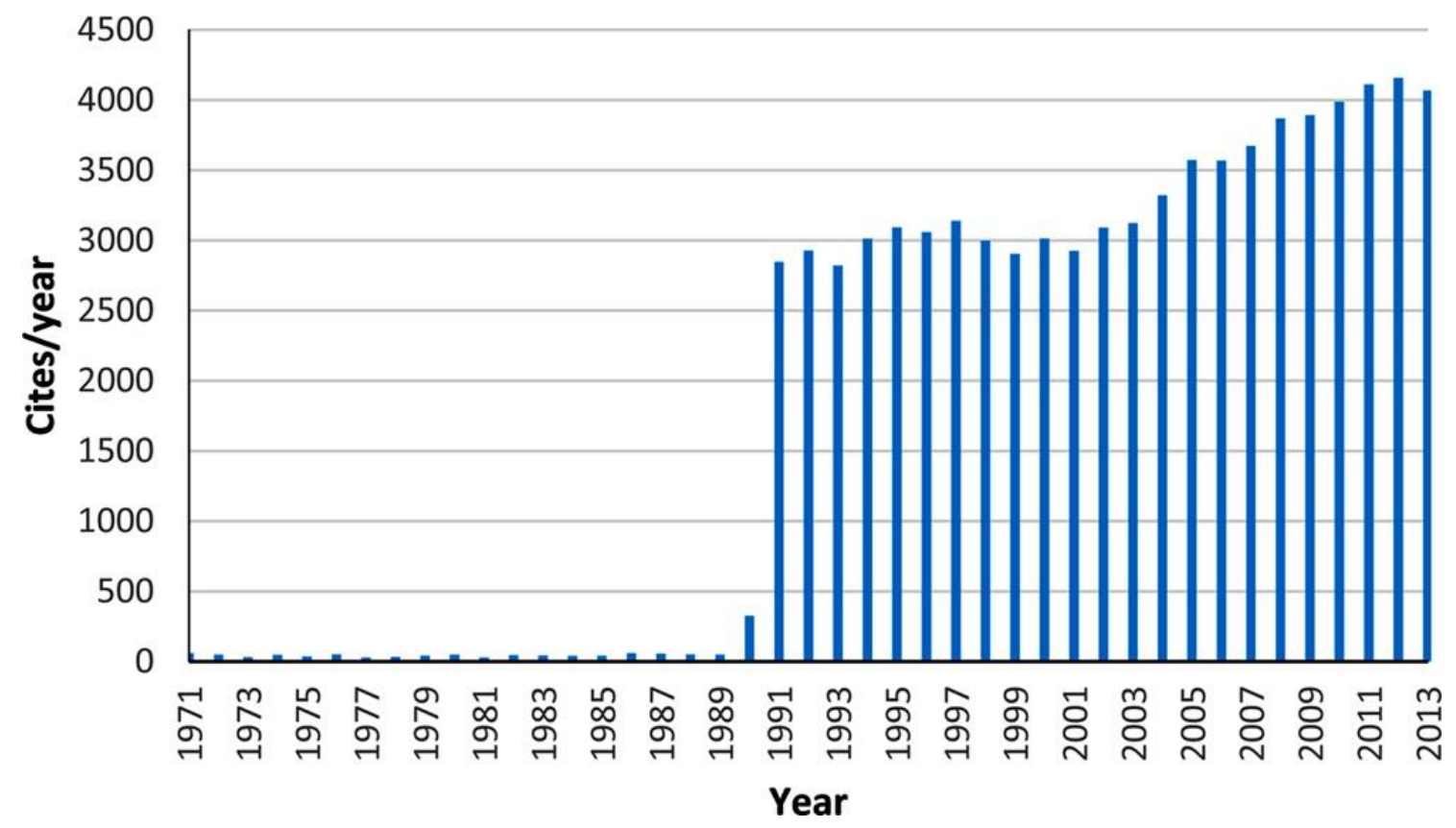

Figure 1.1.5. Histogram of citations by year to molecular gels in the Web of Science. ${ }^{9 b}$ 
Since then, a wide range of molecules have been recognised to form gels with different structures (see Figure 1.1.6). Examples include ureas, amides, nucleobases, phorphyrin derivatives, dendrimers, surfactants, sugars, fatty acid or amino acids among others. ${ }^{4,10}$

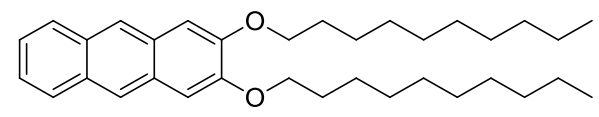

Anthracene derivative

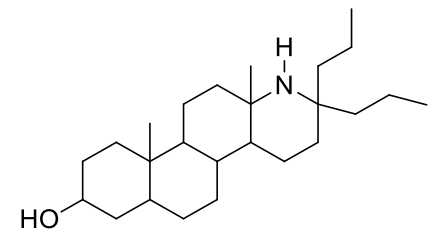<smiles>CCCCCCC(O)CCCCCCCCCCC(=O)O</smiles>

Steroid derivative

Fatty Acid derivative<smiles>CCC(C)[C@H](NC(=O)C(Cc1ccccc1)NC(=O)CN)C(=O)NC(CC(C)C)C(=O)O</smiles>

Peptide derivative

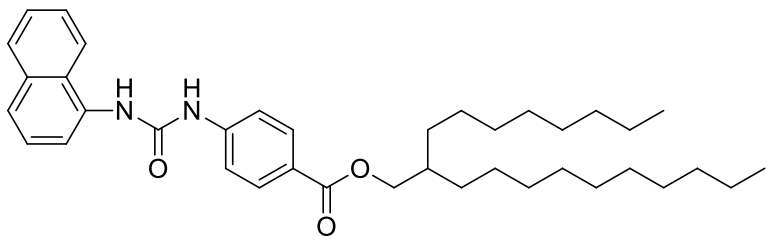

Urea derivative

Figure 1.1.6. Various types of molecules known to act as LMWGs.

This recent attention for molecular gels may be a result of the interesting features that this soft material presents such as the highly reversible nature, dynamic behaviour, high biodegradability and biocompatibility (compared with polymeric gels) or the easy control of functionalization and composition, among others. Additionally, the high organisation inside the supramolecular structure may afford effects such as cooperation, intermolecular interactions between groups or even conformational changes that could mean the emergence of new properties compared with the isolated compound. ${ }^{11}$

Despite the enormous efforts that have been made in the past to understand the basic principles governing self-assembly, these materials are generally discovered by serendipity. The gelation phenomenon is still difficult to predict but a tremendous progression has been made to understand the structural requirements for gelation to occur and the structure-property relationship within the self-assembled structure. ${ }^{12}$ One step further is the functionalization of the conventional LMGWs to develop new materials 
with a desired application. In this sense, gelators could be carefully designed by introducing tailor-made functionalities at the molecular level, which will be reflected in the gel material. ${ }^{9,13}$

\subsubsection{Peptide-Based Molecular Gels: Advances and Applications}

Among all LMWGs used as building blocks for the design of molecular gel materials, those derived from amino acids or peptides are especially attractive due to their inherent biocompatibility and chemical diversity. In nature, peptides have the ability to adopt a structural motif through non-covalent interactions (see Figure 1.1.1.1), moreover they perform a wide variety of functions such as molecular recognition, catalysis, energy storage, etc, that scientists try to mimic.

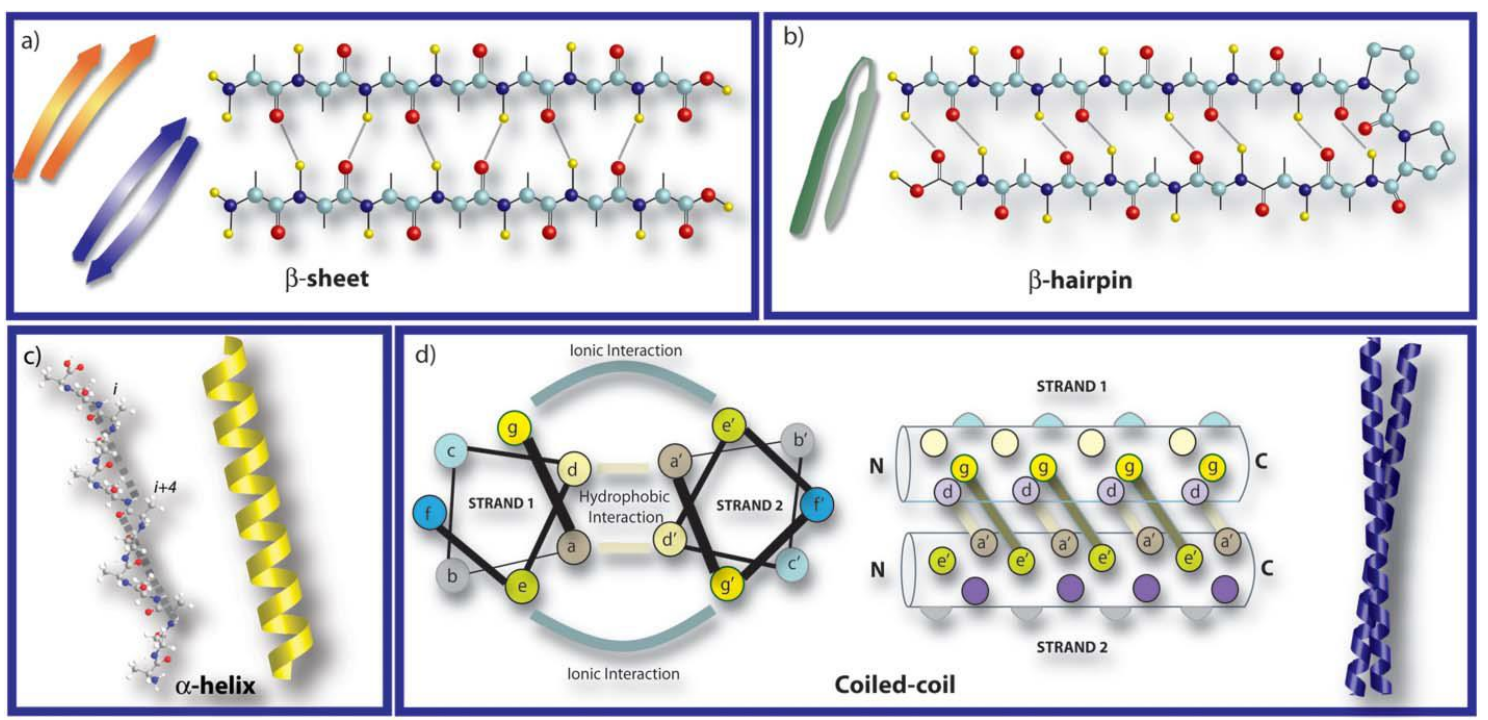

Figure 1.1.1.1. Schematic illustration of the molecular self-assembly involved in the formation of different secondary structures of peptides: (a) $\beta$-sheet, (b) $\beta$-hairpin, (c) $\alpha$-helix and (d) coiled-coil. ${ }^{14}$

Taking advantage of these features (function and assembly) peptide-based gelators can be designed. A small version of these systems may allow an easy way to bring those functionalities to synthetic materials providing many possibilities for the construction of nanomaterials with a desired structure. Self-assembly of peptide derivatives has been reported with several architectures, including fibres, tapes, tubes, sheets and spheres (see Figure 1.1.1.2). ${ }^{15}$ Moreover, peptide derivatives are relatively easy to synthesise and they can also be easily modified chemically and biologically. 


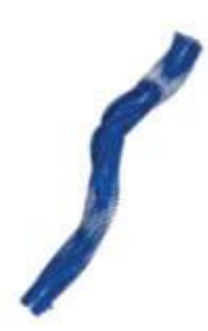

fibre

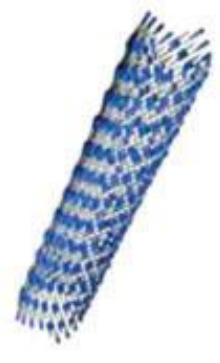

tube

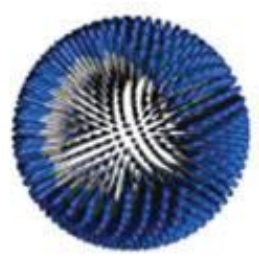

sphere

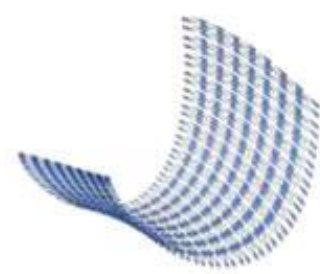

sheet

Figure 1.1.1.2. Supramolecular structures accessible via peptide self-assembly. ${ }^{15}$

A wide range of peptide-derived nanomaterials have been developed for use in Materials Science applications from drug delivery to electronic materials with aromatic amino acids being used as the main motif (see Figure 1.1.1.3). ${ }^{16}$

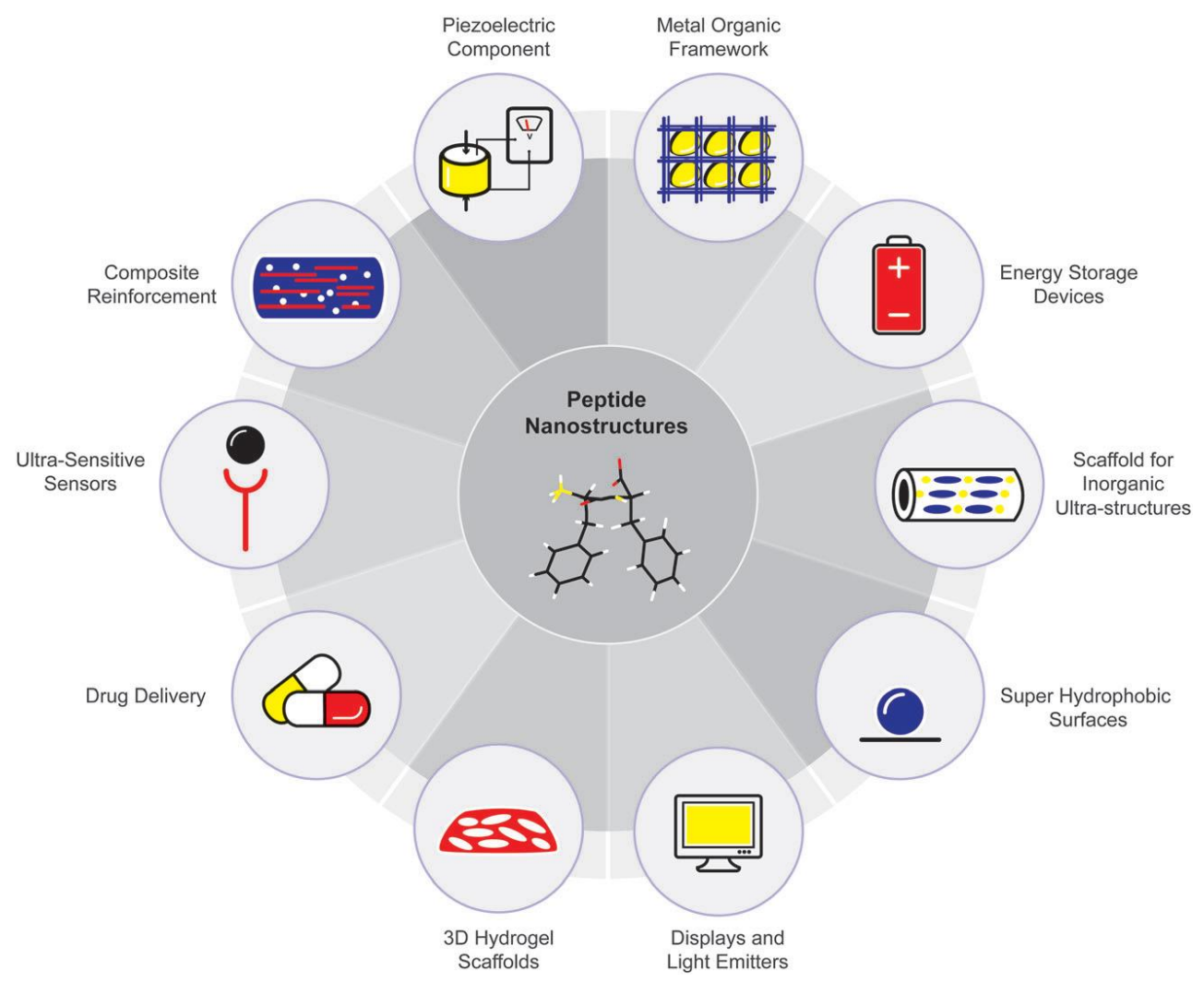

Figure 1.1.1.3. Illustration of the wide range of applications achieved for peptide based nanostructures. ${ }^{16 c}$

Supramolecular peptide-based gels have prompted interest among scientists during last decades and promising applications have been developed using this soft material. ${ }^{14,} 17$ Advances in techniques and methods of characterisation allow an interpretation of the packing mode within the fibre network and provide valuable 
information for the design of better gelators. ${ }^{18}$ In addition to microscopic characterization techniques that reveal pictures of the fibre morphology, complementary tools are employed to obtain a better comprehension of the gel structure and the self-assembly process. Techniques such as small angle electron and neutron scattering can yield useful information about the packing mode and additional spectroscopic tools, such as nuclear magnetic resonance (NMR), UV-vis absorption or fluorescence, are able to identify different aspects of intermolecular interactions that contribute to gelation. Furthermore, advances including novel methods to induce gelation, multi-component gelator systems or responsive-stimuli gels are responsible for such impressive interest and evolution. ${ }^{9 a}$

Despite advances in controlled design of molecular gels with attractive properties, their use in real devices is still a challenge due to some of the problems often found such as low stability at room temperature, the use of volatile solvents or simply problems related with the industrial manufacture. ${ }^{9 b}$ In spite of these problems, scientists continue to investigate molecular gels and interesting uses have been achieved.

With respect to biological applications, some great advances have been made. Biodegradability, biocompatibility, lack of toxicity or modular design has led to the consideration of peptide-based LMWGs as promising new molecules for the construction of nanostructures in drug delivery, tissue engineering or cell culture. ${ }^{19}$ For example, Murayama et al. recently reported cancer cell death via intracellular hydrogelation of a palmitoyl-peptide derivative. In this work, the authors designed a gelator precursor that upon a cancer-enzyme cleavage (matrix metalloproteinase-7, MMP-7) leads to the formation of a gelator molecule that can cross through the cell membrane. Inside the cell the hydrogelation occurs by the self-assembly of the gelator causing the death of the cancer cell by exerting "vital stress" (see Figure 1.1.1.4). The gelator precursor exhibited remarkable cytotoxicity to five different cancer cell lines, while no toxicity was found in normal cells. This work presents a new drug where self-assembly is the mechanism of the cell death, minimising the side effects. ${ }^{20}$ 
A)

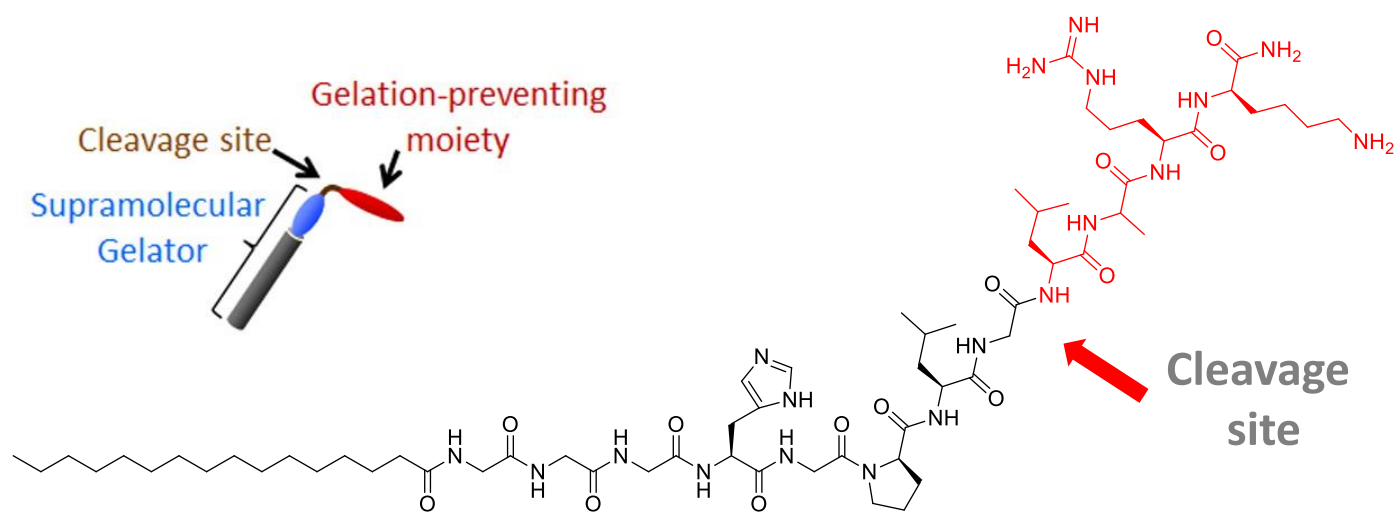

B)
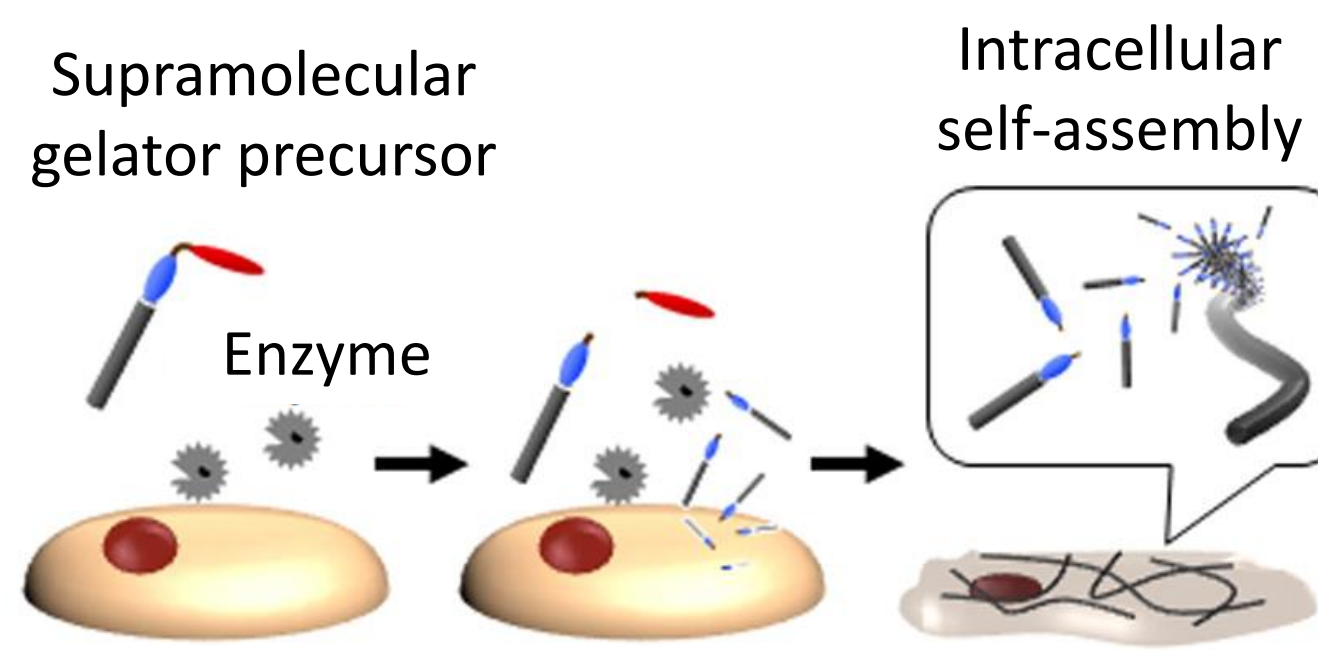

Cancer cell

Cell death

Figure 1.1.1.4. A) Molecular structure of the gelator precursor. B) Illustration of the methodology during the cancer cell death. ${ }^{20}$

Other fields include the use of peptide-based molecular gels as containers for catalytic reactions, ${ }^{21}$ electronics ${ }^{22}$ or as templates for nanostructured materials. ${ }^{23}$ Biomineralization $^{24}$ is an example of the latter, where self-assembly of organic components are used to direct controlled growth and hierarchical organisation of inorganic materials that can be used for the fabrication of functional nanomaterials. Park et al. reported the synthesis of transition metal phosphate nanotubes for its application as a cathode material for rechargeable lithium (Li) ion batteries by using a functionalised peptide hydrogel self-assembly as a template (see Figure 1.1.1.5). ${ }^{25}$ 
a)

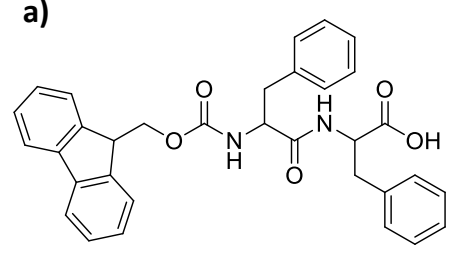

b)

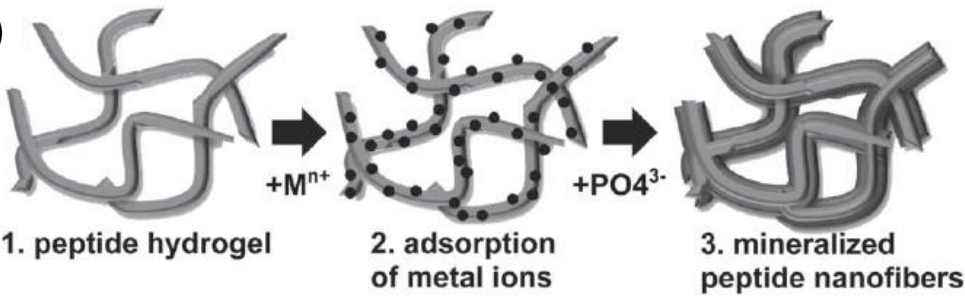

c)
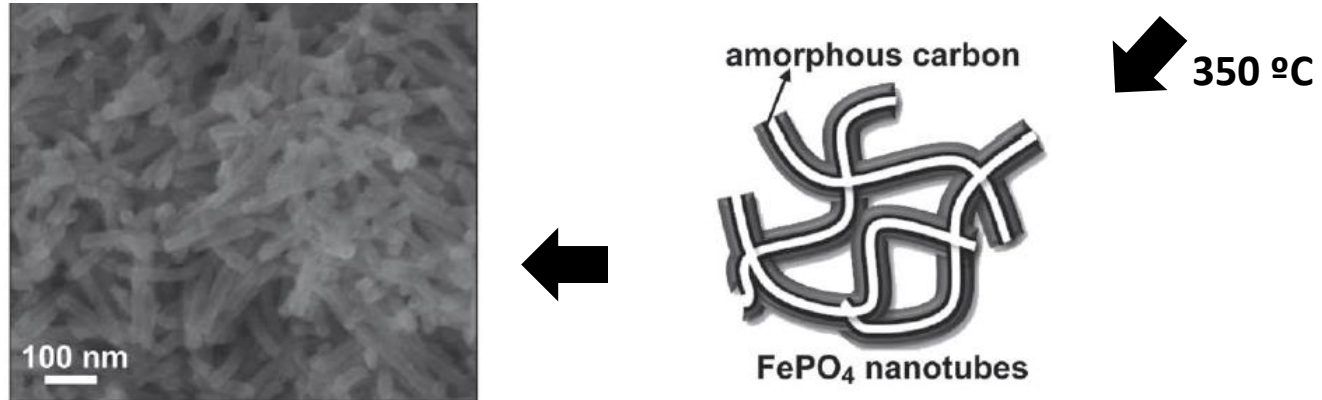

Figure 1.1.1.5. Mineralisation of peptide nanofibres. ${ }^{25}$

Overall these examples represent the multiple potential applications that can be achieved by molecular gels as smart functional materials and are used as a reference for the work reported here. 


\subsection{References and Notes}

1. Lloyd, D. J., "Colloid Chemistry". New YorK, 1926; Vol. 1.

2. Flory, P. J. "Introductory Lecture", Faraday Discussions of the Chemical Society, 1974, 57, 7-18.

3. Estroff, L. A.; Hamilton, A. D. "Water Gelation by Small Organic Molecules", Chem. Rev., 2004, 104, 1201-1218.

4. Terech, P.; Weiss, R. G. "Low Molecular Mass Gelators of Organic Liquids and the Properties of their Gels", Chem. Rev., 1997, 97, 3133-3160.

5. (a) Bradford, S. C. "On the Theory of Gels", Biochem. J., 1921, 15, 553-562; (b) Menger, F. M.; Caran, K. L. "Anatomy of a Gel. Amino Acid Derivatives that Rigidify Water at Submillimolar Concentrations", J. Am. Chem. Soc., 2000, 122, 11679-11691.

6. Hanabusa, K.; Suzuki, M. "Development of Low-Molecular-Weight Gelators and PolymerBased Gelators", Polym. J., 2014, 46, 776-782.

7. Von Lipowitz, A., Liebigs Ann. Chem. Pharm., 1841, 38, 348-355.

8. Meunier, M., J. Ann. Chim. Phys., 1891, 22, 412.

9. (a) Steed, J. W. "Supramolecular Gel Chemistry: Developments Over the Last Decade", Chem. Commun., 2011, 47, 1379-1383; (b) Weiss, R. G. "The Past, Present, and Future of Molecular Gels. What Is the Status of the Field, and Where Is It Going?", J. Am. Chem. Soc., 2014, 136, 7519-7530.

10. Tomasini, C.; Castellucci, N. "Peptides and Peptidomimetics that Behave as Low Molecular Weight Gelators", Chem. Soc. Rev., 2013, 42, 156-172.

11. Lehn, J. M. "Toward Self-Organization and Complex Matter", Science, 2002, 295, 24002403.

12. (a) de Loos, M.; Feringa, B. L.; van Esch, J. H. "Design and Application of Self-Assembled Low Molecular Weight Hydrogels", Eur. J. Org. Chem., 2005, 2005, 3615-3631; (b) Dastidar, P. "Supramolecular Gelling Agents: Can They Be Designed?", Chem. Soc. Rev., 2008, 37, 2699-2715; (c) van Esch, J. H. "We Can Design Molecular Gelators, but Do We Understand them?", Langmuir, 2009, 25, 8392-8394.

13. Dawn, A.; Shiraki, T.; Haraguchi, S.; Tamaru, S.-i.; Shinkai, S. "What Kind of "Soft Materials" Can We Design from Molecular Gels?", Chem. Asian J., 2011, 6, 266-282.

14. Dasgupta, A.; Mondal, J. H.; Das, D. "Peptide Hydrogels", RSC Adv., 2013, 3, 9117-9149.

15. Zelzer, M.; Ulijn, R. V. "Next-Generation Peptide Nanomaterials: Molecular Networks, Interfaces and Supramolecular Functionality", Chem. Soc. Rev., 2010, 39, 3351-3357.

16. (a) Zhang, S. "Fabrication of Novel Biomaterials Through Molecular Self-Assembly", Nat. Biotechnol., 2003, 21, 1171-1178; (b) Gazit, E. "Self-Assembled Peptide Nanostructures: the Design of Molecular Building Blocks and their Technological Utilization", Chem. Soc. Rev., 2007, 36, 1263-1269; (c) Adler-Abramovich, L.; Gazit, E. "The Physical Properties of Supramolecular Peptide Assemblies: from Building Block Association to Technological Applications", Chem. Soc. Rev., 2014, 43, 6881-6893; (d) Fleming, S.; Ulijn, R. V. "Design of 
Nanostructures Based on Aromatic Peptide Amphiphiles", Chem. Soc. Rev., 2014, 43, 8150-8177.

17. (a) Johnson, E. K.; Adams, D. J.; Cameron, P. J. "Peptide Based Low Molecular Weight Gelators", J. Mater. Chem., 2011, 21, 2024-2027; (b) Escuder, B.; Miravet, J. F., Eds., Functional Molecular Gels. RSC, Cambridge, 2014.

18. (a) Caran, K. L.; Lee, D.-C.; Weiss, R. G., "Molecular Gels and their Fibrillar Networks". In Soft Fibrillar Materials, Wiley-VCH Verlag GmbH \& Co. KGaA, 2013; pp 1-75; (b) Nebot, V. J.; Smith, D. K., "CHAPTER 2 Techniques for the Characterisation of Molecular Gels". In Functional Molecular Gels. RSC, Cambridge, 2014; pp 30-66.

19. Skilling, K. J.; Citossi, F.; Bradshaw, T. D.; Ashford, M.; Kellam, B.; Marlow, M. "Insights Into Low Molecular Mass Organic Gelators: a Focus on Drug Delivery and Tissue Engineering Applications", Soft Matter, 2014, 10, 237-256.

20. Tanaka, A.; Fukuoka, Y.; Morimoto, Y.; Honjo, T.; Koda, D.; Goto, M.; Maruyama, T. "Cancer Cell Death Induced by the Intracellular Self-Assembly of an Enzyme-Responsive Supramolecular Gelator", J. Am. Chem. Soc., 2015, 137, 770-775.

21. Escuder, B.; Rodríguez-Llansola, F.; Miravet, J. F. "Supramolecular Gels as Active Media for Organic Reactions and Catalysis", New J. Chem., 2010, 34, 1044-1054.

22. Kim, S. H.; Parquette, J. R. "A Model for the Controlled Assembly of Semiconductor Peptides", Nanoscale, 2012, 4, 6940-6947.

23. Lakshmanan, A.; Zhang, S.; Hauser, C. A. "Short Self-Assembling Peptides as Building Blocks for Modern Nanodevices", Trends Biotechnol., 2012, 30, 155-165.

24. (a) Gungormus, M.; Branco, M.; Fong, H.; Schneider, J. P.; Tamerler, C.; Sarikaya, M. "Self Assembled Bi-Functional Peptide Hydrogels with Biomineralization-Directing Peptides", Biomaterials, 2010, 31, 7266-7274; (b) Chen, C. L.; Rosi, N. L. "Peptide-Based Methods for the Preparation of Nanostructured Inorganic Materials", Angew. Chem. Int. Ed., 2010, 49, 1924-1942.

25. Ryu, J.; Kim, S. W.; Kang, K.; Park, C. B. "Mineralization of Self-Assembled Peptide Nanofibers for Rechargeable Lithium Ion Batteries", Adv. Mater., 2010, 22, 5537-5541. 


\section{CHAPTER 2}

OBJECTIVES 



\subsection{Objectives}

Molecular gels represent an emerging approach for the development of new functional materials. ${ }^{1}$ The hierarchical self-assembly of molecules has been demonstrated to be a powerful tool in Materials Science as it offers unique features like highly ordered systems, synthetic economy, modular architecture or stimuli responsiveness among others. Additionally, supramolecular interactions are governed by weak non-covalent bonds, thus promoting reversibility by means of the construction of dynamic systems with defect correction abilities. Overall, these features make molecular gels good candidates for their use in the design of functionalised materials in fields such as catalysis or stimuli responsive materials (Figure 2.1.1), thus being this the main goal in the present project.

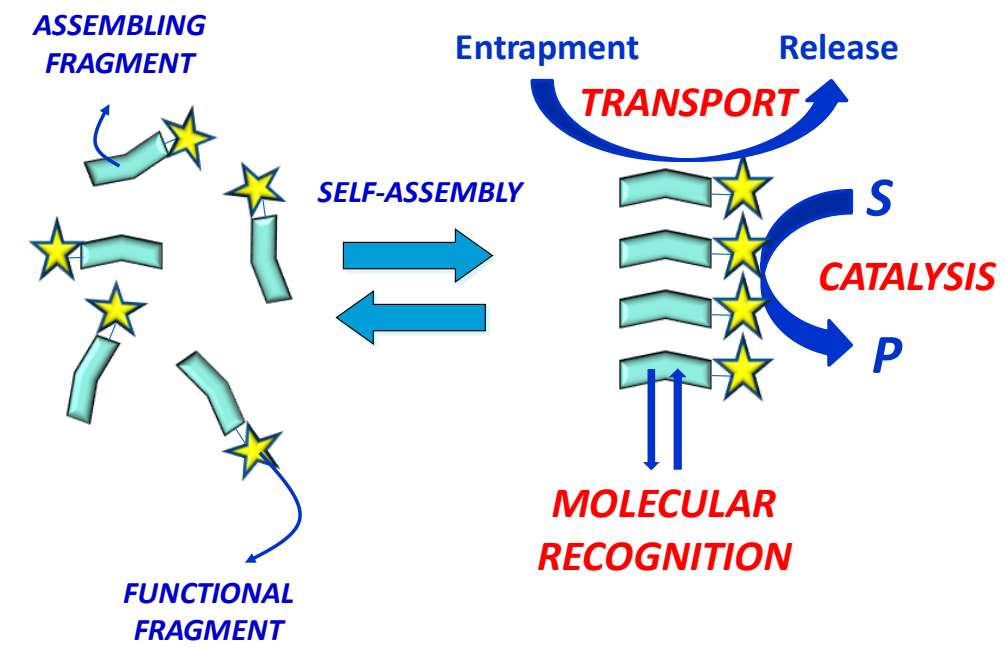

Figure 2.1.1. Scheme of the self-assembly process to obtain molecular functional materials. ${ }^{2}$

The main idea in this project is the design of gelator molecules that will lead to a soft-gel material with additional properties that are not present in the molecule in solution. With this in mind, the design of gelators has been based on a modular approach in which peptide-based structures are slightly modified to produce molecules that combine self-assembling moieties with functional groups that will give the final property (Figure 2.1.2). ${ }^{3}$ In this way, a family of amphiphiles and bolaamphiphiles has been prepared to form gels in organic solvents as well as in aqueous solution. 


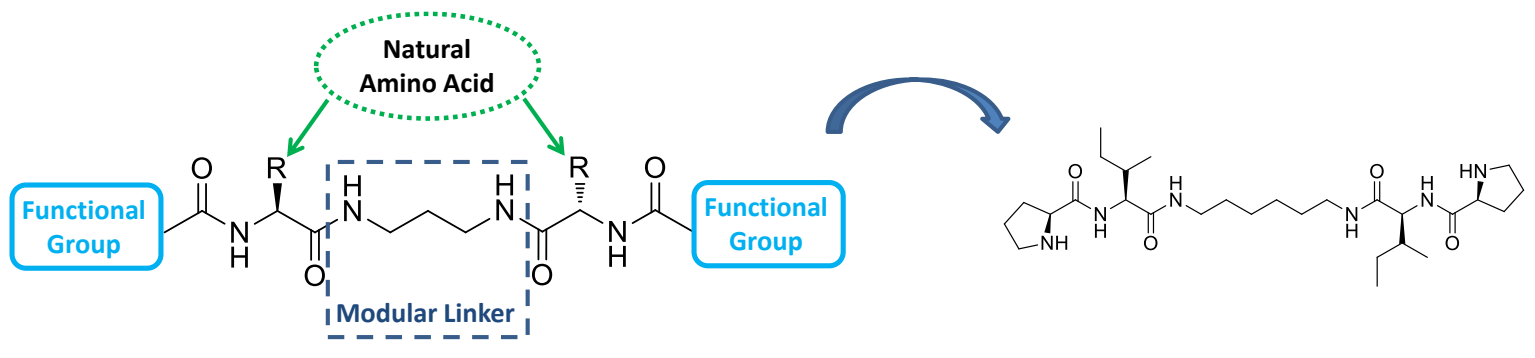

Figure 2.1.2. Representation of the design approach used and a bolaamphiphile structure as an example.

\subsubsection{Specific Objectives}

I. Design and Synthesis of Supramolecular Gelators

Amphiphiles and bolaamphiphiles will be designed directed by the results obtained from structures previously reported in our group. ${ }^{3 c}$ Following a modular approach (see Figure 2.1.2) a series of peptide-based derivatives will be obtained by synthesis in solution.

II. Aggregation Process and Gel Characterisation Study

The gelation ability of the different compounds synthesised will be tested, in organic solvents as well as in water. The self-assembly process will be analysed in order to shed some light on the aggregation rules that govern the gelation properties of the different gelators. Additionally, full characterisation of the gelators will be made from a microscopic to a macroscopic level.

III. Gels as Catalysts

Aimed at the increasing importance of proline derivatives as efficient organocatalysts ${ }^{4}$ and based on previous results reported in our group, ${ }^{2,3 b, c}$ in which this amino acid was incorporated as a functional group for the construction of molecular gelators, a family of L-proline derivatives will be tested as effective catalysts in organic solvent for the Michael reaction.

Furthermore, an amphiphilic proline derivative will be studied as a catalyst in aqueous media for the aldol reaction.

IV. Gels as Stimuli-Responsive Materials

One of the most important fields in Supramolecular Chemistry is the construction of smart materials. ${ }^{5}$ These are materials that are able to change their properties in response to external stimuli. Based on this, the study of $\mathrm{pH}$-responsive gelators will be carried out for a family of molecules with ionisable groups and multi- 
component self-assembled systems will be designed based on their specific response to $\mathrm{pH}$.

V. Dynamic Combinatorial Libraries to Discover Gels with Electronic Properties

The use of dynamic combinatorial chemistry $(D C C)^{6}$ as a powerful tool to discover functional structures will be studied. In this project, naphthalenediimides (NDI) will be used as a functional group in the design of peptide-based molecules capable of showing electronic properties. The best enzymatically self-assembled structure will be obtained from a library of amino acid derivatives in water. Moreover, the incorporation of charge-transfer interactions, by means of the presence of donor-acceptor molecules in the library, will be studied. To finish, a full characterisation of the best structures will be done in order to gain a better understanding of the aggregation process inside the charge-transfer selfassembled structures. 


\subsection{References and Notes}

1. (a) Sangeetha, N. M.; Maitra, U. "Supramolecular Gels: Functions and Uses", Chem. Soc. Rev., 2005, 34, 821-836; (b) Busseron, E.; Ruff, Y.; Moulin, E.; Giuseppone, N. "Supramolecular Self-Assemblies as Functional Nanomaterials", Nanoscale, 2013, 5, 70987140; (c) Ward, M. D.; Raithby, P. R. "Functional Behaviour from Controlled Self-Assembly: Challenges and Prospects", Chem. Soc. Rev., 2013, 42, 1619-1636; (d) Escuder, B.; Miravet, J. F., Eds., Functional Molecular Gels. RSC, Cambridge, 2014; (e) Adler-Abramovich, L.; Gazit, E. "The Physical Properties of Supramolecular Peptide Assemblies: from Building Block Association to Technological Applications", Chem. Soc. Rev., 2014, 43, 6881-6893.

2. Escuder, B.; Rodríguez-Llansola, F.; Miravet, J. F. "Supramolecular Gels as Active Media for Organic Reactions and Catalysis", New J. Chem., 2010, 34, 1044-1054.

3. (a) Miravet, J. F.; Escuder, B. "Pyridine-Functionalised Ambidextrous Gelators: Towards Catalytic Gels", Chem. Commun., 2005, 5796-5798; (b) Rodríguez-Llansola, F.; Escuder, B.; Miravet, J. F. "Switchable Perfomance of an L-Proline-Derived Basic Catalyst Controlled by Supramolecular Gelation", J. Am. Chem. Soc., 2009, 131, 11478-11484; (c) RodríguezLlansola, F.; Miravet, J. F.; Escuder, B. "Supramolecular Catalysis with Extended Aggregates and Gels: Inversion of Stereoselectivity Caused by Self-Assembly", Chem. Eur. J., 2010, 16, 8480-8486.

4. List, B. "Proline-Catalyzed Asymmetric Reactions", Tetrahedron, 2002, 58, 5573-5590.

5. (a) Ulijn, R. V. "Enzyme-Responsive Materials: a New Class of Smart Biomaterials", J. Mater. Chem., 2006, 16, 2217-2225; (b) Yang, X.; Zhang, G.; Zhang, D. "Stimuli Responsive Gels Based on Low Molecular Weight Gelators", J. Mater. Chem., 2012, 22, 38-50; (c) Segarra-Maset, M. D.; Nebot, V. J.; Miravet, J. F.; Escuder, B. "Control of Molecular Gelation by Chemical Stimuli", Chem. Soc. Rev., 2013, 42, 7086-7098.

6. (a) Moulin, E.; Cormos, G.; Giuseppone, N. "Dynamic Combinatorial Chemistry as a Tool for the Design of Functional Materials and Devices", Chem. Soc. Rev., 2012, 41, 10311049; (b) Li, J. W.; Nowak, P.; Otto, S. "Dynamic Combinatorial Libraries: From Exploring Molecular Recognition to Systems Chemistry", J. Am. Chem. Soc., 2013, 135, 9222-9239. 


\section{CHAPTER 3}

SELF-ASSEMBLED L-PROLINE BASED CATALYSTS IN ORGANIC MEDIA 



\subsection{Introduction}

\subsubsection{Organocatalysis with L-Proline}

Since the spatial arrangement within molecules was recognised to be important to the fundamental properties of the substances, chemists have become increasingly interested in enantioselective synthesis of chiral compounds. In that respect, the use of small organic molecules as chiral catalysts, also known as organocatalysis, has become a major topic of research in organic chemistry in the last decades, complementing the traditional organometallic and biological approaches to asymmetric catalysis. These new catalytic methods based on metal-free organic molecules offer some attractive benefits: catalysts are generally nontoxic, readily available and stable. These properties allow most reactions to be performed in wet solvent and in air, which increases reproducibility and operational simplicity. From the time when two seminal reports by List, Lerner and Barbas $^{1}$ and MacMillan et al. on catalysis by chiral secondary amines ${ }^{2}$ showed the potential of this approach, extensive work in this area has been reviewed and examples of organocatalysts can be found, especially aminocatalysis, for a variety of reactions and media. $^{3}$

In this field, peptides have become increasingly popular as asymmetric catalysts for a range of reactions. ${ }^{4}$ Features such as facile synthesis, modularity, and often high selectivity and activity make peptidic catalysts an attractive alternative for organic synthesis.

Among these catalysts, L-proline has become the most successful amino acid studied. ${ }^{5}$ Enzymes are widely recognised as useful catalysts for organic synthesis ${ }^{6}$ showing generally highly chemo-, regio-, diastereo-, and enantioselectivity. Thus, inspired by natural catalysts, enzymes, and with the aim of matching their efficiency and selectivity, L-proline has been used as an enzyme mimic of the metal-free Type I aldolase. ${ }^{7}$ Type I aldolases utilise an enamine-based mechanism involving a catalytically generated enamine intermediate, which can react with various electrophiles. The close relationship between the reaction mechanism of the aldolase type I and the mechanism of the Lproline-catalysed direct aldol reaction is shown in comparison in Figure 3.1.1.1.1 ${ }^{7}$ In both 
cases an enamine represents the key intermediate for the reaction to proceed. Thus, inspired by natural aldolases, several research groups have evaluated peptides bearing $\mathrm{N}$ terminal prolyl residues as catalysts for aldol and conjugate addition reactions. ${ }^{5 \mathrm{~b}, 8}$

A)

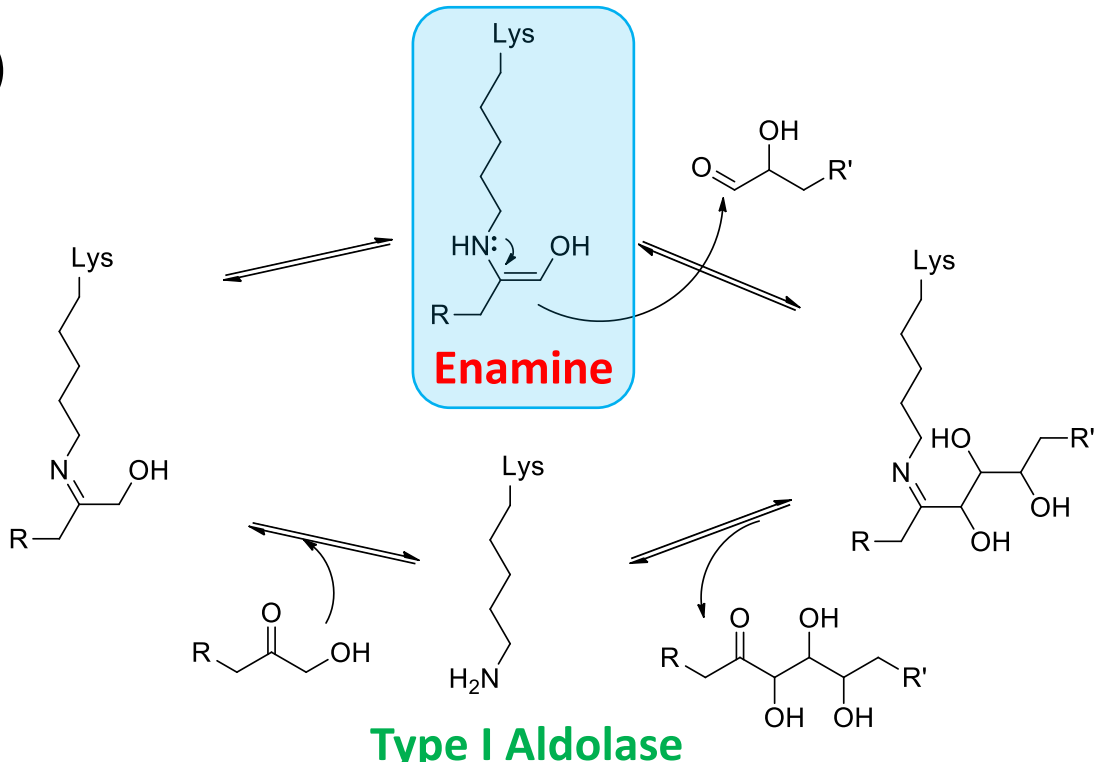

B)

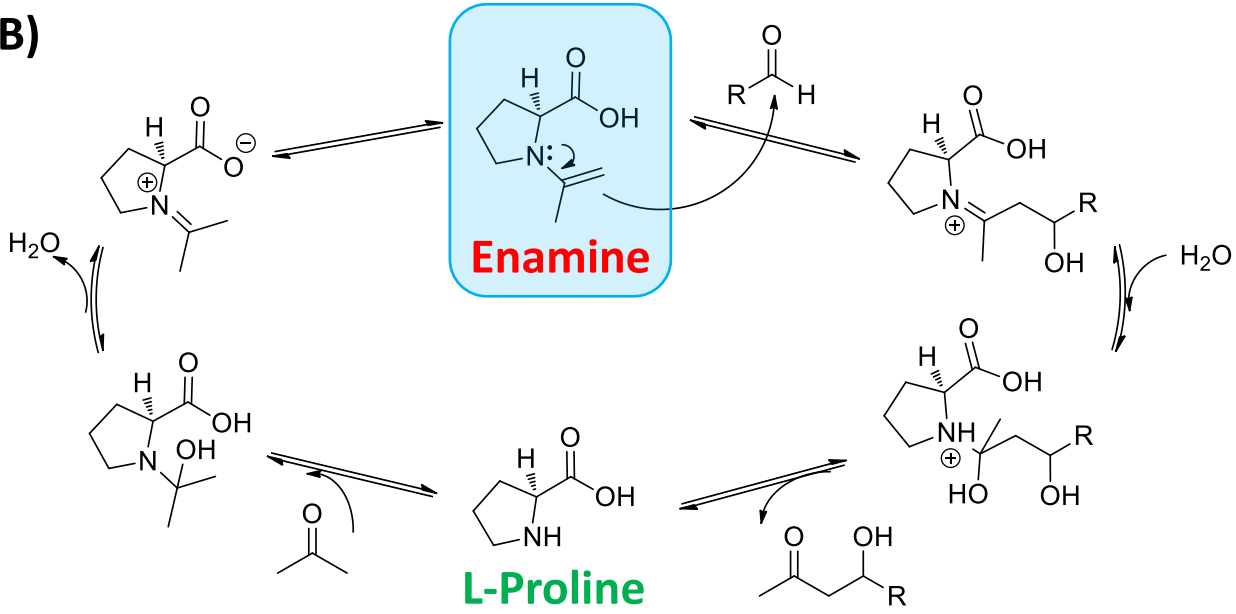

Figure 3.1.1.1. Simplified catalytic cycles for the direct aldol reaction catalysed by aldolase type I (top) and L-proline (bottom).

The asymmetric Michael addition of aldehydes or ketones to nitroolefins is an important method for the synthesis of versatile nitrocarbonyl compounds. These are synthetically useful building blocks because the nitro group can be readily converted into a variety of functional groups including amines and nitrile oxides. The mechanistic similarity between the peptide-catalysed Michael reaction and the corresponding catalysed aldol reaction can be found in the initial formation of the enamine 
intermediate, which subsequently reacts with its electrophilic partner (see Figure $3.1 .1 .2)^{4 a}$

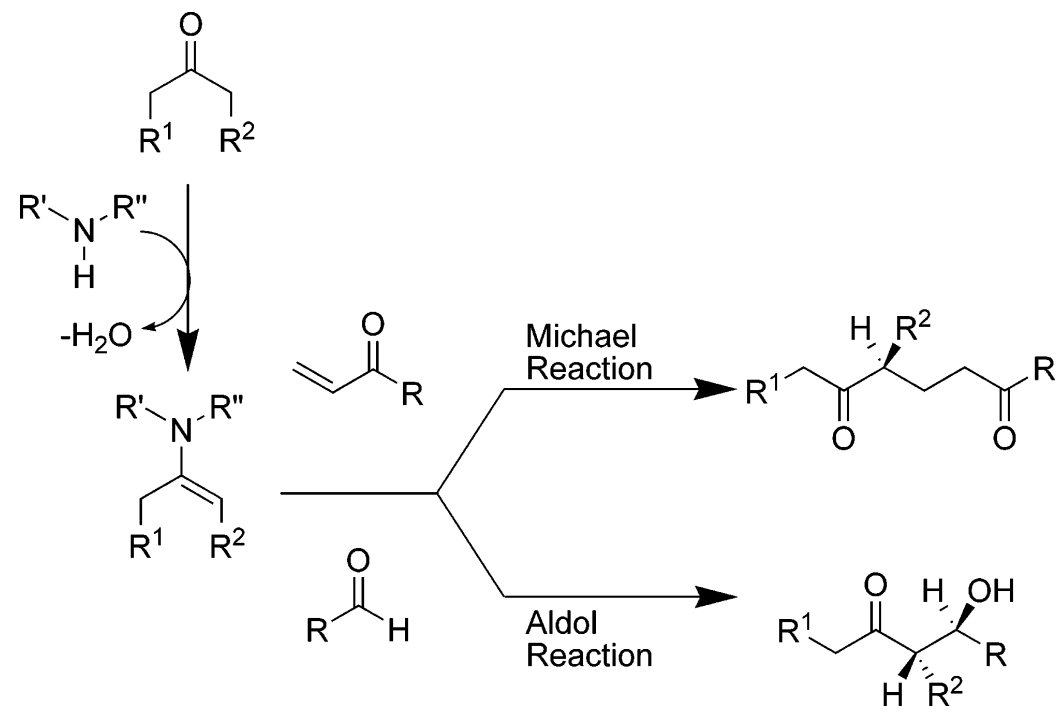

Figure 3.1.1.2. Comparison of amine-catalysed Michael and aldol reactions. ${ }^{4 a}$

Focusing on the Michael reaction, seminal work by List et al. reported the first use of L-proline to efficiently catalyse the conjugated addition of ketones to $\beta$-nitrostyrene. ${ }^{9}$ Some years later the same authors produced a study on $\mathrm{N}$-terminal prolyl peptides, revealing that a series of dipeptides that could catalyse this reaction. It was found that dipeptide $\mathbf{H}-$ Pro-Val-OH gave the Michael product in modest enantiomeric excess and yield. ${ }^{5 b}$

Wennemers et al. on the other hand, studied in detail the peptide catalysed conjugated additions to nitroalkanes with excellent efficiency with respect to conversion and enantioselectivity. ${ }^{10}$ They introduced peptides of general type Pro-Pro-Xaa (where Xaa is an acidic amino acid) as an effective catalyst for enamine catalysis. ${ }^{10 b,} d, 11$ Consequently, rational design based on conformational studies led to the development of H-D-Pro-Pro-Glu- $\mathbf{N H}_{2}$ as an excellent catalyst for the conjugated addition reaction between aldehydes and nitroolefins. ${ }^{10 c}$ Recently, the same group also showed that an immobilized variant of the peptide catalyses the conjugate addition reactions with good conversions allowing efficient reuse for at least 30 times without a significant decrease in reactivity or stereoselectivity (Figure 3.1.1.3). ${ }^{12}$ 

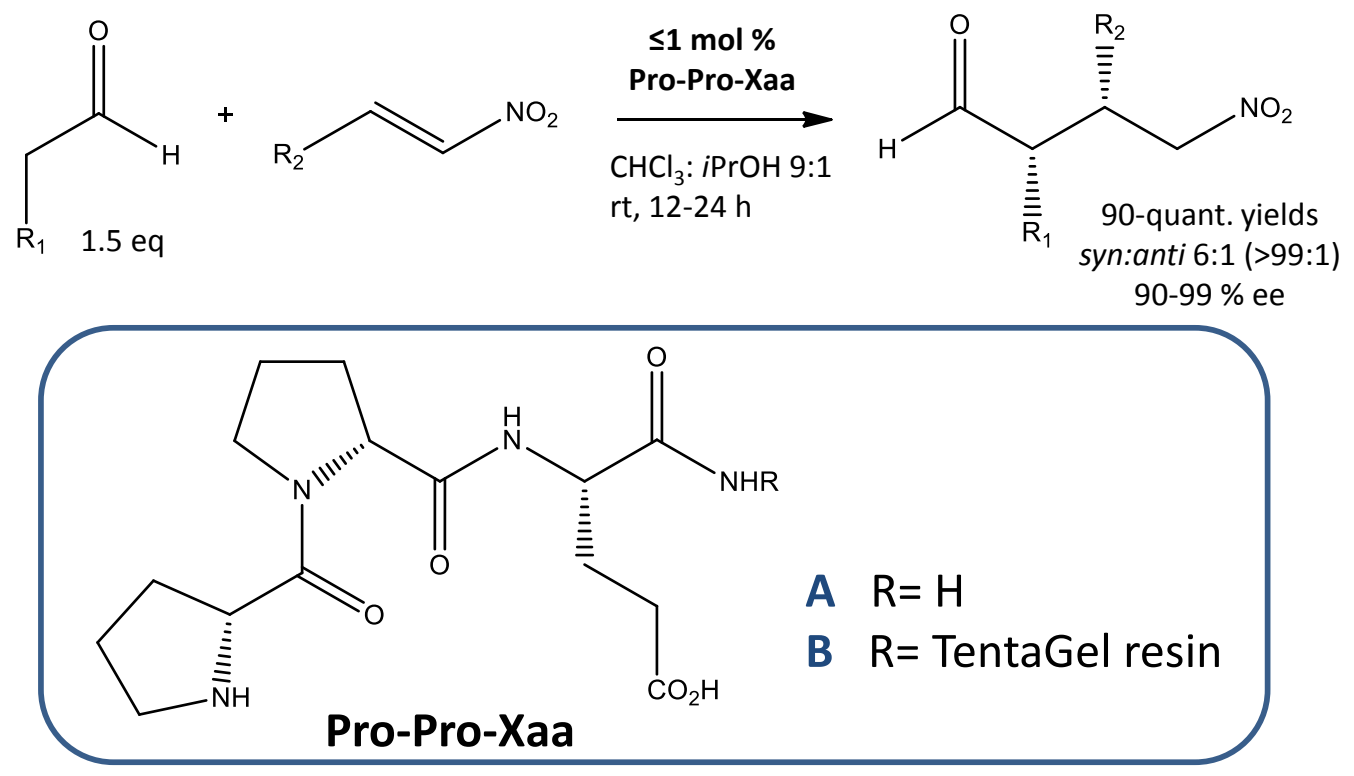

Figure 3.1.1.3. Molecular structure of $\mathbf{H}-\mathbf{D}$-Pro-Pro-Glu- $\mathbf{N H}_{2}$ derivative and its catalytic performance achieved in solution and immobilised in a resin. ${ }^{10 c, 12 a}$

\subsubsection{Supramolecular Catalysis}

Catalysis is one of the main goals of supramolecular chemistry with a pioneering work of Perdersen, Cram and Lehn decades ago. ${ }^{13}$ Since then a supramolecular approach to the catalytic field has attracted the attention of many scientists. ${ }^{14}$ Supramolecular catalysis has been dominated by enzyme-mimic approaches for generating highly-ordered structures with complex architectures. Thus, the properties of the catalyst can be carefully tailored to create an enzyme-like active site. Additionally, in this context, the reversible nature of the supramolecular interactions is ideal for the development of stimuli-controlled catalytic systems as smart materials. Therefore, the function of the material can be triggered by a stimulus that affects the non-covalent binding (the supramolecular interactions), such as temperature, concentration, $\mathrm{pH}$ or solvent polarity among others. Different strategies towards mimicking enzymes have been described ${ }^{15}$ and there are a wide range of examples of catalytic supramolecular assemblies that can be used as a media for chemical reactions such as cyclodextrin derivatives, ${ }^{16}$ micelles, ${ }^{17}$ or capsules $^{18}$ among others (see example in Figure 3.1.2.1). 

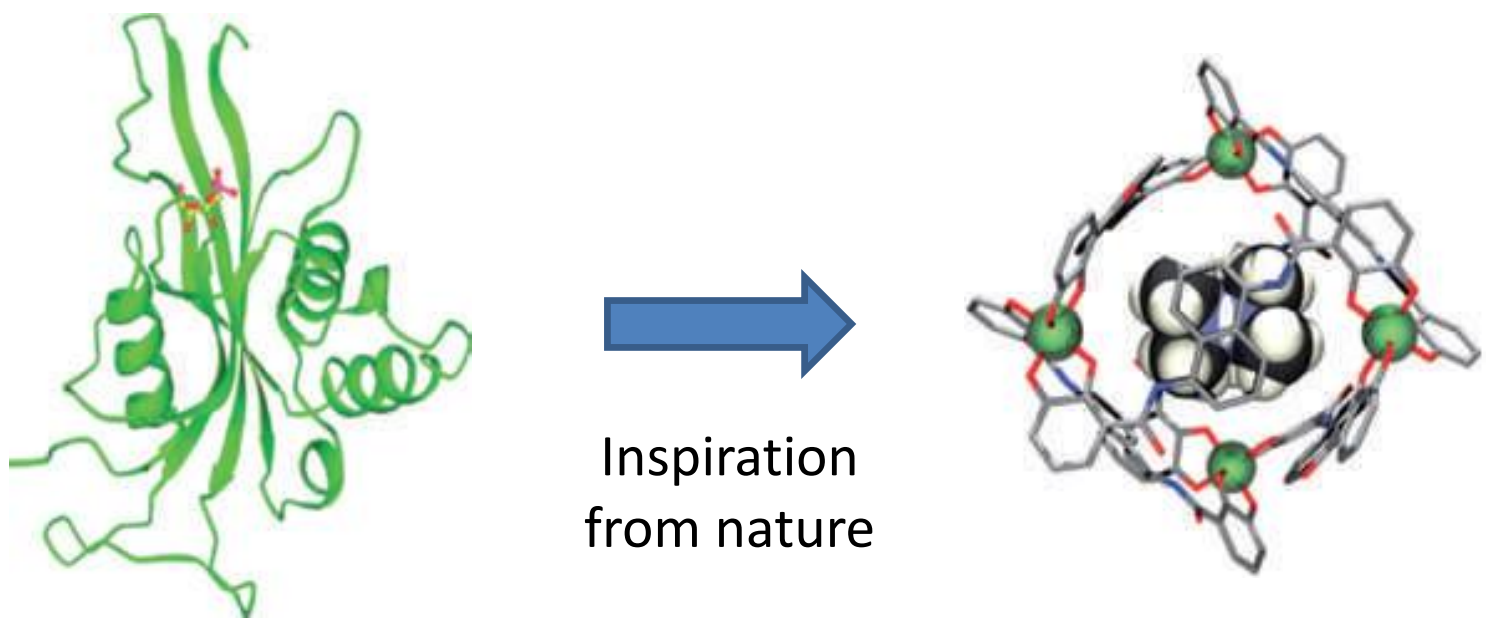

Figure 3.1.2.1. Supramolecular catalyst inspired by Nature. Micro-environment of enzymes (human ADPribose, left) mimicked by a cage compound by Raymond et al. (right). ${ }^{19}$

\subsubsection{Supramolecular Catalytic Gels}

Stimuli-responsive gels constitute an important class of nanostructured and dynamic systems with highly active surface areas and remarkable diffusion properties. Within this group, polymer gels have been traditionally used to obtain catalytic and reactive soft materials. ${ }^{20}$ On the other hand, gels made from low molecular weight compounds represent a more recent and innovative area. ${ }^{21}$

In molecular gels, non-covalent interactions are used for the construction of a multitopic catalyst, in a bottom-up approach. Therefore, gelators can be designed in a modular way by combining an assembling fragment with a functional fragment (catalytic or pre-catalytic) in order to create soft-materials formed by one dimensional selfassembled fibrillar networks (see Figure 3.1.2.1.1). These gels are interesting candidates to act as catalyst due to some important features. Firstly, molecular gels can behave as self-supported catalysts, therefore presenting a large active surface area and can be filtered out of the reaction for reuse; secondly their formation is reversible in most cases (stimuli responsible gels) resulting in a tuneable catalytic system and finally, they present a highly organised self-assembled structure of gelator molecules along the fibre surface, which in the case of catalytic groups results in an enhancement of the efficiency and may generate additional catalytic features such as multivalent interactions and cooperativity. 


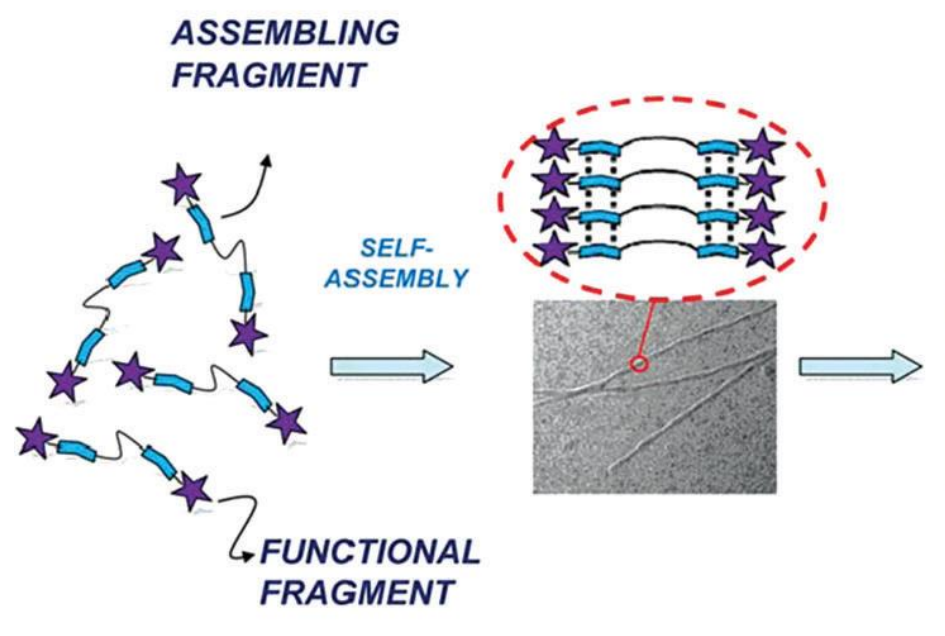

\section{MOLECULAR \\ RECOGNITION}

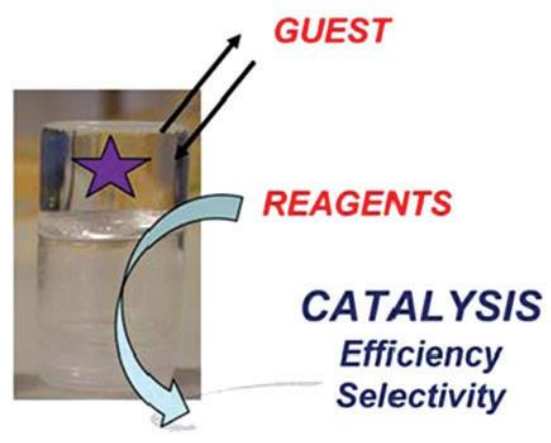

PRODUCTS

Figure 3.1.2.1.1. Strategy for the design of catalytic molecular gels. ${ }^{21 a}$

Using small peptide-based nanofibres to form molecular gels can provide a general approach to achieve the high activity and stability required for mimicking enzymes. For example, Stupp et al. described a hydrogel enzyme model formed by a self-assembled histidine-containing peptide amphiphile (PA). This hydrogel, designed to mimic the hydrolytic ability of esterase, accelerated the hydrolysis of 2,4-dinitrophenyl acetate (DNPA) due to the presence of internally ordered supramolecular nanofibres (Figure $3.1 .2 .1 .2)^{22}$

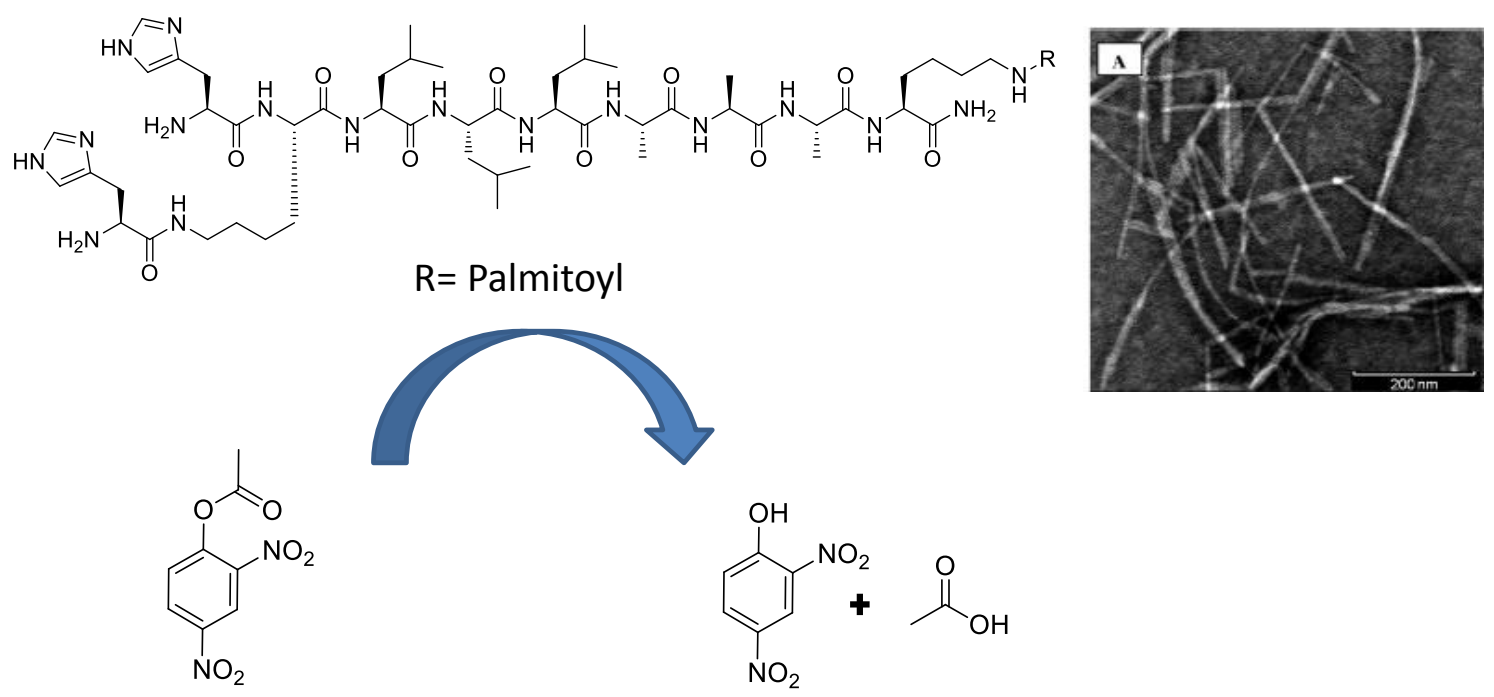

Figure 3.1.2.1.2. Histidine containing PA hydrogel as a catalyst for ester hydrolysis. ${ }^{22}$ 
Liu et al., in another example, described the self-assembly of several L-glutamic acid-based gelators in the presence of $\mathrm{Cu}^{2+}$ ion (Figure 3.1.2.1.3). It was found that, in the case of bolaamphiphilic glutamic acid, $N, N^{\prime}$-hexadecanedioyl-di-L-glutamic acid (L-HDGA), the addition of $\mathrm{Cu}^{2+}$ caused a morphology transition from monolayer nanotube of L-HDGA to a multilayer nanotube. This metallogel was found to be active as catalyst in the asymmetric Diels-Alder cycloaddition between cyclopentadiene and aza-chalcone showing an accelerated reaction rate and enhanced enantiomeric selectivity when compared with non-assembled structures. ${ }^{23}$

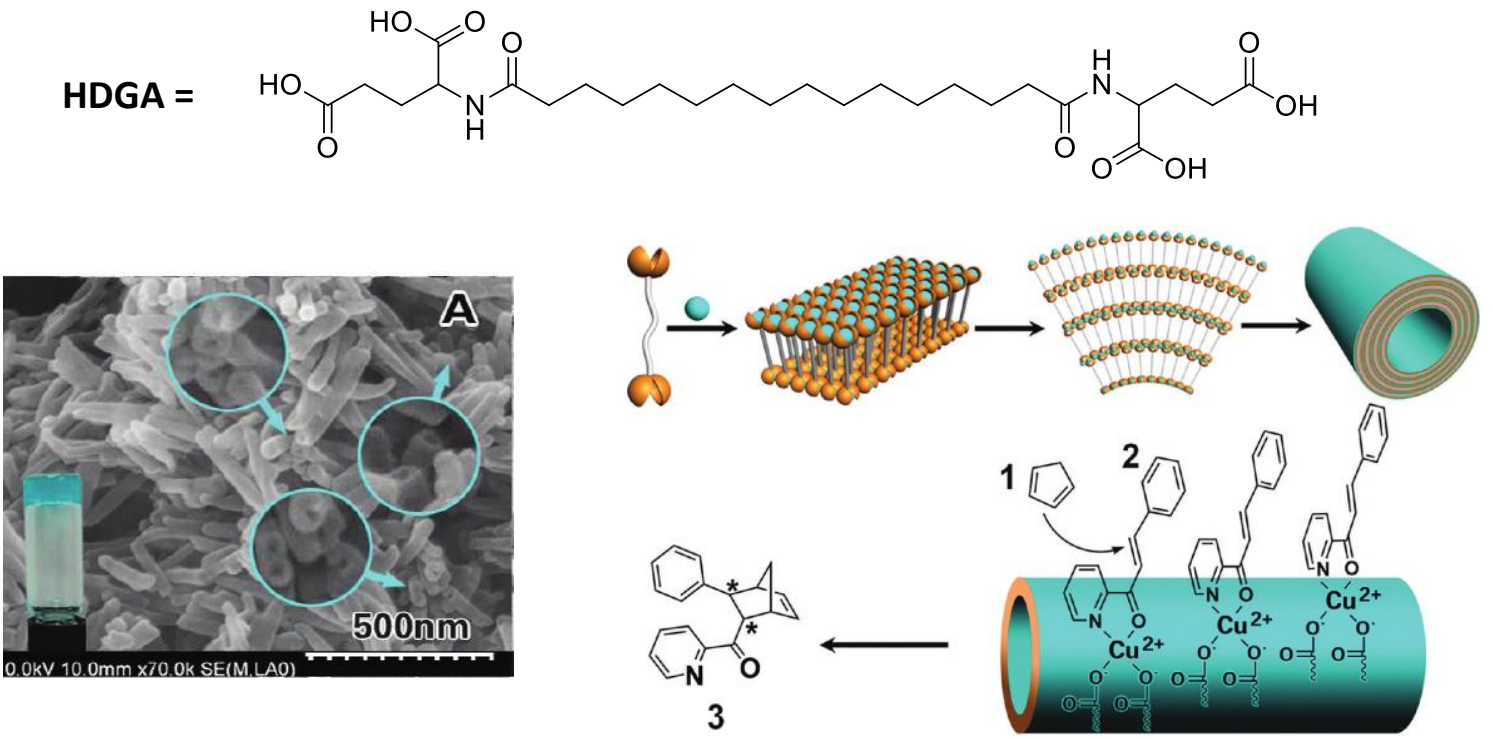

Figure 3.1.2.1.3. Molecular structure of the bolaamphiphilic glutamic acid derivative gelator (L-HDGA) and the microscopic and macroscopic aspect of the gel phase. Schematic representation of the self-assembled structures and its catalytic performance. ${ }^{23}$

In some other cases, molecular gels have also been employed for the confinement of proteins revealing interesting results both on protein structure and function. Xu et al. studied the gel network as a matrix carrier where the enzyme is enclosed and still able to perform its function. The formation of the hydrogel was in situ by the self-assembling of two simple amino acid derivatives (Figure 3.1.2.1.4). The enzyme trapped within the fibres exhibited superior activity and high stability in organic media. ${ }^{24}$ 

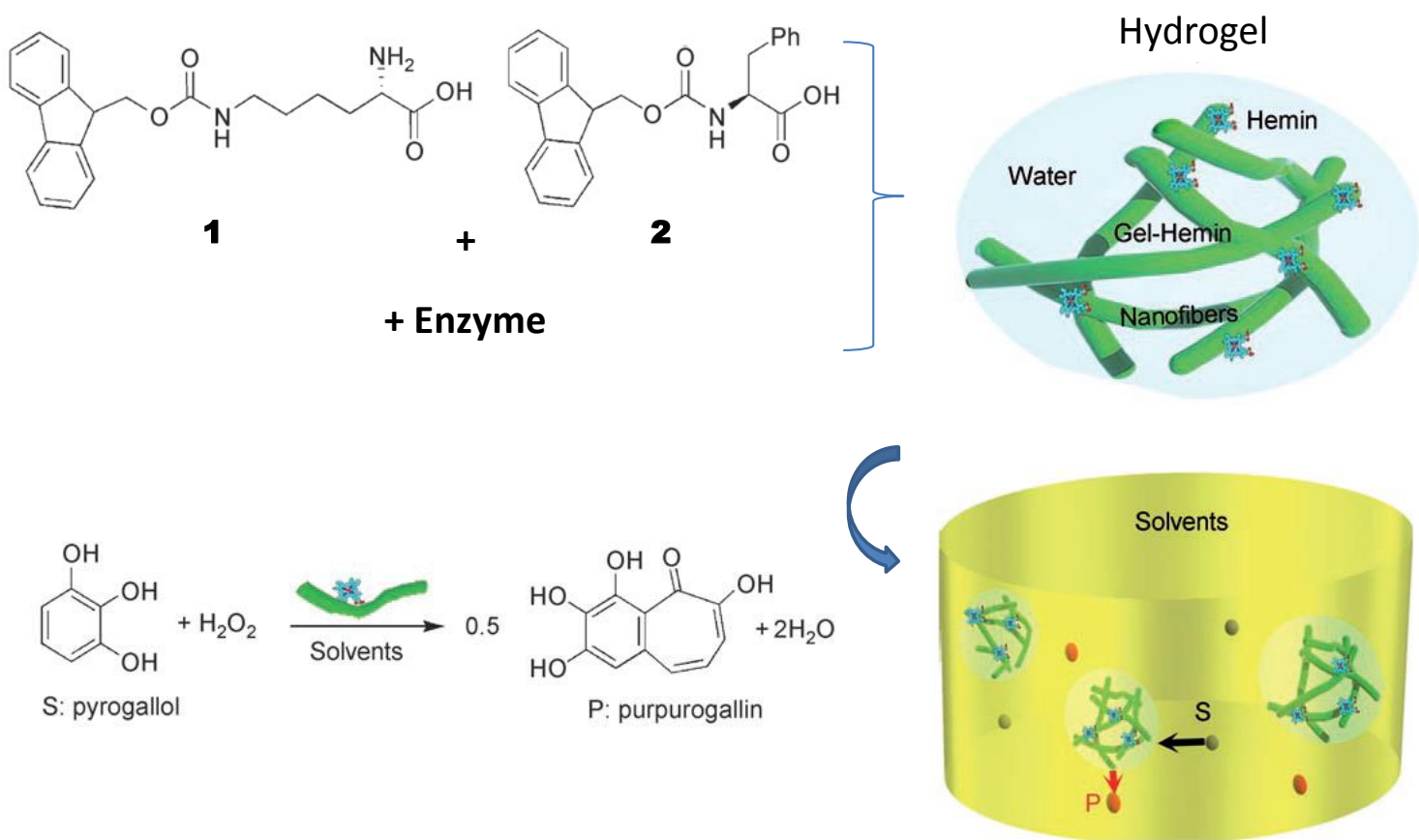

Figure 3.1.2.1.4. Molecular structure of the amino acid derivatives that form the hydrogel where the enzyme is enclosed and the peroxidase reaction that takes place in organic solvents. S: Substrate and P: Products. $^{24 b}$

Moreover, hydrophobic microenvironments created within the fibrillar network of hydrogels have been designed to minimize denaturation of proteins, keeping a wet environment where proteins can remain structurally intact and carry out their biological functions ${ }^{25}$ as well as for the construction of cell architecture mimics. ${ }^{26}$

A previous study reported in our group showed how the aggregation of a bolaamphiphilic organogelator containing L-proline catalytic moieties into fibrillar gel networks in toluene modified the enantioselectivity of the reaction when compared to solution. Gel phase catalysis gave quantitative yields and both good diastereo- and enantioselectivity, a better understanding of the self-assembling process (Figure 3.1.2.1.5) was also achieved. ${ }^{27}$ 

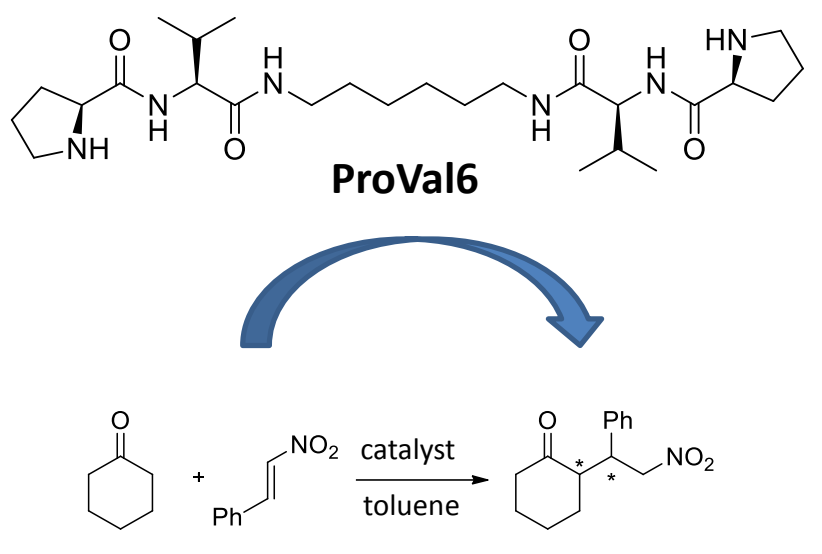

$99 \%$ quant. yield

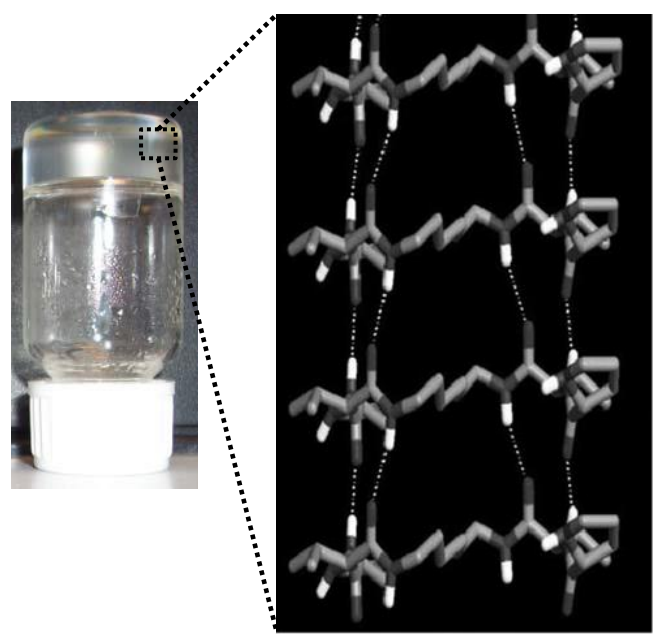

$98: 2$ syn:anti

$34 \%$ e.e. $\left(2 \mathrm{R}, 1^{\prime} \mathrm{S}\right)$

Figure 3.1.2.1.5. Molecular structure of the proline based gelator used in Michael reaction as supramolecular catalyst. ${ }^{27}$

In the same work, the dipeptide ProValPr (3b) (see Figure 3.2.1) was also studied as a catalyst analogue in solution (this compound did not form gels in toluene at the range of concentrations used). Good catalytic conversions and selectivity were found for this compound in the same catalytic reaction, showing good correlation with the gelator derivative ProVal6 results. The use of diverse amino acids in the central fragment could lead to different aggregation abilities as well as a different catalytic behaviour. Thus a screening study where the effect of changing the amino acid attached to L-proline is reported in order to build a gelator structure with improved catalytic activity. 


\subsection{L-Proline Dipeptides: Aggregation Study and its Effect in Organocatalysis}

The design of bolaamphiphilic molecules has been employed successfully in some cases as an effective approach to obtain gelators for organic as well as for aqueous media. ${ }^{28}$ Results reported showed the construction of such soft-materials by the introduction of functional groups to an assembling fragment in order to lead materials with additional functions. Focusing on organocatalysis, the introduction of the L-proline moiety, a well-known catalytic fragment, has been reported to build a family of bolaamphiphilic structures. ${ }^{29}$ Gels formed in this way showed good efficiency and selectivity in different reactions in organic solvents.

In this chapter the use of amino acids building blocks in the bottom-up approach to design self-assembled fragments has been widely studied. It is assumed here that there will be a relationship between the catalytic activity showed by the self-assembled soluble dipeptides studied and the corresponding bolaform gelators. Thus, the amphiphile analogue with the best catalytic activity will be used to build the bolaamphiphile gelator molecule and subsequently, the catalysis in gel-phase will be tested.<smiles>CCCNC(=O)[C@H](C)NC(=O)[C@@H]1CCCN1</smiles>

ProAlaPr (3a)<smiles>CCCNC(=O)[C@H](Cc1ccccc1)NC(=O)[C@@H]1CCCN1</smiles>

ProPhePr (3c)<smiles>CCCNC(=O)[C@@H](NC(=O)[C@@H]1CCCN1)C(C)C</smiles>

ProValPr (3b)<smiles>CCCNC(=O)[C@H](NC(=O)[C@@H]1CCCN1)C(C)CC</smiles>

ProllePr (3d)

Figure 3.2.1. Molecular structure of dipeptide derivatives. 
Here a structure-activity relationship investigation was carried out in order to highlight the importance on the amino acid selection on gelator molecule. A small screening study was performed and four different L-proline dipeptide derivatives were chosen; L-alanine, L-phenylalanine, L-valine and L-isoleucine, all were capped at Cterminus as propylamides (Figure 3.2.1). A detailed study of their conformational preferences in solution and upon aggregation is shown. Catalytic behaviour was evaluated for the 1,4-conjugated addition of cyclohexanone to trans- $\beta$-nitrostyrene

\subsubsection{Synthesis}

The preparation of compounds 3a-d was carried out in an easy manner in grams scale following conventional peptide synthesis in solution. This methodology includes activation of the carboxylic acid required for the peptidic bond formation by aminolysis as well as protection and deprotection of the reactive groups inside the peptide chain.
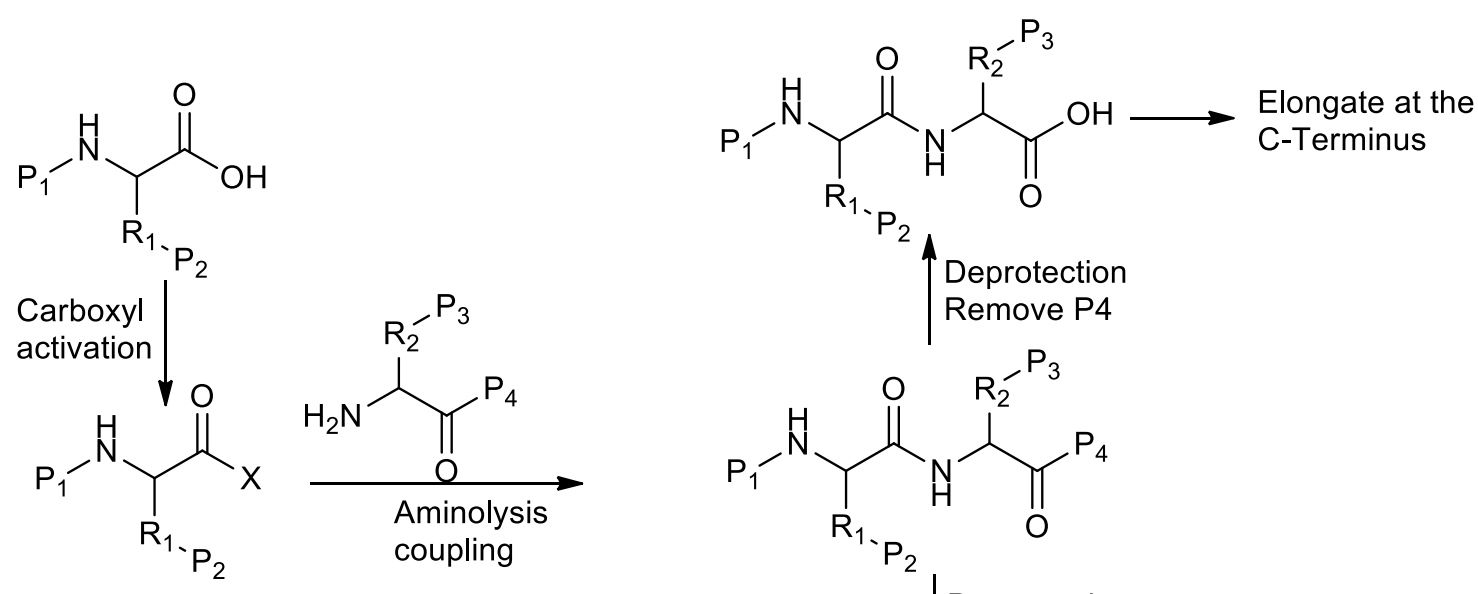<smiles>[R6]C(=O)C([R7])NC(=O)C([R7])N[PH]</smiles>

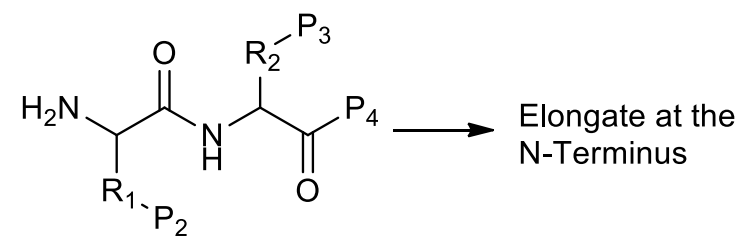

Figure 3.2.1.1. General scheme for peptide synthesis. $P_{n}$ refers to the corresponding protecting group.

Starting from the $\mathrm{N}$-protected L-amino acids (benzyloxycarbonil group, CbZ or Zgroup) activated esters were formed by activation of the carboxylic acid with $N, N^{\prime}$ dicyclohexylcarbodiimide (DCC) and reaction with $\mathrm{N}$-hydroxysuccinimide (3b-d.1 for 
valine, phenylalanine and isoleucine precursors respectively, Scheme 3.2.1.1). In this synthetic step DCU was formed as side product, which precipitated and could be filtered off. The crude active ester was purified by recrystallization in isopropanol.
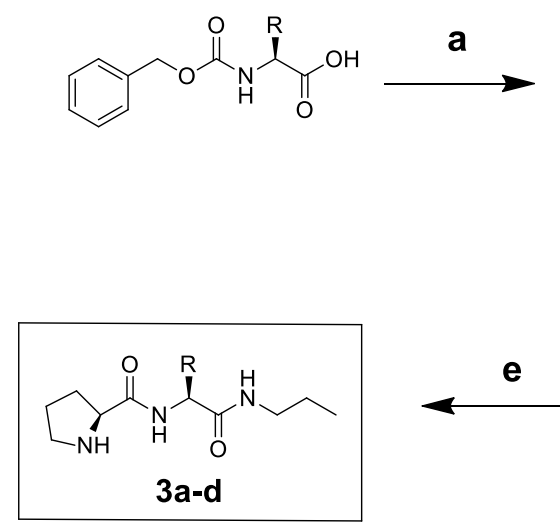<smiles>[2H]C(NC(=O)OCc1ccccc1)C(=O)ON1C(=O)CCC1C</smiles>

3.1

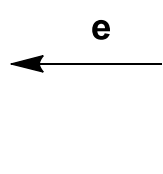

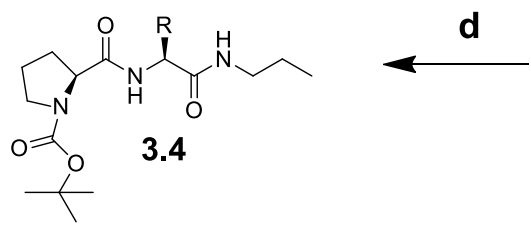

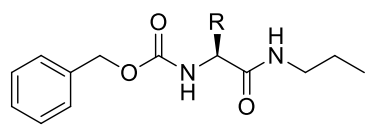

3.2

C

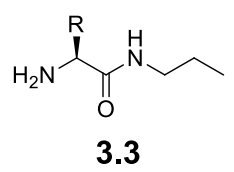

Scheme 3.2.1.1. Schematic synthesis for the dipeptides L-proline derivatives. a) DCC, $N$-hydroxysuccinimide, THF, 0 ㅇ, 3 h (93-87\%); b) Propylamine, DME, rt (18 h) and 50 ㅇ (2 h)(93-89\%); c) $\mathrm{H}_{2}, \mathrm{Pd} / \mathrm{C}, \mathrm{MeOH}, \mathrm{rt} 4 \mathrm{~h}$ (96-86\%); d) Boc-L-Pro-Osu, DME, rt (24 h) and 40 ○C (5 h)(95-60\%); e) TFA/ $\mathrm{CH}_{2} \mathrm{Cl}_{2}, \mathrm{rt}$ (2 h), $\mathrm{NaOH}$ (79-52\%). $\left(\mathrm{R}=-\mathrm{CH}_{3}\right.$ (alanine, 3a), $-\mathrm{CH}\left(\mathrm{CH}_{3}\right)_{2}$ (valine, 3b), $-\mathrm{CH}_{2} \mathrm{Ph}$ (phenylalanine, $3 c$ ) and $-\mathrm{CH}\left(\mathrm{CH}_{3}\right) \mathrm{CH}_{2} \mathrm{CH}_{3}$ (isoleucine, 3d)).

The formation of (N-Cbz-L-aminoacyl) amides (3b-d.2) was carried out by aminolysis of active esters (3b-d.1) with propylamine in DME at room temperature for 18 hours and then warming at $50 \stackrel{\circ}{C}$ for 2 hours. A white solid was obtained in all the cases described in the Scheme 3.2.1.1.

For the L-alanine derivative, 3a, this methodology gave problems in the formation of the active ester, resulting in low yields and recovery of unreacted starting materials. Thus, in this case, the product was obtained by the "mixed anhydrides" method, as is shown in the Scheme 3.2.1.2. Starting from $\mathrm{N}$-Cbz-L-alanine, the mixed anhydride was obtained by reaction with ethyl chloroformate in the presence of triethylamine using THF as solvent and low temperature $0-5 \stackrel{\circ}{ }$ (ice bath). Then the addition of propylamine to the mixture lead to the formation of the corresponding ( $N$-Cbz-L-aminoacyl) amide (3a.2) in good yield after being purified by column chromatography on silica gel (hexane-ethyl acetate; $1: 1)$. 
<smiles>CCCNC(=O)[C@H](C)NC(=O)OCc1ccccc1</smiles>

Scheme 3.2.1.2. Synthetic route for the alanine derivative 3a.2.

Removal of the $\mathrm{N}$-benzyloxycarbonyl protecting group was carried out by hydrogenolysis with palladium on activated carbon as catalyst in $\mathrm{MeOH}$ and under hydrogen atmosphere obtaining the respective (L-aminoacyl) amines (3a-d.3, Scheme 3.2.1.1). The catalyst was removed by filtration through Celite, and the solvent was evaporated under vacuum. The crude oils obtained in an almost quantitative yield were used without further purification for the synthesis of the $N$-tert-butyloxycarbonyl protected proline derivatives (3a-d.4). A solution of the amino amide compound (3a-d.3) in dry dimethoxyethane (DME) was added dropwise over a solution of $t$-Boc-L-proline active ester in dry DME. The mixture was stirred at room temperature for $24 \mathrm{~h}$ and then at $40{ }^{\circ} \mathrm{C}$ for $5 \mathrm{~h}$ to yield the Boc-protected compounds.

Finally, removal of Boc group was performed in dichlorometane after the addition of TFA to the mixture and stirring for 2 hours at room temperature, then the acid was removed under vacuum. The final compounds (3a-d) can be extracted with chloroform from the aqueous phase at basic $\mathrm{pH}(\mathrm{NaOH})$ yielding quantitatively a white solid in all the cases (see Scheme 3.2.1.1). 


\subsubsection{Conformational Changes Associated to the Aggregation of L-Proline Dipeptides}

Here, the self-assembly of the four dipeptide molecules L-proline derivatives 3a-d (see Figure 3.2.1) was studied in toluene from a thermodynamic and structural point of view using NMR experiments. It has been proved that the compound ProValPr (3b) only forms unstable gels in toluene at concentrations above $150 \mathrm{mM}{ }^{27}$ Below this value, a concentration dependant aggregation took place that was not enough to lead the gel formation. By analogy, all the new compounds were fully soluble (the aggregates are not big enough to form 3D networks structures able to entrap the solvent) in the range of concentrations studied $(1-70 \mathrm{mM})$ in this solvent.<smiles>[R]C(C(=O)NCCC)N(C)C(=O)C1CCCN1</smiles>
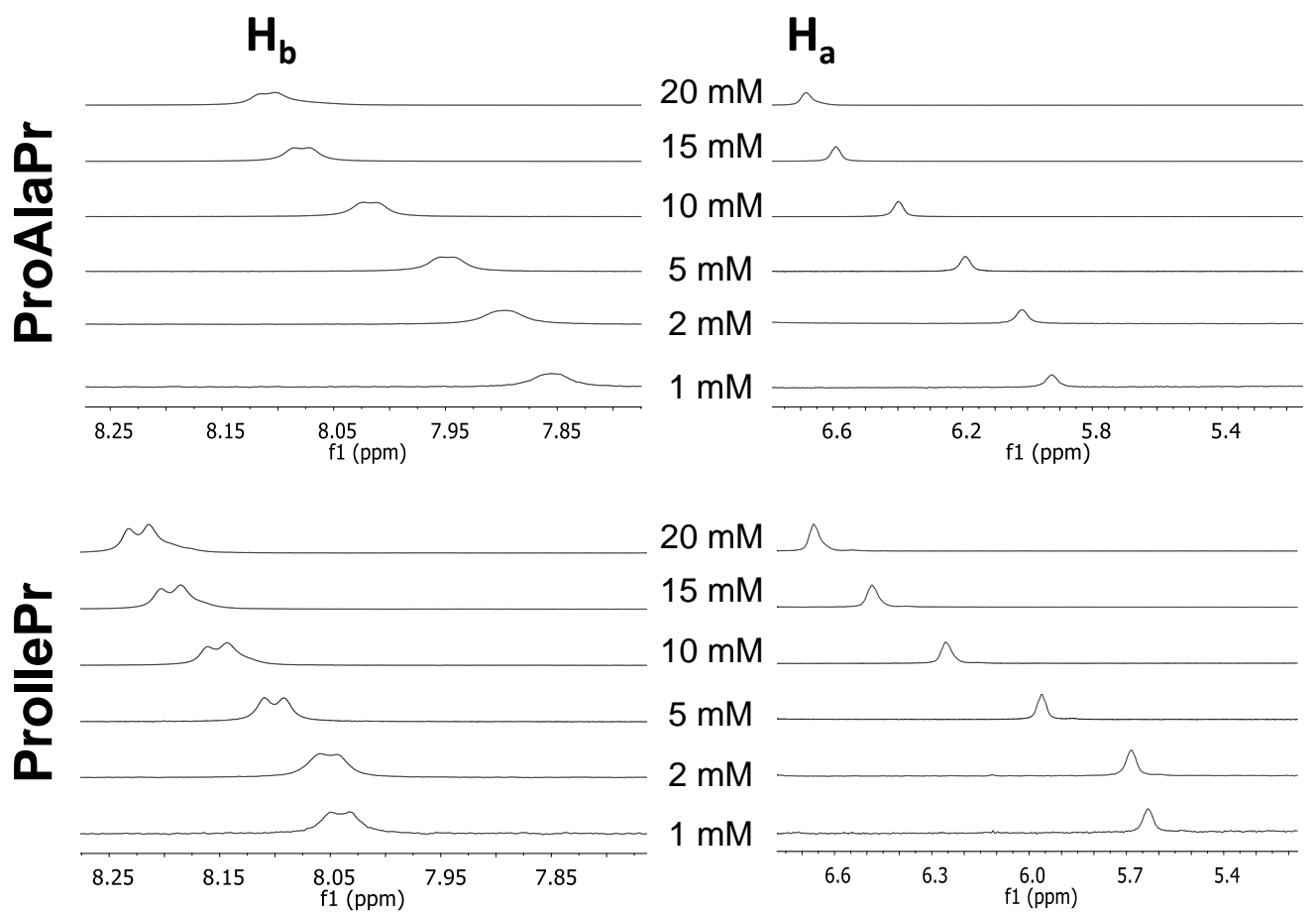

Figure 3.2.2.1. Partial ${ }^{1} \mathrm{H}$ NMR spectra of ProllePr (3d) and ProAlaPr (3a) in $\mathrm{D}_{8}$-toluene at different concentrations.

Firstly, a self-assembly study was carried out by NMR in $D_{8}$-toluene. The chemical shifts of the signals were monitored at different concentrations focussing on the amide region, as they may be involved in the aggregation by means of hydrogen bonds. It can be 
seen in Figure 3.2.2.1 that amide signals $\left(\mathrm{NH}_{\mathrm{a}}\right.$ and $\left.\mathrm{NH}_{\mathrm{b}}\right)$ were shifted downfield as the concentration increases, for both ProllePr (3d) and ProAlaPr (3a). As hypothesised, this behaviour reveals supramolecular association through hydrogen bonds. Similar results were observed for ProValPr (3b) and ProPhePr (3c) (see Figure SI_3.5.1-4).

For a better understanding of the self-assembly process, ${ }^{1} \mathrm{H}$-NMR spectroscopy data corresponding to the shift of the amide $\mathrm{NH}_{\mathrm{a}}$, whose chemical shift had shown to have a strong susceptibility with the concentration, was fitted to a cooperative supramolecular polymerization model. ${ }^{30}$ The results were in agreement with a cooperative behaviour having a dimerization constant $\left(\mathrm{K}_{2}\right)$ and equivalent successive aggregation constants $\left(\mathrm{K}_{\mathrm{n}}\right)$ (See Equation_SI_3.5.1-4 and data in the Supporting Information). The results are shown in Table 3.2.2.1. In all the cases moderate cooperativity was observed being $K_{n}>K_{2}$. Interestingly, the association constants $K_{2}$ found were similar for the four dipeptides but higher values of $K_{n}$ were obtained for ProValPr (3b) and ProllePr (3d), suggesting a high degree of cooperativity for the latter molecules.

Table 3.2.2.1. Thermodynamic constants for the self-aggregation of the catalysts at $30 \circ \mathrm{C}$ in toluene.

\begin{tabular}{ccccc}
\hline Compound & $\mathrm{K}_{\mathbf{2}}$ & $\mathrm{K}_{\mathrm{n}}$ & $\begin{array}{c}\text { Aggregation degree at 1 } \\
\mathbf{m M ~ ( \% ) ~}\end{array}$ & $\begin{array}{c}\text { Aggregation degree at 5 } \\
\mathbf{m M}(\%)^{\text {a }}\end{array}$ \\
\hline ProAlaPr (3a) & 21 & 72 & 4 & 21 \\
ProValPr $^{\text {b (3b) }}$ & 29 & 100 & 6 & 28 \\
ProPhePr (3c) & 17 & 38 & 3 & 16 \\
ProllePr (3d) & 20 & 106 & 4 & 24 \\
\hline
\end{tabular}

[a] Calculated using $\mathrm{K}_{2}$ and $\mathrm{K}_{\mathrm{n}}$ values. Estimated error is ca. $3 \%$.

[b] Constants previously reported. ${ }^{27}$

To measure the amount of aggregated species in the range of concentrations used on the catalytic performance (between 1-10 mM), a species distribution simulation was carried out. In this simulation, the $K_{2}$ and the $K_{n}$ constants were calculated for each individual catalyst molecule used. The results are shown in Table 3.2.2.1 and suggest that despite these differences in the observed $K_{n}$ values, the proportion of aggregated species is maintained in the same order for all the four catalyst molecules. For instance, taking 
ProllePr (3d) as an example, its species distribution diagram was simulated (see Figure 3.2.2.2). The diagram shows that going from $1 \mathrm{mM}$ to $5 \mathrm{mM}$, the dimeric species increase noticeably with a linear trend whereas the oligomeric species increase in an exponential way, in agreement with a cooperative model system.

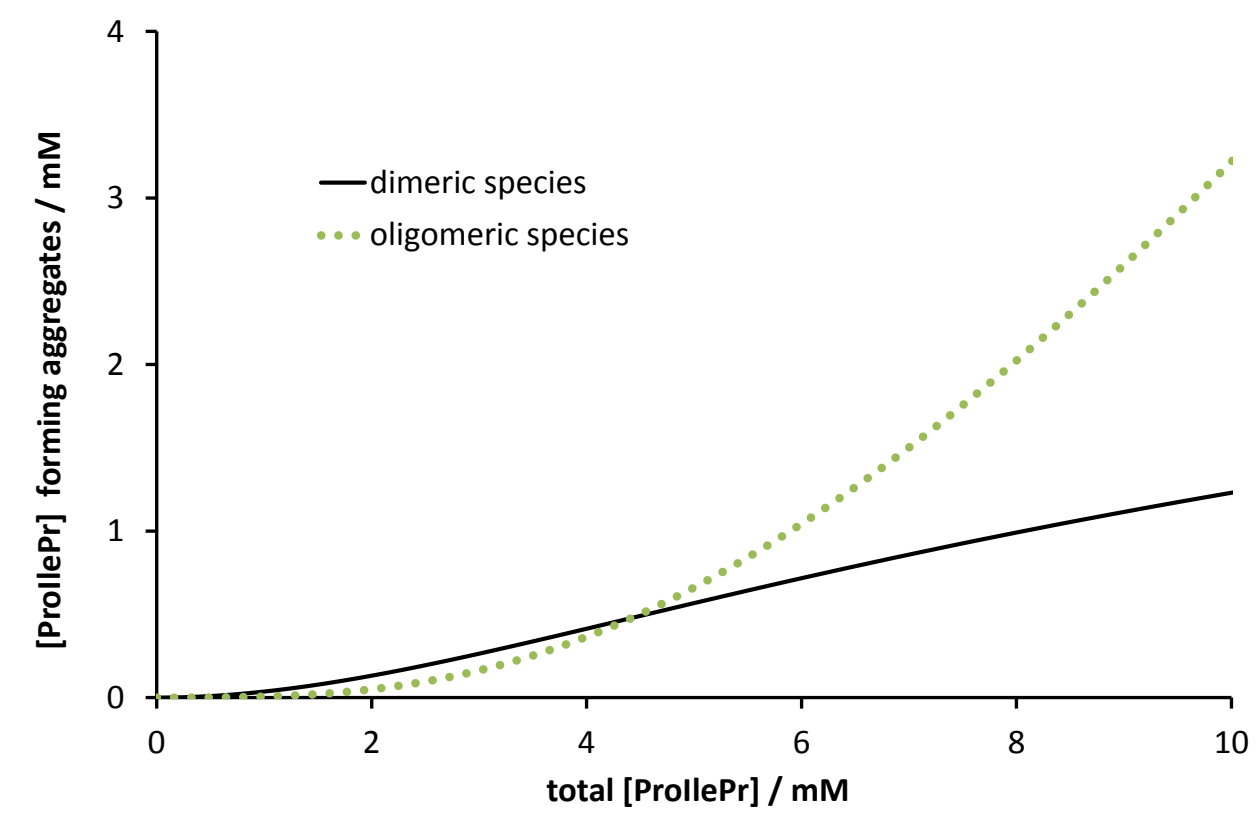

Figure 3.2.2. Species distribution diagram for the aggregation of ProllePr (3d) in toluene.

Molecular mechanics calculations (AMBER* force field, see Experimental Section) for the free catalysts predicted in all the cases two energetically close folded conformations ( $\Delta \mathrm{E} c a .7 \mathrm{~kJ} \mathrm{~mol}^{-1}$ ) near the global minimum, called syn- and anticonformations respectively (see Figure 3.2.2.3). The main difference among the found syn- and anti-conformations is the dihedral angle $\mathrm{N}-\mathrm{C}-\mathrm{C}=\mathrm{O}$ of the proline unit, presenting angles below and above $90^{\circ}$ respectively. In both cases the conformers contain a $\mathrm{H}$-bond between propylamide $\mathrm{NH}\left(\mathrm{NH}_{\mathrm{a}}\right)$ and $\mathrm{CO}$ of the proline moiety, in accordance with the experimental data collected from ${ }^{1} \mathrm{H}-\mathrm{NMR}$ chemical amide shifts described previously (Figure 3.2.2.1). Additionally, the anti-conformation presents an intramolecular hydrogen bond between L-proline amine and the amide $\left(\mathrm{NH}_{\mathrm{b}}\right)$ of the peptidic linkage. The existence of this strong intramolecular hydrogen bond was described previously ${ }^{27,} 31$ and is demonstrated by comparison to the ${ }^{1} \mathrm{H}-\mathrm{NMR}$ spectra with different solvents (see Figure SI_3.5.5). The chemical shift of the $\mathrm{NH}_{b}$, which is adjacent to the proline ring, was revealed to be non-sensitive to solvent change. Thus, suggesting its involvement in $\mathrm{H}$ - 
bonding and no interactions with the solvent molecules, such as these showed to $\mathrm{NH}_{\mathrm{a}}$. On the other hand, folded syn-conformation seemed to present an intramolecular hydrogen bond between L-proline amine and the carbonyl CO unit of the peptide linkage (see Figure 3.2.2.3 and Scheme 3.2.2.1).

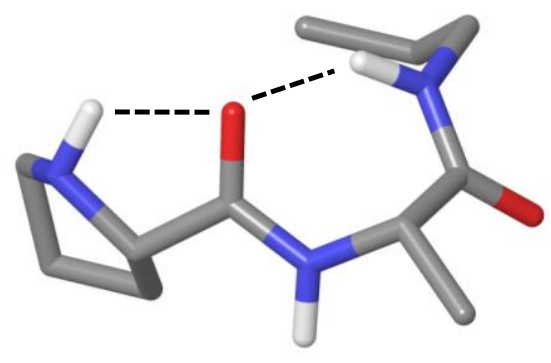

syn

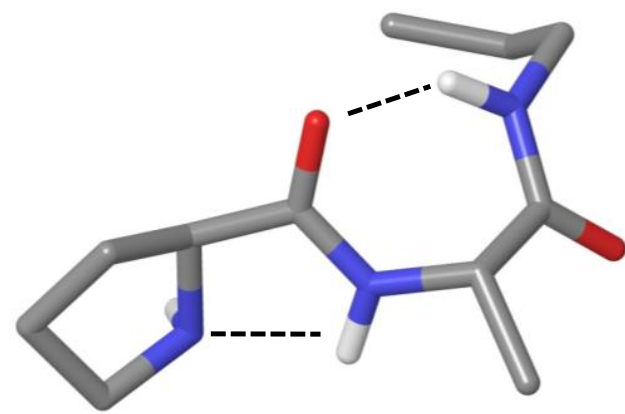

anti

Figure 3.2.2.3. Molecular models (energy minimized, AMBER*) for folded syn- and anti-conformations of ProAlaPr (3a). $E_{a n t i}-E_{s y n}=-7.4 \mathrm{~kJ} \mathrm{~mol}^{-1}$.

To further demonstrate the presence of such conformations in solution, additional studies using NMR were conducted. Firstly, temperature dependant NMR experiments were performed for all compounds at $1 \mathrm{mM}$ (diluted samples where almost no aggregation is present). As can be seen in Figure 3.2.2.4 for ProllePr (3d) derivative both amide signals, showed a variation in the chemical shift with the temperature, pointing to their participation in intramolecular $\mathrm{H}$-bonding. These results are in agreement with the presence of anti-conformations, which possess two intramolecular $\mathrm{H}$-bonding involving both amide signals (see Scheme 3.2.2.1). Similar behaviour was observed for the rest of proline analogues (see Figure SI_3.5.6), suggesting that all are present in the described anti-conformation when they are in solution as diluted samples (see models proposed in Figure 3.2.2.5). 


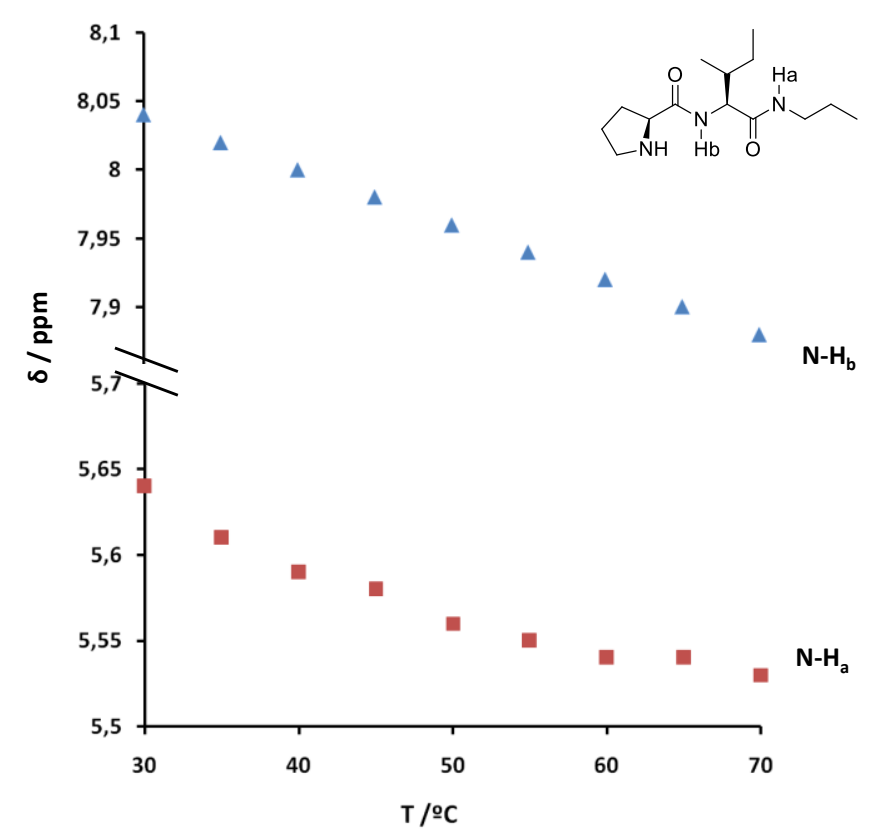

Figure 3.2.2.4. Chemical shift variation with the temperature for amide signals of ProllePr (3d) (1 mM in $D_{8^{-}}$ toluene).

A

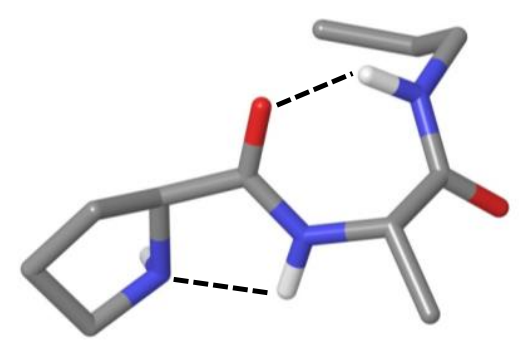

C

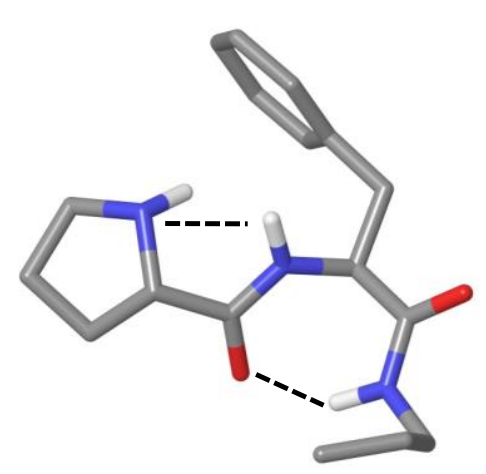

B

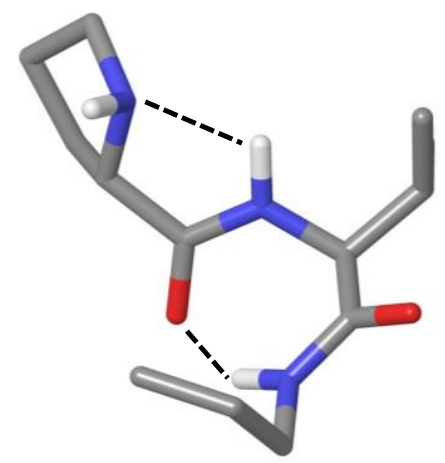

$\mathrm{D}$

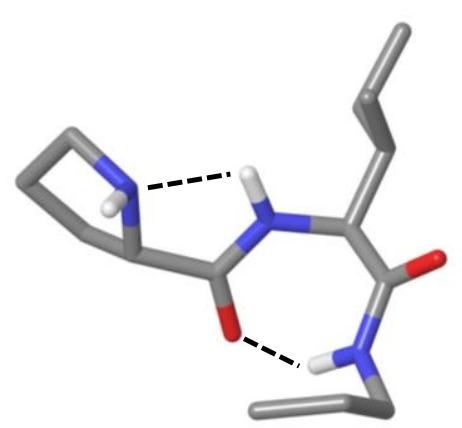

Figure 3.2.2.5. Molecular models for the folded anti-conformers. (A) ProAlaPr (3a), (B) ProValPr (3b), (C). ProPhePr (3c) and (D) ProllePr (3d). 
Besides, it was observed that the signals belonging to the geminal protons in position 3 of the proline ring showed differences in shape as well in the position (Figure 3.2.2.6). The observed splitting could be explained by the conformation adopted by the proline ring forcing the spatial proximity of C3 protons to the carbonyl, experiencing its shielding/deshielding effect to a different degree. This observation also may again point to the presence of the anti-conformation. Although this splitting is common in L-proline rings, its magnitude seems to be related to the particular conformation present and thus suggesting some differences in the structural preferences of these small dipeptides. More differences can be observed between these proline derivatives, such as the chemical shift of the peptidic amide signal $\left(\mathrm{NH}_{\mathrm{b}}\right)$. As shown the Figure 3.2.2.6, signals of ProAlaPr (3a) and ProPhePr (3c) present significantly lower chemical shift values than ProValPr (3b) and ProllePr (3d) (7.85, 7.92, 8.04 and 8.02 ppm respectively). From these NMR results, the splitting degree of the $\mathrm{C} 3$ geminal protons and the chemical shift for the $\mathrm{NH}_{\mathrm{b}}$ suggest that although ProValPr (3b) and ProllePr (3d) present almost exclusively in the anticonformation, it might be possible that ProAlaPr (3a) and ProPhePr (3c) derivatives could present a fast exchange with a small amount of molecules in syn-conformation. This fact would explain the differences in the mentioned NMR data (see Scheme 3.2.2.1).

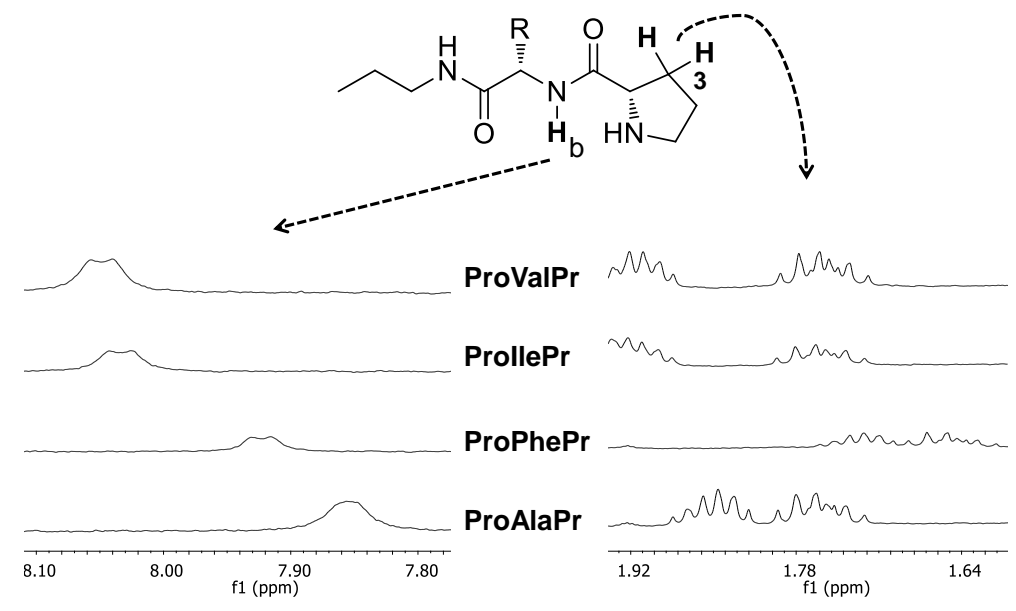

Figure 3.2.2.6. Chemical shift of the amide $\mathrm{NH}_{\mathrm{b}}$ (left) and germinal protons in $\mathrm{C} 3$ of the proline ring (right)

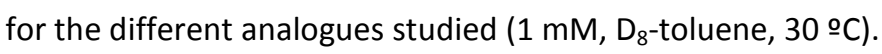




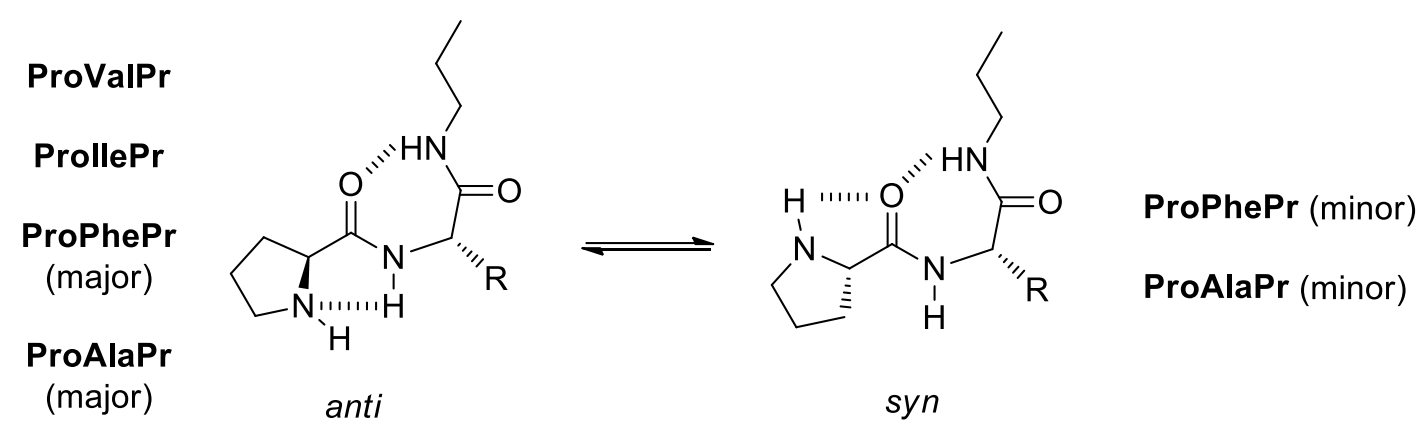

Scheme 3.2.2. Conformational equilibrium for the studied peptides in solution.

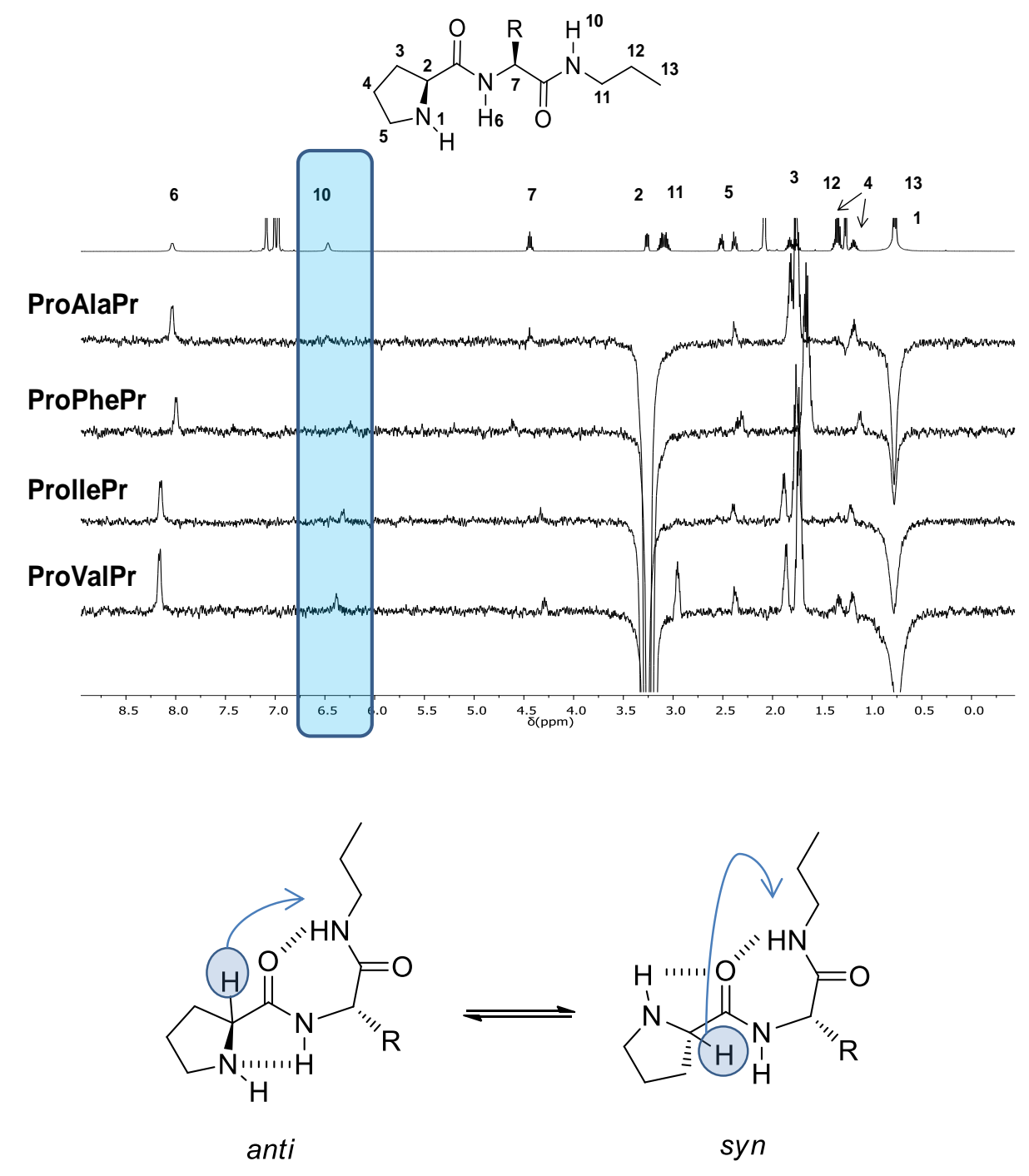

Figure 3.2.2.7. NOESY-1D spectra for all the compounds at $8 \mathrm{mM}$ in $D_{8}$-toluene at $30^{\circ} \mathrm{C}$ 
Finally NOE experiments were carried out for all the analogues studied in solution (see Figure 3.2.2.7). The NOE signal detected between the amide of the propylamide chain $\left(\mathrm{NH}_{\mathrm{a}}\right)$ and the proton of the chiral carbon of proline (position 2 in the caption) also agrees with the presence of anti-conformations in solution for all of them, which presents a shorter distance between these protons in the simulated models ( 3.8 and $4.5 \AA$ for antiand syn-dispositions respectively).

The existence of a fast exchanging syn-and anti-conformations for the alanine and phenylalanine derivatives has been suggested above. A possible explanation for this behaviour could be related with the steric demand of the diverse peptide side chain. On one hand, valine and isoleucine possess bulky groups in both the R and $\mathrm{R}^{\prime}$ positions (see Figure 3.2.2.8). However, alanine and phenylalanine can minimise steric interactions by rotating along the $\mathrm{C} \alpha-\mathrm{C} \beta$ bond. This reasoning explains that ProAlaPr (3a) and ProPhePr (3c) can be present in both syn- and anti-conformations whilst ProValPr (3b) and ProllePr (3d) present only an anti-conformation where the steric repulsion is avoided.

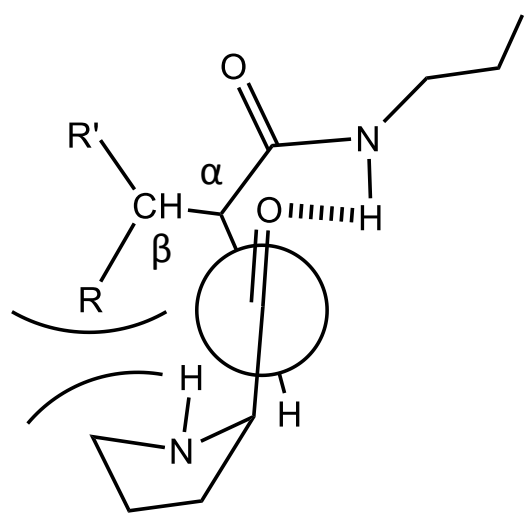

syn

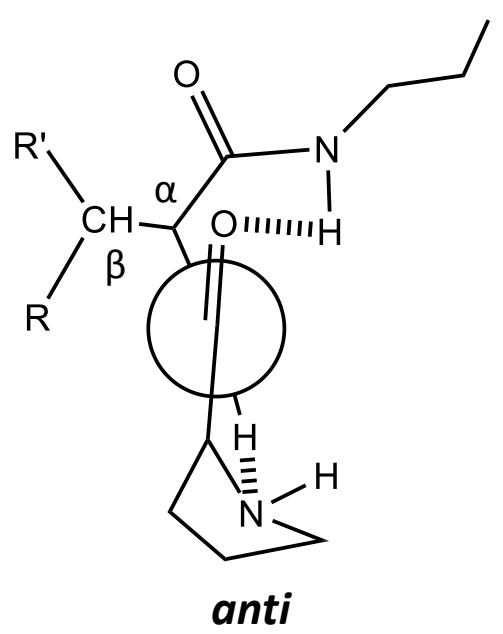

anti

\footnotetext{
ProAlaPr: $\mathrm{R}=\mathrm{H}, \mathrm{R}^{\prime}=\mathrm{CH}_{3}$

ProPhePr: $\mathrm{R}=\mathrm{H}, \mathrm{R}^{\prime}=\mathrm{Bn}$

ProllePr: $\mathrm{R}=\mathrm{CH}_{3}, \mathrm{R}^{\prime}=\mathrm{CH}_{3}-\mathrm{CH}_{2}$

ProValPr: $\mathrm{R}=\mathrm{CH}_{3}, \mathrm{R}^{\prime}=\mathrm{CH}_{3}$
}

Figure 3.2.2.8. Schematic Newman projection of anti- and syn-conformers found for the dipeptides. 


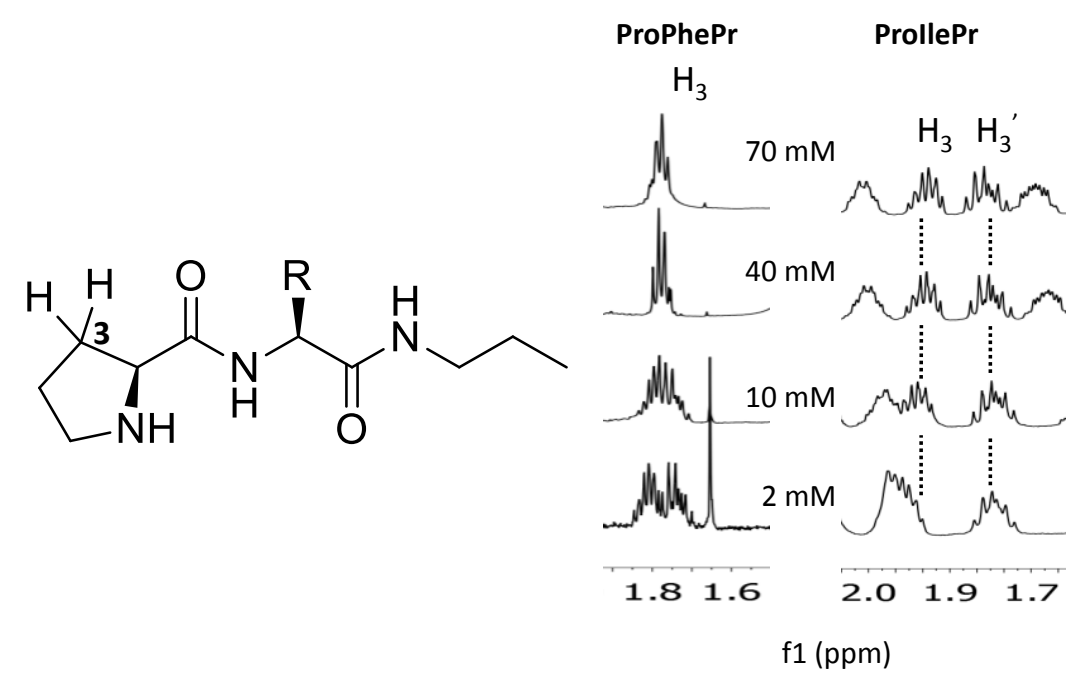

Figure 3.2.2.9. Partial ${ }^{1} \mathrm{H}$ NMR spectra of ProPhePr (3c) and ProllePr (3d) in $\mathrm{D}_{8}$-toluene at different concentrations showing the $\mathrm{H}_{3}$ protons.

To continue with this study, aggregated species were analysed using samples of concentration $70 \mathrm{mM}$ ( $c a .80 \%$ of aggregated species). Once again, here a series of NMR experiments were performed to test the structural preferences. Aside from the already observed shifting in the amide signals (Figure 3.2.2.1), indicative of intermolecular $\mathrm{H}$ bonding, it is noteworthy the evolution of the splitting of the geminal protons on the position 3 of the proline ring. Interestingly, a significant reduction of the splitting was found for the aggregated spectra of ProAlaPr (3a) and ProPhePr (3c) when compared with the diluted samples (see Figure 3.2.2.9). These results indicate a conformational change around the proline ring with the concentration associated with the supramolecular self-assembly process. This suggests a transition from anti- to synconformation upon aggregation. On the other hand, this behaviour was not shown for ProValPr (3b) and ProllePr (3d) (see Figure 3.2.2.9), where the splitting was maintained upon aggregation, indicating the absence of conformational changes during supramolecular self-assembly, as illustrated in the Scheme 3.2.2.2. 


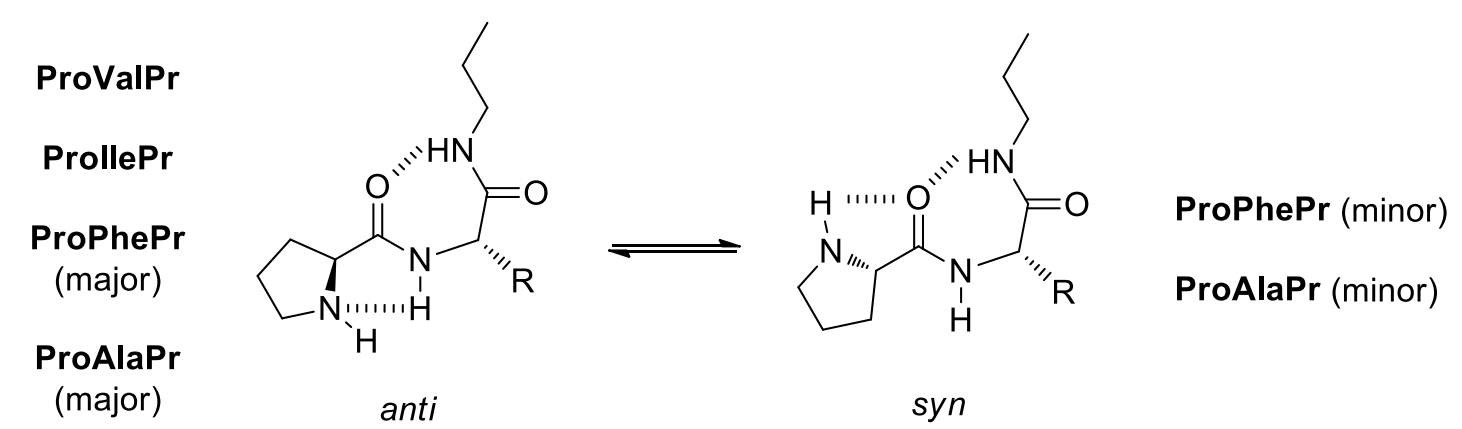

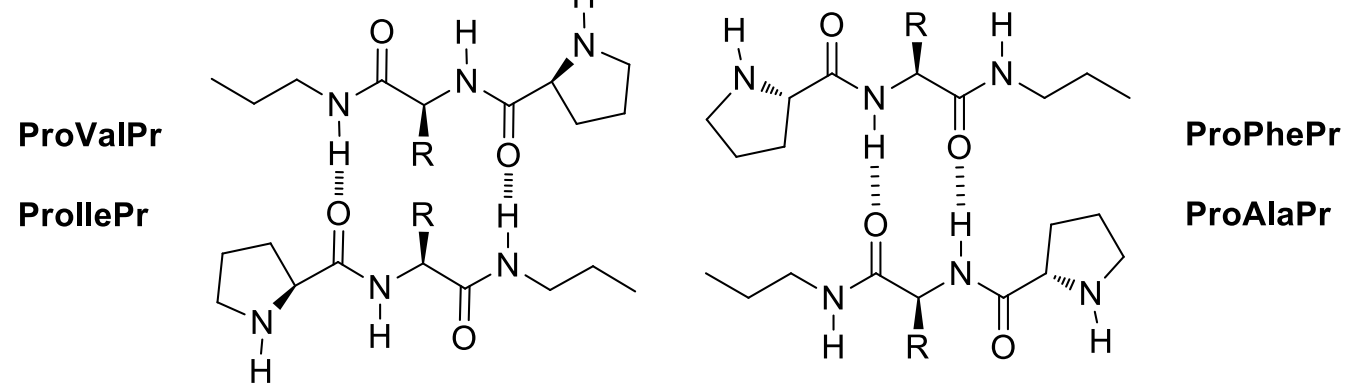

Scheme 3.2.2.2. Schematic representation of the conformational changes associated to aggregation.

Additionally, NOE experiments of the aggregated species showed a correlation between the protons of the propyl chain moiety and the ones on the proline ring. This could be explained with the formation of antiparallel aggregates (see Figure SI_3.5.8-9). In addition, molecular mechanics calculations (AMBER* force field, see Experimental Section) were performed. As a result, energy minimized models were obtained for the aggregated antiparallel dimers revealing several intermolecular hydrogen bonds. In these simulations the distance between the molecules supported the experimental NMR NOE data. The structures obtained for ProllePr (3d) in anti-conformation and ProAlaPr (3a) in syn-conformation are shown in Figure 3.2.2.10. 
A

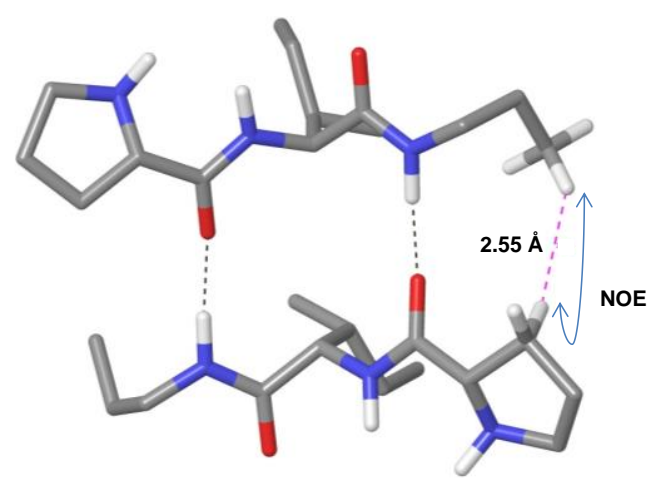

B

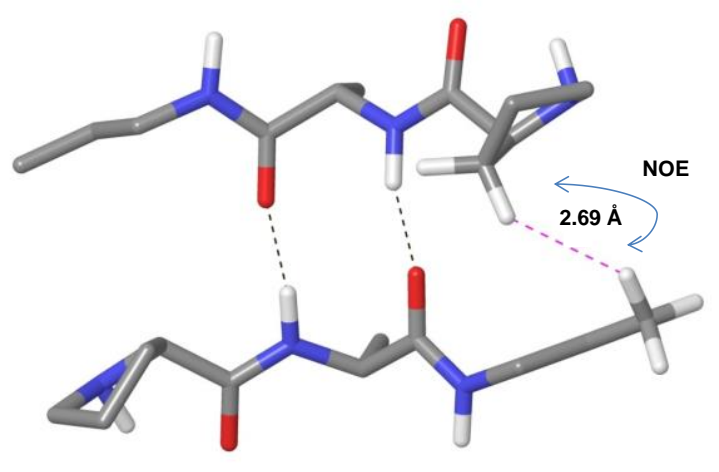

Figure 3.2.2.10. Molecular models for the dimers formed by $\operatorname{ProllePr}(A)$ and $\operatorname{ProAlaPr}(B)$ obtained from molecular mechanics calculations. Non polar hydrogen atoms have been omitted by means of clarity except those involved in NOE contacts.

\subsubsection{Catalytic Studies}

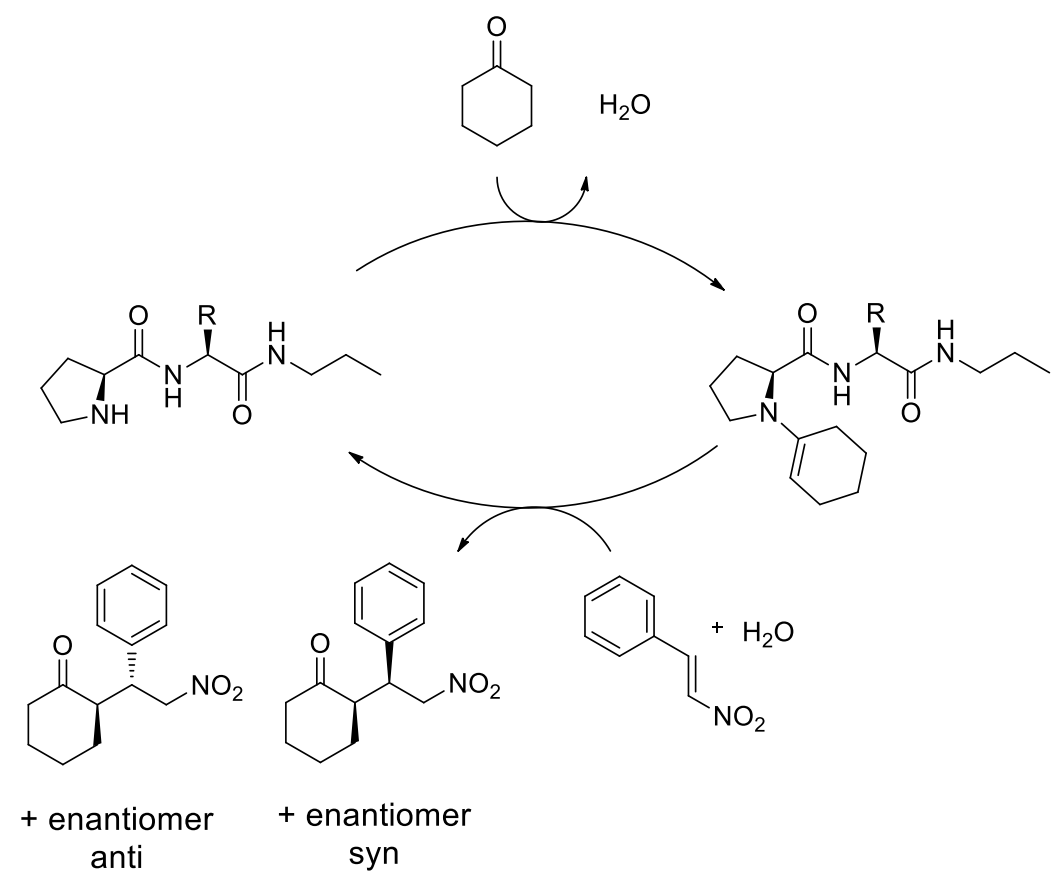

Scheme 3.2.3.1. Catalytic cycle for the conjugated addition of cyclohexanone to trans- $\beta$-nitrostyrene.

Once the structural studies of the different proline dipeptidic catalysts were accomplished, showing the conformational preferences of each molecule, a catalytic study was carried out in order to assess how these conformational preferences and 
aggregation behaviour can affect the catalytic activity. It has been demonstrated that a slight modification in the peptide backbone can influence significantly on the selfassembled structures, and thus, it can potentially affect in the same way their catalytic activity.

To accomplish this, all the catalyst molecules were tested in the conjugated addition of cyclohexanone to $\beta$-trans-nitrostyrene in toluene. It has been previously reported that an improvement of the catalytic performance was observed when didpeptidic structures were compared to a simple L-proline analogue. ${ }^{27}$ This reaction takes place via enamine intermediate as can be seen in Scheme 3.2.3.1 giving rise to synand anti-diastereoisomers and their enantiomers.

In a first test and with the aim to evaluate the effect of the catalyst aggregation, reaction rates were determined. The reaction was carried out at two different catalyst concentrations of $1 \mathrm{mM}$, and $5 \mathrm{mM}$ in which a significant amount of aggregated species can be found (ca. 25\%) (see Table 3.2.2.1). In all the cases the syn-diastereoisomer was the major product.

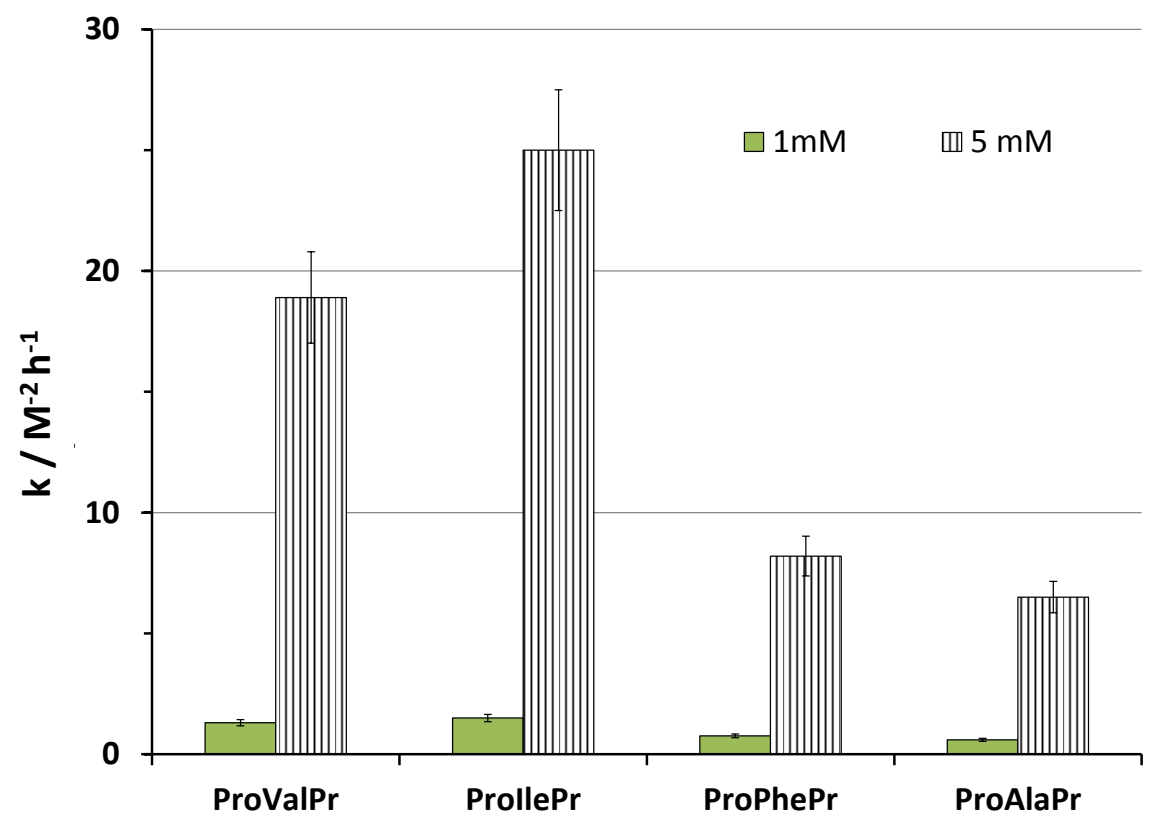

Figure 3.2.3.1. Kinetic constants ( $k$, see Equations_Sl_3.5-7) determined for different concentrations of catalyst in the addition of cyclohexanone to trans- $\beta$-nitrostyrene at $25 \stackrel{\circ}{\circ}$ in toluene. See details in Experimental Section and Supporting Information. 
To evaluate the reaction order, a catalytic reaction using ProllePr (3d) was monitored in situ at regular time intervals by NMR for ca. 60 hours and the data fitted well to a first order kinetic model (see Figure SI_3.5.12). For simplicity the system was analysed in terms of pseudo first order kinetics (see Equation_SI_3.5-7) carrying out the catalytic reaction in the presence of an excess of cyclohexanone.

The catalytic results are summarised in Table SI_3.5.1. Using reaction yields to calculate the alkene concentration, the values for the kinetic constant were obtained (Figure 3.2.3.1). A noticeable increase of the reaction activity can be seen from 1 to $5 \mathrm{mM}$ for all the cases, where the catalyst is almost inactive in diluted (non-aggregated) solutions (ca. 16-fold increase for ProllePr (3d), 10-fold for ProPhePr (3c) and 8-fold increase for ProAlaPr (3a) and ProValPr (3b)). These results point to catalyst activation upon increasing concentration and furthermore agree with the fact that aggregates are much more active than non-aggregated species (aggregation degree of $c a$. $4 \%$ for $1 \mathrm{mM}$ and $25 \%$ for $5 \mathrm{mM})$. A rational explanation for this behaviour might be that upon aggregation, the catalytic amino centre of proline ring is liberated from the intramolecular H-bonding, which prevents its nucleophilic activity (see Scheme 3.2.2.2).

Table 3.2.3.1. Enantioselectivity obtained in the addition of cyclohexanone to trans- $\beta$-nitrostyrene at $25 \stackrel{\circ}{\circ}$ in toluene. ${ }^{a}$

\begin{tabular}{cc}
\hline Catalyst & $\begin{array}{c}\text { Enantiomeric } \\
\text { excess } / \text { \% }^{\mathbf{b}}\end{array}$ \\
\hline ProAlaPr (3a) & 4 \\
ProValPr (3b) & 30 \\
ProPhePr (3c) & 8 \\
ProllePr (3d) & 29 \\
\hline
\end{tabular}

[a] [Cyclohexanone] $=110 \mathrm{mM}$, [trans- $\beta$-nitrostyrene $]=55 \mathrm{mM}$, [catalyst $]=10 \mathrm{mM}$. Reaction time $72 \mathrm{~h}$. [b] syn (2R, 1'S) enantiomer was the major product in all the cases.

In a second test, the catalytic reactions were carried out at $10 \mathrm{mM}$ concentration of catalyst with the aim to compare the enantioselectivity of each dipeptide. The enantiomeric excess results are shown in Table 3.2.3.1. As can be seen only moderate enantioselectivity was reached by the ProVaIPr (3b) or ProllePr (3d) (ca. 30 \% e.e. values), 
on the contrary, ProAlaPr (3a) and ProPhePr (3c) only afforded poor e.e. values (ca. $6 \%$ ). This small difference in the enantioselectivity might be explained by the different preferences for the self-assembling configuration, perhaps the anti-conformation (ProValPr (3b) and ProllePr (3d)) being more selective than the syn-conformation (ProAlaPr (3a) and ProPhePr (3c)). Additionally, enantioselectivity was also measured at different catalyst concentrations in the range from 5 to $30 \mathrm{mM}$ for ProllePr (3d) and ProPhePr (3c) catalysts (see Figure SI_3.5.13). The results showed that the enantioselecivity was maintained for all the concentration values studied in both cases suggesting that upon aggregation the same disposition of the catalytic unit is maintained.

\subsection{L-Proline Derived Bolaamphiphiles: Gel-Phase Study of the Organocatalytic Performance}

\subsubsection{Characterisation of Gels in Toluene}

With the aim to evaluate the different effects on the election of the assembling amino acid on its conformational preference in solution, the aggregation behaviour and its catalytic activity, a small screening study was previously performed in the above section. Using L-proline dipeptide derivatives, among four different amino acids, ProValPr (3b) and ProllePr (3d) structures have been found to show better catalytic properties.

The bolaamphiphilic molecule ProVal6 (see Figure 3.1.2.1.5) has been employed as a good organogelators and remarkable catalytic results were found when was used as a self-assembled gel-phase organocatalyst. This molecule can be thought to be as an analogue of the soluble compound ProValPr (3b). Following the same hypothesis and the modular bottom-up approach, which combines an assembling fragment with a functional fragment (see Figure 3.3.1.1), the bolaamphiphilic molecule Prolle6 (3e) was designed to generate an organogelator that would present enhanced catalytic activity. 


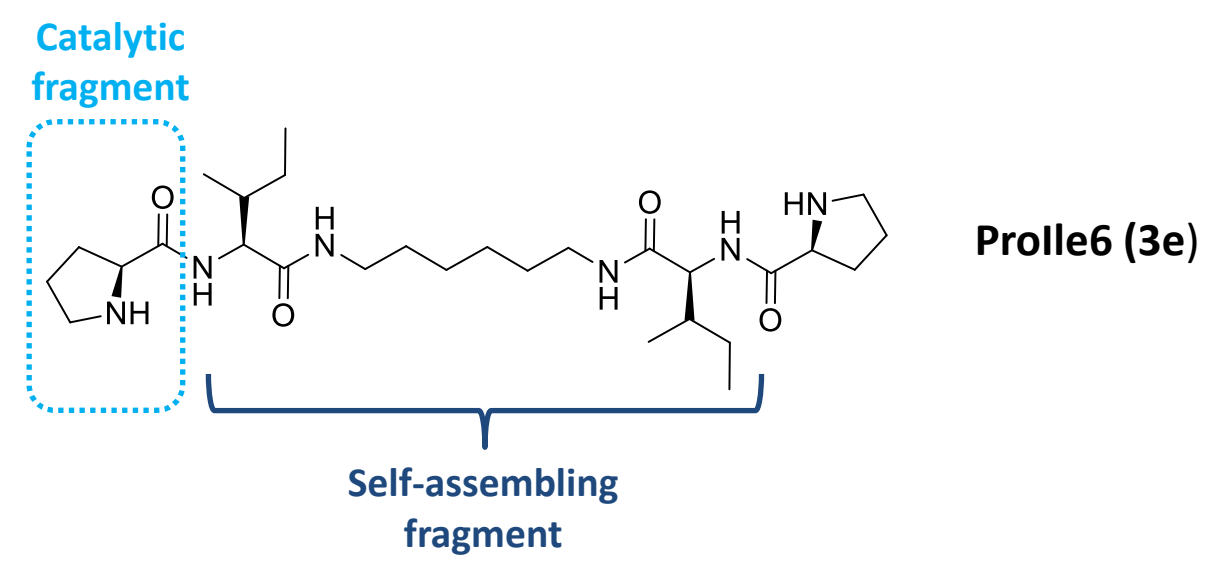

Figure 3.3.1.1. Molecular structure of the compound Prolle6 (3e).

The preparation of compound Prolle6 (3e) was carried out in an efficient manner following the schematic procedures described for peptide synthesis in solution (see Scheme 3.3.1.1). In this case the 1,6-hexanediamine was employed to give the desired bolaamphiphilic molecule in grams scale and with good yield.
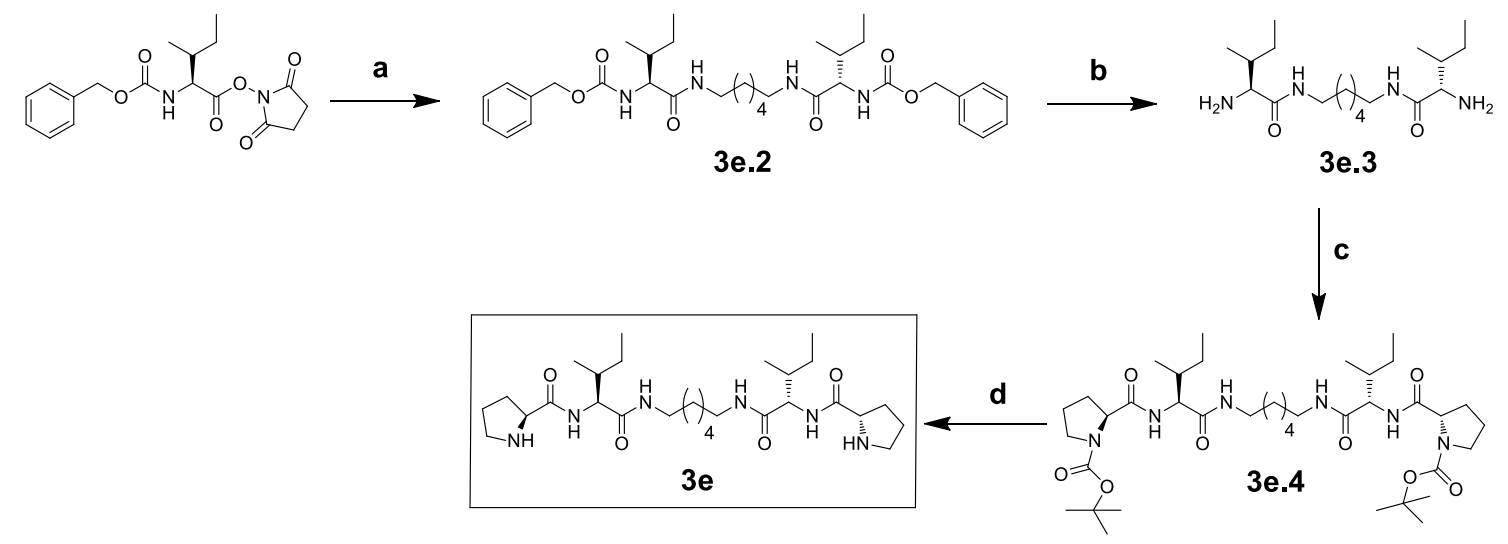

Scheme 3.3.1.1. Schematic synthesis a) 1,6-Hexanediamine, DME, rt (18 h) 50 ㅇ $\mathrm{C}(2 \mathrm{~h})(85 \%) ;$ b) $\mathrm{H}_{2}, \mathrm{Pd} / \mathrm{C}$, $\mathrm{MeOH}, \mathrm{rt} 4 \mathrm{~h}$ (96\%); c) Boc-L-Pro-Osu, DME, rt (24 h) 40 ㅇ (5 h)(95\%); d) TFA/ $\mathrm{CH}_{2} \mathrm{Cl}_{2}$, rt (2 h), $\mathrm{NaOH}(68 \%)$.

As can be seen in Figure 3.3.1.1, the catalyst possesses two polar heads connected through a hydrophobic spacer. It was found that Prolle6 (3e) was able to form molecular gels in toluene resulting in transparent gels as those shown in Figure 3.3.1.2. A study of the gelation properties at macroscopic and microscopic levels was performed. The gelation ability of Prolle6 (3e) in toluene was assessed determining the minimum gelation concentration (MGC) at room temperature. Gels were obtained by following a standard gelation procedure: complete dissolution of the compound in the solvent by gentle 
heating and then cooling down at room temperature. The gels were stabilised during 24 hours (see Experimental Section). Samples with different concentration were prepared and their gelation properties were evaluated by the well-known tube inversion method. A MGC of $7 \mathrm{mM}$ was found after leaving the samples to stabilise overnight. This value follows the trend observed for this kind of molecule as it is similar to the one found for ProVal6 (7 mM) or ProVal8 (11 mM) under the same conditions.

A)<smiles>CCC(C)C(NC(=O)[C@@H]1CCCN1)C(=O)NCCCCCCNC(=O)[C@@H](NC(=O)[C@@H]1CCCN1)C(C)CC</smiles>

B)

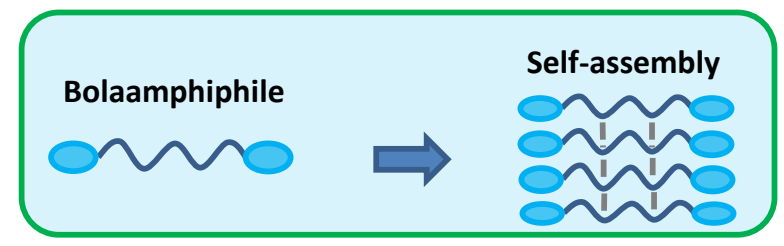

C)

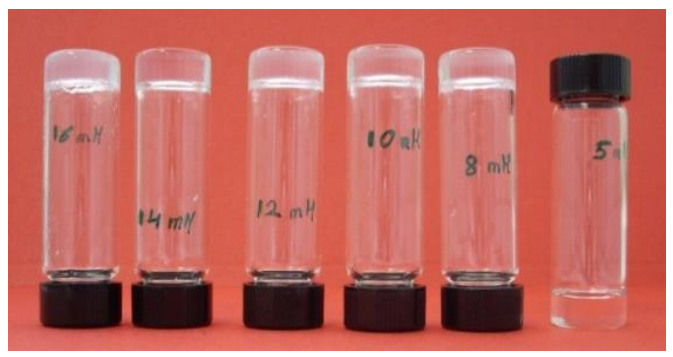

Figure 3.3.1.2. A) Molecular structure of the compound Prolle6 (3e), B) Illustrative representation of bolaamphiphiles and a proposed model for its aggregation and C) Macroscopic aspect of the gels during the determination of the MGC.

Then the analysis of the morphological aspect of the fibrillar network was performed. Transmission electron microscopy (TEM) images of the organogel revealed long twisted fibrils (diameter ca. $20 \mathrm{~nm}$ ) as part of a homogenous and entangled tight network (see Figure 3.3.1.3).
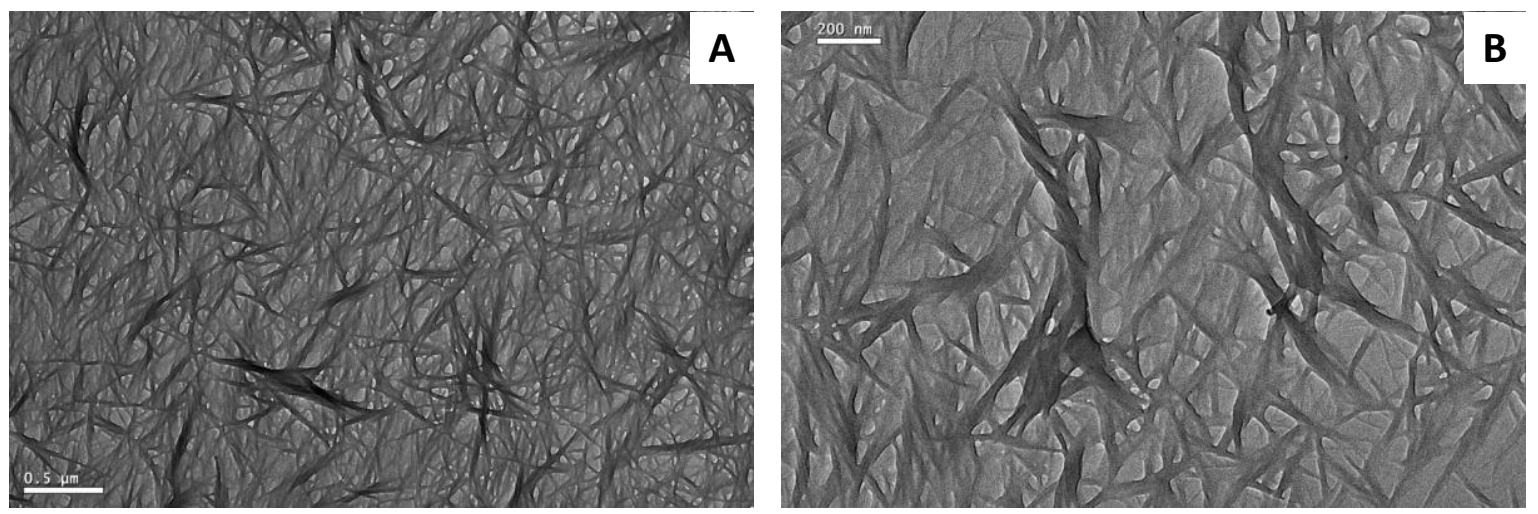

Figure 3.3.1.3. TEM images of Prolle6 (3e) (16 mM in toluene). 
The determination of the gel melting temperatures $\left(T_{\text {gel }}\right)$ using the inversion tube method allows to known the thermal stability of the gel at different gel concentrations. The results showed a gel disassembly $T_{\text {gel }}$ profile in which an increase with the concentration is found ${ }^{32}$ with a maximum $\mathrm{T}_{\text {gel }}$ value of $120 \stackrel{\circ}{ }$, close to the boiling point of the solvent (see Figure 3.3.1.4).

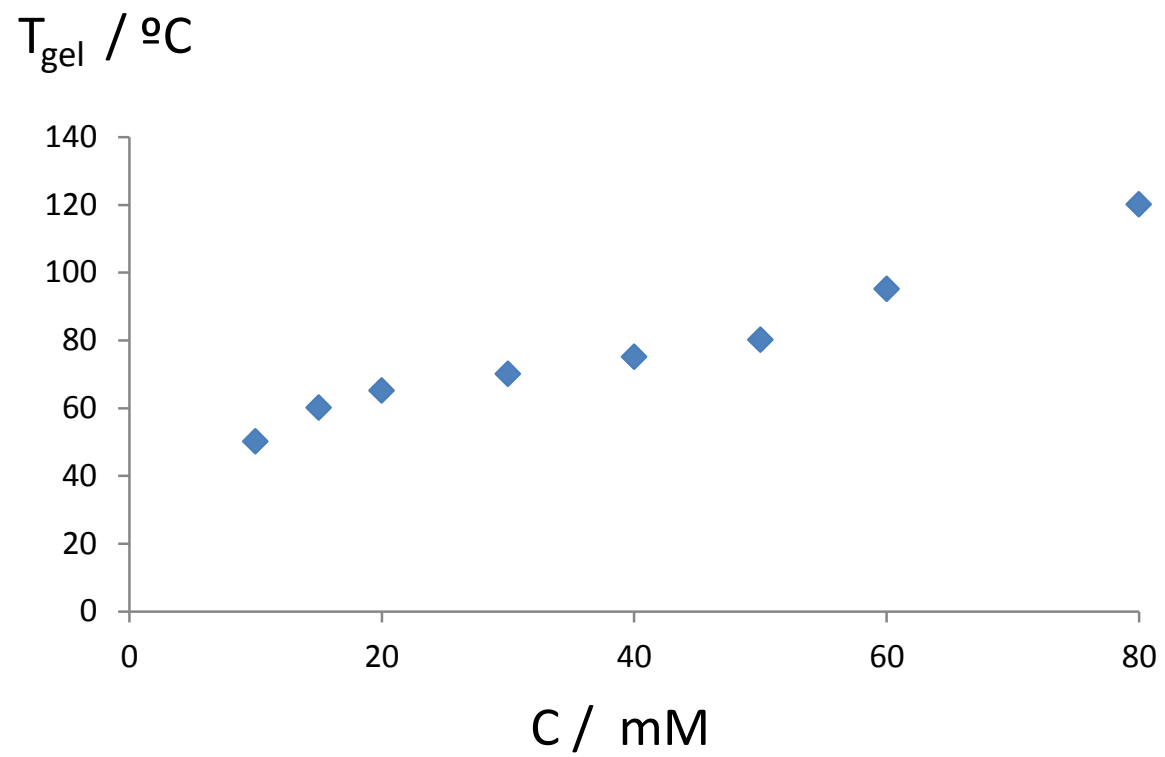

Figure 3.3.1.4. Thermal stability of Prolle6 (3e) gel at different concentrations in toluene.

It is known that supramolecular gels are dynamic systems where an equilibrium is established between monomers (or oligomers) and the solid-like aggregates that constitute the fibrillar network. This equilibrium is governed by a solubility constant $\left(K_{s}\right)$ (see Figure 3.3.1.5). Spectroscopic techniques, such NMR, are potential tools to analyse and study solubility and have been used before to characterise these kinds of systems. ${ }^{32-}$ ${ }^{33}$ In the conventional NMR technique only monomers or small oligomers with suitable relaxation times are observable allowing them to be monitored. On the contrary, large aggregates are NMR silent, as they present a long correlation time (reduced transversal relaxation time $\mathrm{T}_{2}$ ), similar to large macromolecules or proteins. 


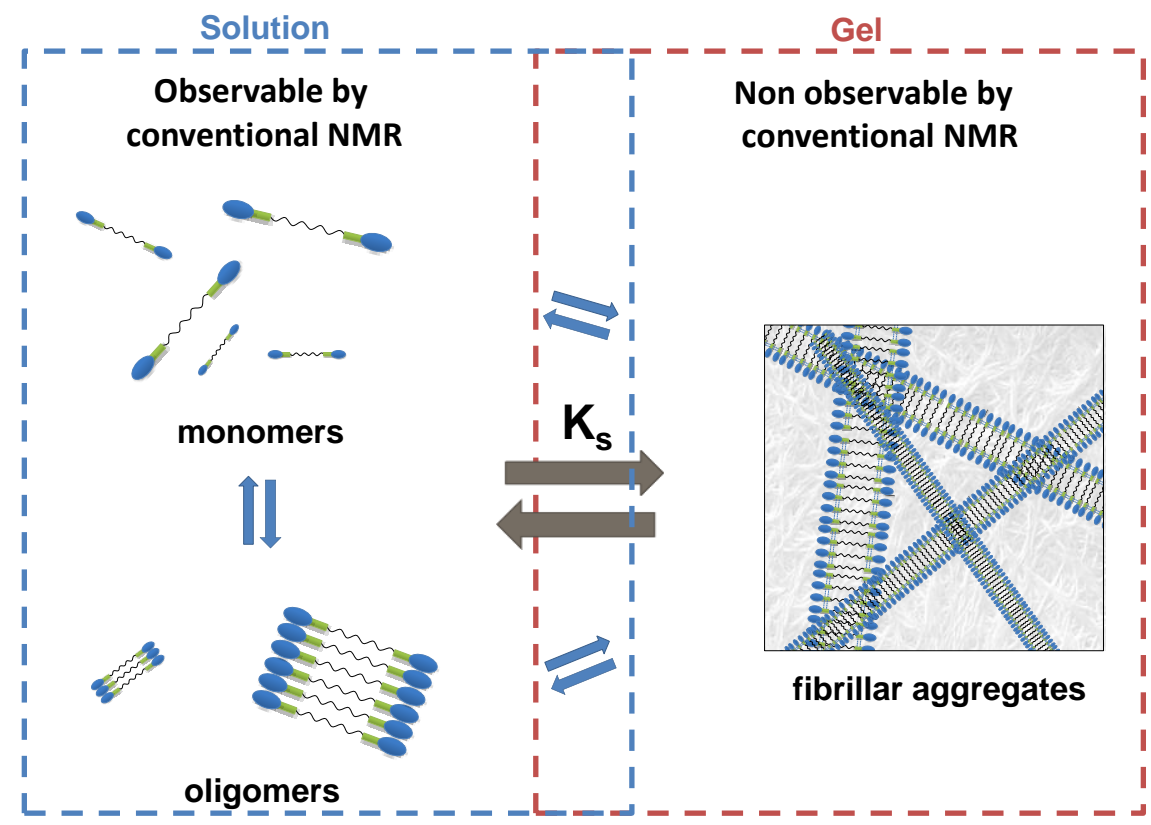

Figure 3.3.1.5. Schematic representation of the dynamic equilibrium between solution and aggregates that takes place in supramolecular fibrillar gels. ${ }^{34}$

In the studied system, $K_{s}$ at room temperature was easily determined by evaluation of the intensity of the NMR signals for different samples with varying concentrations of Prolle6 (3e) in toluene (see Figure 3.3.1.6.A and Experimental Section for procedure). As it can be seen in the graph, at low concentrations $(<M G C)$ the trend follows a straight line (red line) with slope equal to one, being the observed concentration equal to the real one. This result is in agreement with the previous hypothesis indicating that all the material is visible under NMR for samples in solution (non-aggregated). After the MGC, this linear trend is broken and the curve diverges upon increasing concentration until a plateau is reached, suggesting that non-observable NMR species are being formed. At this point all the material added to the sample is incorporated to the fibrillar network and remains NMR silent. The portion of material that is still in solution is the solubility constant $\left(K_{s}\right)$ at a given temperature. In this case for Prolle6 (3e) in toluene the $K_{s}$ found was ca. $2 \mathrm{mM}$ at $30 \stackrel{\circ}{ }$. This value is similar to those found in previous work for molecules such as ProVal6 or ProVal8 in organic solvents ( 3 and $9 \mathrm{mM}$ respectively). 


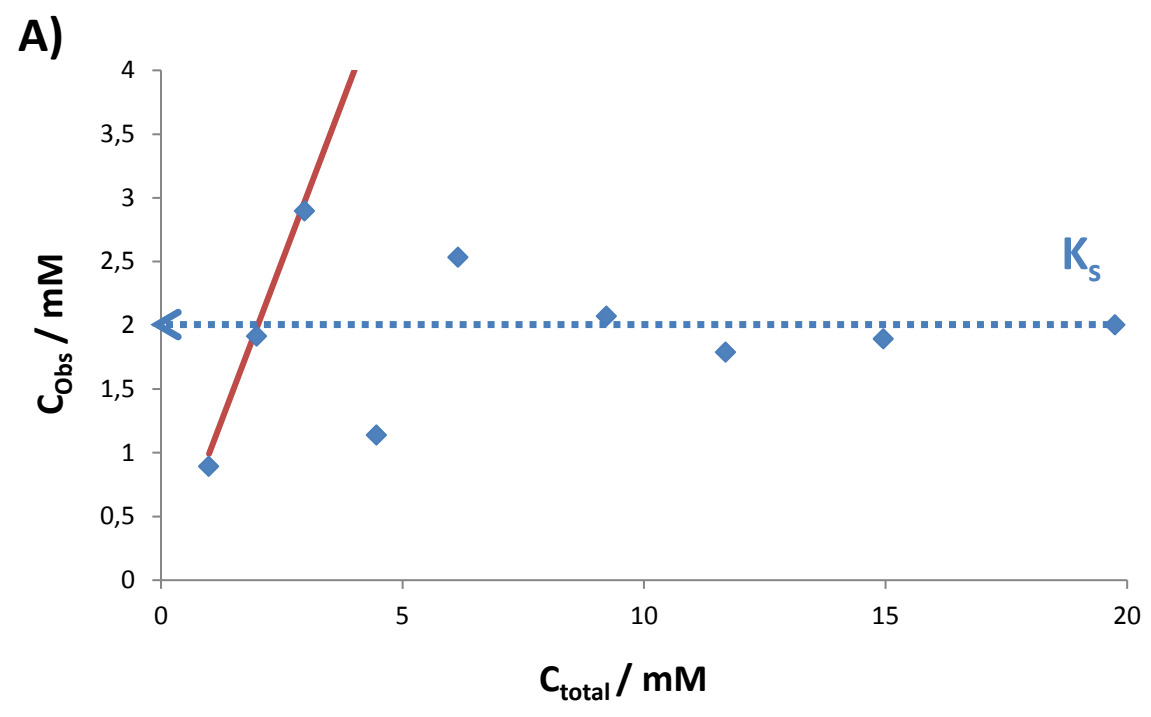

B)

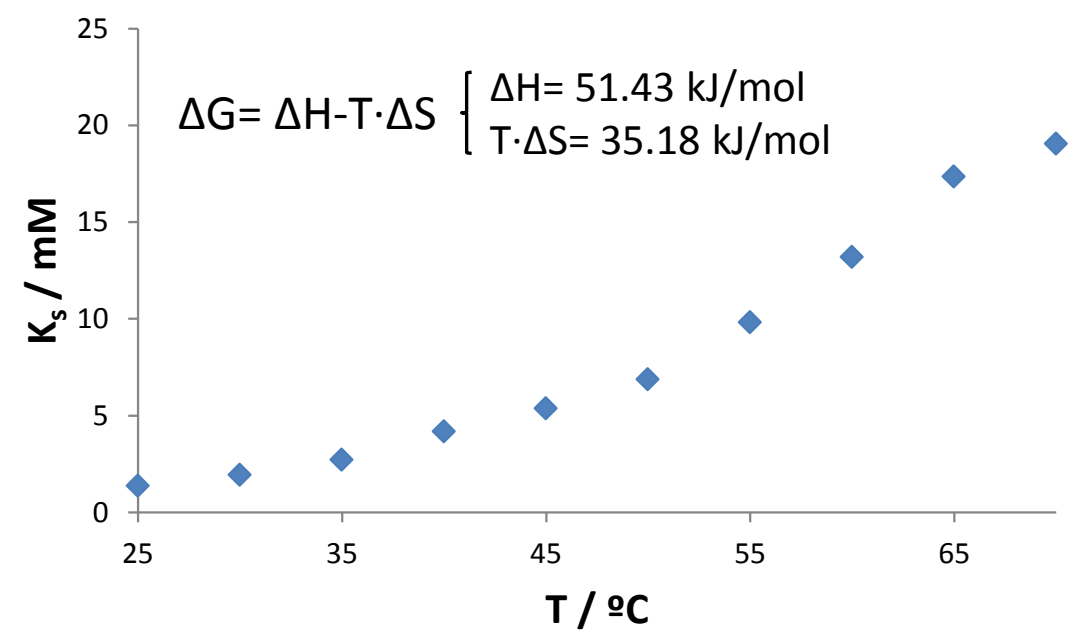

Figure 3.3.1.6. A) Plot of the concentration measured using 1,1,2,2-tetrachloroethane as internal standard against the real concentration calculated by weigh of the samples. The red line indicates case where the observed concentration would be equal to the total concentration and the arrow indicates the $\mathrm{K}_{\mathrm{s}}$ observed. B) Temperature dependence of the solubility data and thermodynamic parameters associated for gel-sol transition in a $20 \mathrm{mM}$ sample at $25 \stackrel{\circ}{\circ} \mathrm{C} . \mathrm{K}_{\mathrm{s}}$ data were obtained from ${ }^{1} \mathrm{H}-\mathrm{NMR}$ analysis.

Then, the variation of the solubility of the self-assembled network with temperature was evaluated. In this case, the integration of the visible NMR signal (nonaggregated) was analysed at different temperatures using an internal standard on a gel sample (ca. $20 \mathrm{mM}$ ). As before, the aggregated species remain NMR-silent and the observable concentration of species is the $\mathrm{K}_{\mathrm{s}}$ value for each different temperature (see Figure 3.3.1.6.B). A higher solubility was observed upon increasing the temperature of the 
gel with an exponential relationship as shown in Figure SI_3.5.14. Thermodynamic parameters were obtained from van't Hoff analysis of the solubility against temperature data (see Figure 3.3.1.6.B). The solubilisation of the fibrillar network resulted in positive values for the enthalpy change (unfavourable) and entropy change (favourable) in agreement with an increment of the degrees of freedom in a gel to sol change.

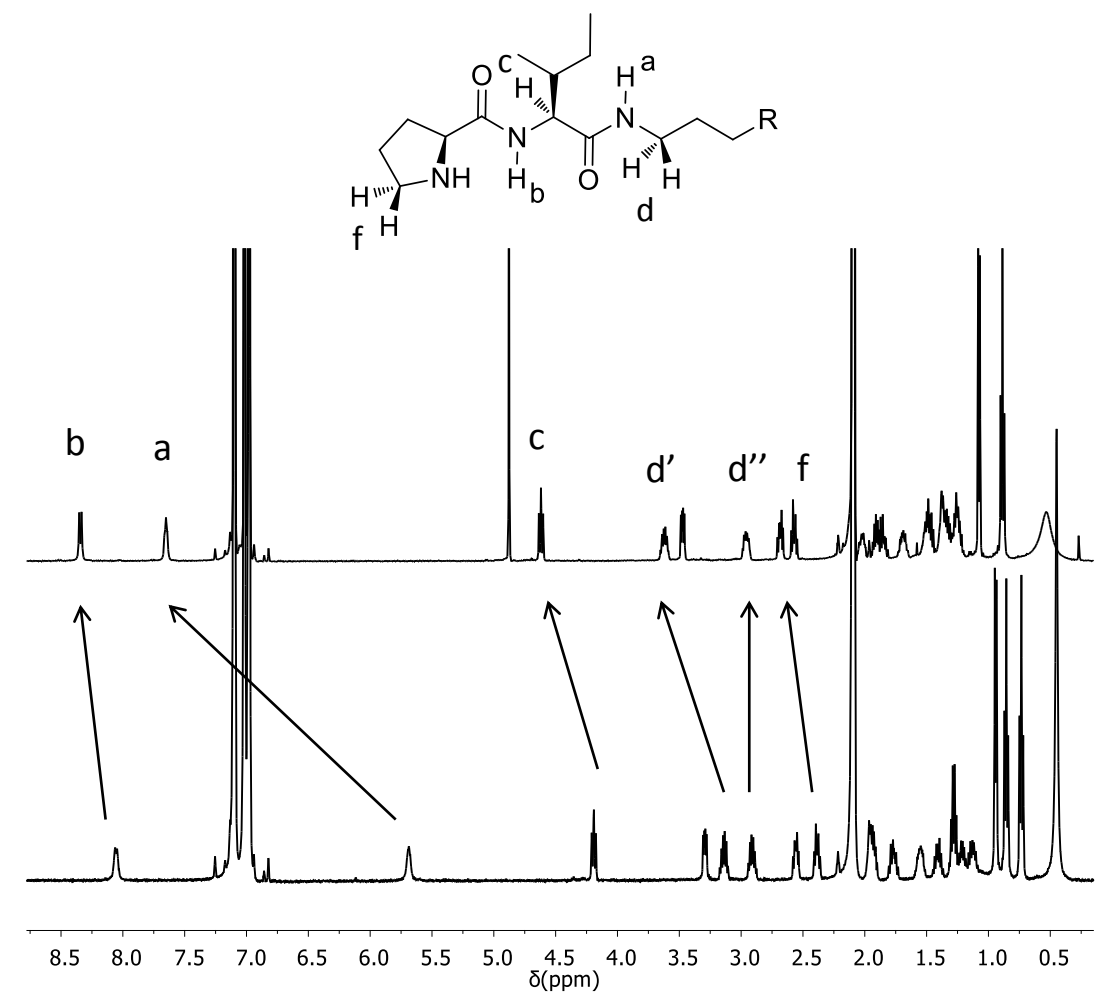

Figure 3.3.1.7. Differences found in the ${ }^{1} \mathrm{H}$-NMR signals of the Prolle6 (3e) (top) and ProllePr (3d) (bottom) $2 \mathrm{mM}$ in $\mathrm{D}_{8}$-toluene. (The signal at $4.9 \mathrm{ppm}$ belongs to the internal standard 1,1,2,2-tetrachloroethane in the top spectrum).

Finally, the self-assembly process was studied to give some insight into how the Prolle6 (3e) molecular structure is packed in the gel state and to propose a possible model of aggregation. To achieve this objective, NMR experiments were performed with detail as it has been done for the molecules in solution. ${ }^{1} \mathrm{H}-\mathrm{NMR}$ spectra of Prolle6 (3e) (bolaamphiphile) and ProllePr (3d) (soluble amphiphile analogue) in diluted solution are compared in Figure 3.3.1.7. Surprisingly, the chemical shifts of both amide signals are shifted downfield. Previously, the involvement of these amides in the self-assembly by means of $\mathrm{H}$-bonding was demonstrated for the compound ProllePr (3d). In addition, the presence of an intramolecular $\mathrm{H}$-bond between L-proline amine and the amide adjacent 
to the proline ring $\left(\mathrm{NH}_{\mathrm{b}}\right)$ was proven. Noticeably, for Prolle6 (3e), the amide signal chemical shift values suggest that not only the amide $\mathrm{NH}_{\mathrm{b}}$ but also $\mathrm{NH}_{\mathrm{a}}$ are involved in intramolecular bonds associated to the folding of the molecule in solution (<MGC). Moreover, the shifts on the signals $\mathbf{c}$, $\mathbf{d}$ and $\mathbf{f}$ suggest a change on the conformational preference of the folded structure.<smiles>CCC(C)[C@H](C(=O)NCCCCCCN(C)C(=O)[C@H](NC(=O)[C@H]1CCCN1)[C@H](C)CC)N(C)C(=O)C1CCCN1</smiles>

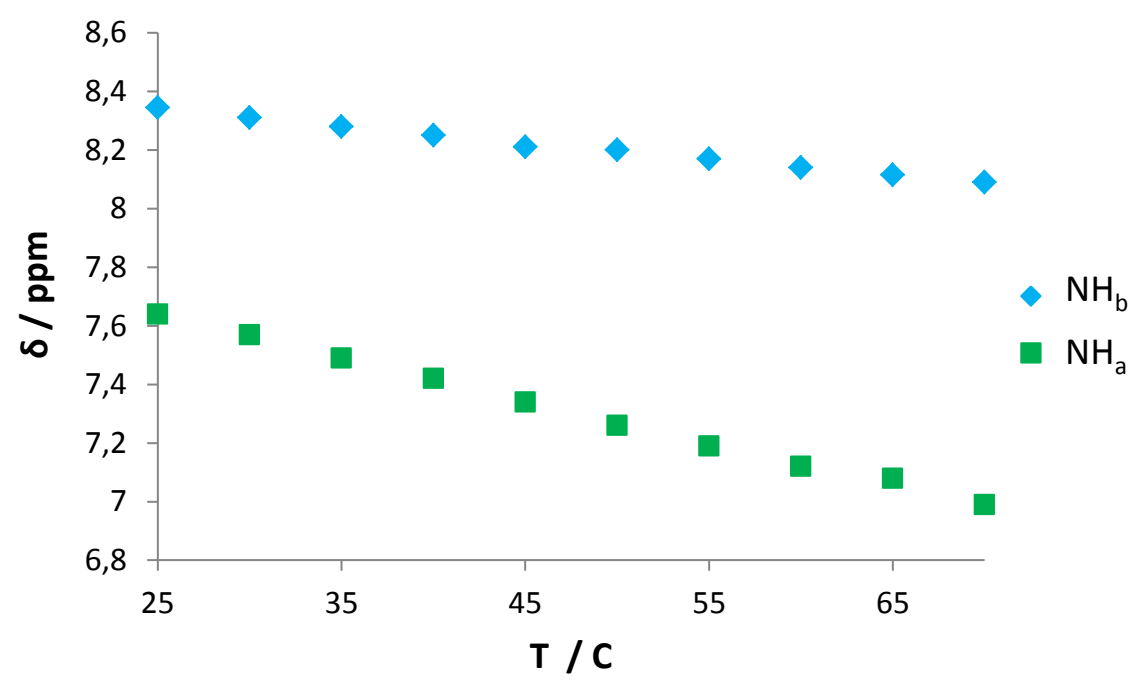

Figure 3.3.1.8. Amide signals shift upon increasing the temperature of $1 \mathrm{mM}$ Prolle6 (3e) in $\mathrm{D}_{8}$-toluene.

NMR studies monitoring the amide signals with the temperature were performed in absence of aggregation (diluted samples). As can be seen in Figure 3.3.1.8, both amide signals revealed a variation upon increasing the temperature from $25 \stackrel{\circ}{ } \mathrm{C}$ to $70 \stackrel{\circ}{ } \mathrm{C}$. This result is in agreement with the presence of intramolecular $\mathrm{H}$-bonds via the two amides when the molecule is in solution. 

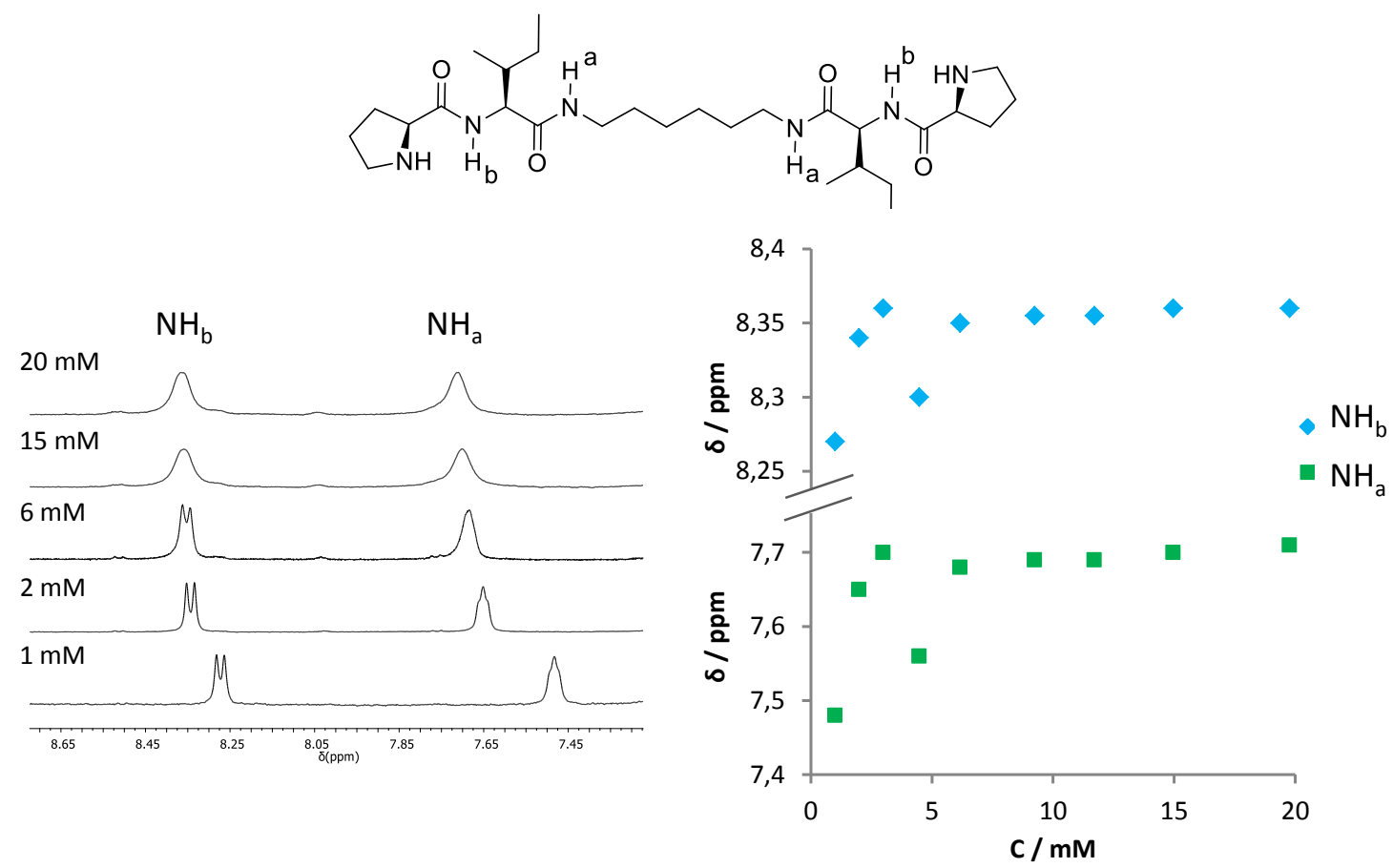

Figure 3.3.1.9. NMR Prolle6 (3e) data in $\mathrm{D}_{8}$-toluene at different concentrations. Partial ${ }^{1} \mathrm{H}-\mathrm{NMR}$ spectra showing the amide signals (left) and representation of the experimental data (right).

Once the involvement of the amides in the folding of the structure in diluted samples via intramolecular bonds has been checked, the self-assembly of the molecule was studied by monitoring the chemical shift of these signals with varying concentration. Figure 3.3.1.9 (left) shows the variation of the Prolle6 (3e) amide chemical shift in toluene, a slight shift downfield can be seen at very small concentrations (<MGC). As the concentration was increased the amide signal chemical shift remained unaltered. In Figure 3.3.1.9 (right), these two different behaviours can be perceived more clearly. These results can be explained by the fact that once the gel is formed, the added molecules remain NMR-silent (as was mentioned before), and only the molecules in solution are NMR-observable. Another observation that points to the formation of aggregates and the gel-phase is the broadening of the signals that appears when the gel is present as a result of an exchange with the fibrillar network (Figure SI_3.5.15).

To conclude, molecular mechanics calculations (AMBER* force filed, see Experimental Section) were carried out for Prolle6 (3e). The models corresponding to the energy minima obtained for both diluted and aggregated states are shown in Figure 3.3.1.10. The model proposed for a diluted sample (non-aggregated state) shows a folded 
structure by means of intramolecular $\mathrm{H}$-bond interactions. This model is in agreement with the experimental data where both amides are involved in intramolecular H-bonds. On the other hand, upon aggregation, the minimised model found showed conformational changes (folded to extended conformation) yielding a stacked onedimensional structure with several intermolecular $\mathrm{H}$-bonds between amides from neighbouring molecules, forming the gel-phase material. In this aggregated conformation L-proline residues are oriented outwards with the nitrogen atom preserving its nucleophilic properties associated to the catalytic activity (see Figure SI_3.5.16).

(A)

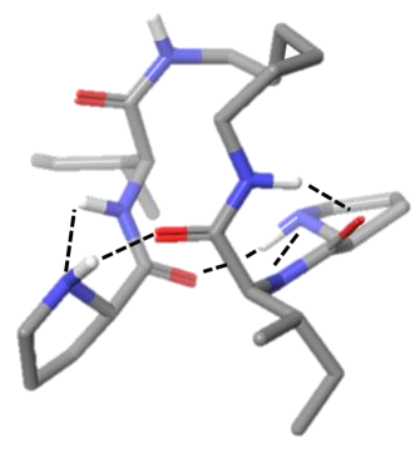

(B)

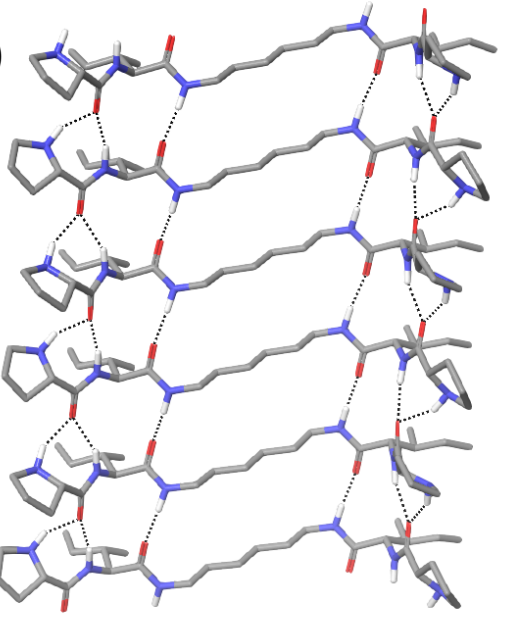

(C)

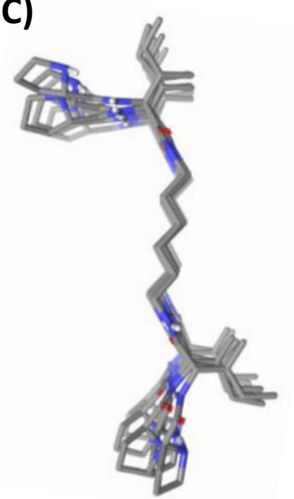

Figure 3.3.1.10. Molecular models for Prolle6 (3e) in solution (A) and forming aggregates in a hexamer 1D stacked network (B and C). Non polar hydrogens have been omitted for clarity.

\subsubsection{Proline Organogels as Catalysts for the Michael Reaction in Toluene}

The final aim of this work was the study of Prolle6 (3e) as an efficient organocatalyst in gel-phase. It was hypothesised that the highly organised self-assembled structures of the catalyst along the fibres would result in an enhancement of the catalytic properties when they are compared with the soluble counterpart. To assess the catalytic activity, 1,4-conjugated addition of cyclohexanone to trans- $\beta$-nitrostyrene has been studied in the gel system in toluene. It has been described before for soluble L-proline derivatives (see Figure 3.2.1), that the reaction takes place via an enamine-type intermediate. The catalytic reaction is illustrated in Scheme 3.2.3.1, with the syndiastereoisomers depicted, as they were the major product for the addition reaction in all 
the cases. The gel was prepared solubilising the gelator molecule by gentle heating in the chosen solvent (toluene) and left to cool to room temperature. Once the gel was obtained and stabilised, a solution of the reagents in toluene was added on top of the gel and left to diffuse into the gel network. After the corresponding reaction time, the gel was disassembled by the addition of acidic water and toluene. In this way the protonated catalyst was pulled apart while the reaction products could be isolated from the organic phase, as illustrated in Figure 3.3.2.1, and analysed.

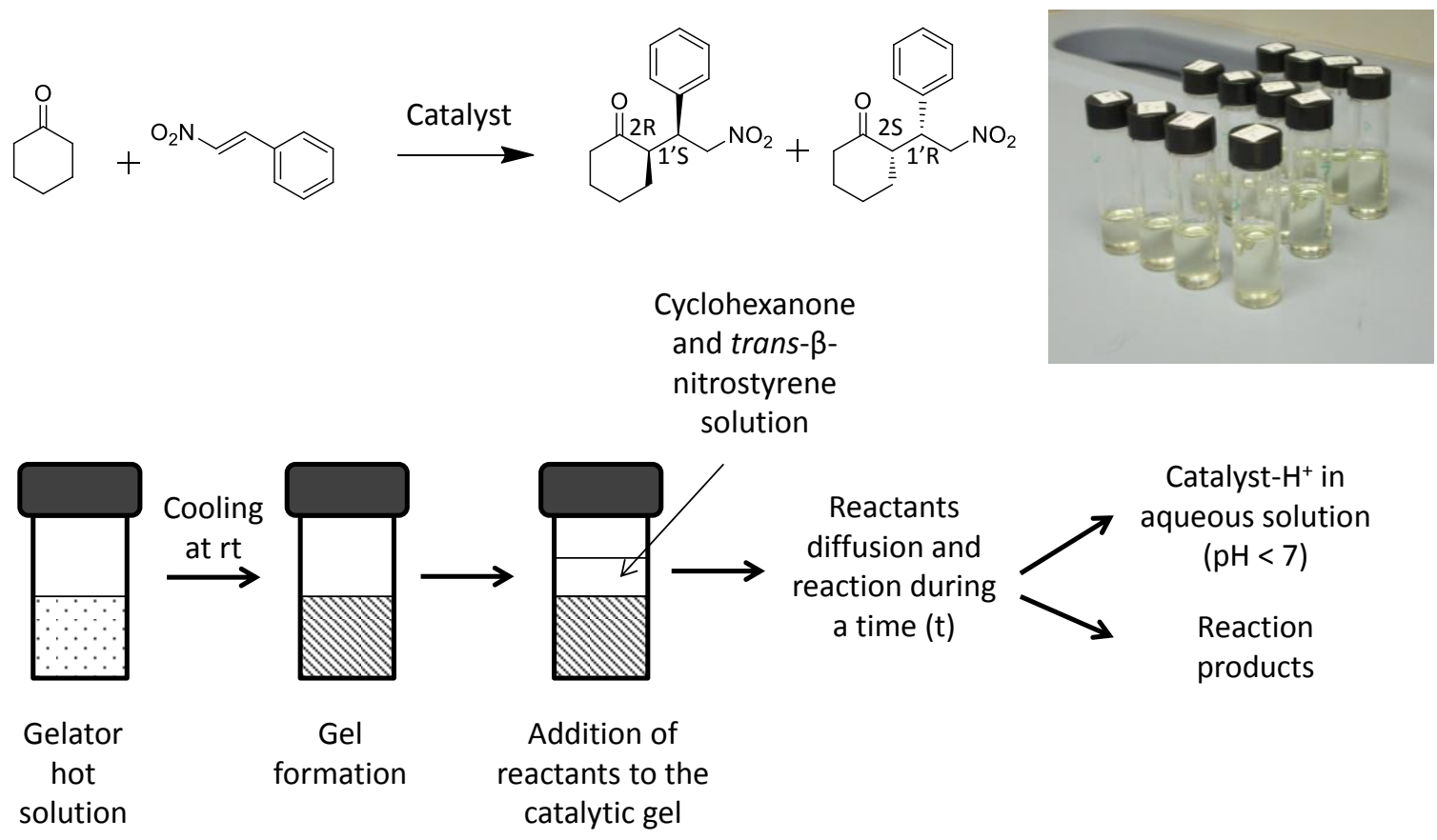

Figure 3.3.2.1. (top) The 1,4-conjugated addition of the cyclohexanone to trans- $\beta$-nitrostyrene used as benchmark reaction to test the catalytic activity showing the syn diastereoisomer as the main product. (bottom) Schematic representation of the procedure followed during the catalysis in gel-phase and a digital photograph with some examples.

Firstly, the evaluation of the gel-phase as catalyst was tested. In order to prove that the catalytic reaction was carried out by the fibres (aggregated species), and not by the molecules of gelator that are in solution in the pools of solvent, the catalytic reaction was performed with different catalyst concentration values in the gel-phase and keeping constant the concentration of the reagents (using different catalyst/reagent ratios). As can be seen in Figure SI_3.5.17, upon going from 10 mol \% to 80 mol \% of proline catalyst (compared with the olefin reagent) the yield obtained increased from $c a .46 \%$ to an almost quantitative conversion ( $c a$. 98\%). These results suggest that the reaction is 
performed in the gel, as the amount of catalyst in the solution remains constant as determined by the solubility constant ( $\mathrm{K}_{\mathrm{s}}$ for Prolle6 (3e) ca. $2 \mathrm{mM}$ ).

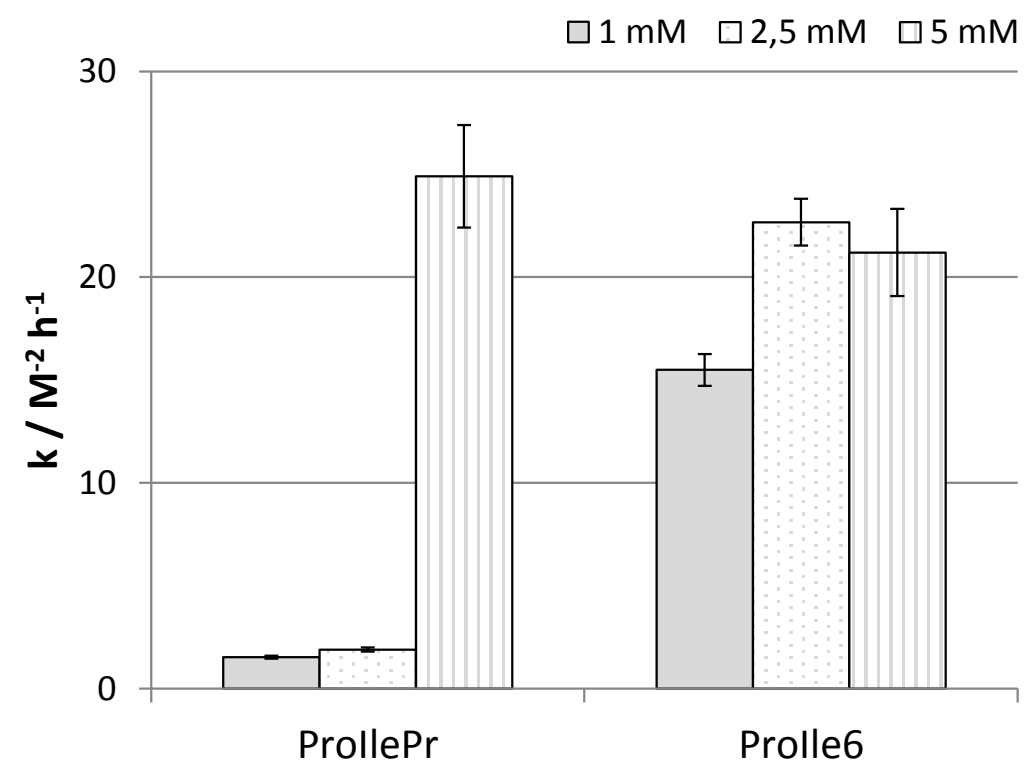

Figure 3.3.2.2. Kinetic constants ( $k$, see Equations_SI_3.5-7) determined for a set of different catalyst concentration samples on the addition of cyclohexanone to trans- $\beta$-nitrostyrene at $25{ }^{\circ} \mathrm{C}$ in toluene. See Table SI_3.5.2 and Supporting Information for more details. Concentration of catalyst in proline equivalents.

To evaluate the catalytic activity of the aggregated bolaamphiphilic species, reaction rates were studied with three different catalyst concentrations. The results were compared with the soluble analogue ProllePr (3d) studied before and are represented in the Figure 3.3.2.2. The system was analysed in terms of pseudo first order kinetics. For the purpose of comparison with soluble analogues, catalyst concentration was considered to be twice that of the bolaamphiphilic compound (two proline units in every gelator molecule). Notably, it was observed that for diluted samples (1 mM and $2.5 \mathrm{mM}$ of catalytic units) the less soluble compound Prolle6 (3e) presented better catalytic activity. This is in agreement with the fact that being less soluble it would form the active aggregated species at a lower concentration. Upon increasing catalyst concentration up to $5 \mathrm{mM}$, the kinetic constant increased considerably for ProllePr (3d) (as seen before, almost 15 fold) and this value was maintained for the bolaamphiphilic gelator. These results indicate that there is no significant loss of the catalytic efficiency when the solid 
species (aggregated fibres) appear. The catalytic proline units are still active after aggregation of the molecule being accessible to react on the fibre surface of the gel.

A comparative study of the catalytic activity of several similar catalysts (in solution and in aggregated gel-phase state) was carried out and summarised in Table 3.3.2.1. In this table, the participation of the second amino acid and the aggregation effect can be analysed. After 3 days of reaction, almost all catalysts presented quantitative conversions, with the exception of the proline amide analogue ProPr, a soluble proline derivative which does not possess an extra amino acid and thus presents more solubility and less aggregation properties. The reactions showed moderate to low enantioselectivity in all the cases. Interestingly, the enantioselectivity was improved with the gels that presented a second amino acid (Prolle6 (3e) and ProVal6 see Figure 3.1.2.1.5) when compared to the soluble analogue ProllePr (3d) suggesting a better arrangement of catalytic units in the aggregated species. Surprisingly, in absence of the second amino acid (ProPr) the main enantiomer was the $\left(2 S, 1^{\prime} R\right)$ instead of $\left(2 R, 1^{\prime} S\right)$ which was major in all the previous cases.

Table 3.3.2.1. Michael 1,4-conjugated addition of cyclohexanone to trans- $\beta$-nitrostyrene catalysed by different supramolecular catalyst. ${ }^{[a]}$

\begin{tabular}{cccc}
\hline Catalyst & Aspect & ${\text { yield } / \%^{[\mathrm{b}]}}^{\text {e.e } / \%^{[\mathrm{c}]}}$ \\
\hline Prolle6 (3e) & gel & $>99$ & $48\left(2 \mathrm{R}, 1^{\prime} \mathrm{S}\right)$ \\
ProllePr (3d) & sol & $>99$ & $29\left(2 \mathrm{R}, 1^{\prime} \mathrm{S}\right)$ \\
ProPr $^{27}$ & sol & 71 & $12\left(2 \mathrm{~S}, 1^{\prime} \mathrm{R}\right)$ \\
ProVal66 $^{27}$ & gel & $>99$ & $34\left(2 \mathrm{R}, 1^{\prime} \mathrm{S}\right)$ \\
\hline
\end{tabular}

[a] Catalyst: $0,032 \mathrm{mmol}$ of proline units in $1 \mathrm{~mL}$ of toluene. Reagents: trans- $\beta$-nitrostyrene $(0,16 \mathrm{mmol})$, cyclohexanone $(3,29 \mathrm{mmol})$ in $2 \mathrm{~mL}$ of toluene 72 hours at 25 ㅇ. $\mathrm{C}$.

[b] Determined by ${ }^{1} \mathrm{H}-\mathrm{NMR}$ spectroscopy.

[c] Enantiomeric excess (e.e.) was determined by chiral-phase HPLC of the syn products.

Additionally, a study of the catalyst concentration effect on the enantiomeric results was performed and disclosed on Figure SI_3.5.18. As can be seen, the results showed no variation with the catalyst concentration. This indicates that upon increasing the concentration no significant change on the conformation of the aggregated species is taking place. 


\subsection{Conclusions}

Four proline derived dipeptidic organocatalysts have been prepared and their aggregation process has been studied. The results show that two different folded conformations can be found when they are in solution (diluted samples in absence of aggregation). These molecular preferences on the disposition of the proline ring lead to the so-called syn- and anti-conformations. The data suggests that ProValPr (3b) and ProllePr (3d) present mainly anti-conformations for both diluted and concentrated samples, whilst for ProAlaPr (3a) and ProPhePr (3c), a fast exchange between anti- and syn-conformations has been demonstrated. Upon aggregation, conformational changes take place from anti- to syn-conformation. In all the cases antiparallel aggregates were found. Additionally, an activation of the catalytic activity with the aggregation was found. The results show that organocatalysis can be rather sensitive to concentration and conformational effects highlighting the importance on minor changes on the molecular structure of the catalyst.

An exhaustive study of the bolaamphiphile Prolle6 (3e), which is a gelator analogue of the ProllePr (3d), has been carried out. This molecule is able to form gels in toluene and its properties have been analysed from a structural and thermodynamic point of view. Studies using NMR shed some light on the aggregation process and a simulated model has been proposed for the self-assembled network by hydrogen bonds through the amides of the neighbouring molecules forming a well-defined 1D fibre. The catalytic activity was tested in the gel-phase and the results indicate that the catalytic activity of the proline unit is maintained upon aggregation in a fibrillar solid-like state, showing quantitative conversion and moderate enantioselectivity.

Overall in this chapter it has been demonstrated that organocatalysis with dipeptide derivatives can be quite sensitive to the concentration and to the conformational disposition of the molecules when aggregation effects are involved. In addition, organocatalysis in gel-phase has been reported showing good conversions and moderate enantioselectivity. These results are encouraging for the study with supramolecular gels as a new type of smart soft-materials to obtain novel catalysts trying to mimic the efficiency and the selectivity of the natural catalysts, enzymes. 


\subsection{Supporting Information}
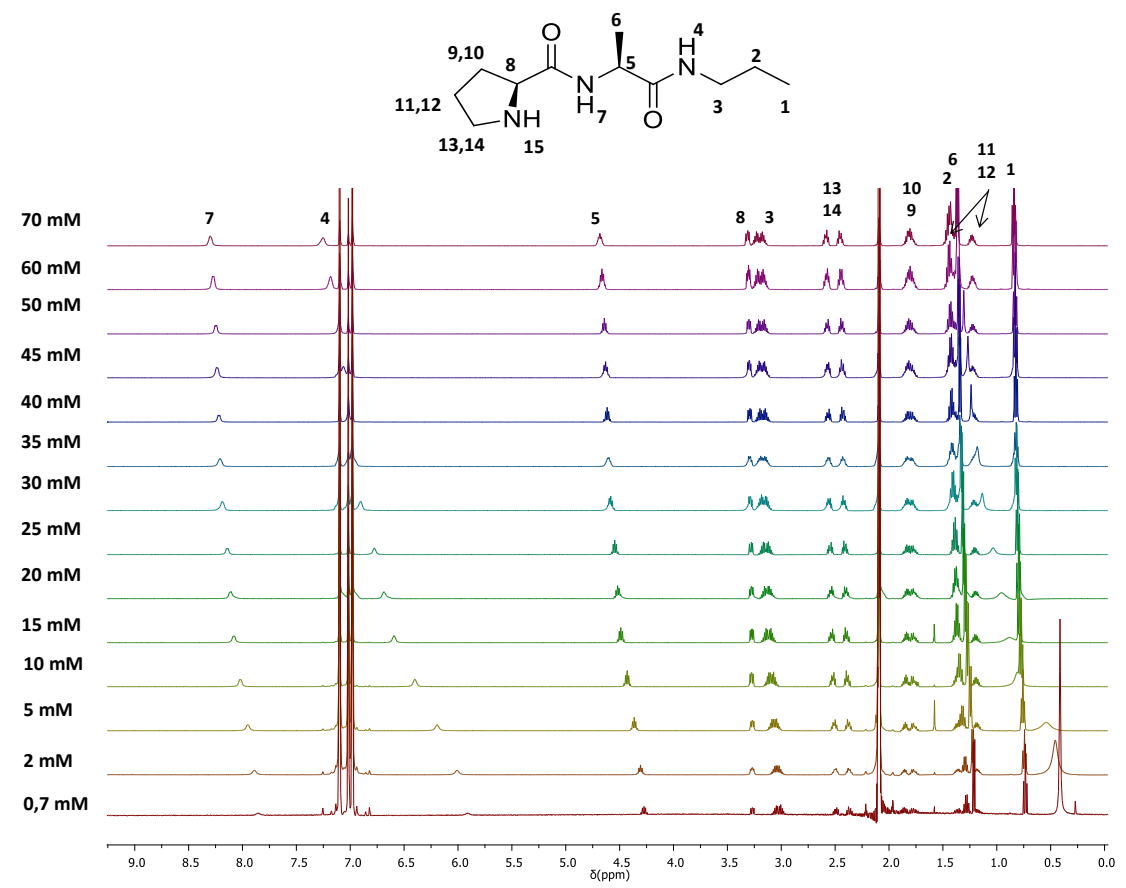

Figure SI_3.5.1. ${ }^{1} \mathrm{H}$-NMR spectra of ProAlaPr (3a) in $\mathrm{D}_{8}$-toluene at different concentrations $(500 \mathrm{MHz}$ at 30 oc).

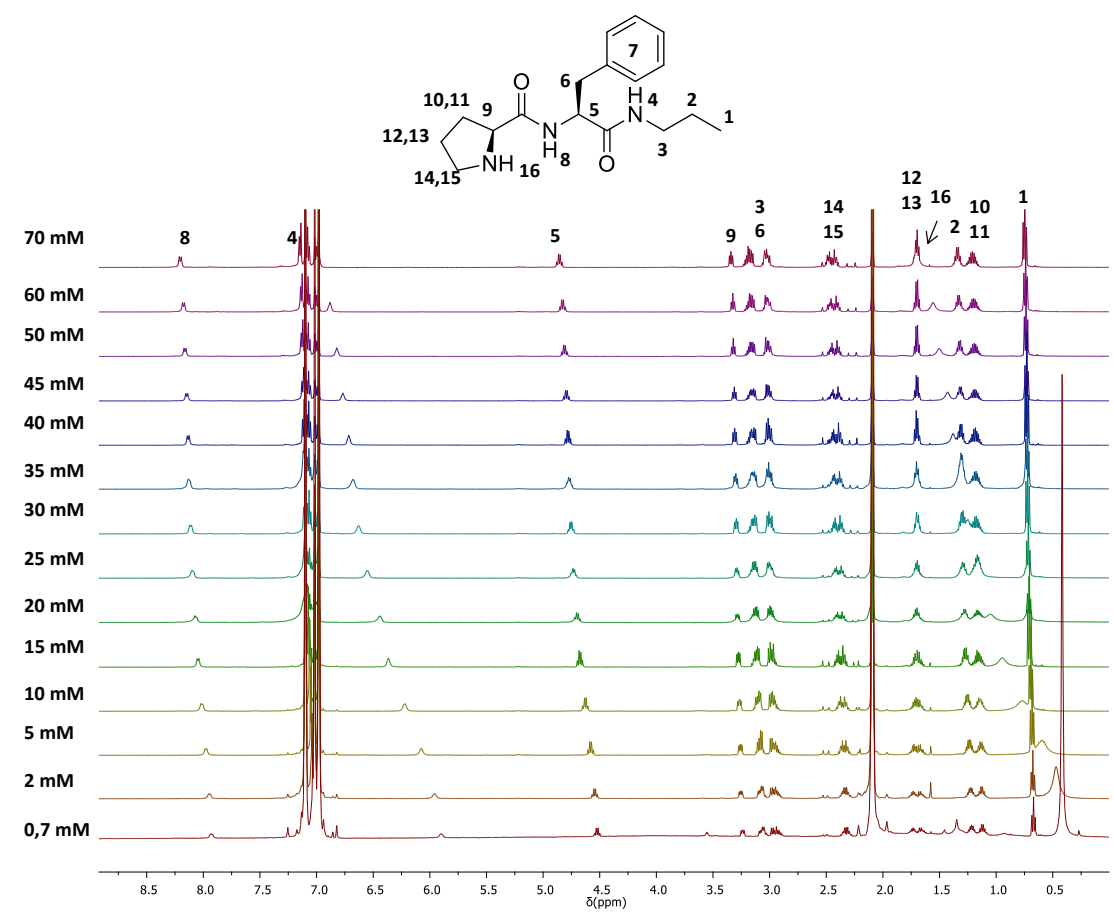

Figure SI_3.5.2. ${ }^{1} \mathrm{H}-\mathrm{NMR}$ spectra of ProPhePr (3c) in $\mathrm{D}_{8}$-toluene at different concentrations $(500 \mathrm{MHz}$ at 30 oc). 


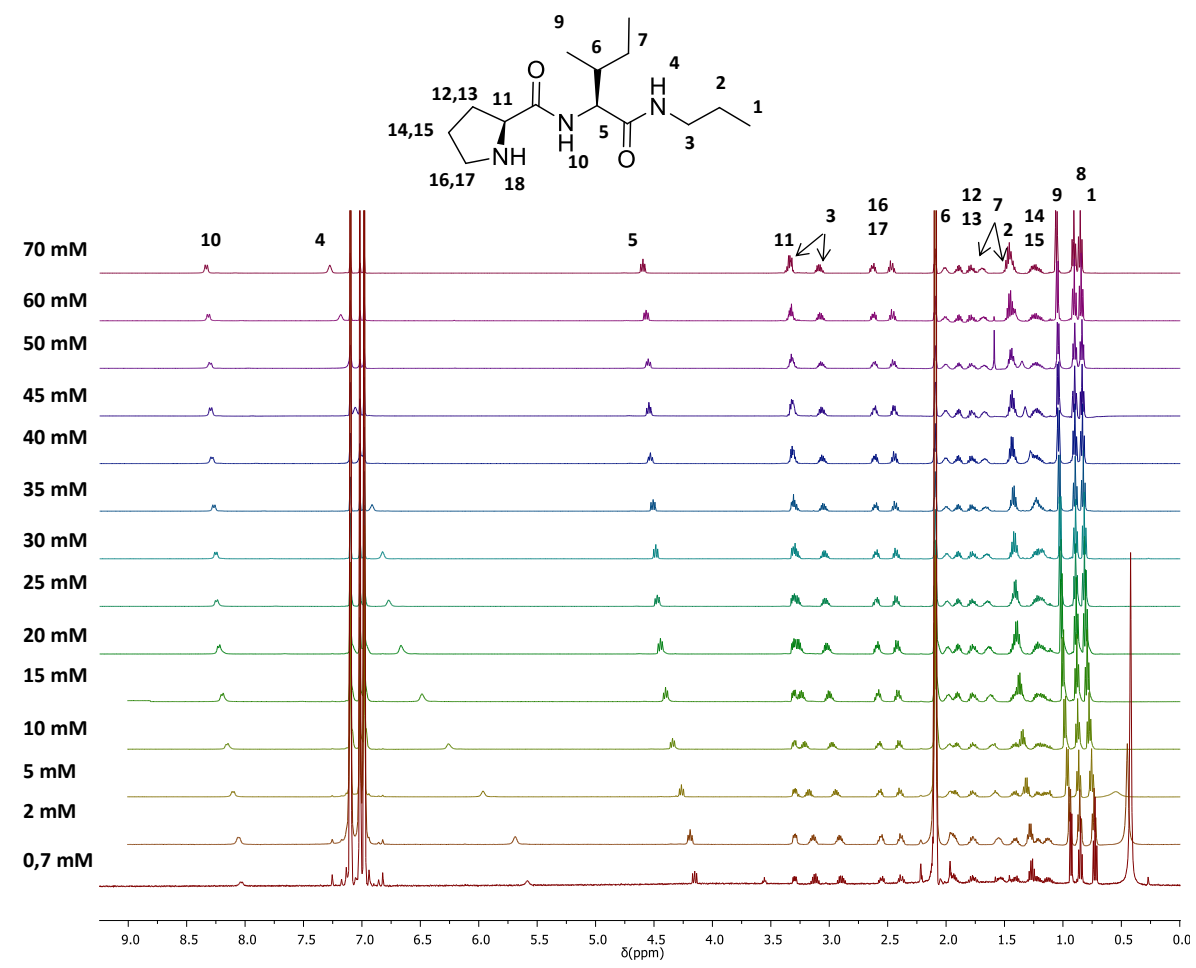

Figure SI_3.5.3. ${ }^{1} \mathrm{H}-\mathrm{NMR}$ spectra of ProllePr (3d) in $\mathrm{D}_{8}$-toluene at different concentrations $(500 \mathrm{MHz}$ at 30 $\stackrel{\circ}{ }$ ).

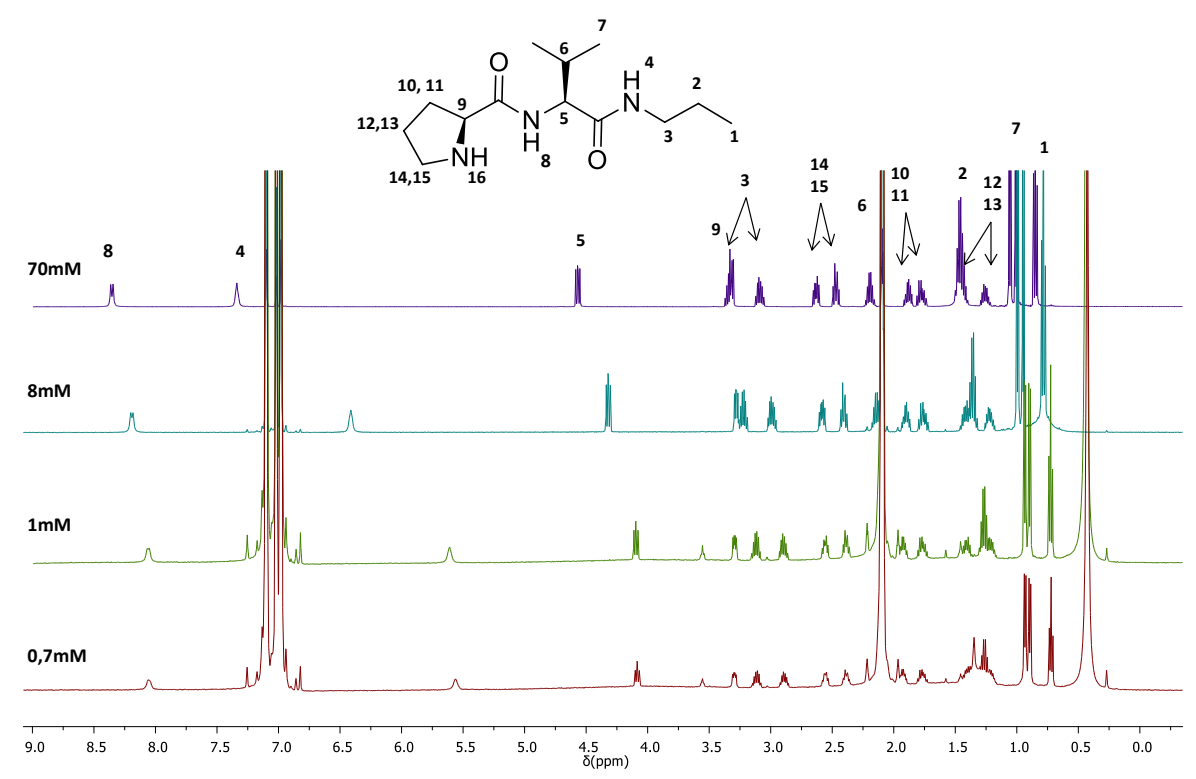

Figure SI_3.5.4. ${ }^{1} \mathrm{H}-\mathrm{NMR}$ spectra of ProValPr (3b) in $\mathrm{D}_{8}$-toluene at different concentrations (500 MHz at 30 oC). 


\section{Determination of aggregation constants}

The chemical shift of $\mathrm{NH}$ from the propylamide moiety was used as a probe and its value was measured at different concentrations. The results were fitted to a cooperative binding model using the equations reported previously in the literature. " $\mathrm{X}$ " in the following Equation_SI_3.1 was minimized by non-linear squares fitting regression with Solver (Microsoft Excel) using $\delta_{\max }$ (maximum chemical shift expected), $\mathrm{K}_{2}$ (dimerization constant, see Equation_SI_3.3), $\mathrm{K}_{\mathrm{n}}$ (successive oligomerization constant, see Equation_SI_3.4) as variables. Experimental data corresponds to $\delta_{\text {exp }}$ (observed chemical shift), c (concentration) and $\delta_{\min }$ (chemical shift in the absence of aggregation, obtained by extrapolation to $c=0$ ).

$$
\begin{aligned}
& X=K_{2}^{1 / 2}+K \frac{P_{f}\left[\left(1-P_{f}\right) c\right]^{1 / 2}}{2 P_{f}-1}-\frac{\left(1-P_{f}\right)^{1 / 2}}{\left(2 P_{f}-1\right) c^{1 / 2}} \\
& P_{f}=\frac{\delta_{\text {max }}-\delta_{\text {exp }}}{\delta_{\text {min }}-\delta_{\text {exp }}} \\
& A+A=A_{2} ; K_{2} \\
& A_{n}+A=A_{n+1} ; K_{n}
\end{aligned}
$$

Equation_SI_3.3

Equation_SI_3.4

Experimental data used for constant determinations and graphical representation of the quality of the fittings.

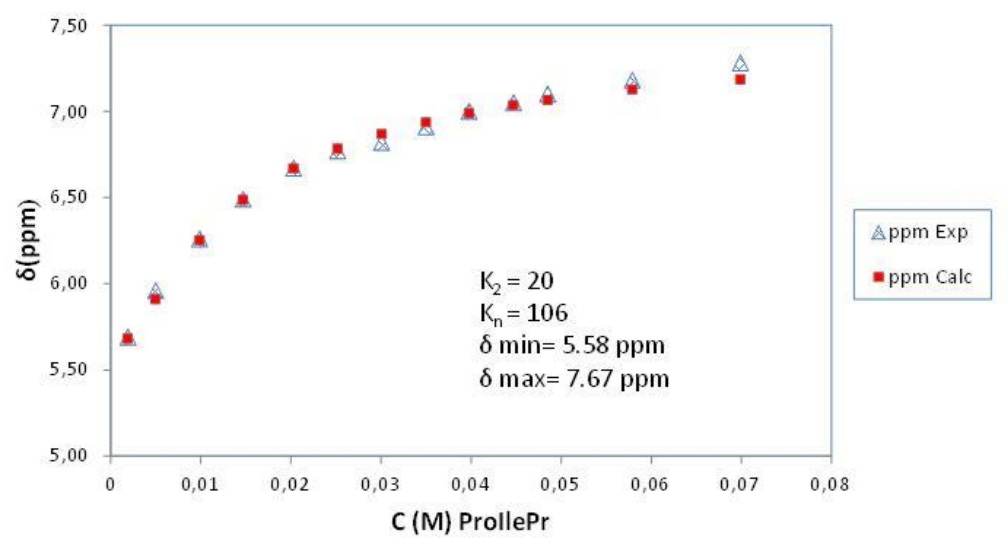

\begin{tabular}{ccc} 
C (M) ProllePr & ppm Exp & ppm Calc \\
\hline 0,0699 & 7,28 & 7,18 \\
0,0579 & 7,18 & 7,13 \\
0,0485 & 7,10 & 7,06 \\
0,0447 & 7,05 & 7,03 \\
0,0398 & 7,00 & 6,99 \\
0,035 & 6,91 & 6,93 \\
0,0301 & 6,82 & 6,87 \\
0,0252 & 6,77 & 6,78 \\
0,0203 & 6,67 & 6,67 \\
0,0147 & 6,49 & 6,48 \\
0,0099 & 6,26 & 6,25 \\
0,005 & 5,96 & 5,91 \\
0,0019 & 5,69 & 5,68
\end{tabular}




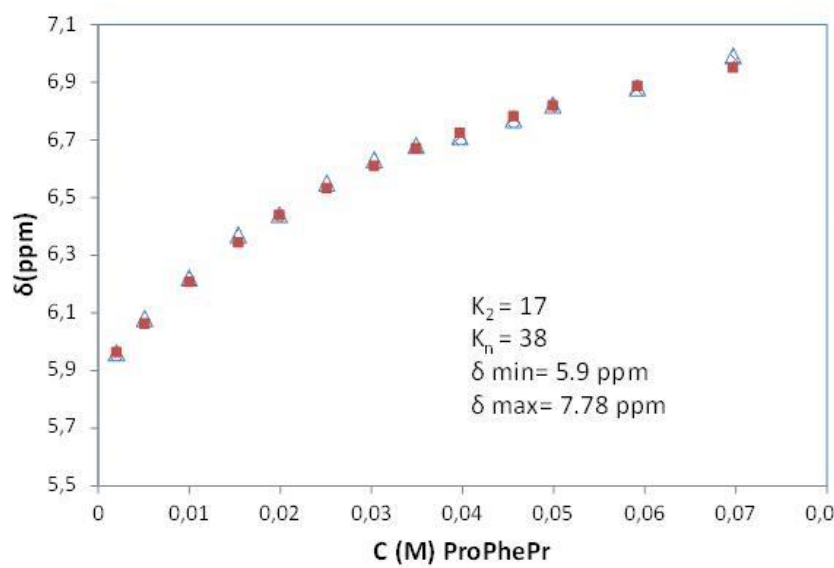

\begin{tabular}{|c|c|c|c|}
\hline & C(M)ProPhePr & ppm Exp & ppm Calc \\
\hline & 0,0697 & 6,99 & 6,95 \\
\hline & 0,0592 & 6,88 & 6,89 \\
\hline & 0,0499 & 6,82 & 6,82 \\
\hline & 0,0456 & 6,77 & 6,78 \\
\hline & 0,0397 & 6,71 & 6,72 \\
\hline \multirow{8}{*}{$\begin{array}{l}\text { Appm Exp } \\
=p p m \text { Calc }\end{array}$} & 0,0349 & 6,68 & 6,67 \\
\hline & 0,0303 & 6,63 & 6,61 \\
\hline & 0,0251 & 6,55 & 6,53 \\
\hline & 0,0199 & 6,44 & 6,44 \\
\hline & 0,0154 & 6,37 & 6,34 \\
\hline & 0,0100 & 6,22 & 6,21 \\
\hline & 0,0051 & 6,08 & 6,06 \\
\hline & 0,0020 & 5,96 & 5,96 \\
\hline
\end{tabular}

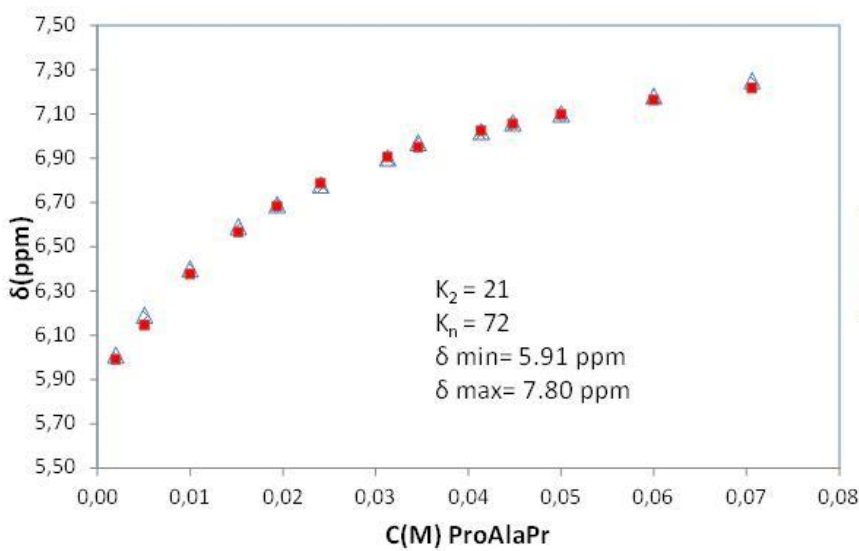

\begin{tabular}{|c|c|c|c|}
\hline & C (M) ProAlaPr & ppm Exp & ppm Cal \\
\hline & 0,0706 & 7,25 & 7,22 \\
\hline & 0,0600 & 7,18 & 7,16 \\
\hline & 0,0500 & 7,10 & 7,10 \\
\hline & 0,0448 & 7,06 & 7,05 \\
\hline & 0,0414 & 7,02 & 7,02 \\
\hline \multirow{2}{*}{$\Delta p p m \operatorname{Exp}$} & 0,0346 & 6,97 & 6,95 \\
\hline & 0,0313 & 6,90 & 6,90 \\
\hline \multirow[t]{6}{*}{ - ppm Calc } & 0,0241 & 6,78 & 6,79 \\
\hline & 0,0194 & 6,69 & 6,68 \\
\hline & 0,0152 & 6,59 & 6,57 \\
\hline & 0,0100 & 6,40 & 6,38 \\
\hline & 0,0051 & 6,19 & 6,15 \\
\hline & 0,0020 & 6,01 & 5,99 \\
\hline
\end{tabular}

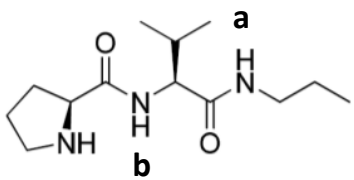

b

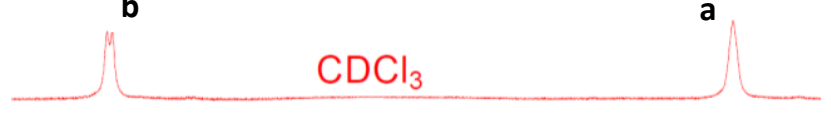

a

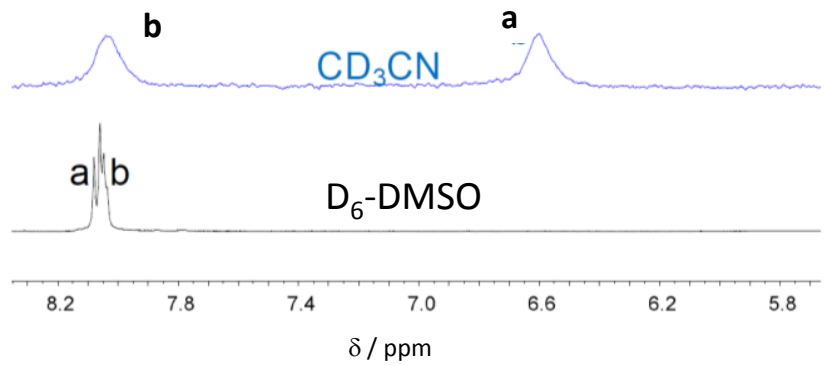

Figure SI_3.5.5. Partial ${ }^{1} \mathrm{H}-\mathrm{NMR}$ spectra of ProValPr (3b) $1 \mathrm{mM}$ in different solvents. 


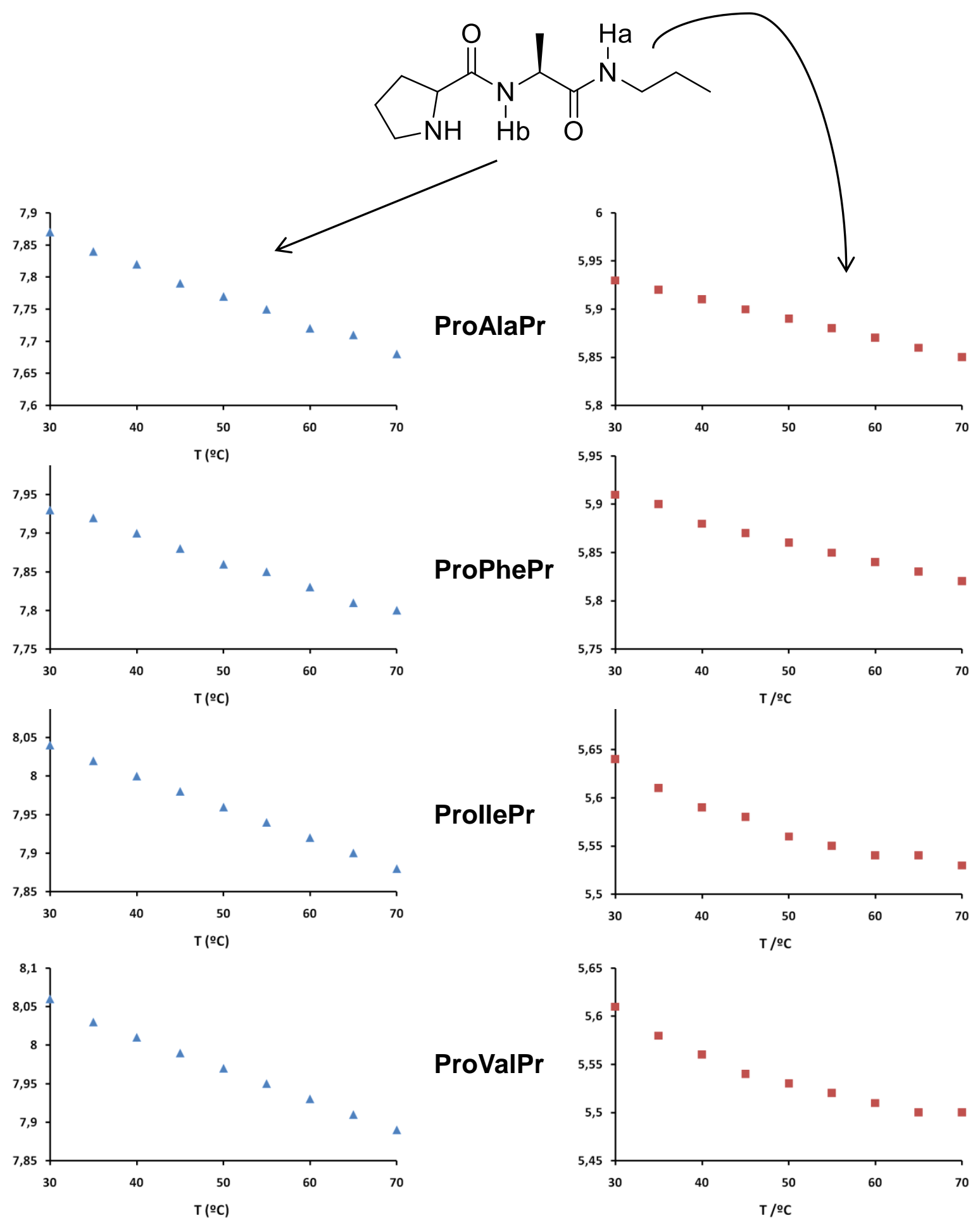

Figure SI_3.5.6. Variation of the amide signals with the temperature. $1 \mathrm{mM}$ samples in $D_{8}$-toluene. 


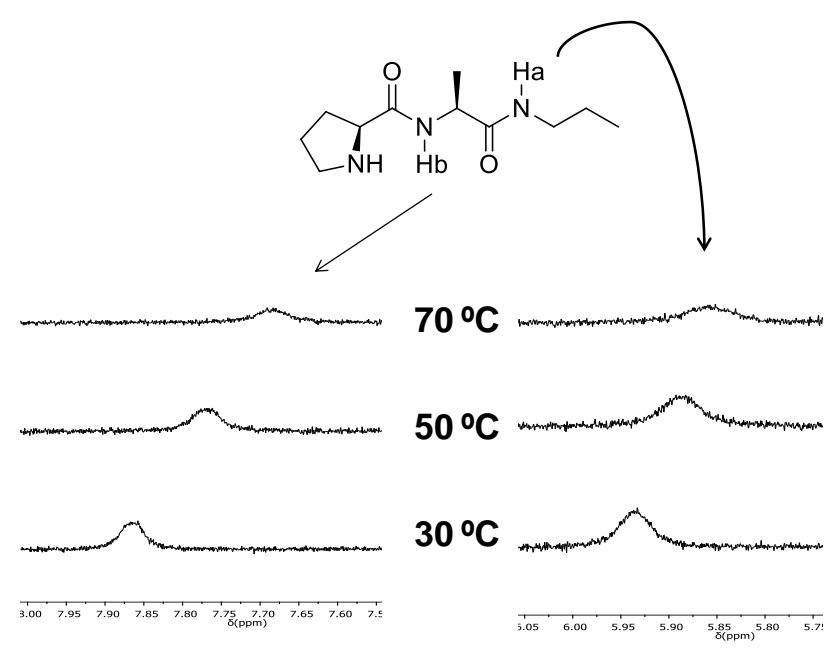

Figure SI_3.5.7. ${ }^{1} \mathrm{H}-\mathrm{NMR}$ variation of the amide signal with the temperature for a sample of ProAlaPr (3a) 1 $\mathrm{mM}$ in $D_{8}$-toluene.

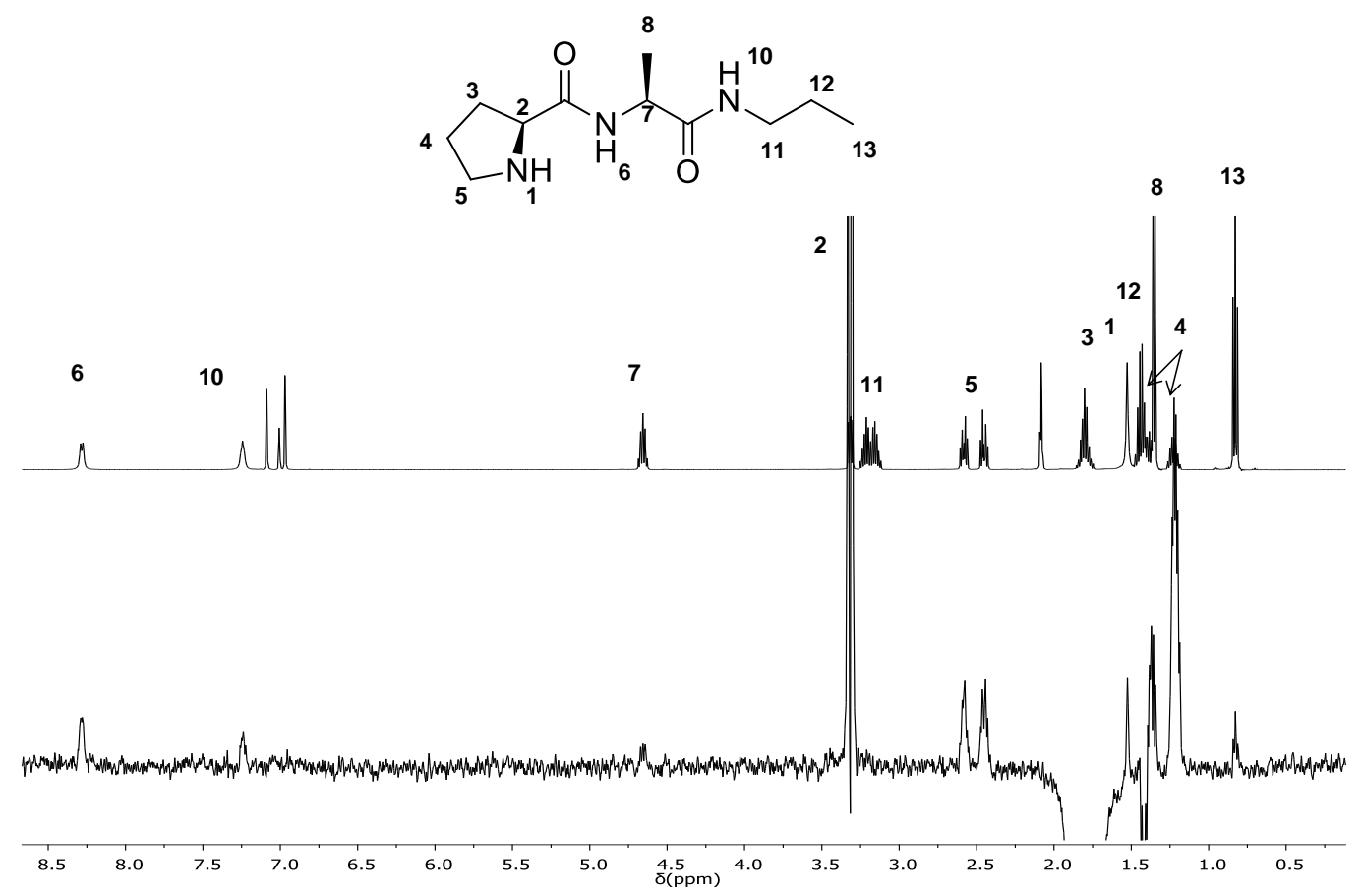

Figure SI_3.5.8. NOESY-1D spectrum for ProAlaPr (3a) at $70 \mathrm{mM}$ in $D_{8}$-toluene at $30 \cong \mathrm{C}$. 


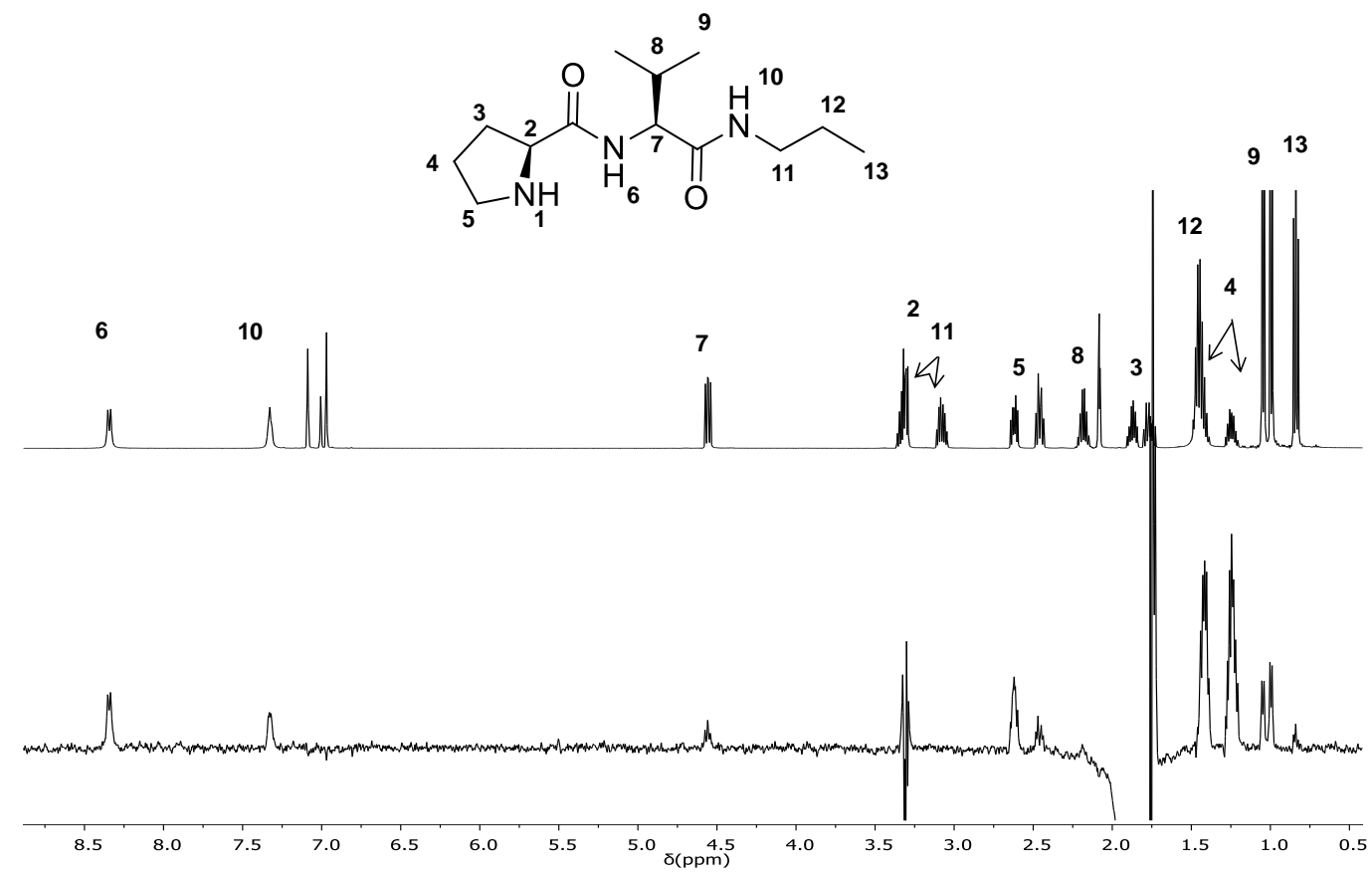

Figure SI_3.5.9. NOESY-1D spectrum for ProValPr (3b) at $70 \mathrm{mM}$ in $\mathrm{D}_{8}$-toluene at $30 \stackrel{\circ}{\circ}$.

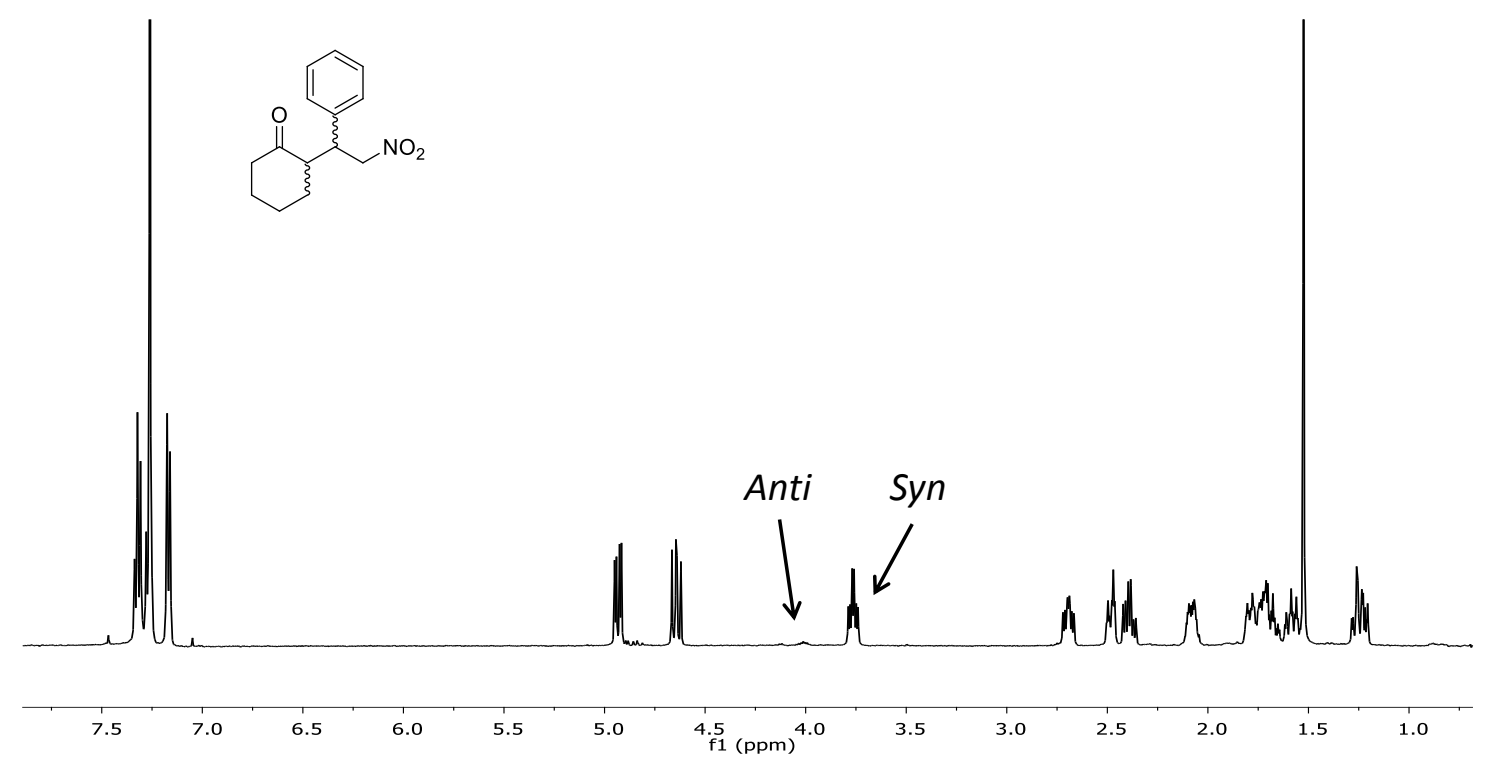

Figure SI_3.5.10. ${ }^{1} \mathrm{H}-\mathrm{NMR}$ of the crude of reaction after column chromatography in $\mathrm{CDCl}_{3}$ (anti= $4.01 \mathrm{ppm}$, syn=3,76 ppm). 
Chrom Type: HPLC Channel : 1

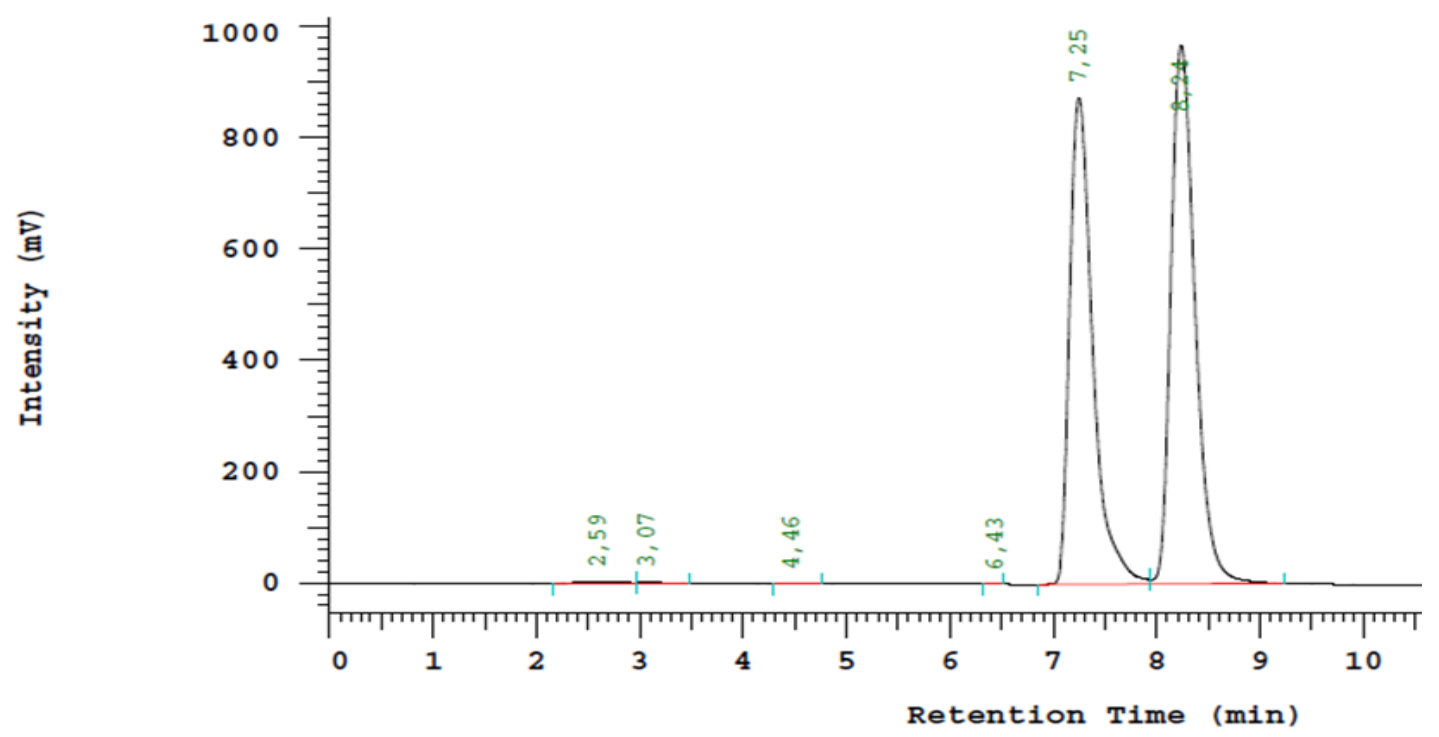

Figure SI_3.5.11. Representative HPLC chromatogram used for determination of enantiomeric excess. Chiral Pack IA column, hexane/IPA (v/v: 85/15), flow rate $=1 \mathrm{~mL} / \mathrm{min}, \lambda=210 \mathrm{~nm}$. $\left(\mathrm{t}_{r}\left(2 \mathrm{R}, 1^{\prime} \mathrm{S}\right)=8,24 \mathrm{~min}, \mathrm{t}_{\mathrm{r}}\left(2 \mathrm{~S}, 1^{\prime} \mathrm{R}\right)=\right.$ $7,25 \mathrm{~min})$.

\section{Determination of kinetic constants}<smiles>O=C1CCCCC1</smiles><smiles>O=[N+]([O-])/C=C/c1ccccc1</smiles><smiles>CC(C)(C)[C@H]1CCCC([C@H](C[N+](=O)[O-])c2ccccc2)C1=O</smiles>

As described in the main text, the reactions were carried out in the presence of an excess of cyclohexanone and therefore for simplicity the system was analysed in terms of pseudo first order kinetics (see Equations_SI_3.5-7; in Equation_SI_3.7 [alkene] $]_{f}=$ concentration at the end of the reaction time, $[\text { alkene }]_{0}=$ initial concentration, $\mathrm{k}^{\prime}=\mathrm{k}$ [ketone][catalyst]). 
$\frac{\mathrm{d}[\text { alkene] }}{\mathrm{dt}}=\mathrm{k}[$ catalyst] [ketone][alkene]

Equation_SI_3.5

$\frac{\mathrm{d}[\text { alkene] }}{\mathrm{dt}}=\mathrm{k}^{\prime}[$ alkene]

Equation_SI_3.6; (pseudo first order kinetics)

$\ln \frac{\text { alkene }_{f}}{[\text { alkene }]_{0}}=k^{\prime} t$

Equation_SI_3.7; (integrated rate equation)

Table SI_3.5.1. Experimental results used for the calculation of the constants cited in the main text for the compounds 3a-d.

\begin{tabular}{ccccccc}
\hline Catalyst & $\begin{array}{c}\text { [catalyst] / } \\
\mathbf{M}\end{array}$ & $\begin{array}{c}\text { [ketone] / } \\
\mathbf{M}\end{array}$ & $\begin{array}{c}\text { [alkene] / } \\
\mathbf{M}\end{array}$ & $\begin{array}{c}\text { reaction } \\
\text { time / } \mathbf{h}\end{array}$ & $\begin{array}{c}\text { yield / } \\
\mathbf{\%}\end{array}$ & $\mathbf{k} / \mathbf{~ M}^{\mathbf{2}} \mathbf{h}^{-\mathbf{1}}$ \\
\hline ProValPr & 0,0058 & 0,580 & 0,029 & 72 & 99 & 18,86 \\
(3b) & 0,001 & 0,548 & 0,026 & 24 & 3 & 2,31 \\
ProllePr & 0,005 & 0,506 & 0,025 & 72 & 99 & 24,97 \\
(3d) & 0,001 & 0,458 & 0,026 & 24 & 2 & 1,53 \\
ProPhePr & 0,005 & 0,506 & 0,025 & 72 & 78 & 8,18 \\
(3c) & 0,001 & 0,548 & 0,026 & 24 & 1 & 0,76 \\
ProAlaPr & 0,005 & 0,506 & 0,025 & 72 & 70 & 6,5 \\
(3a) & 0,001 & 0,548 & 0,026 & 24 & 1 & 0,76 \\
\hline
\end{tabular}

Table SI_3.5.2. Experimental data used for the calculation of the reactions constants cited in the main text.

\begin{tabular}{ccccccc}
\hline Catalyst & $\begin{array}{c}\text { [catalyst] / } \\
\mathbf{M}\end{array}$ & $\begin{array}{c}\text { [ketone] / } \\
\mathbf{M}\end{array}$ & $\begin{array}{c}\text { [alkene] / } \\
\mathbf{M}\end{array}$ & $\begin{array}{c}\text { reaction } \\
\text { time / } \mathbf{h}\end{array}$ & $\begin{array}{c}\text { yield / } \\
\%\end{array}$ & $\mathbf{k} / \mathbf{M}^{-\mathbf{2}} \mathbf{h}^{-\mathbf{1}}$ \\
\hline Prolle6 & 0,0055 & 0,550 & 0,028 & 72 & 99 & 21,19 \\
(3e) & 0,0025 & 0,250 & 0,013 & 72 & 64 & 22,67 \\
& 0,0010 & 0,200 & 0,010 & 72 & 99 & 15,49 \\
ProllePr & 0,0050 & 0,506 & 0,025 & 72 & 99 & 24,97 \\
(3d) & 0,0025 & 1,370 & 0,066 & 24 & 15 & 1,9 \\
& 0,0010 & 0,458 & 0,026 & 24 & 2 & 1,53 \\
\hline
\end{tabular}




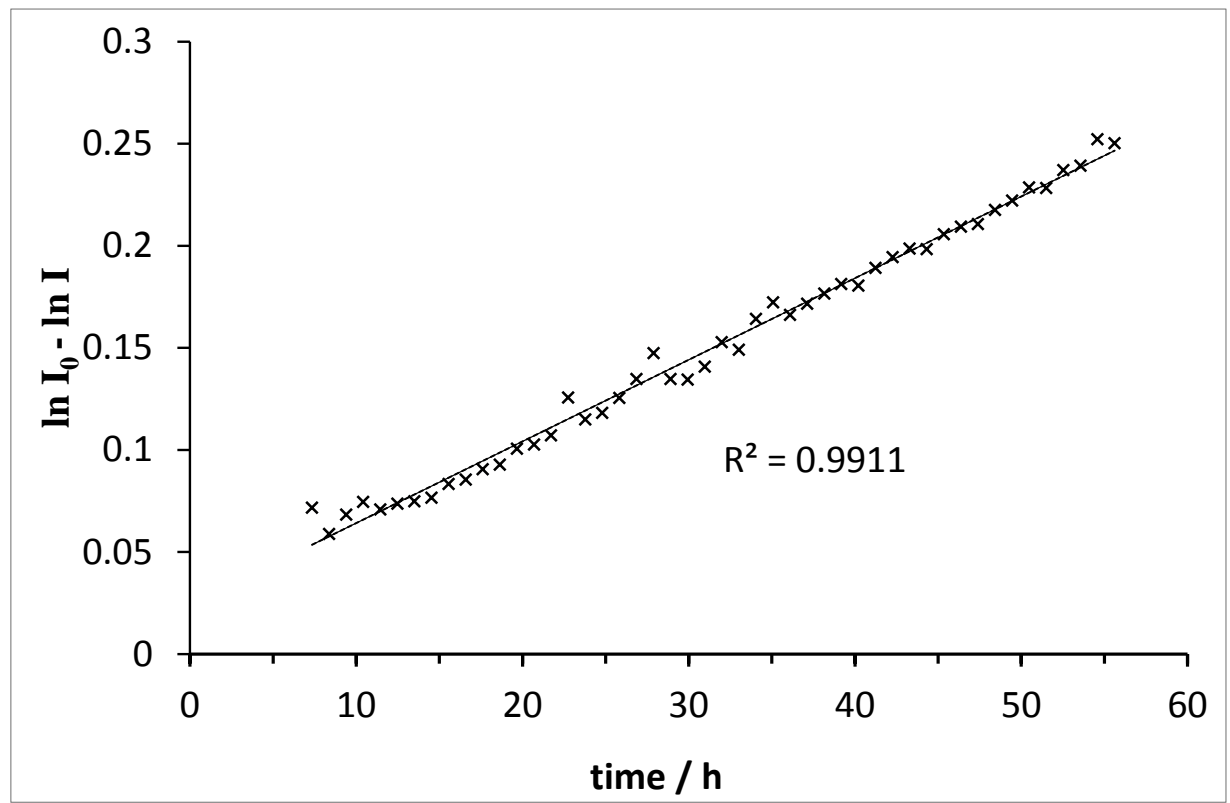

Figure SI_3.5.12. Pseudo first-order fitting of the reaction catalysed by ProllePr (3d). Experimental data correspond to variation of NMR intensity of alkene protons of trans- $\beta$-nitrostyrene with time. [trans- $\beta$ -

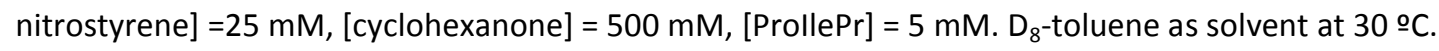

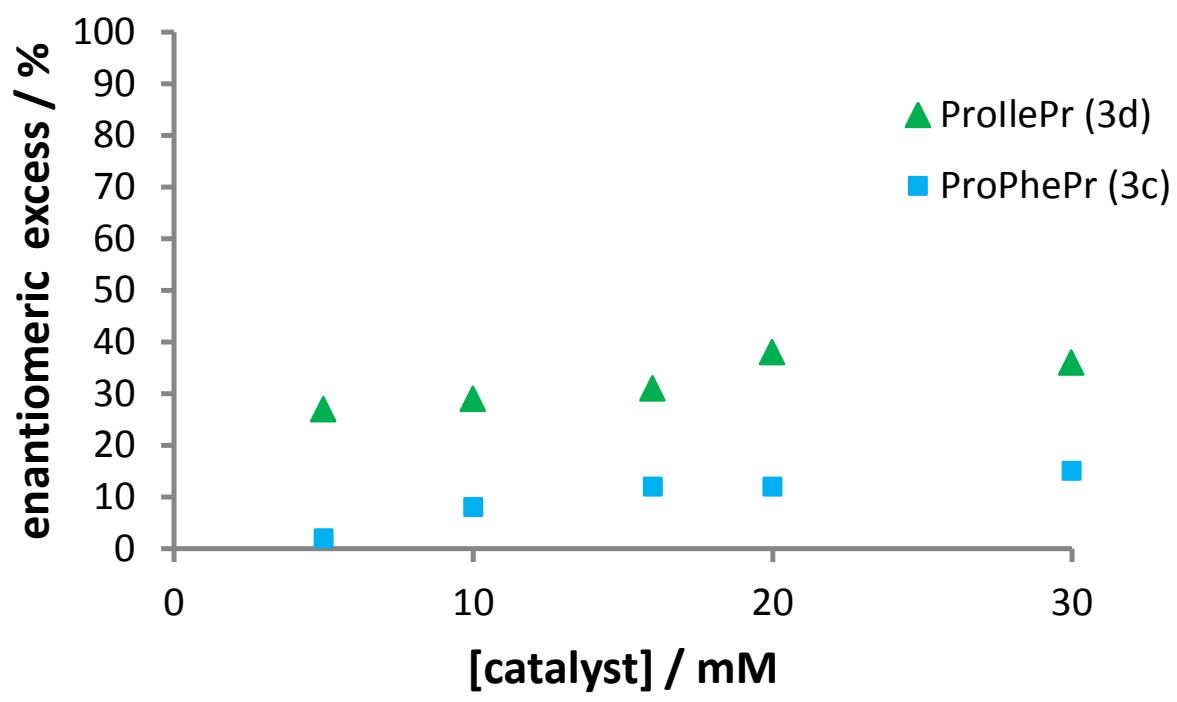

Figure SI_3.5.13. Effect of the concentration of catalyst on the enantiomeric excess. 
A

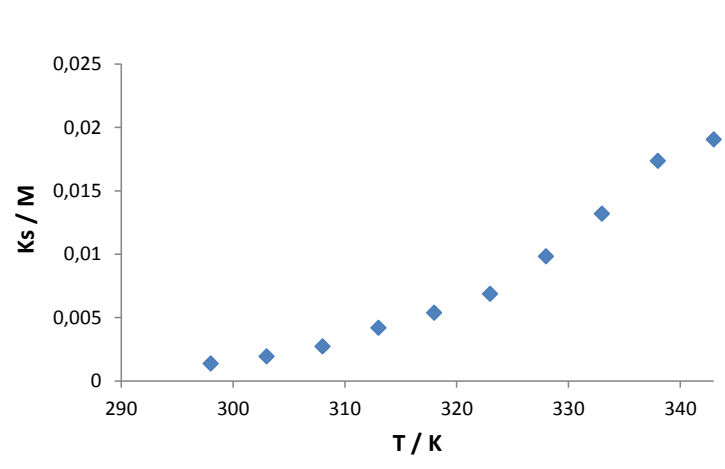

B

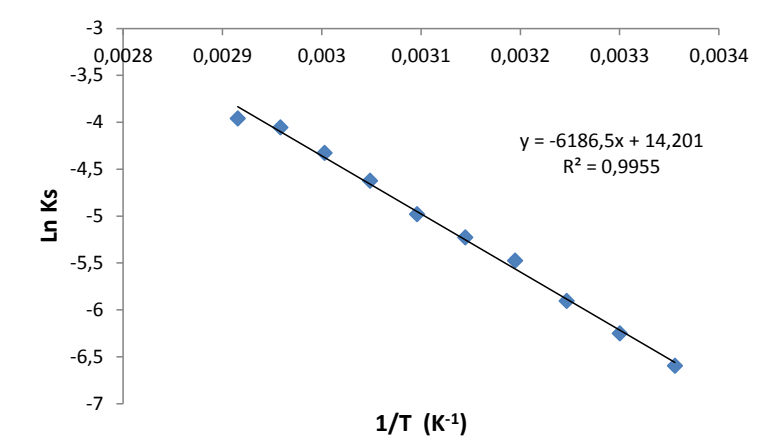

Figure SI_3.5.14. A) Temperature dependence of the solubility for Prolle6 (3e) in the gel phase $(20 \mathrm{mM})$ in $D_{8}$-toluene and $B$ ) Ln Ks vs $1 / T$ data from the same experiment.

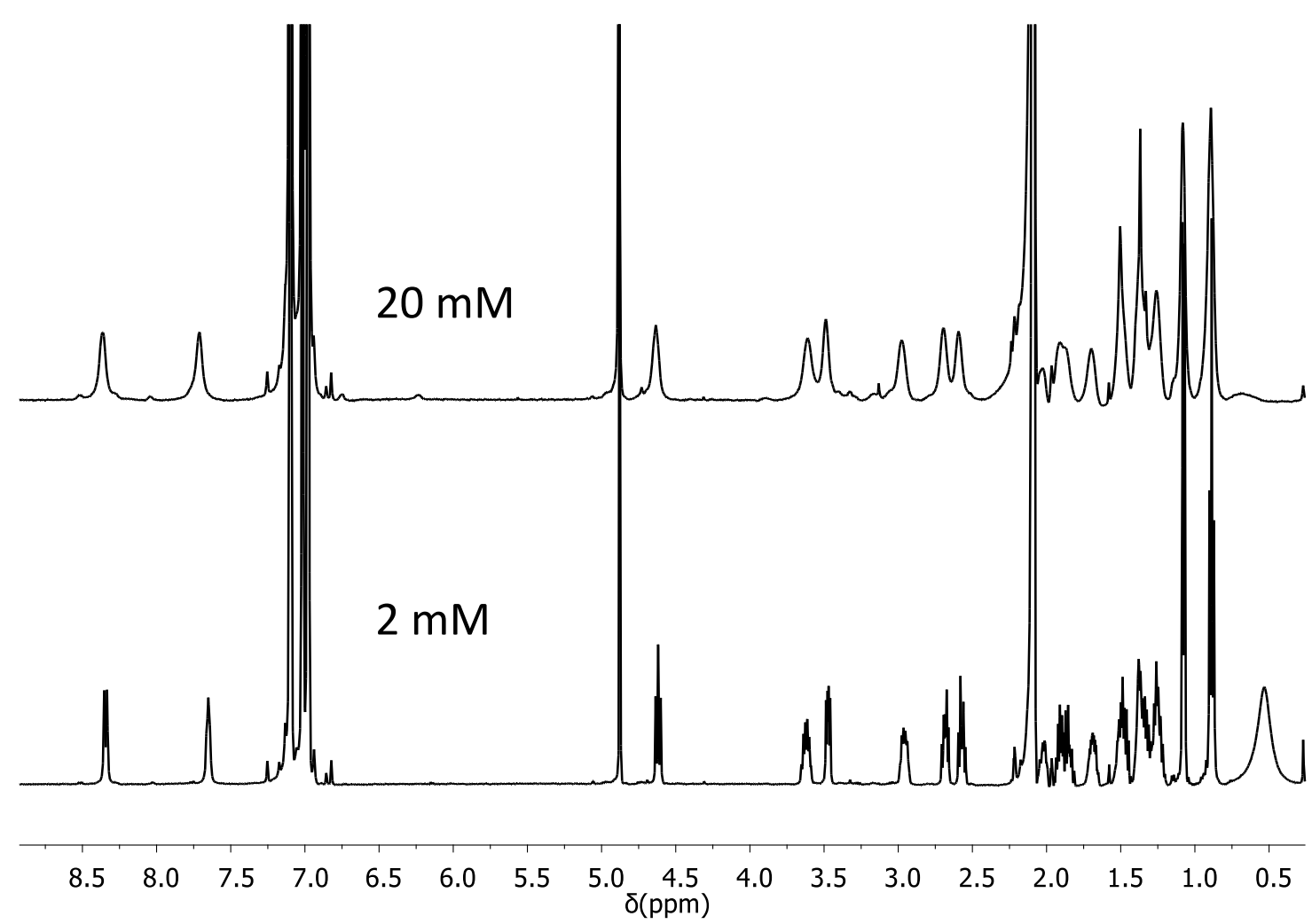

Figure SI_3.5.15. Comparative ${ }^{1} \mathrm{H}$-NMR spectra of Prolle6 (3e) in toluene at different concentrations in solution (bottom) and in gel phase (top). 

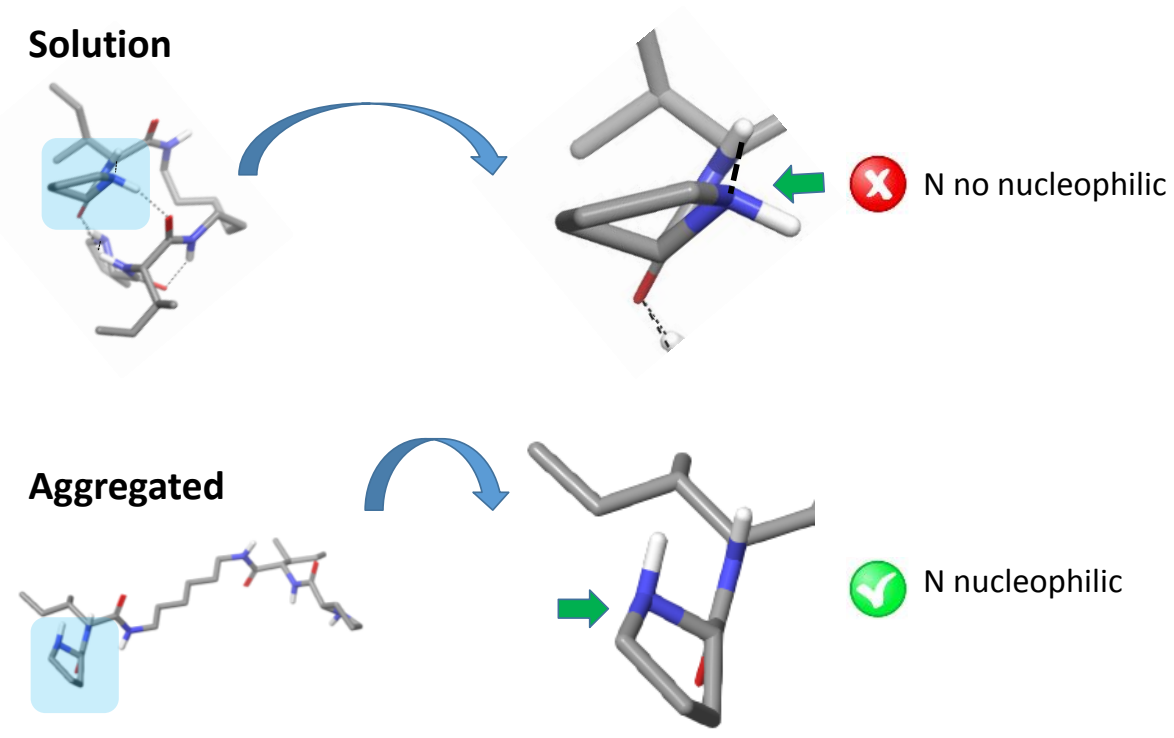

Figure SI_3.5.16. Simulated model of the proline moiety conformation in Prolle6 (3e) in solution (top) and in aggregated state (bottom).

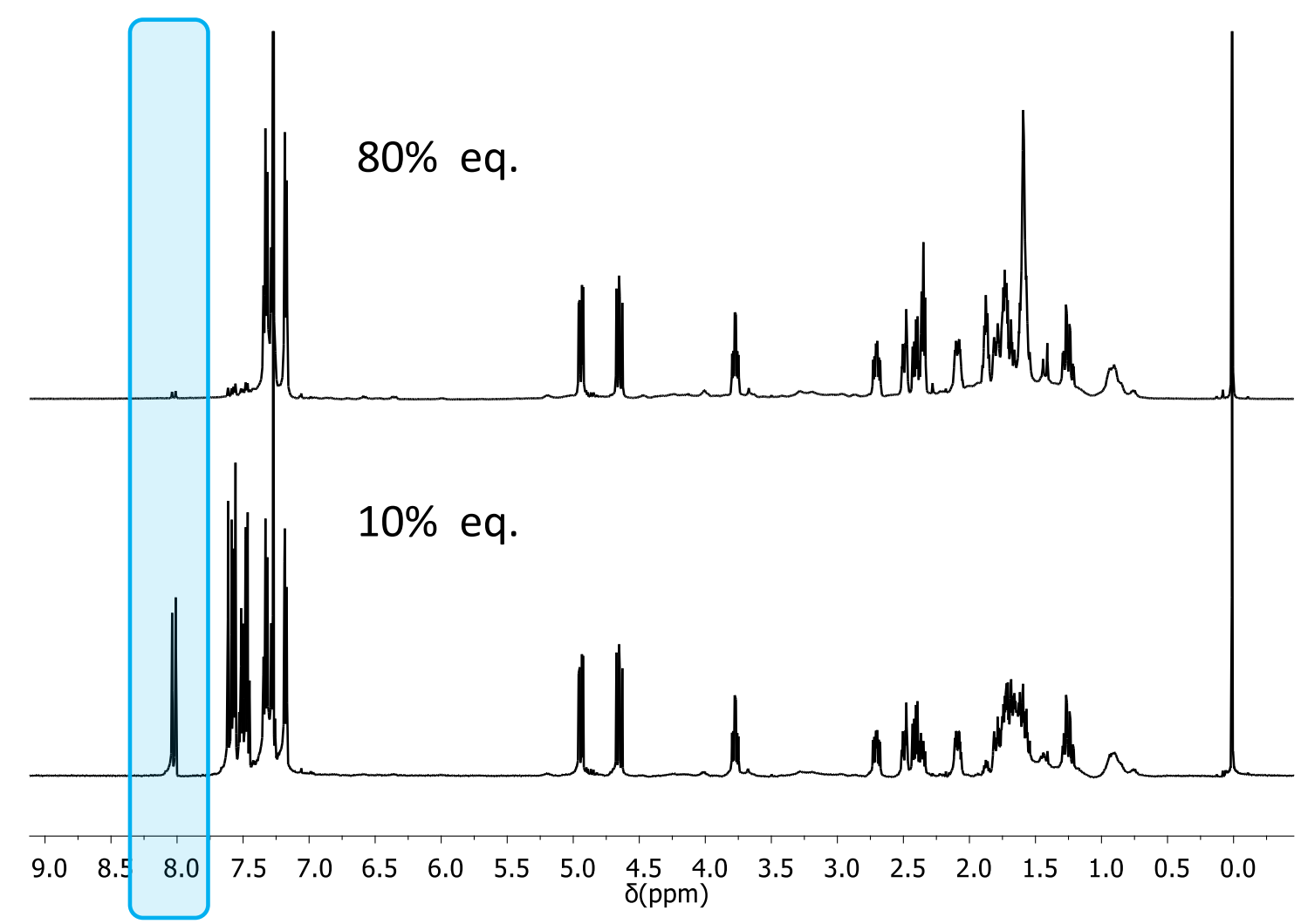

Figure SI_3.5.17. ${ }^{1} \mathrm{H}-\mathrm{NMR}$ spectra in $\mathrm{CDCl}_{3}$ for the Michael addition reaction crudes with a different load of catalyst Prolle6 (3e). Reaction conditions: trans- $\beta$-nitrostyrene $(0,19 \mathrm{mmol})$, cyclohexanone $(1,9 \mathrm{mmol})$ in 2 $\mathrm{mL}$ of toluene at $\mathrm{rt}, 9$ hours. 


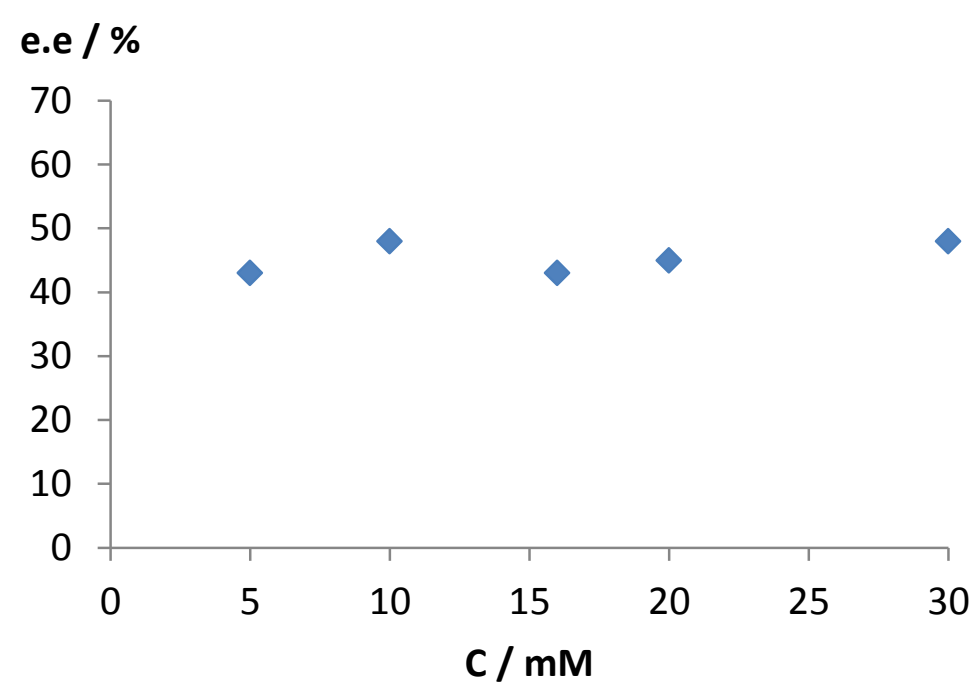

Figure SI_3.5.18. Effect of catalyst concentration on the enantiomeric excess for the Prolle6 (3e) experiment. Concentration expressed in terms of proline equivalent. 


\subsection{References and Notes}

1. List, B.; Lerner, R. A.; Barbas, C. F. "Proline-Catalyzed Direct Asymmetric Aldol Reactions", J. Am. Chem. Soc., 2000, 122, 2395-2396.

2. Ahrendt, K. A.; Borths, C. J.; MacMillan, D. W. C. "New Strategies for Organic Catalysis: The First Highly Enantioselective Organocatalytic Diels-Alder Reaction", J. Am. Chem. Soc., 2000, 122, 4243-4244.

3. (a) Dalko, P. I.; Moisan, L. "Enantioselective Organocatalysis", Angew. Chem. Int. Ed., 2001, 40, 3726-3748; (b) Notz, W.; Tanaka, F.; Barbas, C. F., 3rd "Enamine-Based Organocatalysis with Proline and Diamines: the Development of Direct Catalytic Asymmetric Aldol, Mannich, Michael, and Diels-Alder Reactions", Acc. Chem. Res., 2004, 37, 580-591; (c) Mukherjee, S.; Yang, J. W.; Hoffmann, S.; List, B. "Asymmetric Enamine Catalysis", Chem. Rev., 2007, 107, 5471-5569; (d) Melchiorre, P.; Marigo, M.; Carlone, A.; Bartoli, G. "Asymmetric Aminocatalysis - Gold Rush in Organic Chemistry", Angew. Chem. Int. Ed., 2008, 47, 6138-6171.

4. (a) Davie, E. A. C.; Mennen, S. M.; Xu, Y.; Miller, S. J. "Asymmetric Catalysis Mediated by Synthetic Peptides", Chem. Rev., 2007, 107, 5759-5812; (b) Wennemers, H. "Asymmetric Catalysis with Peptides", Chem. Commun., 2011, 47, 12036-12041; (c) Lewandowski, B.; Wennemers, H. "Asymmetric Catalysis with Short-Chain Peptides", Curr. Opin. Chem. Biol., 2014, 22, 40-46.

5. (a) List, B. "Proline-Catalyzed Asymmetric Reactions", Tetrahedron, 2002, 58, 5573-5590;

(b) Martin, H. J.; List, B. "Mining Sequence Space for Asymmetric Aminocatalysis: NTerminal Prolyl-Peptides Efficiently Catalyze Enantioselective Aldol and Michael Reactions", Synlett, 2003, 2003, 1901-1902.

6. Whitesides, G. M.; Wong, C. H., "Enzymes in Synthetic Organic Chemistry". Pergamon, Oxford, 1994; Vol. Volume 12, p i.

7. (a) Machajewski, T. D.; Wong, C.-H. "The Catalytic Asymmetric Aldol Reaction", Angew. Chem. Int. Ed., 2000, 39, 1352-1375; (b) Gröger, H.; Wilken, J. "The Application of L-Proline as an Enzyme Mimic and Further New Asymmetric Syntheses Using Small Organic Molecules as Chiral Catalysts", Angew. Chem. Int. Ed., 2001, 40, 529-532.

8. (a) Tang, Z.; Yang, Z.-H.; Cun, L.-F.; Gong, L.-Z.; Mi, A.-Q.; Jiang, Y.-Z. "Small Peptides Catalyze Highly Enantioselective Direct Aldol Reactions of Aldehydes with Hydroxyacetone: Unprecedented Regiocontrol in Aqueous Media", Org. Lett., 2004, 6, 2285-2287; (b) Cobb, A. J.; Shaw, D. M.; Longbottom, D. A.; Gold, J. B.; Ley, S. V. "Organocatalysis with Proline Derivatives: Improved Catalysts for the Asymmetric Mannich, Nitro-Michael and Aldol Reactions", Org. Biomol. Chem., 2005, 3, 84-96; (c) Carley, A. P.; Dixon, S.; Kilburn, J. D. "Pyrrolidine-Based Organocatalysts for Enantioselective Michael Addition of Cyclohexanone to trans-beta-Nitrostyrene", Synthesis-Stuttgart, 2009, 2509-2516.

9. List, B.; Pojarliev, P.; Martin, H. J. "Efficient Proline-Catalyzed Michael Additions of Unmodified Ketones to Nitroolefins", Org. Lett., 2001, 3, 2423-2425.

10. (a) Wiesner, M.; Revell, J. D.; Wennemers, H. "Tripeptides as Efficient Asymmetric Catalysts for 1,4-Addition Reactions of Aldehydes to Nitroolefins--a Rational Approach", Angew. Chem. Int. Ed., 2008, 47, 1871-1874; (b) Wiesner, M.; Neuburger, M.; 
Wennemers, H. "Tripeptides of the Type H-D-Pro-Pro-Xaa- $\mathrm{NH}_{2}$ as Catalysts for Asymmetric 1,4-Addition Reactions: Structural Requirements for High Catalytic Efficiency", Chem. Eur. J., 2009, 15, 10103-10109; (c) Wiesner, M.; Wennemers, H. "Peptide-Catalyzed Conjugate Addition Reactions of Aldehydes to Nitroolefins", Synthesis-Stuttgart, 2010, 1568-1571; (d) Kastl, R.; Arakawa, Y.; Duschmale, J.; Wiesner, M.; Wennemers, H. "Peptide-Catalyzed 1,4-Addition Reactions of Aldehydes to Nitroolefins", Chimia, 2013, 67, 279-282.

11. Revell, J. D.; Wennemers, H. "Functional Group Requirements within the Peptide H-ProPro-Asp- $\mathrm{NH}_{2}$ as a Catalyst for Aldol Reactions", Tetrahedron, 2007, 63, 8420-8424.

12. (a) Arakawa, Y.; Wiesner, M.; Wennemers, H. "Efficient Recovery and Reuse of an Immobilized Peptidic Organocatalyst", Adv. Synth. Catal., 2011, 353, 1201-1206; (b) Arakawa, Y.; Wennemers, H. "Enamine Catalysis in Flow with an Immobilized Peptidic Catalyst", ChemSusChem, 2013, 6, 242-245.

13. Lehn, J.-M., "Supramolecular Reactivity and Catalysis". In Supramolecular Chemistry, Wiley-VCH Verlag GmbH \& Co. KGaA, 2006; pp 55-67.

14. (a) Raynal, M.; Ballester, P.; Vidal-Ferran, A.; van Leeuwen, P. W. N. M. "Supramolecular Catalysis. Part 1: Non-Covalent Interactions as a Tool for Building and Modifying Homogeneous Catalysts", Chem. Soc. Rev., 2014, 43, 1660-1733; (b) Raynal, M.; Ballester, P.; Vidal-Ferran, A.; van Leeuwen, P. W. N. M. "Supramolecular Catalysis. Part 2: Artificial Enzyme Mimics", Chem. Soc. Rev., 2014, 43, 1734-1787.

15. (a) Meeuwissen, J.; Reek, J. N. H. "Supramolecular Catalysis Beyond Enzyme Mimics", Nature Chem., 2010, 2, 615-621; (b) Dong, Z.; Yongguo, W.; Yin, Y.; Liu, J. "Supramolecular Enzyme Mimics by Self-Assembly", Curr. Opin. Colloid Interface Sci., 2011, 16, 451-458; (c) Dong, Z.; Luo, Q.; Liu, J. "Artificial Enzymes Based on Supramolecular Scaffolds", Chem. Soc. Rev., 2012, 41, 7890-7908.

16. Yuan, D. Q.; Kitagawa, Y.; Aoyama, K.; Douke, T.; Fukudome, M.; Fujita, K. "Imidazolyl Cyclodextrins: Artificial Serine Proteases Enabling Regiospecific Reactions", Angew. Chem. Int. Ed., 2007, 46, 5024-5027.

17. Zhang, H.; Zhao, W.; Zou, J.; Liu, Y.; Li, R.; Cui, Y. "Aldol Reaction Catalyzed by a Hydrophilic Catalyst in Aqueous Micelle as an Enzyme Mimic System", Chirality, 2009, 21, 492-496.

18. Fraile, J. M.; Garcia, J.; Mayoral, J. "Noncovalent Immobilization of Enantioselective Catalysts", Chem. Rev., 2009, 109, 360-417.

19. Pluth, M. D.; Bergman, R. G.; Raymond, K. N. "Acid Catalysis in Basic Solution: a Supramolecular Host Promotes Orthoformate Hydrolysis", Science, 2007, 316, 85-88.

20. Buchmeiser, M. R., "Polymeric Materials in Organic Synthesis and Catalysis". Wiley-VCH Verlag GmbH \& Co. KGaA, 2003; p i-xxiii.

21. (a) Escuder, B.; Rodríguez-Llansola, F.; Miravet, J. F. "Supramolecular Gels as Active Media for Organic Reactions and Catalysis", New J. Chem., 2010, 34, 1044-1054; (b) Gao, Y.; Zhao, F.; Wang, Q.; Zhang, Y.; Xu, B. "Small Peptide Nanofibers as the Matrices of Molecular Hydrogels for Mimicking Enzymes and Enhancing the Activity of Enzymes", Chem. Soc. Rev., 2010, 39, 3425-3433; (c) Díaz Díaz, D.; Kuhbeck, D.; Koopmans, R. J. "Stimuli-Responsive Gels as Reaction Vessels and Reusable Catalysts", Chem. Soc. Rev., 2011, 40, 427-448. 
22. Guler, M. O.; Stupp, S. I. "A Self-Assembled Nanofiber Catalyst for Ester Hydrolysis", J. Am. Chem. Soc., 2007, 129, 12082-12083.

23. Jin, Q.; Zhang, L.; Cao, H.; Wang, T.; Zhu, X.; Jiang, J.; Liu, M. "Self-Assembly of Copper(II) Ion-Mediated Nanotube and its Supramolecular Chiral Catalytic Behavior", Langmuir, 2011, 27, 13847-13853.

24. (a) Wang, Q.; Yang, Z.; Wang, L.; Ma, M.; Xu, B. "Molecular Hydrogel-Immobilized Enzymes Exhibit Superactivity and High Stability in Organic Solvents", Chem. Commun., 2007, 10321034; (b) Wang, Q.; Yang, Z.; Zhang, X.; Xiao, X.; Chang, C. K.; Xu, B. "A SupramolecularHydrogel-Encapsulated Hemin as an Artificial Enzyme to Mimic Peroxidase", Angew. Chem. Int. Ed., 2007, 46, 4285-4289.

25. Kiyonaka, S.; Sada, K.; Yoshimura, I.; Shinkai, S.; Kato, N.; Hamachi, I. "Semi-Wet Peptide/Protein Array Using Supramolecular Hydrogel", Nature Mater., 2004, 3, 58-64.

26. Brizard, A. M.; van Esch, J. H. "Self-Assembly Approaches for the Construction of Cell Architecture Mimics", Soft Matter, 2009, 5, 1320-1327.

27. Rodríguez-Llansola, F.; Miravet, J. F.; Escuder, B. "Supramolecular Catalysis with Extended Aggregates and Gels: Inversion of Stereoselectivity Caused by Self-Assembly", Chem. Eur. J., 2010, 16, 8480-8486.

28. (a) Fuhrhop, J. H.; Wang, T. "Bolaamphiphiles", Chem. Rev., 2004, 104, 2901-2937; (b) Estroff, L. A.; Hamilton, A. D. "Water Gelation by Small Organic Molecules", Chem. Rev., 2004, 104, 1201-1218.

29. (a) Rodríguez-Llansola, F.; Escuder, B.; Miravet, J. F. "Switchable Perfomance of an LProline-Derived Basic Catalyst Controlled by Supramolecular Gelation", J. Am. Chem. Soc., 2009, 131, 11478-11484; (b) Rodríguez-Llansola, F.; Escuder, B.; Miravet, J. F. "Remarkable Increase in Basicity Associated with Supramolecular Gelation", Org. Biomol. Chem., 2009, 7, 3091-3094; (c) Rodríguez-Llansola, F.; Miravet, J. F.; Escuder, B. "A supramolecular Hydrogel as a Reusable Heterogeneous Catalyst for the Direct Aldol Reaction", Chem. Commun., 2009, 7303-7305.

30. (a) Hunter, C. A.; Anderson, H. L. "What is Cooperativity?", Angew. Chem. Int. Ed., 2009, 48, 7488-7499; (b) De Greef, T. F.; Smulders, M. M.; Wolffs, M.; Schenning, A. P.; Sijbesma, R. P.; Meijer, E. W. "Supramolecular Polymerization", Chem. Rev., 2009, 109, 5687-754.

31. Rodríguez-Llansola, F.; Miravet, J. F.; Escuder, B. "Supramolecular Gel Formation and SelfCorrection Induced by Aggregation-Driven Conformational Changes", Chem. Commun., 2009, 209-211.

32. Hirst, A. R.; Coates, I. A.; Boucheteau, T. R.; Miravet, J. F.; Escuder, B.; Castelletto, V.; Hamley, I. W.; Smith, D. K. "Low-Molecular-Weight Gelators: Elucidating the Principles of Gelation Based on Gelator Solubility and a Cooperative Self-Assembly Model", J. Am. Chem. Soc., 2008, 130, 9113-9121.

33. Escuder, B.; LLusar, M.; Miravet, J. F. "Insight on the NMR Study of Supramolecular Gels and its Application to Monitor Molecular Recognition on Self-Assembled Fibers", J. Org. Chem., 2006, 71, 7747-7752.

34. Nebot, V. J.; Smith, D. K., "CHAPTER 2 Techniques for the Characterisation of Molecular Gels". In Functional Molecular Gels. RSC, Cambridge, 2014; pp 30-66. 
35. de Loos, M.; van Esch, J.; Kellogg, R. M.; Feringa, B. L. "Chiral Recognition in Bis-UreaBased Aggregates and Organogels through Cooperative Interactions ", Angew. Chem. Int. Ed., 2001, 40, 613-616. 



\section{CHAPTER 4}

A SELF-ASSEMBLED L-PROLINE BASED AMPHIPHILIC CATALYST: POLYMORPHISM AND CATALYTIC STUDIES IN WATER 



\subsection{Introduction}

Molecular self-assembly has been of interest in recent years as an approach for the generation of novel nano-scale architectures and its use to fabricate advanced smartmaterials. Among the different strategies used to obtain such nanomaterials, low molecular weight gelators (LMWGs) have attracted the attention of scientists for applications in food science, cosmetics and medicine among others. ${ }^{1}$

Non-covalent interactions between gelator molecules drive self-assembly, which in turn controls the nanostructure favouring the formation of one-dimensional (1D) fibres. Gelation requires the formation of a continuous network structure where cross-links between the fibres are needed to form the matrix of the gel. These cross-links arise from entanglement of fibres, branching of fibres, etc. (see Figure 4.1.1). ${ }^{2}$

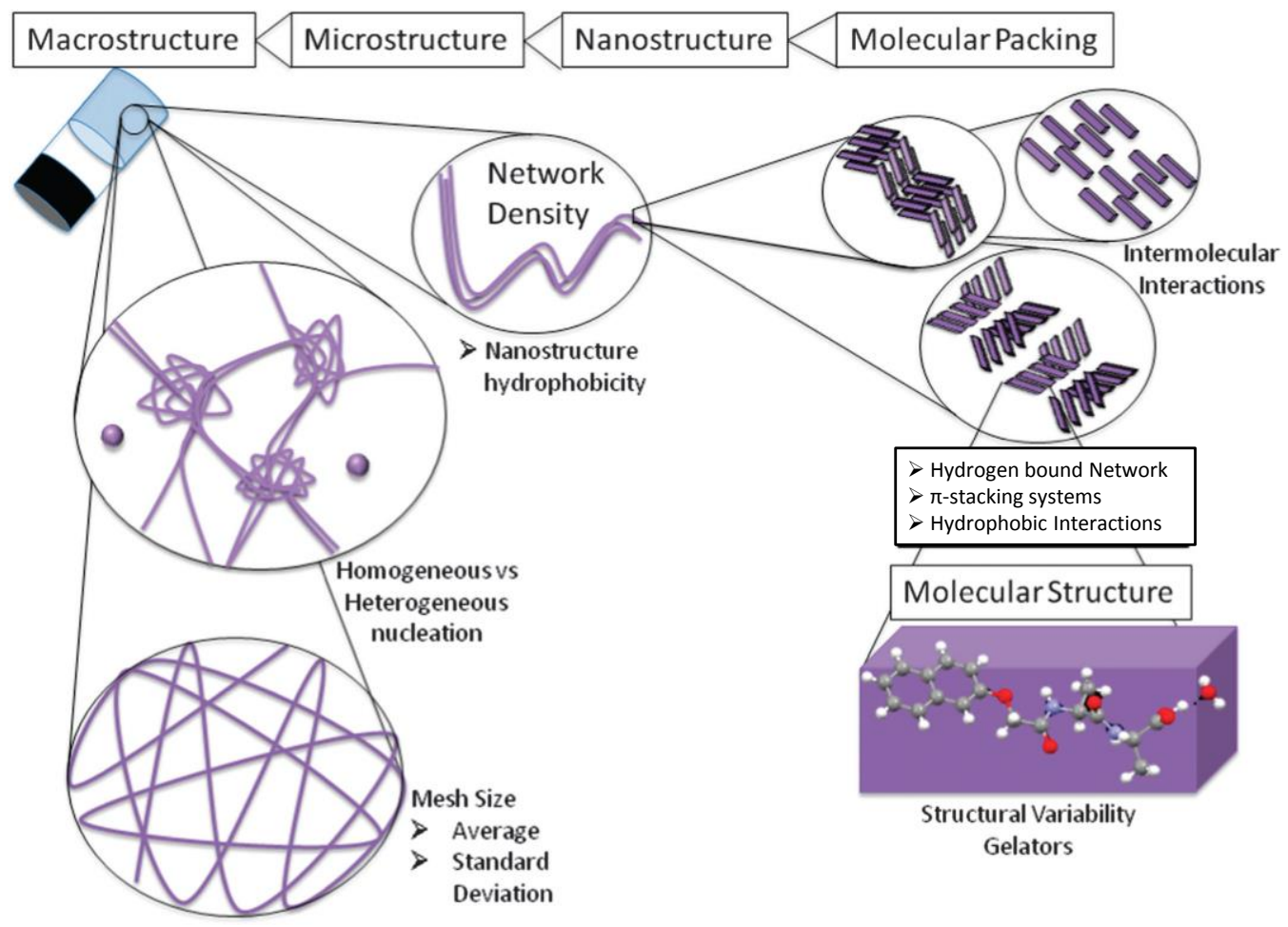

Figure 4.1.1. Schematic representation showing the different levels of assembly leading to gelation with three different molecular packing models that illustrate the possible variability. ${ }^{2}$

Gels with different morphologies have been described including fibres, helices, tapes, tubes and vesicles among others. ${ }^{3}$ Despite efforts by scientists to determine the rules that will produce a particular morphology, in some measure serendipity plays a role 
in leading the final morphology on a particular gelator molecule. Thus, morphology can be influenced by the different environmental parameters as well as by the methodology used for the gelation (temperature, $\mathrm{pH}$, aging, concentration, etc.) and the capture of different metastable (non-equilibrium) aggregates under kinetic control and their selfhealing ability into thermodynamically stable states. ${ }^{4}$

Adams et al. have recently highlighted the relevance of the gelation process (heating-cooling, pH switch, enzymatic control, etc.) on the mechanical properties of low molecular weight hydrogels. ${ }^{2}$ They schematically show how a single precursor could have different pathways for assembly and consequently obtain different gel morphologies (Figure 4.1.2). Changing the process will have a strong effect on the self-assembly pathway, the kinetics of assembly and as a result will directly affect the properties of the resulting gels.

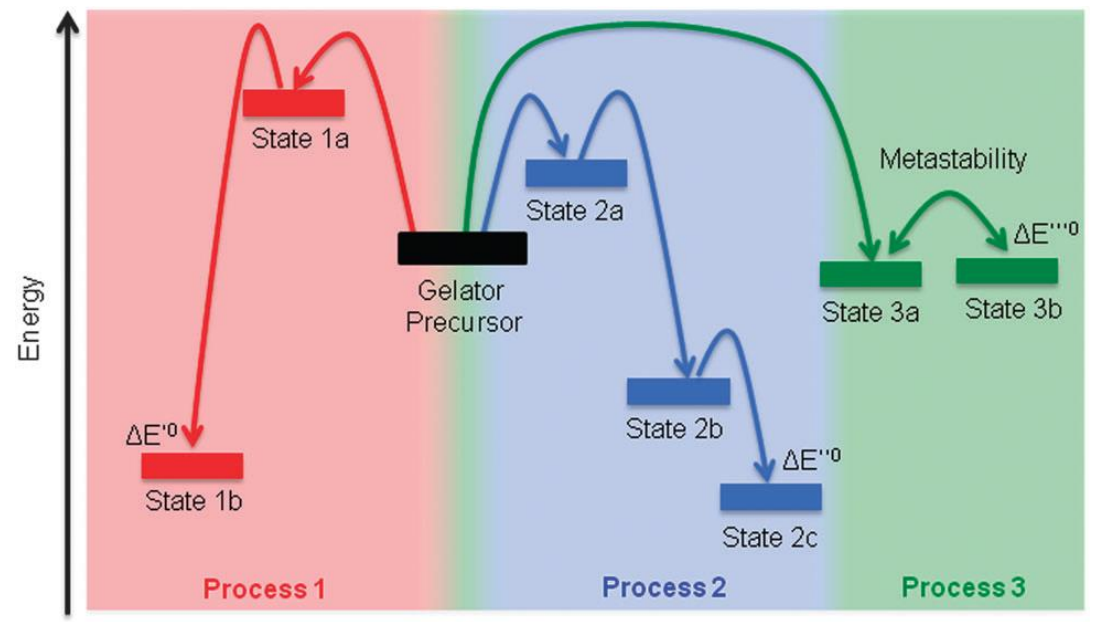

Figure 4.1.2. Different pathways to lead gelation where it can be shown that kinetically trapped states are sometimes formed rather than thermodynamic global minimun. ${ }^{2}$

For example Rybtchinski et al. described the kinetically controlled self-assembly of a single covalent building block in aqueous media, they found a pathway-dependent evolution of diverse molecular nanoscale arrays (fibres, helical structures and tube-like aggregates) based on the amount of water/THF, enabling stepwise assembly. ${ }^{5}$ 
(A)

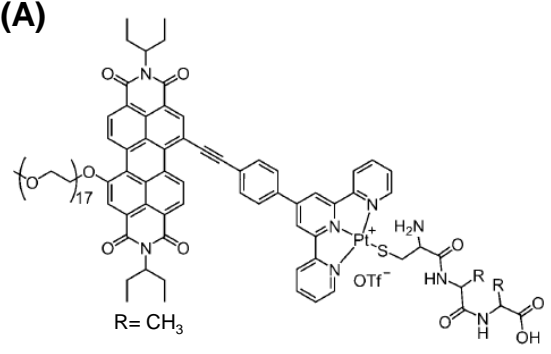

(C)

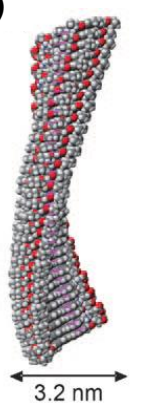

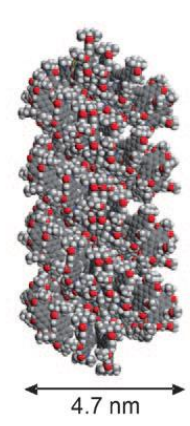

(B)

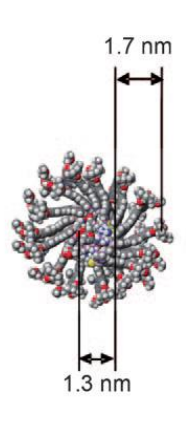

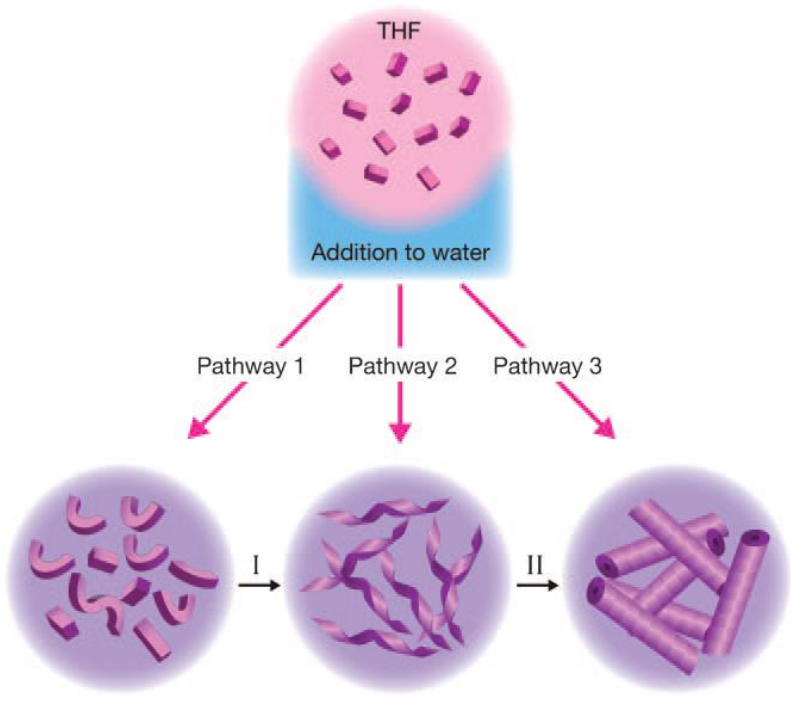

Figure 4.1.3. A) Molecular structure of the amphiphilic perylene diimide (PEG-PDI)/terpyridine platinum complexes with tripeptide Cys-Ala-Ala. B) Schematic illustration of a pathway-dependent self-assembly and C) Molecular models of the nanofibres in a single stack fibre or in a tube-like fibre. ${ }^{5}$

Polymorphism is very important in Chemical and Biochemical Science, especially in Materials Science, where the differences in the packing mode of a molecule can affect its properties as material and thus its final application. For example, there is considerable commercial interest in polymorph prediction from the pharmaceutical industry to avoid dosage, processing, and patent problems. ${ }^{6}$ Paracetamol, also known as acetaminophen, is a widely used antipyretic (fever suppressant) and analgesic (pain killer). Differences in the packing mode within crystal structure between its two metastable polymorphic forms (I and 11$)^{7}$ showed improvement of the compression properties, which is of particular industrial interest because the commercial form I requires binders for tablet formation. ${ }^{8}$

For LMWG, several examples on the influence of preparation procedures on polymorphism have been reported in our group. ${ }^{9}$ Heating-cooling rate, aging time and sample concentration have been shown to play a crucial role in this respect. Moreover, by altering these variables, metastable aggregates may also be formed that could further evolve and self-correct with time. ${ }^{9 b}$ All of these factors therefore could have an enormous effect on the performance of catalytic hydrogels with respect to activity and selectivity. 
(a)

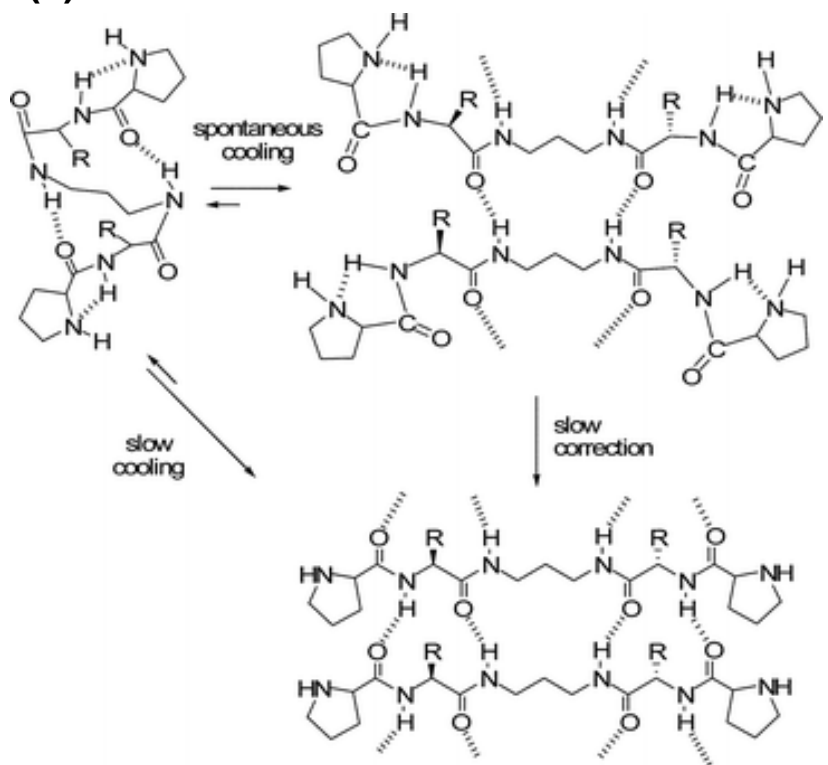

(b)

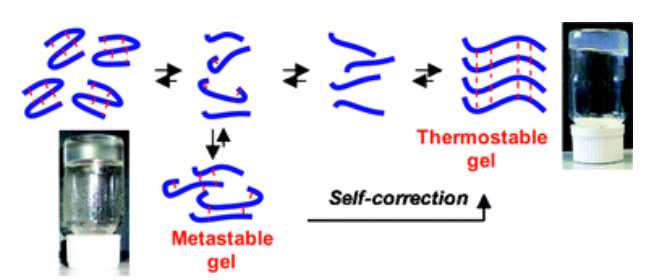

(c)

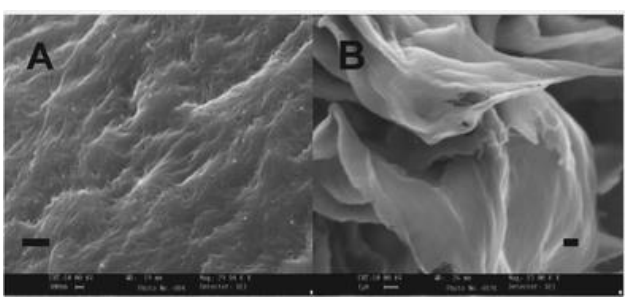

Figure 4.1.4. Scheme of the different self-assembling aggregates cooling-dependent and SEM images of the xerogels obtained in $\mathrm{CH}_{3} \mathrm{CN}$ on $\mathrm{A}$ ) spontaneous cooling and $\mathrm{B}$ ) slow cooling (Bars $\left.1 \mu \mathrm{m}\right)$. $\left(\mathrm{R}=-\mathrm{CH}_{2}\left(\mathrm{CH}_{3}\right)_{2}\right){ }^{9 \mathrm{~b}}$

\subsubsection{Supramolecular Catalysis in Water Mediated by Proline Derivatives}

Following nature and bio-inspired chemistry, the use of water as a versatile solvent for organic reactions has been one of the major challenges in recent years. The development of catalytic systems for organic transformations in aqueous media has prompted enormous interest from environmental, economic and safety points of view. Water is the most inexpensive and benign solvent, ${ }^{10}$ in many cases, due to hydrophobic effects, using water as a solvent not only accelerates reaction rates but also enhances reaction selectivity, even when the reactants are scarcely soluble or insoluble in this medium. ${ }^{11}$

Small-molecule-organocatalysed reactions in water with sufficient asymmetric induction would have immense synthetic utility. Among them, the organocatalysed aldol reaction in water is the most studied..$^{12}$ In the Type I aldolase enzyme, the reaction takes place in water through a hydrophobic active site, diminishing contacts between bulk water and the reactants. In trying to mimic natural enzymes behaviour, equipping the catalyst molecule with appropriate hydrophobic groups should facilitate assembly with organic substrates, protecting them from the aqueous media and providing a 
hydrophobic microenvironment. ${ }^{13}$ Two successful pioneering studies, one by Hayashi et al. ${ }^{12 a}$ (catalysts 1-4 Figure 4.1.1.1) and the other by Barbas et al. ${ }^{12 b}$ (catalyst 5 Figure 4.1.1.1), described very efficiently asymmetric proline-derived chiral amine catalysts, where an enamine intermediate for the aldol condensation in water was proposed. Aggregation of the organocatalyst and the reactants reduces contact with bulk water, by means of the incorporation of hydrophobic groups, as the reaction proceeds. Consequently, creating a hydrophobic pocket where the reaction can proceed will be similar to performing the same reaction in organic solvent. Once again, the use of amino acids for catalysis in water is particularly interesting as they are the building blocks of enzymes, which promote asymmetric reactions in nature. ${ }^{14}$<smiles>[R6]C1CNC(C(=O)O)C1</smiles>

$1 \mathrm{R}=\mathrm{TBS}$

$2 \mathrm{R}=\mathrm{TIBS}$

$3 R=$ TBDPS

$4 \mathrm{R}=\mathrm{H}$

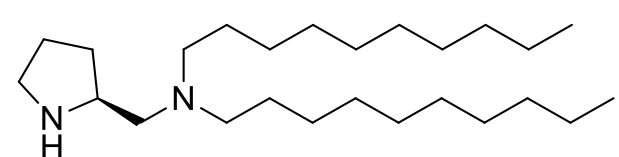

- TFA

5

Figure 4.1.1.1. Different examples of proline derivatives as catalyst for the aldol reaction in water.

In water, in the presence of water, and on water are expressions commonly found in recent publications, and they are often used interchangeably, to describe reactions that proceed under very different conditions. Sometimes, organic co-solvents and/or substrate modifications were employed, as it is assumed that solubility is required for efficient reaction in aqueous media. ${ }^{15}$ On the other hand, the term "on water", firstly defined by Sharpless et al. ${ }^{16}$ refers to organic transformation when insoluble reactant(s) are stirred in aqueous emulsions or suspensions without the addition of any organic cosolvents. Some advantages can be found in carrying out reactions in this manner. Firstly, water is an excellent heat sink because of its large heat capacity, making exothermic processes safer and more selective, especially when they are carried out on a large scale. Secondly, reactions of water-insoluble substrates usually lead to the formation of waterinsoluble products. In such cases, product isolation simply requires filtration of solid 
products (or phase separation in the case of liquids). Finally, the growing list of examples where reactions performed on water are not only faster but also more selective (whether chemo-, regio-, or enantio-) highlights the potential significance of reactions performed in this way. ${ }^{17}$

Therefore not only soluble but also heterogeneous systems have attracted the attention of researchers. For instance, different catalytic organic reactions have been carried out in aqueous media with organic and inorganic polymer supported catalysts, ${ }^{18}$ in micellar dispersions ${ }^{19}$ or emulsions (Figure 4.1.1.2) ${ }^{20}$ among others.

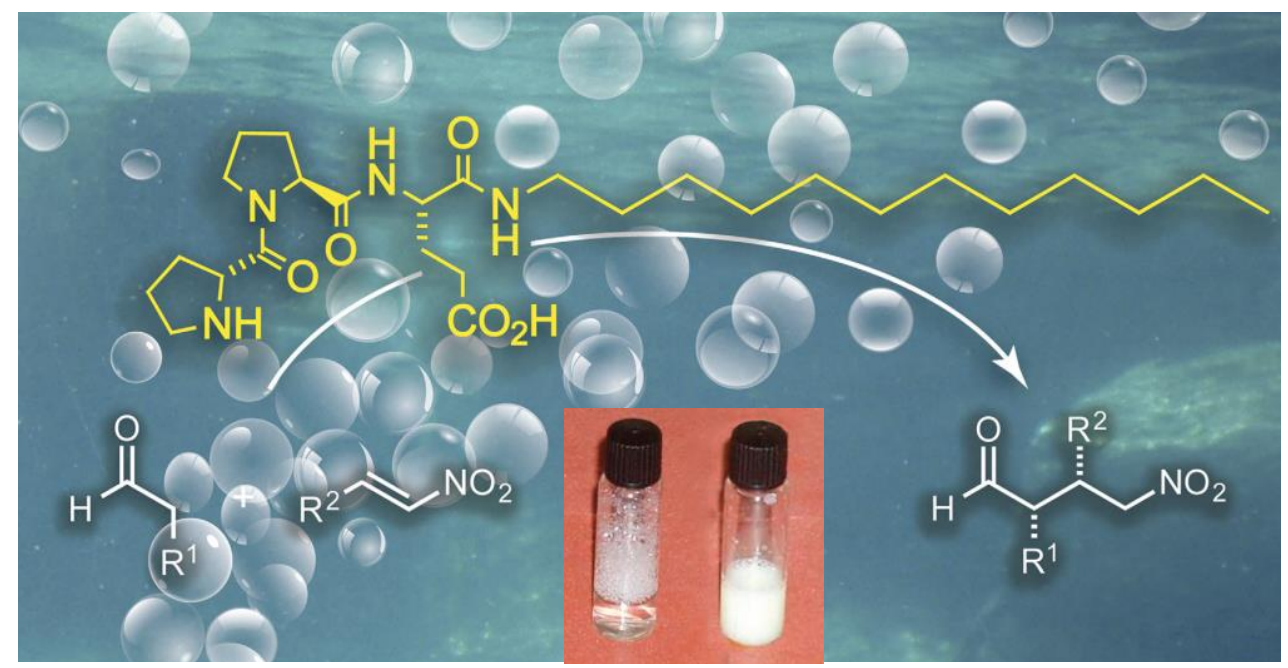

Figure 4.1.1.2. Example of proline amphiphile emulsions used as catalyst in water ${ }^{20}$

Self-assembly has been demonstrated as an effective approach to design catalysts that can work in aqueous media in an effective and selective way. For example the work reported by Liu et al., where the self-assembly of an amphiphilic L-proline derivative to form vesicles was studied in water under compressed $\mathrm{CO}_{2}$. Such structures have been proven to be used as a recyclable organocatalyst for the aldol reaction in water between cyclohexanone and 4-nitrobenzaldehyde with high enantiomeric selectivity (Figure 4.1.1.3). ${ }^{21}$ 


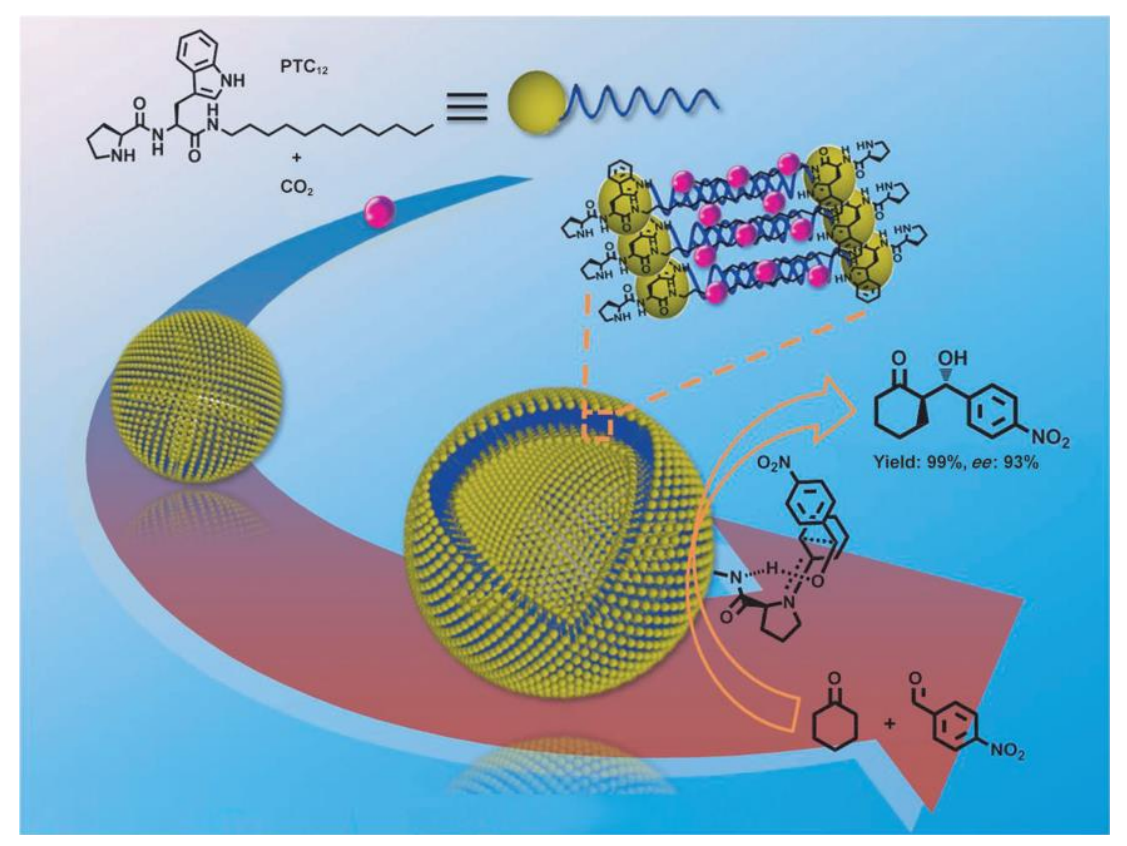

Figure 4.1.1.3. Schematic representation of vesicles formed by the self-assembling of the amphiphilic Lproline derivative under compressed $\mathrm{CO}_{2}$ and a proposed transition-state model for the aldol reaction. ${ }^{21}$

Using the self-assembly of L-proline derivatives as an approach for the design of effective organocatalysts, we propose to investigate the aggregation behaviour and catalytic activity study of a proline-based amphiphile in water. 


\subsection{Study on the Effect of Polymorphism on the Self-Assembly and Catalytic Behaviour of a L-Proline Based Hydrogelator}

Following the rational approach employed before to design new gelators with functionalised properties, compound ProValDoc (4a) was selected. As can be seen in Figure 4.2.1, a catalytic L-proline fragment was chosen and combined with a long chain alkyl fragment, with the aim of building an amphiphilic structure that can self-assemble in aqueous media. $^{22}$

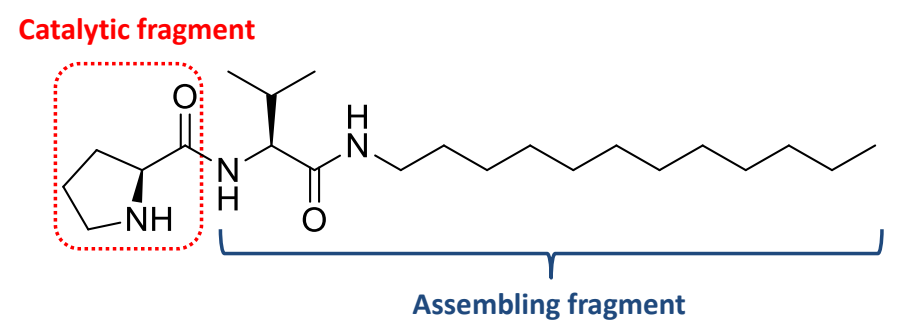

ProValDoc (4a)

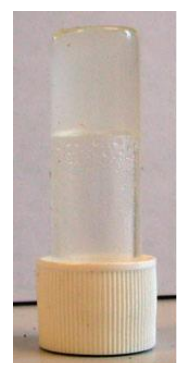

Figure 4.2.1. Molecular structure of the L-proline amphiphile derivative and the macroscopic aspect of the hydrogel (2 mM).

Previous studies performed with hydrogelator ProValDoc (4a) have been reported. ${ }^{23}$ It was found that this amphiphile can form hydrogels above a concentration of $2 \mathrm{mM}\left(0.8 \mathrm{mg} \cdot \mathrm{mL}^{-1}\right)$ upon being heated and then cooled to room temperature accompanied by $1 \mathrm{~min}$ of sonication.

(a)

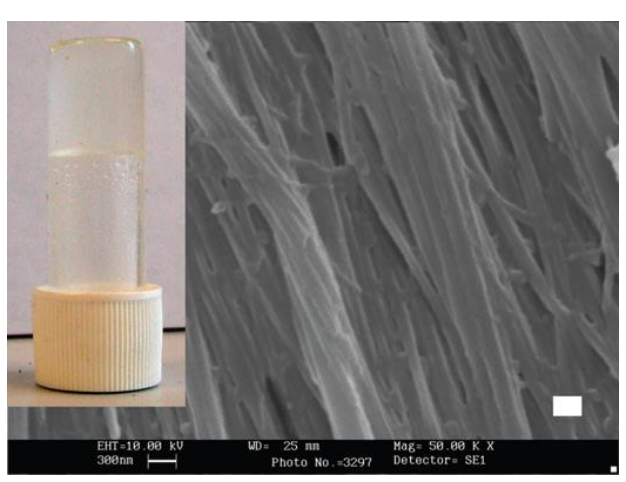

(b)

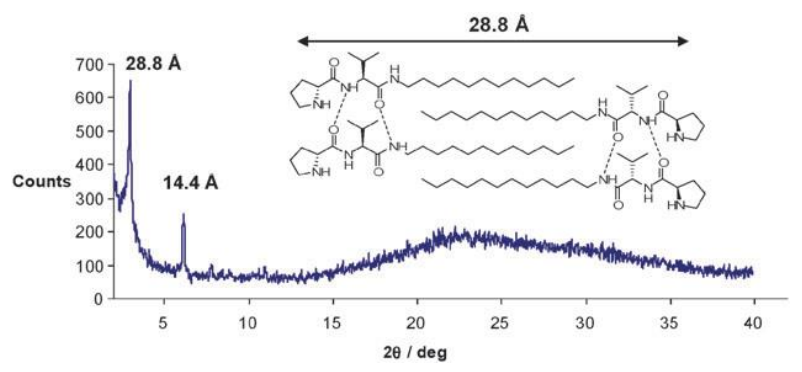

Figure 4.2.2. ProValDoc (4a) hydrogel, (a) Macroscopic and microscopic aspect (bar: $300 \mathrm{~nm}$ ), (b) X-Ray powder diffractogram of a dried sample and a possible bilayer structure. ${ }^{23}$ 
The morphology of the gel revealed the presence of a network of ribbons of several micrometres length. Furthermore, a lamellar structure in which compound ProValDoc (4a) forms a bilayer with intercalation of the alkyl tails was found in the X-Ray powder diffraction of the xerogel (see Figure 4.2.2).

In the same work, its behaviour as an efficient catalyst in the direct aldol reaction between cyclohexanone and 4-nitrobenzaldehyde was demonstrated. The hydrogel performed the reaction in toluene leading to quantitative yields of the aldol product with high diastereo and enantioselectivity even after 3 runs (Figure 4.2.3).<smiles>O=C1CCCCC1</smiles><smiles>O=C(I)c1ccc([N+](=O)[O-])cc1</smiles>

Hydrogel $4 a$ toluene<smiles>O=C1CCCC[C@H]1[C@H](O)c1ccc([N+](=O)[O-])cc1</smiles>

\begin{tabular}{cccc}
\hline Entry & Yield (\%) & anti:syn & e.e. ${ }^{*}$ (\%) \\
\hline Run 1 & 98 & $92: 8$ & 88 \\
Run 2 & $>99$ & $93: 7$ & 87 \\
Run 3 & $>99$ & $92: 8$ & 90 \\
\hline
\end{tabular}

(*) major enantiomer S,R-anti

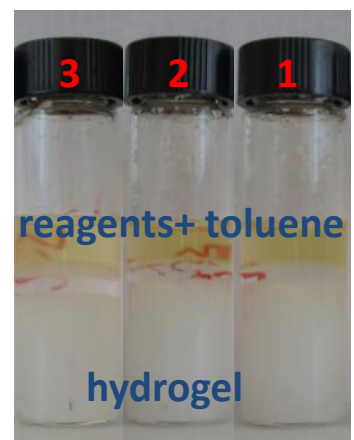

Figure 4.2.3. Previous catalytic results obtained for the ProValDoc (4a) as hydrogelator in the direct aldol reaction. $^{23}$ 


\subsubsection{Self-Assembly Studies}

During the previous studies of compound ProValDoc (4a) it was noticed that the gelation process sometimes failed, apparently under similar experimental conditions leading to weak hydrogels, dispersions or precipitates. These results prompted us to develop a detailed revision on the influence of the gelation process on the self-assembly and the morphologies resulted from varying slightly the procedures such as heating temperature, aging time, ultrasounds and $\mathrm{pH}$ switching.

To study the different self-assembled structures for the compound ProValDoc (4a), it was decided to keep the concentration fixed at $2 \mathrm{mM}$ in water $(3 \mathrm{mg} \cdot$ in $4 \mathrm{~mL}$ ), in this way concentration effects are avoided and only external stimuli would be responsible of the different patterns obtained. The whole set of experiments performed are summarised in Table 4.2.1.1. The procedure in all cases was to solubilise the compound in a silicone oil bath, controlling the heating temperature and the heating time. Additionally, experiments where a change in $\mathrm{pH}$ helps to solubilise the compound were also carried out, taking advantage of the ability of the compound to be protonated and deprotonated by means of the proline ring. After dissolution, the samples were left to reach equilibrium over a specific aging time at room temperature. Finally, ultrasound was applied in some samples as an additional stimulus (see Figure 4.2.1.1).

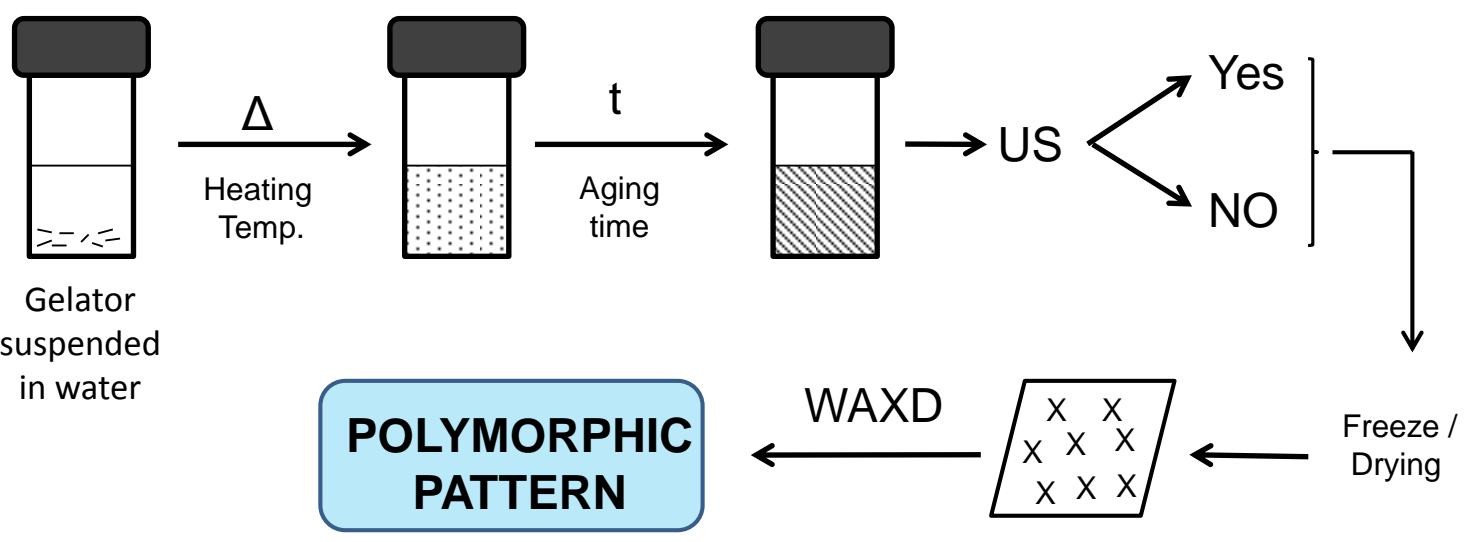

Figure 4.2.1.1. Scheme of the procedure used. 
Table 4.2.1.1. Summary of the aggregation behaviour of compound $4 a^{a}$

\begin{tabular}{|c|c|c|c|c|c|c|c|}
\hline $\begin{array}{c}\text { Entry } \\
(*)\end{array}$ & $\begin{array}{c}4 a \\
(\mathrm{mM})\end{array}$ & $\begin{array}{c}\text { Heating } \\
\text { Temp (으) }\end{array}$ & $\begin{array}{l}\text { Heating } \\
\text { time }\end{array}$ & $\begin{array}{l}\text { Aging } \\
\text { time }\end{array}$ & $\begin{array}{l}\text { US } \\
\text { time }\end{array}$ & Polymorph & Aspect $^{b, c}$ \\
\hline 1 & 2 & 100 & $2 \mathrm{~h}$ & $10 \mathrm{~min}$ & - & $B+a$ & Disp \\
\hline 2 & 2 & 100 & $2 \mathrm{~h}$ & - & - & B & Disp \\
\hline 3 & 2 & 100 & $16 \mathrm{~h}$ & $10 \mathrm{~min}$ & - & B & Disp \\
\hline 4 & 2 & 100 & $16 \mathrm{~h}$ & - & - & B & Disp \\
\hline 5 & 2 & 100 & $2 \mathrm{~h}$ & - & $10 \mathrm{~min}$ & $A+b$ & Disp \\
\hline 6 & 2 & 100 & $2 \mathrm{~h}$ & - & $2 \mathrm{~h}$ & A & Disp \\
\hline 7 & 2 & 100 & $2 \mathrm{~h}$ & $2 \mathrm{~h}$ & $1 \mathrm{~min}$ & $A+B$ & WG \\
\hline 8 & 2 & 100 & $2 \mathrm{~h}$ & $2 \mathrm{~h}(80 \circ \mathrm{C})$ & - & B & $\mathrm{S}$ \\
\hline 9 & 2 & 80 & $2 \mathrm{~h}$ & $10 \min$ & - & $A+C+b$ & $\mathrm{~S}$ \\
\hline 10 & 2 & 80 & $15 \mathrm{~min}$ & - & - & $A+b$ & $\mathrm{~S}$ \\
\hline 11 & 2 & 80 & $2 \mathrm{~h}$ & - & - & $B+a$ & $S$ \\
\hline 12 & 2 & 80 & $15 \mathrm{~min}$ & - & $10 \mathrm{~min}$ & $A+C$ & $\mathrm{~S}$ \\
\hline 13 & 2 & - & - & - & $2 \mathrm{~h}$ & $A$ & Disp \\
\hline 14 & - & - & - & - & - & $A+b$ & solid \\
\hline 15 & - & Melt (A) & & & & $A+B$ & solid \\
\hline 16 & $2(\mathrm{pH} 7)$ & - & - & $10 \mathrm{~min}$ & - & D & $\mathrm{S}$ \\
\hline 17 & $2(\mathrm{pH} 8)$ & - & - & $10 \mathrm{~min}$ & - & D & $\mathrm{S}$ \\
\hline 18 & $4(\mathrm{pH} 8)$ & - & - & $18 \mathrm{~h}$ & - & A & G \\
\hline 19 & $5(\mathrm{pH} 8)$ & - & - & $10 \mathrm{~min}$ & - & $A+d$ & $\mathrm{G}$ \\
\hline
\end{tabular}

[a] Major component polymorphs: A, B, C and D; minor component polymorphs: a, b, c and d. See ESI for WAXD graphs.

[b] Macroscopic aspect: G, gel; WG: weak gel; Disp: dispersion; S: suspension.

[c] Dispersions are converted into weak gels after long time (16-18h).

$\left.{ }^{*}\right)$ The number on the entry corresponds to the number of WAXD pattern in Supporting Information (see Figure SI_4.5.1-4).

The macroscopic aspect of the samples was then studied. The samples obtained resulted in dispersions, suspensions or gels. Afterwards, all samples were frozen in liquid nitrogen and lyophilised to obtain a xerogel that was studied by wide-angle X-ray diffraction (WAXD). As can be seen in the Table 4.2.1.1, four different patterns of polymorphs were found (A-D) mainly as a mixture. In some cases it was possible to isolate single polymorphs to be characterised individually, as shown in Figure 4.2.1.2. 
(a)

(c)
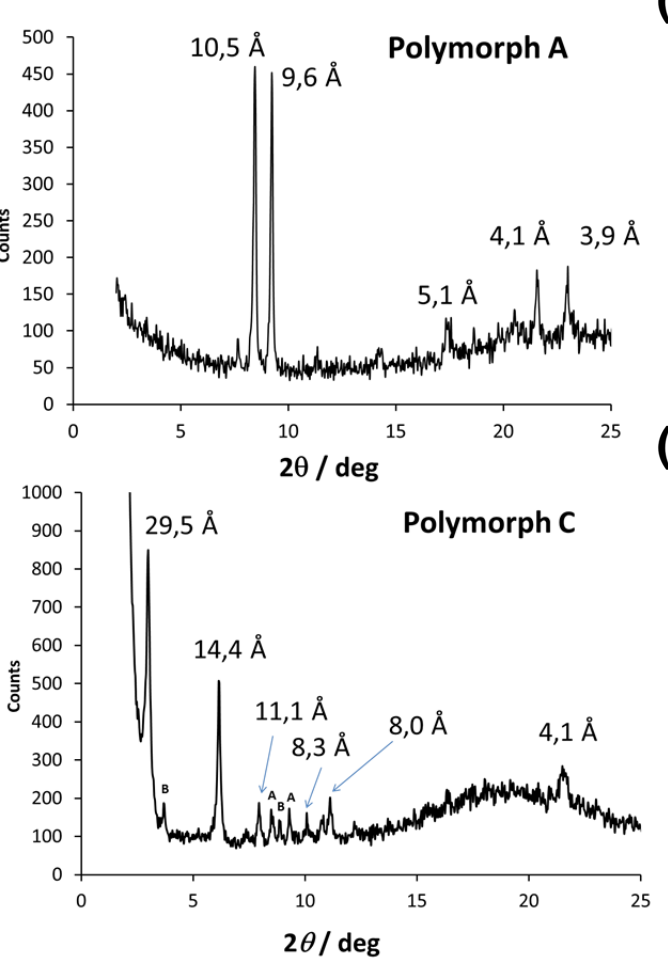

(b)
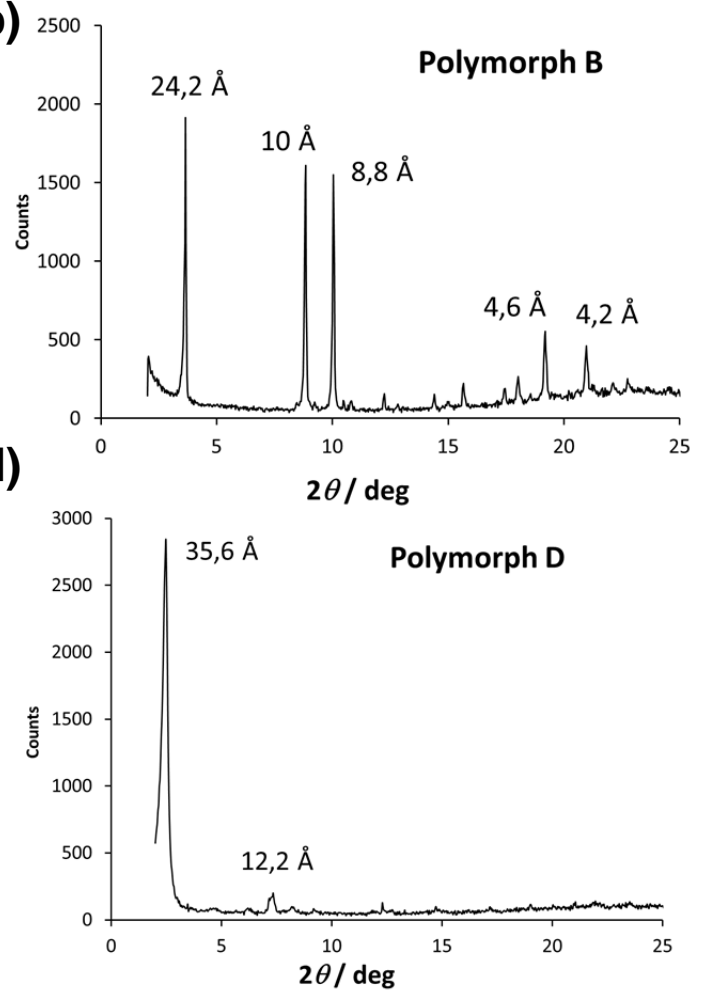

Figure 4.2.1.2. Wide-angle X-ray diffraction (WAXD) patterns for polymorphs A-D.

To study how slight changes in the experimental procedure affect the aggregation packing inside the gel network, a set of samples were prepared in water, heating at 100 oc (Table 4.2.1.1 Entries 1-8). Heating was maintained for either $2 \mathrm{~h}$ or $16 \mathrm{~h}$ and then samples were lyophilised immediately or after being aged at 25 으 for a period of time. The macroscopic aspect of the samples was that of a translucent dispersion (Entries 1-4, Figure SI_4.5.8) resulting mainly in the formation of polymorph B (Figure 4.2.1.2.b). Additionally, heating the sample during the aging time for $2 \mathrm{~h}$ did not lead to any change in the preference for the polymorph obtained, also B in this instance (Entry 8). Once the samples were introduced to ultrasound for an additional period of time, a jelly-like suspension was observed (Entries 5-7) and polymorph A appeared (Figure 4.2.1.2.a). These results suggest that polymorph $\mathbf{B}$ is favoured by long heating time and polymorph $\mathbf{A}$ appears as the major component after 10 minutes of ultrasound. Experimental conditions could be set up to obtain samples with pure A (Entry 6) and B (Entries 2-4 and 8).

Secondly, the heating temperature was reduced to 80 ㄷ (Entries 9-12) and under certain conditions a new polymorph appeared (Entry 9), polymorph C (Figure 4.2.1.2.c) 
together with additional polymorphs. Again, long heating times without aging at 25 o $\mathrm{C}$ favoured polymorph B (Entry 11) whereas short heating times and ultrasound favoured polymorph $\mathbf{A}$ (Entries 10 and 12 ). All samples remained as jelly suspensions.

It seems reasonable to propose that polymorph $\mathbf{A}$ is the one favoured under thermodynamic control (long aging time) whereas polymorph $\mathbf{B}$ is favoured at high temperatures (long heating time). Therefore B polymorph would be kinetically trapped, as a metastable structure, by fast cooling either to 25 o C (Entry 3) or freezing with liquid nitrogen (Entry 4). On the other hand polymorph $\mathbf{C}$ could not be found in its pure form as it is a metastable structure with a small energy barrier, thus it always appears as a mixture of polymorphs with $\mathbf{A}$ and $\mathbf{B}$.

The effect of ultrasound alone was also studied. Sonochemical effects have being widely described in self-assembled systems with unexpected changes in packing/crystallization resulting in different crystalline polymorphs or in nanomaterials with variable shape and size. ${ }^{24}$ Ultrasound can induce the process of crystallographic nucleation and growth, breaking the existing aggregates into "infecting" seeds that act as templates for the growth of new aggregates. In the current case, ultrasound could not only led to pure polymorph A (Entry 13) but could also provide enough energy to overcome the kinetic barrier for the conversion between polymorphs B and A (for example comparing Entry 2 with Entry 6).

The pure starting solid was also analysed by WAXD directly from the synthesis batch (Entry 14) giving mainly polymorph $\mathbf{A}$ with a small amount of $\mathbf{B}$, and after a meltingcooling cycle (melting on a glass slide and fast cooling to room temperature, Entry 15), the amount of kinetically trapped B increased considerably.

Finally, a set of tests triggering gelation by means of changing the $\mathrm{pH}$ was performed. To accomplish this, ProValDoc (4a) was dissolved by the addition of 1 equivalent of $\mathrm{HCl}$ with slight heating. These samples were further diluted with buffer solutions at $\mathrm{pH} 7$ and 8 (Entries 16-19). At low concentration (2 mM) a suspension was formed (see Figure SI_4.5.8) and a new diffraction pattern was observed, polymorph D (Figure 4.2.1.2.d). This last pattern was predominant at either $\mathrm{pH} 7$ and 8 (Entries 16 and 17). Higher concentrations were required for gelation at $\mathrm{pH} 8(>4 \mathrm{mM})$ but the gelation 
process gave rise to the formation of polymorph $\mathbf{A}$ at the expense of polymorph $\mathbf{D}$ (Entries 18-19).

Remarkably, polymorph $\mathbf{D}$ was obtained at $\mathrm{pH}$ values close to the $\mathrm{pK}_{\mathrm{a}}$ of the molecule. The $\mathrm{pK}_{\mathrm{a}}$ of the compound ProValDoc (4a) was determined by potentiometric titration and it was found to be 6.4 at $1 \mathrm{mM}$ (below MGC) and 6.0 at $5 \mathrm{mM}$ (above MGC) (Figure SI_4.5.9). These values are considerably shifted from what is expected for the proline amino acid unit $\left(\mathrm{pK}_{\mathrm{a}} \mathrm{ca}\right.$. 10.6). Additional studies with a soluble propylamide analogue of ProValDoc (4a), the compound ProValPr (3b) were performed. Potentiometric titration showed a $\mathrm{pK}_{\mathrm{a}}$ value of $c a$. 8.8 for a solution of ProValPr (3b) in water. This shifted $\mathrm{pK}_{\mathrm{a}}$ may be explained as a consequence of a decrease in the solubility and thus an increase in the hydrophobic interactions for the compound with a long twelve carbon tail.

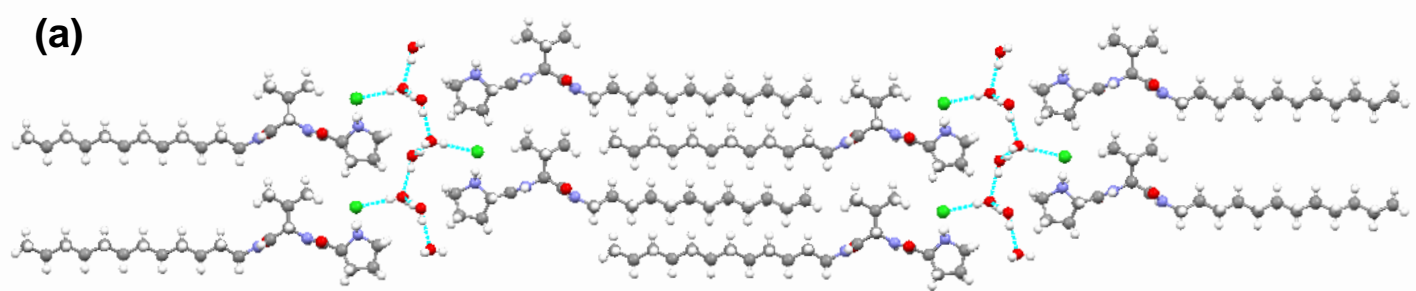

(b)

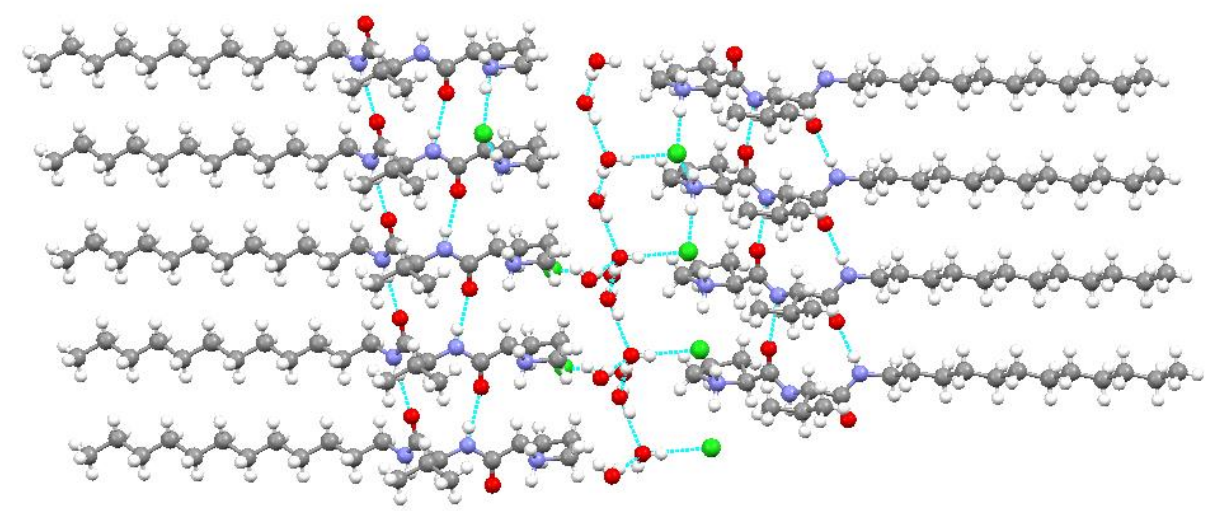

Figure 4.2.1.3. Single crystal X-Ray packing of ProValDoc (4a) $\cdot \mathbf{H C l} \cdot 2 \mathrm{H}_{2} \mathrm{O}$.

Single crystals of ProValDoc (4a) were obtained for the hydrochloride dihydrate species (Figure 4.2.1.3 and Figure SI_4.5.5), although the catalyst appears protonated, valuable information on the structural characteristics of the gel phase may be extracted. An intercalated bilayer structure was formed in which the alkyl chains appeared closely 
packed by van der Waals and hydrophobic interactions (Figure 4.2.1.3.a). Moreover, polar dipeptidic heads form a parallel $\beta$-sheet-like structure with intermolecular $\mathrm{H}$-bonds between alkyl amide $\mathrm{NH}$ and valine carbonyl group $(\mathrm{N}-\mathrm{H} \cdots \mathrm{O}=\mathrm{C}, 2,837 \AA, 173,17 \AA$ ) and between valine amide $\mathrm{NH}$ and proline carbonyl group ( $\mathrm{N}-\mathrm{H} \cdots \mathrm{O}=\mathrm{C}, 2,910 \AA ⿻$, 164,68 $\AA$ ). Packing is completed by chloride counter ions and a layer of water molecules that forms an interface with neighbouring bilayers (Figure 4.2.1.3.b). This layered structure resembles that proposed previously from powder diffraction data of xerogels of ProValDoc (4a) (see Figure 4.2.2) and, although the conformation of the head groups is likely to be different, the hydrophobic inner regions should be similar.

Interestingly, it was found that the main low angle diffraction peak of polymorph D (Figure 4.2.1.2.d) was coincident with the 001 diffraction plane distance found for the simulation of the powder diffraction pattern from the X-Ray single crystal (Figure SI_4.5.6) of compound ProValDoc (4a) $\cdot \mathbf{H C l} \cdot 2 \mathrm{H}_{2} \mathrm{O}$ in aqueous acidic solution. It could be estimated that at $\mathrm{pH} 7$ there is about a $20 \%$ of compound ProValDoc (4a) in a protonated state. These results may suggest that few of those crystals, formed by the compound in the protonated state, could act as seeds for the aggregation growth within the fibrillar network in a similar structure, leading to polymorph $\mathbf{D}$, highly oriented in the direction of the 001 diffraction plane. A similar effect was observed at pH 8 at $2 \mathrm{mM}$ (Entry 17 Table 4.2.1.1). On the contrary, polymorph $\mathbf{D}$ tended to disappear at higher concentrations (Entries 19 and 20 Table 4.2.1.1) where the seeding effect of the crystals would be less effective by means of aggregation effects toward the formation of the polymorph $\mathbf{A}$ that leads to a hydrogel at these concentrations.

Regarding the thermal interconversion between polymorphs $\mathbf{A}$ and $\mathbf{B}$, polymorphs are classified into two types, monotropes and enantiotropes, depending on the range of temperatures and pressures that they are stable. If there are different polymorphs, each one stable at a different temperature range and pressure, then the polymorphs are said to be enantiotropes. On the other hand, sometimes only one polymorph is stable at all temperatures (below the melting point) with all the other polymorphs being unstable. These polymorphs are said to be monotropes. Both types of polymorphs can be easily understood by looking at the Figure 4.2.1.4. For an enantiotropic system (Figure 4.2.1.4.a), polymorph $I$ is stable below the transition temperature $\left(T_{t}\right)$, since its free 
energy $\left(G_{l}\right)$ is lower than that of polymorph II $\left(G_{\|}\right)$. For temperatures above $T_{t}$, a reversible transition can be observed at which the free energy curves cross, before the melting point is reached, and free energy of polymorph II becomes less than that for polymorph I $\left(G_{\| \mid}<G_{1}\right)$. Whereas for monotropic systems (Figure 4.2.1.4.b), there is a stable polymorph (polymorph I) that has a lower free energy throughout the temperature range below the melting point. For this system, the free energy curves do not cross, and therefore no reversible transition can be observed, the polymorph with the higher free energy curve and solubility is the unstable polymorph (polymorph II). ${ }^{25}$

a) Enantiotropic system

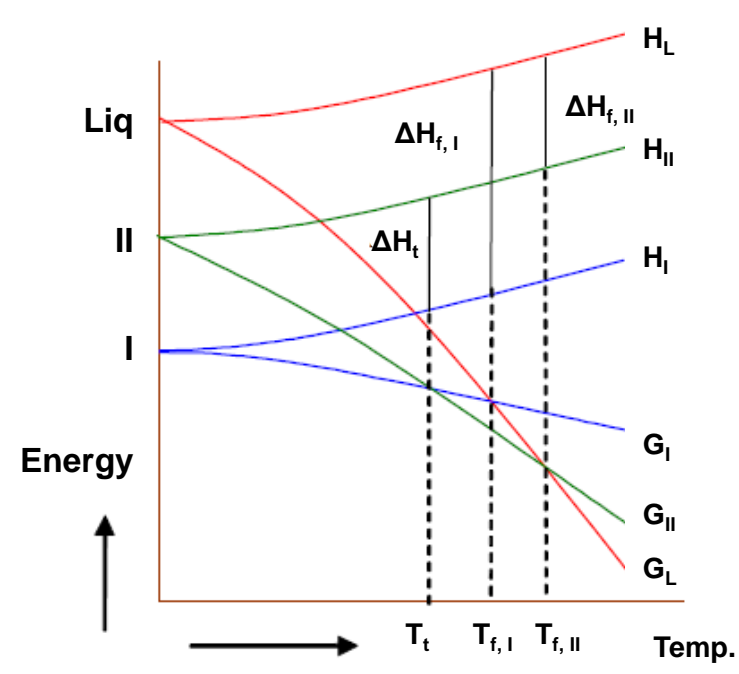

b) Monotropic system

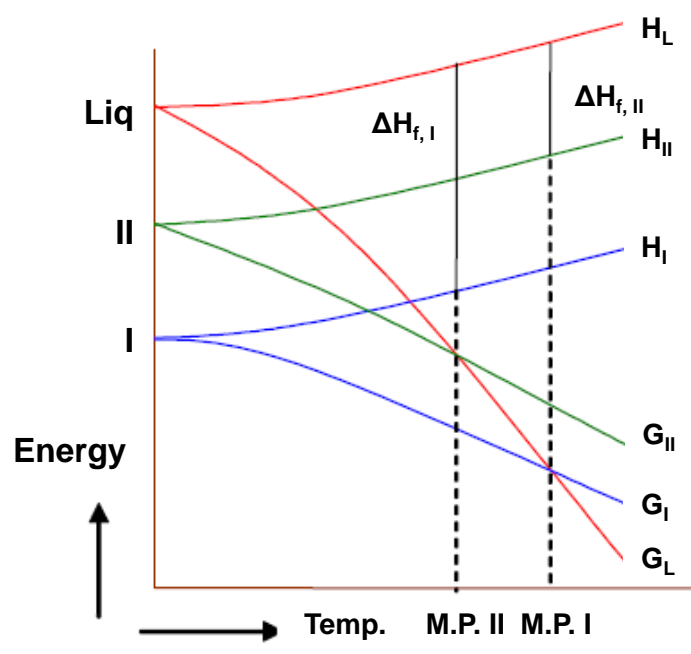

Figure 4.2.1.4. Variation of energy with temperature for enantiotropic and monotropic systems $\left(\Delta \mathrm{H}_{\mathrm{f}, \mathrm{l}}\right.$ and $\Delta \mathrm{H}_{\mathrm{f}, \mathrm{II}}$ represents enthalpy of fusion and $\Delta \mathrm{H}_{\mathrm{t}}$ represents enthalpy of transformation. ${ }^{25 b}$

With respect to thermodynamics, for a polymorphic phase transition, the thermal event can be either endothermic or exothermic depending on the nature of the transition. Enantiotropic transitions are reversible and are normally observable as endothermic events in the heating cycle and exothermic events in the cooling cycle. Monotropic transitions are irreversible and can be observed as exothermic events. ${ }^{25 a}$

The thermodynamics of interconversion between polymorphs $\mathbf{A}$ and $\mathbf{B}$ were studied by Differential Scanning Calorimetry (DSC). As can be seen in Figure 4.2.1.5 for polymorph $\mathbf{A}$, the heating curve showed an endothermic transition at $71 \stackrel{\circ}{\circ}$ and a melting peak at 101 o $\mathrm{C}$ (a melting point requires energy flowing to the sample and is thus 
endothermic too). After cooling, the transition peak (exothermic) cannot be seen due to hysteresis (refers to systems that have memory, and therefore the dependence of the response in a system not only in the current action, but also on its history of past actions and as a consequences in a delay). This type of endothermic transition is therefore related to enantiotropic transition between polymorphs $\mathbf{A}$ and $\mathbf{B}^{25 a}{ }^{26}$ These two polymorphs are in equilibrium and their relative population depends on temperature, polymorph $\mathbf{B}$ being formed at high temperature and polymorph $\mathbf{A}$ obtained after slow cooling or equilibration at room temperature.
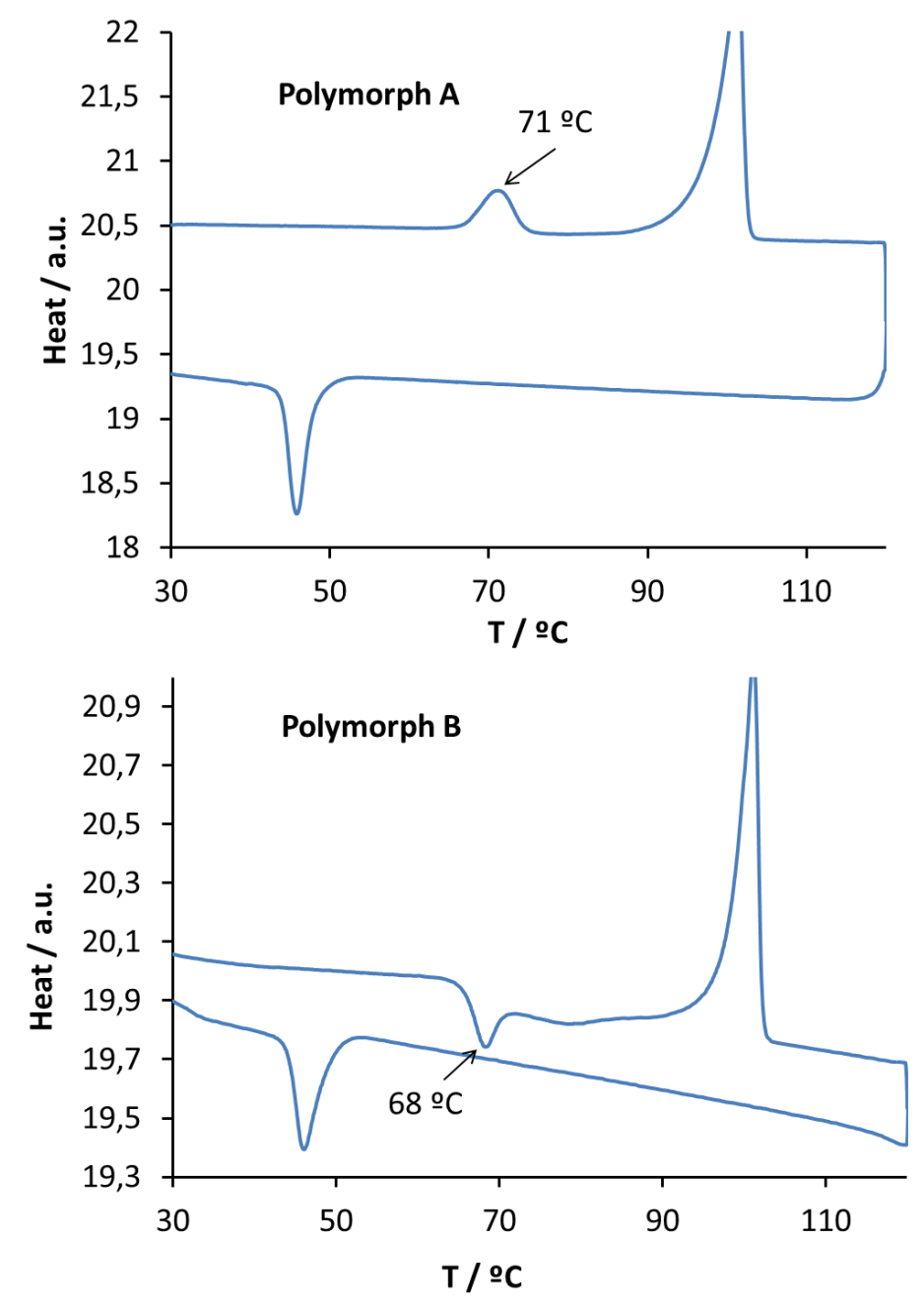

Figure 4.2.1.5. Differential Scanning Calorimetry (DSC) analysis for polymorphs $\mathbf{A}$ and $\mathbf{B}$.

On the other hand, the DSC diagram of pure polymorph B, kinetically captured at high temperature, showed a heating curve with an exothermic transition at $c a .68 \stackrel{\circ}{\circ}$ and a melting peak at around $101 \stackrel{\circ}{\circ}$. This transition corresponds to the conversion of 
polymorph $\mathbf{B}$ back to polymorph $\mathbf{A}$ after overcoming the energetic barrier between $\mathbf{B}$ and A. This can be proven by analysis of a polymorph B sample captured in the DSC study in

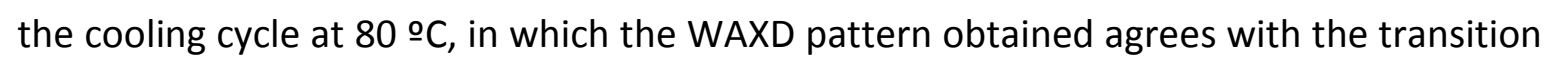
to the polymorph $\mathbf{A}$ (see Figure SI_4.5.7). In this case the transition is exothermic because polymorph $\mathbf{A}$ is more stable at low temperatures and so the system loses energy when it goes from polymorph $\mathbf{B}$ to $\mathbf{A}$. The exothermic peak that appeared at $c a .45 \stackrel{\circ}{ } \mathrm{C}$ and in both graphs belongs to the transition from liquid to solid state shifted by hysteresis.

Finally, a full spectroscopic characterization was carried out for the different polymorphs. Spectroscopic techniques -circular dichroism and IR-have been used to find structural differences between polymorphs. CD spectra showed no main difference between polymorph $\mathbf{A}$ and $\mathbf{B}$ suggesting a similar environment for the amide chromophore head in both polymorphs (Figure 4.2.1.6). This result is in agreement with the FTIR, where the absorption in the amide I region $(C=0)$ was monitored (Figure 4.2.1.7 bottom). It was found that both polymorphs present similar bands at $1640 \mathrm{~cm}^{-1}$. However, the $\mathrm{CH}_{2}$ stretching vibration bands revealed some slight differences between polymorph A and $\mathbf{B}$ (see Figure 4.2.1.7 top), pointing to different packing modes of the alkyl tails. ${ }^{27}$

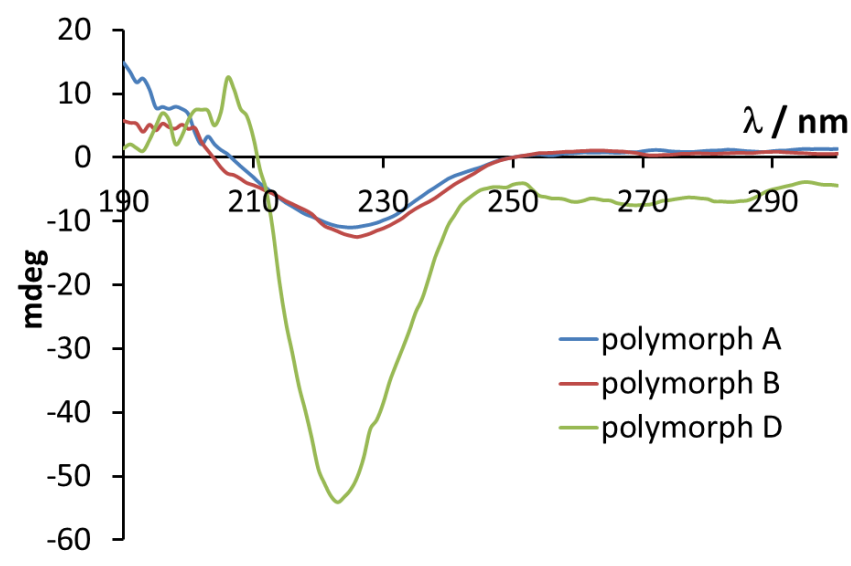

Figure 4.2.1.6. Circular Dichroism (CD) of $\mathrm{KBr}$ pellets of polymorphs $\mathbf{A}, \mathbf{B}$ and $\mathbf{D}$.

On the contrary, the analysis of polymorph $\mathbf{D}$ showed alkyl packing similar to polymorph B (similar $\mathrm{CH}_{2}$ stretching vibrational bands in Figure 4.2.1.7 bottom) but slight differences in the position of the amide I region band $(\mathrm{C}=\mathrm{O}$ stretching band Figure 4.2.1.7 top) that was shifted $5 \mathrm{~cm}^{-1}$ to higher wavelengths suggesting weaker hydrogen bonds. 
Additionally, a stronger CD effect was observed for polymorph $\mathbf{D}$, pointing to a higher rigidity in the dipeptide region for these aggregates (Figure 4.2.1.6).
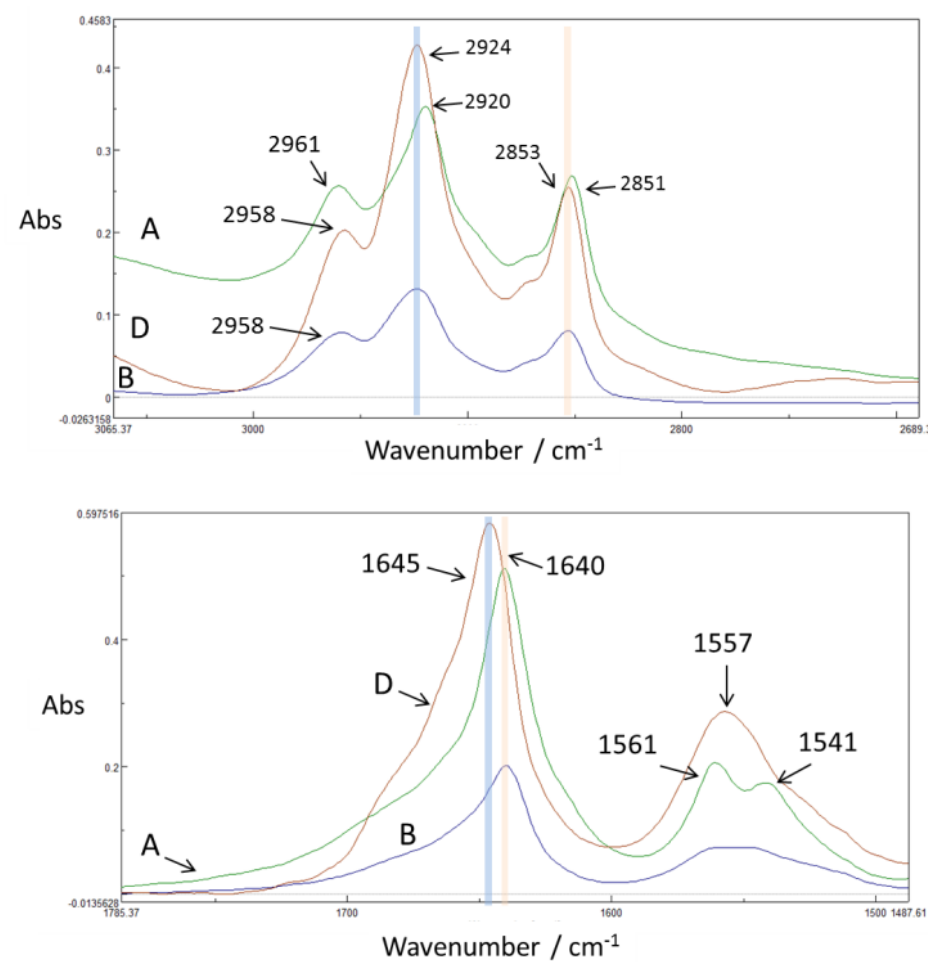

Figure 4.2.1.7. FTIR of $\mathrm{KBr}$ pellets of polymorphs $\mathbf{A}$, B and $\mathbf{D}$.

The structural differences between polymorphs were revealed the microscopic level by TEM and SEM. Polymorph A (Figure 4.2.1.8.A and B) showed a network of long and wide fibres of $c a .30$ nanometres width. On the contrary, polymorph B (Figure 4.2.1.8.C and D) showed a highly dense network made of very thin and short fibres of $c a$. $10 \mathrm{~nm}$ of width. These slight microscopic differences could influence on the ability of each polymorph to form hydrogels: polymorph $\mathbf{A}$ forms gels immediately after sonication whereas polymorph B needs additional aging time to give a self-sustainable gel. Microscopy images of polymorph D (Figure 4.2.1.8.E and F) showed the presence of long and flat aggregated structures, tens of micrometres in length formed by the alignment of thin tape shaped aggregates of up to 10 nanometres in width following a preferred direction. In this case the fibrillar network is not strong enough to hold the solvent and the gelation process does not take place (see Figure SI_4.5.8). 

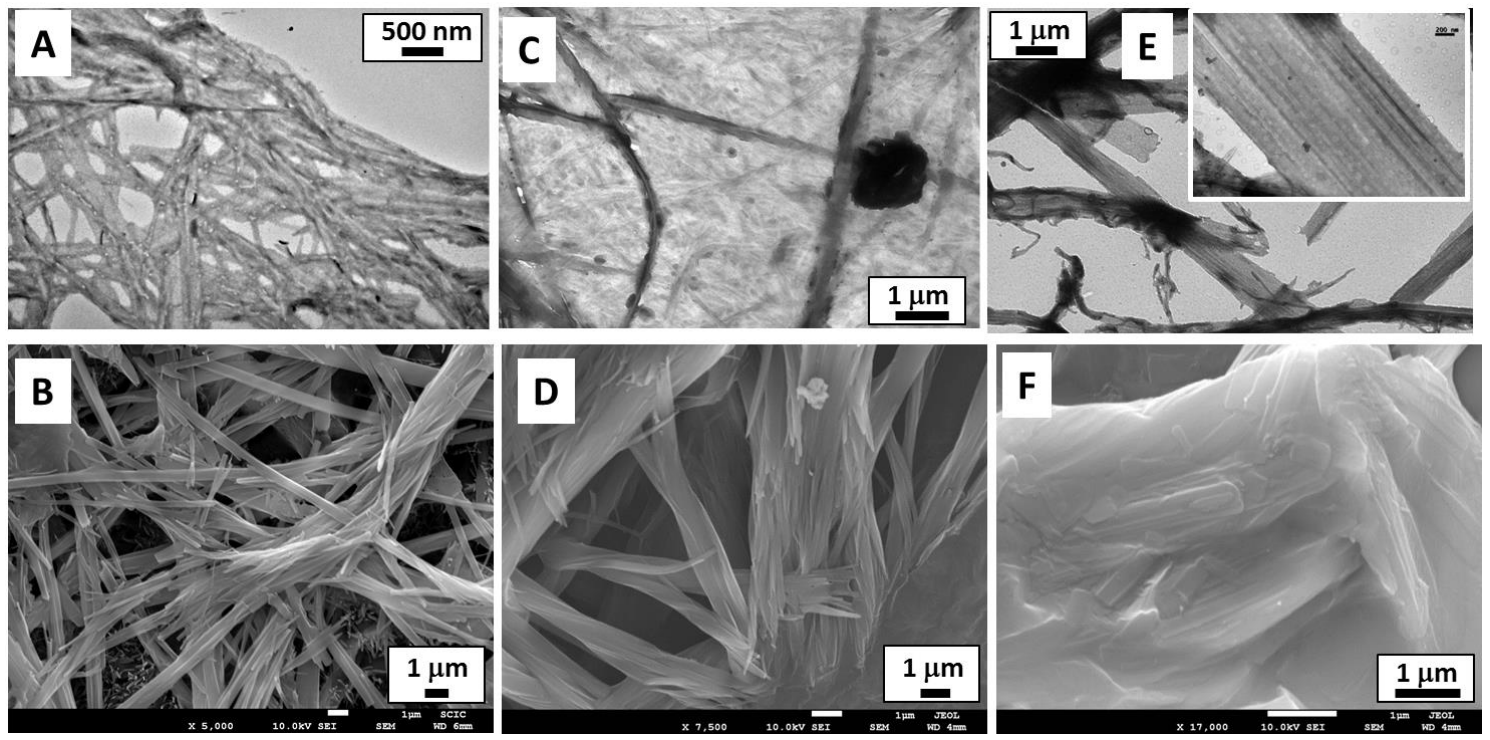

Figure 4.2.1.8. TEM (top) and FESEM (bottom) images of polymorphic aggregates $\mathbf{A}$ ( $A$ and $B$ ), $\mathbf{B}$ ( $C$ and $D)$ and $\mathbf{D}(\mathrm{E}$ and $\mathrm{F})$.

\subsubsection{Effect of Polymorphism in Catalysis}

The next step in this study was to check the catalytic performance of the different polymorphs found and characterised (A, B and D) in the coupling reaction of cyclohexanone and 4-nitrobenzaldehyde (see Figure 4.2.2.1).<smiles>O=C1CCCCC1</smiles><smiles>O=Cc1ccc([N+](=O)[O-])cc1</smiles><smiles>[GeH3]O[GeH3]</smiles><smiles>O=C1CCCC[C@H]1[C@H](O)c1ccc([N+](=O)[O-])cc1</smiles>

Figure 4.2.2.1. Scheme of the direct aldol reaction studied.

The aggregated catalysts were prepared following the conditions of Entries 6 (polymorph A), Entry 3 (polymorph B) and Entry 16 (polymorph D) in Table 4.2.1.1 respectively. The reactants were then added on the top of the gel (see Experimental Section for details) and the reactions were left $24 \mathrm{~h}$ at room temperature yielding the aldol product. 
Table 4.2.1.2. Summary of the catalytic results at $25^{\circ} \mathrm{C}$ after $24 \mathrm{~h}^{\mathrm{a}}$

\begin{tabular}{cccc}
\hline Polymorph & Yield (\%) $^{\text {b }}$ & $\begin{array}{c}\text { d.r. } \\
\text { (anti:syn) }^{\text {b }}\end{array}$ & e.r. $^{\text {c }}$ \\
\hline A & $>95$ & $86: 14$ & $85: 15$ \\
B & $>95$ & $85: 15$ & $79: 21$ \\
D & $>95$ & $80: 20$ & $78: 22$ \\
\hline
\end{tabular}

[a] Hydrogel: $0.008 \mathrm{mmol}$ of $4 \mathrm{a}(0.2 \mathrm{eq})$ in water $(4 \mathrm{~mL})$; Reagents: 4-nitrobenzaldehyde (1 eq), ketone (10 eq).

[b] Determined by NMR.

[c] Determined by chiral-phase HPLC of the anti-product (major enantiomer: S,R-anti).

The obtained results are summarised in Table 4.2.1.2. As can be seen quantitative yields were obtained for the different polymorphs and only slight differences in diastereo and enantioselectivity were found, suggesting that the catalytic proline ring is active in all the polymorphs but the subtle differences seen in the packing mode results in small shifts in the observed selectivity. Further catalytic experiments to check the yield at shorter reaction times showed some differences in the reaction rates (Figure 4.2.2.2). Polymorph A showed quantitative conversion after $2 \mathrm{~h}$ of reaction whereas yields of polymorphs $\mathbf{B}$ and $\mathbf{D}$ after this time were only $70 \%$ and $35 \%$ respectively. The sample with polymorph $\mathbf{B}$ as catalyst gave full conversion after $4 \mathrm{~h}$ and in the case of polymorph $\mathbf{D}$ the yield increased up to $85 \%$ only after $15 \mathrm{~h}$.

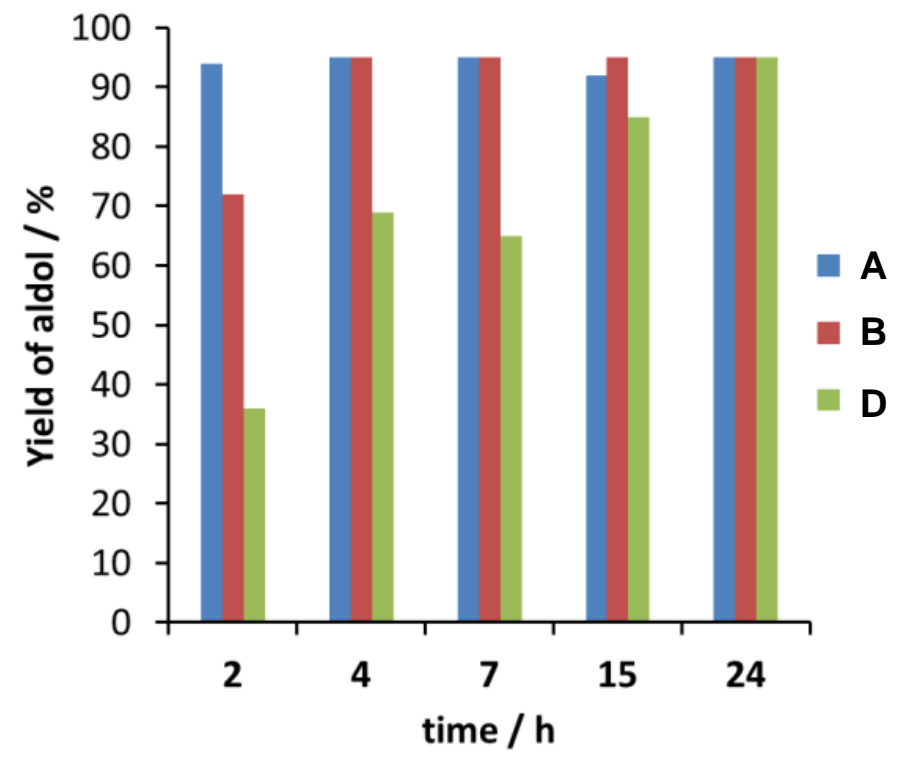

Figure 4.2.2.2. Catalytic performance of polymorphs $\mathbf{A}, \mathbf{B}$ and $\mathbf{D}$ at different reaction times. 
These results are in agreement with the fact that although all the polymorphs for the compound ProValDoc (4a) are catalytically active for the aldol reaction between the cyclohexanone and the 4-nitrobenzaldehyde, small differences in the aggregation packing mode can affect the catalytic performance, probably due to differences in the accessibility of the catalytic site.

\subsection{The Role of the Hydrophobic Effect in Catalysis}

After the positive results obtained in the use of proline based hydrogel ProValDoc (4a) as catalyst for the direct aldol reaction (see also Figure 4.2.3), ${ }^{23}$ it was hypothesised that the hydrophobic effect would be the main driving force for both self-assembly of the catalyst and orientation of the substrates to the catalytic centre mimicking the hydrophobic pocket in the enzymes. With this idea in mind and in trying to shed some light on the role of hydrophobicity on the reaction mechanism, the catalytic performance of the hydrogel formed by this amphiphile was tested in the direct aldol reaction for a series of linear alkyl ketones (Figure 4.3.1).<smiles>O=Cc1ccc([N+](=O)[O-])cc1</smiles><smiles>[R]C(=O)C[C@H](O)c1ccc([N+](=O)[O-])cc1</smiles>

Figure 4.3.1. Schematic representation of the catalytic aldol reaction performed. $\left(\mathrm{R}=-\left(\mathrm{CH}_{2}\right)_{n} \mathrm{CH}_{3} ; \mathrm{n}=0-9\right)$

In order to test the influence of the hydrophobic effect on catalytic behaviour, aliphatic ketones of different chain length were chosen. Catalytic hydrogel samples were prepared at the minimum gel concentration $(2 \mathrm{mM})$. As previously stated, the gelation process can define the assembly of a molecule, and therefore, dictate the final properties in the gel. Taking this into account, all the hydrogel samples were prepared in order to give the polymorph $\mathbf{A}$. To accomplish this, samples were heated in a silicon oil bath, controlling the heating time and the temperature. Afterwards, samples were sonicated for $1 \mathrm{~min}$ and stabilised for $24 \mathrm{~h}$ prior to the addition of the reactants. The results of the catalytic performance are summarised in Table 4.3.1. 
Table 4.3.1. Catalytic results of the aldol reaction of aliphatic ketones with 4-nitrobenzaldehyde. ${ }^{a}$

\begin{tabular}{ccc}
\hline Ketone & ${\text { Yield }(\%)^{\mathbf{b}}}$ & e. . $^{\mathbf{d}^{\mathbf{1}}}$ \\
\hline 1,3-dihydroxy-acetone & $<1$ & - \\
Acetone & $2^{\mathrm{c}}$ & - \\
2-butanone & $<1$ & - \\
2-pentanone & 22 & $65: 35$ \\
2-hexanone & 42 & $70: 30$ \\
2-heptanone & 68 & $70: 30$ \\
2-octanone & 40 & $70: 30$ \\
2-nonanone & 88 & $70: 30$ \\
2-dodecanone & 98 & $65: 35$ \\
\hline
\end{tabular}

[a] Hydrogel: $0.008 \mathrm{mmol}$ of $4 \mathrm{a}(0.2 \mathrm{eq})$ in water $(4 \mathrm{~mL})$; Reagents: 4-nitrobenzaldehyde (1 eq), ketone (10 eq), $25 \stackrel{\circ}{\circ}, 24 \mathrm{~h}$.

[b] Determined by NMR, average of at least 3 reaction samples.

[c] $68 \%$ after 11 days.

[d] Determined by chiral-phase HPLC. Enantiomers not assigned.

As can be seen in Table 4.3.1, after $24 \mathrm{~h}$ of reaction, products for short chain ketones like acetone and 2-butanone were almost undetected. Yields were moderate for ketones with 5 and 6 carbon atoms and increased considerably for longer chains such as 2-nonanone and 2-dodecanone. Thus, a clear trend was observed where higher yields were obtained upon increasing the hydrophobicity of the ketone. On the other hand, was also noticed that the enantiomer ratios (e.r.) were maintained for all the series suggesting that the steric demands of the reaction site are the same in all the cases.

A further study was carried out to assess the kinetic profiles of the different ketones. The yield at several reaction times was determined as can be seen in Figure 4.3.2. Different behaviours were observed and a clear correlation with the alkyl chain length was suggested. Conversion of ketones with chains of 5 to 8 carbon atoms was significantly slower than for those with 9 or 12 carbon atoms. A clear trend is observed that reveals an important role of the hydrophobicity of the ketone. Indeed, a clear correlation of reactivity and log $\mathrm{P}$ values can be observed even at short reaction times (5 to $10 \mathrm{~h}$ ) as can be seen in Figure 4.3.3. More polar ketones like 1,3-dihydroxyacetone (log P: -1.86), acetone ( $\log P: 0.2)$ or 2-butanone (log P: 0.86) did not react, ketones of 
intermediate polarity $(1<\log \mathrm{P}<2.5)$ had a low yield and less polar ketones such as 2 nonanone or 2-dodecanone ( $\log \mathrm{P}>3$ ) showed high yields after $10 \mathrm{~h}$.

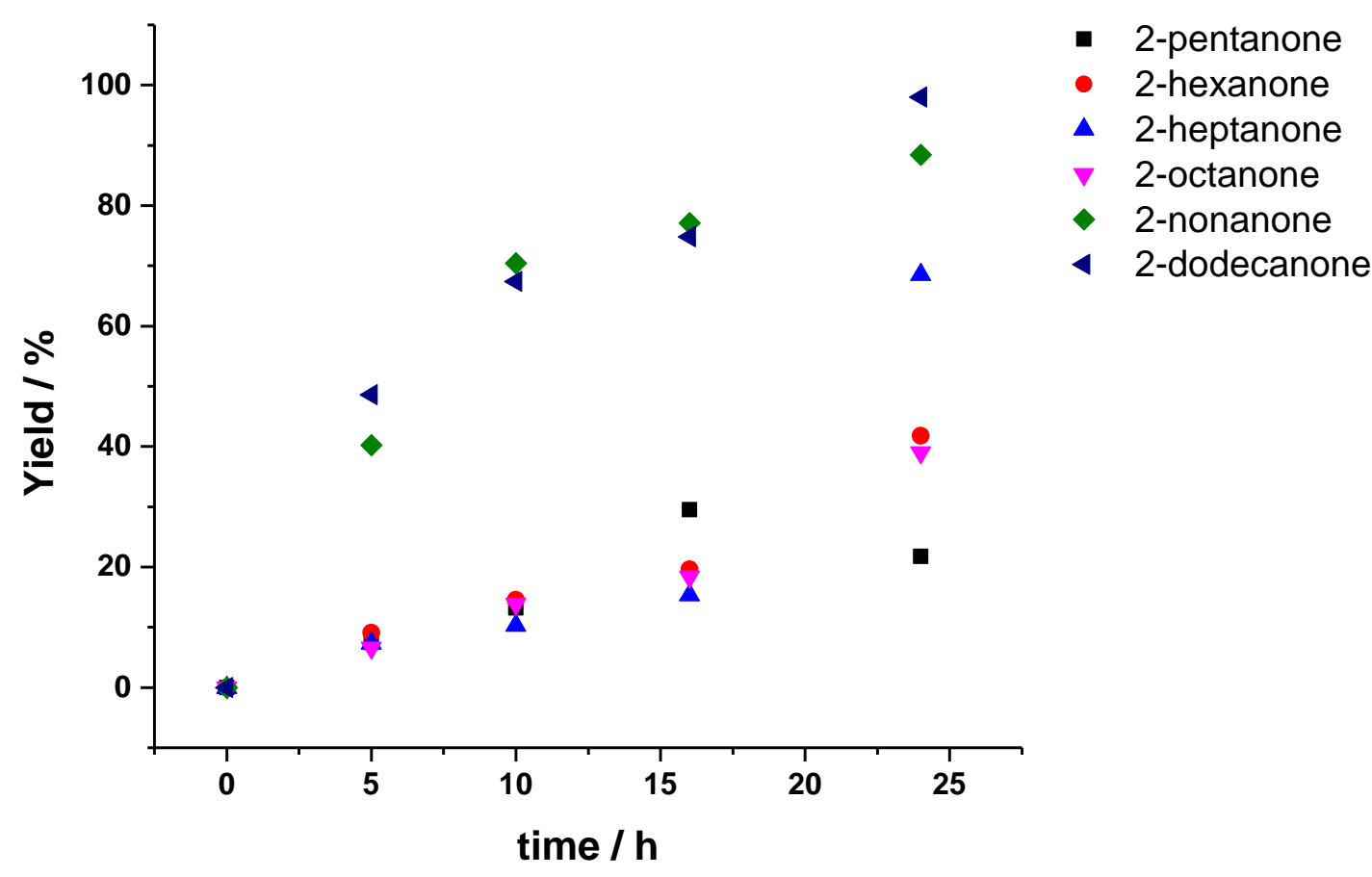

Figure 4.3.2. Yield of the aldol product vs reaction time.

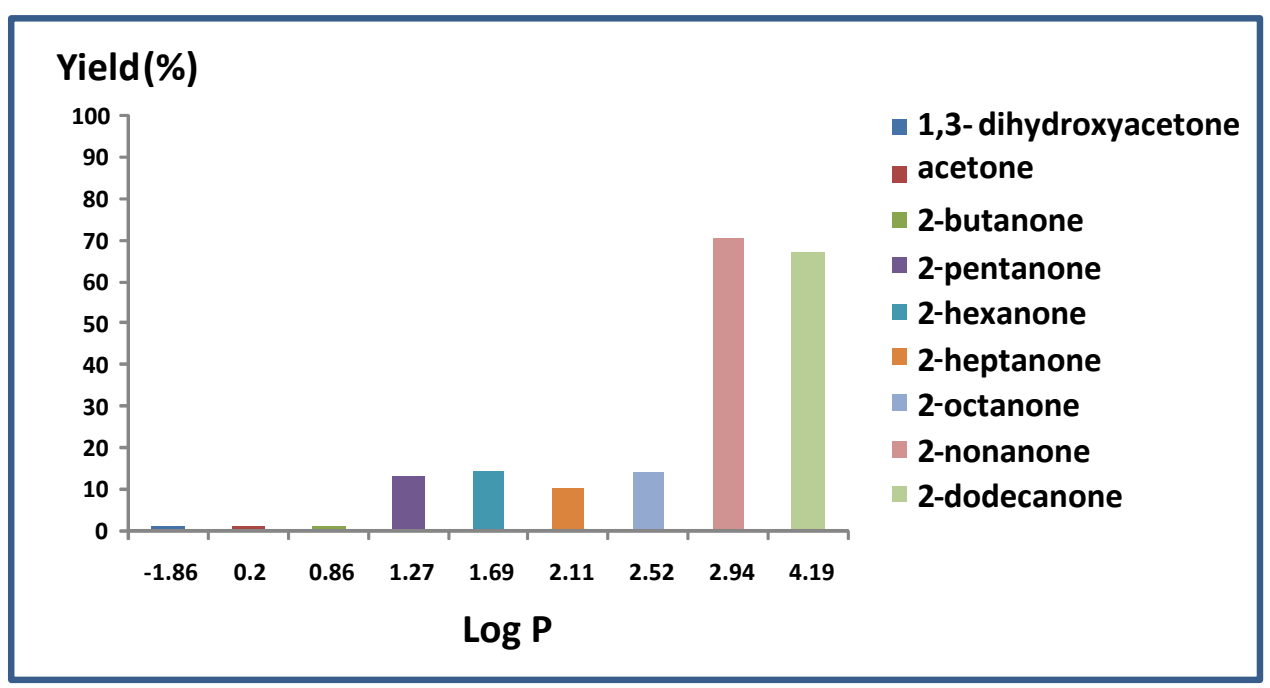

Figure 4.3.3. Yield of aldol product vs log $\mathrm{P}$ of ketone after $10 \mathrm{~h}$ of reaction.

The next step was to study the reaction mechanism for the direct aldol reaction when the proline moiety is used as organocatalyst. The accepted mechanism involves the reaction of the proline catalyst and the ketone to form an enamine intermediate that 
subsequently adds to the aldehyde carbonyl group (see Figure 3.1.1.1 bottom). In the current case, since the proline derivative is not soluble in water and remains as a separated gel phase, the ketone must be placed within the gel aggregates in order to react with compound ProValDoc (4a). Therefore, it seems reasonable that the more hydrophobic ketones will accumulate into the gel phase, minimising interactions with the water molecules, and reacting with the catalyst there. Studies to detect the catalystketone enamine intermediates were performed. Unluckily, the enamine intermediates were not visible by NMR in solution but surprisingly could however be detected by mass spectrometry (MS) for a sample of hydrogel and 2-docecanone (0.2:10) stabilized for $6 \mathrm{~h}$ at room temperature (Figure 4.3.4) supporting the hypothesis that the reaction takes places via enamine for the proline catalyst.

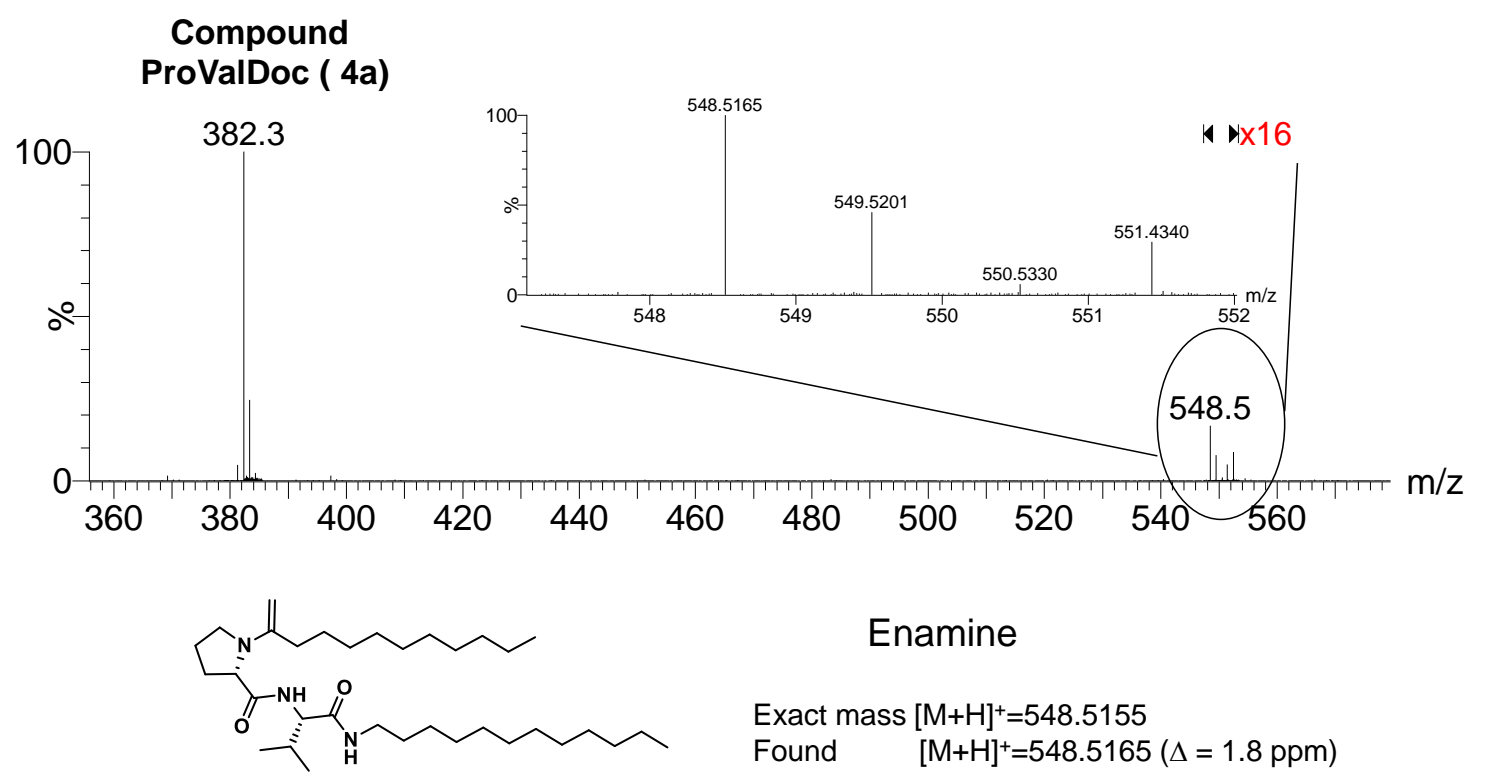

Figure 4.3.4. MS graph for the mixture of ProValDoc (4a) hydrogel with 2-dodecanone showing the enamine formation.

Additionally, a water soluble analogue proline catalyst, the compound ProValPr (3b), where the alkyl chain of 12 carbon atoms was substituted for a shorter, less hydrophobic unit with only 3 carbon atoms (propyl tail), (Figure 4.3.1. $n=2$ ). This analogue was not able to form aggregated structures in water meaning the catalyst remains in solution during the reaction. The aldol reaction was performed with highly soluble ketones, like acetone, and with less soluble ketones, like with 2-dodecanone. Remarkably, the reaction does not proceed in the absence of the hydrogel as catalytic phase for any of 
the ketones. This highlights the importance of the formation of the supramolecular catalytic aggregates to achieve their catalytic role, meaning that the catalytic proline fragment can only successfully act as a catalyst upon forming supramolecular aggregates.

Further studies were carried out to test the selectivity of the catalyst ProValDoc (4a) for the different ketones. Here the aldol reaction was carried out in competition with two alkyl ketones that differ in the tail length and hence hydrophobicity and solubility in water. The first study involved the ketones 2-heptanone and 2-dodecanone with 4nitrobenzaldehyde. After $24 \mathrm{~h}$, the reaction was analysed by ${ }^{1} \mathrm{H}-\mathrm{NMR}$ showing $89 \%$ of conversion. Mass spectrometry revealed that only the product of the longer and less soluble ketone was formed (2-dodecanone product). These results point to the importance of the hydrophobic effect in this direct aldol reaction where only the more hydrophobic ketones were able to be placed inside the reaction site and react with the catalyst (Figure 4.3.5). An additional experiment was carried out involving a more soluble ketone, 2-pentanone, in competition with the 2-dodecanone showing similar results. Here only $77 \%$ conversion was found and the mass spectra showed that only the more hydrophobic ketone was able to yield the aldol product (Figure SI_4.5.10).

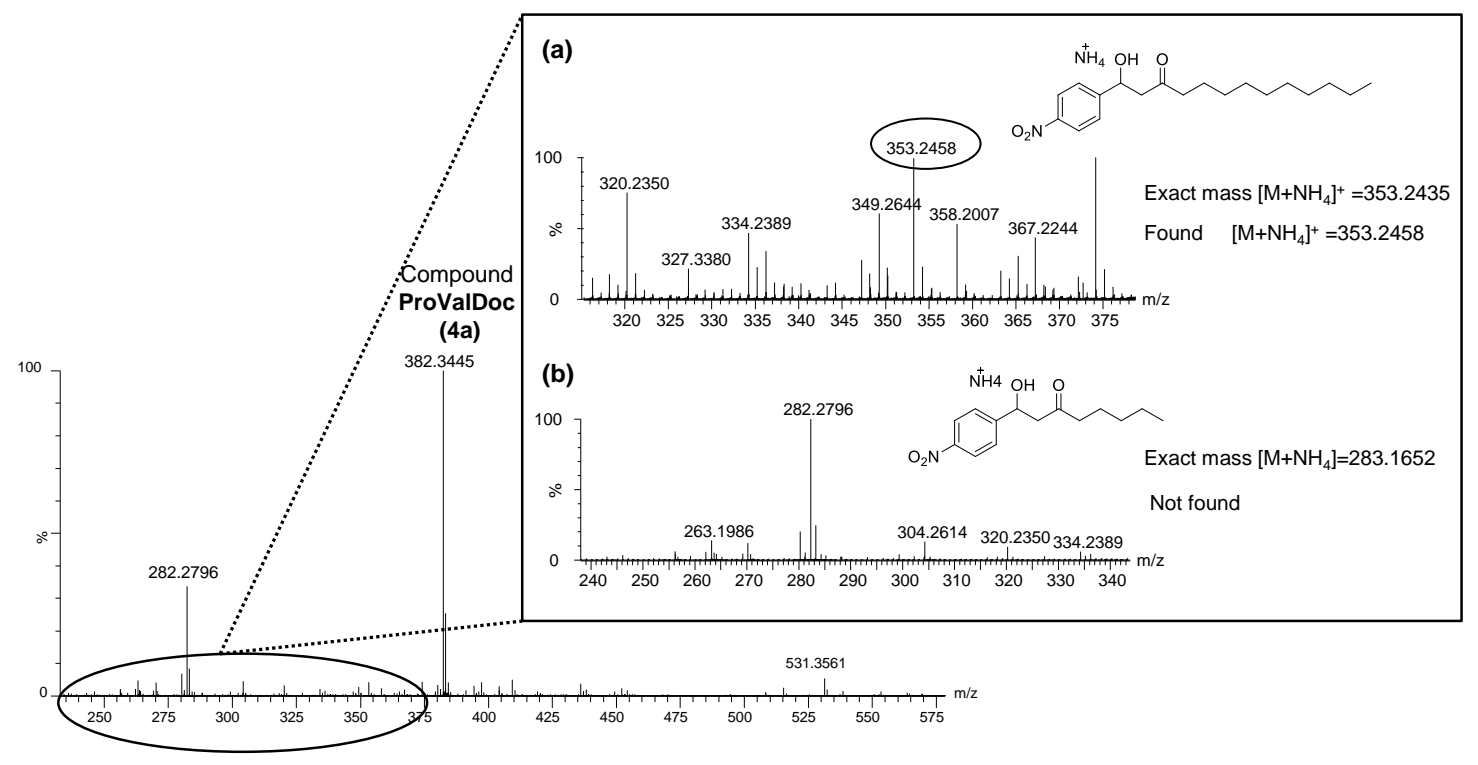

Figure 4.3.5. MS graph for a competition test between two ketones in the aldol addition to 4nitrobenzaldehyde in the presence of the hydrogel $4 a$. The inset shows the area for the products for the reaction with (a) 2-dodecanone and (b) 2-heptanone. Reaction conditions: Hydrogel: 0.008mmol of 4a (0.2 eq) in water ( $4 \mathrm{~mL}$ ); Reagents: 4-nitrobenzaldehyde (1 eq), ketone (5 eq each), 25 으, $24 \mathrm{~h}$. 
In light of this and with information on the aggregation packing given by the crystal X-ray structure shown before (see Figure 4.2.1.3 and Figure SI_4.5.5), a schematic reaction site can be hypothesised for the hydrogel system (see Figure 4.3.6) where ketones bearing a long tail can be anchored into the bilayer by van der Waals and hydrophobic interactions to then react in close proximity with the catalyst forming the required enamine intermediate. We have already reported that the hydrogel ProValDoc (4a) reacts with aldehydes according to their hydrophobicity which in the studied cases triggered the hydrogel disassembly. ${ }^{28}$ In the current case, it appears that 4nitrobenzaldehyde that is highly insoluble in water, could form insoluble iminium intermediates that remain anchored to the hydrogel phase where they react with enamine intermediates.

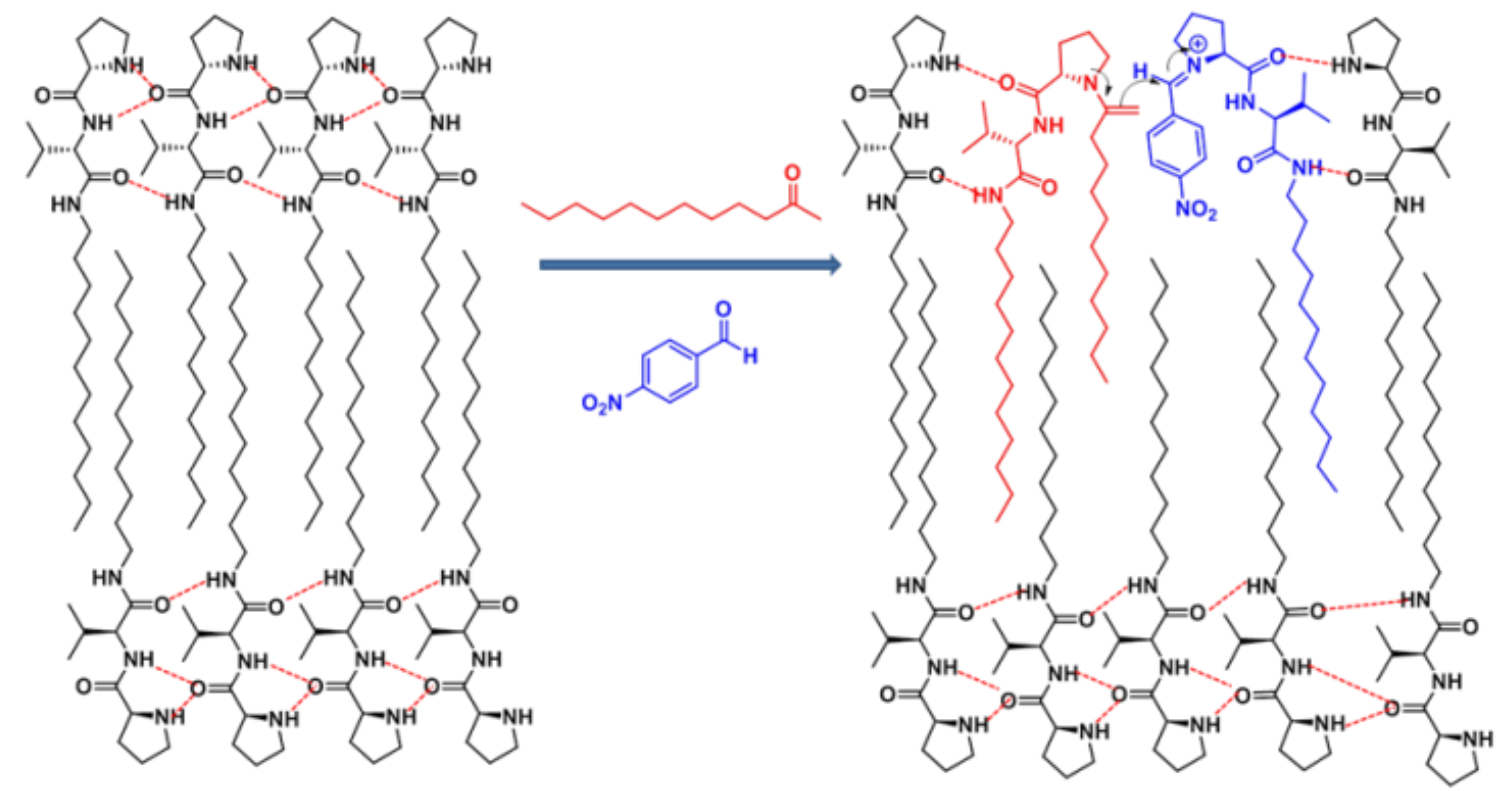

Figure 4.3.6. Proposed scheme of the hydrogel reaction site. 


\subsection{Conclusions}

In summary, an example of polymorphism where a simple low molecular weight gelator, upon modification of the environmental parameters (temperature, aging time, ultrasound, etc.), may result in the formation of different packing arrangements has been studied. As a consequence, the differences on the packing mode of the aggregates are reflected in its functional behaviour. In this case, four different polymorphs of the amphiphilic ProValDoc (4a) hydrogelator were found and characterised. In addition, the catalytic performance of the different polymorphs was tested on the benchmark direct aldol reaction of cyclohexanone with 4-nitrobenzaldehyde in so called "on water reactions". This work highlights the importance of the supramolecular self-assembly study within such complex systems in order to avoid unexpected and/or irreproducible results. Moreover, the current work exemplifies that the role of polymorphism is a transversal issue with relevance not only in crystal engineering and pharmaceutical science but also in soft matter and in catalysis.

\section{Hydrophobicity}

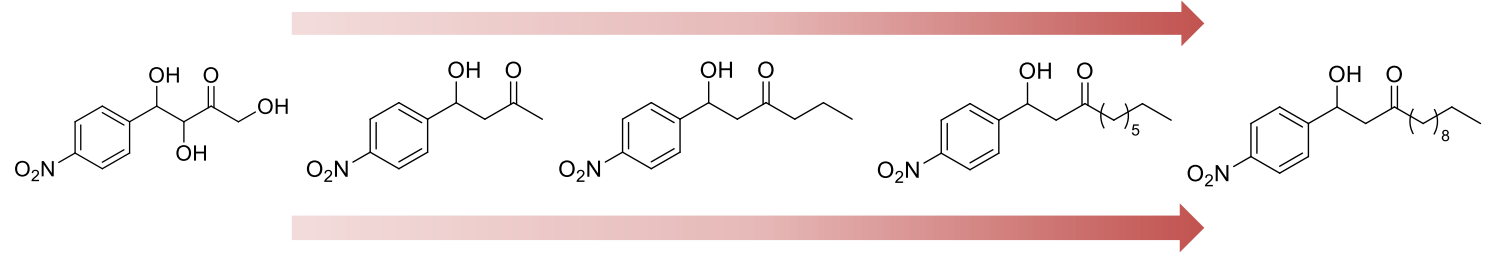

Catalytic System Reactivity

Figure 4.4.1. Schematic representation of the results obtained for the ketone reactivity in the aldol reaction performed by the hydrogelator ProValDoc (4a).

A further study with this ProValDoc (4a) hydrogelator as a catalyst showed that its aggregation is driven by the hydrophobic effect resulting in the emergence of the catalytic behaviour. Moreover, this investigation has shed some light on the mechanism of the proline catalysed aldol reaction forming an enamine intermediate with the ketone inside hydrogel active site. This hydrophobic active site mimics the one found in the enzymes 
combining a recognition centre based on hydrophobic and van der Waals interactions with a reaction centre for covalent catalysis. The role of the hydrophobic effect for this hydrogelator and its activity as a selective organocatalyst for the hydrophobic substrates in the aldol reaction has been highlighted (see Figure 4.4.1). 


\subsection{Supporting Information}

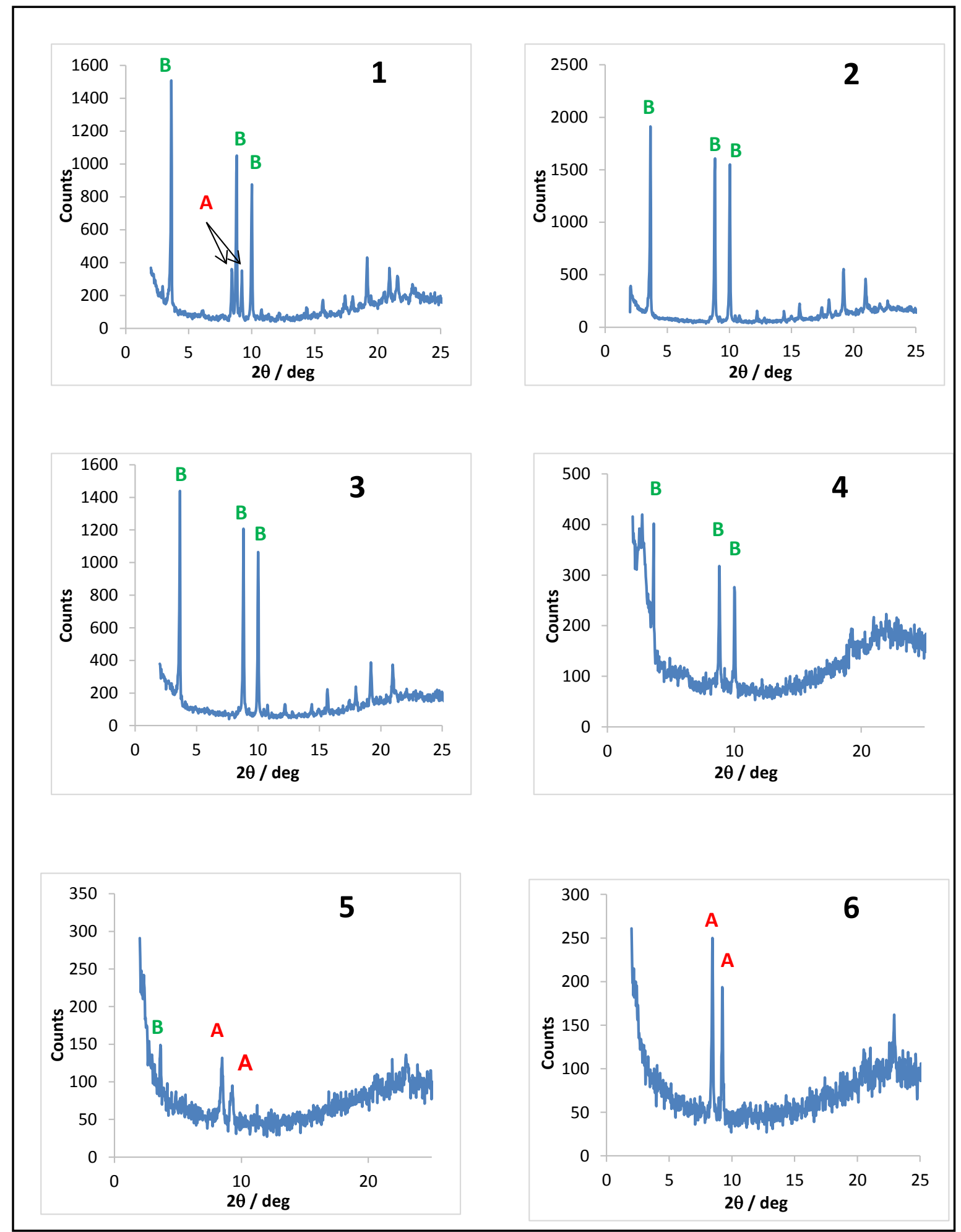

Figure SI_4.5.1. X-Ray powder diffractograms corresponding to Entries 1-6 on Table 4.2.1.1. 

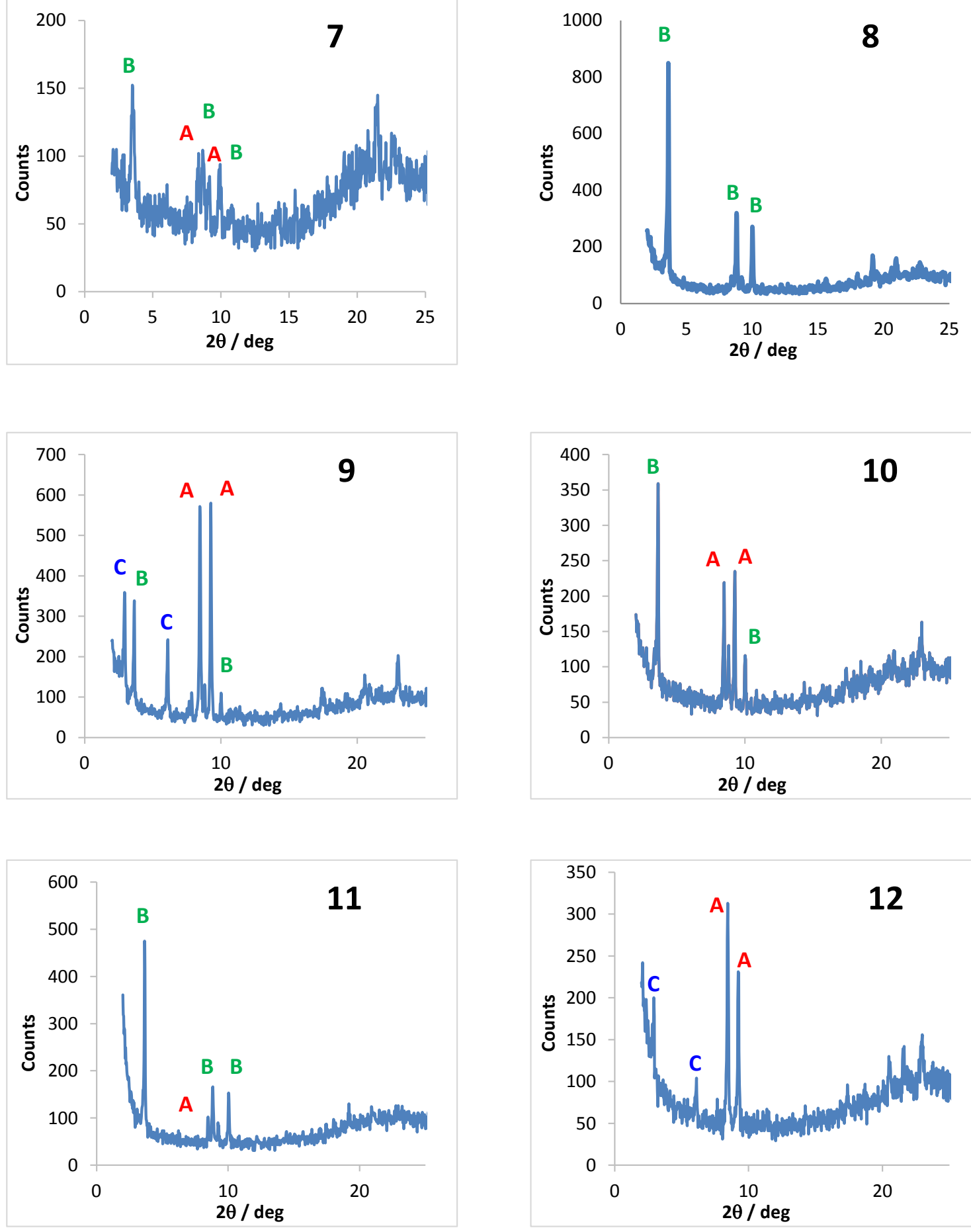

Figure SI_4.5.2. X-Ray powder diffractograms corresponding to Entries 7-12 on Table 4.2.1.1. 

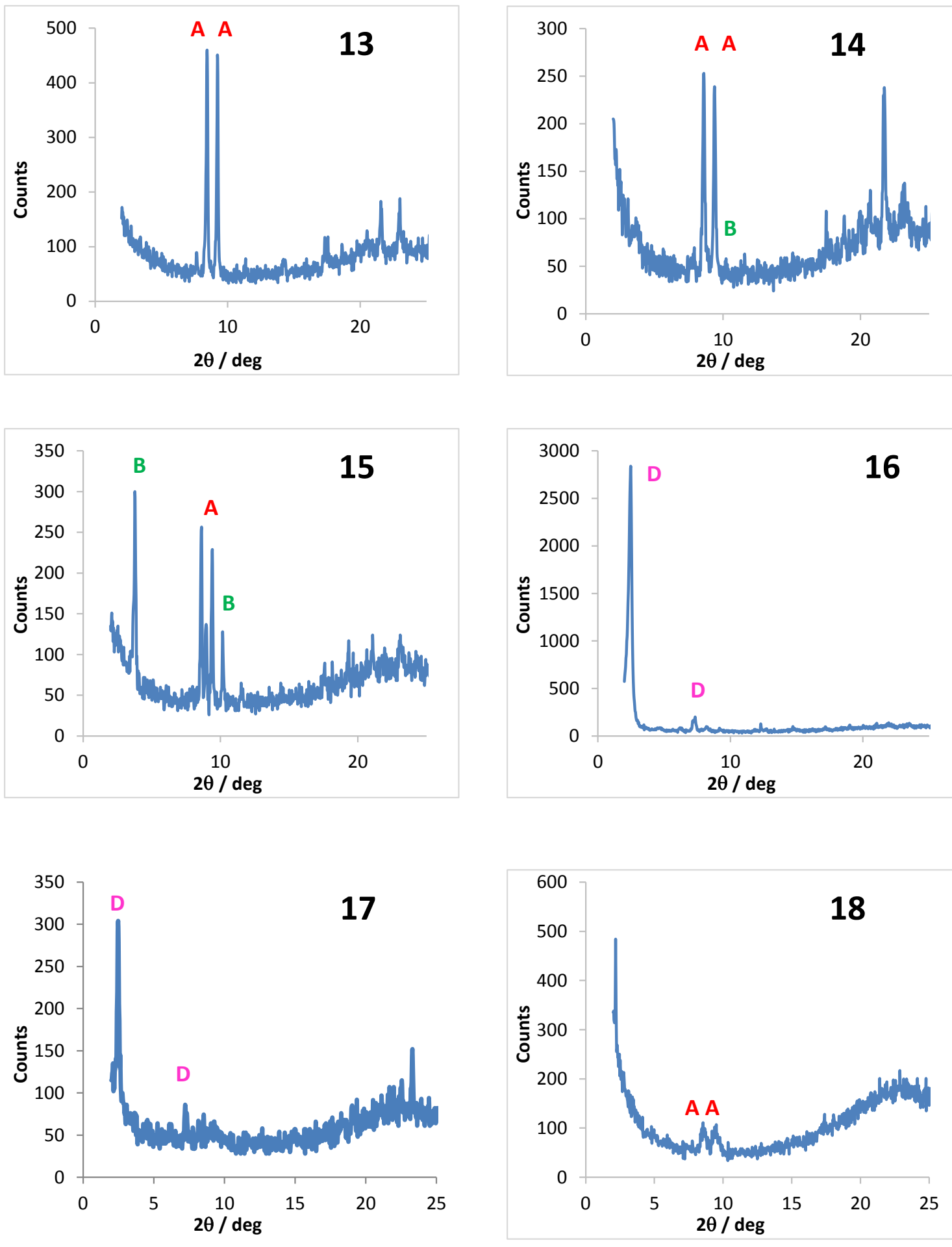

Figure SI_4.5.3. X-Ray powder diffractograms corresponding to Entries 13-18 on Table 4.2.1.1 


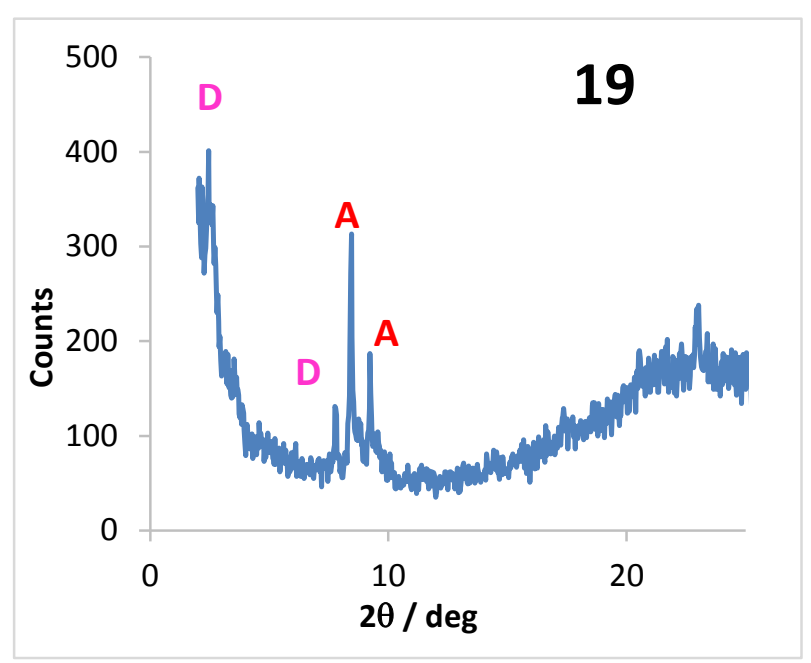

Figure SI_4.5.4. X-Ray powder diffractograms corresponding to Entry 19 on Table 4.2.1.1

(a)

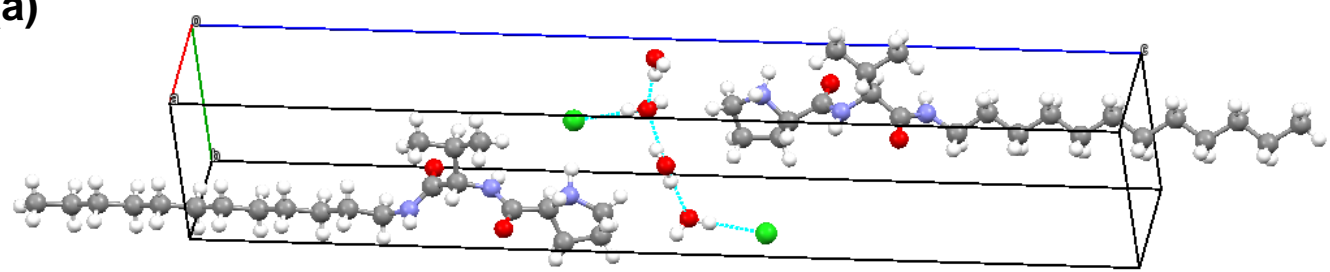

(b)

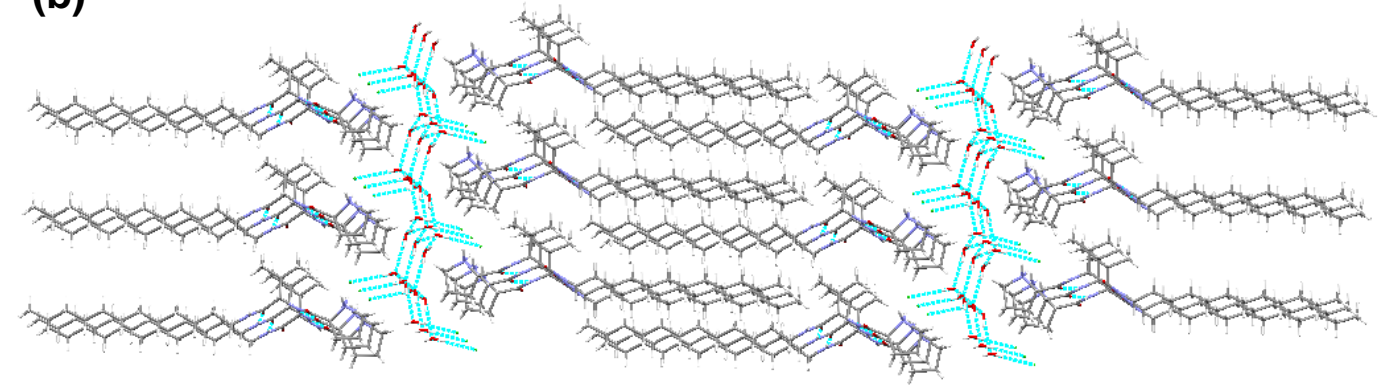

Figure SI_4.5.5. Single crystal X-ray structure of compound ProValDoc $(\mathbf{4 a}) \cdot \mathbf{H C l} \cdot 2 \mathbf{H}_{2} \mathrm{O}$ where it can be seen a) the unit cell of the crystal and b) stacked molecules forming a bilayer structure. 


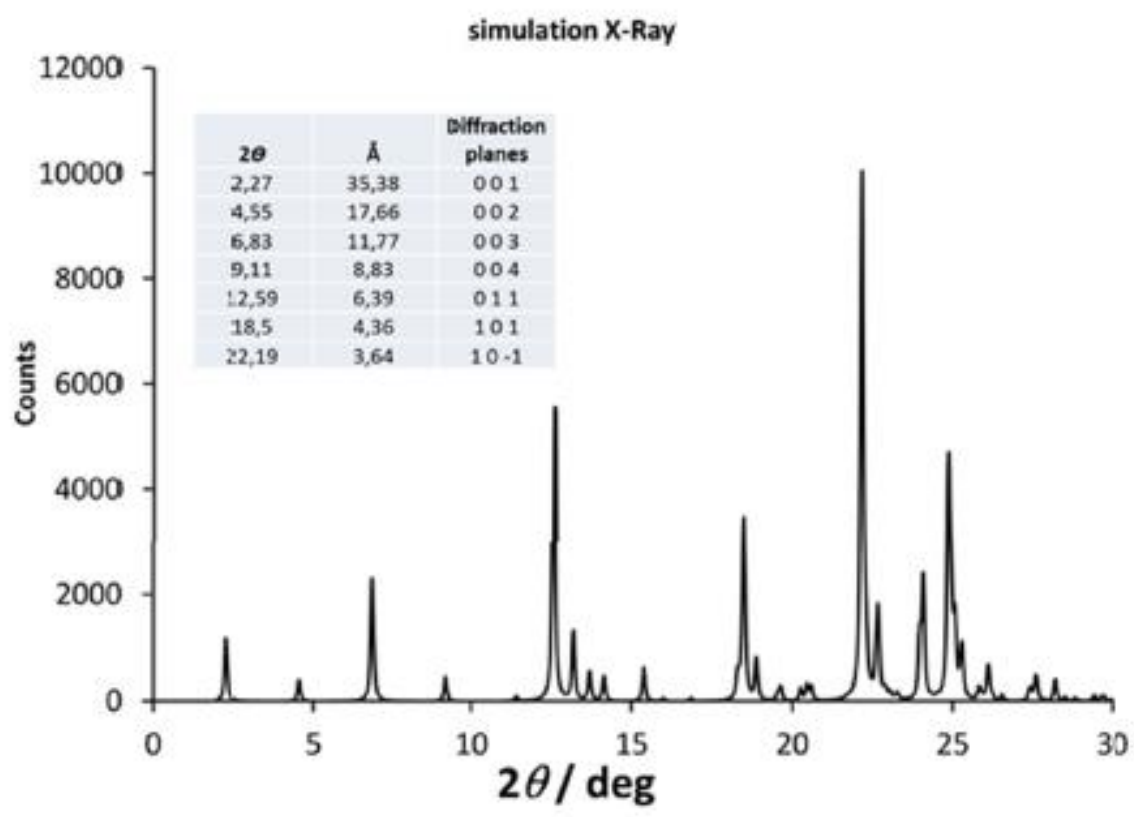

Figure SI_4.5.6. X-Ray powder difractogram pattern of compound ProValDoc (4a) $\cdot \mathbf{H C l} \cdot 2 \mathrm{H}_{2} \mathbf{O}$ simulated from single crystal data. ${ }^{29}$

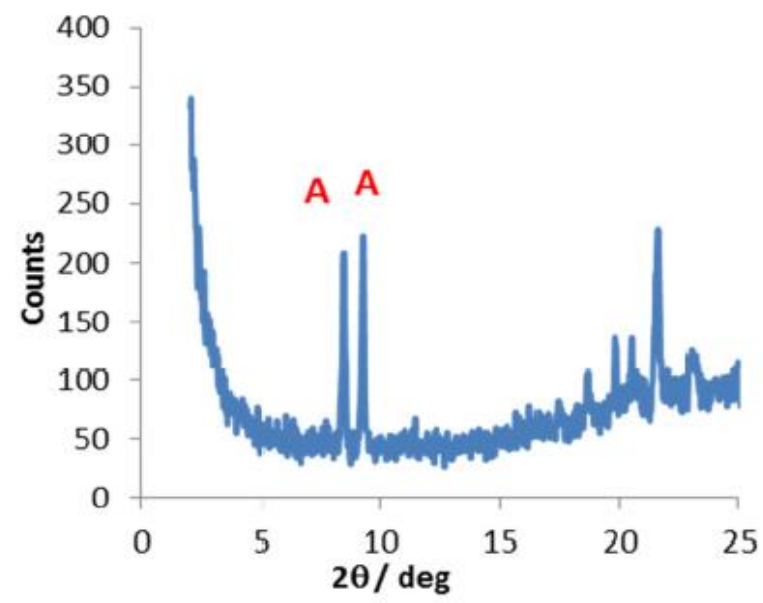

Figure SI_4.5.7. X-Ray powder diffractogram corresponding to the DSC sample of polymorph B captured

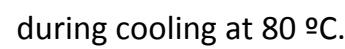


A

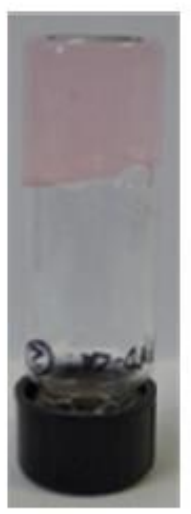

B

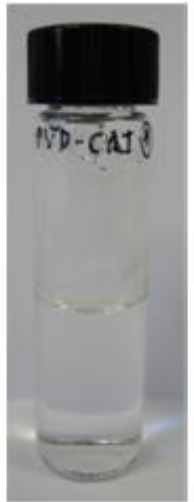

D

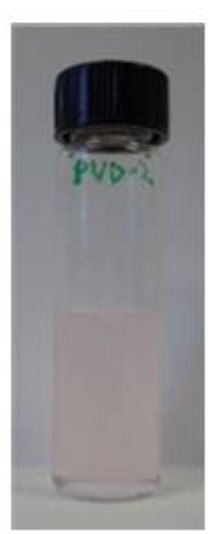

Figure SI_4.5.8. Macroscopic aspect of samples with polymorph A, B and D.

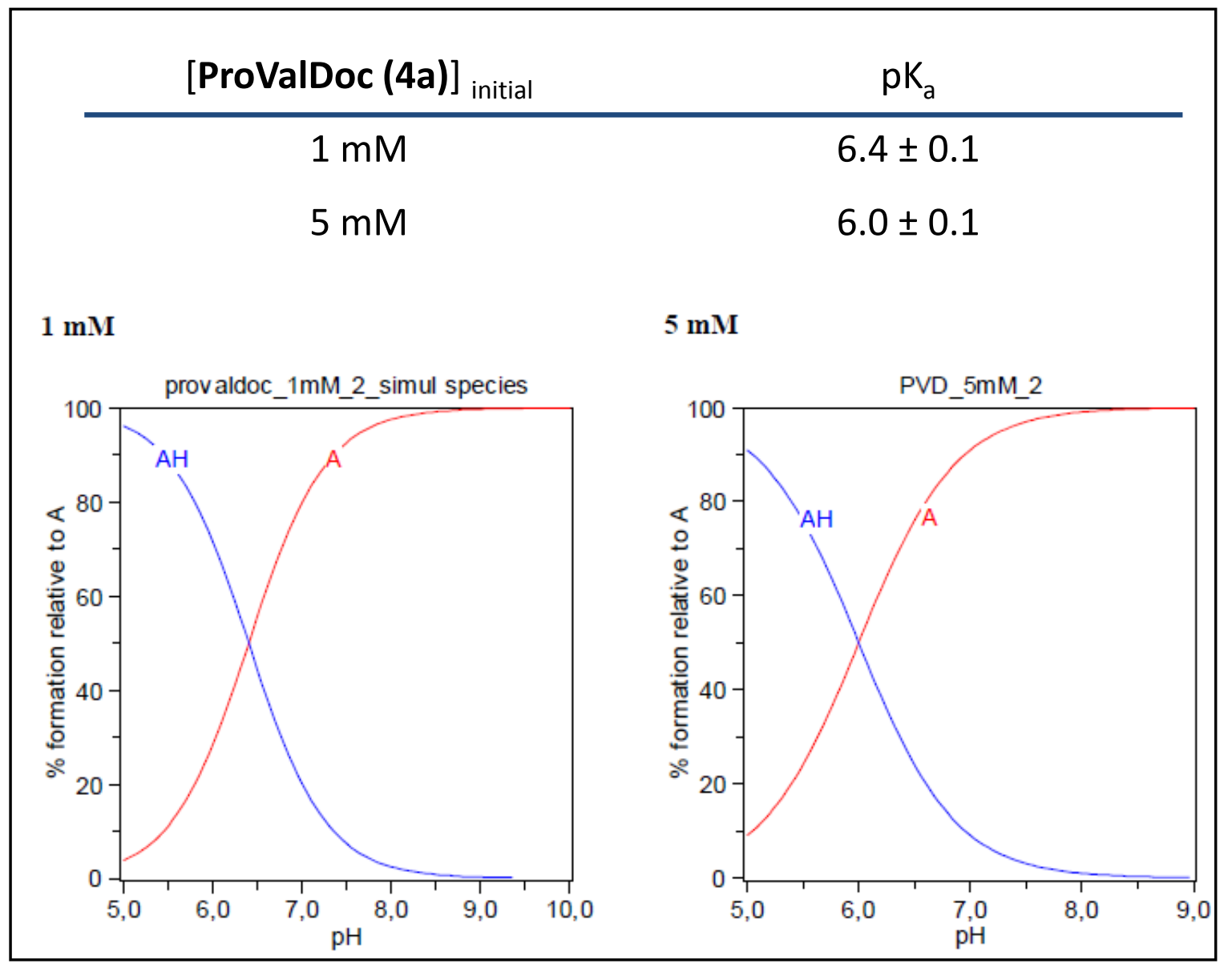

Figure SI_4.5.9. $\mathrm{pK}_{\mathrm{a}}$ and species distribution diagrams as a function of $\mathrm{pH}$ for compound ProValDoc (4a) at two different concentrations. For $\mathrm{pK}_{\mathrm{a}}$ determinations see Experimental Section 


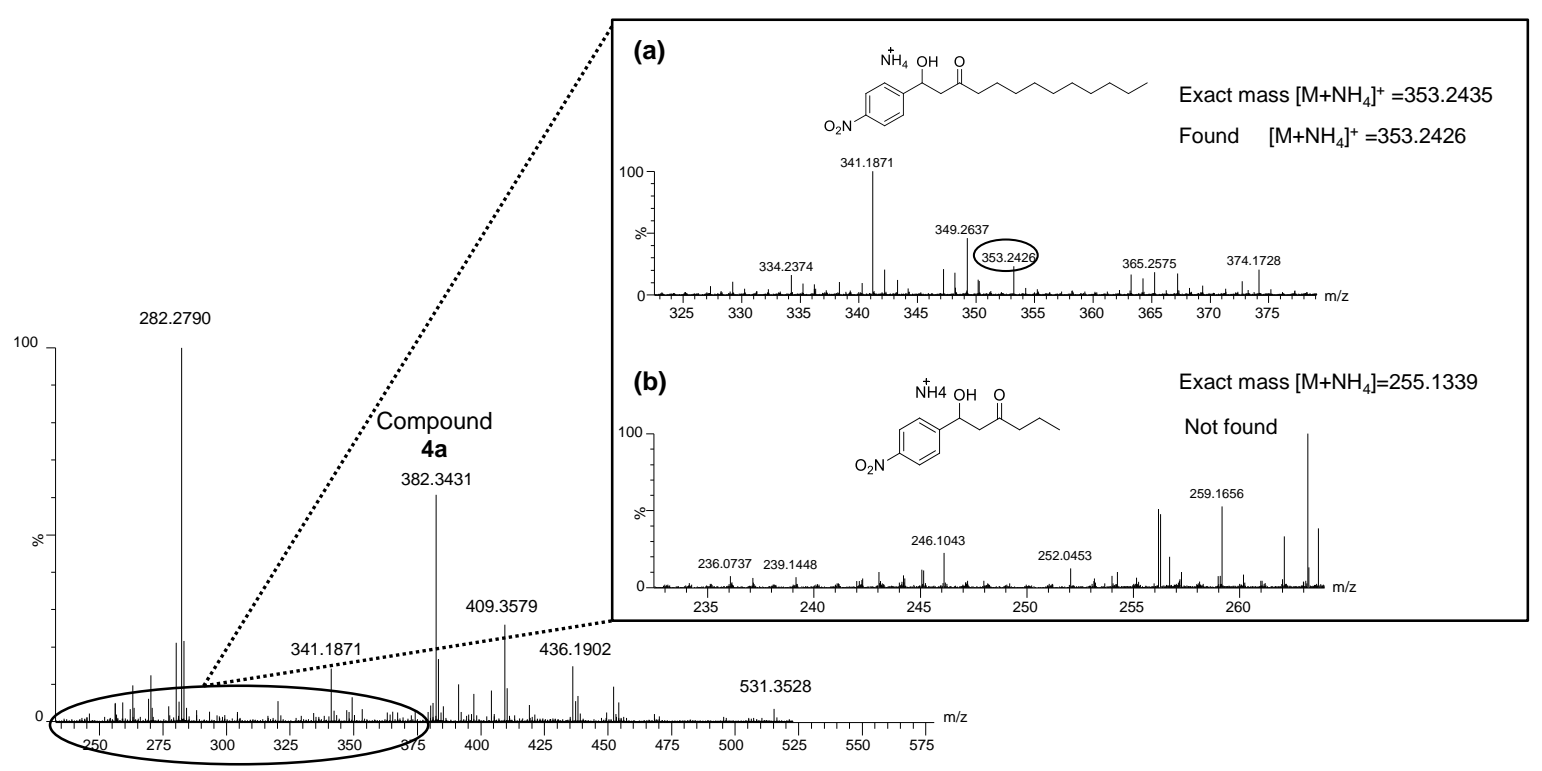

Figure SI_4.5.10. MS graph for a competition sample test in the presence of hydrogel 4a. The inset shows the amplified area where products should be when 4-nitrobenzaldehyde reacts with; (a) 2-dodecanone and (b) 2-pentanone. Reaction conditions: Hydrogel: $0.008 \mathrm{mmol}$ of $4 \mathrm{a}$ (0.2 eq) in water (4 mL); Reagents: 4nitrobenzaldehyde (1 eq), ketone (5 eq each), 25 으, $24 \mathrm{~h}$.

\section{Characterisation of aldol products:}

\section{1-(4-nitrophenyl)-1-hydroxy-3-butanone}<smiles>CC(=O)CC(O)c1ccc([N+](=O)[O-])cc1</smiles>

${ }^{1} \mathrm{H}$ NMR $\left(300 \mathrm{MHz}, \mathrm{CDCl}_{3}\right) \delta(\mathrm{ppm}): 2.22(\mathrm{~s}, 3 \mathrm{H}), 2.86(\mathrm{~d}, J=1.8 \mathrm{~Hz}, 1 \mathrm{H}), 2.88(\mathrm{~s}, 1 \mathrm{H})$ $3.70(\mathrm{~s}, 1 \mathrm{H}), 5.27(\mathrm{dd}, J=2.9,3.3 \mathrm{~Hz}, 1 \mathrm{H}), 7.54(\mathrm{~d}, J=8.8 \mathrm{~Hz}, 2 \mathrm{H}), 8.21(\mathrm{~d}, J=8.8 \mathrm{~Hz}, 2 \mathrm{H}) ;{ }^{13} \mathrm{C}$ NMR $\left(75 \mathrm{MHz}, \mathrm{CDCl}_{3}\right) \delta$ (ppm): 30.6, 51.4, 68.8, 123.7, 126.3, 147.3, 149.9, 208.2. 


\section{1-(4-nitrophenyl)-1-hydroxy-3-pentanone}<smiles>CCC(=O)CC(O)c1ccc([N+](=O)[O-])cc1</smiles>

${ }^{1} \mathrm{H}$ NMR (300 MHz, CDCl $\left.{ }_{3}\right) \delta(p p m): 1.09(\mathrm{t}, J=7.3 \mathrm{~Hz}, 3 \mathrm{H}), 2.48(\mathrm{q}, J=7.3 \mathrm{~Hz}, 2 \mathrm{H}$ ), $2.82(\mathrm{~m}, 2 \mathrm{H}), 3.67$ (d, J=3.3 Hz, 1H), 5.27 (dt, J=3.5 Hz, 7.7, 1H), 7.53 (d, J=8.6 Hz, 2H), 8.21 $(\mathrm{d}, J=9.8 \mathrm{~Hz}, 2 \mathrm{H}) ;{ }^{13} \mathrm{C} \mathrm{NMR}\left(75 \mathrm{MHz}, \mathrm{CDCl}_{3}\right) \delta$ (ppm): 7.4, 36.8, 50.2, 69.1, 123.7, 126.4, $147.3,150.1,211.4$.

\section{1-(4-nitrophenyl)-1-hydroxy-3-hexanone}<smiles>CCCC(=O)CC(O)c1ccc([N+](=O)[O-])cc1</smiles>

${ }^{1} \mathrm{H}$ NMR (500 MHz, CDCl $\left.{ }_{3}\right) \delta(\mathrm{ppm}): 8.21(\mathrm{~d}, J=8.7 \mathrm{~Hz}, 2 \mathrm{H}), 7.54(\mathrm{~d}, J=8.7 \mathrm{~Hz}, 2 \mathrm{H})$, $5.32-5.21(\mathrm{~m}, 1 \mathrm{H}), 3.62(\mathrm{~d}, J=3.3 \mathrm{~Hz}, 1 \mathrm{H}), 2.81(\mathrm{~m}, 2 \mathrm{H}), 2.43(\mathrm{t}, J=7.3 \mathrm{~Hz}, 2 \mathrm{H}), 1.63(\mathrm{~h}, J$ $=7.4 \mathrm{~Hz}, 2 \mathrm{H}), 0.93(\mathrm{t}, J=7.4 \mathrm{~Hz}, 3 \mathrm{H}) ;{ }^{13} \mathrm{C} \mathrm{NMR}\left(100 \mathrm{MHz}, \mathrm{CDCl}_{3}\right) \delta(\mathrm{ppm}): 211.0,150.2$, $147.2,126.4,123.7,69.0,50.5,45.4,17.0,13.6$.

HPLC conditions Chiralpak IA ( $n$-hexane/THF: 80/20, $1.0 \mathrm{~mL} / \mathrm{min}, \lambda=250 \mathrm{~nm}$ ), $t_{\mathrm{R}}=11.63 \mathrm{~min}$ (minor), $t_{\mathrm{R}}=20.10 \mathrm{~min}$ (major).

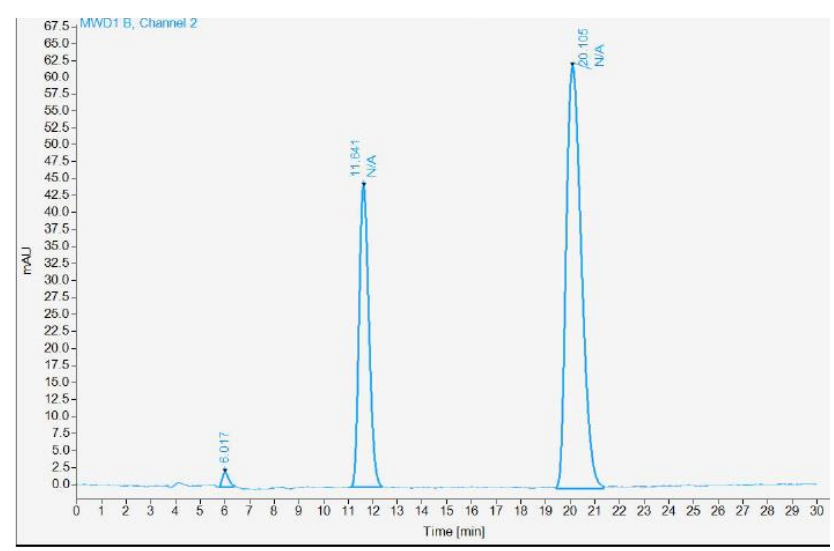

Figure SI_4.5.11. Chromatogram of a mixture of 1-(4-nitrophenyl)-1-hydroxy-3-hexanone enantiomers. 


\section{1-(4-nitrophenyl)-1-hydroxy-3-heptanone}<smiles>CCCCC(=O)CC(O)c1ccc([N+](=O)[O-])cc1</smiles>

${ }^{1} \mathrm{H}$ NMR $\left(500 \mathrm{MHz}, \mathrm{CDCl}_{3}\right) \delta(\mathrm{ppm}): 8.21(\mathrm{~d}, J=8.6 \mathrm{~Hz}, 2 \mathrm{H}), 7.54(\mathrm{~d}, J=8.4 \mathrm{~Hz}, 2 \mathrm{H})$, $5.29-5.24(\mathrm{~m}, 1 \mathrm{H}), 3.63(\mathrm{~d}, J=3.0 \mathrm{~Hz}, 1 \mathrm{H}), 2.82(\mathrm{~m}, 2 \mathrm{H}), 2.45(\mathrm{t}, J=7.4 \mathrm{~Hz}, 2 \mathrm{H}), 1.59(\mathrm{~m}$, $2 \mathrm{H}), 1.32(\mathrm{~m}, 2 \mathrm{H}), 0.91(\mathrm{t}, J=7.3 \mathrm{~Hz}, 2 \mathrm{H}) ;{ }^{13} \mathrm{C} \mathrm{NMR}\left(100 \mathrm{MHz}, \mathrm{CDCl}_{3}\right) \delta(\mathrm{ppm}): 211.0,150.2$, $147.2,126.4,123.7,69.0,50.5,43.3,25.5,22.2,13.8$.

HPLC conditions Chiralpak IA ( $n$-hexane/THF: 80/20, $1.0 \mathrm{~mL} / \mathrm{min}, \lambda=250 \mathrm{~nm}$ ), $t_{\mathrm{R}}=11.57 \mathrm{~min}$ (minor), $t_{\mathrm{R}}=23.19 \mathrm{~min}$ (major).

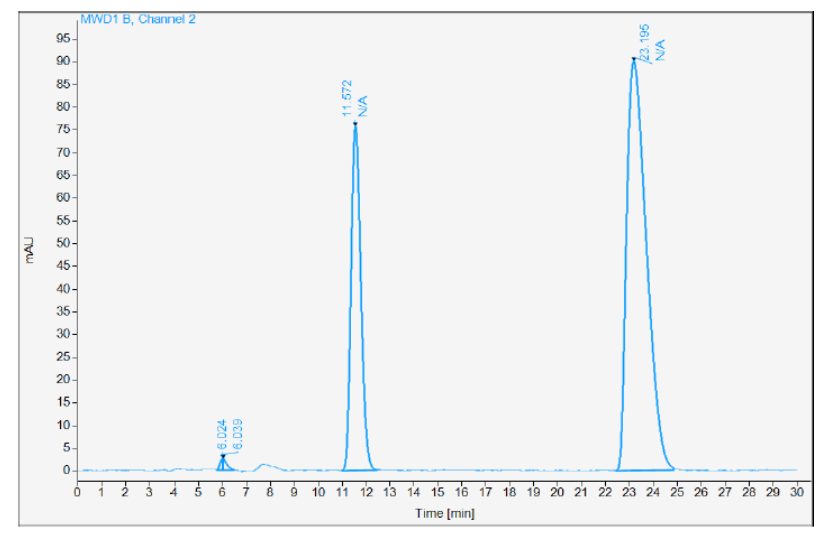

Figure SI_4.5.12. Chromatogram of a mixture of 1-(4-nitrophenyl)-1-hydroxy-3-heptanone enantiomers.

\section{1-(4-nitrophenyl)-1-hydroxy-3-octanone}<smiles>CCCCCC(=O)CC(O)c1ccc([N+](=O)[O-])cc1</smiles>

${ }^{1} \mathrm{H}$ NMR $\left(500 \mathrm{MHz}, \mathrm{CDCl}_{3}\right) \delta(\mathrm{ppm}): 8.21(\mathrm{~d}, J=8.8 \mathrm{~Hz}, 2 \mathrm{H}), 7.54(\mathrm{~d}, J=8.8 \mathrm{~Hz}, 2 \mathrm{H})$, $5.29-5.23(\mathrm{~m}, 1 \mathrm{H}), 3.63(\mathrm{~d}, J=3.3 \mathrm{~Hz}, 1 \mathrm{H}), 2.87-2.75(\mathrm{~m}, 2 \mathrm{H}), 2.44(\mathrm{t}, J=7.4 \mathrm{~Hz}, 2 \mathrm{H})$, $1.60(\mathrm{dt}, J=14.9,7.4 \mathrm{~Hz}, 2 \mathrm{H}), 1.35-1.19(\mathrm{~m}, 4 \mathrm{H}), 0.89(\mathrm{t}, J=7.1 \mathrm{~Hz}, 3 \mathrm{H}):{ }^{13} \mathrm{C}$ NMR $(126$ $\left.\mathrm{MHz}, \mathrm{CDCl}_{3}\right) \delta$ (ppm): 211.1, 150.0, 126.4, 123.7, 69.1, 50.5, 43.6, 31.2, 23.2, 22.3, 13.8; (ESI-TOF, positive mode) $\mathrm{m} / \mathrm{z}$ exp $[\mathrm{M}+\mathrm{Na}]^{+}$calcd for $\mathrm{C}_{14} \mathrm{H}_{19} \mathrm{NNaO}_{4}{ }^{+}$288.1206; found, $288.1210[\mathrm{M}+\mathrm{Na}]^{+},(\Delta=0.7 \mathrm{ppm})$. 
HPLC conditions Chiralpak IA (n-hexane/THF: 80/20, $1.0 \mathrm{~mL} / \mathrm{min}, \lambda=250$ $\mathrm{nm}), t_{\mathrm{R}}=10.73 \mathrm{~min}$ (minor), $t_{\mathrm{R}}=12.84 \mathrm{~min}$ (major).

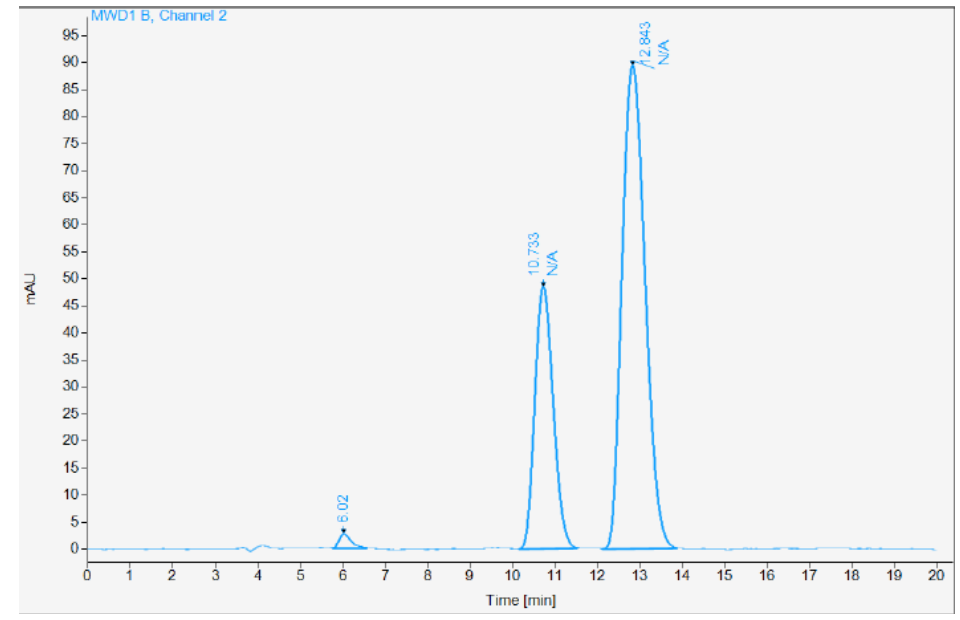

Figure SI_4.5.13. Chromatogram of a mixture of 1-(4-nitrophenyl)-1-hydroxy-3-octanone enantiomers.

\section{1-(4-nitrophenyl)-1-hydroxy-3-nonanone}<smiles>CCCCCCC(=O)CC(O)c1ccc([N+](=O)[O-])cc1</smiles>

${ }^{1} \mathrm{H}$ NMR $\left(500 \mathrm{MHz}, \mathrm{CDCl}_{3}\right) \delta(\mathrm{ppm}): 8.22(\mathrm{~d}, J=8.7 \mathrm{~Hz}, 2 \mathrm{H}), 7.55(\mathrm{~d}, J=8.8 \mathrm{~Hz}, 2 \mathrm{H})$, $5.28(\mathrm{~m}, 1 \mathrm{H}), 3.65(\mathrm{~s}, 1 \mathrm{H}), 2.83(\mathrm{~m}, 2 \mathrm{H}), 2.45(\mathrm{t}, J=7.5 \mathrm{~Hz}, 2 \mathrm{H}), 1.67-1.56(\mathrm{~m}, 2 \mathrm{H}), 1.29(\mathrm{~s}$, $6 \mathrm{H}), 0.89$ (t, $J=5.5 \mathrm{~Hz}, 3 \mathrm{H}) ; 13 \mathrm{C} \mathrm{NMR}(\mathrm{CDCl} 3,75 \mathrm{MHz}) \delta$ (ppm): 211.3, 150.2, 147.4, 126.5, $123.8,69.0,50.5,43.6,31.4,28.7,23.4,22.3,13.8$.

HPLC conditions Chiralpak IA (n-hexane/THF: 80/20, $1.0 \mathrm{~mL} / \mathrm{min}, \lambda=250$ $\mathrm{nm}), t_{\mathrm{R}}=11.26 \mathrm{~min}$ (minor), $t_{\mathrm{R}}=14.91 \mathrm{~min}$ (major). 


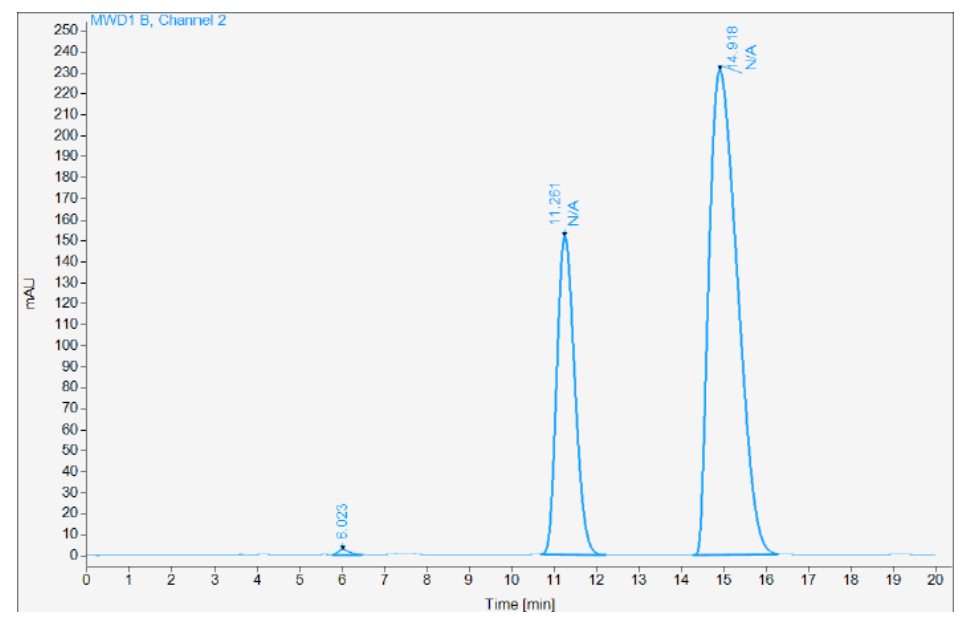

Figure SI_4.5.14. Chromatogram of a mixture of 1-(4-nitrophenyl)-1-hydroxy-3-nonanone enantiomers

\section{1-(4-nitrophenyl)-1-hydroxy-3-decanone}<smiles>CCCCCCCC(=O)CC(O)c1ccc([N+](=O)[O-])cc1</smiles>

${ }^{1} \mathrm{H}$ NMR $\left(500 \mathrm{MHz}, \mathrm{CDCl}_{3}\right) \delta(\mathrm{ppm}): 8.21(\mathrm{~d}, J=7.2 \mathrm{~Hz}, 2 \mathrm{H}), 7.54(\mathrm{~d}, J=7.5 \mathrm{~Hz}, 2 \mathrm{H})$, $5.26(\mathrm{~m}, 1 \mathrm{H}), 3.64(\mathrm{~s}, 1 \mathrm{H}), 2.82(\mathrm{~m}, 2 \mathrm{H}), 2.44(\mathrm{t}, J=7.4 \mathrm{~Hz}, 2 \mathrm{H}), 1.60(\mathrm{~m}, 2 \mathrm{H}), 1.28(\mathrm{~m}, 8 \mathrm{H})$, $0.88(\mathrm{t}, J=6.7 \mathrm{~Hz}, 3 \mathrm{H}) .{ }^{13} \mathrm{C} \mathrm{NMR}\left(75 \mathrm{MHz}, \mathrm{CDCl}_{3}\right) \delta(\mathrm{ppm}): 209.6,150.8,147.4,126.4$, 123.7, 69.0, 50.5, 43.65, 31.6, 29.7, 23.5, 22.5, 14.0; (ESI-TOF, positive mode) m/z exp $[\mathrm{M}+\mathrm{Na}]^{+}$calcd for $\mathrm{C}_{16} \mathrm{H}_{23} \mathrm{NNaO}_{4}{ }^{+} 316.1519 ;$ found, $316.1529[\mathrm{M}+\mathrm{Na}]^{+},(\Delta=1.3 \mathrm{ppm})$.

HPLC conditions Chiralpak IA ( $n$-hexane/THF: 80/20, $1.0 \mathrm{~mL} / \mathrm{min}, \lambda=250$ $\mathrm{nm}$ ), $t_{\mathrm{R}}=9.23 \mathrm{~min}$ (minor), $t_{\mathrm{R}}=11.44 \mathrm{~min}$ (major). 


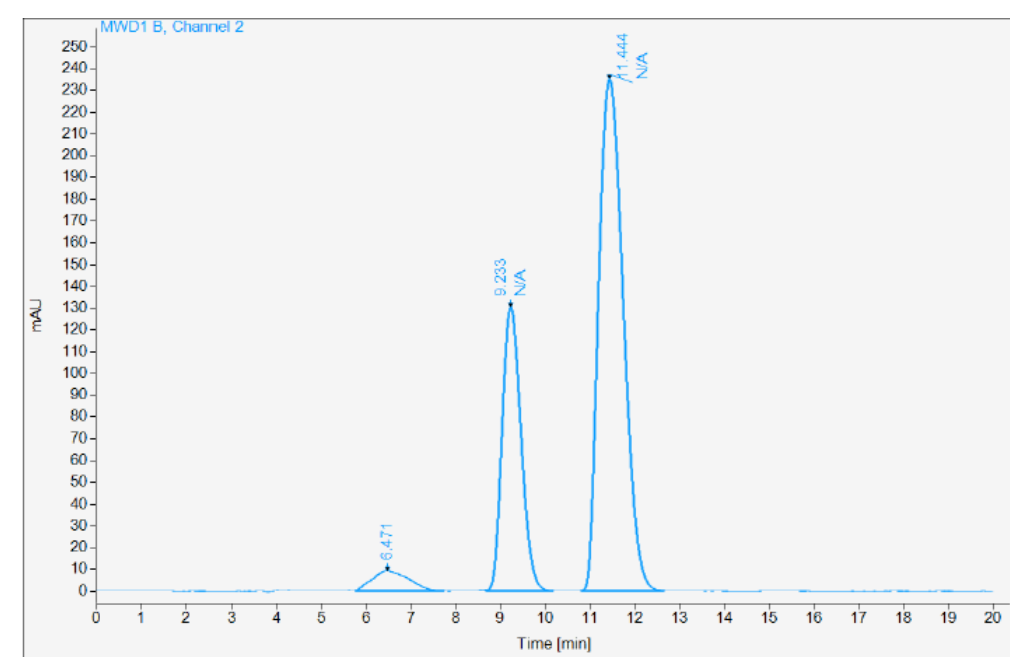

Figure SI_4.5.15. Chromatogram of a mixture of 1-(4-nitrophenyl)-1-hydroxy-3-decanone enantiomers.

\section{1-(4-nitrophenyl)-1-hydroxy-3-tridecanone}<smiles>CCCCCCCCCCC(=O)CC(O)c1ccc([N+](=O)[O-])cc1</smiles>

${ }^{1} \mathrm{H}$ NMR (500 MHz, $\left.\mathrm{CDCl}_{3}\right) \delta(\mathrm{ppm}): 8.21(\mathrm{~d}, J=8.3 \mathrm{~Hz}, 2 \mathrm{H}), 7.54(\mathrm{~d}, J=8.1 \mathrm{~Hz}, 3 \mathrm{H})$, $5.26(\mathrm{~m}, 1 \mathrm{H}), 3.64(\mathrm{~s}, 1 \mathrm{H}), 2.81(\mathrm{~m}, 2 \mathrm{H}), 2.44(\mathrm{t}, J=7.4 \mathrm{~Hz}, 2 \mathrm{H}), 1.63-1.56(\mathrm{~m}, 2 \mathrm{H}), 1.26$ $(\mathrm{m}, 14 \mathrm{H}), 0.88(\mathrm{t}, J=6.7 \mathrm{~Hz}, 3 \mathrm{H}) . ;{ }^{13} \mathrm{C} \mathrm{NMR}\left(\mathrm{CDCl}_{3}, 125 \mathrm{MHz}\right) \delta(\mathrm{ppm}): 210.8,150.1,147.4$, $126.2,123.5,68.9,50.4,43.5,31.7,29.3,29.2,29.1,29.1,28.9,23.4,13.9$.

HPLC conditions Chiralpak IA (n-hexane/THF: 80/20, $1.0 \mathrm{~mL} / \mathrm{min}, \lambda=250$ $\mathrm{nm}), t_{\mathrm{R}}=7.71 \mathrm{~min}$ (minor), $t_{\mathrm{R}}=10.84 \mathrm{~min}$ (major). 


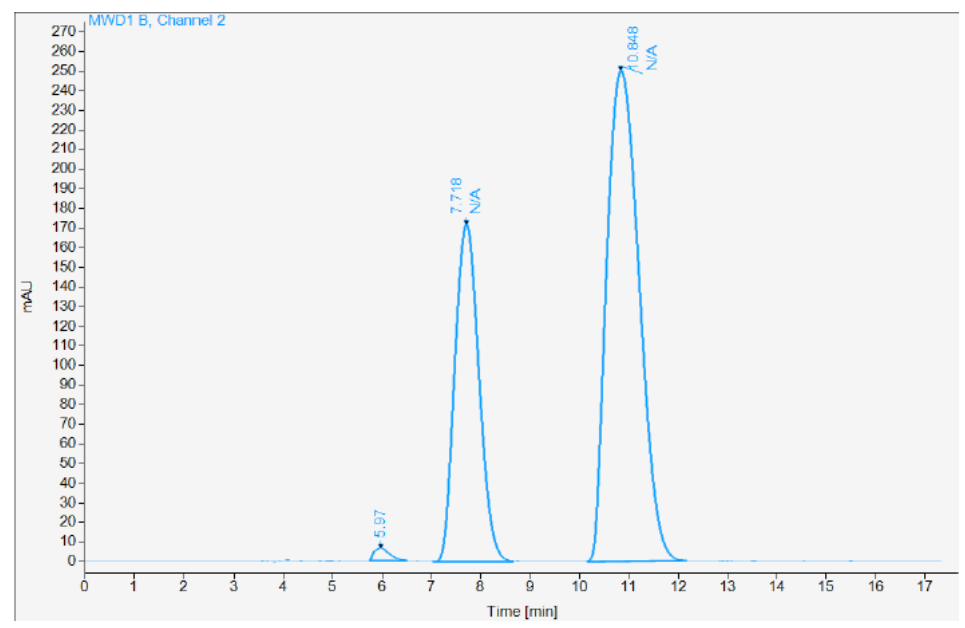

Figure SI_4.5.16. Chromatogram of a mixture of 1-(4-nitrophenyl)-1-hydroxy-3-tridecanone enantiomers. 


\subsection{References and Notes}

1. (a) Sangeetha, N. M.; Maitra, U. "Supramolecular Gels: Functions and Uses", Chem. Soc. Rev., 2005, 34, 821-836; (b) Xu, B. "Gels as Functional Nanomaterials for Biology and Medicine", Langmuir, 2009, 25, 8375-8377; (c) Escuder, B.; Miravet, J. F., Eds., "Functional Molecular Gels", RSC, Cambridge, 2014.

2. Raeburn, J.; Zamith Cardoso, A.; Adams, D. J. "The Importance of the Self-Assembly Process to Control Mechanical Properties of Low Molecular Weight Hydrogels", Chem. Soc. Rev., 2013, 42, 5143-5156.

3. (a) Palmer, L. C.; Stupp, S. I. "Molecular Self-Assembly into One-Dimensional Nanostructures", Acc. Chem. Res., 2008, 41, 1674-1684; (b) Saito, N.; Shigeno, M.; Yamaguchi, M. "Two-Component Fibers/Gels and Vesicles Formed from Hetero-DoubleHelices of Pseudoenantiomeric Ethynylhelicene Oligomers with Branched Side Chains", Chem. Eur. J., 2012, 18, 8994-9004.

4. (a) Huang, X.; Raghavan, S. R.; Terech, P.; Weiss, R. G. "Distinct Kinetic Pathways Generate Organogel Networks with Contrasting Fractality and Thixotropic Properties", J. Am. Chem. Soc., 2006, 128, 15341-15352; (b) Coates, I. A.; Smith, D. K. "Controlled Self-AssemblySynthetic Tunability and Covalent Capture of Nanoscale Gel Morphologies", Chem. Eur. J., 2009, 15, 6340-6344; (c) Liu, Z.-X.; Feng, Y.; Yan, Z.-C.; He, Y.-M.; Liu, C.-Y.; Fan, Q.-H. "Multistimuli Responsive Dendritic Organogels Based on Azobenzene-Containing Poly(aryl ether) Dendron", Chem. Mater., 2012, 24, 3751-3757.

5. Tidhar, Y.; Weissman, H.; Wolf, S. G.; Gulino, A.; Rybtchinski, B. "Pathway-Dependent SelfAssembly of Perylene Diimide/Peptide Conjugates in Aqueous Medium", Chem. Eur. J., 2011, 17, 6068-6075.

6. (a) Yu, L.; Furness, M. S.; Raw, A.; Outlaw, K. W.; Nashed, N.; Ramos, E.; Miller, S. F.; Adams, R.; Fang, F.; Patel, R.; Holcombe, F., Jr.; Chiu, Y.-y.; Hussain, A. "Scientific Considerations of Pharmaceutical Solid Polymorphism in Abbreviated New Drug Applications", Pharm. Res., 2003, 20, 531-536; (b) Brittain, H. G., "Polymorphism in Pharmaceutical Solids". second ed., Informa Healthcare, 2009.

7. (a) Haisa, M.; Kashino, S.; Maeda, H. "The Orthorhombic Form of p-Hydroxyacetanilide", Acta Crystallogr., Sect. B, 1974, 30, 2510-2512; (b) Haisa, M.; Kashino, S.; Kawai, R.; Maeda, H. "The Monoclinic Form of p-Hydroxyacetanilide", Acta Crystallogr., Sect. B, 1976, 32, 1283-1285.

8. Beyer, T.; Day, G. M.; Price, S. L. "The Prediction, Morphology, and Mechanical Properties of the Polymorphs of Paracetamol", J. Am. Chem. Soc., 2001, 123, 5086-5094.

9. (a) Tsekova, D. S.; Sáez, J. A.; Escuder, B.; Miravet, J. F. "Solvent-Free Construction of SelfAssembled 1D Nanostructures from Low-Molecular-Weight Organogelators: Sublimation vs. Gelation", Soft Matter, 2009, 5, 3727-3735; (b) Rodríguez-Llansola, F.; Miravet, J. F.; Escuder, B. "Supramolecular Gel Formation and Self-Correction Induced by AggregationDriven Conformational Changes", Chem. Commun., 2009, 209-211; (c) Fontanillo, M.; Angulo-Pachón, C. A.; Escuder, B.; Miravet, J. F. "In Situ Synthesis-Gelation at Room Temperature vs. Heating-Cooling Procedure. Fine Tuning of Molecular Gels Derived from Succinic Acid and L-Valine", J. Colloid Interface Sci., 2013, 412, 65-71; (d) Nebot, V. J.; DíazOltra, S.; Smets, J.; Fernandez Prieto, S.; Miravet, J. F.; Escuder, B. "Freezing Capture of 
Polymorphic Aggregates of Bolaamphiphilic (L)-Valine-Based Molecular Hydrogelators", Chemistry, 2014, 20, 5762-5767.

10. (a) Li, C.-J.; Chen, L. "Organic Chemistry in Water", Chem. Soc. Rev., 2006, 35, 68-82; (b) Brogan, A. P.; Dickerson, T. J.; Janda, K. D. "Enamine-Based Aldol Organocatalysis in Water: Are they Really "All Wet"?", Angew. Chem. Int. Ed., 2006, 45, 8100-8102.

11. Rideout, D. C.; Breslow, R. "Hydrophobic Acceleration of Diels-Alder Reactions", J. Am. Chem. Soc., 1980, 102, 7816-7817.

12. (a) Hayashi, Y.; Aratake, S.; Okano, T.; Takahashi, J.; Sumiya, T.; Shoji, M. "Combined Proline-Surfactant Organocatalyst for the Highly Diastereo- and Enantioselective Aqueous Direct Cross-Aldol Reaction of Aldehydes", Angew. Chem. Int. Ed., 2006, 45, 5527-5529; (b) Mase, N.; Nakai, Y.; Ohara, N.; Yoda, H.; Takabe, K.; Tanaka, F.; Barbas, C. F., 3rd "Organocatalytic Direct Asymmetric Aldol Reactions in Water", J. Am. Chem. Soc., 2006, 128, 734-735; (c) Zhao, J.-F.; He, L.; Jiang, J.; Tang, Z.; Cun, L.-F.; Gong, L.-Z. "OrganoCatalyzed Highly Diastereo- and Enantio-Selective Direct Aldol Reactions in Water", Tetrahedron Lett., 2008, 49, 3372-3375; (d) Mlynarski, J.; Paradowska, J. "Catalytic Asymmetric Aldol Reactions in Aqueous Media", Chem. Soc. Rev., 2008, 37, 1502-1511.

13. Schmidt, J.; Ehasz, C.; Epperson, M.; Klas, K.; Wyatt, J.; Hennig, M.; Forconi, M. "The Effect of the Hydrophobic Environment on the Retro-Aldol Reaction: Comparison to a Computationally-Designed Enzyme", Org. Biomol. Chem., 2013, 11, 8419-8425.

14. Paradowska, J.; Stodulski, M.; Mlynarski, J. "Catalysts Based on Amino Acids for Asymmetric Reactions in Water", Angew. Chem. Int. Ed., 2009, 48, 4288-4297.

15. Grieco, P. A., "Organic Synthesis in Water". Blackie Academic and Professional, 1998.

16. Narayan, S.; Muldoon, J.; Finn, M. G.; Fokin, V. V.; Kolb, H. C.; Sharpless, K. B. "'On Water": Unique Reactivity of Organic Compounds in Aqueous Suspension", Angew. Chem. Int. Ed., 2005, 44, 3275-3279.

17. (a) Jung, Y.; Marcus, R. A. "On the Theory of Organic Catalysis "on Water"", J. Am. Chem. Soc., 2007, 129, 5492-5502; (b) Chanda, A.; Fokin, V. V. "Organic Synthesis "On Water"", Chem. Rev., 2009, 109, 725-748.

18. Minakata, S.; Komatsu, M. "Organic Reactions on Silica in Water", Chem. Rev., 2009, 109, 711-724.

19. Zhang, H.; Zhao, W.; Zou, J.; Liu, Y.; Li, R.; Cui, Y. "Aldol Reaction Catalyzed by a Hydrophilic Catalyst in Aqueous Micelle as an Enzyme Mimic System", Chirality, 2009, 21, 492-496.

20. Duschmale, J.; Kohrt, S.; Wennemers, H. "Peptide Catalysis in Aqueous Emulsions", Chem. Commun., 2014, 8109-8112.

21. Qin, L.; Zhang, L.; Jin, Q.; Zhang, J.; Han, B.; Liu, M. "Supramolecular Assemblies of Amphiphilic L-Proline Regulated by Compressed $\mathrm{CO}_{2}$ as a Recyclable Organocatalyst for the Asymmetric Aldol Reaction", Angew. Chem. Int. Ed., 2013, 52, 7761-7765.

22. (a) Hartgerink, J. D.; Beniash, E.; Stupp, S. I. "Self-Assembly and Mineralization of PeptideAmphiphile Nanofibers", Science, 2001, 294, 1684-1688; (b) Hartgerink, J. D.; Beniash, E.; Stupp, S. I. "Peptide-Amphiphile Nanofibers: A Versatile Scaffold for the Preparation of Self-Assembling Materials", Proc. Natl. Acad. Sci. U. S. A., 2002, 99, 5133-5138; (c) Estroff, 
L. A.; Hamilton, A. D. "Water Gelation by Small Organic Molecules", Chem. Rev., 2004, 104, 1201-1218; (d) Zhang, J.; Zhao, Y.; Han, S.; Chen, C.; Xu, H. "Self-Assembly of Surfactant-Like Peptides and their Applications", Sci. China Chem., 2014, 57, 1634-1645.

23. Rodríguez-Llansola, F.; Miravet, J. F.; Escuder, B. "A Supramolecular Hydrogel as a Reusable Heterogeneous Catalyst for the Direct Aldol Reaction", Chem. Commun., 2009, 7303-7305.

24. (a) Naota, T.; Koori, H. "Molecules that Assemble by Sound: an Application to the Instant Gelation of Stable Organic Fluids", J. Am. Chem. Soc., 2005, 127, 9324-9325; (b) Wu, J.; Yi, T.; Shu, T.; Yu, M.; Zhou, Z.; Xu, M.; Zhou, Y.; Zhang, H.; Han, J.; Li, F.; Huang, C. "Ultrasound Switch and Thermal Self-Repair of Morphology and Surface Wettability in a Cholesterol-Based Self-Assembly System", Angew. Chem. Int. Ed., 2008, 47, 1063-1067; (c) Cravotto, G.; Cintas, P. "Molecular Self-Assembly and Patterning Induced by Sound Waves. The Case of Gelation", Chem. Soc. Rev., 2009, 38, 2684-2697.

25. (a) Burger, A.; Ramberger, R. "On the Polymorphism of Pharmaceuticals and Other Molecular Crystals. I", Microchim. Acta, 1979, 72, 259-271; (b) Purohit, R.; Venugopalan, P. "Polymorphism: an Overview", Resonance, 2009, 14, 882-893.

26. Henck, J.-O.; Kuhnert-Brandstatter, M. "Demonstration of the Terms Enantiotropy and Monotropy in Polymorphism Research Exemplified by Flurbiprofen", J. Pharm. Sci., 1999, 88, 103-108.

27. Escuder, B.; Martí, S.; Miravet, J. F. "Organogel Formation by Coaggregation of Adaptable Amidocarbamates and their Tetraamide Analogues", Langmuir, 2005, 21, 6776-6787.

28. Rodríguez-Llansola, F.; Miravet, J. F.; Escuder, B. "Aldehyde Responsive Supramolecular Hydrogels: towards Biomarker-Specific Delivery Systems", Chem. Commun., 2011, 47, 4706-4708.

29. Berdugo, C.; Miravet, J. F.; Escuder, B. "Substrate Selective Catalytic Molecular Hydrogels: the Role of the Hydrophobic Effect", Chem. Commun., 2013, 49, 10608-10610. 

CHAPTER 5

\section{IONISABLE HYDROGELS: STUDY AND APPLICATIONS}





\subsection{Introduction}

The creation of smart materials has attracted the attention of scientists in recent years for their applications in fields such as drug release, biomedicine and electronic materials among others. ${ }^{1}$ Inspired by nature, the design of stimuli responsive materials has been a desirable target in Materials Chemistry. Supramolecular chemistry, governed by reversible, weak and non-covalent interactions, is an interesting approach to the construction of such materials as the self-assembling interactions responsible for the properties of these structures are sensitive to external stimuli. ${ }^{2}$

Supramolecular gels have raised interest in this field showing promising applications, most of them referent to supramolecular polymeric gels. ${ }^{3}$ On the contrary, stimuli responsive molecular gels, ${ }^{4}$ formed by low-molecular weight gelators (LMWGs), have been scarcely developed in the past but are receiving increased attention nowadays as they offer various advantages over traditional polymer gels, for example, they are thermoreversible and they are easier for the body to degrade.

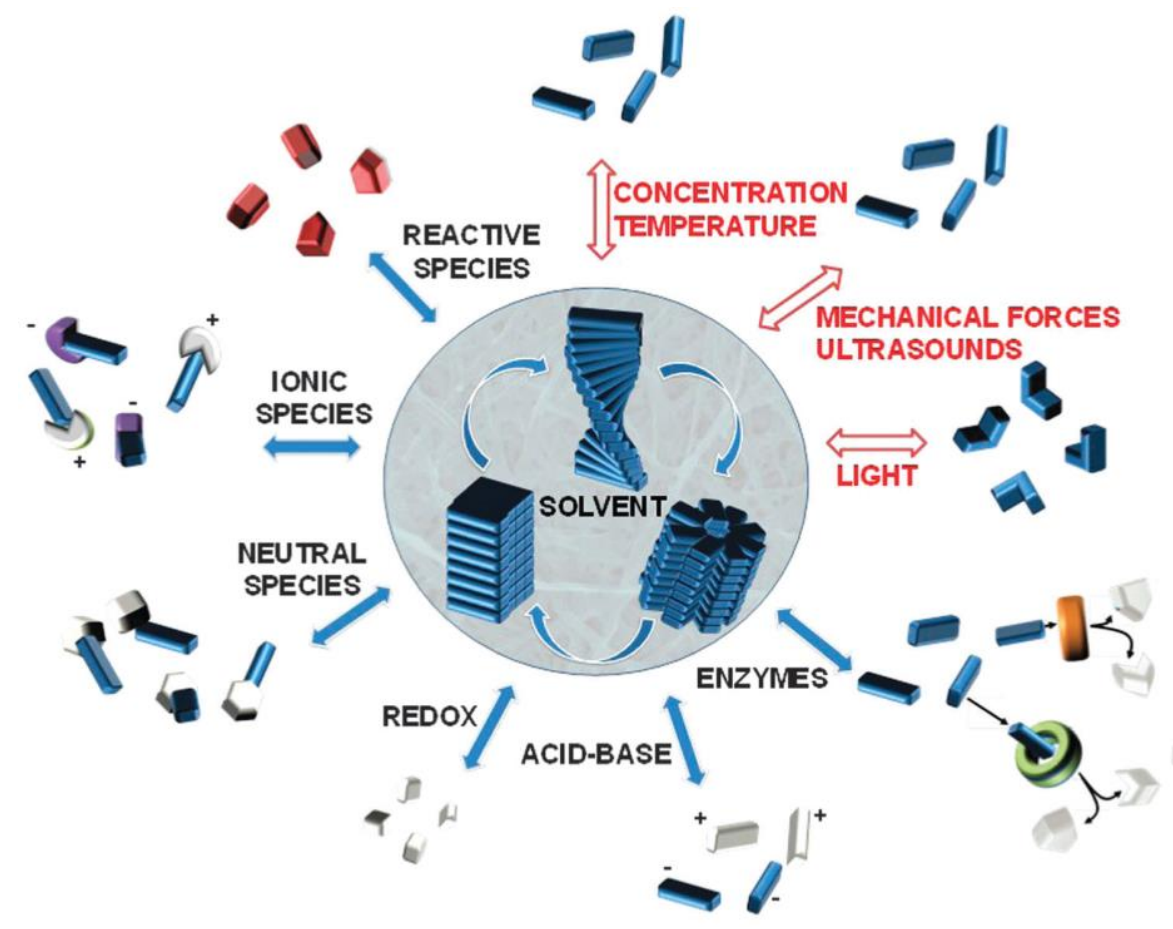

Figure 5.1.1. Illustrative classification of the different stimuli that can regulate molecular gel formation (physical in red and chemical in blue). ${ }^{4 b}$ 
The introduction of functional groups within the gelator structure that possess specific responses allows gelation to be switched-on by a large number of external stimuli. External stimuli can be classified into two main groups: physical and chemical. Physical stimuli such as UV-vis light, ultrasound or temperature, and chemical; $\mathrm{pH}$, redox or selectivity are used as triggers for morphological transitions (see Figure 5.1.1).

Using $\mathrm{pH}$ to induce gelation has been used as an interesting approach to control gel formation. In this way, with the aim of preparing homogeneous and reproducible gel systems, the gelation process can be controlled by means of an acid-base reaction. Examples include the system reported by Hayes et al., where a urea derivative gelator molecule bearing a carboxylic acid was studied. In that case, the gelation process took place after the addition of $\mathrm{HCl}$ to a solution of the compound dissolved in basic media. ${ }^{5}$ Following this study a family of $\mathrm{pH}$-tuneable hydrogelators were found that presented interesting applicability in water purification (see Figure 5.1.2). ${ }^{6}$

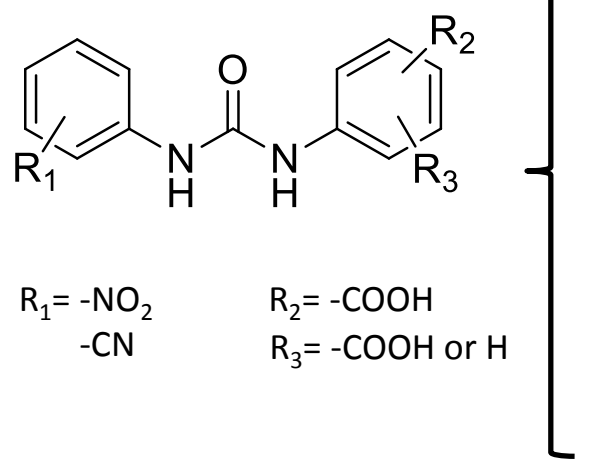<smiles>O=C(Nc1ccc([N+](=O)[O-])cc1)Nc1cc(C(=O)O)cc(C(=O)O)c1</smiles>

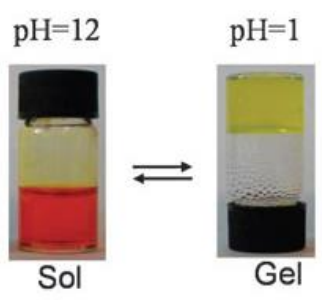

Figure 5.1.2. Urea derivatives described by Hayes et al. and one example of gelation induced by $\mathrm{pH}$-change.

With the same idea, Adams et al. reported the introduction of glucono- $\delta$-lactone (GdL) hydrolysis to gluconic acid in water to progressively change the $\mathrm{pH}$ and obtain hydrogels of Fmoc-dipetides, avoiding poor reproducibility of the gel properties often found by conventional acidification procedures. (Figure 5.1.3). ${ }^{7}$ 
A)
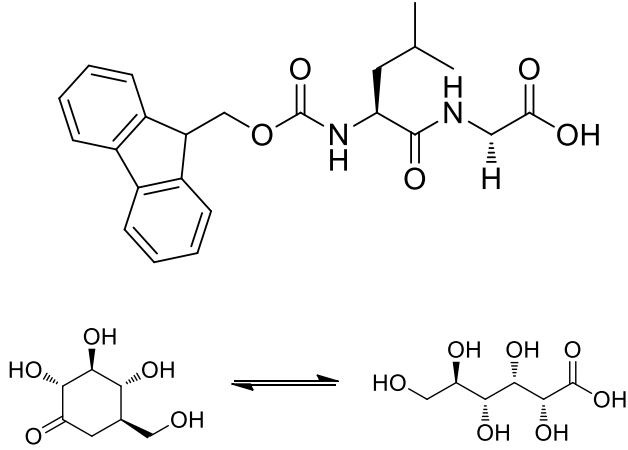

B)

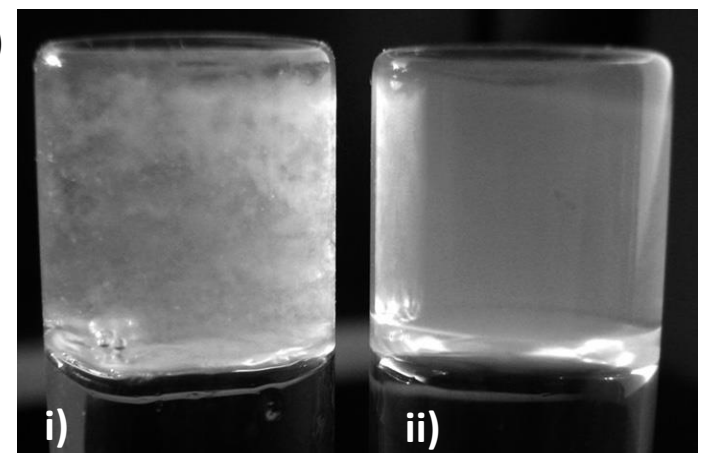

Figure 5.1.3. A) Fmoc-protected dipeptide used as a hydrogelator and the reaction of $\delta$-gluconolactone (GdL) hydrolysis used to generate acidic conditions. B) Photographs of hydrogels prepared by i) addition of aqueous $\mathrm{HCl}$ (turbid) and ii) hydrolysis of GdL (transparent and uniform gels).

One step further in complexity is the study of self-assembly in multicomponent supramolecular systems, ${ }^{8}$ reminiscent of the level of complexity found in natural systems. The multicomponent system approach leads to the presence of more than one stimuliresponsive group, offering a facile way to tailor the properties of the gel. A variety of two component systems based on non-covalent interactions has been reported. ${ }^{9}$ The mixing of two or more gelators to form a two-component hydrogel is one of the possibilities to produce materials with good microstructural tuneability, where for example, changing the molar ratio of the two components could induce dramatic morphological changes. ${ }^{10}$

When the components in a solution of more than one gelator molecule selfassemble they can either co-assemble, by intermolecular interactions between both types of gelator in the same fibrillar network, or self-sort. In the latter case the two types of gelator molecules self-assemble orthogonally and form two different assemblies that coexist in the same system. It is reasonable to expect co-assembly, being the predominant mechanism, for gelators with similar structural features and self-sorting for structurally diverse gelators.

The co-assembly of tetrapeptides to yield a $\mathrm{pH}$ responsive hydrogel (see Figure 5.1.4) has recently been reported in our group. ${ }^{11}$ In this work, the ionic interactions between the two peptides are likely responsible of hydrogel formation. Additionally, these ionisable groups are used to modulate the gelation components with $\mathrm{pH}$ changes, showing potential application in selective release. 


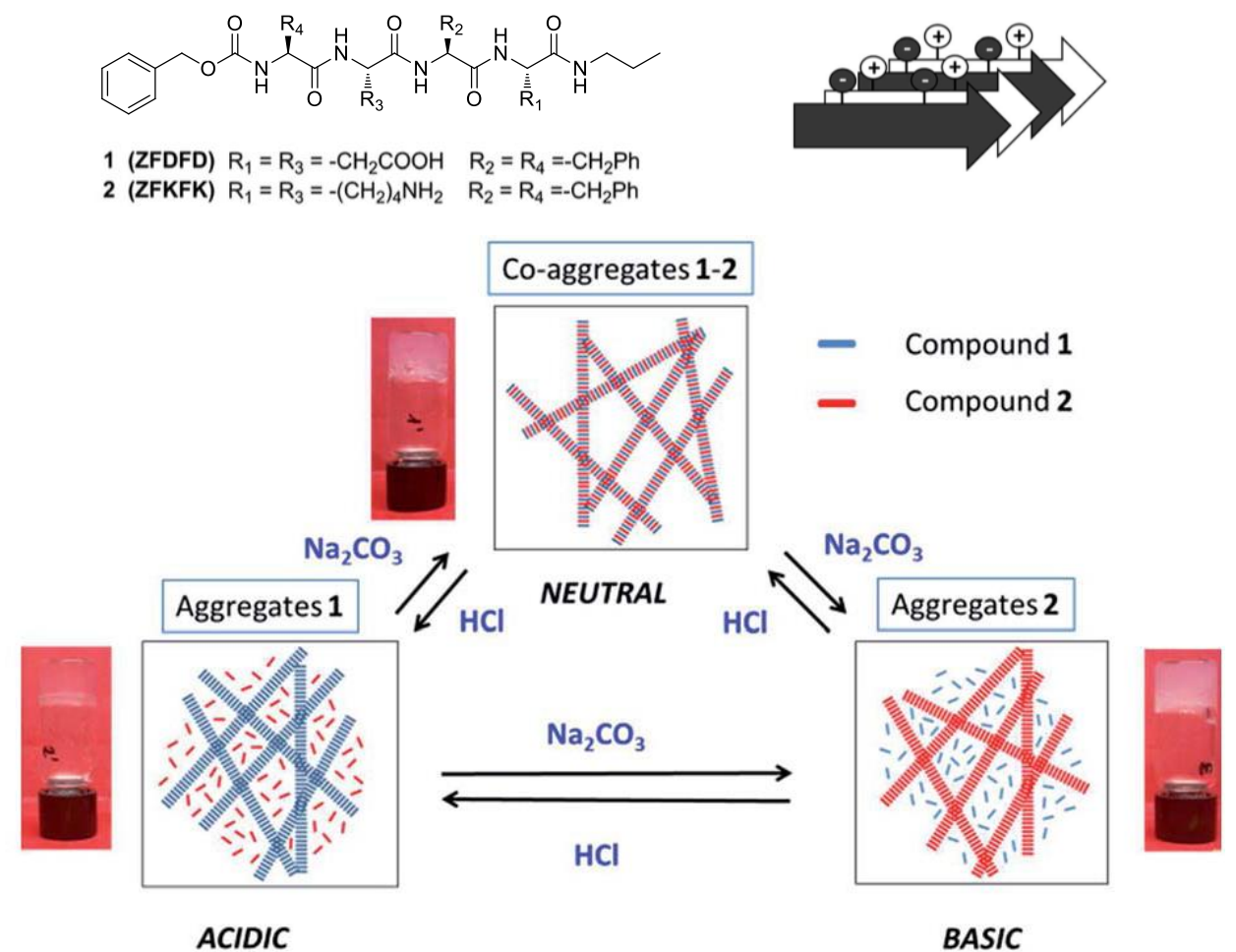

Figure 5.1.4. Molecular structure of the tetrapeptides involved in a co-assembly process that affords hydrogel (top) and schematic view of the $\mathrm{pH}$-responsiveness of the hydrogel system (bottom). ${ }^{11}$

Adams et al. not long ago reported the first $\mathrm{pH}$-controlled self-sorting of two Fmoc-dipeptides derivatives, taking advantage of their different $\mathrm{pK}_{\mathrm{a}}$ values (see Figure 5.1.5). ${ }^{12}$ Progressively reducing the $\mathrm{pH}$ by means of $\delta$-gluconolactone hydrolysis led to selective gelation of the more basic molecule first.<smiles>CC(=O)NC(=O)C(C)NC(=O)COc1ccc2ccccc2c1</smiles>
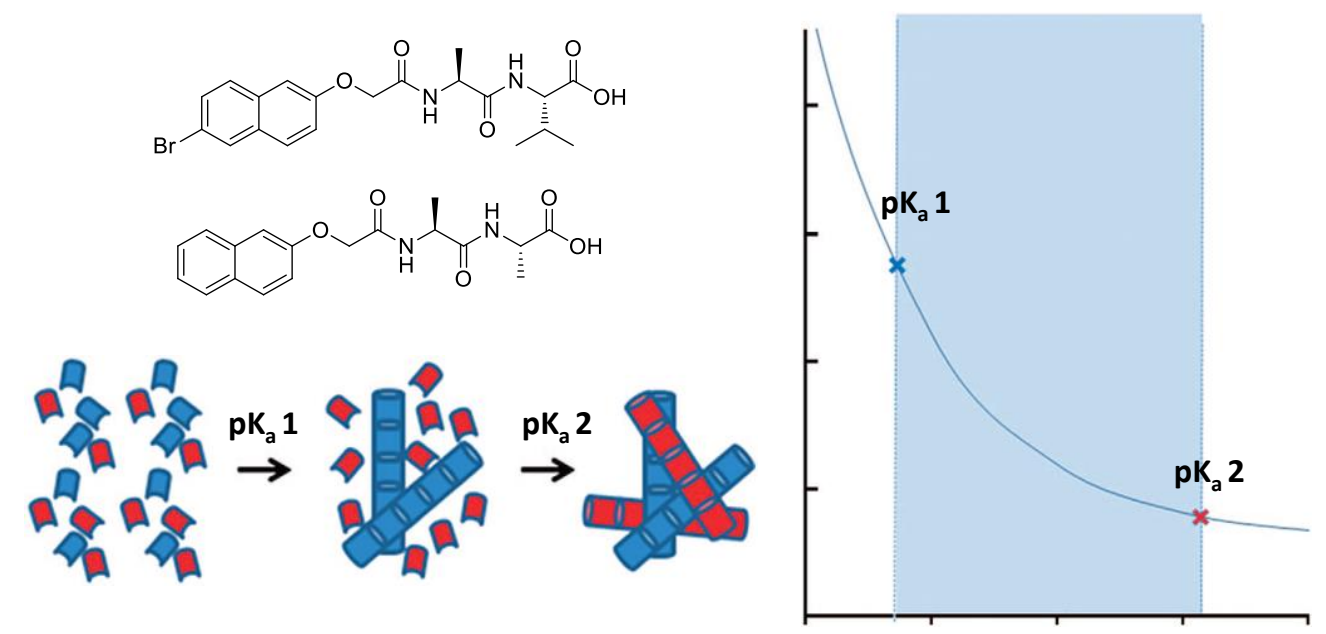

Figure 5.1.5. Schematic cartoon of a self-sorting gelation process depending on the $\mathrm{pK}_{\mathrm{a}}$. 


\subsection{Ionisable Hydrogelators}

With the aim of designing functional materials capable of responding to the influence of different stimuli, a family of compounds bearing a monoamide of succinic acid was studied (see Figure 5.2.1). Both amphiphilic and bolaamphiphilic compounds bearing terminal ionisable groups were considered.

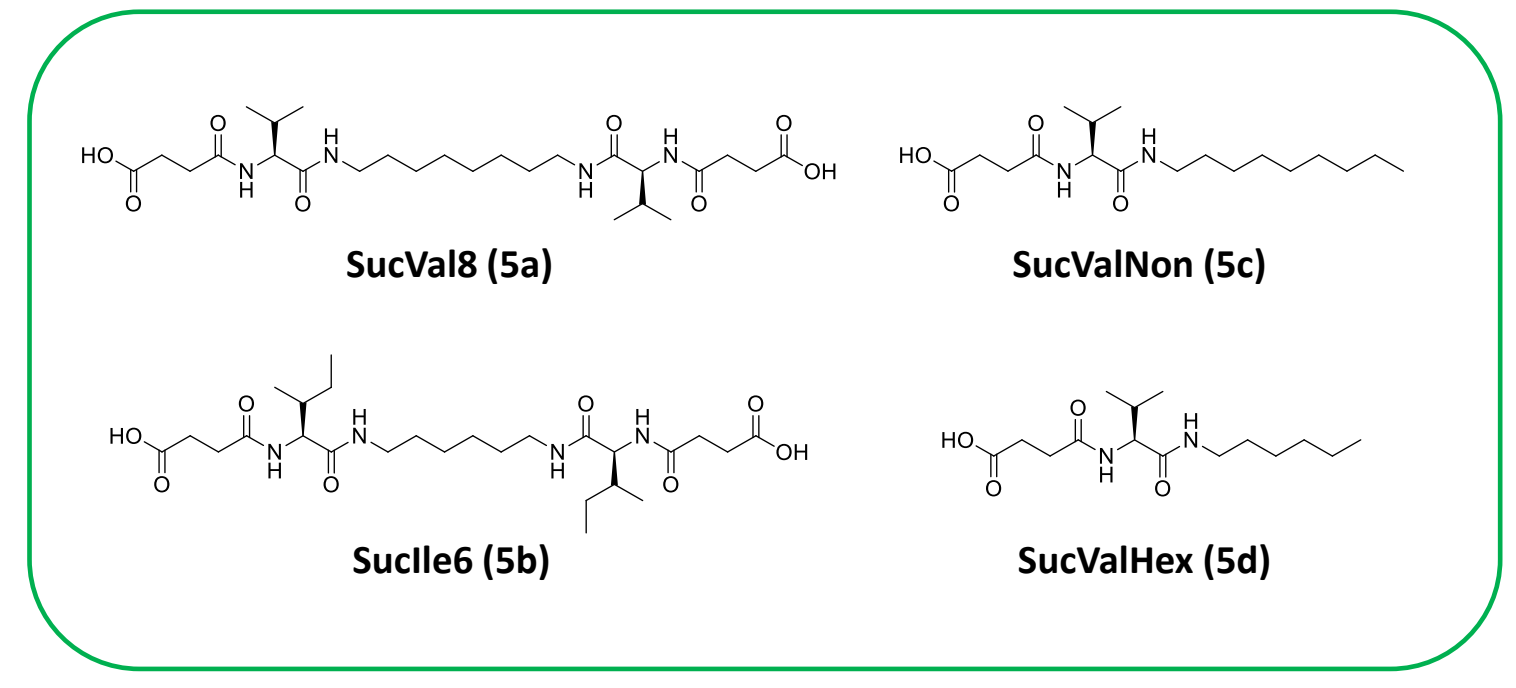

Figure 5.2.1. Molecular structure of succinic acid derivatives tested as hydrogelators.

All molecules were synthesised in an easy manner following conventional peptide synthesis in solution (see Scheme 5.1.1 and Scheme 5.1.2).<smiles>[R]C(NC(=O)OCc1ccccc1)C(=O)ON1C(=O)CCC1=O</smiles>

5a-b.1

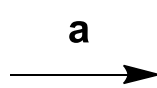<smiles>[R]C(NC(=O)CNC(=O)NCCNC(=O)C([R])NC(=O)OCc1ccccc1)C(=O)NC(=O)OCc1ccccc1</smiles>

5a-b.2

b
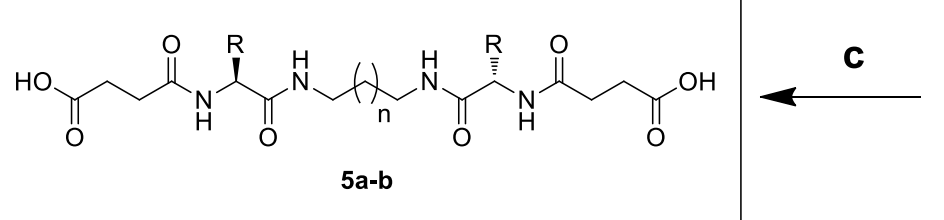<smiles>[R]C(N)C(=O)NCC1CCC(CNC(=O)C([R])N)C1</smiles>

Scheme 5.1.1. Synthesis of bolaamphiphiles $5 \mathrm{a}^{13}$ and 5 b. a) Diamine, DME, rt $(18 \mathrm{~h})$ and 50 ㅇ $(2 \mathrm{~h})(85-$ 95\%); b) $\mathrm{H}_{2}, \mathrm{Pd} / \mathrm{C}, \mathrm{MeOH}, \mathrm{rt}, 4 \mathrm{~h}$ (95-99\%); c) Succinic anhydride, THF, reflux, 16 h, 80-90\%. 


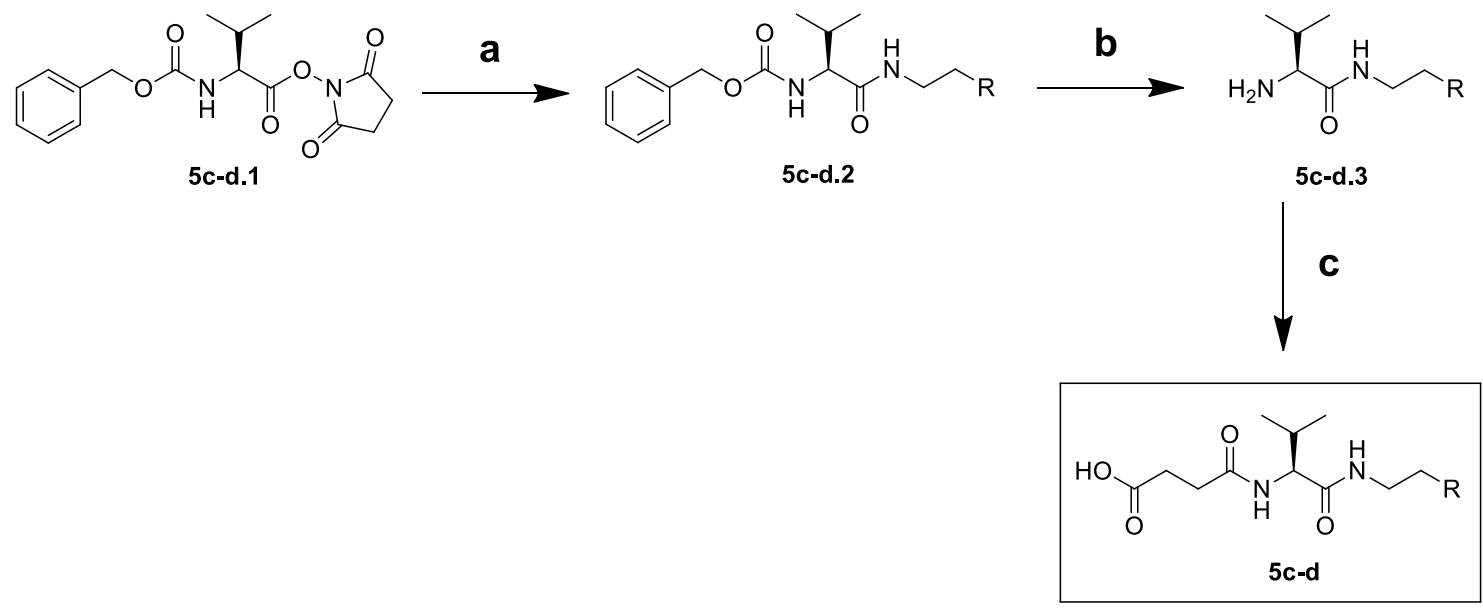

Scheme 5.1.2. Synthesis of amphiphiles $\mathbf{5 c}$ and $\mathbf{5 d}$. a) Amine, THF, 50 ㅇ․ $(5 \mathrm{~h})(96 \%) ;$ b) $\mathrm{H}_{2}, \mathrm{Pd} / \mathrm{C}, \mathrm{MeOH}, \mathrm{rt}$ (4-6 h) (95-97\%); c) Succinic anhydride, $\mathrm{THF}, \mathrm{Na}_{2} \mathrm{CO}_{3}$, rt (16 h)( 93-95\%).

A previous study with the compound SucVal8 (5a) has been reported showing its gelation behaviour with different organic solvents as well as in water. Moreover, a full characterization of the gels was performed at both macroscopic and microscopic levels. In that study an in situ synthesis-gelation procedure was described and compared with the common heating-cooling procedure, finding different polymorphic gels. ${ }^{13}$

In this work in first place, the gelation ability of these compounds in water was tested. The results are summarised in Table 5.2.1, showing the minimum gelator concentration values (MGC) obtained after a heating-cooling cycle and with the tube inversion approach. Moreover, the solubility constant $\left(K_{s}\right)$ was measured at room temperature by NMR. Only three of the four compounds tested were able to retain the liquid and form a gel material, the two bolaamphiphiles SucVal8 (5a) and Suclle6 (5b) as well as the amphiphile SucValNon (5c). For the compound SucValHex (5d), presumably the interactions involved in the aggregation were not strong enough to form a gel, as a consequence a viscous solution containing aggregates was found. 
Table 5.2.1. Hydrogelation study for compounds 5a-d with a heating-cooling procedure.

\begin{tabular}{ccc}
\hline Compound & MGC / mM & Ks / mM \\
\hline SucVal8 (5a) & 6 & 2,5 \\
Suclle6 (5b) & 15 & 6 \\
SucValNon (5c) & 7 & 1 \\
SucValHex (5d) & No gel/Aggregates & --- \\
\hline
\end{tabular}

5.2.1. $\mathrm{pH}$-Sensitive Hydrogelators: Potentiometric Determination of their Acid-Base Properties

In order to find a softer methodology to obtain gels which would avoid heatingcooling cycles and with the final aim to produce stimuli-responsive materials, the use of $\mathrm{pH}$ change to induce gelation was studied. Analysis of the thermodynamic constants associated to acid-base equilibria is necessary to properly design and use molecular hydrogelators with ionisable functional groups. Potentiometric titrations represent a very convenient way to address this issue. Once the thermodynamic parameters in a given system are characterised, a clear prediction of the different species present at different $\mathrm{pH}$ values can be made, allowing for a controlled $\mathrm{pH}$ tuning of hydrogel formation.

$$
\begin{gathered}
\mathrm{A}^{2-} \stackrel{\mathrm{K}_{1}}{\rightleftharpoons} \mathrm{AH}^{-} \stackrel{\mathrm{K}_{2}}{\rightleftharpoons} \mathrm{AH}_{2} \\
K_{1}=\frac{\left[A H^{-}\right]}{\left[A^{2-}\right]\left[H^{+}\right]} \\
K_{2}=\frac{\left[A H_{2}\right]}{\left[A H^{-}\right]\left[H^{+}\right]} \\
p K_{a}=-\log _{10} K_{a}
\end{gathered}
$$

Scheme 5.2.1.1. Studied equilibria and thermodynamic constants. 
In first instance the ionisation of the bolaamphiphilic compounds SucVal8 (5a) and Suclle6 (5b) was studied. Both molecules present two terminal ionisable carboxylic acid moieties. The acid dissociation constants (see Scheme 5.2.1.1 for equations) were determined by acidic titration after dissolving the compounds in aqueous $\mathrm{NaOH}$ (see titration curve for Sucval8 (5a) 1 mM in Figure 5.2.1.1 top) and protonation constants for diluted samples ( $1 \mathrm{mM}$, no gel formation) were calculated and are summarised in Table 5.2.1.1.

Table 5.2.1.1. Protonation constants for diprotic compounds in diluted solutions (concentration $<1 \mathrm{mM}$ ).

\begin{tabular}{ccc}
\hline Compound & $\mathrm{pK}_{\mathrm{a}} 1$ & $\mathrm{pK}_{\mathrm{a}} 2$ \\
\hline SucVal8 (5a) & $5,4(0,1)$ & $2,6(0,1)$ \\
Suclle6 (5b) & $4,8(0,1)$ & $4,3(0,1)$ \\
\hline
\end{tabular}

[a] Calculated errors in parenthesis.

Analysis of the $\mathrm{pK}_{\mathrm{a}}$ values for these compounds reveals a significant difference in the second protonation constant $\left(\mathrm{pK}_{\mathrm{a}} 2\right)$. The neutral species $\left(\mathrm{AH}_{2}\right)$ of SucVal8 (5a) is notably more acidic than in the case of Suclle6 (5b). A species distribution diagram can be obtained from those constants (see Figure 5.2.1.1 left).

The species distribution diagram for SucVal8 (5a) reveals that the species $\mathrm{AH}^{-}$is predominant in the $\mathrm{pH}$-range from 2 to 6 . The stabilisation of this $\mathrm{AH}^{-}$species for SucVal8 (5a) in comparison to Suclle6 (5b) may be linked to the favourable formation of ionic aggregates or stabilization of the carboxylate by means of intramolecular $\mathrm{H}$-bonding. Interestingly, the titration curve of SucVal8 (5a) reveals a $\mathrm{pH}$ jump for values around 8 , possibly associated to the partial protonation of aggregates formed by $A^{2-}$ species. For Suclle6 (5b), this $\mathrm{pH}$ jump in the titration curve is also present (see Figure 5.2.1.1 right), but to a minor degree indicating a less favourable partial protonation or less favourable formation of charged aggregated species (see species distribution diagram in Figure 5.2.1.1 left). 
A)
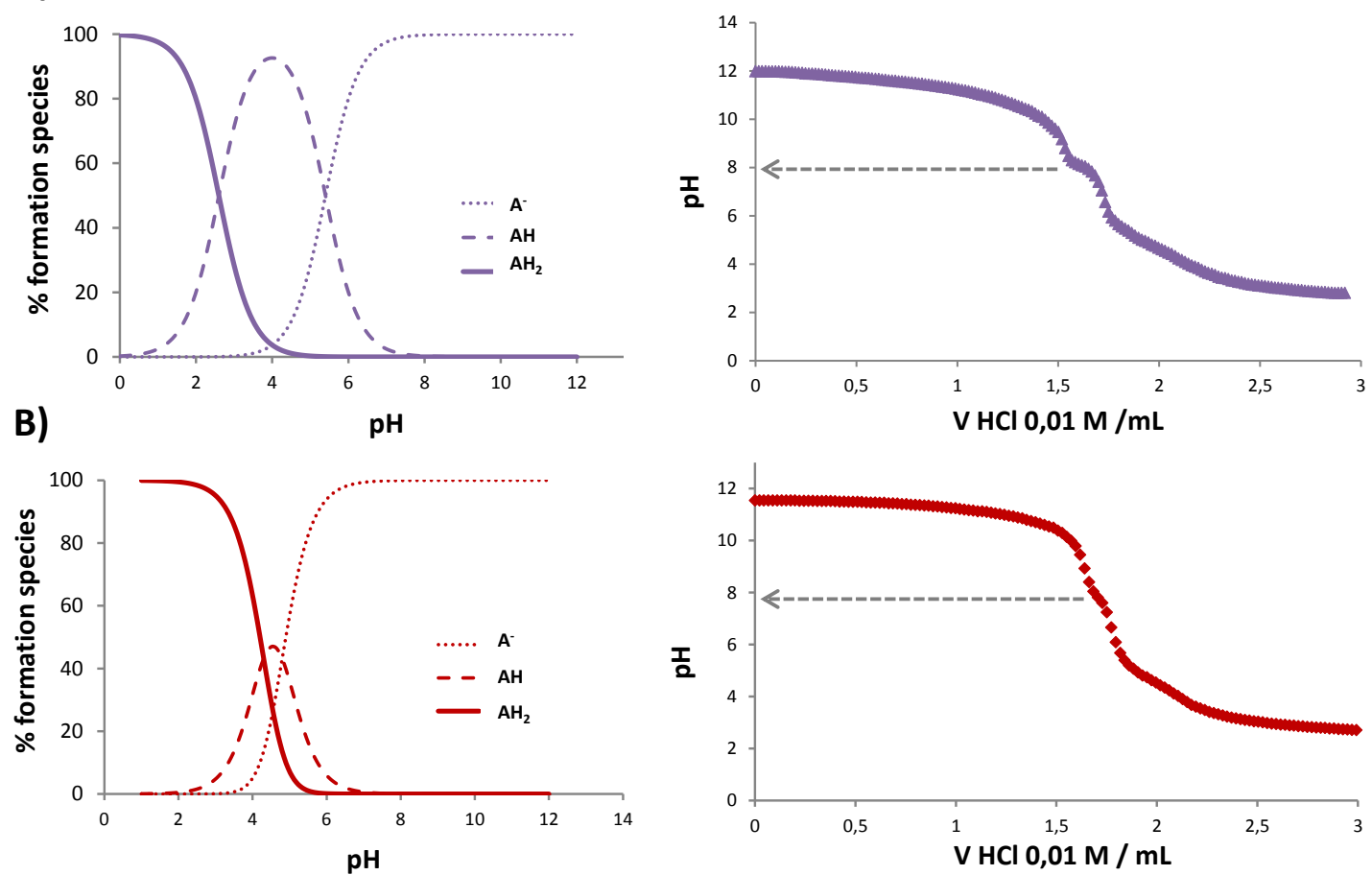

Figure 5.2.1.1. Species distribution diagram (left) and titration curve (right) for diluted samples of A) SucVal8 (5a) and B) Suclle6 (5b).

A proposed scheme showing how this intermediate species, corresponding to partially protonated structures, can be stabilised by means of the formation of some type of aggregates such as micelles or partially protonated vesicles is shown in Figure 5.2.1.2. The example illustrates how relatively small molecular changes significantly affect the acid-base properties for these molecules.

To test if the acid-base equilibrium is shifted when gelation is involved, a new titration experiment was first carried out on a sample above the MGC for the SucVal8 (5a) (see Table 5.2.1.2). As can be seen the effect of the formation of fibres in the acidity constants was more evident in the second equilibrium constant, whilst there is no variation for the first one. $\mathrm{A}$ big increase in the basicity of $\mathrm{AH}^{-}$species $\left(\mathrm{pK}_{\mathrm{a}} 2\right.$ changes from 2,6 to 4,9 from diluted to aggregated samples) was obtained as a result of the gelation for $\mathrm{AH}_{2}$ in the concentrated sample. In the case of Suclle6 (5b) (see Table 5.2.1.2) an increase of the basicity is also observed, but in this case it is less intense ( $\mathrm{pK}_{\mathrm{a}} 2$ changes from 4.3 to 4.9) pointing to weaker intermolecular interactions in the gel fibres. 


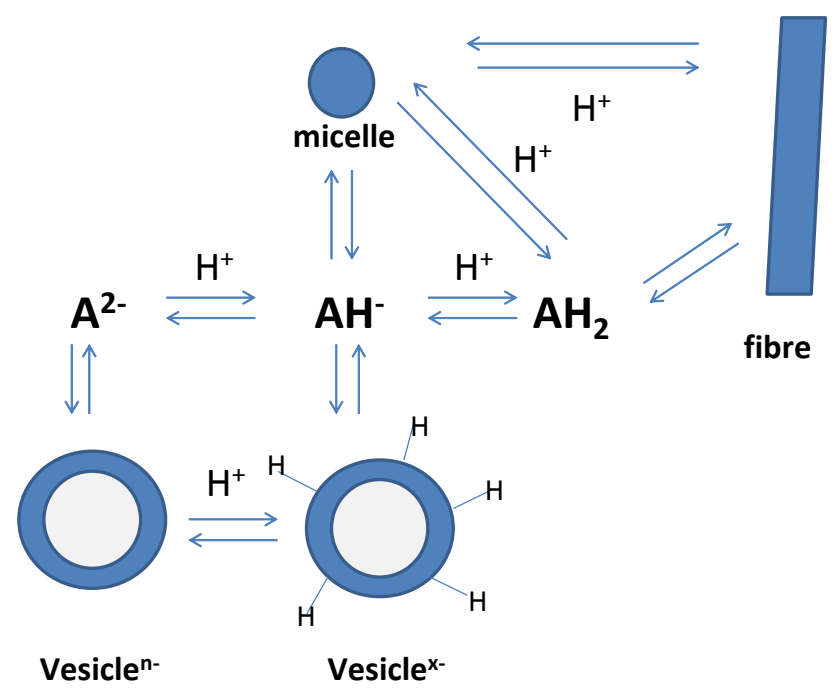

Figure 5.2.1.2. Schematic illustration of aggregated species involved during the acid-base equilibria.

Interestingly, the presence of partially protonated species that are able to form different aggregated structures were also found in the concentrated titration curve samples (see Figure SI_5.5.1). To further check the presence of these species, additional experiments were performed. Dynamic light scattering (DLS) analysis of a concentrated sample of SucVal8 (5a) in $\mathrm{NaOH}$ solution showed the formation of large aggregates of about $90 \mathrm{~nm}$ (see Figure SI_5.5.3) in agreement with the formation of vesicles as such depicted in Figure 5.2.1.2.

Table 5.2.1.2. Protonation constants for bolaamphiphiles in diluted solutions $(<1 \mathrm{mM})$ and concentrated solutions (> $16 \mathrm{mM}) .^{a}$

\begin{tabular}{cccc}
\hline Compound & Constant & Diluted & Aggregated \\
\hline SucVal8 & $\mathbf{p K}_{\mathrm{a}} \mathbf{1}$ & $5,4(0,1)$ & $5,4(0,1)$ \\
& $\mathbf{p K}_{\mathrm{a}} \mathbf{2}$ & $2,6(0,1)$ & $4,9(0,1)^{*}$ \\
\hline Suclle6 & $\mathbf{p K}_{\mathrm{a}} \mathbf{1}$ & $4,8(0,1)$ & $5,1(0,1)$ \\
& $\mathbf{p K}_{\mathrm{a}} \mathbf{2}$ & $4,3(0,1)$ & $4,9(0,1)^{*}$ \\
\hline
\end{tabular}

[a] Calculated errors in parenthesis.

*Estimated from the $\mathrm{pH}$ value at the plateau in the titration curve.

It is worthy to say that kinetic effects involved in the protonation of these molecules are important. A comparative titration curve of samples under the same 
conditions, but varying titration rate (from 0,05 to $0,003 \mathrm{~mL} / \mathrm{min}$ ) is illustrated in Figure 5.2.1.3. As can be seen, a significant difference in the shape was observed and consequently, differences in the ionisation constant can be found. This result points to the importance of the optimisation of the conditions in the potentiometric study when aggregation equilibria are present.

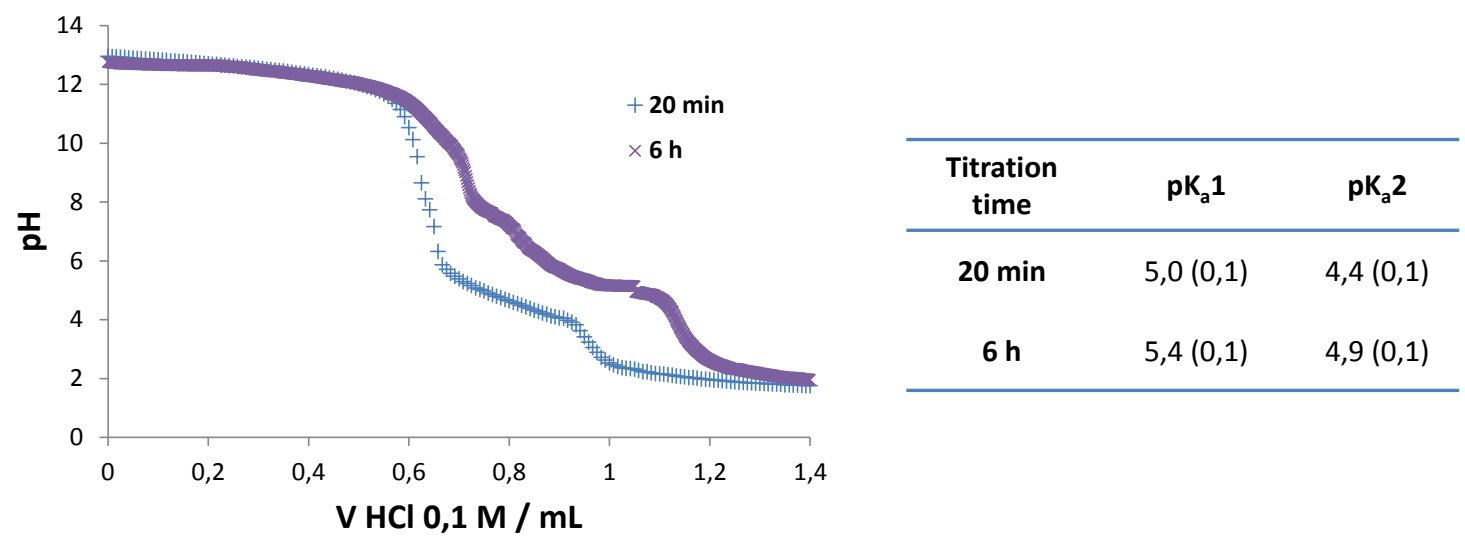

Figure 5.2.1.3. Comparative study of SucVal8 (5a) titration in concentrated samples (16 mM) at different rates $(0,05 \mathrm{~mL} / \mathrm{min}$ for the $20 \mathrm{~min}$ sample and $0,003 \mathrm{~mL} \min$ for the $6 \mathrm{~h}$ sample).

The ionisation of the amphiphilic compounds SucValNon (5c) and SucValHex (5d) was then studied. These molecules present a terminal ionisable carboxylic acid moiety and were capped at C-terminus with a long hydrocarbon tail of 9 or 6 carbon atoms. The acid dissociation constant of SucValNon (5c) was first determined and found to be 5,5 for a $1 \mathrm{mM}$ sample (<MGC, no gel formation) (see Table 5.2.1.3). The acid-base equilibrium for SucValNon (5c) as well as the titration curve and the species distribution diagram are presented in Figure 5.2.1.4. Under gelation conditions (sample above MGC) the new $\mathrm{pK}_{\mathrm{a}}$ value was found to be $c a$. 5,6. These results suggest no significant differences in acidity constants upon increasing concentration, revealing less intense intermolecular interactions in the fibrillar networks when compared to the diprotic compounds (see Table 5.2.1.2). The same study was then performed for the other amphiphilic molecule SucValHex (5d) (see Table 5.2.1.3) having $\mathrm{pK}_{\mathrm{a}}$ values of 4,4 in diluted and 4,6 in the aggregated sample. These values indicate that the additional hydrophobic effect produced by increasing in three the carbon atoms of the chain length from SucValHex $(5 d)$ to SucValNon $(5 c)$ results in $c a$. one unit increase in the $\mathrm{pK}_{\mathrm{a}}$ value. 


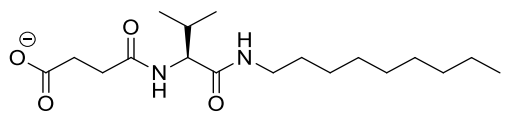

$\mathbf{A}^{-}$

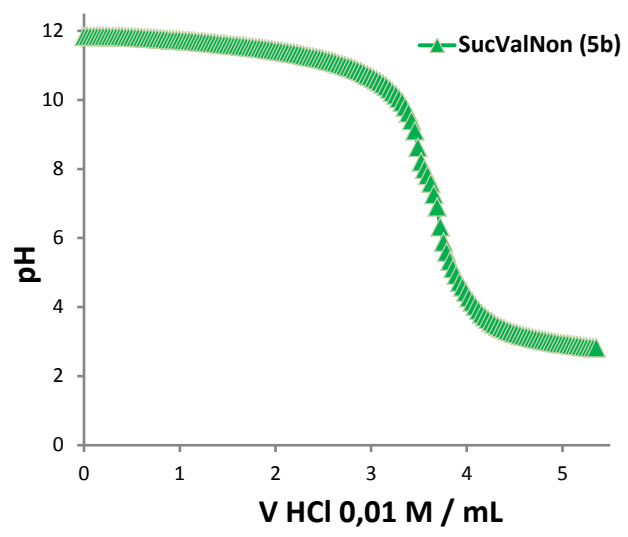

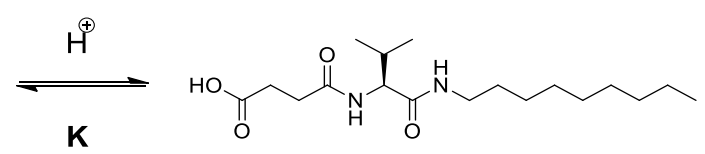

AH

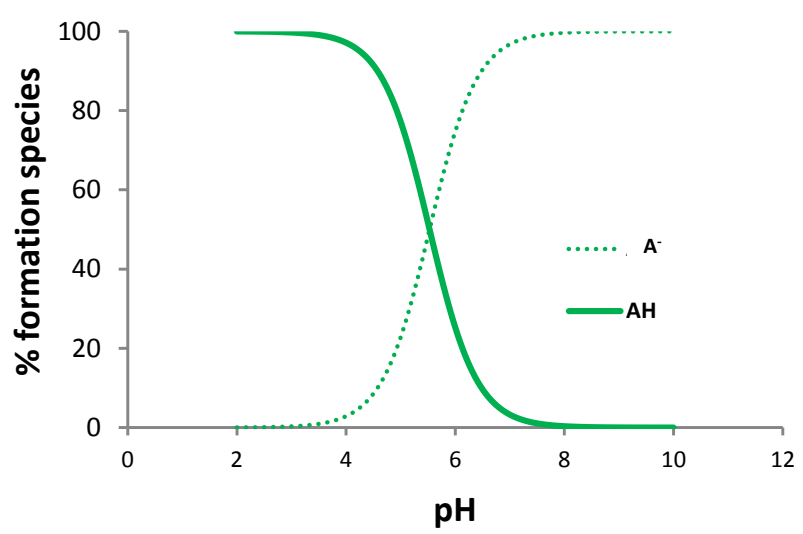

Figure 5.2.1.4. Titration curve (left) and species diagram (right) for SucValNon (5c) $1 \mathrm{mM}$ sample.

Table 5.2.1.3. Protonation constant for monoprotic compounds in diluted samples $(<1 \mathrm{mM})$ and concentrated samples (> $25 \mathrm{mM})$.

\begin{tabular}{ccc}
\hline Compound & $\mathrm{pK}_{\mathrm{a}} /$ Diluted & $\mathrm{pK}_{\mathrm{a}} /$ Aggregated \\
\hline SucValNon (5c) & $5,5(0,1)$ & $5,6(0,1)$ \\
SucValHex (5d) & $4,4(0,1)$ & $4,6(0,1)^{*}$ \\
\hline
\end{tabular}

[a] Calculated errors in parenthesis.

*Estimated from the $\mathrm{pH}$ value at the plateau in the titration curve.

Interestingly, for these compounds an intermediate jump in the titration curve was also found at $\mathrm{pH}$ ca. 8 for both molecules, suggesting in this case the involvement of the $\mathrm{AH}^{-}$species into partially protonated, in this case the charged amphiphile could form micelles (see Figure 5.2.1.4 right for SucValNon (5c) and Figure SI_5.5.1.D for SucValHex (5d)).

In summary a brief study on the thermodynamic constants associated to acid-base equilibria for a family of four molecular hydrogelators with ionisable functional groups has been described. Some difficulties associated to the interplay of acid-base equilibria and self-assembly have also been found. For example, acidity constants can be modified as a result of the formation of vesicles, micelles, etc. Also, it has been found that the self- 
assembly process associated to hydrogel formation can produce remarkable shifts in the acid-base constants. These results indicate that rational design of $\mathrm{pH}$-responsive hydrogels requires careful characterization of acid-base constants.

\subsection{Two-Component pH-Responsive Hydrogels}

A similar study with another ionisable functional group was performed, in this case a proline bolaamphiphile derivative Prolle6 (3e) was chosen (Figure 5.3.1). The proline unit is a well-known amino acid with acid-base behaviour $\left(\mathrm{pK}_{\mathrm{a}}\right.$ of the ammonium unit around 10,9$)^{14}$ and therefore could be used for the preparation of $\mathrm{pH}$-responsive hydrogelators.
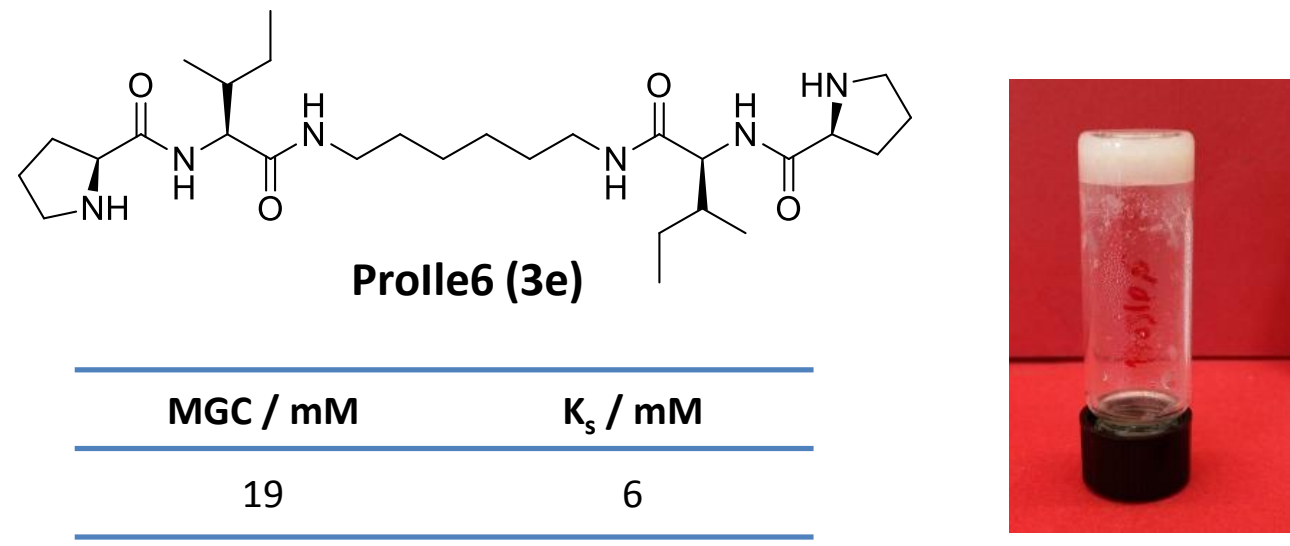

Figure 5.3.1. Molecular structure Prolle6 (3e), photograph of the hydrogel and hydrogelation parameters.

Compound Prolle6 (3e) has been already studied and was found to form organogels in toluene with organocatalytic activity (see Chapter 3). Here, its gelation ability was tested in water. A white-opaque hydrogel was obtained (Figure 5.3.1) above a gelator concentration of $19 \mathrm{mM}$ after a heating-cooling cycle (see Experimental Section). In order to evaluate the thermodynamic parameters of the gelation process, the solubility of the hydrogel was studied by means of NMR measurements, ${ }^{15}$ as done previously for

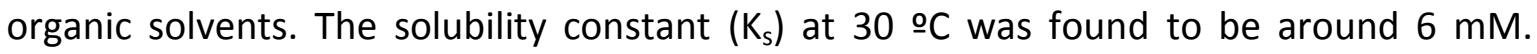
Additionally, the variation of the solubility of the self-assembled networks with temperature was evaluated. As can be seen in Figure 5.3.2 left, the typical pattern commonly observed for different gelators, showing an exponential relationship of the solubility with the temperature, was not found contrary to the same compound in toluene (see chapter 3 Figure 3.3.1.5). In this case, the solubility of the gelator in water 
showed a lower dependence with the temperature, with a decrease in solubility upon increasing the temperature, suggesting that hydrophobic interactions are the main driving force for the aggregation. ${ }^{16}$ Additionally, a van't Hoff analysis (see Experimental Section) of the data (see Figure 5.3.2 right) gave a negative value for the enthalpy of gel to solution transformation ( $\Delta \mathrm{H}=-11,6 \mathrm{~kJ} / \mathrm{mol}$, favourable), which was compensated by a negative entropy value for the same process $(T \cdot \Delta S=-23,8 \mathrm{~kJ} / \mathrm{mol}$, unfavourable). The latter factor is associated with an increase in order of water molecules related with the gelator dissolution. ${ }^{17}$ Negative entropy values of solubilisation have been observed before in aqueous media for hydrogelators which additionally present a decrease in solubility with increasing temperature. ${ }^{18}$ These results confirm the presence of an important hydrophobic contribution in the self-assembly process. Therefore, an entropically driven aggregation process is observed in water ${ }^{19}$ contrary to the enthalpically driven process found for the same gelator in organic solvents (see Chapter 3, Figure 3.3.1.5).
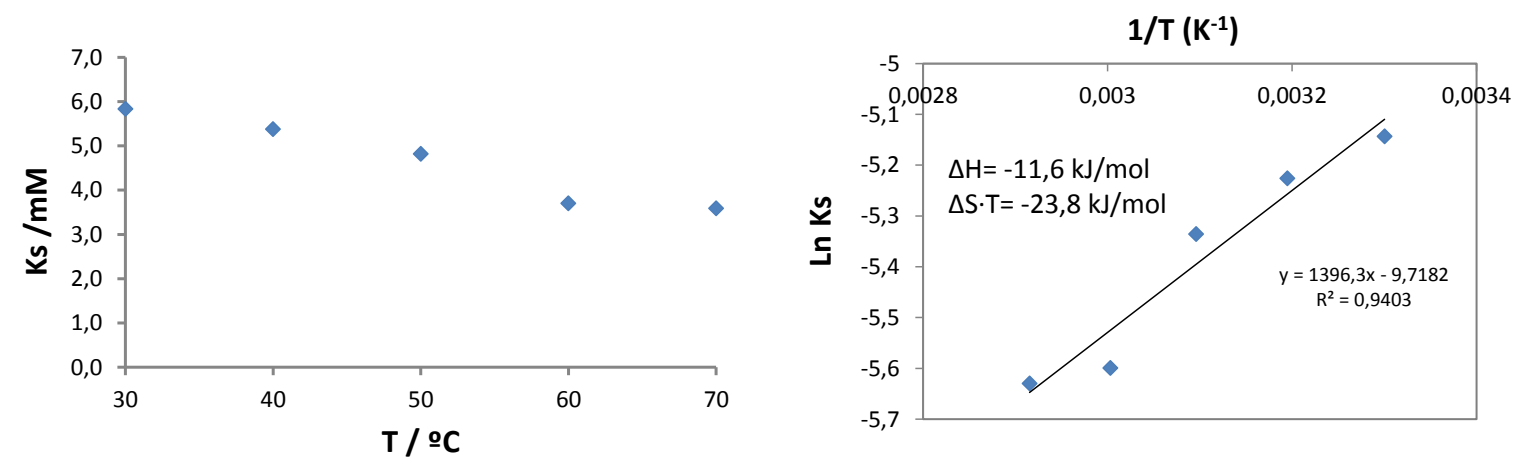

Figure 5.3.2. Temperature dependence of the solubility of Prolle6 (3e) using a $20 \mathrm{mM}$ sample (left). Ln Ks vs $1 / T$ data plots with the thermodynamic parameters associated (enthalpy and the entropy) in the gel-sol transition (right). The $\mathrm{K}_{\mathrm{s}}$ data were obtained from NMR analysis.

Next, the analysis of the morphological aspect of the hydrogel was performed. Electron microscopy images revealed short, tape shaped fibrils, highly entangled and straight with diameters between 200-300 nm (see SEM in Figure 5.3.3 and further images in Figure SI_5.5.4). 

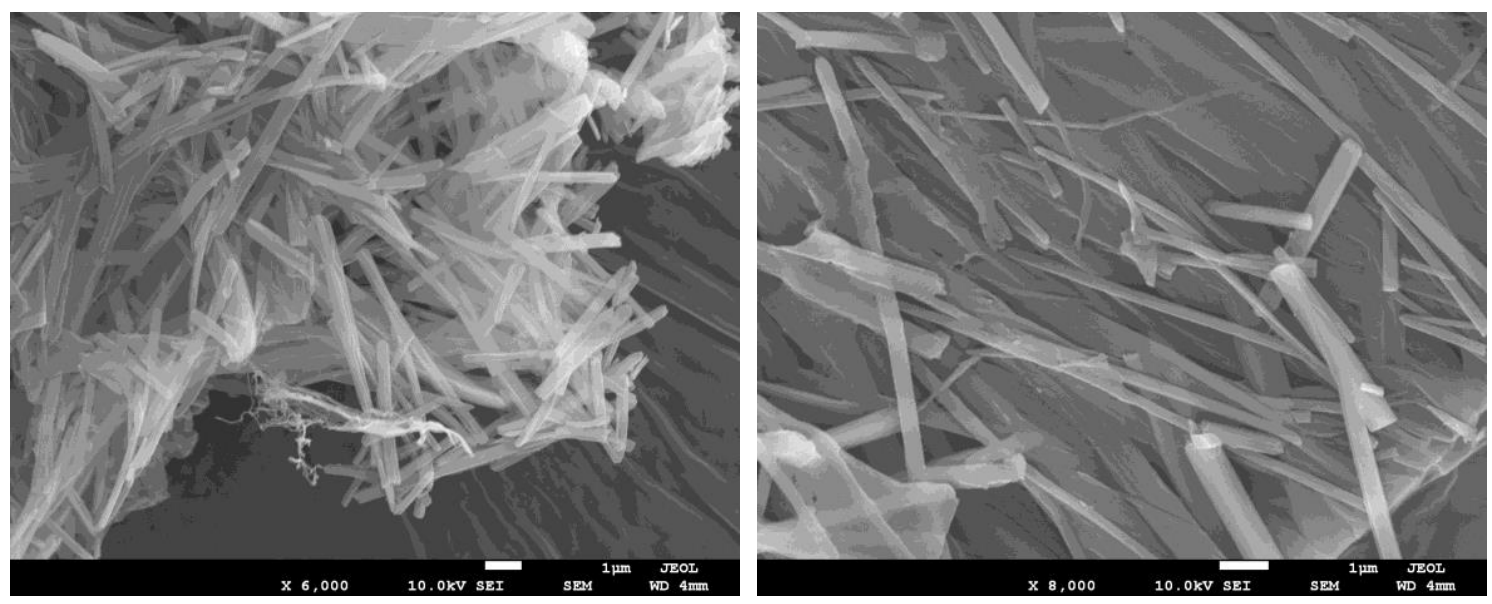

Figure 5.3.3. SEM images of Prolle6 (3e) hydrogel sample at $20 \mathrm{mM}$.

Finally, thermodynamic constants for acid-base equilibria were analysed. Titrations in aqueous $\mathrm{HCl}$ solution were carried out at different concentrations (see titration curve in Figure SI_5.5.5). First, the protonation constants for a diluted sample (concentration < MGC) were studied revealing a difference for $\mathrm{pK}_{\mathrm{a}}$ values between first and second ionization of almost one unit (see table in Figure 5.3.4). This suggests the stabilization of the intermediate monoprotonated $\mathbf{B H}^{+}$species that are predominant in a wide $\mathrm{pH}$ range. (Figure SI_5.5.5). The effect of the self-assembly process was then studied in a concentrated sample. A decrease in the value of the first protonation constant (Table on Figure 5.3.4) was found upon increasing concentration, indicating a higher stability of the neutral B species as a consequence of its aggregation and gel formation.

\begin{tabular}{ccc} 
Prolle6 (3e) & Solution & Aggregated \\
\hline $\mathbf{p k a}_{\mathbf{a}} \mathbf{n}$ & $9,3(0,1)$ & $8,7(0,1)$ \\
$\mathbf{p k a}^{2}$ & $8,1(0,1)$ & $8,3(0,1)$
\end{tabular}

Figure 5.3.4. Representation of the acid-base equilibria for Prolle6 (3e) and table with the protonation constants of a diluted sample $(2,5 \mathrm{mM})$ and a concentrated sample $(33 \mathrm{mM}$, calculated errors in parenthesis). 
Following this, a two-component hydrogel with tuneable properties based on $\mathrm{pH}$ changes was designed. A pyridine-functionalised molecule (5e) was chosen to be used in conjunction with Prolle6 (3e). Ionization constants for PyrVal3 (5e) were found to be 3,9 and 3,1 for the first and the second protonation constant respectively (see Figure 5.3.5).<smiles>CC(C)[C@H](NC(=O)c1ccncc1)C(=O)NCCCNC(=O)[C@@H](NC(=O)c1ccncc1)C(C)C</smiles>

PyrVal3 (5e)

\begin{tabular}{ccc}
\hline MGC / mM & $\mathbf{K}_{\mathbf{s}} / \mathbf{m M}$ & $\mathbf{p K _ { \mathbf { a } }}$ \\
\hline 17 & 9 & $\begin{array}{l}\mathrm{pK}_{\mathrm{a}}(1)=3,9 \\
\mathrm{pK}_{\mathrm{a}}(2)=3,1\end{array}$ \\
\hline
\end{tabular}

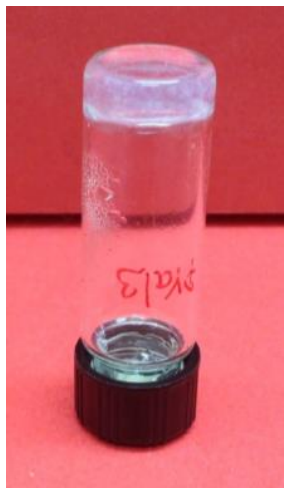

Figure 5.3.5. Molecular structure of the hydrogelator PyrVal3 (5e) with some of its features ${ }^{19}$ (left) and hydrogel image (right).

PyrVal3 (5e) had been studied before in our group as an ambidextrous gelator, as it can form gels in organic solvents as well as in water. ${ }^{20}$ Additionally, its gelation behaviour has been fully characterised from a thermodynamic perspective. ${ }^{19}$ Some of the gelation properties are summarised in the table on Figure 5.3.5, showing a MGC of 17 $\mathrm{mM}$ in water, $^{19}$ similar to that obtained for the proline derivative Prolle6 (3e) and a solubility constant of $9 \mathrm{mM}$ at $25^{\circ} \mathrm{C}$. TEM imaging of the gel was also carried out, revealing an entangled network of fibres, microns in length, as can be seen in Figure 5.3.6 (additional images in Figure SI_5.5.6).
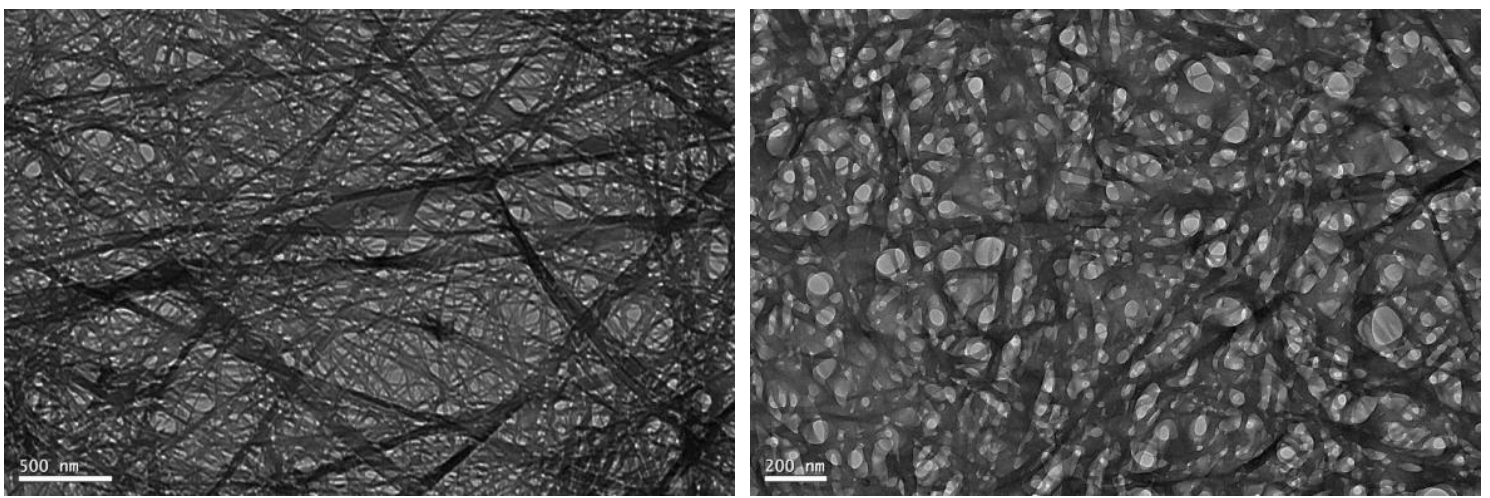

Figure 5.3.6. TEM images of PyrVal3 (5e) hydrogel sample $24 \mathrm{mM}$. 
The two gelators were used to create a pH-responsive complex system and using the acid dissociation constants obtained for both compounds, a species distribution diagram was simulated between a pH-range from 1 to 12 (Figure 5.3.7). As can be seen, this system is expected to be a solution at $\mathrm{pH}$ values below $c a$. 3 , where the two main species are diprotonaded in each compound $\left(\mathbf{A H}_{\mathbf{2}}{ }^{2+}\right.$ for the pyridine derivative and $\mathbf{B H}_{\mathbf{2}}{ }^{2+}$ for the proline derivative). Above this $\mathrm{pH}$ value, the concentration of neutral species $\mathbf{A}$, which is responsible for hydrogelation, should increase progressively with the $\mathrm{pH}$. As a result, a gel from PyrVal3 (5e) should be formed, and the diprotonated species $\mathbf{B H}_{2}{ }^{2+}$, corresponding to the proline derivative, would remain the major component up to $\mathrm{pH} c a$. 8. Above pH 8 neutral species B should start to appear. As a consequence, gel formation by Prolle6 (3e) should take place above this $\mathrm{pH}$ value. At this point a complex system formed by two different gelators could be reached. It was hypothesised that in this case when the two gelators are together in basic conditions they would assemble as independent networks (self-sorted) due to their differences in the molecular structures.
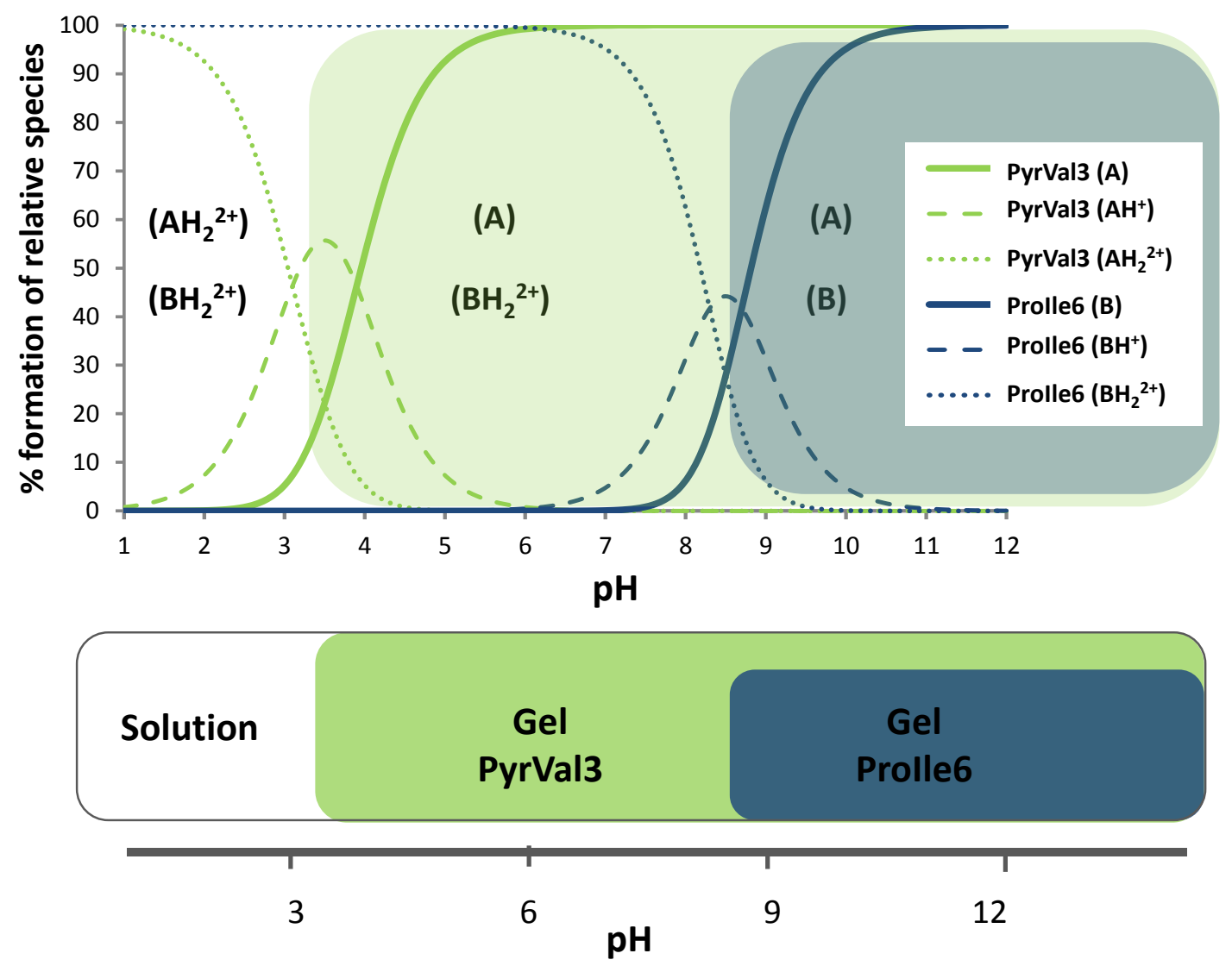

Figure 5.3.7. Illustrative scheme of the species distribution diagram expected for the two-component system at different $\mathrm{pH}$ values. 
To evaluate the predicted behaviour, the described two-component system was studied. It was decided to perform the experiments at concentrations above the MGC (17 $\mathrm{mM}$ for PyrVal3 (5e) and $19 \mathrm{mM}$ for Prolle6 (3e)) and in equimolar ratio (1:1). First, a two-component hydrogel ( $24 \mathrm{mM}$ of each compound) was formed after a heating-cooling procedure (see Experimental Section). The microscopic features of the gel revealed an entangled network of long and thin fibres of 20-30 nm of diameter (see Figure 5.3.8 and Figure SI_5.5.10).
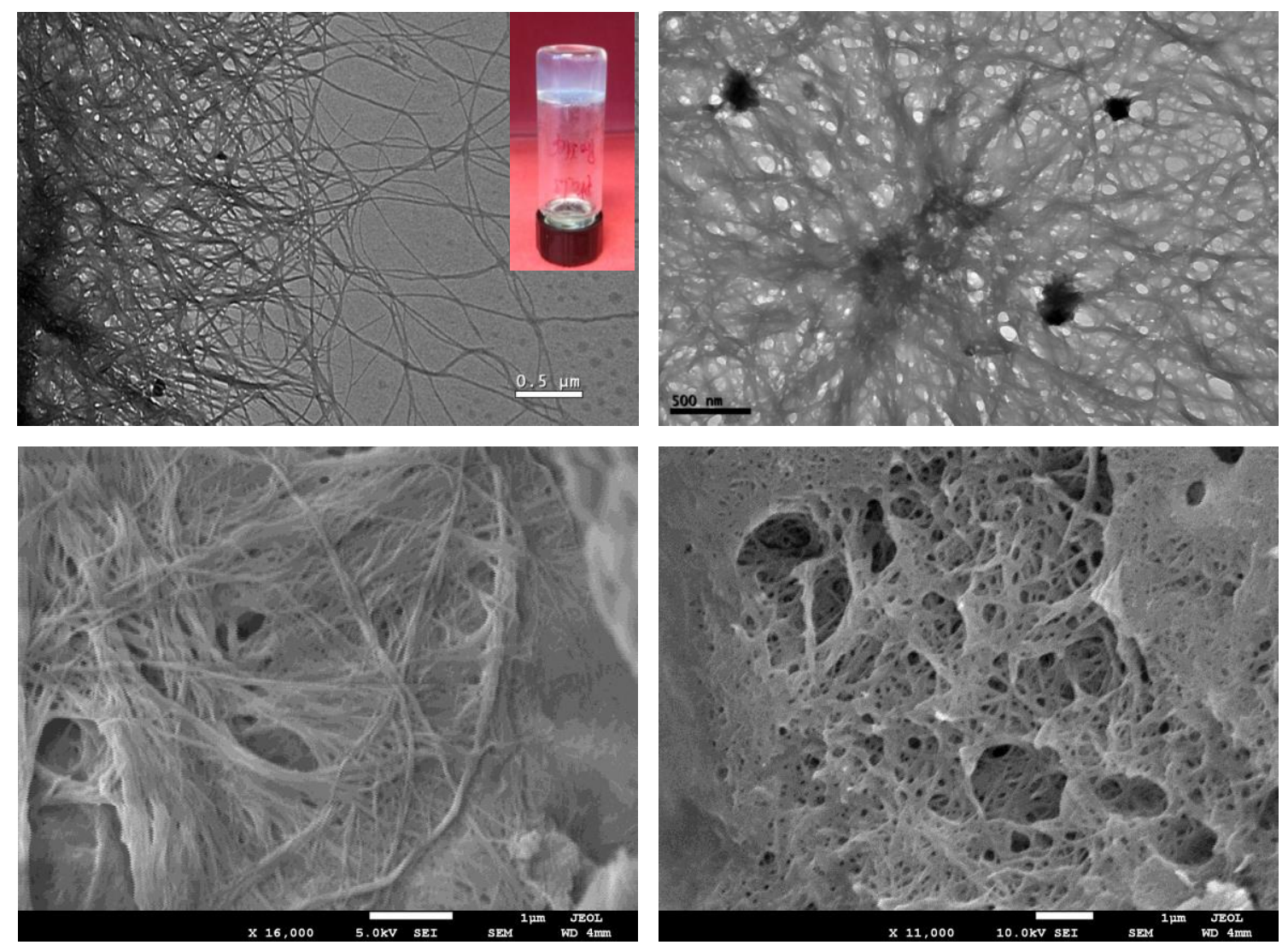

Figure 5.3.8. TEM (top) and SEM (bottom) images of hydrogel formed by the sample Prolle6 (3e) and PyrVal3 (5e) (1:1) 24 mM mixture. The inset shows a macroscopic image of the hydrogel.

In order to test the $\mathrm{pH}$-responsiveness, an experiment using acetic acid $1 \mathrm{M}$ as a media to change the $\mathrm{pH}$ was performed. According to the $\mathrm{pK}_{\mathrm{a}}$ values described above, the controlled addition of acetic acid should lead to the protonation only of the proline units, whilst the pyridine moieties remain neutral. Consequently, the addition of the amount of acetic acid required to protonate the proline units should result in the disassembling of the Prolle6 (3e) hydrogel network, however, the PyrVal3 (5e) gel network should keep its integrity. Surprisingly, it was found that when acetic acid (2 moles per mol of proline 
units) was added to the mixture, the gel suddenly disassembled to reach a solution, clearly implying the dissolution of the gel network formed by PyrVal3 (5e). Unexpectedly, further addition of acetic acid resulted in the transformation of the solution into a gel (see Figure 5.3.9 bottom). To gain insight on the reasons for this behaviour, the solubility of both gelators was monitored by NMR upon addition of increasing amounts of acetic acid $1 \mathrm{M}$. It is important to note that the molecules forming part of the gel network are NMR silent and only the fraction of molecules that are in solution will be NMR visible. The results for the variation of the NMR intensity for PyrVal3 (5e) are presented in Figure 5.3.9 top. It can be seen, PyrVal3 (5e) is solubilised in parallel with the addition of acetic acid until a maximum at $50 \mu \mathrm{L}$. At this point the system becomes a solution with the selfassembled network dissolved. Beyond this point, the solubility of PyrVal3 (5e) decreases, resulting in gel formation. On the contrary, in this experiment Prolle6 (3e) was fully solubilised upon addition of $20 \mu \mathrm{L}$ of acetic acid $1 \mathrm{M}$, as indicated by NMR, remaining in solution upon further addition of acetic acid (see Figure SI_5.5.9).
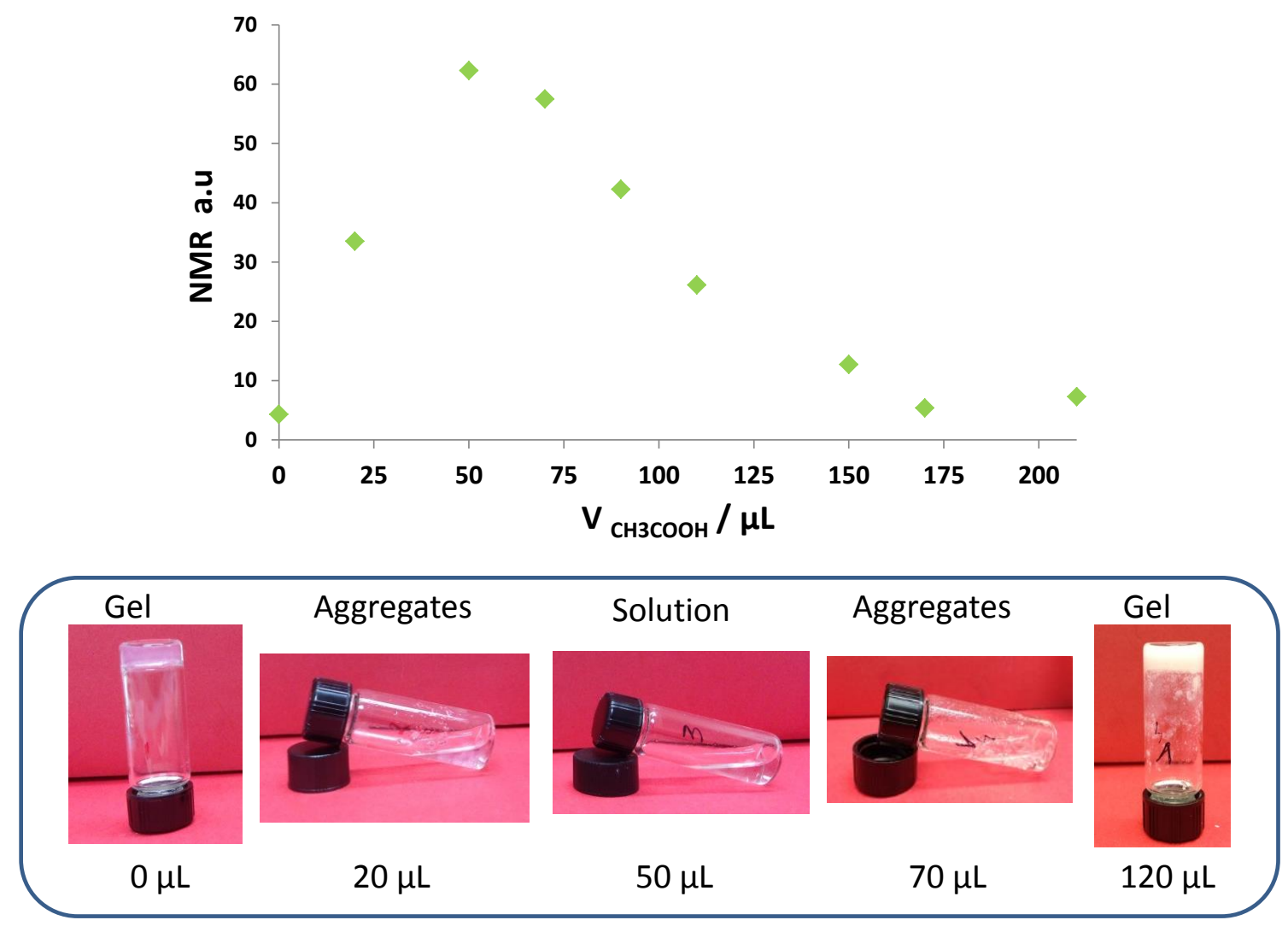

Figure 5.3.9. A plot of PyrVal3 (5e) ${ }^{1} \mathrm{H}-\mathrm{NMR}$ intensity signal during the addition of acetic acid $1 \mathrm{M}$ in the coaggregated sample gel (top) and images illustrating the solubilisation process indicating the amount of added acid (bottom). 
This behaviour may be rationalised by the self-assembly of the monoprotonated Prolle6 (3e) molecules. As can be seen Figure 5.3.10, the initial fibrillar network formed in the Prolle6 (3e) hydrogel is disassembled after the ionisation of one of its proline rings. These species can behave as a cationic amphiphiles and aggregate into spherical aggregates such as micelles possessing a hydrophobic centre. These aggregates could interact with the PyrVal3 (5e) and progressively solubilise neutral PyrVal3 (5e), thus disassembling its hydrogel network. The addition of more acid leads to the protonation of the second proline ring generating diprotonated species that can no longer form micelles, resulting in the liberation of PyrVal3 (5e). This compound would then give rise to the formation of a fibrillar network resulting again in a hydrogel. In support of this rationale it is noted that studies reported previously have shown inhibition of PyrVal3 (5e) gelation properties in the presence of sodium dodecyl sulfate (SDS) micelles. ${ }^{21}$

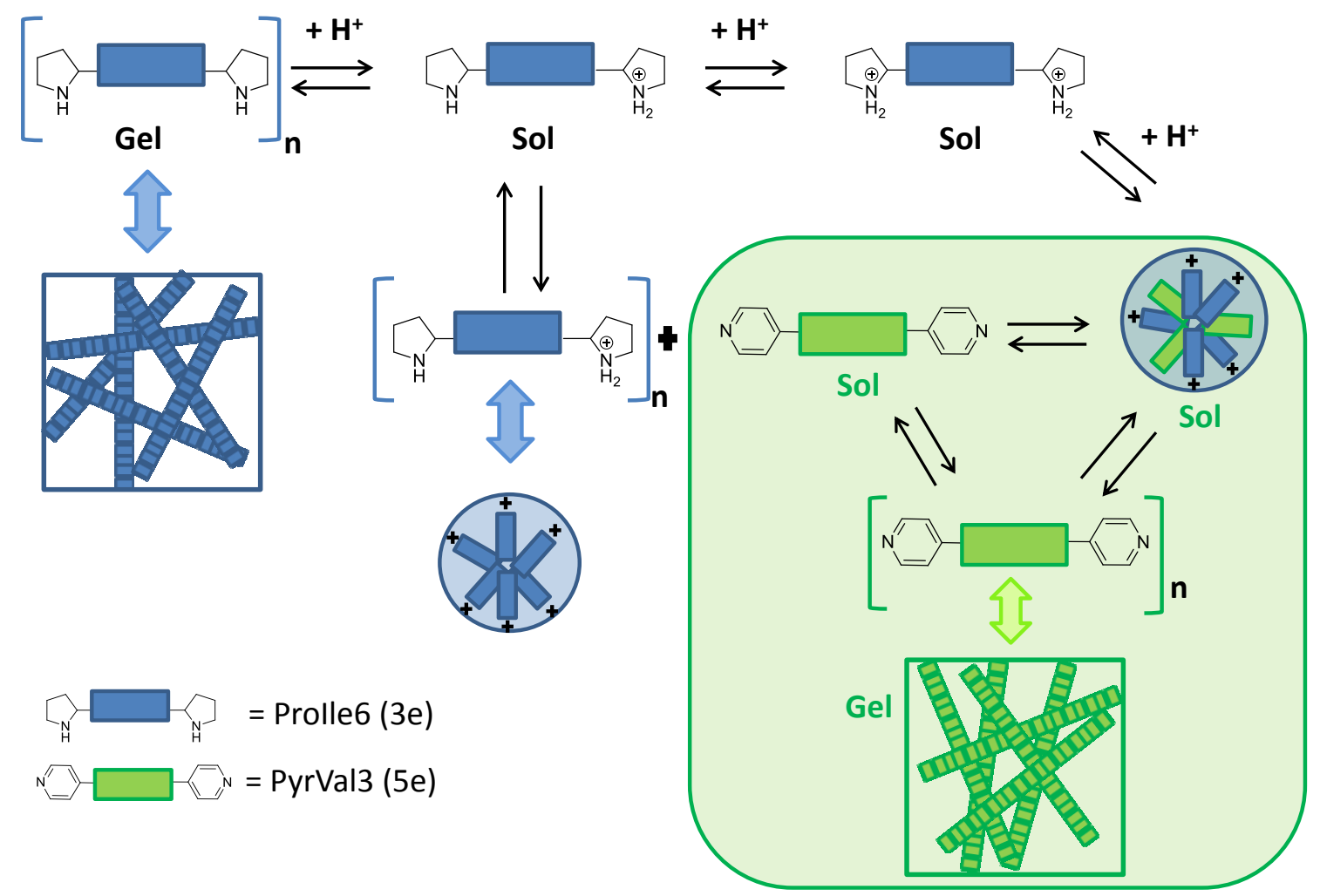

Figure 5.3.10. Schematic representation of the equilibria involved in the solubilisation of the PyrVal3 (5e) hydrogel in the presence of Prolle6 (3e).

Electron microscopy images for this system with different degrees of protonation are shown in Figure 5.3.11. As can be seen, the solubilisation of the hydrogel was 
demonstrated by a change of morphology; showing only a small percentage of thin fibres with some broken network (Figure 5.3.11 A-B and Figure SI_5.5.14) compared with the entangled system of fibres found when the two-component hydrogel was present (Figure 5.3.8).
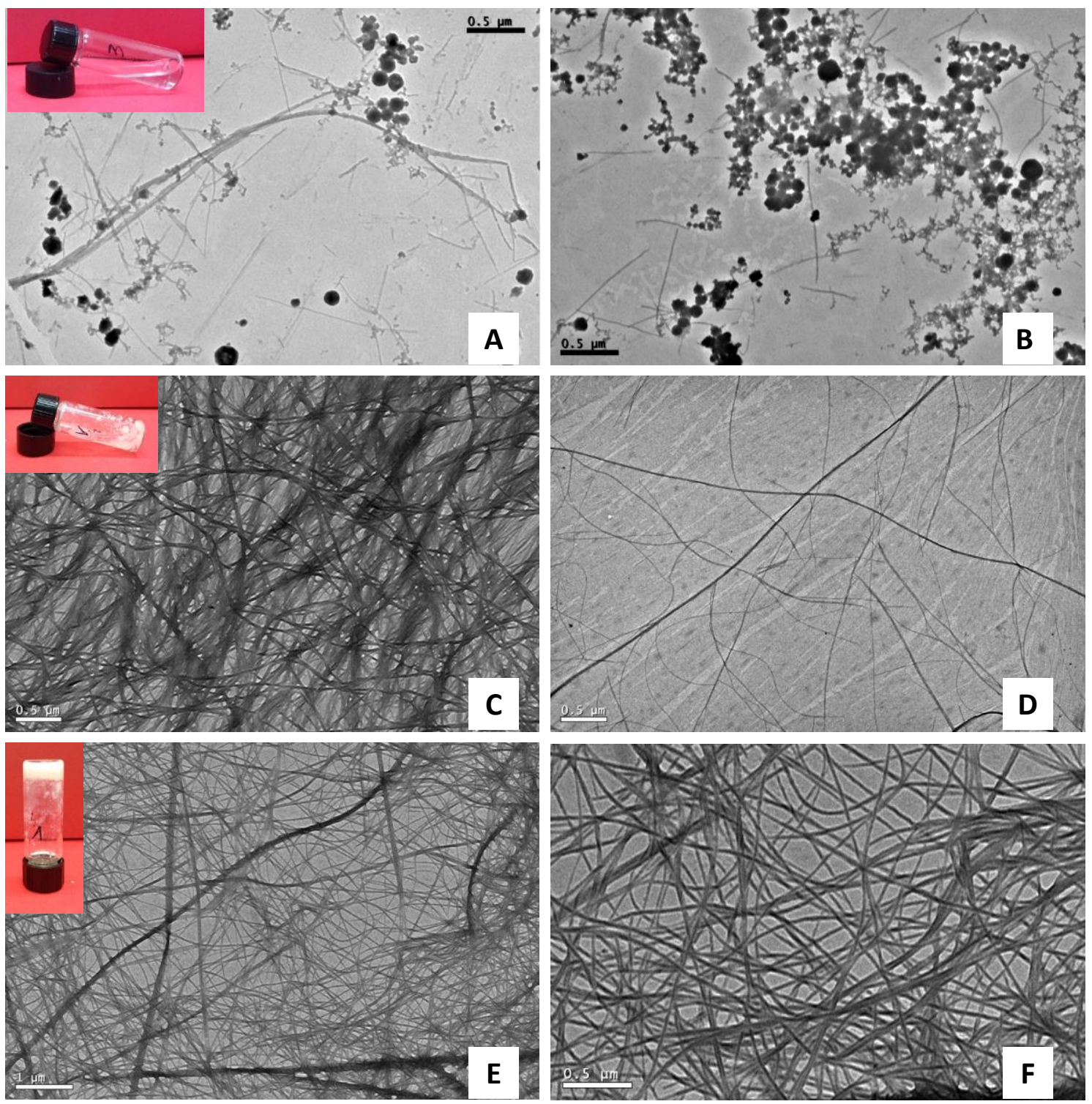

Figure 5.3.11. TEM images of the sample Prolle6 (3e) and PyrVal3 (5e) (1:1) 24mM mixture hydrogel samples after the addition of acetic acid 1M. A-B) $50 \mu \mathrm{L}$ (solution sample). C-D) $70 \mu \mathrm{L}$ (aggregates in the sample). E-F) $120 \mu \mathrm{L}$ (gel sample). Insets: photo of the different samples.

Further addition of acetic acid led to the macroscopic emergence of aggregates and a fibrillar network formed by thin, long and entangled fibres (Figure 5.3.11 C-D and Figure SI_5.5.15), similar to those observed for a gel at pH 5 (Figure 5.3.11 E-F and Figure 
SI_5.5.16), where long fibres of $c a .20 \mathrm{~nm}$ of diameter were seen. The effect of acetic acid was studied by electron microscopy for both hydrogelators separately. For a Prolle6 (3e) $24 \mathrm{mM}$ hydrogel, the presence of acetic acid in small amounts (10 $\mu \mathrm{L})$ provoked the solubilisation of the self-assembled fibres shown in Figure 5.3.3, and an amorphous material was obtained upon solvent removal (see Figure SI_5.5.11, Figure SI_5.5.12). On the contrary, the addition on $30 \mu \mathrm{L}$ of acetic acid to a PyrVal3 (5e) hydrogel $24 \mathrm{mM}$ did not show any variation on the microscopic aspect of the fibres (Figure SI_5.5.13), in agreement with the species distribution diagram that showed neutral species at this $\mathrm{pH}$ and hence molecules that are able to gel.

Further experiments were performed via ${ }^{1} \mathrm{H}-\mathrm{NMR}$ with the goal of a better understanding of this complex system when the $\mathrm{pH}$ was used to trigger the aggregation behaviour. Diffusion ordered spectroscopy (DOSY) experiments have been used before in literature to study self-assembly. ${ }^{16,22}$ The measurement of diffusion is carried out by observing the attenuation of the NMR signals during a pulsed field gradient experiment following an exponential decay (see example in Figure SI_5.5.17). The degree of attenuation occurs at a rate proportional to the diffusion coefficient (D) and thus its slope was used to calculate the diffusion coefficient for a molecule. The diffusion coefficient (D) provides information related with the size and shape of the molecule, as well as physical properties of the surrounding environment such as viscosity, temperature, etc., of each chemical species in solution, therefore the magnitude of the diffusion coefficient is related to changes associated with, for instance, micelle formation. ${ }^{21,23} \mathrm{~A}$ first experiment was performed using Prolle6 (3e) samples that had been acidified with acetic acid up to $\mathrm{pH} 8$ at a different concentrations. All the samples were a solution due to the protonation of one of the two proline rings at this $\mathrm{pH}$. As can be seen in Table 5.3.1, a decrease of the diffusion coefficient was observed when the concentration was increased from $1 \mathrm{mM}$ to $20 \mathrm{mM}$ pointing to a self-assembling process that may be related with the formation of micelles for the monoprotonated species (as is illustrated in Figure 5.3.10). In other words, the large hydrodynamic volume of self-assembling structures, such as micelles, compare with the free molecule in solution provokes a diminution of the diffusion coefficient suggesting the formation of charged-micelles for the Prolle6 (3e) at this $\mathrm{pH}$. 
Table 5.3.1. Diffusion coefficients obtained for a protonated Prolle6 (3e) sample at a different concentrations in $\mathrm{H}_{2} \mathrm{O}$.

\begin{tabular}{ccc}
\hline Prolle6 (3e) /mM & pH & $\mathrm{D} / 10^{-10} \mathrm{~m}^{2} \mathrm{~s}^{-1}$ \\
\hline 20 & 8 & $3,41(0,07)$ \\
10 & 8 & $3,64(0,07)$ \\
5 & 8 & $3,72(0,09)$ \\
\hline
\end{tabular}

[a] Calculated errors in parenthesis.

The diffusion coefficients of PyrVal3 (5e) under different conditions were compared (Table 5.3.2.). Interestingly, a marked decrease of the diffusion coefficient of PyrVal3 (5e) from $4,96 \cdot 10^{-2} \mathrm{~m}^{2} \mathrm{~s}^{-1}$ to $4,10 \cdot 10^{-2} \mathrm{~m}^{2} \mathrm{~s}^{-1}$ was observed in the two-component sample at $\mathrm{pH} 8$ when compared with the diffusion coefficient of the free compound at 5 $\mathrm{mM}$ in solution (at a concentration below $\mathrm{K}_{\mathrm{s}}$ in water) (Entries 1 and 3). This implies the incorporation of PyrVal3 (5e) in micelles at this $\mathrm{pH}$. It should be noted that the diffusion coefficient reported here reflects and averaged value for the free and the micellar species. When the same species were measured at $\mathrm{pH} 5$ within the gel phase, the diffusion coefficient again returned to the value found for PyrVal3 (5e) in the initial hydrogel, without the presence of acetic acid (Entries 4 and 2), demonstrating the absence of micelles at this $\mathrm{pH}$, and in agreement with the disassembly of the micelles at lower $\mathrm{pH}$ due to the formation of diprotonated species of Prolle6 (3e).

Table 5.3.2. Diffusion coefficients obtained for the different systems to study in $\mathrm{H}_{2} \mathrm{O}$.

\begin{tabular}{|c|c|c|c|c|c|c|}
\hline Entry & $\begin{array}{l}\text { Prolle6 } \\
\text { (3e) } / \mathrm{mM}\end{array}$ & $\begin{array}{l}\text { PyrVal3 } \\
\text { (5e) /mM }\end{array}$ & pH & Aspect & $\begin{array}{c}D_{\text {Prolle6 }} / \\
10^{-10} \mathrm{~m}^{2} \mathrm{~s}^{-1}\end{array}$ & $\begin{array}{c}D_{\text {PyrVal3 }} / \\
10^{-10} \mathrm{~m}^{2} \mathrm{~s}^{-1}\end{array}$ \\
\hline 1 & -- & 5 & -- & Sol & -- & $4,95(0,05)$ \\
\hline 2 & 24 & 24 & -- & Gel & $3,1(0,1)$ & $3,37(0,08)$ \\
\hline 3 & 24 & 24 & 8 & Sol & $3,84(0,03)$ & $4,10(0,02)$ \\
\hline 4 & 24 & 24 & 5 & Gel & $3,4(0,1)$ & $3,5(0,1)$ \\
\hline
\end{tabular}

[a] Calculated errors in parenthesis. 
The two-component pH-responsive hydrogel system behaviour was tested using buffer solutions at different $\mathrm{pH}$ values. First, the gelation ability of the mixture $24 \mathrm{mM}$ $(1: 1)$ in the presence of Bromothymol Blue, a pH indicator that changes its colour from yellow at $\mathrm{pH}$ below 6,0 to blue at $\mathrm{pH}$ above 7,6 , was tested in the presence of buffer solutions $(1 \mathrm{mM}$ of Tris/ $\mathrm{HCl}$ buffer solution at $\mathrm{pH} 7,4$ or $1 \mathrm{mM}$ of acetic acid/acetate at $\mathrm{pH}$ 5). The samples were left to stabilise for 24 hours. Additionally, samples of the individual components were also prepared for comparison (see Figure SI_5.5.18). Surprisingly, contrary to the previous experiments, two-component hydrogels were obtained for all $\mathrm{pH}$ values tested, however some differences in the kinetics of gelation were found. It was noted that the sample with buffer at $\mathrm{pH} 7,4$ did not form a gel until it was left to stabilise for 24 hours, whilst with water a gel is formed within one hour and at $\mathrm{pH} 5$ after 3 hours. One possible explanation for the formation of a gel in the sample at $\mathrm{pH} 7,4$, which was a solution in the previous experiments performed with acetic acid addition, could be related to the slow gelation kinetics for PyrVal3 (5e). It has been shown before that the MGC for this compound decreases with time and therefore the amount of PyrVal3 (5e) in solution (not taking part or the mixed micelles formation) at this $\mathrm{pH}$ can reach the MGC after $24 \mathrm{~h}$ leading to a gel after long times. The results for the samples containing the individual compounds showed that for Prolle6 (3e), only the sample with water leads to a

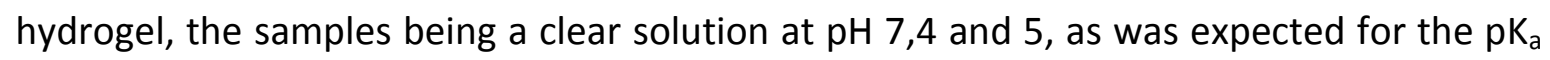
values obtained. On the contrary, for PyrVal3 (5e) all the samples lead to a hydrogel formation at all $\mathrm{pH}$ values tested.

The behaviour of the assembled structures when a buffer solution at different $\mathrm{pH}$ values is added over an aqueous solution was studied. For this purpose, two-component hydrogel samples at $24 \mathrm{mM}(1: 1)$ loaded with Bromothymol Blue were prepared in water and left to stabilise over 24 hours. Blue gel samples were obtained in all the cases. After this time, $100 \mu \mathrm{L}$ of buffer solution $(1 \mathrm{mM}$ of Tris/HCl buffer solution at $\mathrm{pH} 7,4$ or $1 \mathrm{mM}$ of acetic acid/acetate at $\mathrm{pH} 5$ ) were added on the top of the gel and left to diffuse. A blank with the addition of $500 \mu \mathrm{L}$ of water was also prepared (Figure SI_5.5.19) and the gel samples were monitored over time (Figure 5.3.12). As can be seen for the two-

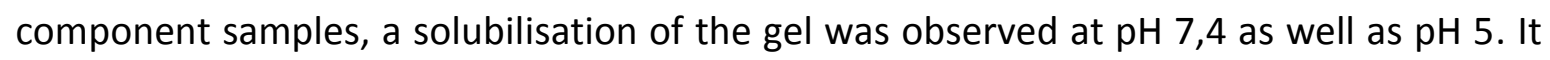
should to be noted that the blank sample with water maintained the hydrogel network 
for hours, thus a solubilisation due to a decrease in the concentration may be discarded. At $\mathrm{pH} 7,4$, the sample was slowly disassembled with time, the complete solubilisation of the sample was not observed and a gel reformed after 24 hours.

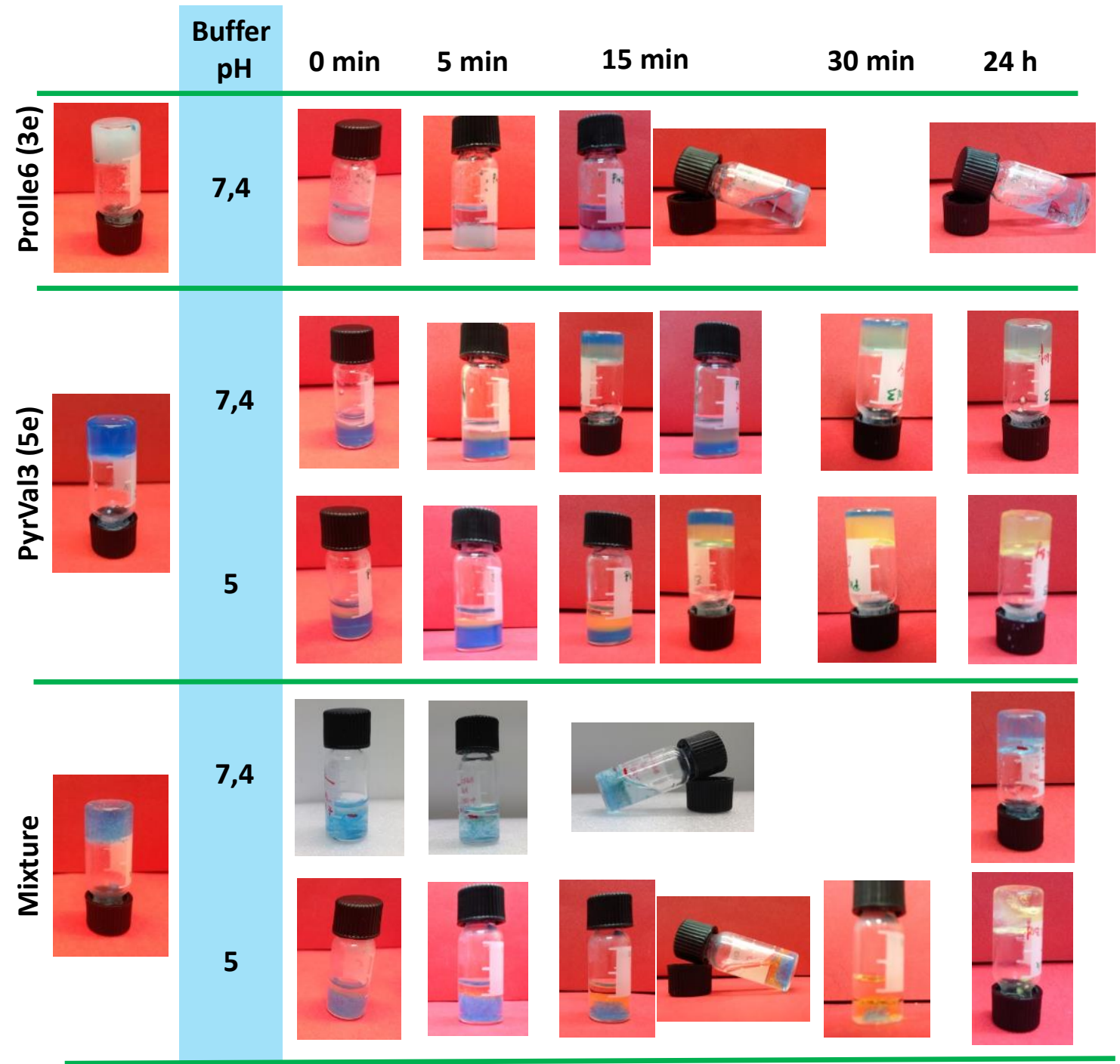

Figure 5.3.12. Images showing the addition of buffer solution to the samples over time. (Mixture: sample Prolle6 (3e) + PyrVal3 (5e) (1:1))

For the sample at $\mathrm{pH} 5$, a change in colour from blue to yellow was observed with the diffusion of the buffer. Here on the contrary, a quicker solubilisation of the hydrogel network was found, with almost all in solution within $30 \mathrm{~min}$, but again a gel was reformed after 24 hours. At $\mathrm{pH} \mathrm{5,} \mathrm{it} \mathrm{was} \mathrm{hypothesised} \mathrm{that} \mathrm{no} \mathrm{micelle} \mathrm{formation} \mathrm{occurs}$ from Prolle6 (3e) (see species distribution diagram in Figure 5.3.7). However, the disassembling of the gel at this $\mathrm{pH}$ value indicates a solubilisation of PyrVal3 (5e) under 
these conditions. In this regard it should be considered that the presence of salts in high concentration (1 $\mathrm{M}$ of buffer solution) may change the acid-base self-assembly species distribution and could result in the presence of micelles of Prolle6 (3e) in saline environment at $\mathrm{pH}$ 5. The effect of the salts in the self-assembly of gelators (Hofmeister effect) and how they can modify the aggregation behaviour has been reported previously. ${ }^{24}$ Nevertheless, work related with the effect of salts in these types of systems remains pending. The formation of the gel after 24 hour at $\mathrm{pH} 7,4$ as well as at $\mathrm{pH} 5$, as before could be explained by slow gelation kinetics of PyrVal3 (5e). For the Prolle6 (3e) sample, solubilisation at $\mathrm{pH}$ 7,4 was fast during the first 15 min with no gel formation in this time. In contrast, for the PyrVal3 (5e) samples, no solubilisation took place at $\mathrm{pH} 7,4$ nor $\mathrm{pH} 5$, where a gel was still present after 24 hours, in agreement with the species distribution diagram described above.

To summarise, the preparation of a two-component hydrogel system formed by bolaamphiphilic hydrogelators Prolle6 (3e) and PyrVal3 (5e) in equimolar ratio has been demonstrated. The hydrogel is found to be $\mathrm{pH}$-responsive in an remarkable way, giving rise to gel to solution transitions at $\mathrm{pH}$ values around 7 and then to a solution to gel transition at $\mathrm{pH}$ values around 5. The solubilisation of the PyrVal3 (5e) hydrogel network via its incorporation in micelles formed by monoprotonated Prolle6 (3e) species present at $\mathrm{pH} 8$ is used to rationalize this unexpected behaviour.

\subsubsection{Application in Controlled Release}

The construction of stimuli-responsive smart materials can be of interest in the development of localised drug release systems with enhanced specificity and reduced toxicity. In this field, supramolecular gels capable of triggering gelation when a stimulus is applied have been used in the design of controlled drug release systems. ${ }^{25}$ Here the evaluation of the two-component $\mathrm{pH}$-responsive hydrogel described above as a candidate for the development of a controlled release system has been performed. For this purpose, Methyl Orange was chosen as a model drug and its release was studied at different $\mathrm{pH}$ values. Methyl Orange (Figure 5.3.1.1) is a dye well-known by its use as pH indicator in titrations having a $\mathrm{pK}_{\mathrm{a}}$ of 3,47 in water at $25 \stackrel{\circ}{\circ} \mathrm{C}$ and thus showing reddish 
colour below $\mathrm{pH} \mathrm{3,1} \mathrm{and} \mathrm{yellowish} \mathrm{above} \mathrm{pH} 4,4$, it has also been used as an anionic model compound for drug delivery study in gels. ${ }^{26}$

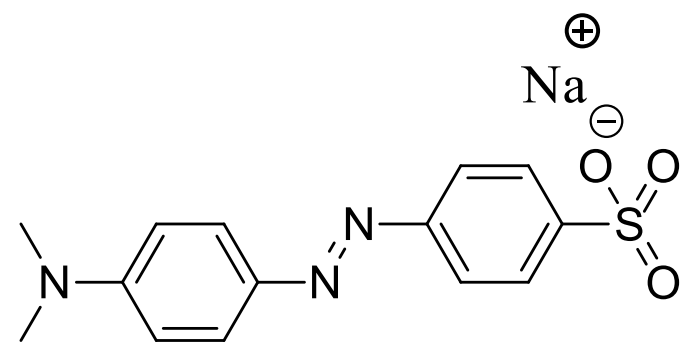

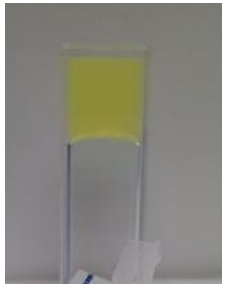

PyrVal3

(5e)

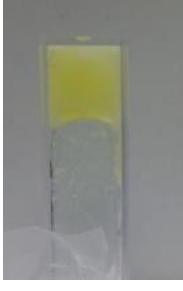

Prolle6

(3e)

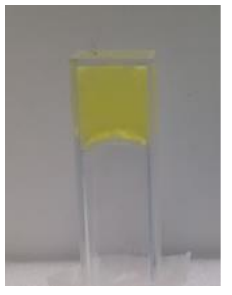

Mixture

Figure 5.3.1.1. Chemical structure of Methyl Orange and the hydrogel formed loaded with the Methyl Orange used in the controlled release experiments.

In order to evaluate the release behaviour, two-component hydrogel samples (1 mL) $24 \mathrm{mM} \mathrm{(1:1;} \mathrm{Prolle6} \mathrm{(3e)/PyrVal3} \mathrm{(5e))} \mathrm{loaded} \mathrm{with} \mathrm{Methyl} \mathrm{Orange} \mathrm{(30} \mu \mathrm{L}$ of stock solution $1 \mathrm{mg} / 5 \mathrm{~mL}$ ) were prepared by the conventional heating-cooling method and then transferred into a quartz cuvette $(1 \mathrm{~cm}$ path length). After stabilisation of the hydrogel for $24 \mathrm{~h}$, a buffer solution was added on the top $(1 \mathrm{~mL})$ and the release of the Methyl Orange from the gel was monitored with time in situ, following the absorbance at $\lambda=464 \mathrm{~nm}$ of the supernatant solution.

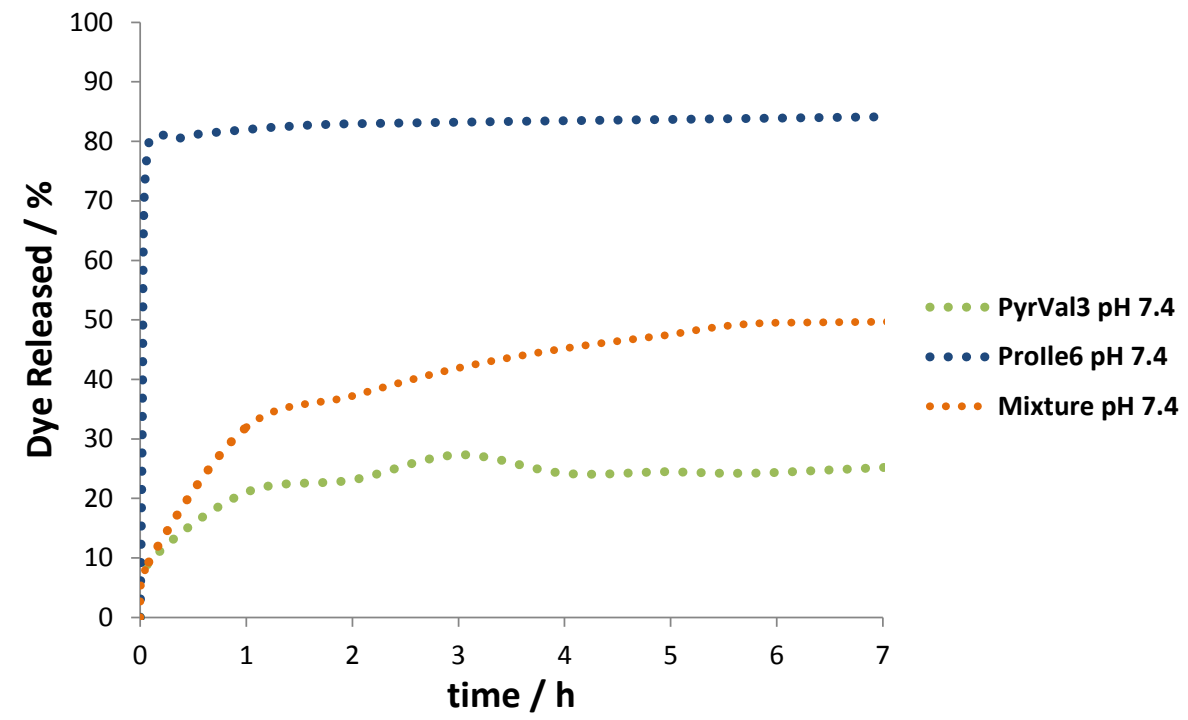

Figure 5.3.1.2. Release profile of Methyl Orange from the different hydrogels samples at $\mathrm{pH} 7.4$. 
The release profile of the hydrogel mixture was analysed at pH 9,4 $(1 \mathrm{M} \mathrm{Tris} / \mathrm{HCl}$ buffer solution); where both hydrogelators will remain as a neutral species and the hydrogel maintains its fibrillar network for both hydrogel components. Additionally, Prolle6 (3e) and PyrVal3 (5e) dye-loaded hydrogels were also prepared individually (24 $\mathrm{mM}$ ) in the same way (see Figure 5.3.1.1). The release profiles were measured for 7 hours. As can be seen in Figure SI_5.5.20 the three different systems showed a similar release profiles for the Methyl Orange, suggesting non-specific interactions of the dye with any of the hydrogel networks and additionally demonstrating that no solubilisation process take place at this specific $\mathrm{pH}$.

The release rate at $\mathrm{pH} 7,4(1 \mathrm{M}$ Tris/ $\mathrm{HCl}$ buffer solution) was then measured. At this $\mathrm{pH}$ the proline units are partially protonated in Prolle6 (3e), solubilising the PyrVal3 (5e) hydrogel network. Prolle6 (3e) and PyrVal3 (5e) dye-loaded hydrogels were also prepared with the aim of comparing the results with the two-component gel. After the addition of the buffer solution on the top of the gel, the release profiles of the three systems were analysed over 7 hours (see Figure 5.3.1.2) and three different profiles were found. On one hand, the PyrVal3 (5e) hydrogel was found to have a similar behaviour as at $\mathrm{pH} \mathrm{9,5} \mathrm{indicating} \mathrm{that} \mathrm{at} \mathrm{this} \mathrm{pH}$, the fibrillar network remains unvaried as no protonation takes place within the hydrogelator. For Prolle6 (3e), the solubilisation of the hydrogel took place by means of the protonation of the proline ring as proven by a fast release within the first few minutes. On the other hand, the two-component sample showed an intermediate behaviour demonstrating the solubilisation of the PyrVal3 (5e) hydrogel. 


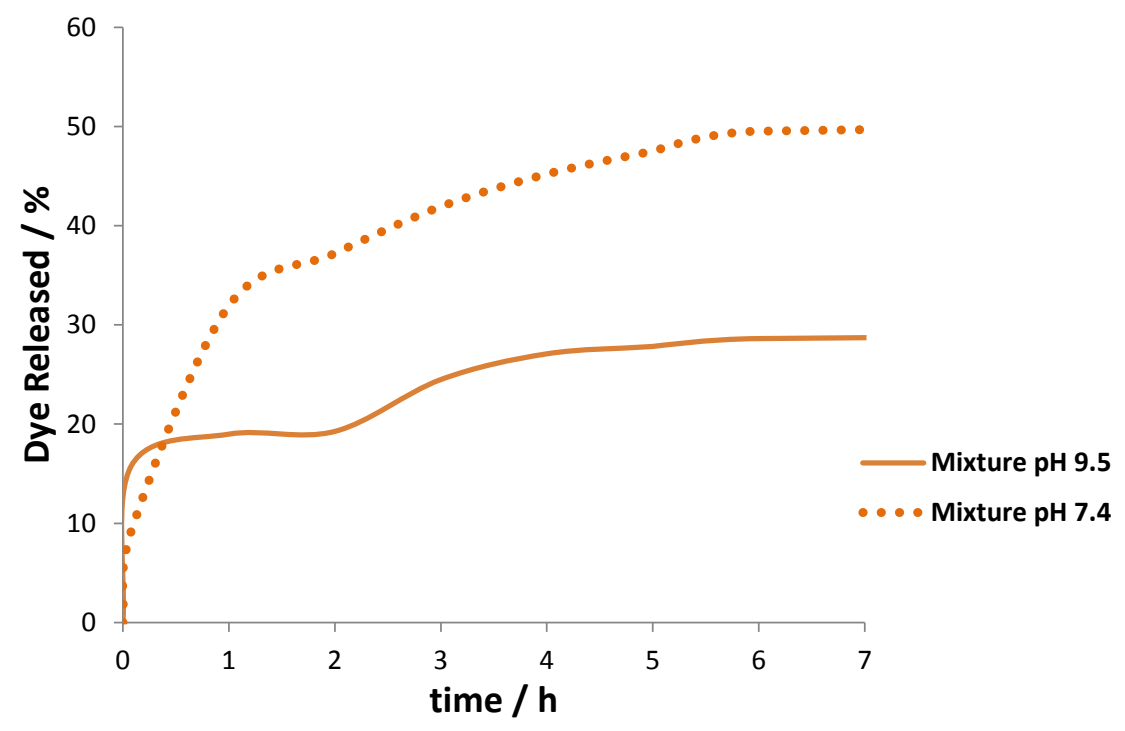

Figure 5.3.1.3. Release profile of Methyl Orange from the co-assembled hydrogels at different $\mathrm{pH}$.

Two different release profiles were found for the two-component hydrogel (Prolle6 (3e)/PyrVal3 (5e); 1:1) after a pH-change was used as a stimulus (see Figure 5.3.1.3), demonstrating the possible application of such $\mathrm{pH}$-responsive systems formed by different ionisable functionalised hydrogelators as candidates for drug delivery gel systems. 


\subsection{Conclusions}

In this chapter, with the aim to find an alternative gelation approach to the heating-cooling procedure, and with the final goal to generate $\mathrm{pH}$-responsive materials, $\mathrm{a}$ family of molecules bearing an ionisable group has been studied from a thermodynamic perspective. All the molecules were synthesised as hydrogelators containing amino acid derivatives with at least one succinic acid moiety, responsible of the acid-base behaviour. Some differences in the acid dissociation constant related with the aggregation ability have been found. The comparative study of samples above and below the MGC showed that the gelation process can produce remarkable shifts on the acid-base constants related with the higher stability of the neutral species involved in the self-assembly. Moreover, some additional equilibria involving charged self-assembled species in solution have been proposed involving micelles and vesicles. Additionally it has been found that the kinetics of the self-assembly process during the titration is a key factor to be considered in order to obtain reproducible results.

Finally, a two-component hydrogel has been designed in order to create a complex stimuli-responsive system. Two different hydrogelators with different acid-base properties have been chosen and a hydrogel has been prepared which presents an unusual $\mathrm{pH}$-responsive behaviour forming solutions upon going from basic to neutral solutions and then returning to gel state upon further acidification to $\mathrm{pH} 5$. Additionally, its use as a $\mathrm{pH}$-tuneable delivery system has been shown to have potential application in controlled drug release. 


\subsection{Supporting Information}

A)

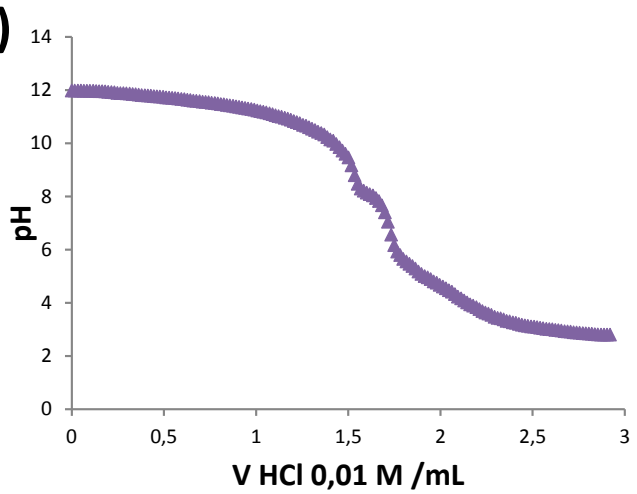

B)

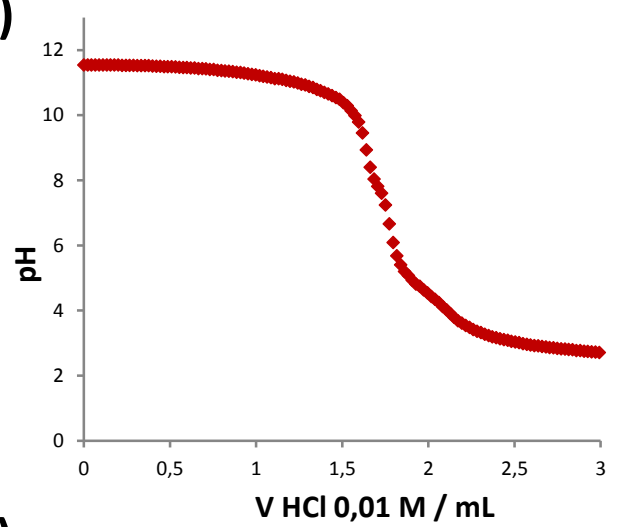

C)

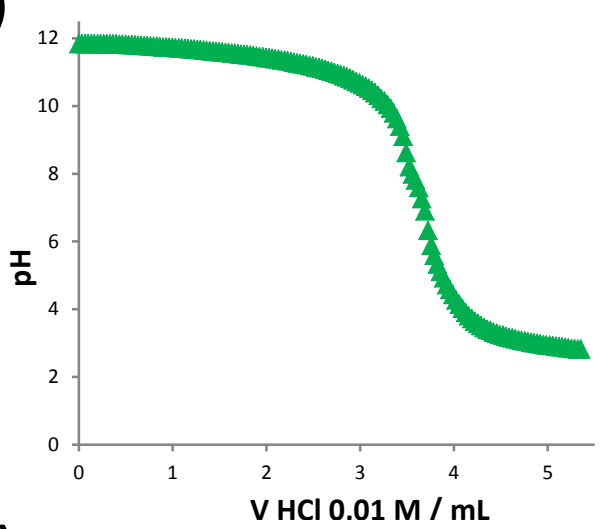

D)

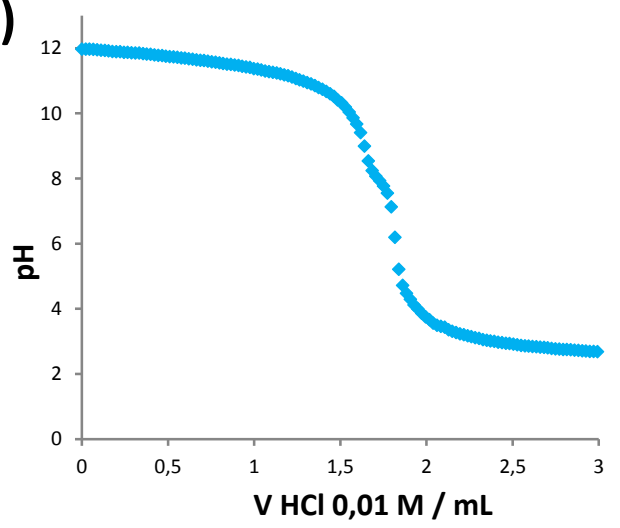

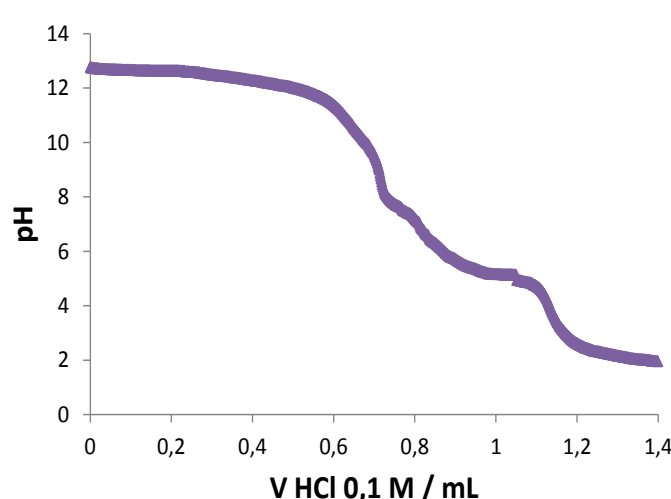
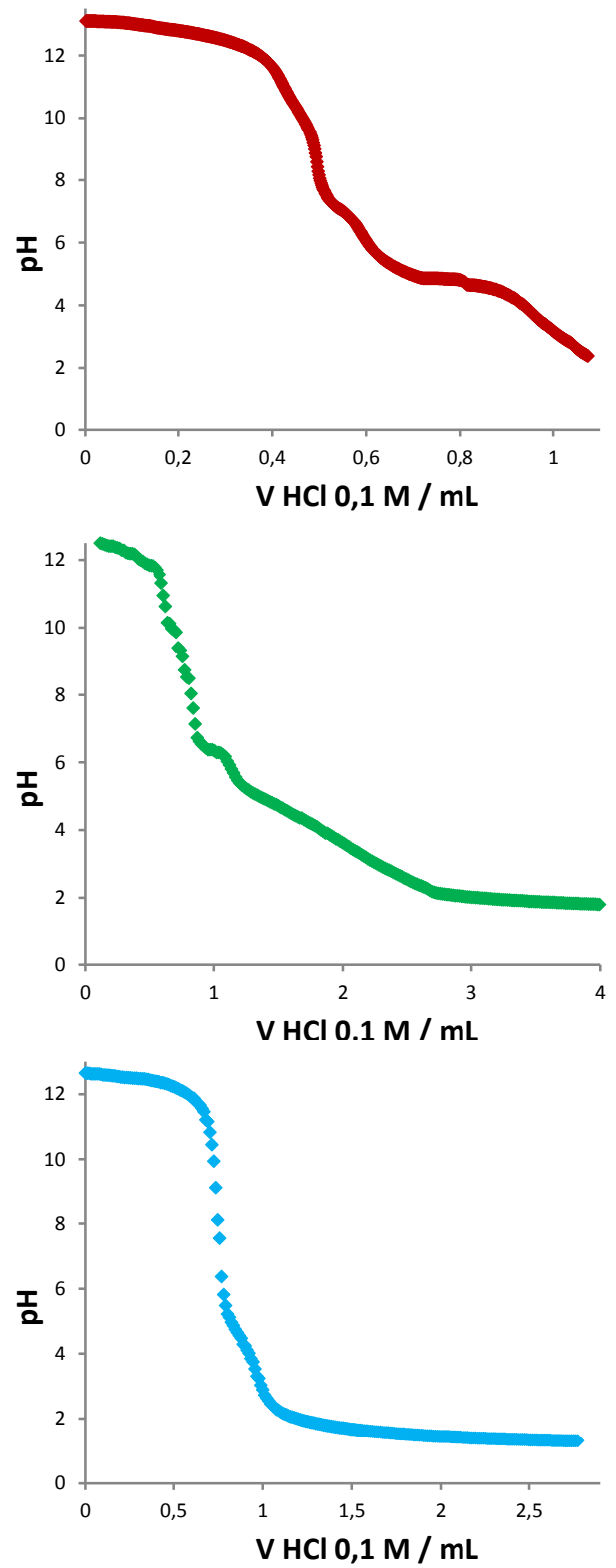

Figure SI_5.5.1. Titration curves of A) SucVal8 (5a), B) Suclle6 (5b), C) SucValNon (5c) and D) SucValHex (5d) of diluted (left) and concentrated (right) samples. 
A)

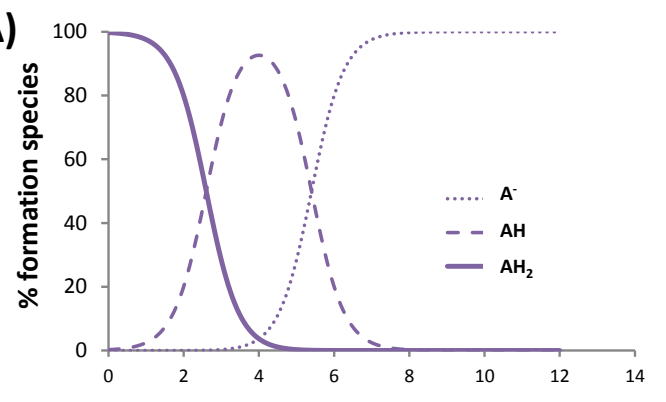

B)
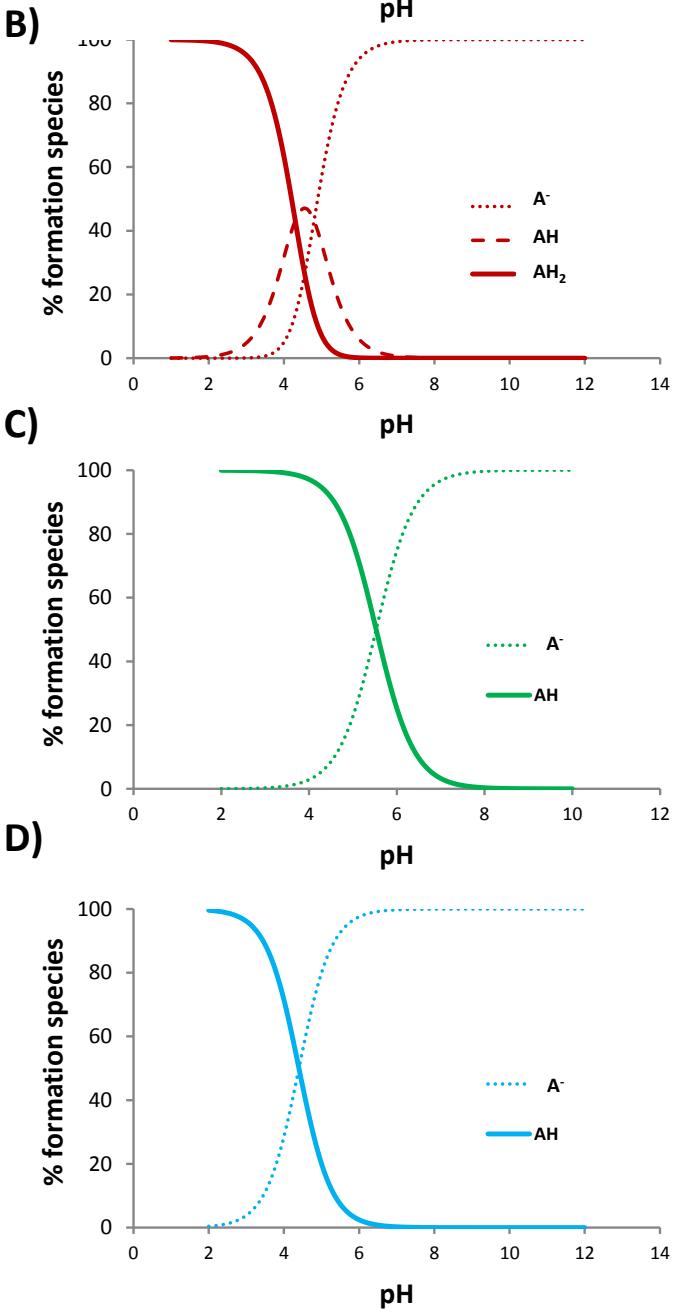
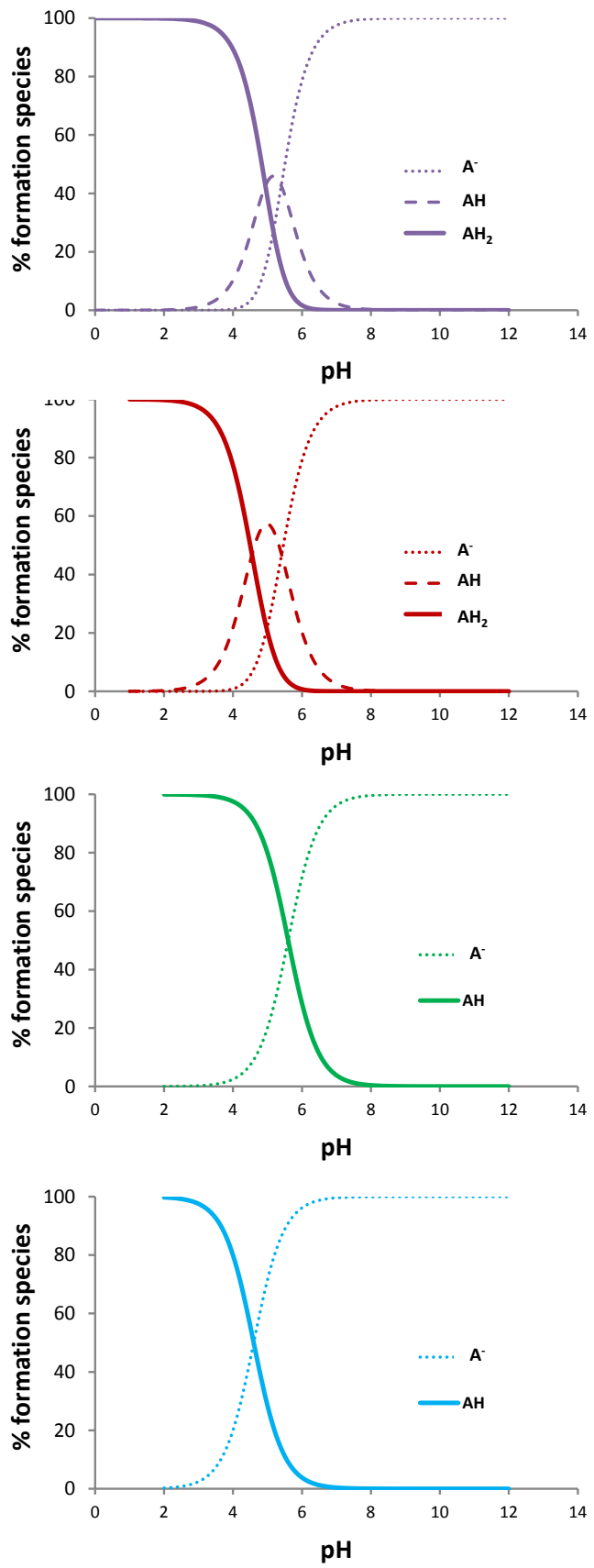

Figure SI_5.5.2. Species distribution diagram of A) SucVal8 (5a), B) Suclle6 (5b), C) SucValNon (5c) and D) SucValHex (5d) of diluted (left) and concentrated (right) samples. 
Size Distribution by Volume

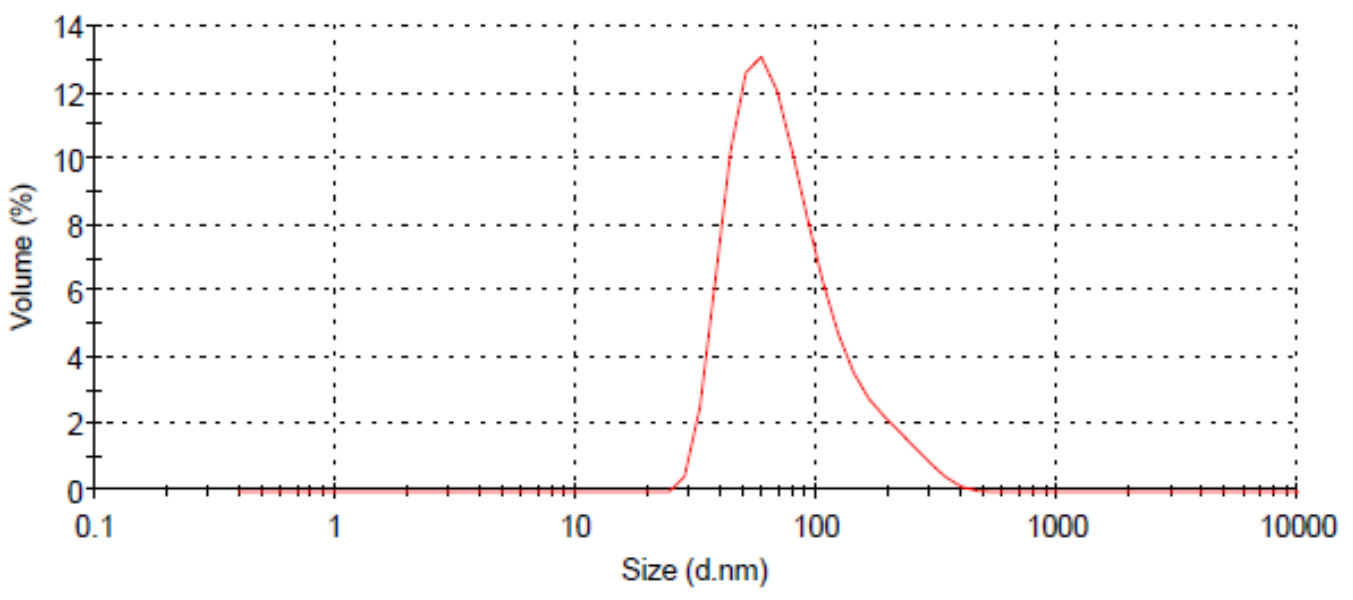

Figure SI_5.5.3. Dynamic light scattering (DLS) of a sample $16 \mathrm{mM}$ of SucVal8 (5a) in aqueous $\mathrm{NaOH}$ solution (average $d=94,47 \mathrm{~nm}$ ).

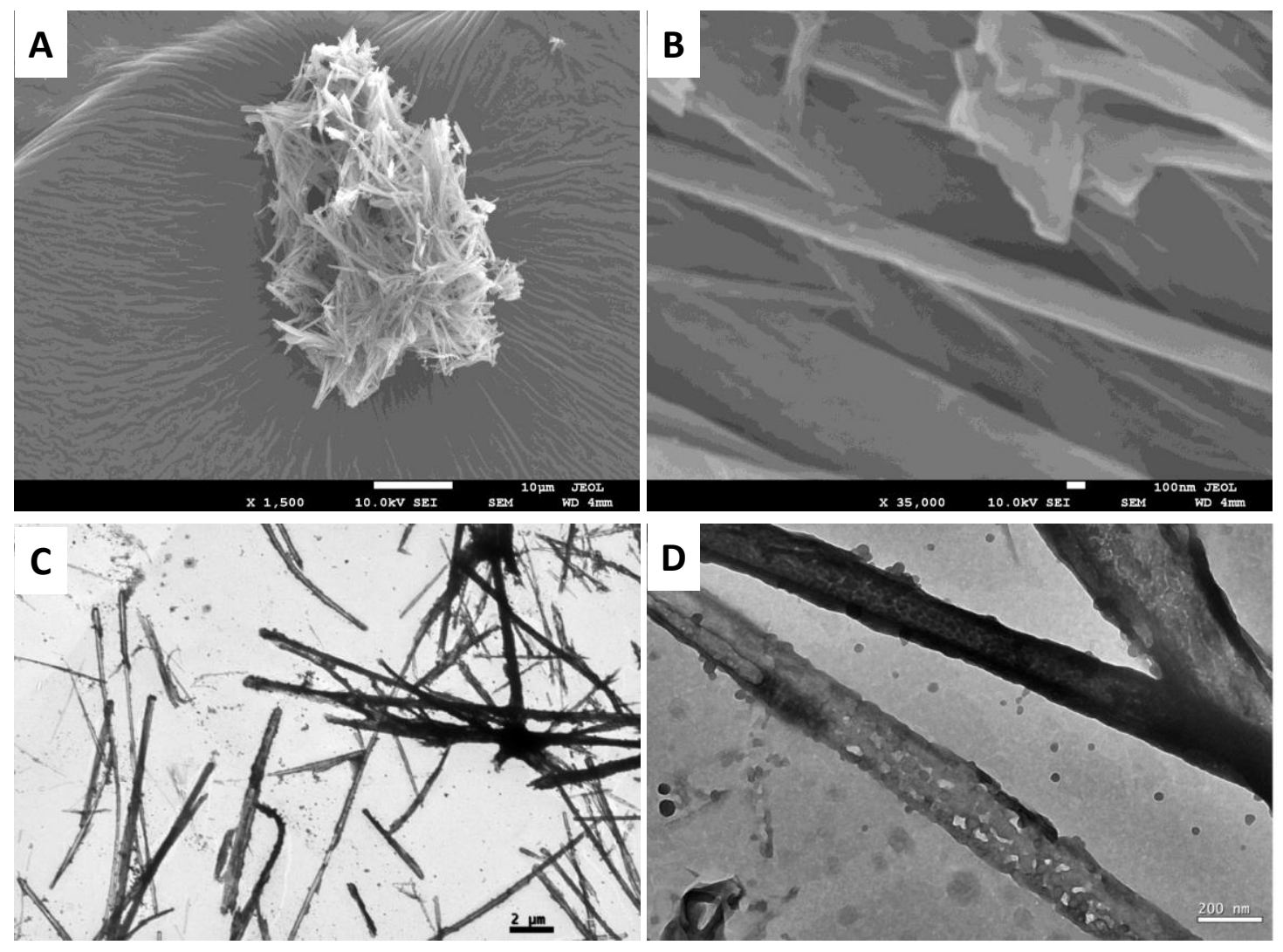

Figure SI_5.5.4. SEM (A-B) and TEM (C-D) images of a Prolle6 (3e) hydrogel sample 20 mM. 
A)

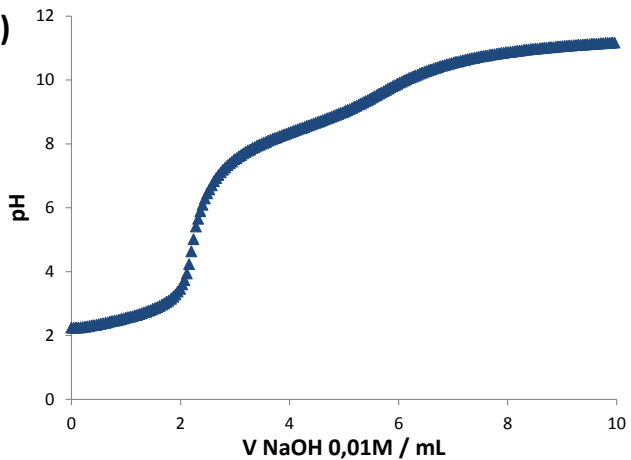

B)

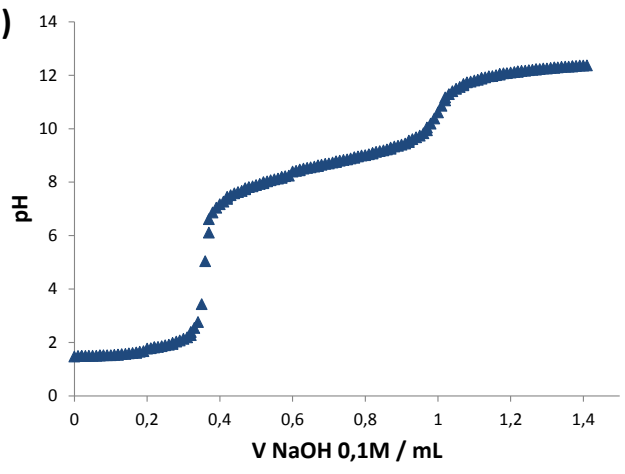

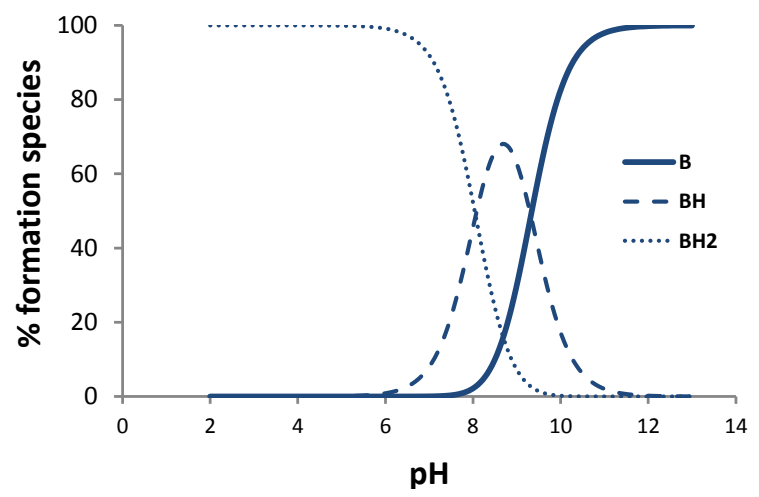

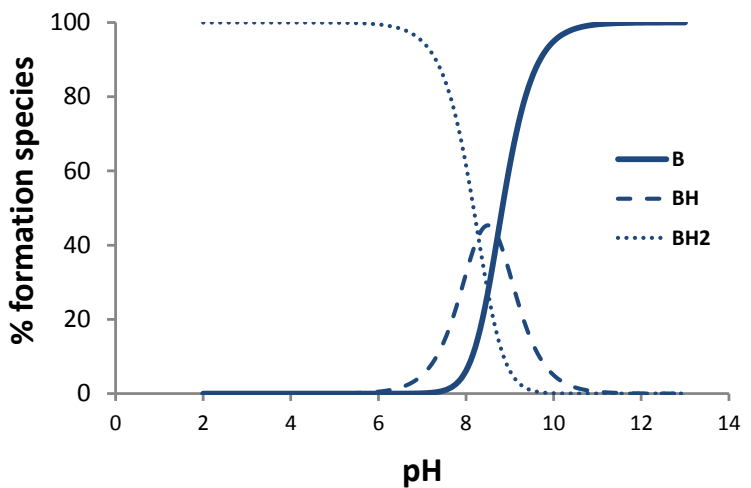

Figure SI_5.5.5. Titration curve (left) and species distribution diagram (right) of Prolle6 (3e) at A) 2,5 mM and B) $33 \mathrm{mM}$.
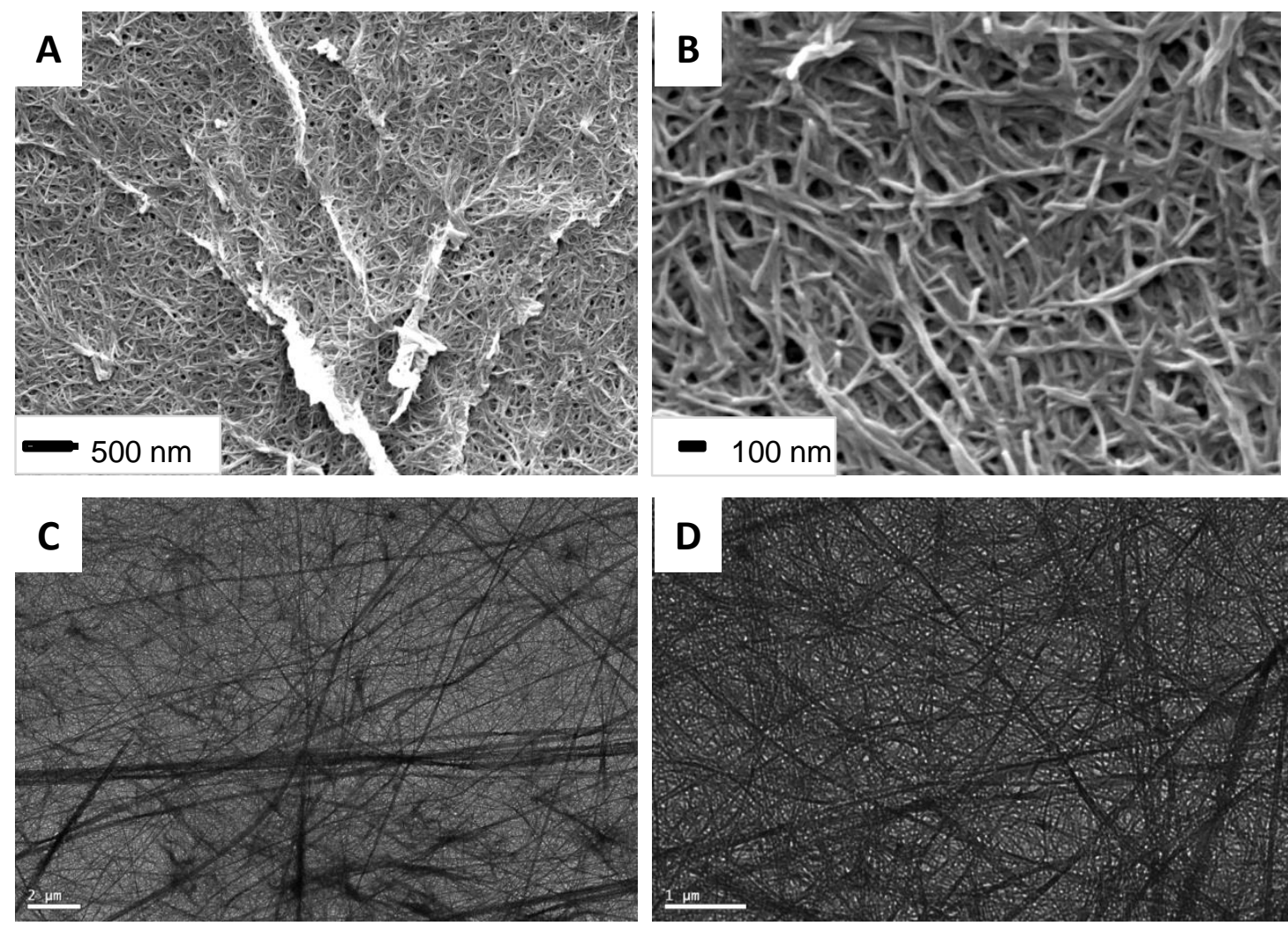

Figure SI_5.5.6. PyrVal3 (5e) hydrogel SEM images (A-B) 19 mM and TEM (C-D) 24 mM. 


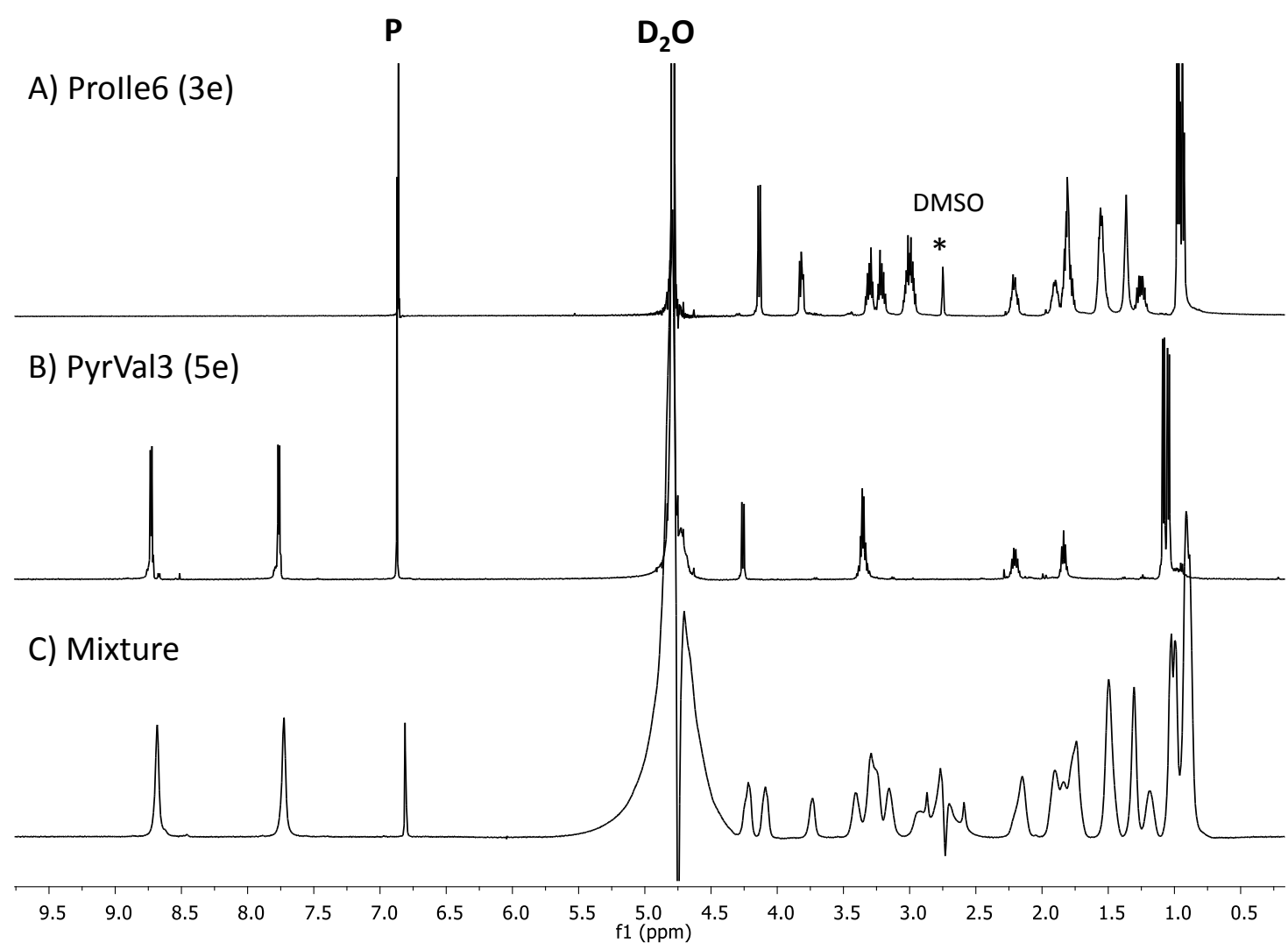

Figure SI_5.5.7. ${ }^{1}$ H.NMR spectra of A) $20 \mathrm{mM}$ of Prolle6 (3e), B) $20 \mathrm{mM}$ of PyrVal3 (5e) and C) sample Prolle6 (3e) + PyrVal3 (5e) (1:1) 24 mM mixture in $\mathrm{D}_{2} \mathrm{O}$.

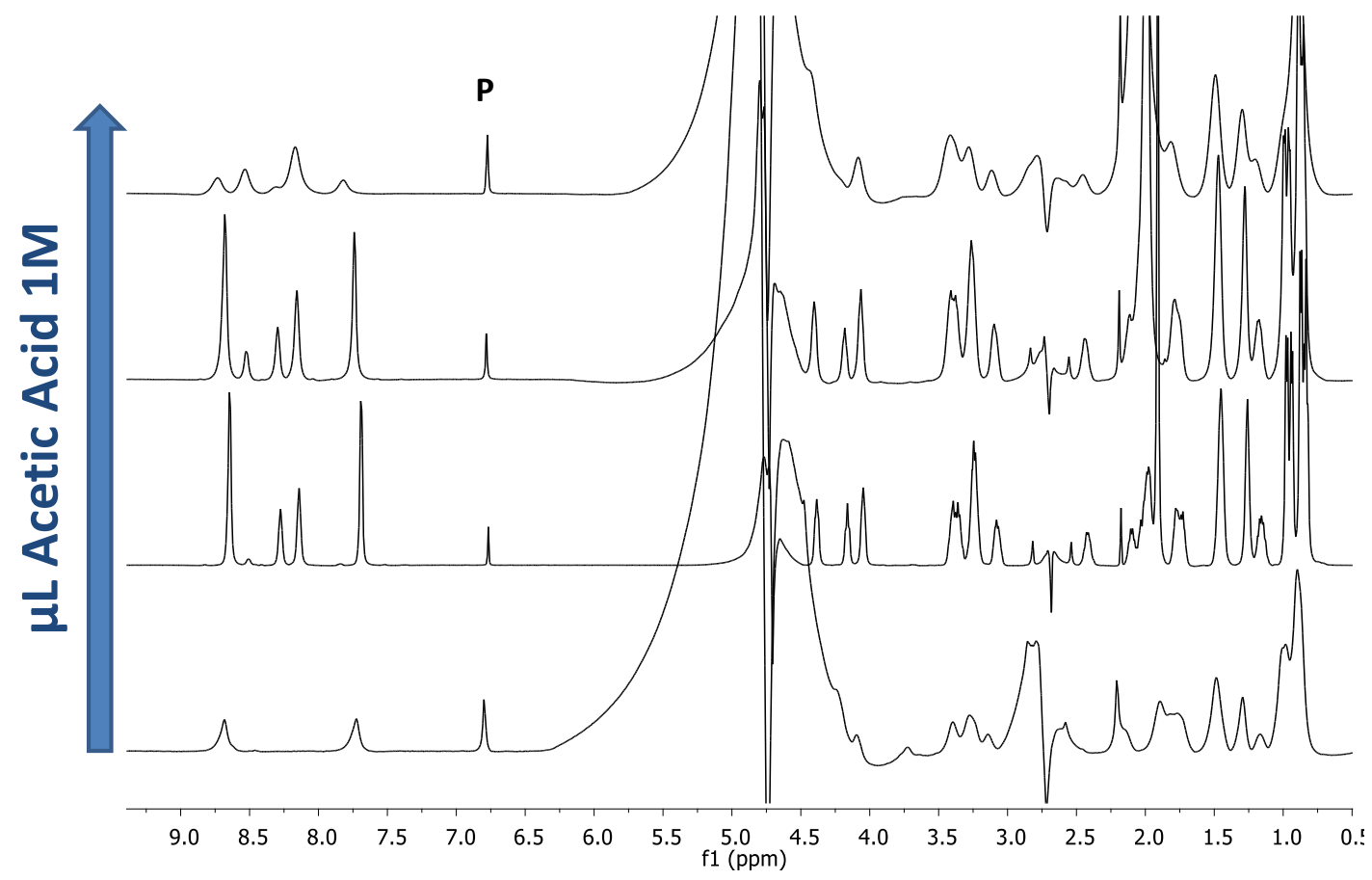

Figure SI_5.5.8. Stacked ${ }^{1}$ H-NMR spectra of the sample Prolle6 (3e) + PyrVal3 (5e) 24 mM mixture with increasing volumes of acetic acid $1 \mathrm{mM}$. 


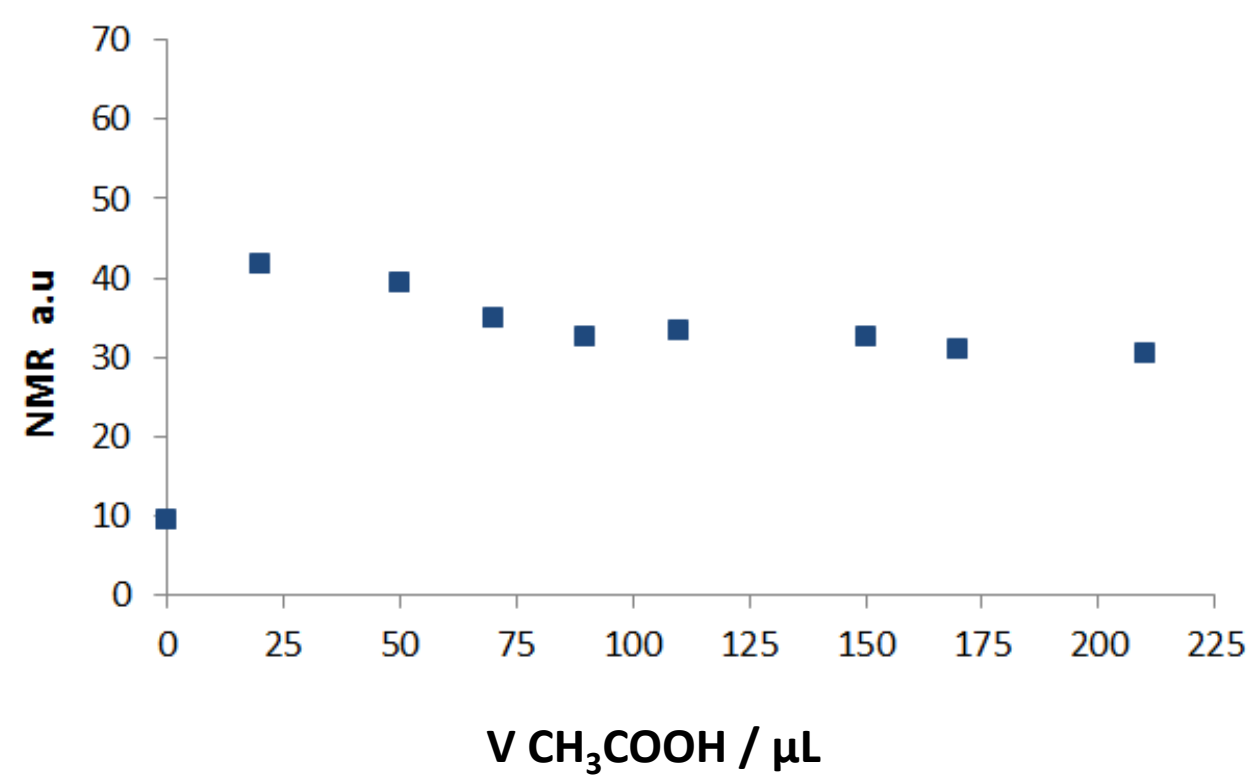

Figure SI_5.5.9. Plot of Prolle6 (3e) ${ }^{1}$ H-NMR intensities for the sample Prolle6 (3e) + PyrVal3 (5e) (1:1) 24 $\mathrm{mM}$ mixture during the progressive addition of acetic acid $1 \mathrm{M}$ showing the solubilisation of the gel network. 

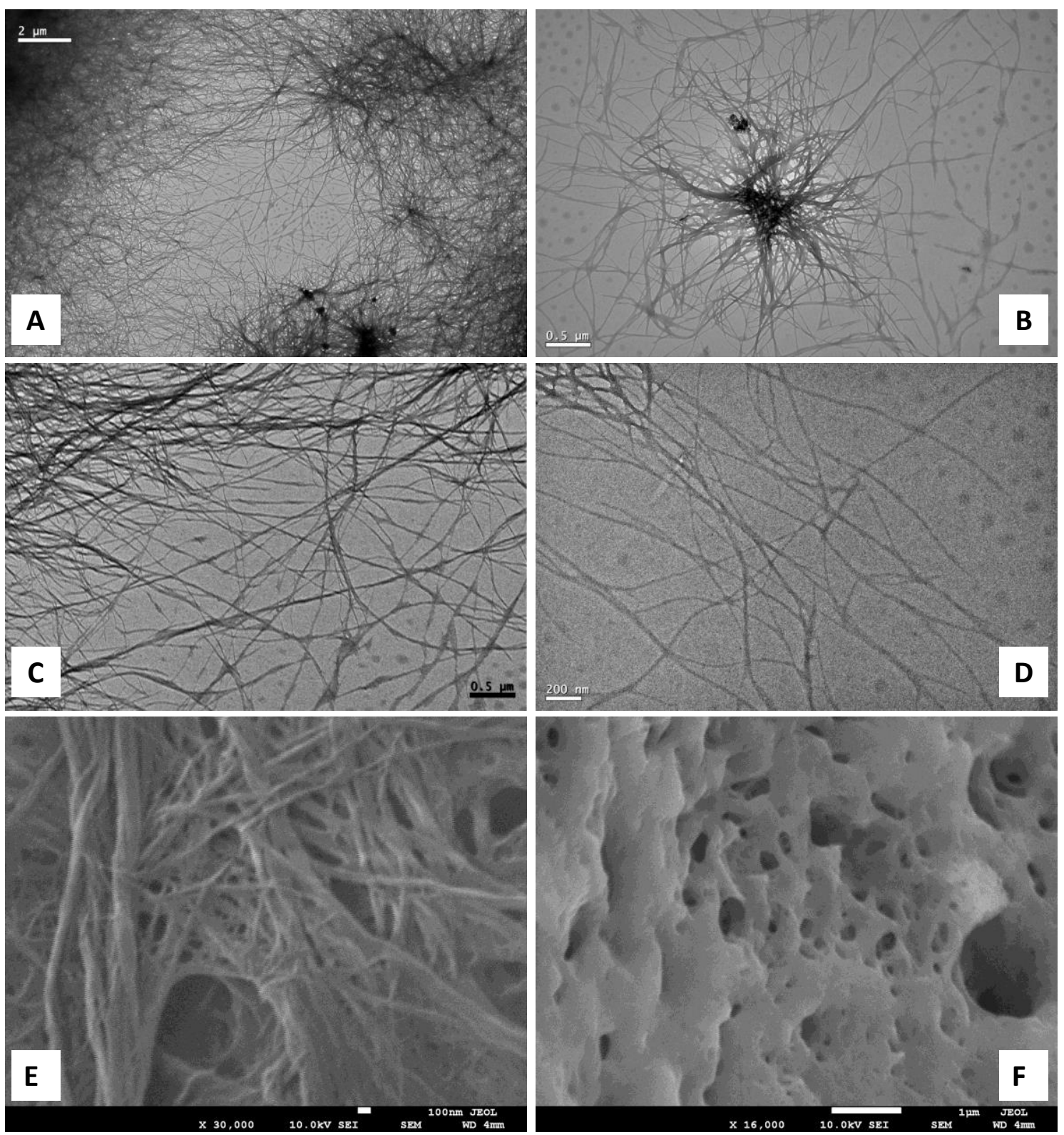

Figure SI_5.5.10. TEM (A-D) and SEM (E-F) images of the two-component hydrogel sample Prolle6 (3e) + PyrVal3 (5e) (1:1) 24 mM mixture. 

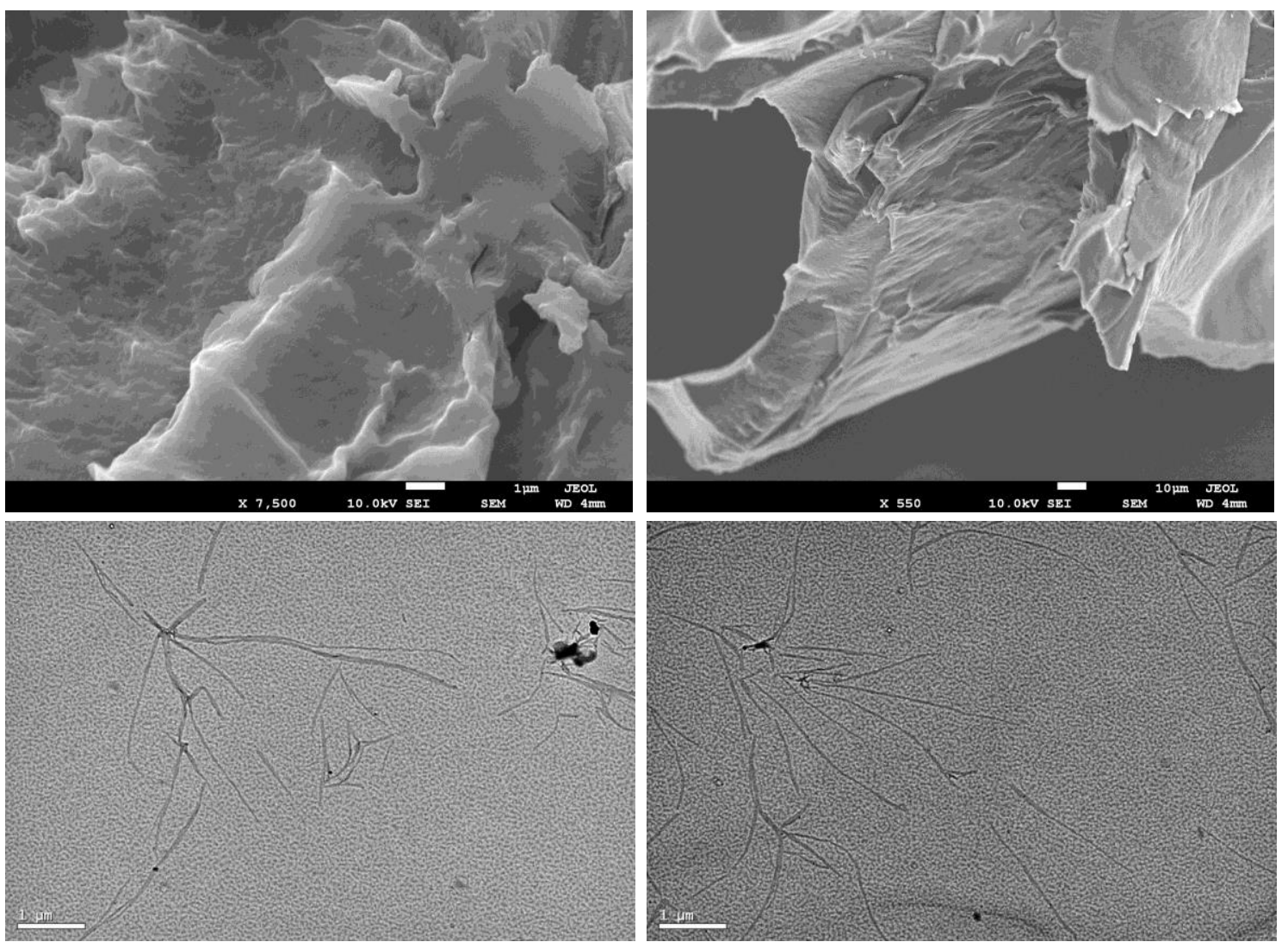

Figure SI_5.5.11. SEM (top) and TEM (bottom) images of Prolle6 (3e) hydrogel sample 24 mM with acetic acid $1 \mathrm{M}(10 \mu \mathrm{L})$.
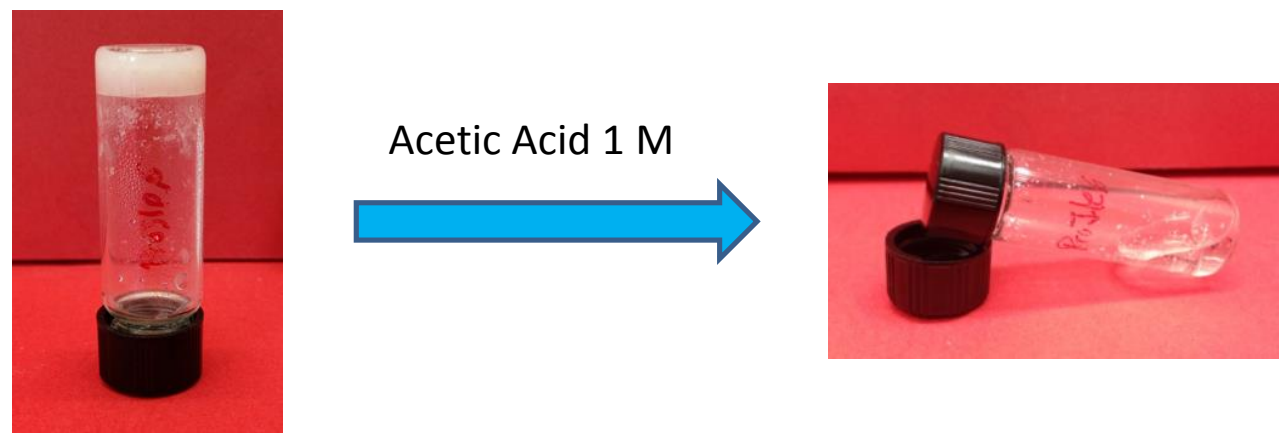

Figure SI_5.5.12. Images showing the solubilisation of a Prolle6 (3e) hydrogel in the presence of acetic acid $1 \mathrm{M}$. 

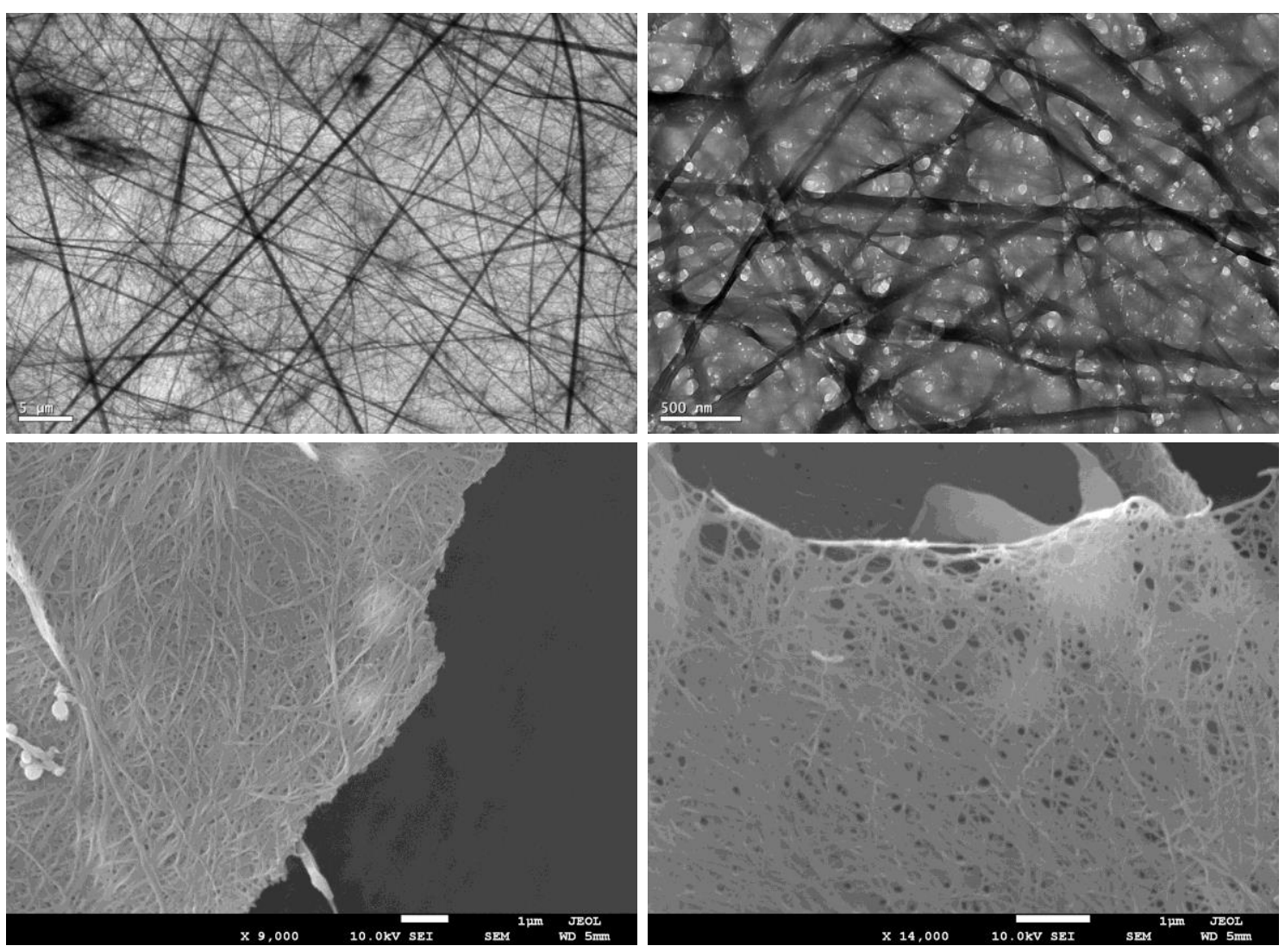

Figure SI_5.5.13. TEM (top) and SEM (bottom) images of PyrVal3 (3e) hydrogel sample $24 \mathrm{mM}$ with the presence of acetic acid $1 \mathrm{M}(30 \mu \mathrm{L})$.

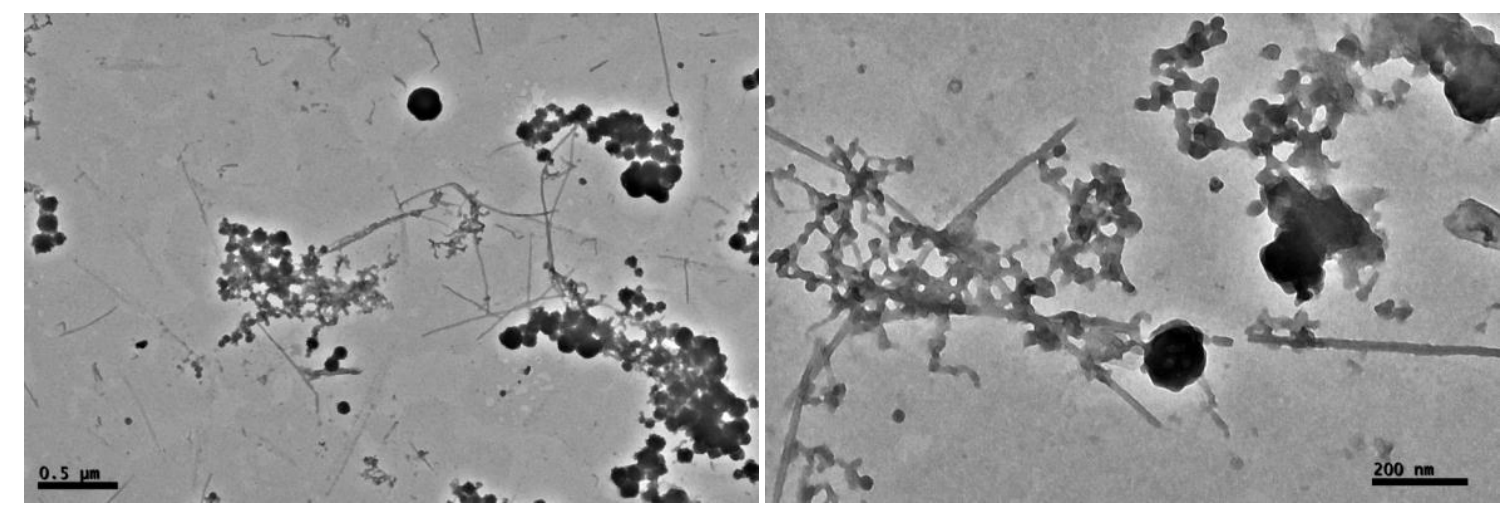

Figure SI_5.5.14. TEM images of the sample Prolle6(3e) +PyrVal3 (5e) (1:1) 24 mM mixture with acetic acid $1 \mathrm{M}$ once the gel has been solubilised. 

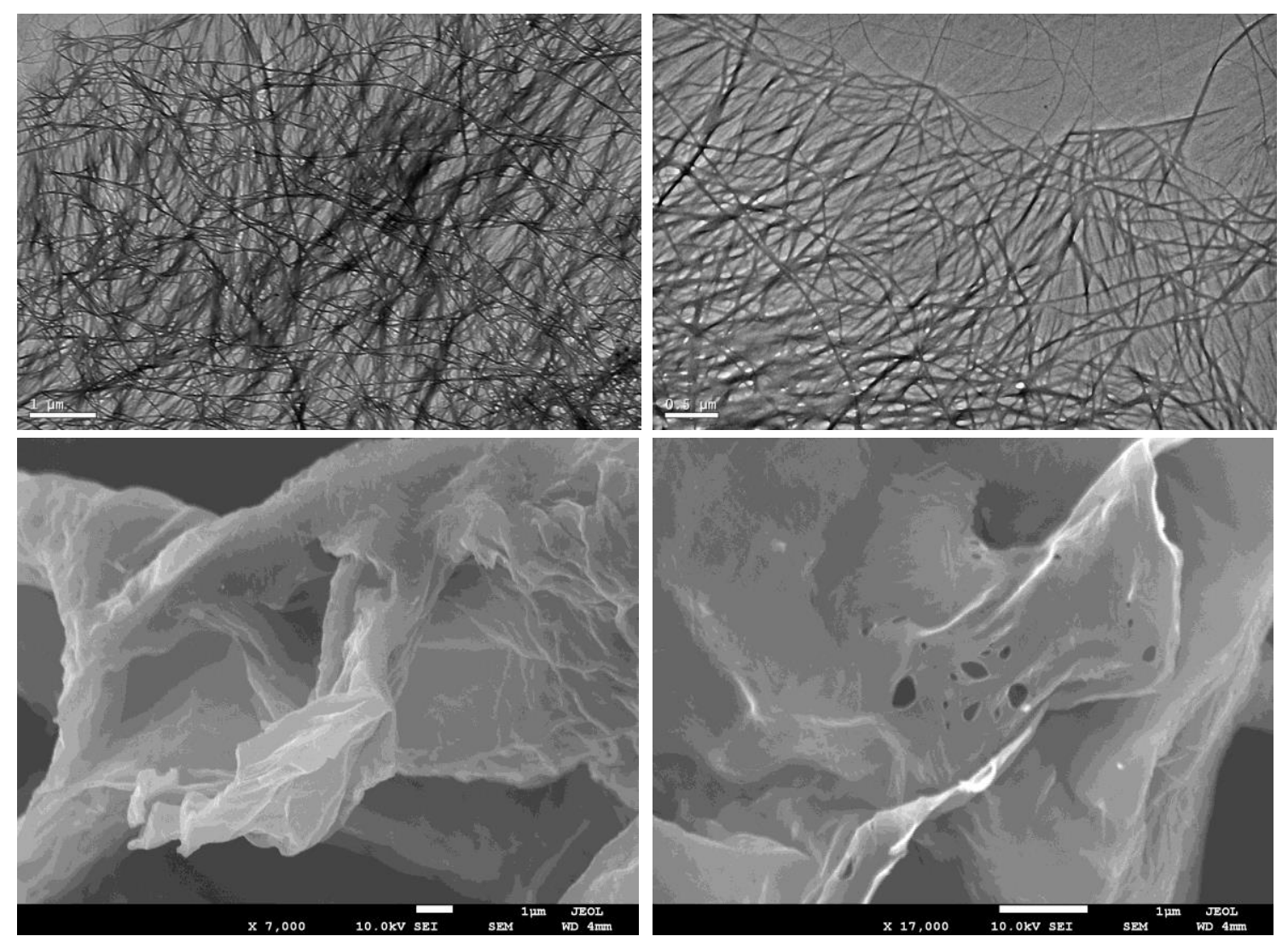

Figure SI_5.5.15. TEM (top) and SEM (bottom) images of the sample Prolle6(3e) +PyrVal3 (5e) (1:1) 24 mM mixture with acetic acid $1 \mathrm{M}$ having macroscopic aggregates in the sample. 

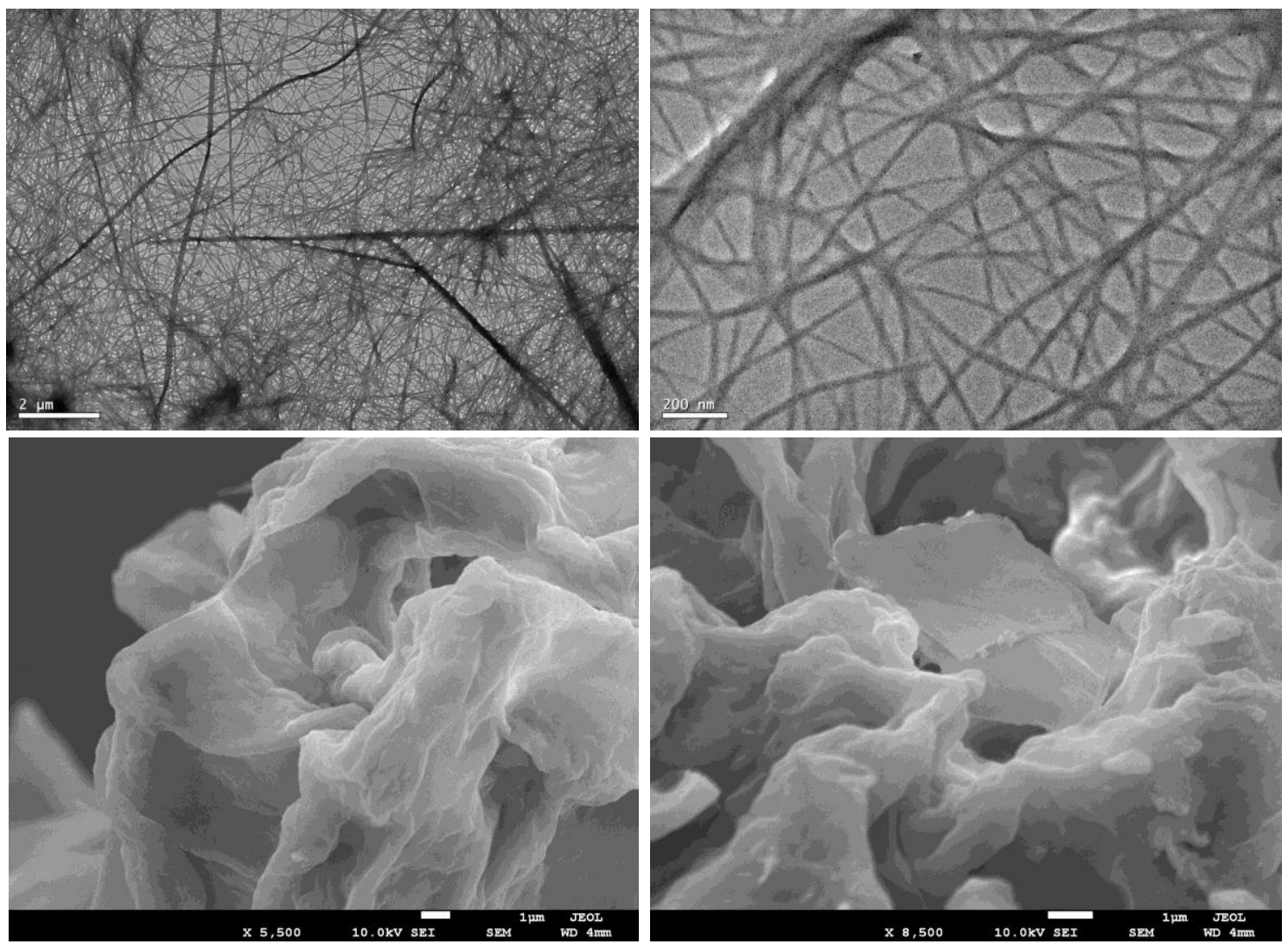

Figure SI_5.5.16. TEM (top) and SEM (bottom) images of the sample Prolle6(3e) +PyrVal3 (5e) (1:1) 24 mM mixture with acetic acid $1 \mathrm{M}$ once the gel has been recovered.

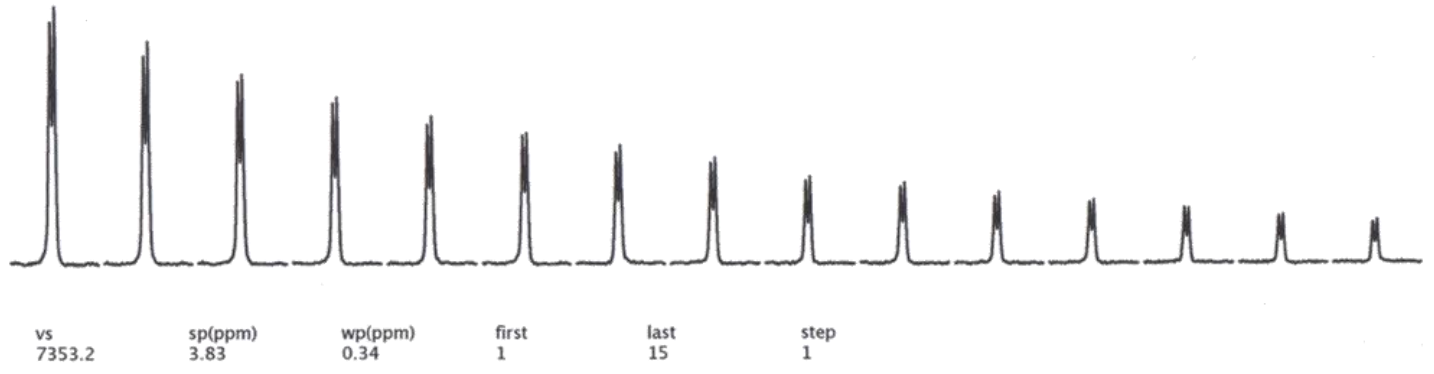

Figure SI_5.5.17. Example of the signal attenuation experienced during the DOSY experiments for a Prolle6 (3e) sample $20 \mathrm{mM}$ with acetic acid at $\mathrm{pH} 8$. 


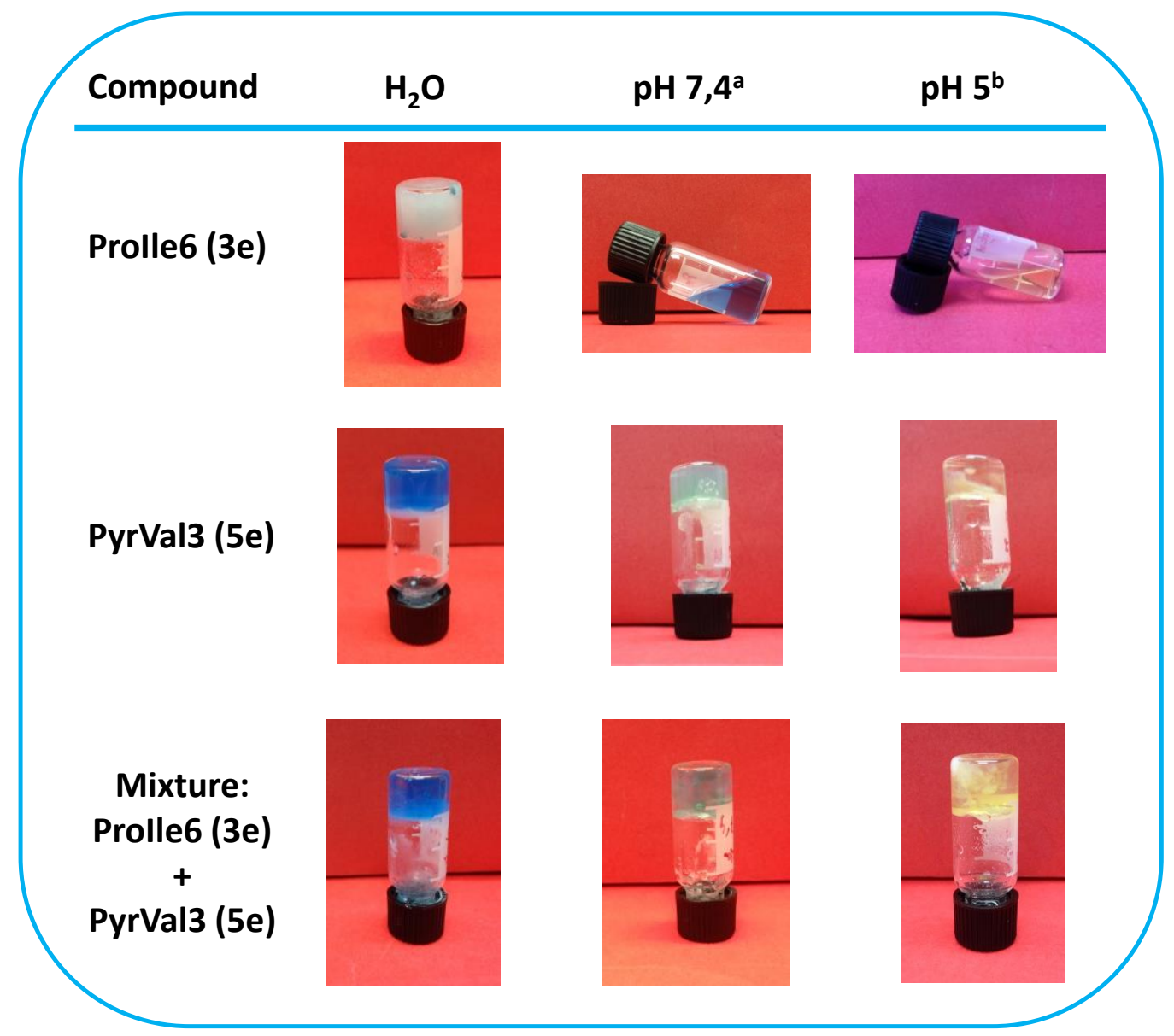

Figure SI_5.5.18. Gelation test with the mixture two-component hydrogel sample and samples of the individual components loaded with Bromothymol Blue indicator at different $\mathrm{pH}$ using buffer solutions
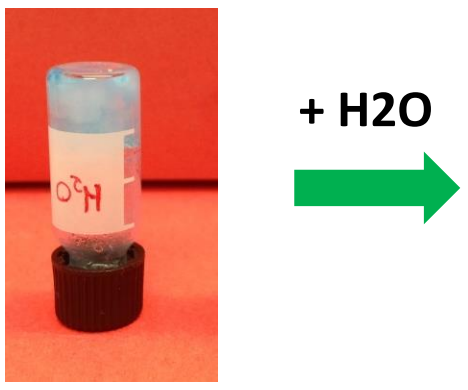

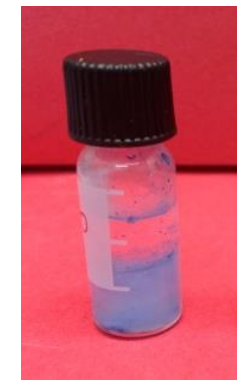

$0 \min$

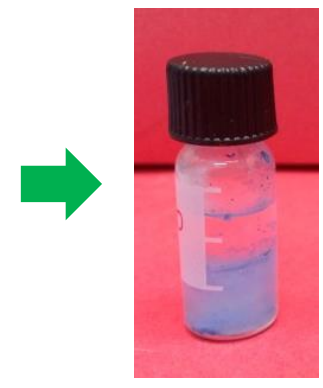

$15 \min$

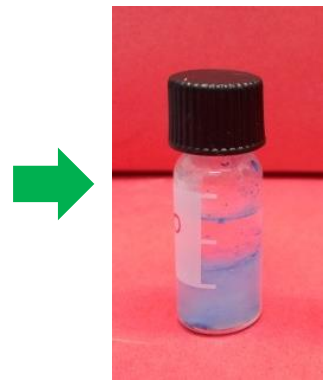

$30 \min$

Figure SI_5.5.19. Blank experiment of the sample Prolle6 (3e) + PyrVal3 (5e) (1:1) 24 mM mixture when 500 $\mu \mathrm{L}$ of water were added on the top of the gel and left it to diffuse over time. 


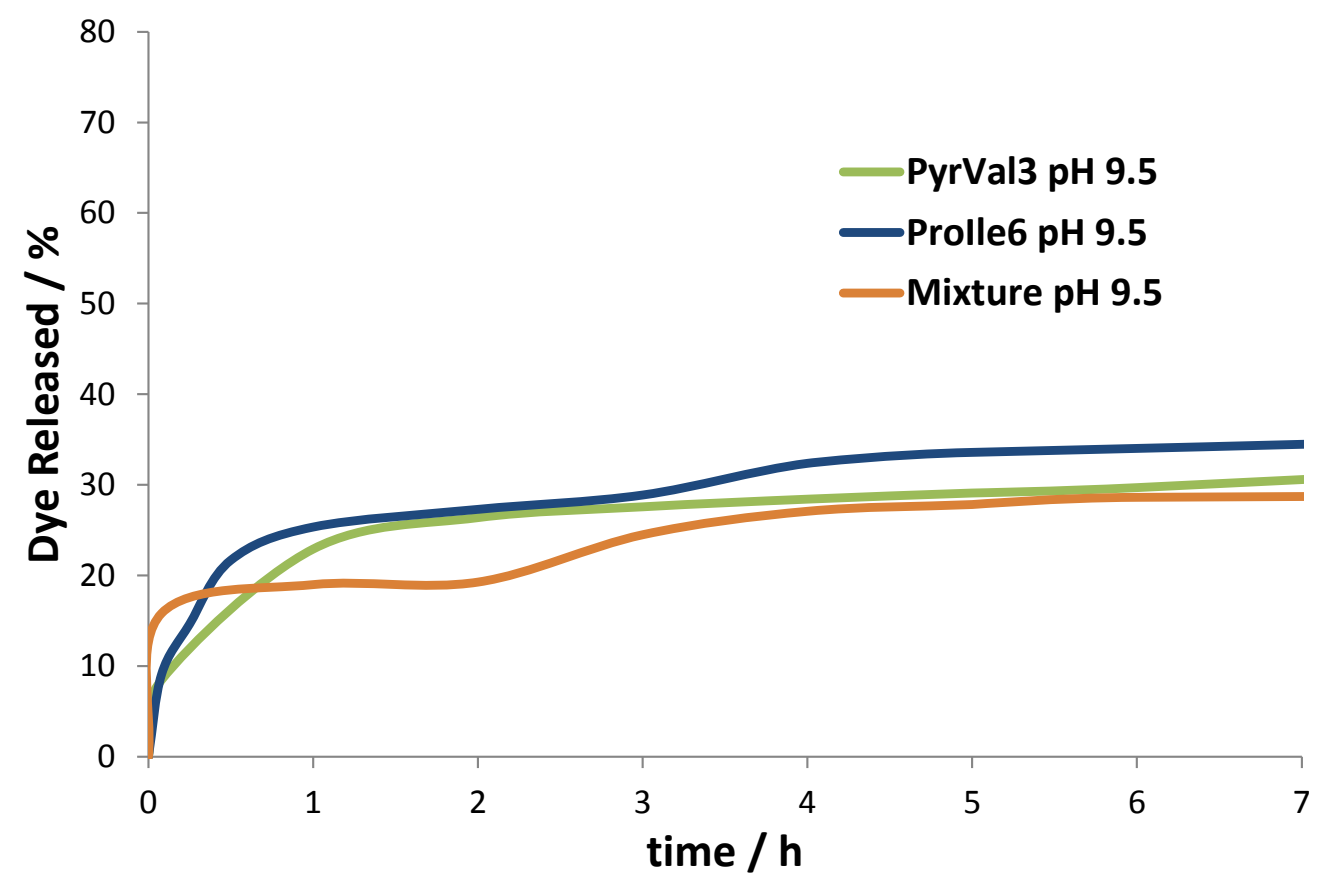

Figure SI_5.5.20. Release profiles of hydrogels loaded with Methyl Orange at pH 9.5. 


\subsection{References and Notes}

1. (a) Ulijn, R. V. "Enzyme-Responsive Materials: a New Class of Smart Biomaterials", J. Mater. Chem., 2006, 16, 2217-2225; (b) Lowik, D. W.; Leunissen, E. H.; van den Heuvel, M.; Hansen, M. B.; van Hest, J. C. "Stimulus Responsive Peptide Based Materials", Chem. Soc. Rev., 2010, 39, 3394-3412.

2. Busseron, E.; Ruff, Y.; Moulin, E.; Giuseppone, N. "Supramolecular Self-Assemblies as Functional Nanomaterials", Nanoscale, 2013, 5, 7098-7140.

3. (a) de Las Heras Alarcon, C.; Pennadam, S.; Alexander, C. "Stimuli Responsive Polymers for Biomedical Applications", Chem. Soc. Rev., 2005, 34, 276-285; (b) Ahn, S.-k.; Kasi, R. M.; Kim, S.-C.; Sharma, N.; Zhou, Y. "Stimuli-Responsive Polymer Gels", Soft Matter, 2008, 4, 1151-1157.

4. (a) Yang, X.; Zhang, G.; Zhang, D. "Stimuli Responsive Gels Based on Low Molecular Weight Gelators", J. Mater. Chem., 2012, 22, 38-50; (b) Segarra-Maset, M. D.; Nebot, V. J.; Miravet, J. F.; Escuder, B. "Control of Molecular Gelation by Chemical Stimuli", Chem. Soc. Rev., 2013, 42, 7086-7098; (c) Xiong, M.; Wang, C.; Zhang, G.; Zhang, D., "CHAPTER 3 Molecular Gels Responsive to Physical and Chemical Stimuli". In Functional Molecular Gels. RSC, Cambridge, 2014; pp 67-94.

5. Rodríguez-Llansola, F.; Escuder, B.; Miravet, J. F.; Hermida-Merino, D.; Hamley, I. W.; Cardin, C. J.; Hayes, W. "Selective and Highly Efficient Dye Scavenging by a pH-Responsive Molecular Hydrogelator", Chem. Commun., 2010, 46, 7960-7962.

6. Wood, D. M.; Greenland, B. W.; Acton, A. L.; Rodríguez-Llansola, F.; Murray, C. A.; Cardin, C. J.; Miravet, J. F.; Escuder, B.; Hamley, I. W.; Hayes, W. "pH-Tunable Hydrogelators for Water Purification: Structural Optimisation and Evaluation", Chem. Eur. J., 2012, 18, 26922699.

7. Adams, D. J.; Butler, M. F.; Frith, W. J.; Kirkland, M.; Mullen, L.; Sanderson, P. "A New Method for Maintaining Homogeneity During Liquid-Hydrogel Transitions Using Low Molecular Weight Hydrogelators", Soft Matter, 2009, 5, 1856-1862.

8. Buerkle, L. E.; Rowan, S. J. "Supramolecular Gels Formed from Multi-Component Low Molecular Weight Species", Chem. Soc. Rev., 2012, 41, 6089-6102.

9. (a) Brizard, A.; Stuart, M.; van Bommel, K.; Friggeri, A.; de Jong, M.; van Esch, J. "Preparation of Nanostructures by Orthogonal Self-Assembly of Hydrogelators and Surfactants", Angew. Chem. Int. Ed., 2008, 47, 2063-2066; (b) Hirst, A. R.; Miravet, J. F.; Escuder, B.; Noirez, L.; Castelletto, V.; Hamley, I. W.; Smith, D. K. "Self-Assembly of TwoComponent Gels: Stoichiometric Control and Component Selection", Chemistry, 2009, 15, 372-379; (c) Tam, A. Y.-Y.; Yam, V. W.-W. "Recent Advances in Metallogels", Chem. Soc. Rev., 2013, 42, 1540-1567.

10. Hirst, A. R.; Smith, D. K.; Feiters, M. C.; Geurts, H. P.; Wright, A. C. "Two-Component Dendritic Gels: Easily Tunable Materials", J. Am. Chem. Soc., 2003, 125, 9010-1.

11. Tena-Solsona, M.; Alonso-de Castro, S.; Miravet, J. F.; Escuder, B. "Co-Assembly of Tetrapeptides into Complex pH-Responsive Molecular Hydrogel Networks", J. Mater. Chem. B, 2014, 2, 6192-6197. 
12. Morris, K. L.; Chen, L.; Raeburn, J.; Sellick, O. R.; Cotanda, P.; Paul, A.; Griffiths, P. C.; King, S. M.; O'Reilly, R. K.; Serpell, L. C.; Adams, D. J. "Chemically Programmed Self-Sorting of Gelator Networks", Nat. Commun., 2013, 4, 1480.

13. Fontanillo, M.; Angulo-Pachon, C. A.; Escuder, B.; Miravet, J. F. "In Situ Synthesis-Gelation at Room Temperature vs. Heating-Cooling Procedure. Fine Tuning of Molecular Gels Derived from Succinic Acid and L-Valine", J. Colloid Interface Sci., 2013, 412, 65-71.

14. Nelson, D. L.; Cox, M. M., "Principles of Biochemistry". fourth ed.

15. Escuder, B.; LLusar, M.; Miravet, J. F. "Insight on the NMR Study of Supramolecular Gels and its Application to Monitor Molecular Recognition on Self-Assembled Fibers", J. Org. Chem., 2006, 71, 7747-7752.

16. Hamley, I. W.; Nutt, D. R.; Brown, G. D.; Miravet, J. F.; Escuder, B.; Rodríguez-Llansola, F. "Influence of the Solvent on the Self-Assembly of a Modified Amyloid Beta Peptide Fragment. II. NMR and Computer Simulation Investigation", J. Phys. Chem. B, 2010, 114, 940-951.

17. Privalov, P. L.; Gill, S. J. "The Hydrophobic Effect: a Reappraisal", Pure \& Appl. Chem., 1989, 61, 1097-1104,.

18. Vinther, J. M.; Kristensen, S. M.; Led, J. J. "Enhanced Stability of a Protein with Increasing Temperature", J. Am. Chem. Soc., 2011, 133, 271-278.

19. Nebot, V. J.; Armengol, J.; Smets, J.; Prieto, S. F.; Escuder, B.; Miravet, J. F. "Molecular Hydrogels from Bolaform Amino Acid Derivatives: A Structure-Properties Study Based on the Thermodynamics of Gel Solubilization", Chem. Eur. J., 2012, 18, 4063-4072.

20. Miravet, J. F.; Escuder, B. "Pyridine-Functionalised Ambidextrous Gelators: Towards Catalytic Gels", Chem. Commun., 2005, 5796-5798.

21. Nebot, V. J.; Escuder, B.; Miravet, J. F.; Smets, J.; Fernández-Prieto, S. "Interplay of Molecular Hydrogelators and SDS Affords Responsive Soft Matter Systems with Tunable Properties", Langmuir, 2013, 29, 9544-9550.

22. (a) Yang, K.; Cheng, Y.; Feng, X.; Zhang, J.; Wu, Q.; Xu, T. "Insights into the Interactions between Dendrimers and Multiple Surfactants: 5. Formation of Miscellaneous Mixed Micelles Revealed by a Combination of ${ }^{1} \mathrm{H}$ NMR, Diffusion, and NOE Analysis", J. Phys. Chem. B, 2010, 114, 7265-7273; (b) Nonappa; Saman, D.; Kolehmainen, E. "Studies on Supramolecular Gel Formation Using DOSY NMR", Magn. Reson. Chem., 2015, 53, 256260.

23. Menger, F. M.; Lu, H.; Lundberg, D. "A-B-A-B-A Block Amphiphiles. Balance between Hydrophilic and Hydrophobic Segmentation", J. Am. Chem. Soc., 2007, 129, 272-273.

24. Nebot, V. J.; Ojeda-Flores, J. J.; Smets, J.; Fernández-Prieto, S.; Escuder, B.; Miravet, J. F. "Rational Design of Heat-Set and Specific-Ion-Responsive Supramolecular Hydrogels Based on the Hofmeister Effect", Chem. Eur. J., 2014, 20, 14465-14472.

25. (a) Uhrich, K. E.; Cannizzaro, S. M.; Langer, R. S.; Shakesheff, K. M. "Polymeric Systems for Controlled Drug Release", Chem. Rev., 1999, 99, 3181-3198; (b) Truong, W. T.; Lewis, L.; Thordarson, P., "CHAPTER 6 Biomedical Applications of Molecular Gels". In Functional Molecular Gels. RSC, Cambridge, 2014; pp 157-194. 
26. Ruiz, J.; Mantecón, A.; Cádiz, V. "Investigation of Loading and Release in PVA-Based Hydrogels", J. Appl. Polym. Sci., 2002, 85, 1644-1651. 


\section{CHAPTER 6}

\section{BIOCATALYTIC GELATION-DRIVEN DYNAMIC COMBINATORIAL LIBRARY TO DISCOVER CHARGE-TRANSFER PEPTIDE NANOSTRUCTURES}

Performed during a placement at the University of Strathclyde Glasgow under the supervision of the Prof. R. V. Ulijn in the department of Pure and Applied Chemistry (June - December 2013) 


\subsection{Introduction}

\subsubsection{Supramolecular Electronics}

The design of electronic devices based on organic semiconductors ${ }^{1}$ offers an attractive alternative to conventional inorganic devices due to potentially lower costs, simpler packaging and compatibility with flexible substrates. Exciting results have been obtained with organic light-emitting diodes (OLEDs) with the first polymeric material now commercially available. ${ }^{2}$ Nowadays, organic electronics are being used in the manufacture of OLED displays (e.g., TV and cell phone displays), solar cells, and transistors (see Figure 6.1.1.1). Material scientists have obtained new molecular structures with useful properties, using supramolecular electronic chemistry, ${ }^{3}$ which combines features of polymeric materials; ${ }^{4}$ are easy to synthesise, manipulate and incorporate into devices, with organic materials; have precise ordering, which supports the high charge-carrier (electron and hole) mobility essential to electronic performance. In this way, supramolecular electronics implies materials that can self-assemble, therefore are easier to process than crystalline materials with a high ordered system, and suitable for the desired electronic functions.

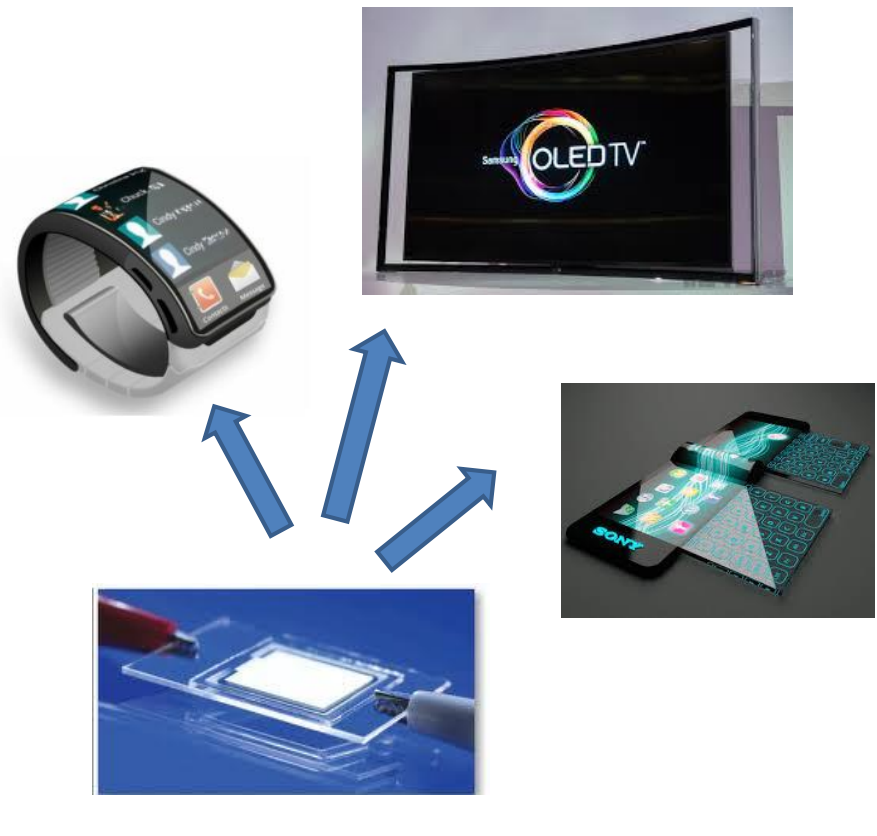

OLEDs

(Organic light-emitting diodes)
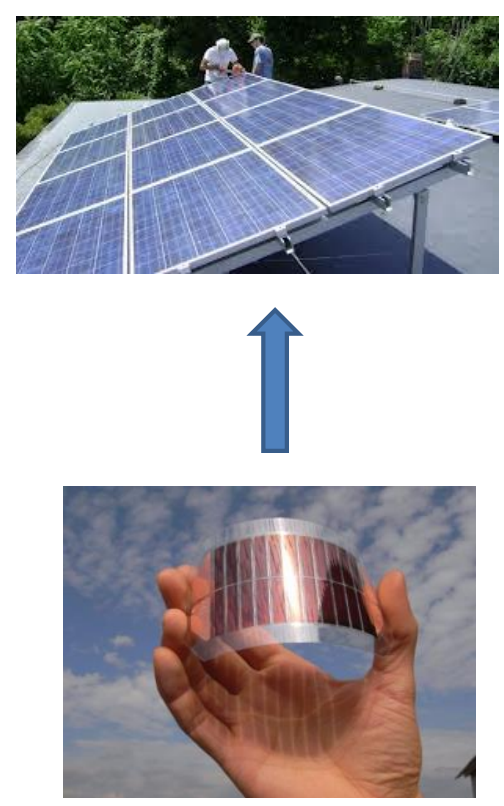

OPVs

(Organic photovoltaics)

Figure 6.1.1.1. Examples of some applications in organic electronics. 
The bottom-up approach has been reported for inorganic nanowires ${ }^{5}$ and carbon nanotubes ${ }^{6}$ showing promising electrical properties. Taking advantage of this approach, organic materials that self-assemble have been designed with the aim of producing a new generation of opto(electronic) nanodevices. ${ }^{7}$

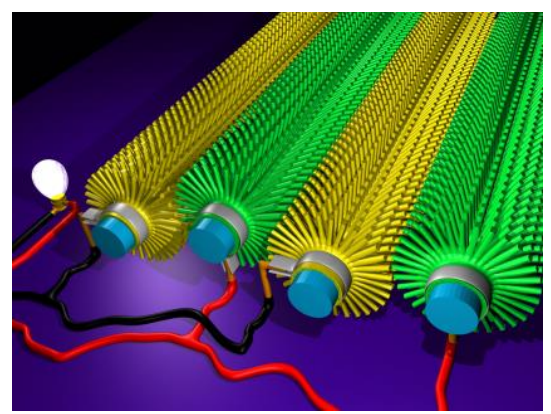

Inorganic nanowires

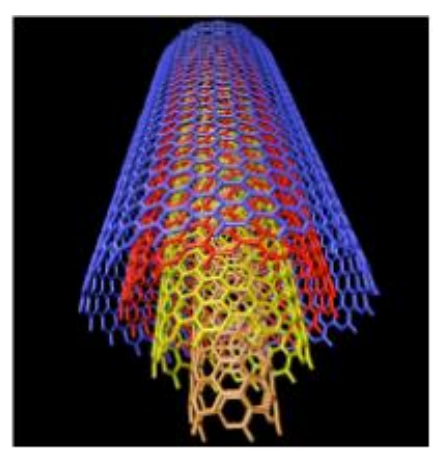

\section{Carbon} nanotubes

Figure 6.1.1.2. Examples of supramolecular organization for inorganic nanowires ${ }^{8}$ (left) and carbon nanotubes ${ }^{9}$ (right).

The introduction of $\pi$-conjugated systems, which possess delocalised electrons, that allow fabrication of well-defined electroconducting nanostructures with controllable length and morphology has been advantageous. ${ }^{10}$ These materials contain electrons that are not involved in individual covalent bonds, so can travel freely over the molecular structure and therefore display high electron mobility, a feature desired in these kind of materials.

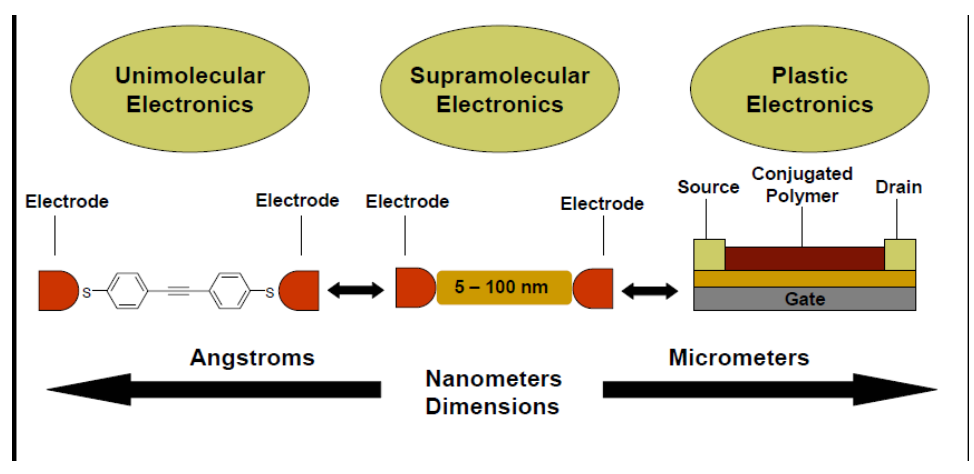

Figure 6.1.1.3. Schematic representation showing the difference, in length scale, between molecular electronics, supramolecular electronics and plastic electronics. ${ }^{7}$ 
The field of supramolecular electronics is based on the construction of assemblies of $\pi$-conjugated nanowire systems on the scale of 5-100 $\mathrm{nm}$. Self-assembly is an efficient strategy to allocate the $\pi$-conjugated chromophore moiety in a well-defined position via $\pi$-stacking, as it enables a high degree of spatial organization, proximity and face to face packing mode, thus facilitating the formation of highly organised, one dimensional (1D) nanostructures. In these systems the charge can be carried over intermolecular $\pi$ electron clouds along the length of the nanostructure, thus devices would possess favourable electron mobility, resulting from a combination of small dimensions and high structural order. ${ }^{7}$

An attractive approach to create arrays of $\pi$-electron chromophores is the use of short peptides to predictably direct the assembly into 1D nanofibres, taking advantage of its simplicity, variety and chemical accessibility. Peptide systems where aggregation generates 1D nanowire structures have been studied widely. ${ }^{11}$ As an example, Tovar et al. designed a bithiophene oligopeptide derivative where the $\pi-\pi$ stacking of the bithiophene structure drives the formation of supramolecular 1D fibres in a hydrogel, thus generating nanowire-like structures with promising electronic properties (see Figure 6.1.1.4).
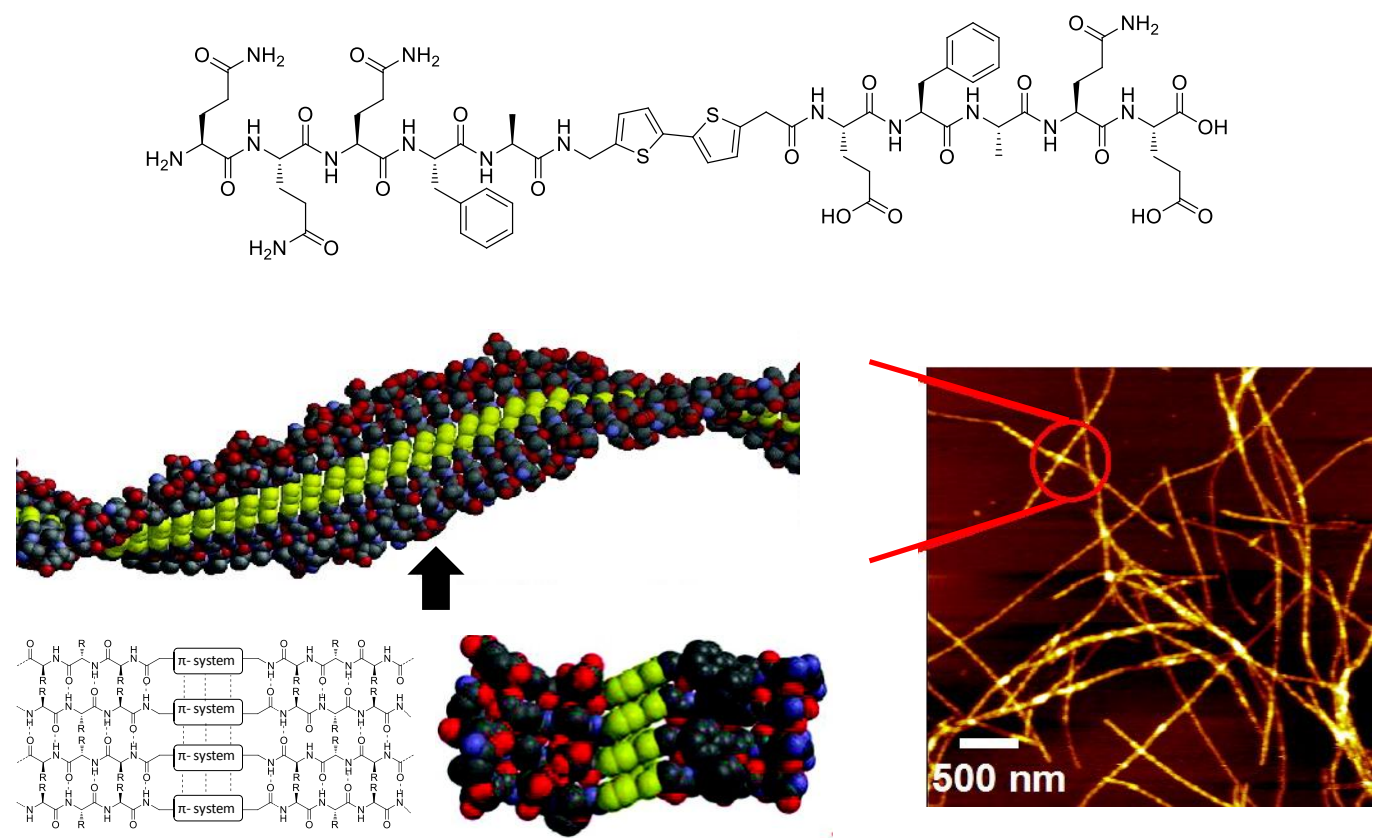

Figure 6.1.1.4. Example of 1D nanofibres formed by aggregation of $\pi$-chromophores functionalised peptide. $^{11 a}$ 
In this context, gelation has emerged as an excellent strategy with important developments in the field of soft materials with electronic properties, ${ }^{12}$ due to the ability of molecular gels to convert information at the molecular level to the nanoscopic and macroscopic levels within stable and shape-persistent materials. Gels provide precise organisation in well-defined geometries, e.g. 1D fibrillar structures, and thus are a useful tool in the fabrication of nanomaterials with strong electronic mobility features. ${ }^{13} \mathrm{~A}$ number of gels have been reported with electronic conductivity and charge-carrier mobility that may be applied in organic field-effect transistors (OFETs) and organic solar cells (OSCs). Some of the relevant features of these soft-gel materials are the tuneable sizes and shapes, optoelectronic properties and the capability of the gel matrix to accommodate various components to achieve efficient charge transport properties.

\subsubsection{Supramolecular Charge-Transfer Nanostructures}

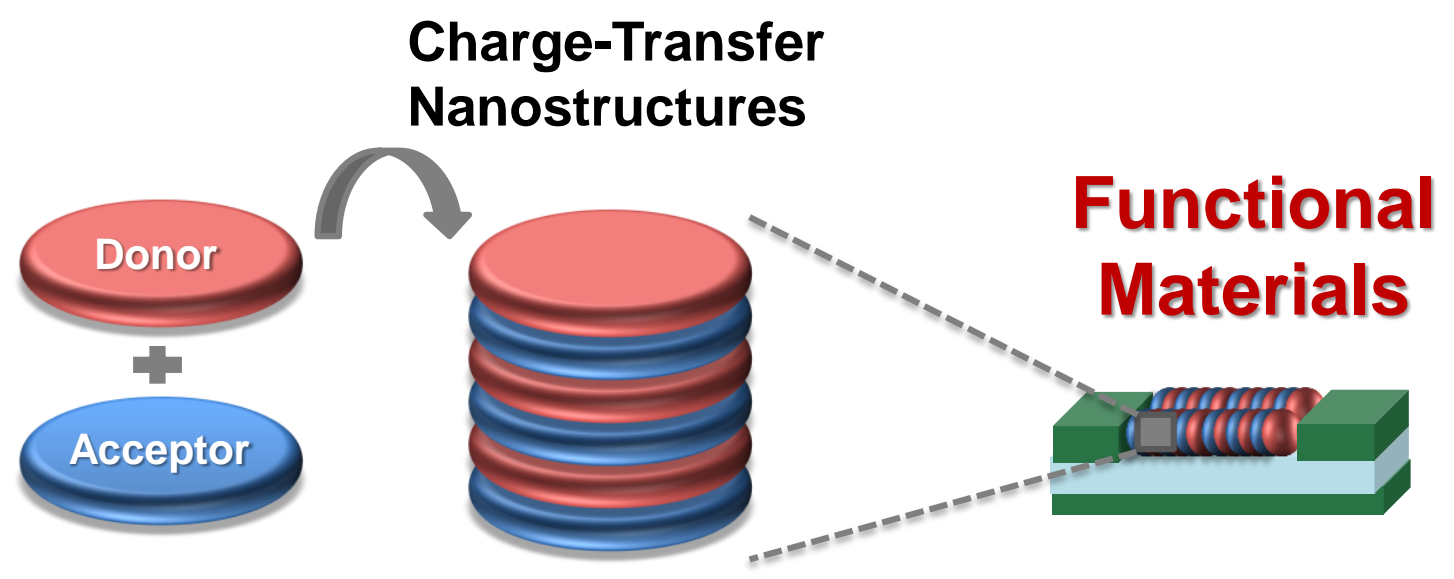

Figure 6.1.2.1. Illustration of alternated co-stacking of acceptors and donors to form charge-transfer nanostructures for being used in devices.

To obtain electroconductive properties, external doping and new molecular designs are being developed for improved electronic functionality of these supramolecular materials. The supramolecular charge-transfer strategy has gained importance in the field of electronics as it can be effectively employed to the formation of 1D nanostructures with alternate co-stacking of electron rich donors (D) and electron poor acceptors (A) in a highly ordered fashion, promoting electron mobility from the donor to the acceptor, through the nanomaterial. ${ }^{14}$ 
The first mixed stack charge-transfer (MS-CT) assemblies utilised conducting properties in charge-transfer crystals formed between tetrathiafulvalene (TTF) and tetracyanoquinodimethane (TCNQ) in 1973 (see Figure 6.1.2.2). ${ }^{15}$ In a TTF-TCNQ crystal, the base molecules are arranged independently in separate parallel-aligned stacks, where electron transfer occurs from donor (TTF) to acceptor (TCNQ) stacks. The bottom-up supramolecular approach with charge-transfer structures ${ }^{14,16}$ is the latest contribution to this field and has been used successfully as a motif for the synthesis of different aggregated structures such as polymers, ${ }^{17}$ liquid crystals ${ }^{18}$ and catenanes ${ }^{19}$ among others, in recent years.<smiles>[R]C1=C([Y])SC(=C2SC(c3ccc([R])s3)=C([R])C([R])S2)S1</smiles>

TTF
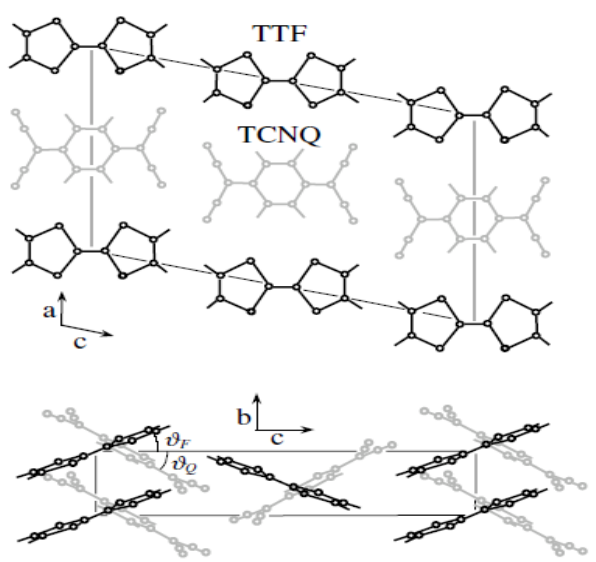

Figure 6.1.2.2. Crystal structure of TTF-TCNQ. ${ }^{15 b}$

Additional features are obtained with gel-phase materials being used as stimuliresponsive smart materials for various sensing applications. ${ }^{13 b}$ Maitra et al. published one of the first examples of gelation driven by charge-transfer in $1999 .{ }^{20}$ In their work they reported a two-component gelator system based on the co-assembly of bile acidappended aromatic donors in the presence of trinitrofluorenone (TNF) acceptor in organic solvents in a fibrillar-like network (see Figure 6.1.2.3). 

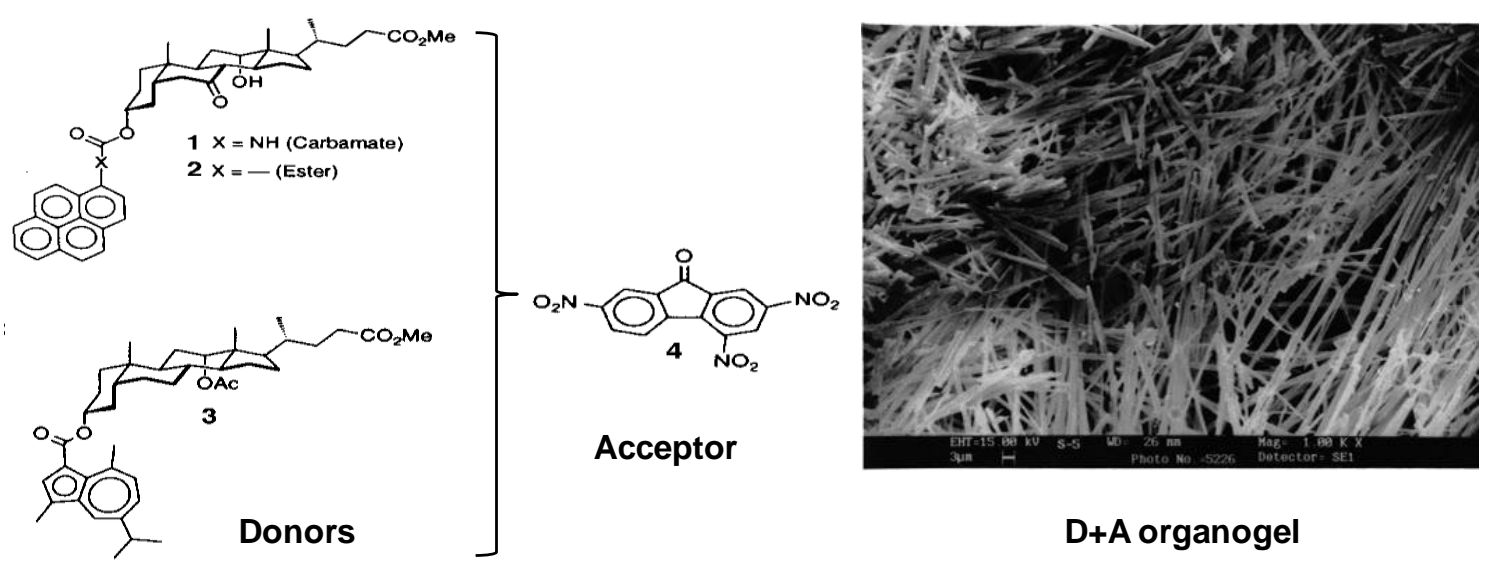

Figure 6.1.2.3. Molecular structures of the different donors with an acceptor studied with charge-transfer properties and a scanning electron microscopy image of a dried gel derived from $3 / 4$ in $n$-octanol $(3 \% \mathrm{w} / \mathrm{w})$ showing a fibrous network. ${ }^{20}$

In most cases, these charge-transfer gels have shown improved mechanical strength and lower critical gelator concentration compared to the individual components. These results highlight the key role of charge-transfer interactions in the supramolecular arrangement leading to the gel formation. ${ }^{21}$

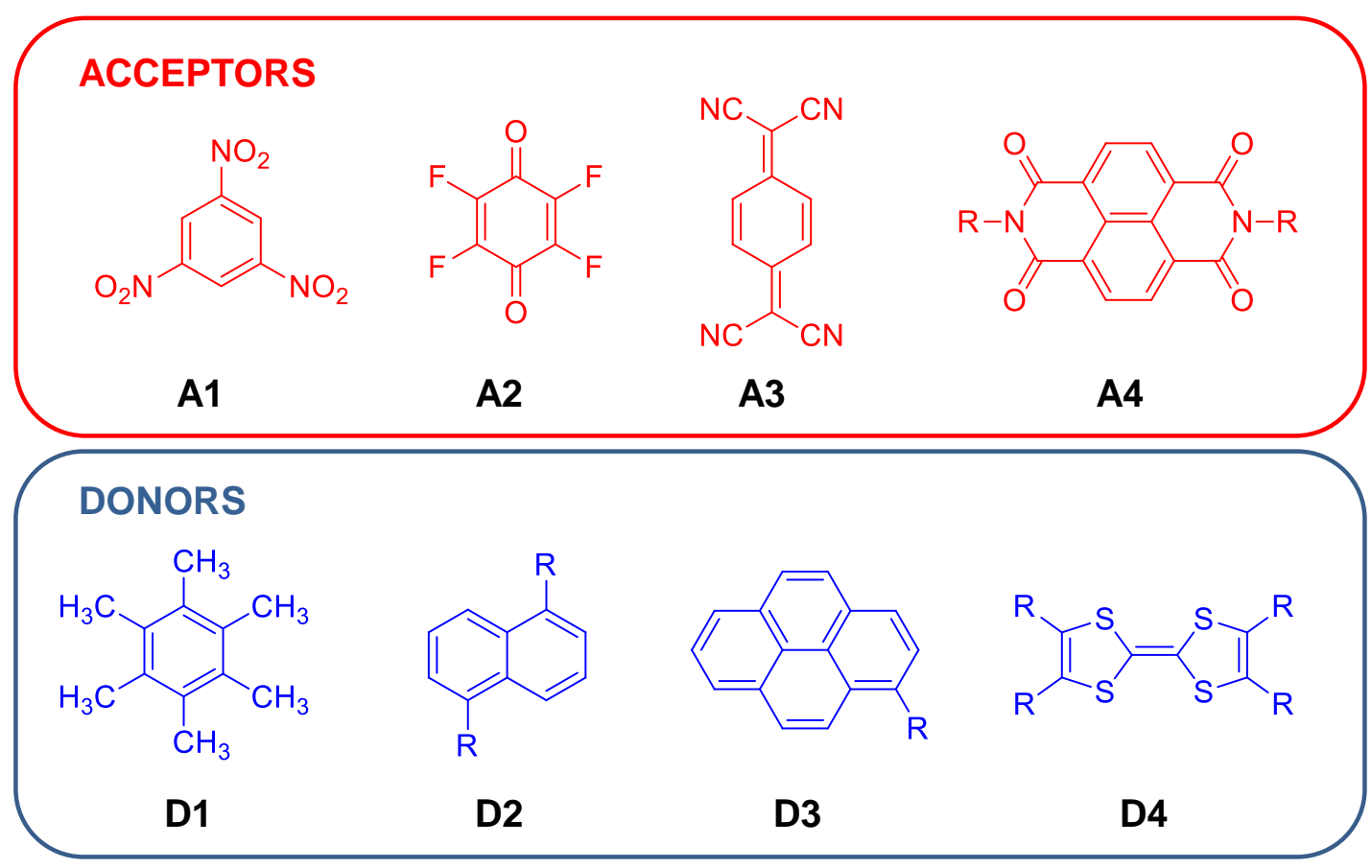

Figure 6.1.2.4. Examples of acceptors $(A 1=1,3,5$-trinitrobenzene, $A 2=$ tetrafluoro- $p$-benzoquinone, $A 3=$ 7,7,8,8-tetracyano-p-quinodimethane (TCNQ), $A 4=$ naphthalenediimide (NDI)) and donors (D1= hexamethylbenzene, D2= naphthalene derivative, D3= pyrene derivatives, D4= tetrathiafulvalene (TTF) derivatives). 
Among the candidate molecules for charge-transfer systems are naphthalenediimides (NDIs) or trinitrofluorenones (TNFs), which have been studied as acceptors. It is known that they can form effective charge-transfer complexes with polyaromatic hydrocarbons like anthracene, naphthalene or pyrene derivatives (see examples in Figure 6.1.2.4).

Naphthalenediimides (NDI) and their derivatives have been investigated intensively for organic optoelectronic materials. NDIs are a compact, electron deficient class of aromatic compounds capable of self-organisation and incorporation into larger multi-component assemblies through intercalation. ${ }^{22}$ NDIs have proved useful in the evaluation of design principles because of their facile synthesis and electron accepting properties, as well as the similarity of these properties to the naturally occurring acceptors of plant and bacterial photosynthetic reaction centres.

Recent reports have demonstrated the self-assembly of NDI-appended peptides into various nanoscales aggregates, such as $1 \mathrm{D}$ nanotubes, ${ }^{23}$ ribbons, $^{24}$ belts $^{25}$ and fibres. ${ }^{26}$ Furthermore, the use of NDIs derivatives as acceptors in charge-transfer complexes, with various electron rich donors such as pyrene ${ }^{27}$, and naphthalene derivatives, is well known. ${ }^{28}$ Figure 6.1.2.5 illustrates one such example and shows these molecular structures as useful candidates in the fabrication of self-assembling functional materials.

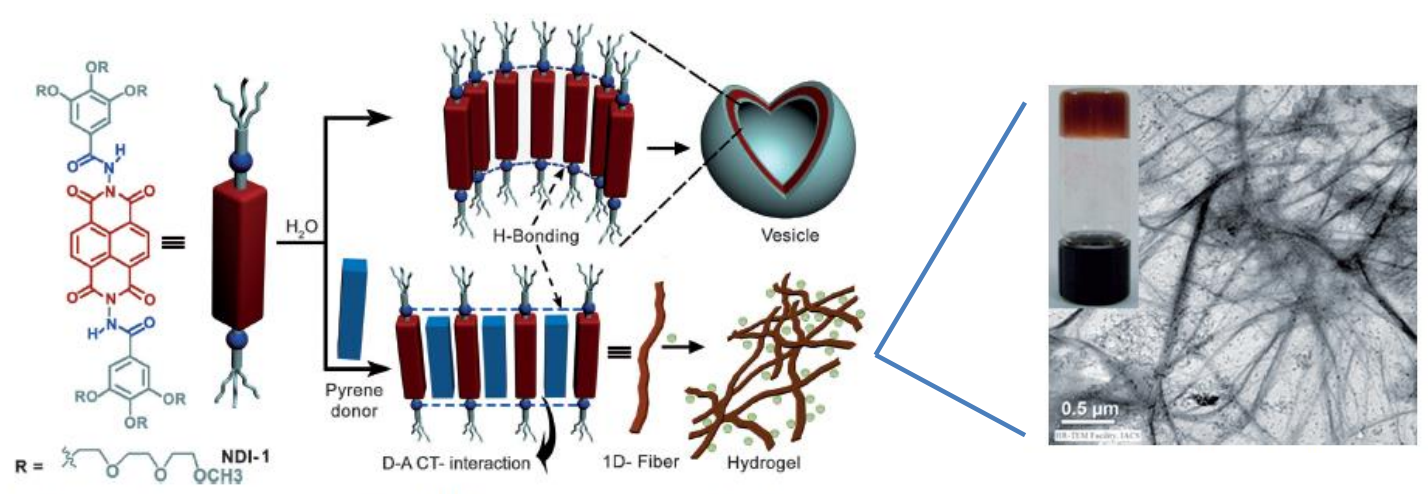

Figure 6.1.2.5. Scheme of NDI derivative self-assembly and illustrative morphological transition from vesicular aggregates of NDI alone (top) to fibres (bottom) mediated by charge-transfer interactions (2D-to1D vesicle-to-fibre) and gelation when pyrene intercalation occurs in water. TEM image showing the fibrillar gel network, the inset shows the digital photograph of the charge-transfer gel sample. ${ }^{27 a}$ 


\subsubsection{Dynamic Combinatorial Chemistry}

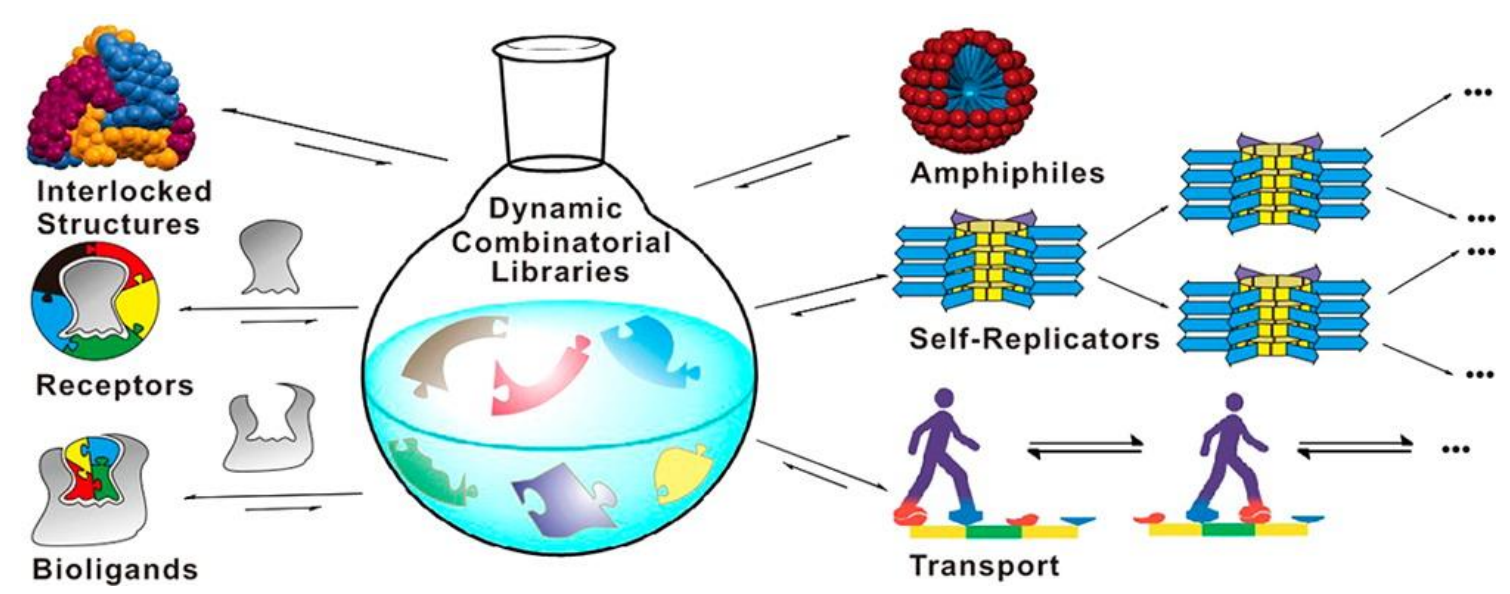

Figure 6.1.3.1. Illustrative representation with some examples where DCL are involved. ${ }^{29}$

There is an increased interest for scientists to discover design rules for molecules that self-assemble with additional functions in order to create new functional materials. For this purpose, dynamic combinatorial chemistry $(D C C)^{30}$ was developed in the mid1990s as an approach to identify individual molecules that effectively bind to each other in a reversible (dynamic) way. DCC has become a powerful technique in cases where molecular recognition events are involved. In dynamic combinatorial libraries (DCLs), the members of the library interconvert continuously by exchanging building blocks with each other through reversible bonds, either covalently or non-covalently. As a result, the product distribution is governed by thermodynamics, ${ }^{31}$ and therefore the population of an individual member is dictated by its relative stability. DCLs are responsive, meaning that the system is able to evolve under the action of external influences or stimuli. An alteration in the experimental conditions will shift the relative stabilities of the library members and thus change the library distribution towards the formation of the more stable structure (see Figure 6.1.3.2). ${ }^{32}$

In the past decade, DCLs have been used as a tool for discovering new structures with attractive properties in the functional materials field ${ }^{32}$ that would not be achieved

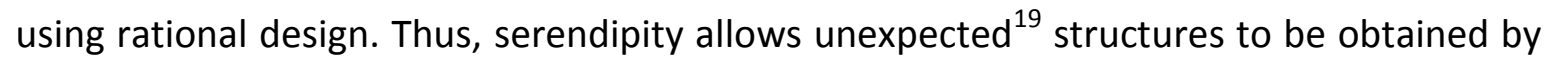
screening of a wide range of molecules simultaneously. In this respect, the DCC approach can shed some light on what structure fits best for a desired purpose. 

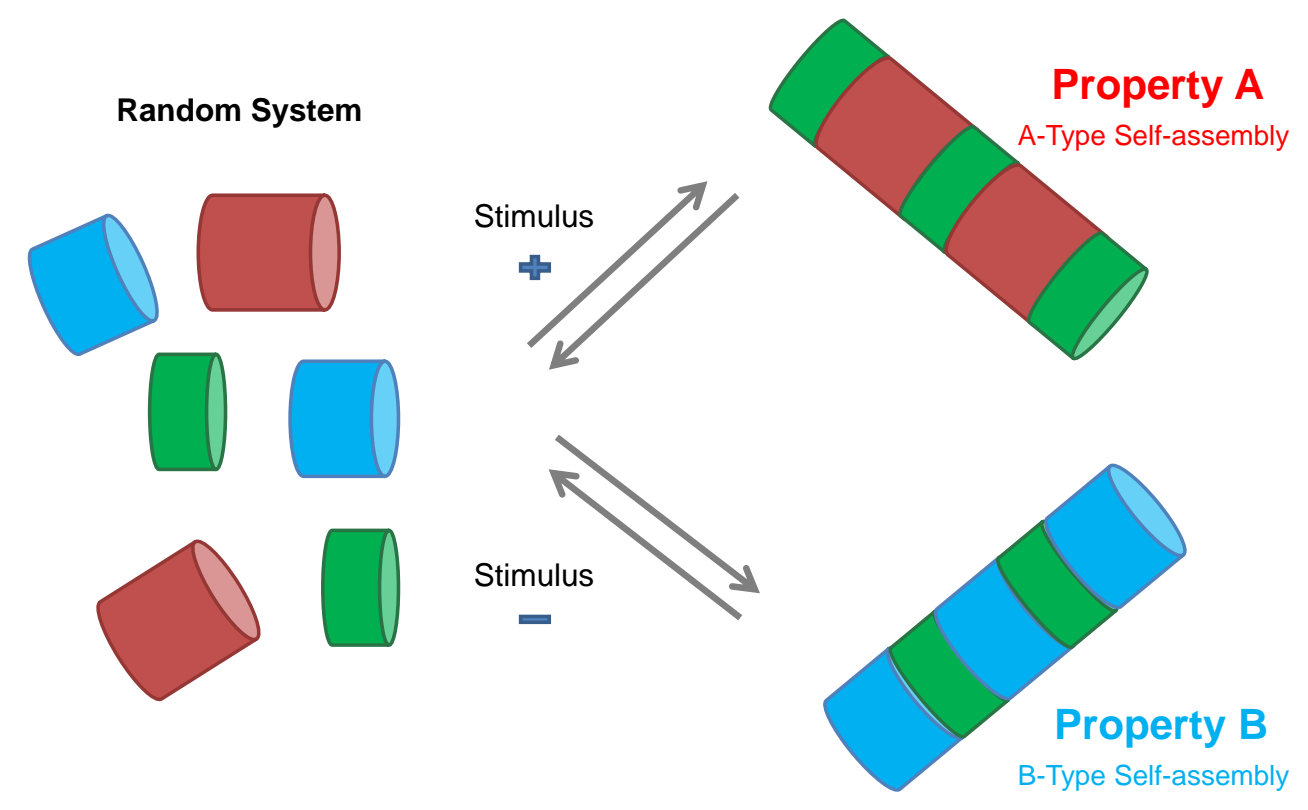

Figure 6.1.3.2. Evolution of a dynamic system under the influence of different stimuli. ${ }^{32}$

Recently, DCLs containing peptide derivatives have been reported in the study of stable supramolecular assemblies ${ }^{33}$ and their associated properties. Changing the sequence in a peptidic moiety can trigger the structure towards new properties and so the resulting functionality can be manipulated. The attraction of DCLs for peptide nanomaterial discovery is that they reduce the time and the synthetic effort required by testing all the possible combinations of amino acids. ${ }^{34}$. This approach opens up the possibility of exploiting the versatility of peptides for the discovery of new structures with new properties and applications.

\section{REVERSIBLE CHEMISTRY}

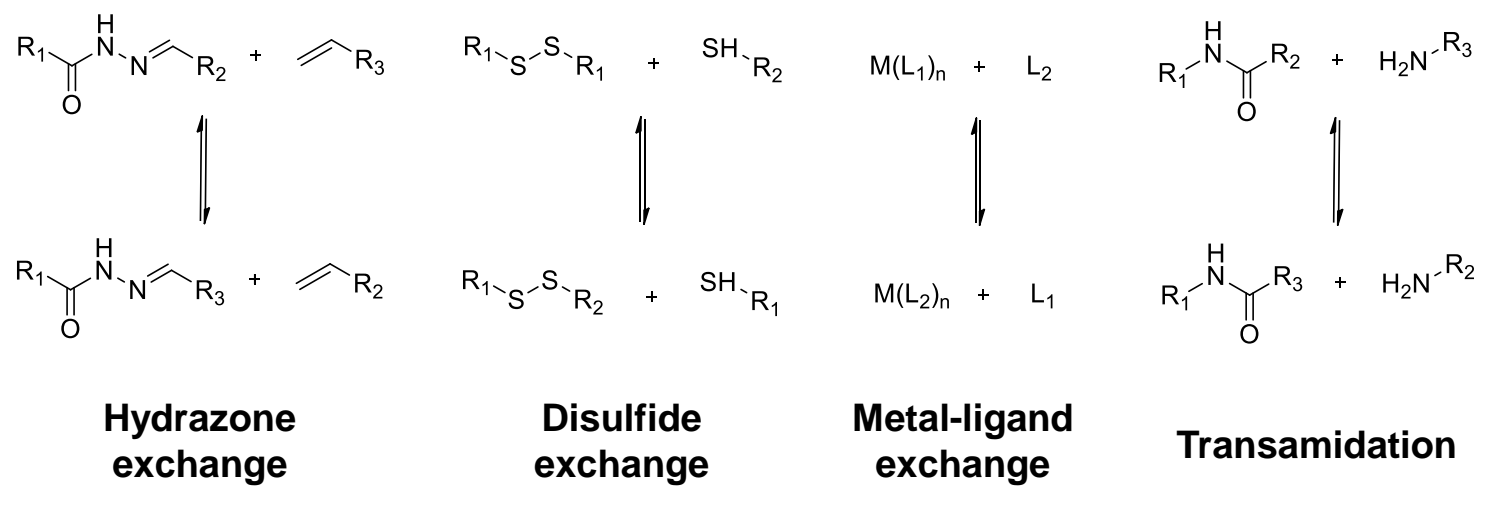

Figure 6.1.3.3. Some examples of reversible reactions that can take place in DCL of peptides. ${ }^{33}$ 
Examples of peptide libraries include those developed using hydrazone, ${ }^{35}$ disulphide, ${ }^{36}$ metal binding ${ }^{37}$ and amide exchange reactions ${ }^{34}$ among others (see Figure 6.1.3.3). In this work, a fully reversible enzymatic amide bond exchange approach of short peptides has been used, allowing the study of peptide sequence in gelation-driven DCLs.

\subsubsection{Enzyme Driven Gelation}

Self-assembly plays an important role in nature and is involved in many biomolecular processes. Nanostructures such as vesicles, micelles, microtubules or filaments are examples of these self-assembled systems. Scientists are able to synthesise small molecules that can behave in a similar way. These self-assembled structures can sometimes lead to gel formation, normally through physical or chemical stimuli. In aqueous media, another way to trigger gelation to form a hydrogel is through a biocatalytic approach, in which an enzyme catalytically converts a precursor to a hydrogelator that can self-assembles to form a hydrogel, indeed enzymatic gelation of small organic molecules has been utilised to generate different biomaterials. ${ }^{38}$ Here, the enzyme catalyses bond formation (and cleavage) and thus a self-assembling structure emerges from non-assembling precursors.

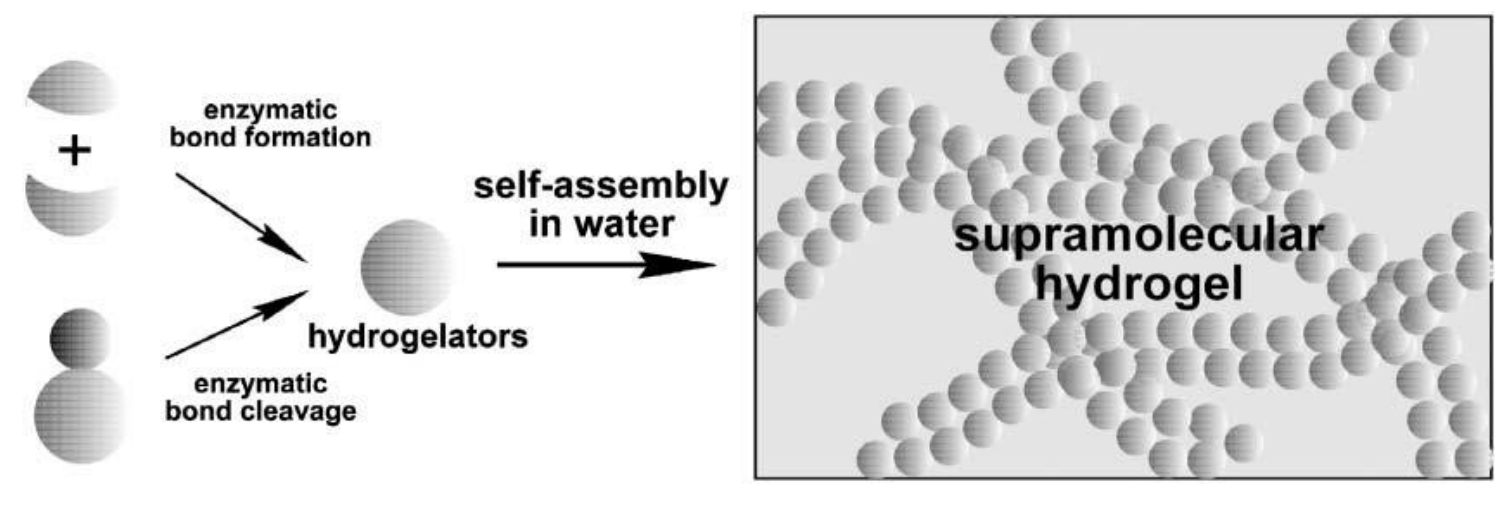

Figure 6.1.4.1. Illustrative representation of biocatalytic gel process. ${ }^{39}$

The use of enzymes as a selective stimuli to provoke the formation of supramolecular structures has a number of advantages such as mild and constant conditions, $^{40}$ as well as highly selectivity. Furthermore, the study of enzymes allows a better understanding of healthy and diseased pathways and thus is useful for the development of biomedical materials. ${ }^{41}$ In this way, these biocatalytically produced 
systems include features like spatiotemporal control of nucleation and growth, the possibility of controlling mechanical properties, defect correction and component selection, and the capabilities of a system that operates under thermodynamic control. ${ }^{42}$ A wide range of enzyme-catalysed reactions have been used for this purpose, these include strategies using protein cross-linking enzymes such transglutaminase or enzymatic production of gelators using protease, esterase or phosphatase, among others $^{43}$ (see Figure 6.1.4.2).

(A)

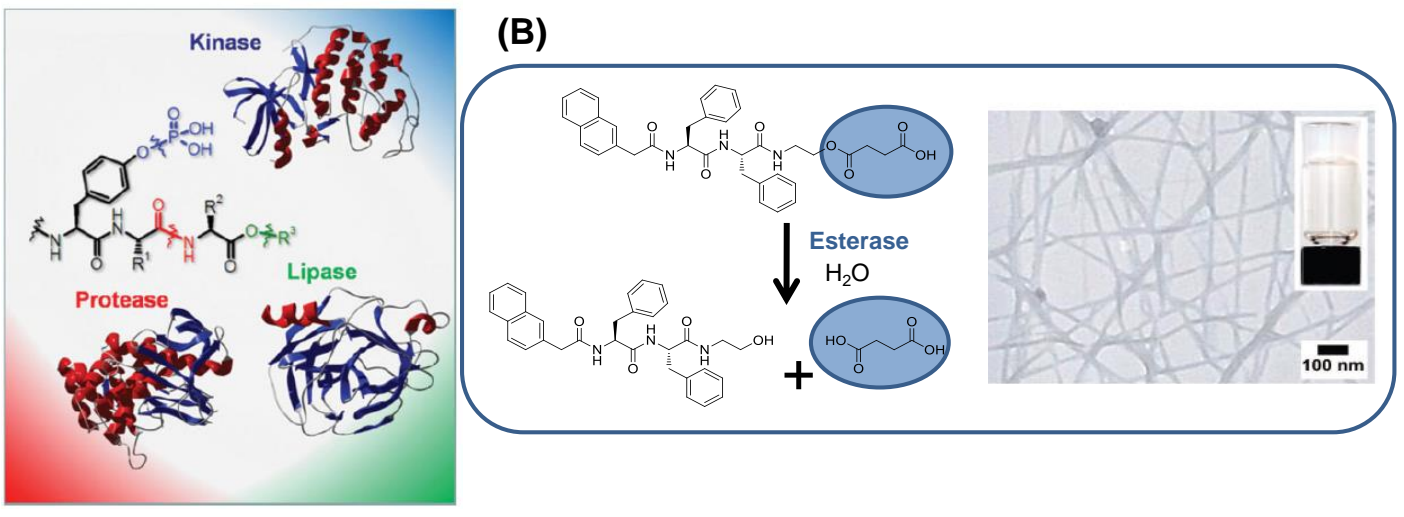

(C)

\begin{tabular}{llcl}
\hline \multicolumn{1}{c}{ Enzymes } & \multicolumn{1}{c}{ Substrate } & mgc (wt\%) & \multicolumn{1}{c}{ Application } \\
\hline alkaline phosphatase & Fmoc- $\mathrm{Y}-\mathrm{P}(\mathrm{O})(\mathrm{OH})_{2}$ & 2,0 & Biomineralization \\
acid phosphatase & Fmoc- $\mathrm{Y}-\mathrm{P}(\mathrm{O})(\mathrm{OH})_{2}$ & 0,5 & Inhibitor screening \\
3-lactamase & Nap-FF-NH $\left(\mathrm{CH}_{2}\right)_{2} \mathrm{~S}-\mathrm{ACLH}$ & 0,1 & Assay bacteria \\
thermolysine & Fmoc-FF & 0,22 & Tissue engineering \\
esterase & Nap-FF-NH $\left(\mathrm{CH}_{2}\right) \mathrm{OCO}\left(\mathrm{CH}_{2}\right) \mathrm{COOH}$ & 0,08 & Control fat cells \\
\hline
\end{tabular}

Figure 6.1.4.2. (A) Different enzymes involved in hydrogelation processes. ${ }^{42}$ (B) Example of biocatalytic gelation via esterase. ${ }^{44}$ (C) Table with examples with enzymes and its corresponding substrates with the minimal gelation concentration and the potential applications. ${ }^{38}$

Proteases, a family of enzymes well known for their ability to hydrolyse peptidic bonds in aqueous media, are used to catalyse amide condensation to produce a peptidic hydrogelator that self-assembles. The free energy change of amide bond formation/hydrolysis is relatively small and therefore the reaction may be reversed by stabilization of the peptide formation over the hydrolysis products. Organic co-solvents, ${ }^{45}$ highly concentrated substrate suspensions ${ }^{46}$ and solid-phase methods with immobilised reactants ${ }^{47}$ have been used previously to shift the reaction equilibrium from hydrolysis to synthesis. Another approach is coupling protease catalysis with molecular self-assembly, 
where self-assembly drives the shift in equilibrium to amide condensation, giving rise to a fully reversible system that operates under thermodynamic control. These highly dynamic systems have attractive features such as defect correction and self-selection of the most stable structures. Here, we take advantage of these properties to carry out DCL studies and thus, select and amplify the most thermodynamically stable structure through a biocatalytic self-assembly process in order to discover the best charge-transfer nanostructures.

\subsection{Synthesis}

\subsubsection{Synthesis of NDI-Tyr Acceptor}

The chemistry of NDI is considered simple and it can be summarised in Scheme 6.2.1.1..$^{22}$

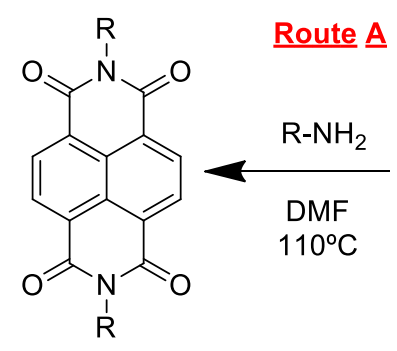<smiles>O=C1OC(=O)c2ccc3c4c(ccc1c24)C(=O)OC3=O</smiles>

Route $\underline{B}$
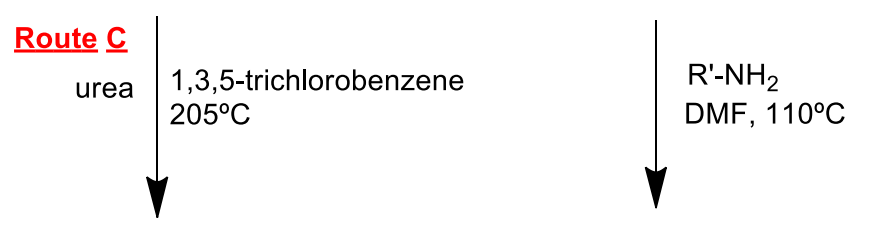<smiles>O=C1NC(=O)c2ccc3c4c(ccc1c24)C(=O)NC3=O</smiles><smiles>[R]N1C(=O)c2ccc3c4c(ccc(c24)C1=O)C(=O)N([R])C3=O</smiles>

Scheme 6.2.1.1. General synthetic procedure for preparing symmetric and $N, N^{\prime}$-disubstituted NDIs. ${ }^{22}$

The synthesis of symmetric NDI compounds is an efficient one step procedure in which commercially-available 1,4,5,8-naphthalenetetracarboxylic dianhydride NDA is 
condensed with the appropriate primary amine in a high boiling solvent, usually DMF (see Scheme 6.2.1.1). This method is clean and high yielding for a variety of amines.

Naphthalenediimides bearing two different substituents on the diimide nitrogens $\left(N, N^{\prime}\right.$-disubstituted NDIs) can be prepared by a method developed by Ghadiri which requires careful control of $\mathrm{pH}$ (Scheme 6.2.1.1. Route B). The difference in reactivity of anhydride and dicarboxylates is more satisfactory than application of a statistical approach. ${ }^{48}$ Thus after complete hydrolysis of a suspension of the dianhydride NDA with $\mathrm{KOH}$, re-acidification to $\mathrm{pH} 6.2$ with $\mathrm{H}_{3} \mathrm{PO}_{4}$ leads to the formation of naphthalenetetracarboxylic acid monoanhydride. Addition of one equivalent of a primary amine to the same reaction vessel, followed by overnight heating at reflux, yields naphthalene monoimides in good isolated yield and purity by a simple filtration workup. It is found that the dicarboxylic acid moieties could be directly converted into imides by condensation with primary amines in DMF at temperatures above $110 \stackrel{\circ}{\circ}$ without the need to isolate the corresponding anhydrides first.

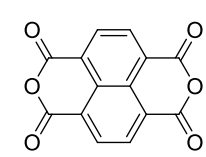

NDA

(6a.1)

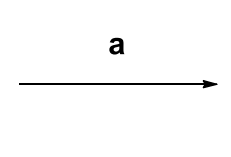

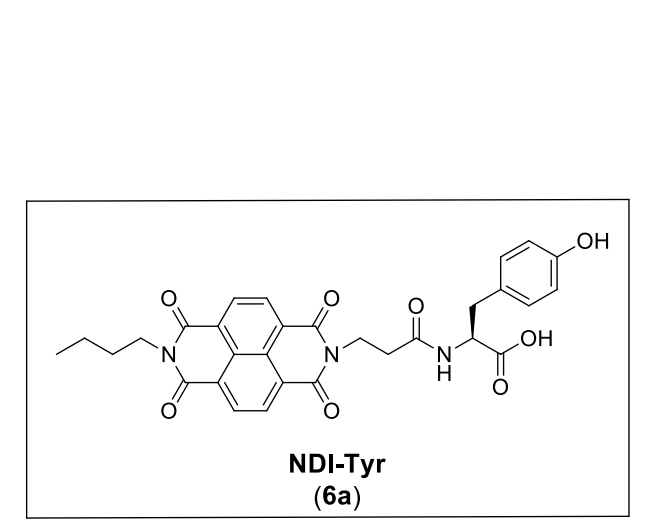

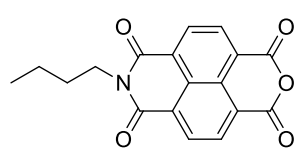

n-Bu-NDI

$(6 a .2)$

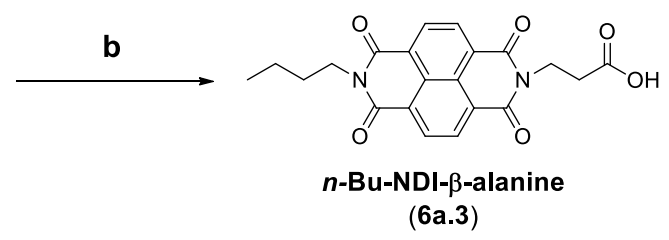

(6a.3)

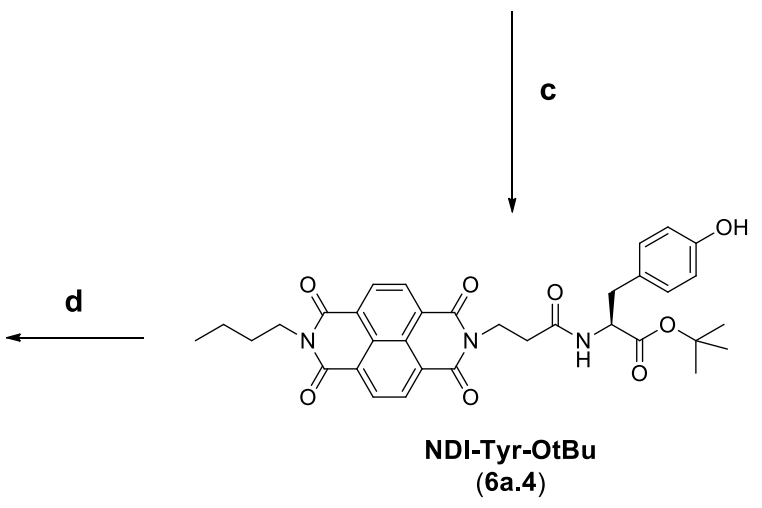

Scheme 6.2.1.2. Synthesis of NDI-Tyr (6a). a) KOH, water, $n$-butyl amine, reflux (18 h)(73\%); b) $\beta$-alanine, DMF, DIPEA, reflux (18 h), (78\%); c) L-tyrosine-tert-butyl ester, HBTU, DMF, DIPEA, rt (18 h)(56\%); d) TFA, rt $(18 \mathrm{~h})(85 \%)$.

Following this procedure, NDI tyrosine appended derivative NDI-Tyr (6a) has been synthesised. In order to reach the desired compound, 1,4,5,8-naphthalenetetracarboxylic acid dianhydride NDA (6a.1) was firstly hydrolysed with $\mathrm{KOH}$. Then $n$-butyl amine was 
added once the solution was acidified to $\mathrm{pH} \mathrm{6.2,} \mathrm{the} \mathrm{mixture} \mathrm{was} \mathrm{left} \mathrm{overnight,} \mathrm{heating}$ at reflux to give the monoimide $\boldsymbol{n}$-Bu-NDI (6a.2). In the next step, a direct condensation with $\beta$-alanine in DMF using DIPEA was carried out heating at reflux overnight to give the naphthalenediimide $\boldsymbol{n}$-Bu-NDI- $\boldsymbol{\beta}$-alanine (6a.3). To continue, L-tyrosine-tert-butyl ester was coupled in a DMF solution with DIPEA and HTBU as coupling agent to then remove the protecting tert-butyl group with TFA to yield the NDI-Tyr (6a) compound as is shown in Scheme 6.2.1.2.

\subsubsection{Synthesis of Dialkoxy Naphthalene Donors}

The two alkoxy isomers of naphthalene derivative 1,5-DAN and 2,6-DAN were synthesised following the same procedure. Starting from a dihydroxy napthalene solution in acetone, ethyl bromobutyrate was added in the presence of $\mathrm{K}_{2} \mathrm{CO}_{3}$. The mixture was heated to reflux for 8 hours to obtain the dialkoxy ester derivative. Then the dialkoxy napththalene (DAN) was obtained after dissolution of the ester in THF and a $\mathrm{NaOH}$ solution for 8 additional hours (See Scheme 6.2.2.1).

A)<smiles>Oc1cccc2c(O)cccc12</smiles>

$1,5-\mathrm{DHN}$

B)

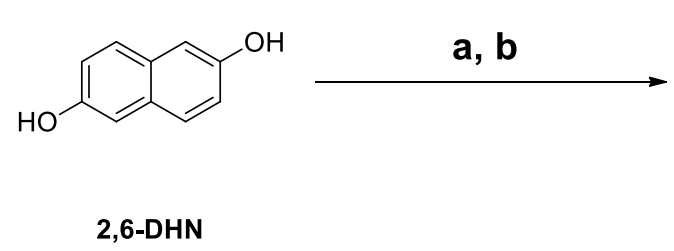<smiles>N#CCOc1cccc2c(OCCCC(=O)O)cccc12</smiles>

1,5-DAN<smiles>O=C(O)CCCOc1ccc2cc(OCCCC(=O)O)ccc2c1</smiles>

2,6-DAN

Scheme 6.2.2.1. Synthetic route for 1,5-DAN (90\%) and 2,6-DAN (95\%) a) ethyl bromobutyrate, $\mathrm{K}_{2} \mathrm{CO}_{3}$, acetone, reflux ( $8 \mathrm{~h}), \mathrm{b})$ aq $\mathrm{NaOH}, \mathrm{THF} /$ water, $8 \mathrm{~h}$. 


\subsection{Biocatalytic Gelation-Driven Dynamic Combinatorial Library as a Tool to Discover New Charge-Transfer Systems}

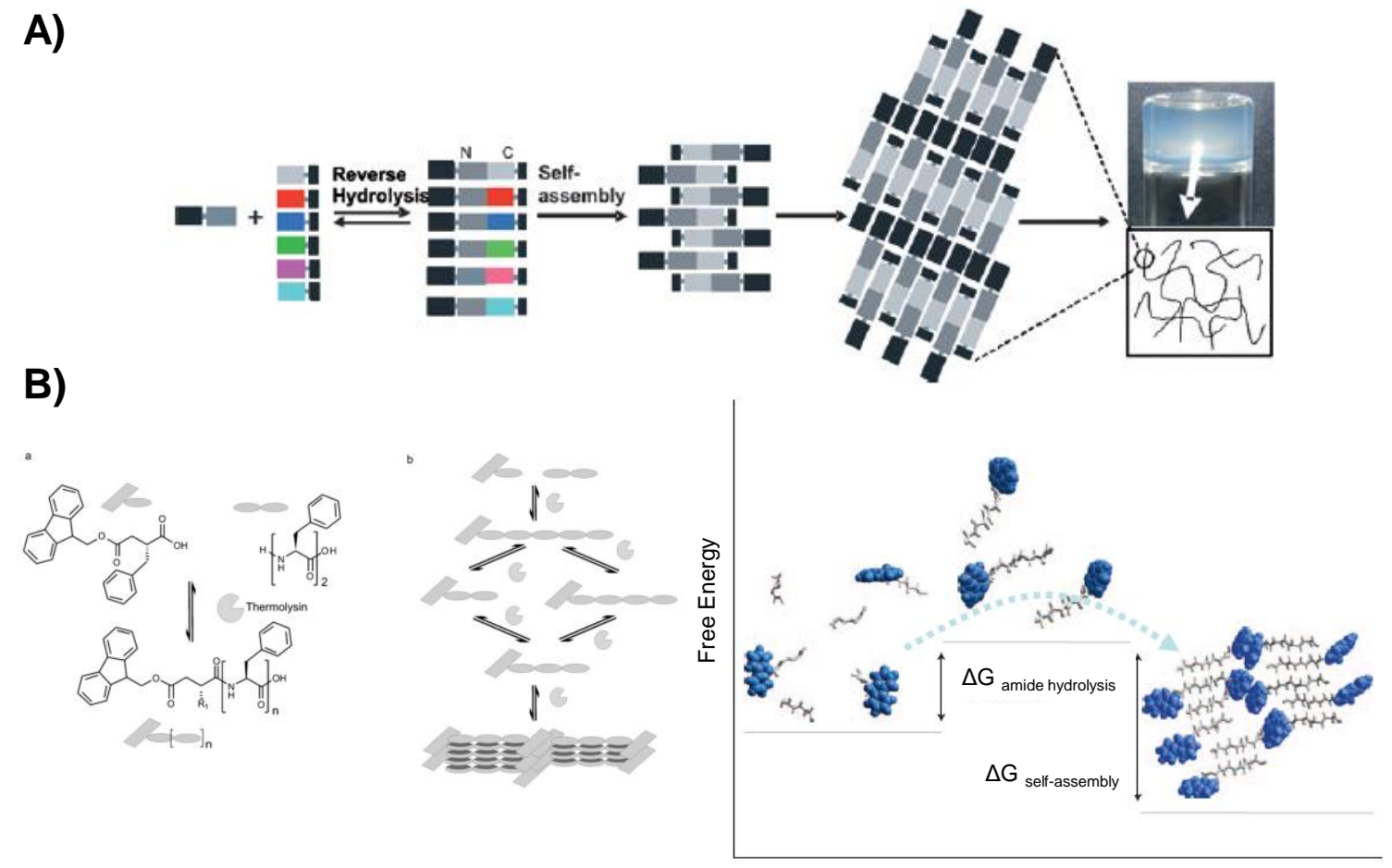

Figure 6.3.1. Examples of biocatalytic self-assembly DCLs using amide condensation reaction. ${ }^{31 a, 34}$

Peptide-based DCLs have been used as a powerful tool to find structural materials, however its use to discover functionalised devices has not yet been widely explored in the literature. Some examples include studies by Ulijn et al. (see Figure 6.3.1), ${ }^{31 a, 34}$ where an enzymatic gelation-driven approach is employed. In the case of biocatalytic assembly, supramolecular aggregation is the driving force to shift the enzyme action toward the amide condensation in a reversible and thus dynamic way, generating the hydrogelator structure that predominates in the final mixture. Consequently, the most thermodynamically stable member is selected and amplified among all the library components.

Following the previous work, where biocatalytic DCL methodology is used to discover the electronically functionalised napththalene dipeptide tyrosine hydrogelator (Nap-YX-NH $\mathbf{N H}_{\mathbf{2}}$ ) (Figure 6.3.2), it was found that the napthathalene derivative can produce energy transfer interactions when a water-soluble dansyl- $\beta$-alanine (DA) compound is 
introduced in the system as an acceptor. ${ }^{49}$ Moreover, the selection and amplification of the most effective energy transfer nanostructure from a mixture of peptide derivatives in aqueous medium, was demonstrated. In the current work, the 1,4,5,8napthalenetetracarboxylic acid diimide tyrosine appended conjugate derivative (NDI-Tyr (6a)) has been chosen as a precursor (Figure 6.3.3) to generate a new biocatalytic gelation-driven DCL with potential electronic properties. It is known that the NDI molecule is a $\pi$-chromophore, which can self-assemble in a face-to-face packing mode, giving electron deficient systems. These kinds of compounds are widely used as n-type semiconductors and as acceptors in charge-transfer complexes.

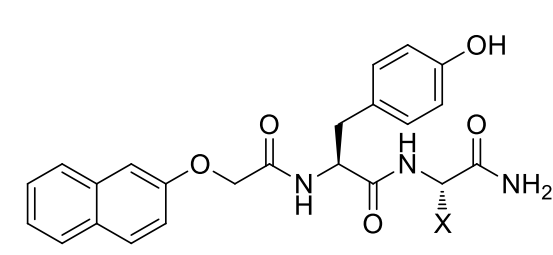

Nap-YX-NH

D

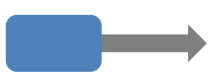

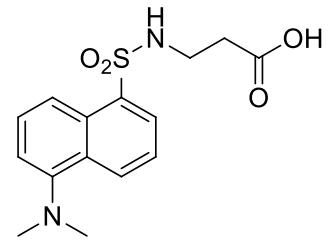

DA (Dansyl- $\beta$-alanine)

A

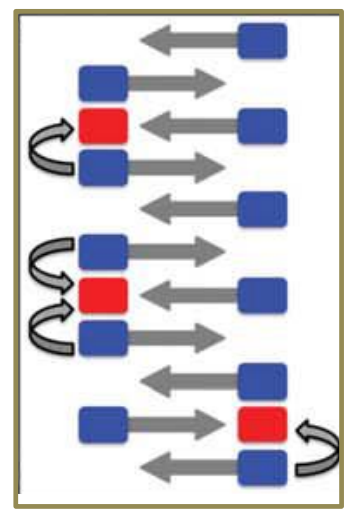

ET

Figure 6.3.2. Molecular structures and schematic self-assembly representation used in the energy-transfer (ET) DCL study ( $\mathrm{Y}=$ Tyrosine, $\mathrm{X}=$ different amino acid nucleophiles). ${ }^{49}$

To produce the library, the NDI-Tyr (6a) derivative was added to a collection of

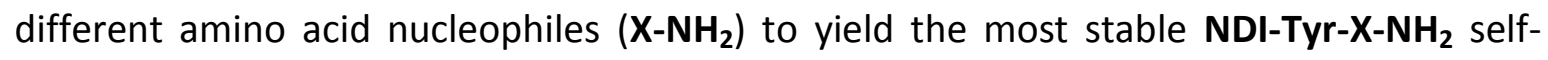
assembled acceptor hydrogel structure under thermodynamic control and mild conditions, after the addition of the enzyme via amide condensation. The non-specific endoprotease thermolysin from Bacillus thermoproteolyticus rokko ${ }^{31 a}$ was employed to catalyse the reaction between the NDI-Tyr (6a) conjugate and the different amidated amino acids derivatives $\left(\mathbf{X}-\mathbf{N H}_{\mathbf{2}}\right.$ ) as nucleophiles (Figure 6.3.3). In order to check how the hydrophilic/hydrophobic balance could influence the enzyme action, different amino acids were used: tyrosine (Tyr), phenylalanine (Phe), leucine (Leu), valine (Val), alanine (Ala) and glycine (Gly). 


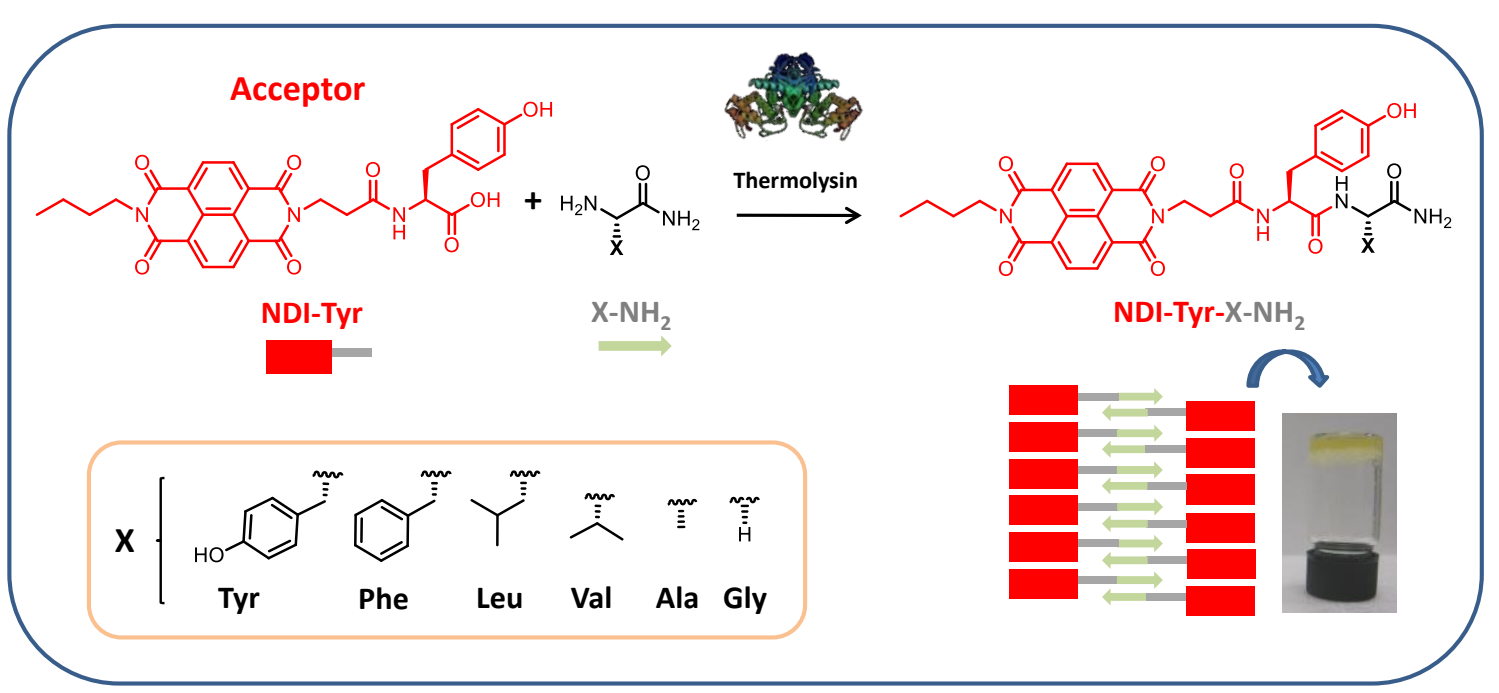

Figure 6.3.3. Thermolysin-catalysed condensation reaction between $\mathbf{N D I - T y r}(6 \mathrm{6})$ and $\mathbf{X}-\mathbf{N H}_{2}$ to form NDITyr-X- $\mathbf{N H}_{\mathbf{2}}$ bioconjugates and schematic representation showing the resulting aggregated hydrogelator system.<smiles>CCCN1C(=O)c2ccc3c4c(ccc(c24)C1=O)C(=O)N(CCC(=O)NC(Cc1ccc(O)cc1)C(=O)N[C@@H](Cc1ccc(O)cc1)C(N)=O)C3=O</smiles>

NDI-Tyr-Tyr-NH ${ }_{2}$ (6b)<smiles>CCCN1C(=O)c2ccc3c4c(ccc(c24)C1=O)C(=O)N(CCC(=O)NC(Cc1ccc(O)cc1)C(=O)N[C@H](CC(C)C)C(N)=O)C3=O</smiles>

NDI-Tyr-Leu-NH ${ }_{2}(6 \mathrm{~d})$

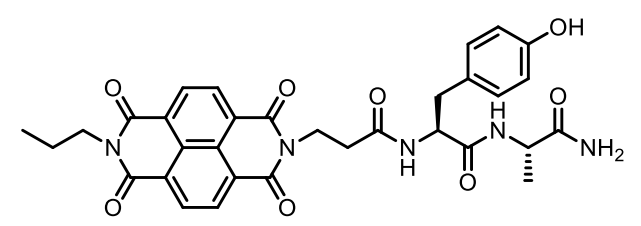

NDI-Tyr-Ala-NH $\mathrm{N}_{2}(6 f)$<smiles>CCCN1C(=O)c2ccc3c4c(ccc(c24)C1=O)C(=O)N(CCC(=O)NC(Cc1ccc(O)cc1)C(=O)N[C@H](Cc1ccccc1)C(N)=O)C3=O</smiles>

NDI-Tyr-Phe- $\mathrm{NH}_{2}(6 \mathrm{c})$<smiles>CCCN1C(=O)c2ccc3c4c(ccc(c24)C1=O)C(=O)N(CCC(=O)NC(Cc1ccc(O)cc1)C(=O)N[C@@H](C(N)=O)C(C)C)C3=O</smiles>

NDI-Tyr-Val-NH ${ }_{2}(6 e)$<smiles>CCCN1C(=O)c2ccc3c4c(ccc(c24)C1=O)C(=O)N(CCC(=O)NC(Cc1ccc(O)cc1)C(=O)N[C@@H](C)C(N)=O)C3=O</smiles>

NDI-Tyr-Gly-NH ${ }_{2}(6 \mathrm{~g})$

Figure 6.3.4. Molecular structures of the NDI-Tyr-X-NH acceptors formed inside the DCL system.

It was found that the NDI-Tyr (6a) precursor forms a strong yellow-orange gel at $10 \mathrm{mM}$ in phosphate buffer $(100 \mathrm{mM})$ at pH 8 within 2 minutes, with the gel being stable for several months. Transmission electron microscopy (TEM) images of the hydrogel 
revealed formation of twisted nanofibre structures with length in the micrometre scale (Figure 6.3.5).

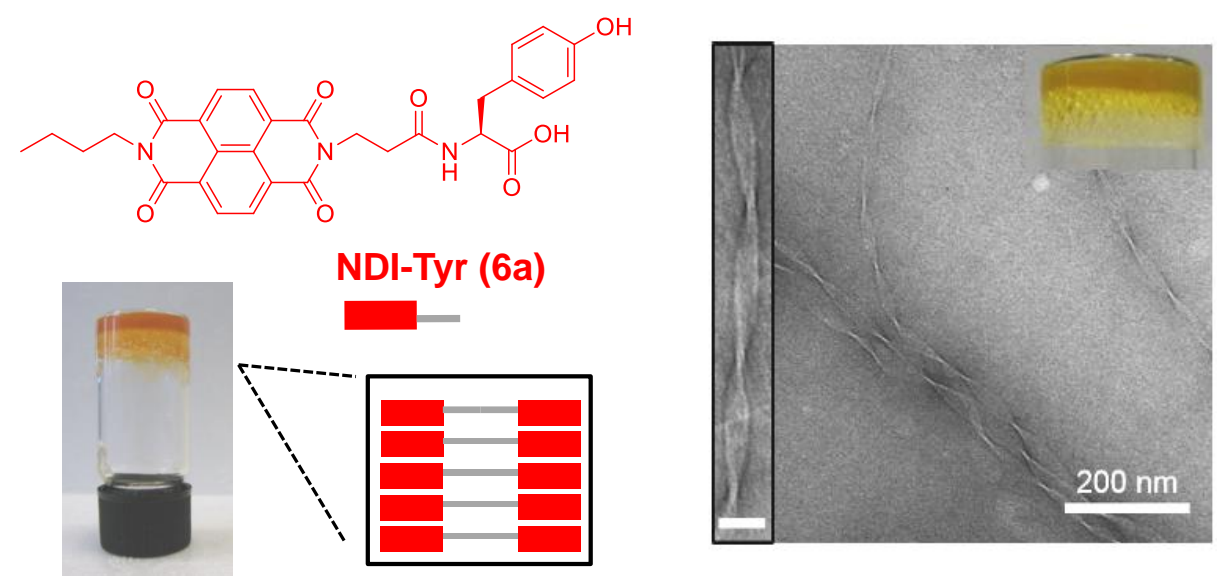

Figure 6.3.5. NDI-Tyr (6a) molecular structure and a photography of a gel sample $10 \mathrm{mM}$ in buffer and TEM image of the fibres. Inset: the magnified image of twisted nanofibres (scale bar: $40 \mathrm{~nm}$ ).

Before studying the library system, the individual formation of the different NDITyr-X-NH $\mathbf{N H}_{\mathbf{2}}$ product from each possible was studied in isolation. The dynamics of the enzymatic reaction were analysed using high performance liquid chromatography (HPLC). Time-dependent results (Figure 6.3.6) after the addition of thermolysin $\left(1 \mathrm{mg} \cdot \mathrm{mL}^{-1}\right)$ to the mixture of NDI-Tyr (6a) (10 mM) and two fold excess of $\mathbf{X}-\mathbf{N H}_{\mathbf{2}}$ showed some moderate conversions for the conjugates NDI-Tyr-Phe-NH $\mathbf{N H}_{2}$ (6c) (65\%) and NDI-Tyr-Leu-NH $\mathbf{2}$ (6d) $(45 \%)$, but others like tyrosine (6b), alanine (6f) or glycine $(\mathbf{6 g})$ derivatives showed very low conversions, 6,4 and $0.1 \%$ respectively, after 48 hours. ${ }^{50}$ This indicates that the enzymatic self-assembly of NDI-Tyr-Phe-NH $\mathbf{N H}_{2} \quad(6 \mathrm{c})$ and NDI-Tyr-Leu-NH $\mathbf{N H}_{2} \quad(\mathbf{6 d})$ is thermodynamically favoured. Conversely, the rest $(\mathbf{6 b}, \mathbf{6 f}$ and $\mathbf{6 g}$ ) could not be formed in an enzymatic way, where the weak self-assembling properties cannot overcome the bias for amide hydrolysis.

It is interesting to note that although NDI-Tyr-X-NH $\mathbf{N H}_{\mathbf{2}}$ structures are difficult to obtain using normal synthesis in solution, due to their low solubility in common solvents, the NDI-Tyr-Phe- $\mathbf{N H}_{\mathbf{2}}(\mathbf{6 c})$ derivative was synthesised and purified successfully. Its gelation behaviour was then tested and it was found that the compound quickly formed a precipitate at room temperature after heating the solution. This highlights the importance of the biocatalytically self-assembled compounds that cannot be formed 
otherwise through common procedures, due to its low solubility. On the contrary, the precursors are soluble enough to react with the enzyme and form the hydrogelator compound (Figure SI_6.6.1).

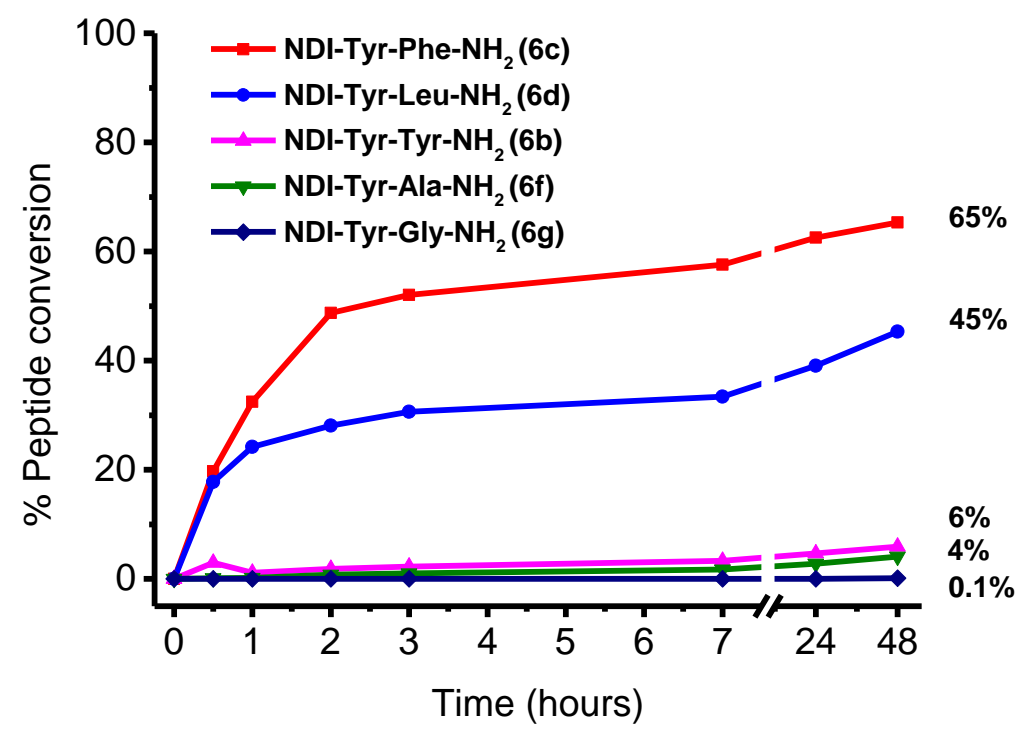

Figure 6.3.6. Time-dependant HPLC percentage of peptide conversion from each combination NDI-Tyr (6a) $+\mathrm{X}-\mathrm{NH}_{2}$ in isolation.

Remarkably, a self-supporting yellow-orange hydrogel was obtained in all the cases after enzyme addition (Figure SI_6.6.2). However, slight colour changes were observed, implying alterations in chromophore organization and in the NDI-Tyr-X-NH $\mathbf{N}_{\mathbf{2}}$ conversion.

Having established that each of the peptide candidates could be formed using thermolysin, the dynamic combinatorial library experiment was carried out. Here a peptide library of all six amino acid nucleophiles $\left(\mathbf{X}-\mathbf{N H}_{\mathbf{2}}\right.$ where $\mathbf{X}=\mathbf{T} \mathbf{y r}$, Phe, Leu, Val, Ala and Gly) were added, each in 2-fold excess (20 mM), to the NDI-Tyr (6a) precursor (10 $\mathrm{mM}$ ) in the presence of the enzyme, together in one-pot and thereby allowing the enzyme-catalysed condensation reaction to proceed in competition. Time-dependent species distribution is shown in Figure 6.3.7. After 48 hours it was found that the NDI-TyrPhe- $\mathrm{NH}_{2}(6 \mathrm{c})$ derivative was formed preferentially in a good yield of $48 \%$, corresponding

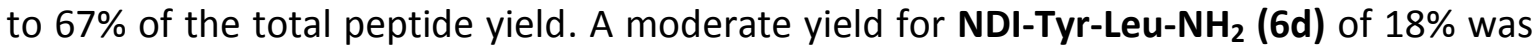
obtained, while NDI-Tyr-Tyr-NH $\mathbf{N H}_{2}$ (6b), NDI-Tyr-Ala-NH $\mathbf{N H}_{\mathbf{2}}$ (6f) and NDI-Tyr-Gly- $\mathbf{N H}_{\mathbf{2}}$ (6g) were formed with a poor conversion (less than $1 \%$ ). In this competition DCL experiment, 
NDI-Tyr-Phe- $\mathbf{N H}_{\mathbf{2}}(\mathbf{6 c})$ was identified as the most stable structure, representing the lowest state of the free energy landscape. It was also demonstrated that the self-assembly of this structure is the more favourable as it is able to shift the enzyme action to the reverse hydrolysis.

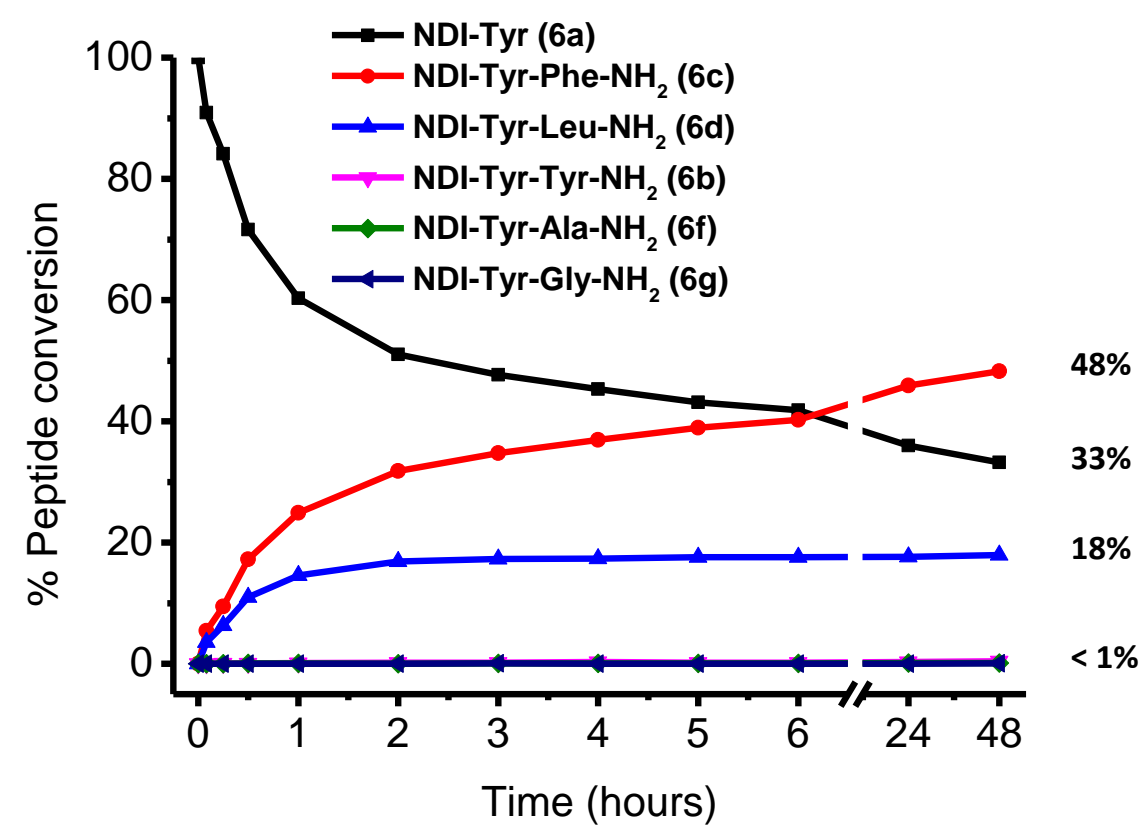

Figure 6.3.7. Time-dependent HPLC graph of the system DCL without donor.

The DCL system forms an orange gel less than $1 \mathrm{~h}$ after the enzyme addition. $\mathrm{A}$ colour change to a yellowish gel is observed after $24 \mathrm{~h}$, indicating a change in the organization of the aggregated structures and the formation of new molecules (NDI-Tyr$\mathbf{X}-\mathrm{NH}_{2}$ ). The system was stable for several days (see Figure SI_6.6.4 for colour changes).

The next step in this study was to test the electronic properties of the new acceptor structures formed inside the library system. Different $\pi$-electron rich donors: dialkoxy naphthalenes (1,5-DAN and 2,6-DAN), dihydroxy naphthalenes (1,5-DHN and 2,6-DHN), 1-pyrene acetic acid (1-PAA) and 1,5-diamino naphthalene (1,5-DAmN) were chosen to form effective charge-transfer complexes with the NDI molecule (Figure 6.3.8). 
(a)

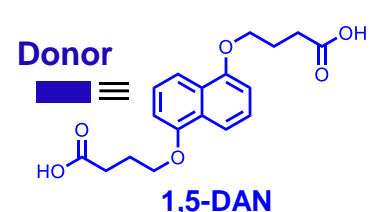<smiles>O=C(O)CCOc1ccc2cc(OCCC(=O)O)ccc2c1</smiles>

2,6-DAN<smiles>Oc1cccc2ccccc12</smiles>

1,5-DHN<smiles>Oc1ccc2cc(O)ccc2c1</smiles>

2,6-DHN

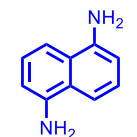

1,5-DAmN

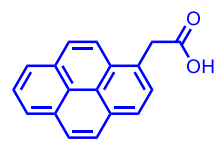

1-PAA (b)

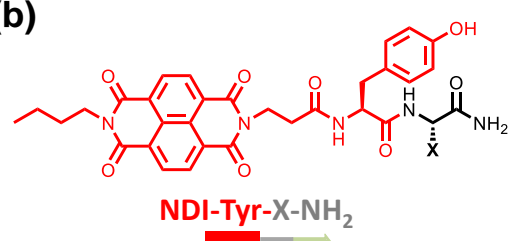

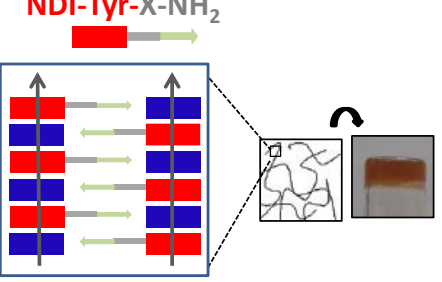

Figure 6.3.8. a) Molecular structure of the different donors studied. b) Schematic representation of D-A coassembly showing charge-transfer properties.

In all the cases a broad-band, typical of charge-transfer complex formation, was observed between 400 and $700 \mathrm{~nm}^{27 a, 51}$ in the UV-vis absorption spectra (Figure 6.3.9.a) in diluted samples of NDI-Tyr (6a) in the presence of each donor..$^{52}$ Additionally, a change in colour of these NDI-Tyr(6a)/donor diluted samples (Figure 6.3.9.b) were obtained with a variety of colours ranging from yellow-orange to green or plum, suggesting the presence of donor-acceptor interactions between NDI and the corresponding donor ring.

a)

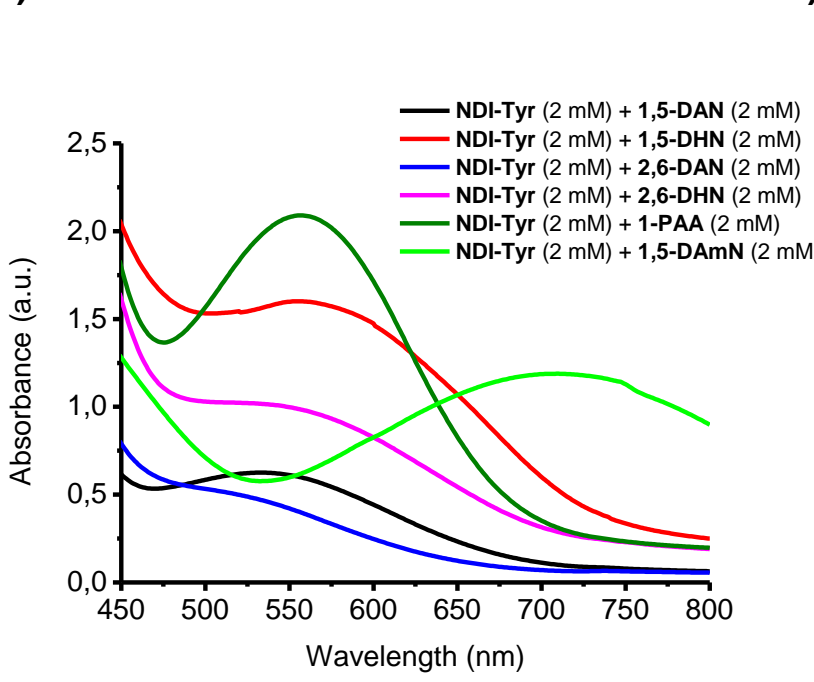

b)

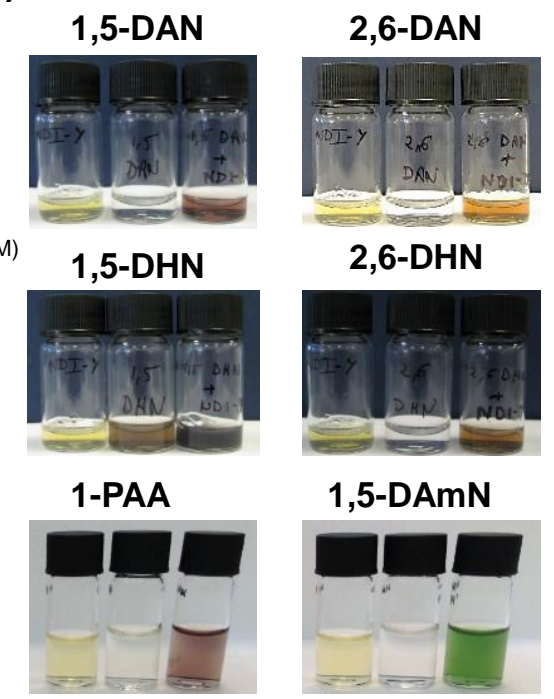

Figure 6.3.9. (a)UV-vis absorption spectra of the charge-transfer complex between NDI acceptors and various donors (2 $\mathrm{mM}$ each). (b) Digital photographs of the corresponding solution samples showing change in colour due to charge-transfer complex formation. The vials represent the solutions of the NDI-Tyr sample, the corresponding donor and the corresponding mixed charge-transfer donor-acceptor solution. 

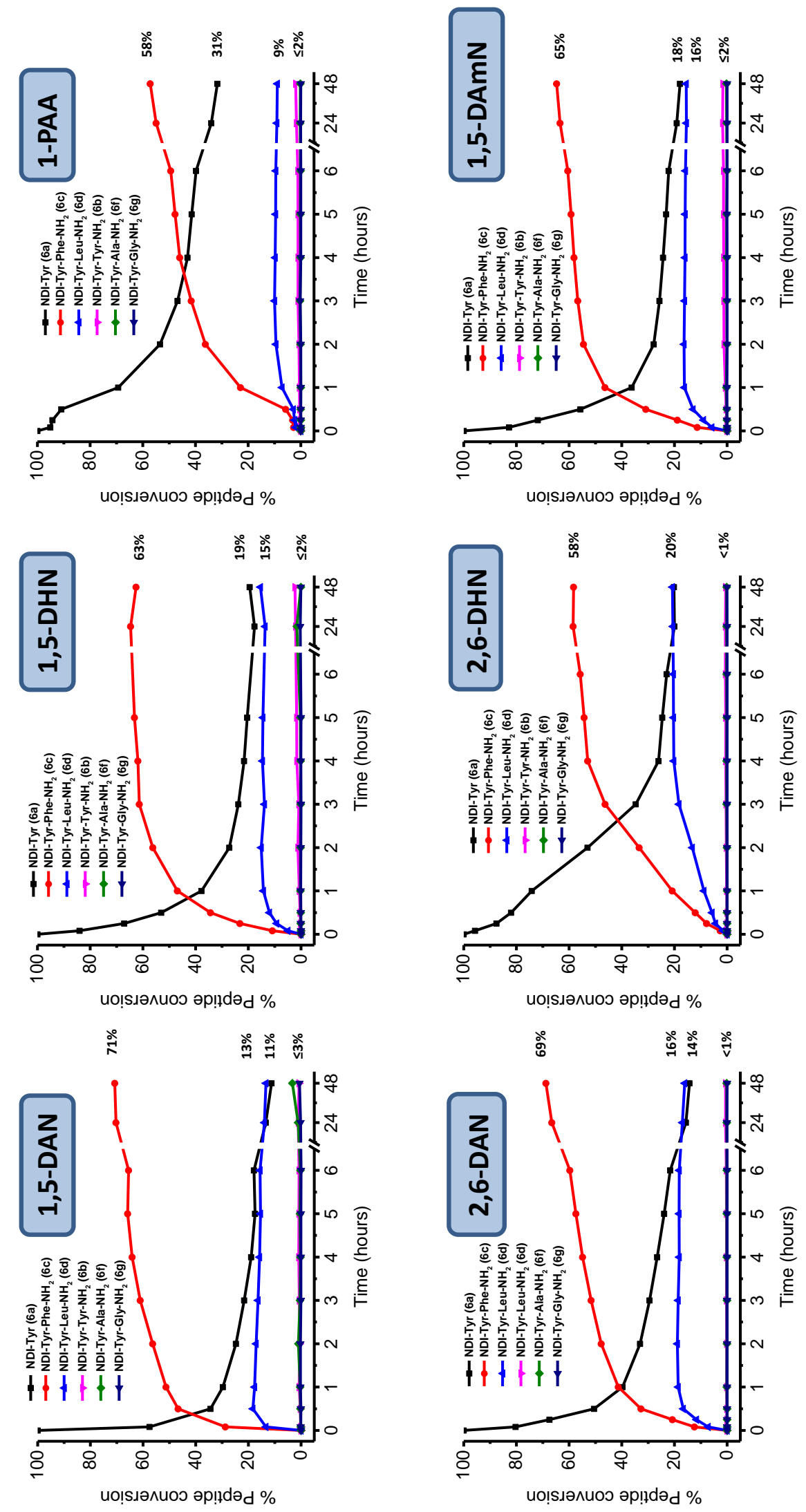

Figure 6.3.10. Time-dependent HPLC conversion of NDI-Tyr- $\mathbf{X}-\mathbf{N H}_{2}$ in the DCLs systems in the presence of donors. 
Peptide-based DCLs with the presence of donors were then prepared. A mixture of the six amino acid nucleophiles $\left(\mathbf{X}-\mathbf{N H}_{\mathbf{2}}\right)$, each in 2-fold excess, was added to the NDI-Tyr (6a) $(10 \mathrm{mM})$ together in the presence of donor solution in a 1:1 ratio. A colour change was observed in all systems, pointing to the charge-transfer complexation in all cases. After the addition of thermolysin, a hydrolgel was formed in all systems. The evolution of all peptide derivatives was analysed over time by HPLC (Figure 6.3.10). Time-dependent conversion showed a change in the DCL distribution in all the systems upon addition of donor. Good conversions of NDI-Tyr-Phe- $\mathbf{N H}_{2}(6 \mathrm{c})$ were obtained for all the studied systems, 71\% 1,5-DAN, 69\% 2,6-DAN, 65\% 1,5-DAmN, 63\% 1,5-DHN, 58\% 2,6-DHN and $57 \%$ 1-PAA. These results, together with the moderate or low conversion for the rest of nucleophiles $(\mathbf{6 b}, \mathbf{6 d}, \mathbf{6 f}$ and $\mathbf{6 g}$ ) demonstrate an amplification and selection for the most stable structure, $\mathrm{NDI}-\mathrm{Tyr}-\mathrm{Phe}-\mathrm{NH}_{2}(\mathbf{6 c})$.

Noticeably, the difference between the yields obtained for NDI-Tyr-Phe- $\mathrm{NH}_{\mathbf{2}}(\mathbf{6 c})$ in the absence ( $48 \%$ conversion without donor) and in the presence of donor ( $71 \%$ in the case of 1,5-DAN) in DCL indicates that the presence of suitable donors and acceptors in DCL provide the additional energy through charge-transfer interactions. This causes the biocatalytic self-assembly of $\mathbf{N D I}-\mathbf{T y} \mathbf{Y}-\mathbf{X}-\mathbf{N H}_{\mathbf{2}}$ conjugates to be more favourable and eventually allows the system to reach the lowest minimum state of the free energy

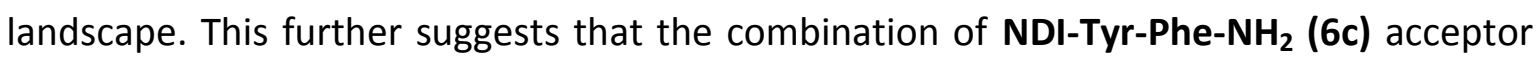
and 1,5-DAN donor corresponds to the most stable charge-transfer hydrogel system where amplification of up to $20 \%$ was found.

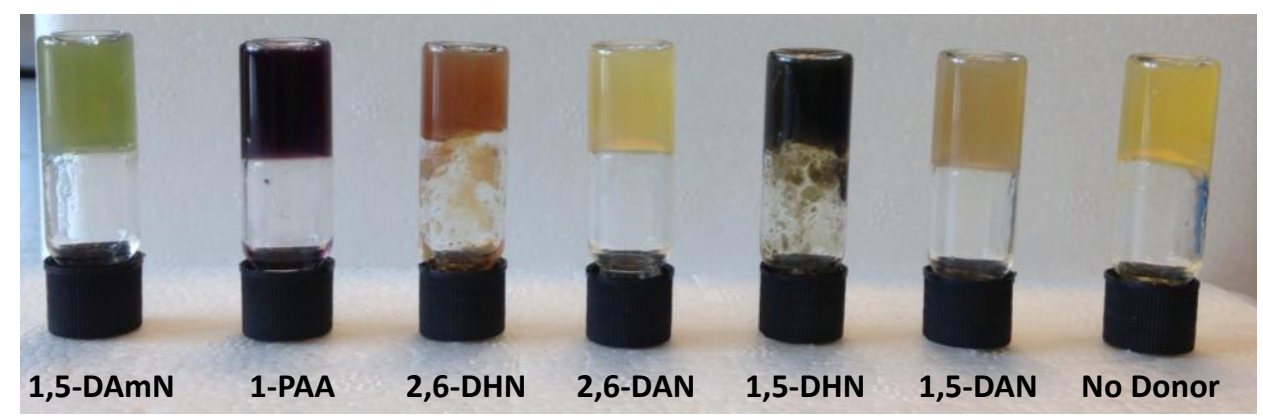

Figure 6.3.11. Digital photograph showing the different colours of the gels upon charge transfer complexation of NDI-Tyr-X-NH $\mathbf{N H}_{\mathbf{2}}$ in DCLs in the presence of several $\pi$-electron-rich donors used in this study. 
The evolution of the gel $\mathrm{DCL}$ systems after the addition of thermolysin were followed over time. In all the cases a change in colour was found, pointing to the formation of new structures inside the library with a new supramolecular organization with charge transfer interactions (

Figure 6.3.11 and Figure SI_6.6.4).

It was hypothesised that the extra interaction provided by the donor-acceptor assembly would allow the system to select and amplify the most stable self-assembled structure in the DCL to a high degree. To demonstrate this, a new DCL experiment was carried out where the donor-acceptor ratio was modified for both 1,5-DAN and 2,6-DAN (Figure SI_6.6.5). For instance, the relative yields of NDI-Tyr-Phe- $\mathbf{N H}_{\mathbf{2}}(\mathbf{6 c})$ in the DCL after $48 \mathrm{~h}$ are 57, 68, 71 and 73\% for 1,5-DAN donor system and 59, 63, 69 and 69\% for 2,6DAN donor system at 1:10, 1:2, 1:1 and 2:1 donor-acceptor ratios respectively, while the other peptide derivatives remain unchanged or show a decrease in the yield. These results confirm that the product distribution in the library is clearly dependent on the nature of and the extent of the interactions present and is entirely thermodynamically driven by the more favourable charge transfer co-assembly between donors and acceptors in the library.

Furthermore, in order to test that the system inside the DCL using the enzymeassisted gelation is in equilibrium under thermodynamic control, and to further verify the fully reversible nature of the thermolysin condensation reaction, a competition experiment was carried out. Initially the NDI-Tyr (6a) precursor and Leucine (Leu-NH $\mathbf{N H}_{\mathbf{2}}$ derivative were mixed in the presence of thermolysin. After $24 \mathrm{~h}$ the NDI-Tyr-Leu- $\mathbf{N H}_{\mathbf{2}}$ (6d) product was obtained in $37 \%$ yield (Figure 6.3.12). At this point, $\mathbf{P h e}-\mathbf{N H}_{\mathbf{2}}$ together with 1,5-DAN were added to the system and left for $192 \mathrm{~h}$ to reach equilibrium in competition. HPLC conversions showed that, NDI-Tyr-Phe- $\mathbf{N H}_{2}$ (6c) was produced preferentially in $82 \%$ yield replacing NDI-Tyr-Leu- $\mathbf{N H}_{\mathbf{2}}(\mathbf{6 d})$ which was significantly reduced to $12 \%$, suggesting that when charge-transfer interactions are present, not only the selection, but also the amplification of the most favourable NDI-Tyr-Phe- $\mathbf{N H}_{\mathbf{2}}(\mathbf{6 c})$ selfassembled structure is a consequence of the less stable self-assembled derivative. These results demonstrate that the enzyme-assisted self-assembly of donor-acceptor systems is 
fully reversible and equilibrium driven. These observations were again confirmed when the control experiment involving the addition of only Phe- $\mathbf{N H}_{\mathbf{2}}$ (without 1,5-DAN, indicating the absence of charge-transfer) to the NDI-Tyr-Leu- $\mathbf{N H}_{\mathbf{2}}(\mathbf{6 d})$ system at $24 \mathrm{~h}$ showed that after $192 \mathrm{~h}$, an equilibrium state was reached with leucine (6d) and phenylalanine (6c) derivatives forming in only 42 and $24 \%$ respectively.

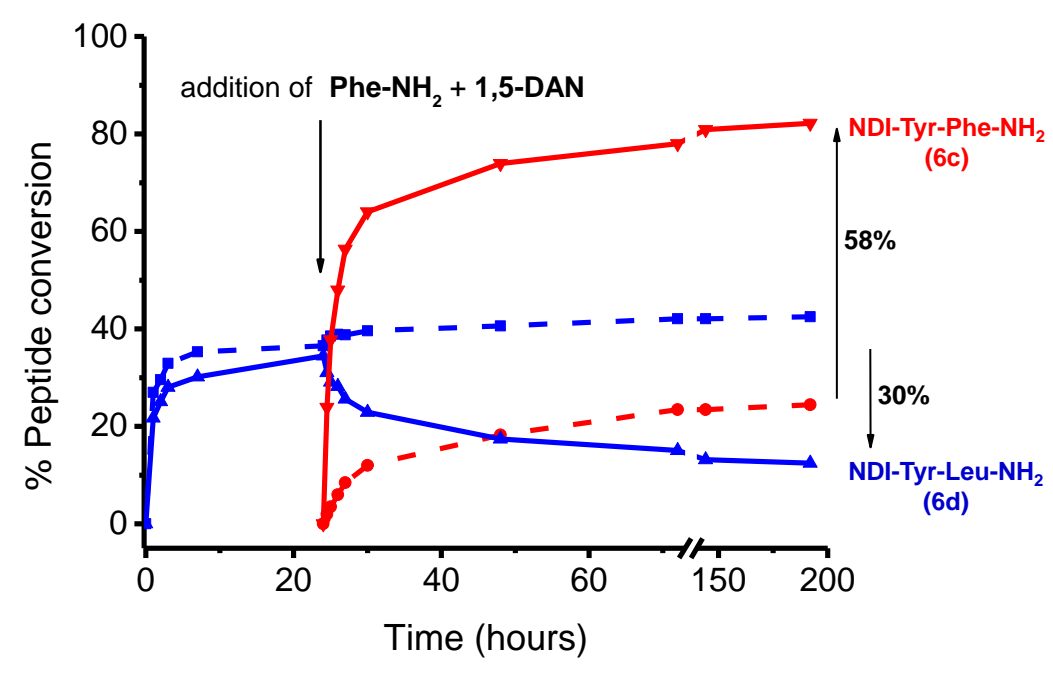

Figure 6.3.12. Time-dependent HPLC conversion in a competition test. NDI-Tyr-Leu- $\mathbf{N H}_{\mathbf{2}}$ (6d) (blue) and NDI-Tyr-Phe- $\mathrm{NH}_{2}(\mathbf{6 c})$ (red) in absence (dashed traces) and in the presence of donor (solid traces).
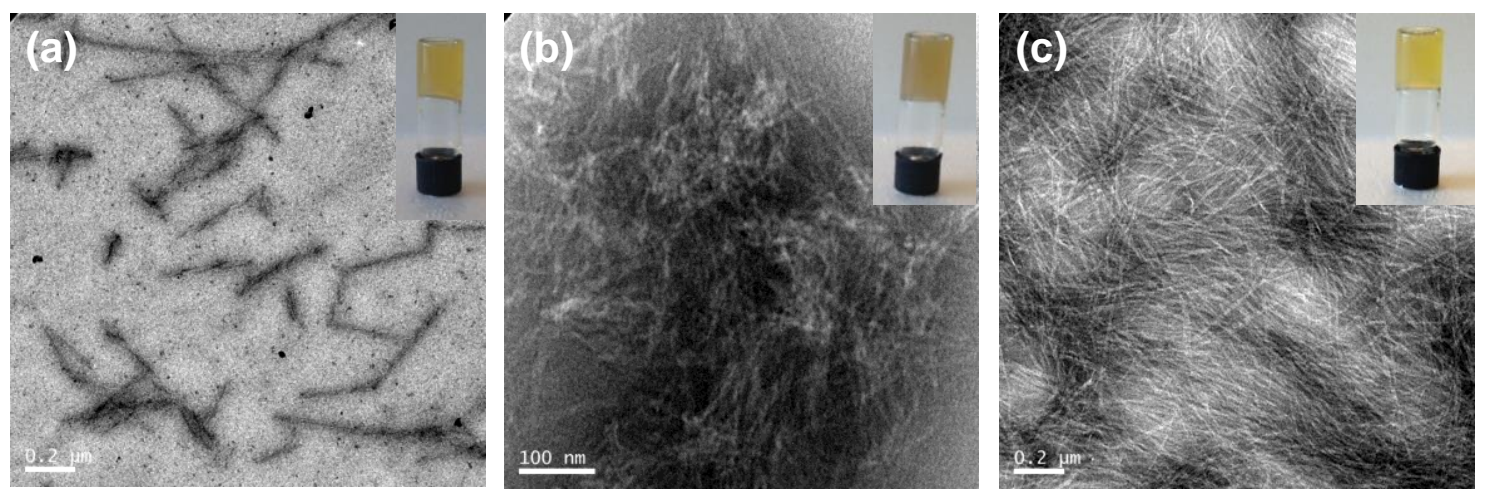

Figure 6.3.13. TEM images of DCL hydrogels at $24 \mathrm{~h}$ after exposing to thermolysin in the absence and presence of charge transfer. (a) In the absence of any donor, (b) In the presence of 1,5-DAN donor and (c) In the presence of 2,6-DAN donor. Inset: Digital photographs of the corresponding functional hydrogels.

The two best acceptor-donor DCL systems, together with the DCL system in absence of donor were chosen to be characterised further. Firstly, morphological and stiffness changes were measured $24 \mathrm{~h}$ after thermolysin addition. TEM imaging of dried 
DCL gel samples revealed the formation of short nanofibres of 1-2 $\mu \mathrm{m}$ in length for the system in absence of donor (Figure 6.3.13.a). When the donor interactions are present, a dense network of 1D functional charge-transfer twisted nanofibres of several micrometres in length for 1,5-DAN (Figure 6.3.13.b) as well as 2,6-DAN donor systems were observed (Figure 6.3.13.c).

The mechanical properties of these systems were measured by rheology. A stiffness value of $470 \mathrm{~Pa}$ was observed for when the donor is not present and an enhancement to either $850 \mathrm{~Pa}$ or $1100 \mathrm{~Pa}$ when the donor is present (1,5-DAN and 2,6DAN respectively, Figure 6.3.14). These results point to extra charge-transfer being responsible for the stronger interactions in the self-assembly, resulting in the formation of a dense 3D network in the reinforced hydrogels.

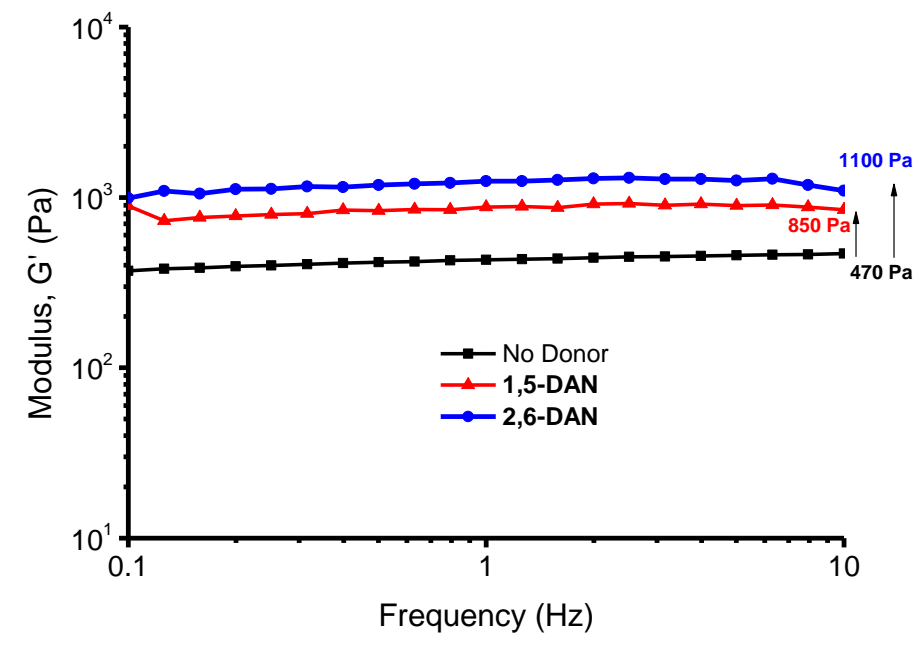

Figure 6.3.14. Stiffness of DCLs hydrogels in absence (no donor) and in presence of 1,5-DAN and 2,6-DAN donors, as measured by rheology.

A study of the structural changes of the self-assembly inside the $D C L$ with fluorescence emission spectroscopy was then performed. A diluted sample of NDI-Tyr (6a) in DMSO (non-assembling state) was used as a reference. The fluorescence spectra of this species showed a maximum at $410 \mathrm{~nm}$ and a low energy shoulder at $430 \mathrm{~nm}$, as has been referenced before for monomeric $N, N^{\prime}$-dialkyl-substituted NDIs ${ }^{53}$ (Figure SI_6.6.3). Time-dependent fluorescence spectra of the hydrogel DCL systems were also analysed, in the absence of donor (Figure 6.3.15.a) prior to enzyme addition ( $0 \mathrm{~min}$ ), a red-shifted 
broad band emission maximum at $548 \mathrm{~nm}$ was observed with two weak shoulders at 438 and $467 \mathrm{~nm}$, consistent with inter-NDI electronic communication in the excited state between closely packed NDI groups. ${ }^{24,26}$ The addition of thermolysin resulted in a gradual increase in the intensity associated with self-assembly in the aggregation induced emission (AIE). ${ }^{54}$ A broadening of the prominent peak at $548 \mathrm{~nm}$ in the spectra and the emergence of a band at $467 \mathrm{~nm}$ gradually increased over time indicating a change in the morphology with the formation of NDI-Tyr-X-NH $\mathbf{N H}_{\mathbf{2}}$ acceptor derivative gelator structures.

(a) DCL with no donor

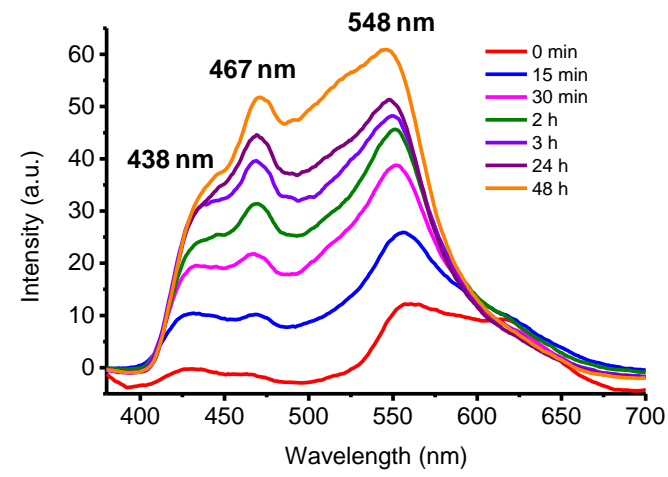

(b)

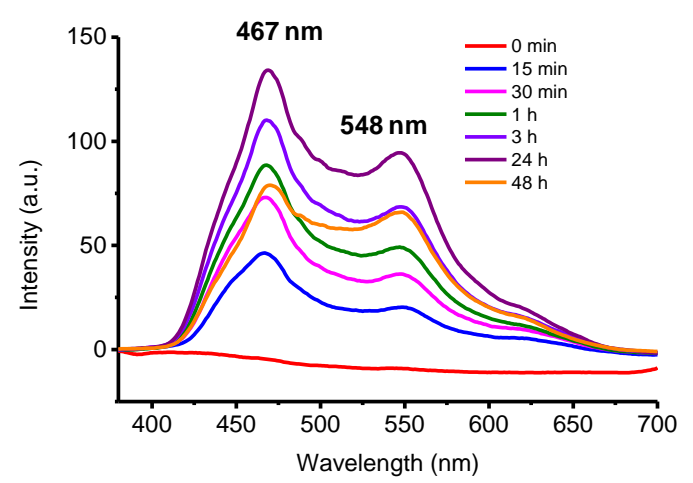

(c) DCLin presence of 2,6-DAN

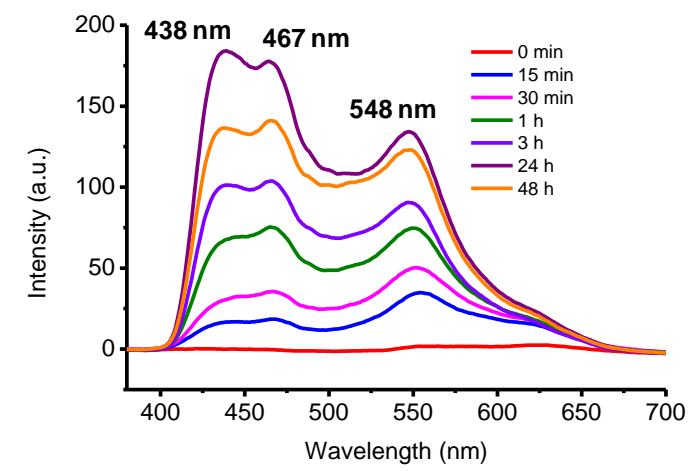

Figure 6.3.15. Time-dependent fluorescence emission spectra of DCL hydrogels before and after the addition of thermolysin. (a) In the absence of any donor, (b) In the presence of 1,5-DAN donor and (c) In the presence of 2,6-DAN donor.

When donors were present in 1,5-DAN and 2,6-DAN DCL systems, before the addition of thermolysin $(\min 0)$, the spectra revealed that the fluorescence was completely quenched and no emission was observed when either, the NDI $\left(\lambda_{\text {exc }}=360 \mathrm{~nm}\right.$, Figure 6.3.15) or the DAN donor ( $\lambda_{\mathrm{exc}}=296 \mathrm{~nm}$, Figure 6.3.16) were excited. This indicates the presence of charge-transfer interactions inside the gel system. Interestingly, the 
addition of thermolysin to the DCL immediately enhanced the emission and the peaks reappear at 467 and $548 \mathrm{~nm}$ for 1,5-DAN, and at 438, $467 \mathrm{~nm}$ together with a band at 548 $\mathrm{nm}$ for 2,6-DAN within 15 minutes. As before, these bands increased gradually over time with the intensity of the peak at $467 \mathrm{~nm}$ (in 1,5-DAN) or $438 \mathrm{~nm}$ (in 2,6-DAN) dominating over the peak at $538 \mathrm{~nm}$. An increase in the emission at $24 \mathrm{~h}$ and a decrease in the emission after $48 \mathrm{~h}$ were similarly observed in both systems when the donor is present. These observations can be attributed to the morphological changes leading to the formation of more ordered, charge-transfer aggregates within the hydrogel system.
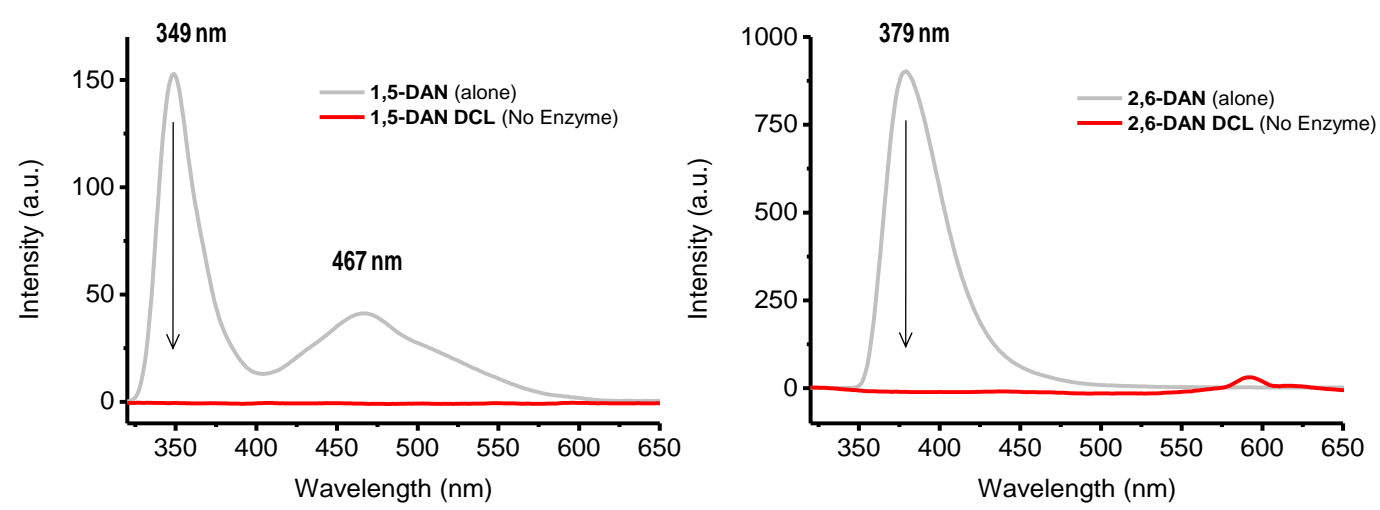

Figure 6.3.16. Fluorescence emission spectra of the $D C L$ system after the addition of thermolysin together with the emission of a sample of the donor solution alone $\left(\lambda_{\mathrm{exc}}=296 \mathrm{~nm}\right)$ a) 1,5-DAN and b) 2,6-DAN.

\subsection{Study of a Supramolecular Nanostructure on NDI-Dipeptide in a Charge-Transfer Complex}

We decided to further study the best acceptor functionalised peptide-based charge-transfer nanostructure found previously using the dynamic combinatorial library approach as a tool to discover materials in a biocatalysed fully reversible amide condensation reaction in a thermodynamically optimised way. Therefore, NDI-Tyr-Phe$\mathbf{N H}_{2}$ (6c) was chosen as the best NDI-peptide acceptor. A full study of the enzymatic condensation and self-assembly to produce energy-minimised electronically functionalised nanostructures was performed in absence and in the presence of chargetransfer interactions in water.

As before, naphthalendiimide tyrosine appended amino acid conjugated (NDI-Tyr, 6a) was used as a precursor for the biocatalytic synthesis of $\mathbf{N D I}-\mathbf{T y r}-\mathbf{P h e}-\mathbf{N H}_{\mathbf{2}}(\mathbf{6 c})$ under 
thermodynamic control. The condensation and self-assembly of this dipeptide nanostructure was studied (Figure 6.4.1.a and c).

(1)

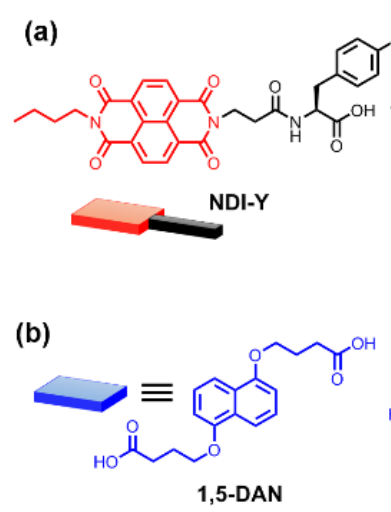

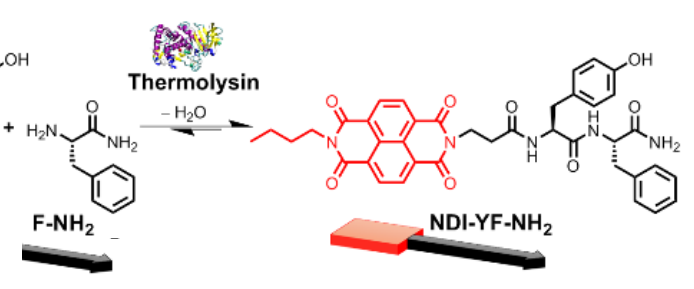
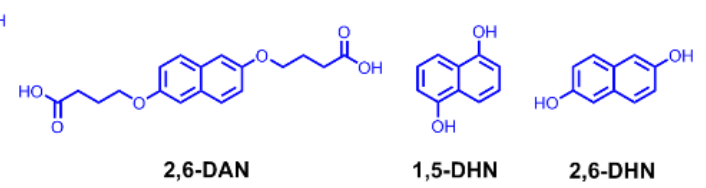

(c)
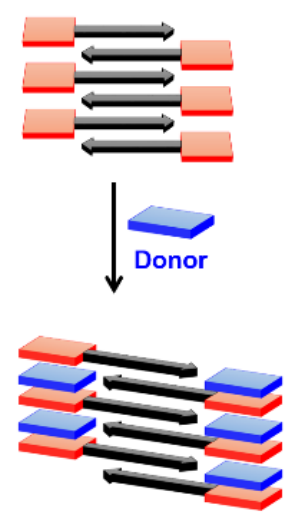

Figure 6.4.1 a) Thermolysin-catalysed condensation reaction showing the formation of the NDI-Tyr-Phe--NH (6c) acceptor structure. b) Molecular structures of various dialkoxy/ dihydroxy naphthalene derivative donor used in this study. c) Schematic illustration of the proposed supramolecular aggregation in absence (top) and in presence of donor (bottom).

To accomplish this, NDI-Tyr (6a) precursor (10 mM) was added in a two-fold excess to phenylalanine nucleophile derivative $\left(\mathbf{P h e}-\mathbf{N H}_{2}\right)$ in $100 \mathrm{mM}$ phosphate buffer $(\mathrm{pH} 8)$ solution. It was found that when the Phe- $\mathbf{N H}_{\mathbf{2}}$ was present $(20 \mathrm{mM})$, a slight colour change in the gel to orange was observed (compared with the gel sample of NDI-Tyr (6a)

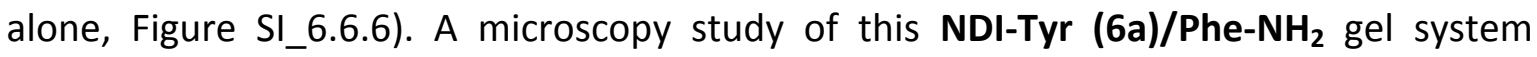
revealed twisted nanofibres with lengths in the micrometre scale with TEM. AFM also showed the formation of an entangled nanofibre network of several micrometres in length (Figure 6.4.2.a and c). Upon the addition of thermolysin, the gel colour turned to a pale yellow after about $3 \mathrm{~h}$. A morphological change was also observed when the enzyme was present. TEM and AFM imaging of the gel sample showed the formation of a dense network of chiral nanofibres (Figure 6.4.2.b and d). As was mentioned before, time-

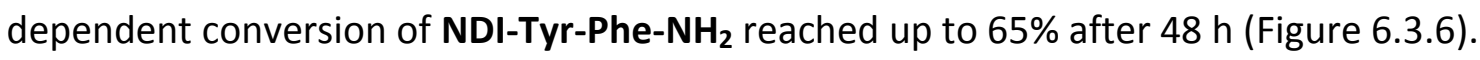

Charge-transfer interactions were then investigated when the NDI-amino acid derivative acceptor was mixed with electron rich dialkoxy (DAN) or dihydroxy (DHN) naphthalene derivative donors. To accomplish this, 1,5-DAN, 2,6-DAN, 1,5 DHN and 2,6 DHN (Figure 6.4.1.b) were selected as possible candidate donors based on the previous results with the DCL systems. 

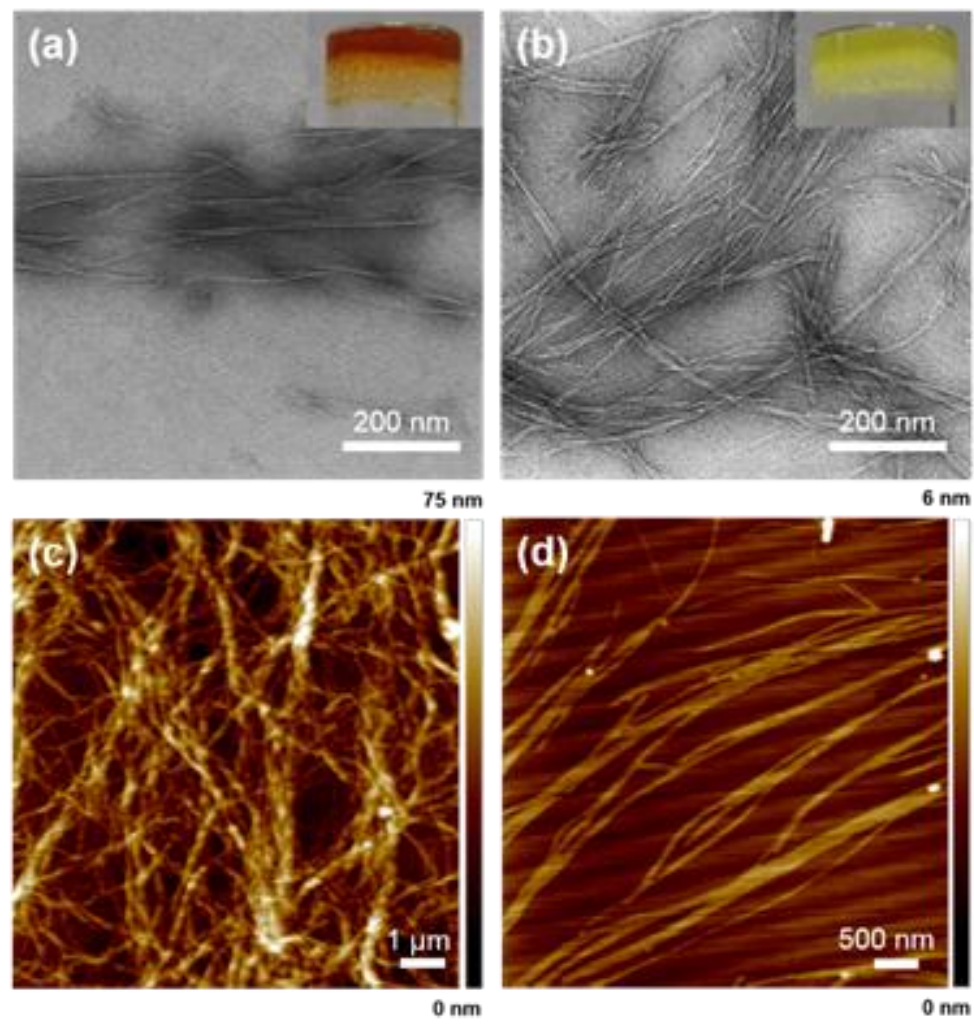

Figure 6.4.2. Microscopy images of NDI-Tyr (6a) / Phe- $\mathbf{N H}_{2}$ hydrogel samples. TEM images before (a) and after (b) the addition of thermolysin; insets show the digital photographs of the corresponding gel samples. Tapping-mode AFM height images of hydrogels before (c) and after (d) the addition of thermolysin.

A change in colour was observed when the donor was present in the system for all cases (Figure SI_6.6.6) suggesting the formation of the charge-transfer complexes between the NDI $\pi$-electron deficient chromophore ring and the $\pi$-electron rich naphthalene derivative ring shown previously. These observations were also confirmed as before with UV-vis absorbance, where the typical charge-transfer band appeared between 450 and $700 \mathrm{~nm}$ (Figure 6.3.9).

Surprisingly, the addition of 1,5-DAN donor (10 mM) to NDI-Tyr (6a) acceptor (10 $\mathrm{mM}$ ) completely transformed the self-supported yellow-orange hydrogel system into a viscous dark plum solution. The gel-sol transition was also investigated by microscopy. TEM and SEM images of the NDI-Tyr (6a)/Phe-NH $\mathbf{N H}_{2}$ 1,5-DAN sample showed the complete transition from chiral nanofibres of the NDI-Tyr (6a) aggregated structure (Figure 6.3.5 and Figure 6.4.2) to spherical aggregates (Figure 6.4.5.a and d), similar to the ones found only for the 1,5-DAN donor solution alone (Figure SI_6.6.11.b). The gel-sol 
transition indicates that the interactions between the NDI moieties in the supramolecular gel can be disrupted by the intermolecular charge-transfer interactions and thus provoking this disassembly effect (see Figure 6.4.3 route B). Dynamic light scattering (DLS) experiments further confirmed the presence of spherical aggregates (Figure SI_6.6.11). ${ }^{55}$

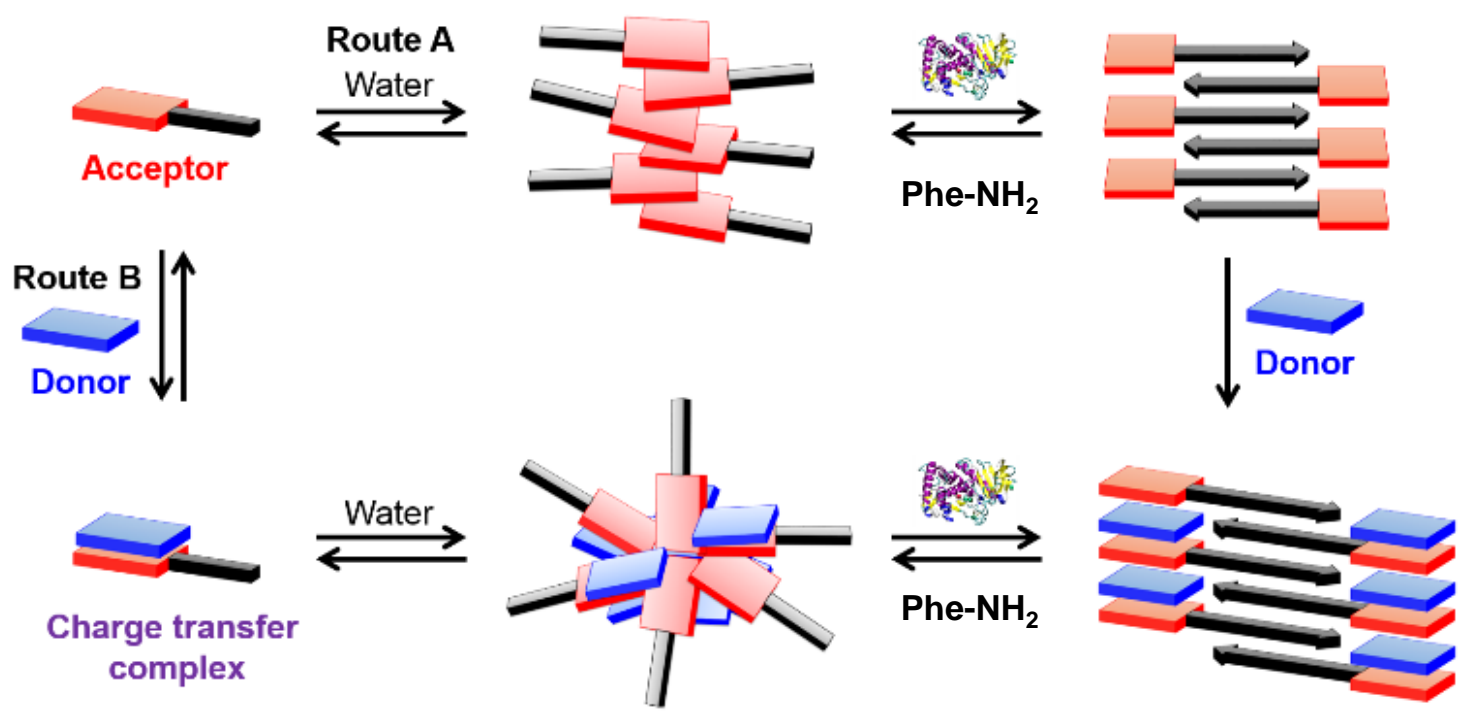

Figure 6.4.3. Schematic illustration showing the proposed differential supramolecular organization of NDITyr (6a) / NDI-Tyr-Phe- $\mathbf{N H}_{2}(6 \mathrm{c})$ acceptors.in the absence and presence of various DAN/DHN donors and the formation of highly ordered chiral 1D supramolecular charge transfer nanofibres.

Time-dependent conversion was analysed with HPLC over time (Figure 6.4.4). It was found that when the donor is present an enhancement of the NDI-Tyr-Phe- $\mathbf{N H}_{\mathbf{2}}(\mathbf{6 c})$ conversion was reached for all systems. For instance, for the 1,5-DAN system, the conversion was amplified up to $94 \%$ after $48 \mathrm{~h}$. Similar results were obtained in the presence of the other donors: 88,85 and $86 \%$ conversions for 2,6-DAN, 1,5-DHN and 2,6DHN respectively. As in the DCL systems, these results indicate that for the aggregationdriven enzymatic amide condensation of the NDI-dipeptide conjugate, the presence of extra charge-transfer interactions allow the system to reach a more stable minimum under thermodynamic control. Thus, provoking an enhancement on the formation of the NDI-Tyr-Phe- $\mathbf{N H}_{2}(\mathbf{6 c})$ acceptor nanostructure. A change in colour over time was found for all the systems, supporting the results that point to the formation of a new selfassembled NDI-Tyr-Phe- $\mathbf{N H}_{2}$ (6c) structure (Figure SI_6.6.6). 


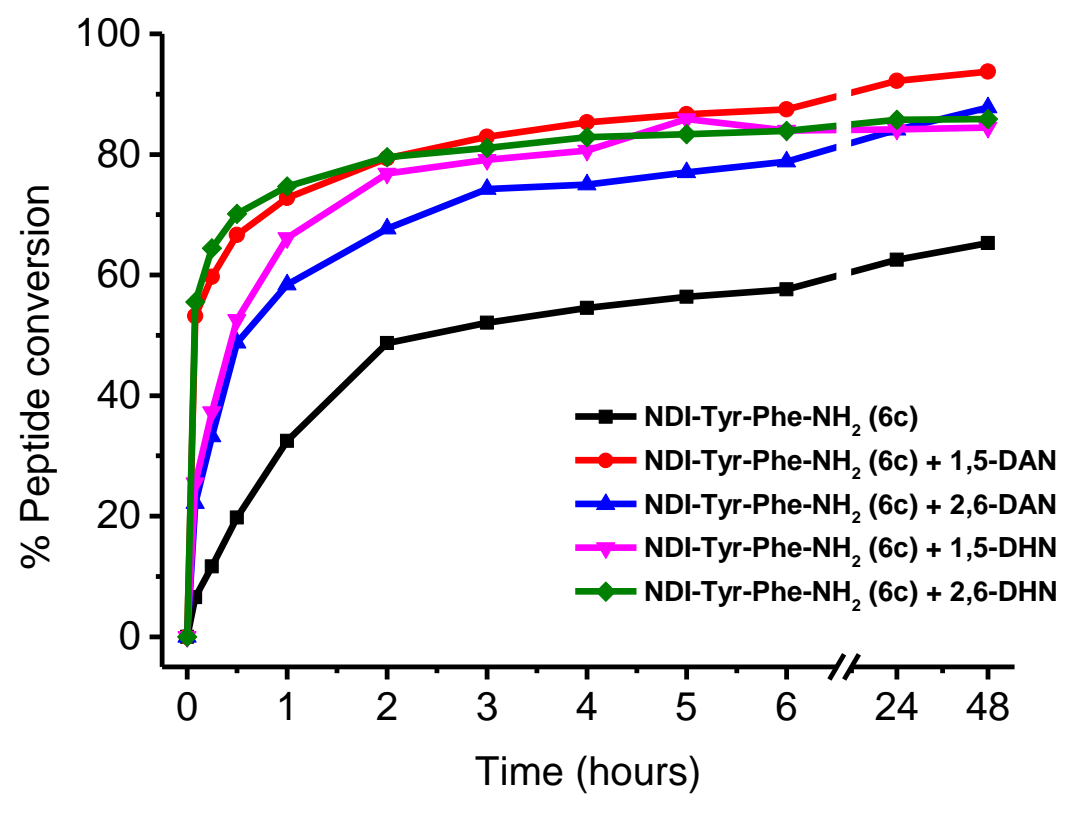

Figure 6.4.4. Time-dependent HPLC conversion of NDI-Tyr-Phe- $\mathbf{N H}_{\mathbf{2}}(\mathbf{6 c})$ in absence and in the presence of the wide range of donors studied.
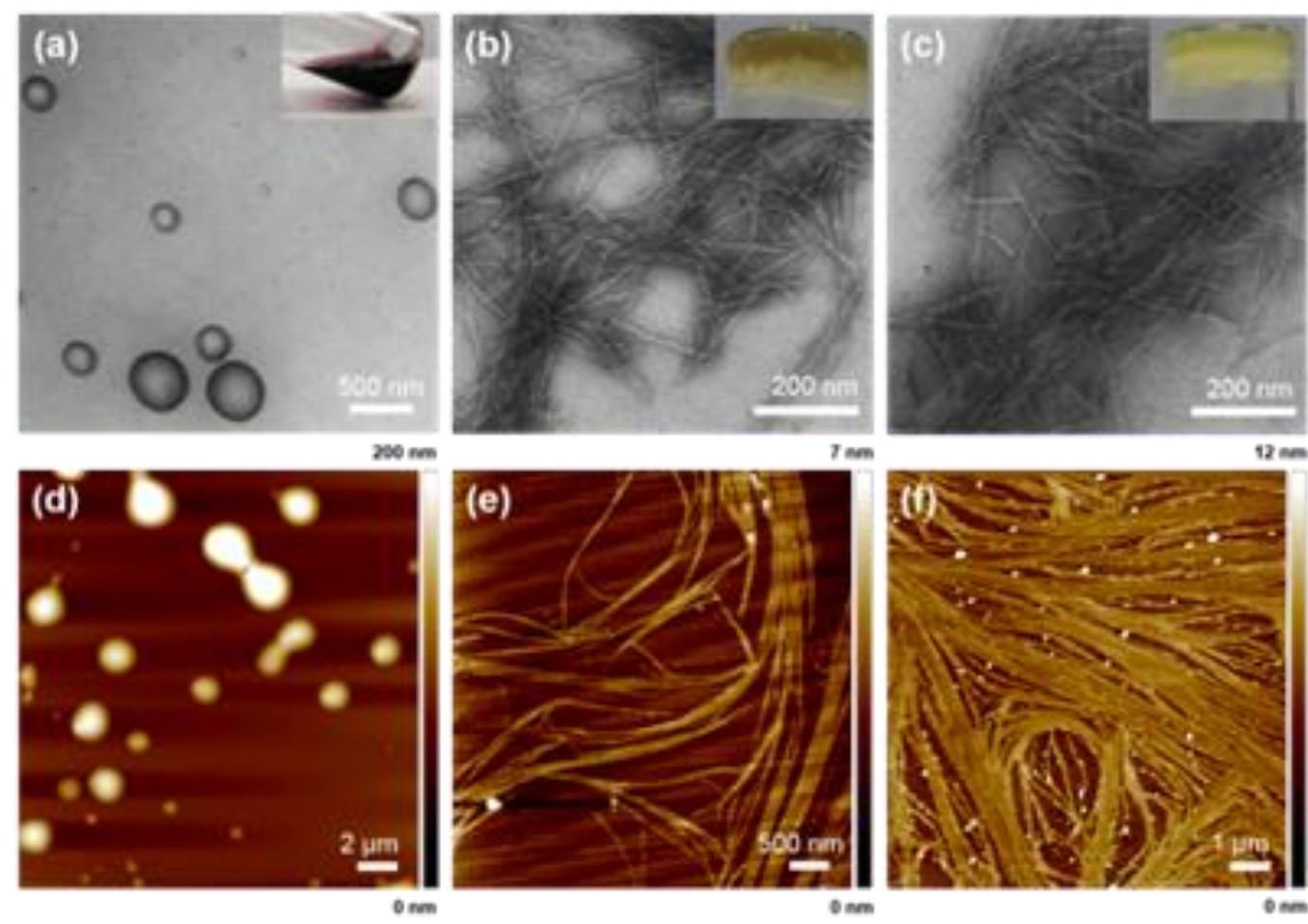

Figure 6.4.5. TEM images ( $a$ and $b$ ) and AFM height images ( $d$ and e) of NDI-Tyr (6a) / Phe-NH $/$ / 1,5-DAN (1:1) acceptor / donor mixture before ( $a$ and $d$ ) and after ( $b$ and e) exposing to thermolysin for $24 \mathrm{~h}$. The corresponding TEM (c) and AFM (f) images of the charge-transfer hydrogel of NDI-Tyr (6a)/Phe- $\mathbf{N H}_{2} / \mathbf{2 , 6 -}$ DAN mixture (1:1) after exposing to thermolysin for $24 \mathrm{~h}$. Insets in (a-c) show the digital photographs of the corresponding gel / sol samples. 
TEM and AFM images for these charge-transfer systems showed a change after the addition of thermolysin from spherical aggregates to a dense network of nanofibres for the 1,5-DAN sample (Figure 6.4.5.a-b, d-e) as was described in Figure 6.4.3 for route B. Similar fibrillar morphologies were also shown for all donors studied (Figure 6.4.5.c and $f$ and Figure SI_6.6.12) also presented in Figure 6.4.3, route A. where a fibrillar chargetransfer network is formed.

The mechanical properties of the gel samples were analysed by rheology. A stiffness of $250 \mathrm{~Pa}$ was observed for the NDI-Tyr (6a) gel sample alone with an increase up to 1200 Pa upon formation of the NDI-Tyr-Phe- $\mathbf{N H}_{\mathbf{2}}$ (6c) supramolecular structure. In the presence of donors, the stiffness of the hydrogels increased significantly, up to 3000 and $4000 \mathrm{~Pa}$ for 1,5-DAN and 2,6-DAN, respectively (Figure 6.4.6), as charge-transfer interactions reinforce the network of the fibres.

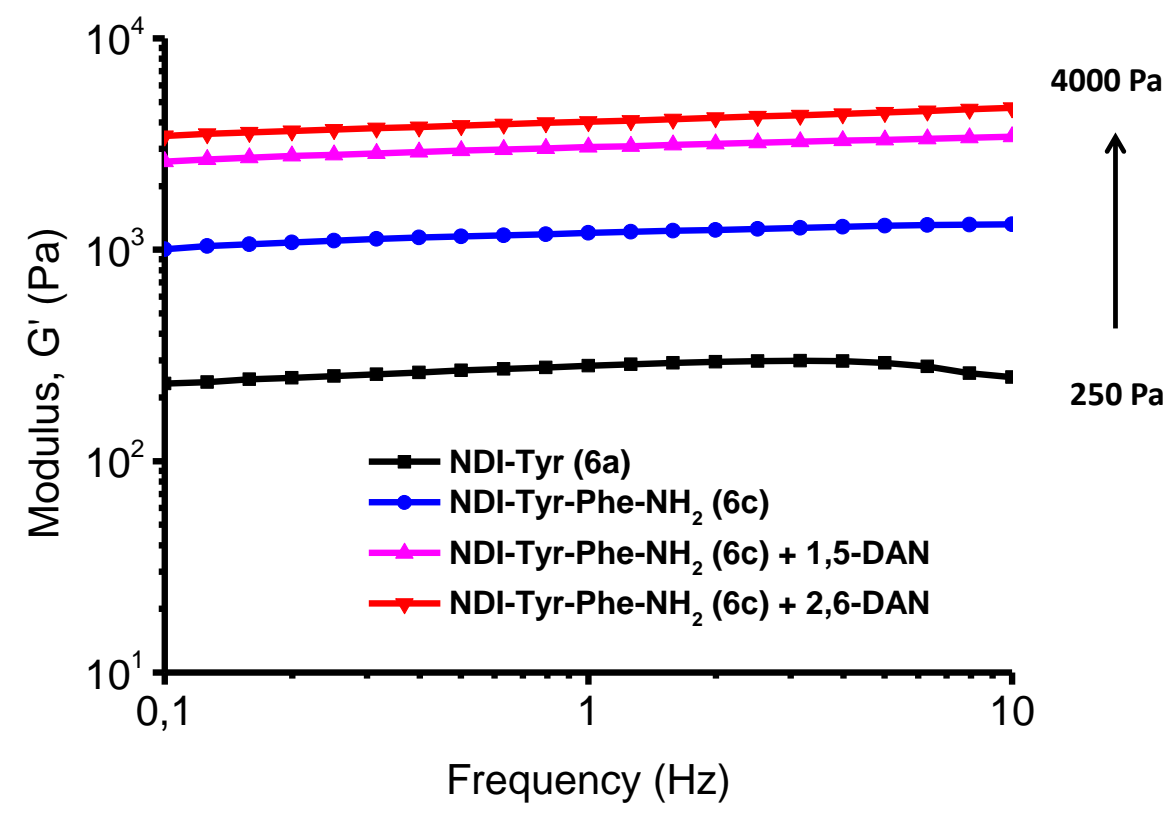

Figure 6.4.6. Stiffness of acceptor hydrogels alone and charge-transfer donor-acceptor hydrogels, as measured by rheology.

Finally, to study the involvement of supramolecular aggregation in the NDIderivative, further analysis with fluorescence emission, Fourier-transform infrared (FTIR) and circular dichroism were performed on the hydrogels before and after the addition of thermolysin. 
Firstly, the sample without donor (in absence of charge-transfer interactions) was analysed. Taking diluted sample of NDI-Tyr (6a) in DMSO as a reference (Figure SI_6.6.3) fluorescence emission of the NDI-Tyr (6a) + Phe- $\mathbf{N H}_{2}$ mixture before the enzyme addition (0 $\mathrm{min}$ ) showed a red-shifted broad band emission with a maximum at $548 \mathrm{~nm}$ and two weak shoulder peaks at 432 and $468 \mathrm{~nm}$, characteristic to the intermolecular excimer emission from closely packed NDI chromophores in the fibre. The addition of thermolysin induced an increase in the intensity of these peaks over time, with the peak at $468 \mathrm{~nm}$ dominating the emission spectra after 24 h (Figure SI_6.6.9). These observations support the results obtained in the $D C L$ hydrogel system when no donor is present with the

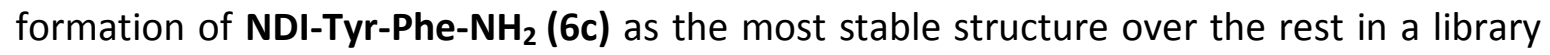
of components.

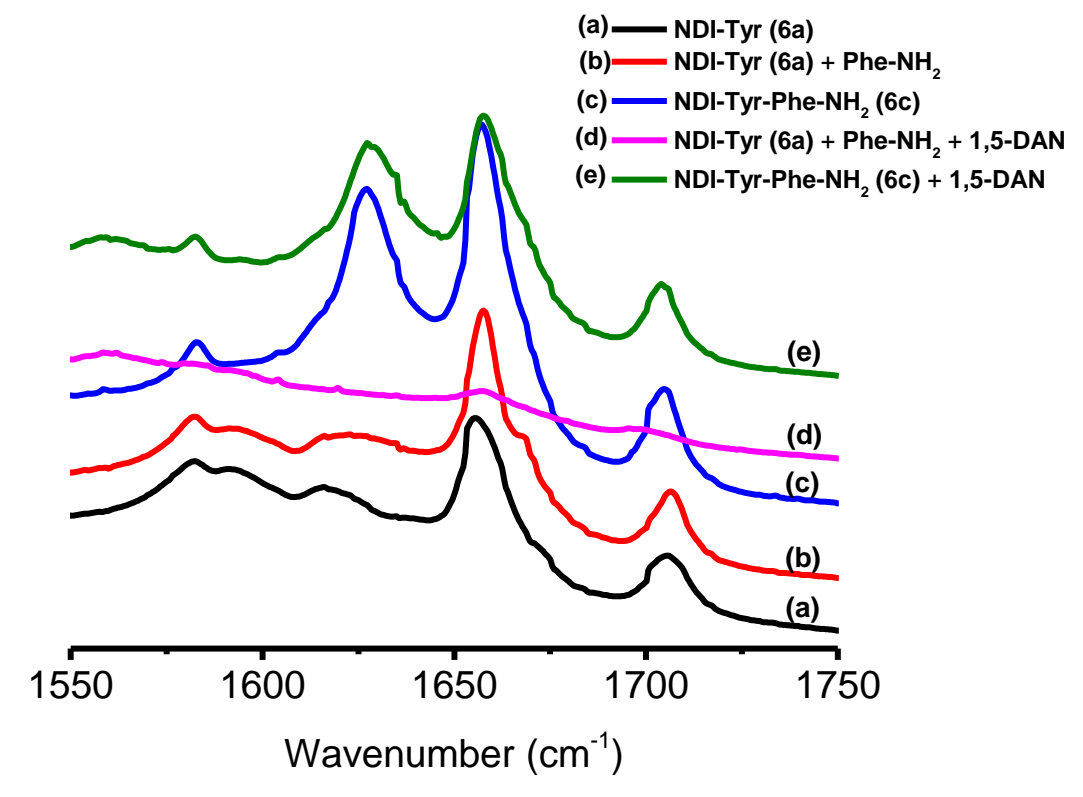

Figure 6.4.7. FTIR spectra in the amide I region of the corresponding gel/sol samples in $\mathrm{D}_{2} \mathrm{O}$.

Experiments preformed with FTIR showed the possible role of the hydrogen bonding in the different structures as well as the changes in the assembly. The absorption of the gels were analysed in the amide I region of the spectra in $\mathrm{D}_{2} \mathrm{O}$. As it is shown in Figure 6.4.7, the NDI-Tyr (6a) precursor sample showed a weak peak at $1616 \mathrm{~cm}^{-1}$. Similar results were obtained for $\mathbf{N D I}-\mathbf{T y r}(6 \mathbf{6})+\mathbf{P h e}-\mathbf{N H}_{\mathbf{2}}$ when no enzyme is present. Interestingly, the new structure NDI-Tyr-Phe- $\mathbf{N H}_{\mathbf{2}}(\mathbf{6 c})$ formed by the biocatalytic reaction, showed an intense peak at $1627 \mathrm{~cm}^{-1}$, typical from $\beta$-sheet intermolecular $\mathrm{H}$-bonding 
network inside the fibre. ${ }^{25}$ The additional peaks at 1583, 1657 and $1704 \mathrm{~cm}^{-1}$ found in all the gels correspond to the symmetric and asymmetric stretching of the imide carbonyl of NDI chromophores. ${ }^{24}$.

CD spectroscopy of the NDI-Tyr (6a) precursor gel sample displayed a weak signal in the band I region (300-400 nm) of $\pi-\pi^{*}$ transitions polarized along the long $z$-axis, while a strong positive Cotton effect in the band II region (220-260) was shown, corresponding to $\pi-\pi^{*}$ transitions polarized along the short $y$-axis of NDI chromophores. ${ }^{56}$ On the contrary, the NDI-Tyr-Phe- $\mathbf{N H}_{\mathbf{2}}(\mathbf{6 c})$ gel sample remarkably showed negative Cotton effects in both band I and band II regions (Figure SI_6.6.8), suggesting a left-handed, M-type chiral orientation of NDI chromophores ${ }^{57}$ within the nanofibre. The combined microscopic and spectroscopic observations consistently indicate that the biocatalytic assembly of NDI-dipeptide conjugated led to the formation of more ordered assemblies of chiral nanofibres inside the hydrogel sample.

Finally, the presence of charge-transfer interactions on the sample by the addition of donors was analysed. For example, when the 1,5-DAN donor was present in the sample, fluorescence emission was completely quenched and no emission was observed when either NDI (Figure SI_6.6.9) acceptor or DAN donor (Figure SI_6.6.10) were excited before the addition of the enzyme. The gel-sol transition shown before by microscopy and DLS was confirmed by FTIR experiments. FTIR spectra did not show any peaks in the amide I region (1610 to $1640 \mathrm{~cm}^{-1}$ ), suggesting the absence of a $\beta$-sheet-like hydrogen bonding network (Figure 6.4.7) and thus the transition to a disassembled solution by disruption of the supramolecular interactions. The same behaviour was observed for the rest of donors in the FTIR spectra, and no peaks were found in the amide I region (Figure SI_6.6.7). Similarly, no signals were observed in both band I and band II regions of the CD spectra (Figure SI_6.6.8). ${ }^{58}$ All these observations consistently indicate that chargetransfer complex formation between NDI-Tyr (6a) acceptor and 1,5-DAN donor pair clearly lacks the ability to form the elongated supramolecular reinforced nanofibres.

After the addition of thermolysin in the 1,5-DAN system, the reorganization of the morphology with the formation of the NDI-Tyr-Phe- $\mathbf{N H}_{\mathbf{2}}(\mathbf{6 c})$ fibres was verified by fluorescence emission with the instantaneous enhancement of emission as the peaks at 
467 and $548 \mathrm{~nm}$ reappeared within $5 \mathrm{~min}$. Replacing 1,5-DAN by 2,6-DAN led to similar observations, with more pronounced enhancement of emission observed (Figure SI_6.6.9). CD spectra of the gel in the presence of 1,5-DAN showed strong negative Cotton effects in both band I and band II regions, pointing to the return of the chiral arrangement of NDI-Tyr-Phe- $\mathbf{N H}_{2}$ (6c) acceptors and 1,5-DAN donors within the selfassembled nanofibres (Figure SI_6.6.8). FTIR spectra confirm this result with a dramatically enhanced peak at $1627 \mathrm{~cm}^{-1}$. All these results once again consistently indicate that the presence of charge-transfer interactions between DAN/DHN donors and NDI-Tyr-Phe- $\mathbf{N H}_{2}(\mathbf{6 c})$ acceptor within the self-assembled nanofibres giving rise to supramolecular chiral-charge transfer nanofibres.

\subsection{Conclusions}

To summarise, peptide-based functional DCLs have been successfully developed. In this case, the self-assembly of NDI-dipeptides was performed through a fully reversible thermolysin-catalysed amide condensation under thermodynamic control, which leads to the formation of hydrogel soft-materials. The library allows selection and amplification of the most stable self-assembled structure in a mixture of peptide derivatives, highlighting its use as a powerful tool for the discovery of new structures that cannot be discovered by rational design. Moreover, the biocatalytic approach described here provides a pathway for in situ formation of NDI-dipeptide derivatives, driven by their aggregation in the hydrogel, which are difficult to obtain using direct self-assembly. Further experiments confirm the dynamic and reversible behaviour of the process that ensures defectcorrection.

The electronic properties of the new NDI acceptor structures have been tested inside the DCL system by means of suitable donors such as naphthalene or pyrene derivatives. Furthermore, it has been demonstrated that the presence of additional charge-transfer interactions allows for enhanced amplification of the most stable functional peptide nanostructures, which show higher conversions. Based on thermolysin-catalysed condensation yields, 1,5-DAN is the best donor for the NDI-TyrPhe- $\mathrm{NH}_{2}(6 \mathrm{c})$ acceptor structure where the co-assembly of these two components form 
the most stable highly ordered 1D functional aqueous charge-transfer peptide hydrogel nanostructure. The DCL methodology proves to be an effective tool to open up new possibilities for the discovery of functionalised nanomaterials.

Finally, a full characterization of the best structure NDI-Tyr-Phe- $\mathbf{N H}_{\mathbf{2}}(\mathbf{6 c})$ found in the library has been performed, wherein its acceptor properties have been tested with various DAN/DHN donors. The formation of 1D chiral charge-transfer nanofibres in aqueous media through a biocatalytically amide condensation approach has been proven. This approach holds great promise for the development of functional nanomaterials with enhanced long-range charge transfer properties and fewer defects. 


\subsection{Supporting Information}

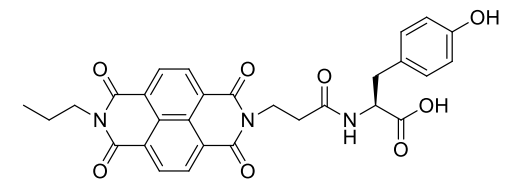

NDI-Tyr (6a)
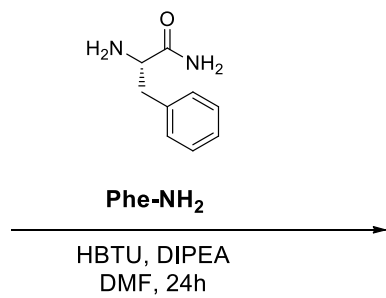

DMF, 24h

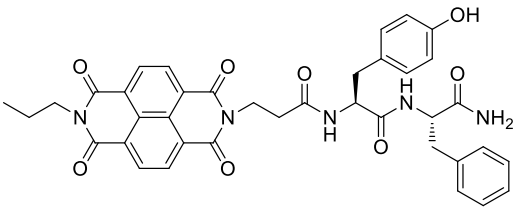

NDI-Tyr-Phe- $\mathrm{NH}_{2}(6 \mathrm{c})$

It was noticed that the NDI-Tyr-Phe- $\mathbf{N H}_{\mathbf{2}}(\mathbf{6 c})$ dipeptide derivative was extremely hard to purify and characterize because of its extreme poor solubility in most of the common solvents tested, which in itself provides justification of its in situ formation using biocatalysis. However, surprisingly NDI-Tyr-Phe- $\mathrm{NH}_{2}$ (6c) was successfully synthesised with the ${ }^{1} \mathrm{H}-\mathrm{NMR}$ spectrum in DMSO showing that further purification was not necessary for gelation test experiments. The obtained compound ( $>95 \%$ pure) has been tested for gelation at the required concentrations $(20 \mathrm{mM})$ both in the absence and presence of 1,5DAN donor. It was obtained amorphous precipitation (see following picture), and thus no gelation upon cooling a heated solution in buffer $(\mathrm{pH}=8)$. This fact highlights the significant role of the enzyme needed for the formation of self-supporting hydrogels in this case.

(a)

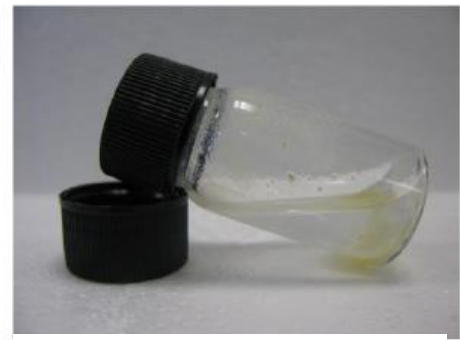

NDI-Tyr-Phe- $\mathrm{NH}_{2}$ (b)

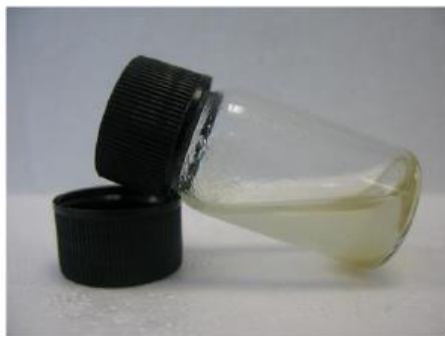

NDI-Tyr-Phe- $\mathrm{NH}_{2}$ + 1,5-DAN

Figure SI_6.6.1. Synthesis and gelation experiments for chemically synthesized NDI-Tyr-Phe- $\mathbf{N H}_{\mathbf{2}}$ (6c) acceptor molecule, Digital Photographs showing the precipitation of chemically synthesized NDI-Tyr-Phe$\mathbf{N H}_{\mathbf{2}}(\mathbf{6 c})$ acceptor molecule $(20 \mathrm{mM})$ both in the (a) absence and (b) presence of 1,5-DAN donor (1:1). 

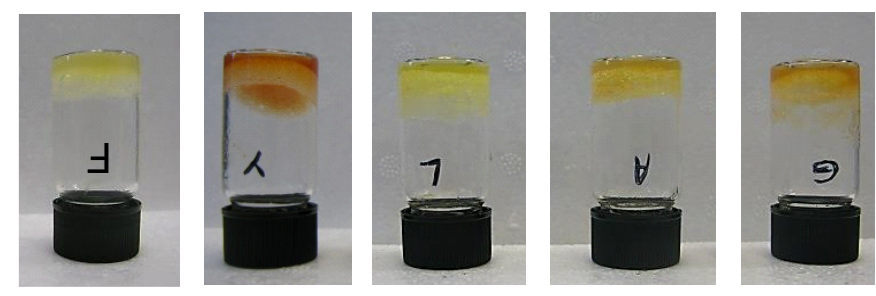

Figure SI_6.6.2. Digital photographs of the hydrogels from each combination of NDI-Tyr (6a) $+\mathbf{X}^{-\mathbf{N H}_{2}}$ in isolation to produce $\mathbf{N D I}-\mathbf{T y r}-\mathbf{X}-\mathbf{N H}_{2} \mathbf{2} \mathrm{h}$ after the addition of the enzyme.

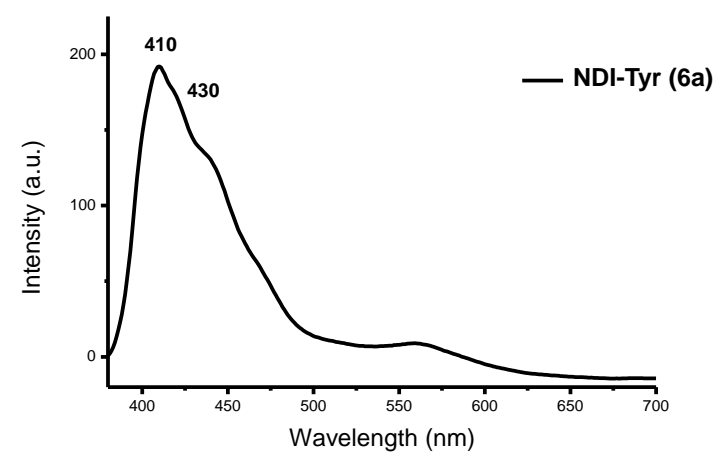

Figure SI_6.6.3. Fluorescence emission spectra of a diluted sample of NDI-Tyr (6a) $(250 \mu \mathrm{M})$ in DMSO when was excited at the acceptor excitation wavelength $\left(\lambda_{\text {exc }}=360 \mathrm{~nm}\right)$. 


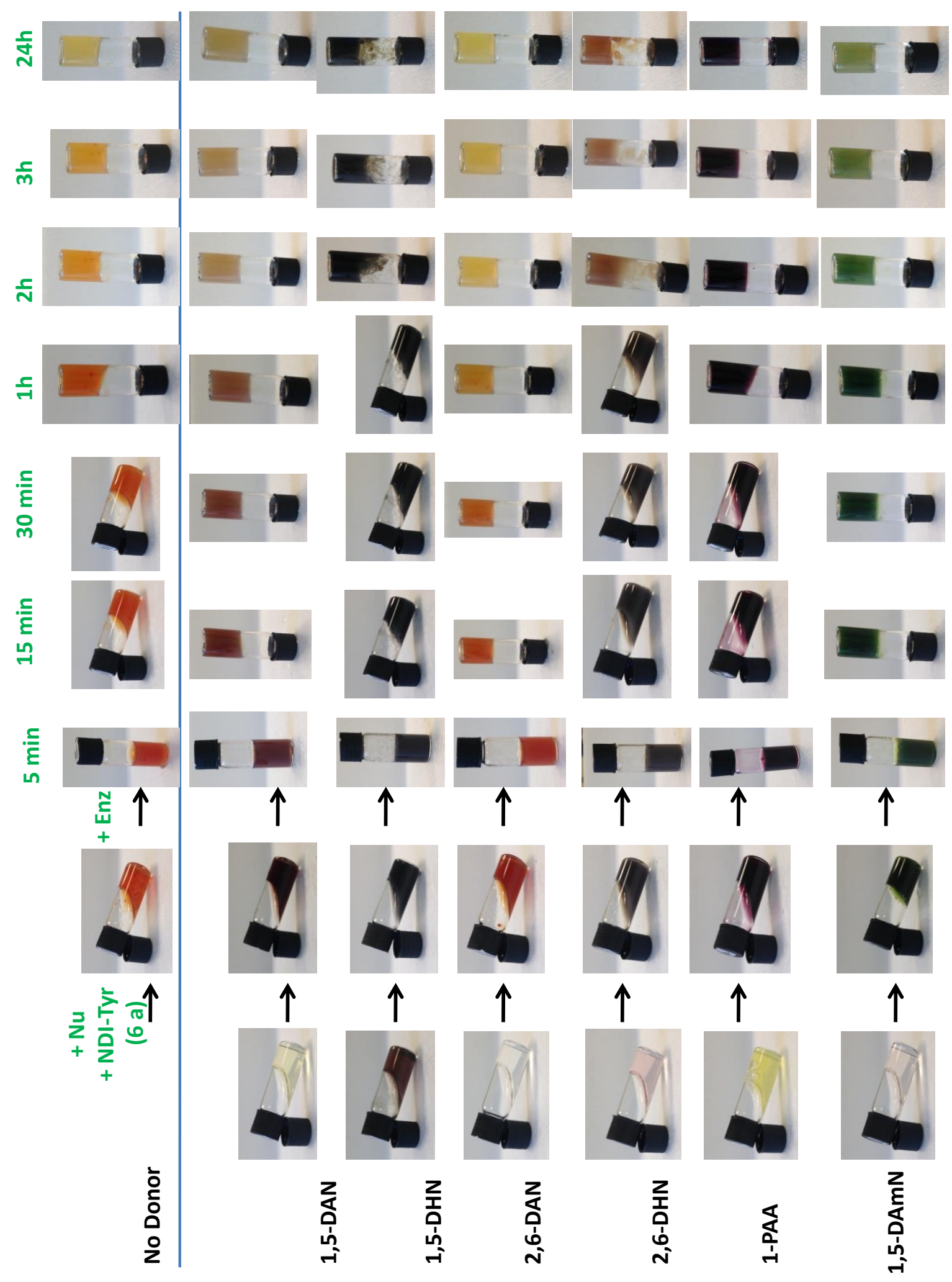

Figure SI_6.6.4. Photographs of the corresponding gel / sol DCL samples of NDI-Tyr-X-NH $\mathbf{N H}_{2}$ acceptor conjugates in absence / or presence of donors over time. $(\mathrm{Nu}=$ the different amino acid nucleophiles $\mathbf{X}$ $\mathrm{NH}_{2}$ ). 

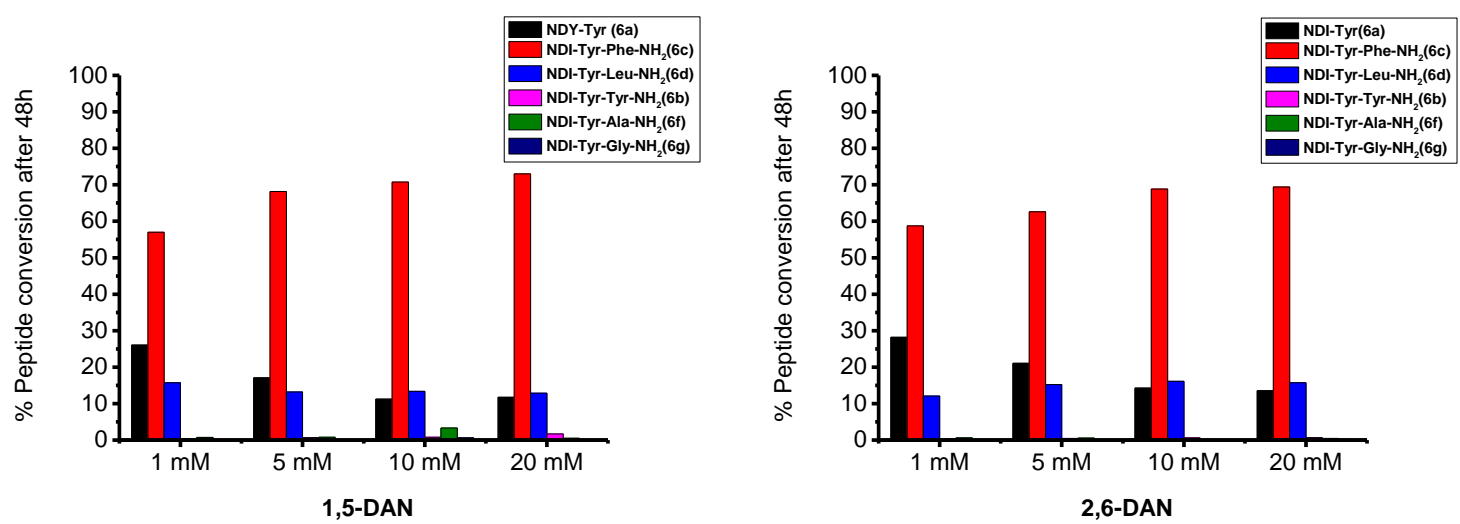

\begin{tabular}{|c|c|c|c|c|c|c|c|c|}
\hline & $C_{\text {Donor }}$ & $\begin{array}{l}\text { Ratio } \\
\text { A/D }\end{array}$ & $\begin{array}{c}\text { NDI-Tyr } \\
\text { (6a) }\end{array}$ & $\begin{array}{c}\text { NDI-Tyr } \\
\text { Phe-NH, }{ }_{2}(6 \mathrm{c})\end{array}$ & $\begin{array}{c}\text { NDI-Tyr- } \\
\text { Leu-NH (6d) }\end{array}$ & $\begin{array}{c}\text { NDI-Tyr- } \\
\text { Tyr-NH }{ }_{2} \text { (6b) }\end{array}$ & $\begin{array}{c}\text { NDI-Tyr- } \\
\text { Ala-NH, }(6 f)\end{array}$ & $\begin{array}{c}\text { NDI-Tyr- } \\
\text { Gly-NH, }(6 \mathrm{~g}) \\
\end{array}$ \\
\hline \multirow[t]{4}{*}{ 1,5DAN } & $1 \mathrm{mM}$ & 10:01 & 26,03 & 57,01 & 15,73 & 0,41 & 0,73 & 0,09 \\
\hline & $5 \mathrm{mM}$ & 2:01 & 17,06 & 68,19 & 13,19 & 0,67 & 0,76 & 0,13 \\
\hline & $10 \mathrm{mM}$ & 1:01 & 11,22 & 70,72 & 13,36 & 0,79 & 3,29 & 0,61 \\
\hline & $20 \mathrm{mM}$ & 1:02 & 11,76 & 73,02 & 12,86 & 1,7 & 0,53 & 0,13 \\
\hline \multirow[t]{4}{*}{$2,6 \mathrm{DAN}$} & $1 \mathrm{mM}$ & 10:01 & 28,17 & 58,72 & 12,08 & 0,3 & 0,64 & 0,09 \\
\hline & $5 \mathrm{mM}$ & 2:01 & 21,03 & 62,6 & 15,22 & 0,43 & 0,58 & 0,14 \\
\hline & $10 \mathrm{mM}$ & 1:01 & 14,28 & 68,81 & 16,09 & 0,63 & 0,18 & 0,01 \\
\hline & $20 \mathrm{mM}$ & 1:02 & 13,51 & 69,41 & 15,72 & 0,7 & 0,47 & 0,19 \\
\hline
\end{tabular}

Figure SI_6.6.5. Percentage of peptide conversion NDI-Tyr- $\mathbf{X}-\mathrm{NH}_{2}$ in the DCL at various ratios Acceptor/Donor measured $48 \mathrm{~h}$ after the addition of thermolysin in the presence of donors a) 1,5-DAN, b) 2,6-DAN.

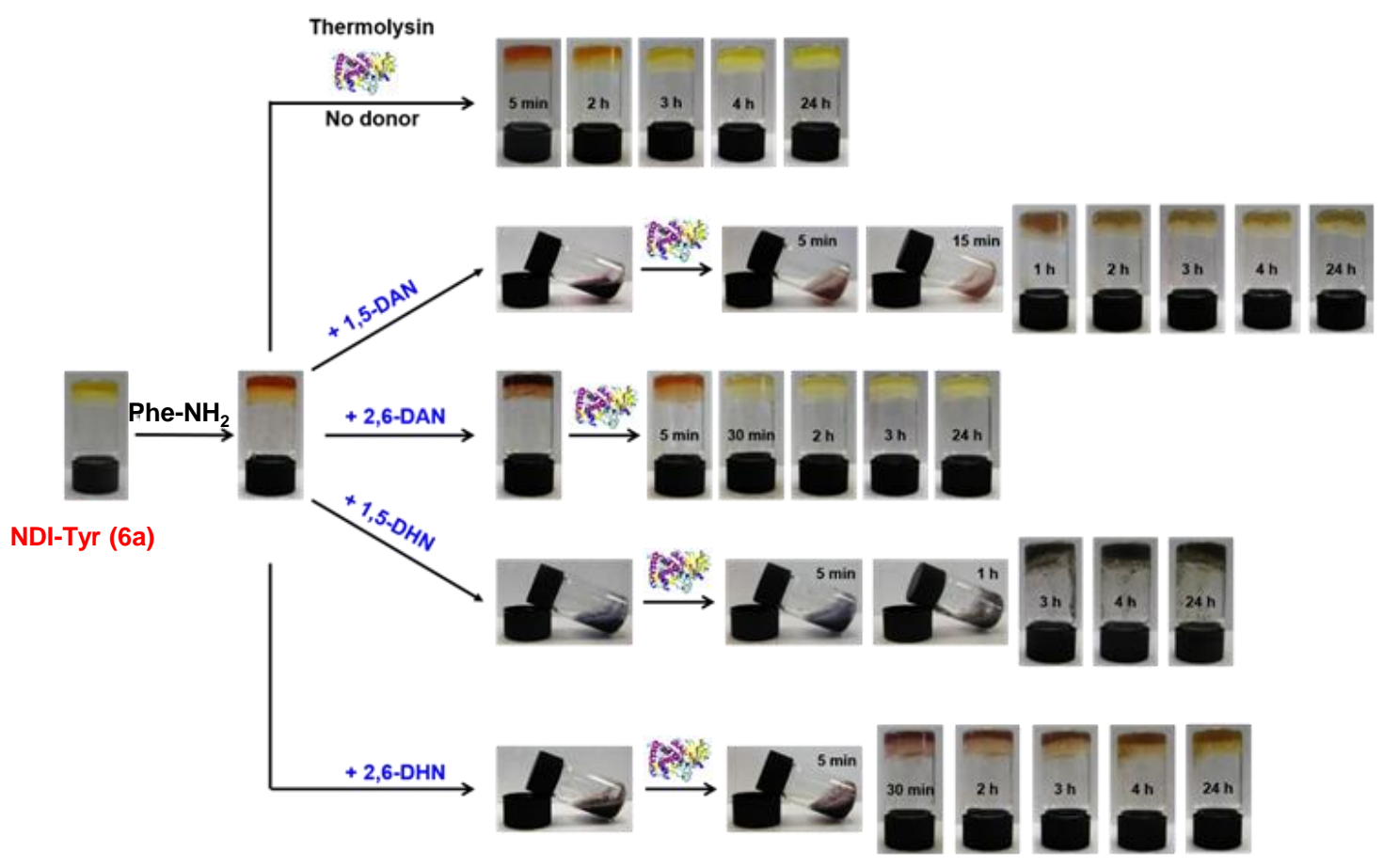

Figure SI_6.6.6. Digital photographs of the corresponding gel/sol samples of NDI-Tyr (6a)/NDI-Tyr-Phe-NH (6c) acceptor conjugates in the presence of various DAN/DHN donors: the obvious colour changes observed were monitored over time after the addition of thermolysin to the corresponding samples in $100 \mathrm{mM}$ phosphate buffer ( $\mathrm{pH} 8)$. 


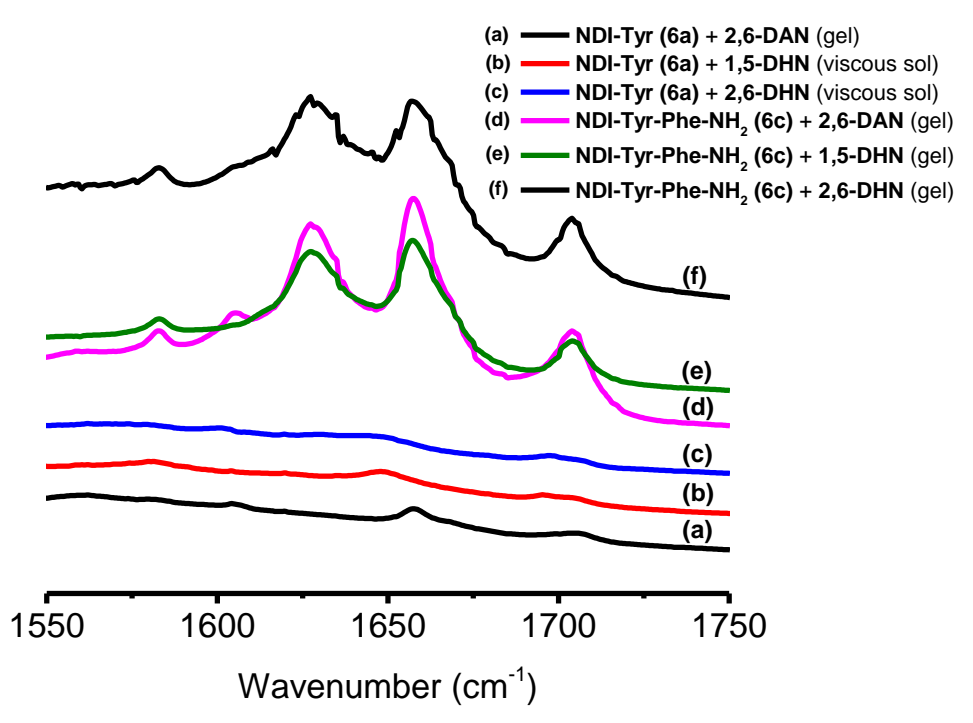

Figure SI_6.6.7. FTIR spectra revealing the information about the presence of intermolecular $\beta$-sheet-like structures in the presence of various DAN/DHN donors before and after the addition of thermolysin.

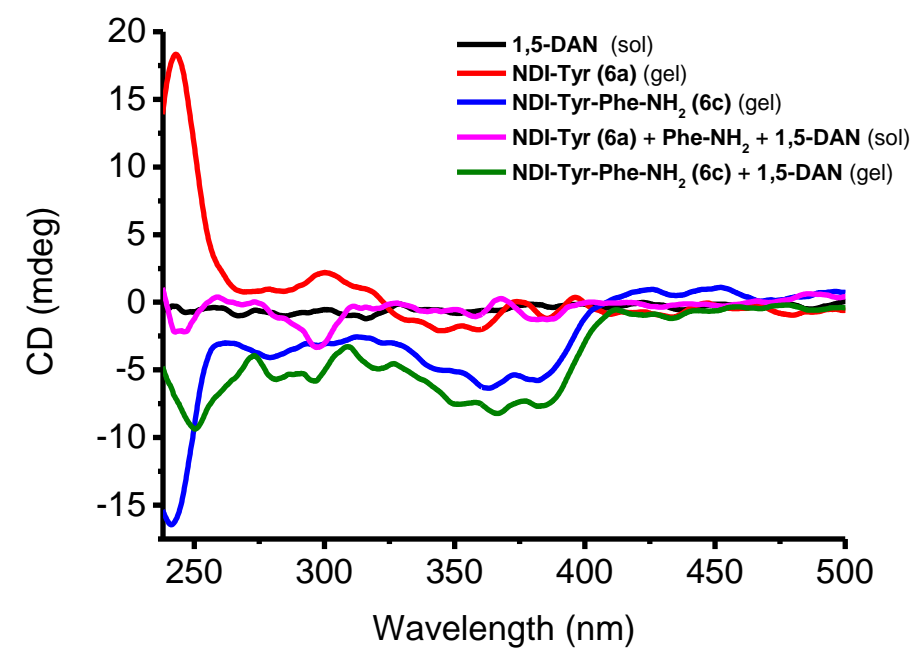

Figure SI_6.6.8. Circular dichroism (CD) spectra of the corresponding gel/sol samples of NDI-Tyr (6a)/NDITyr-Phe- $\mathbf{N H}_{\mathbf{2}}(\mathbf{6 c})$ conjugate acceptors in the absence and presence of 1,5-DAN donor. 


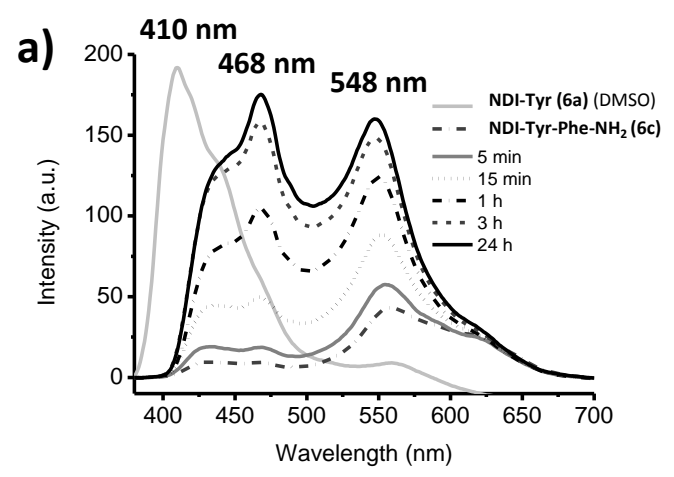

b)

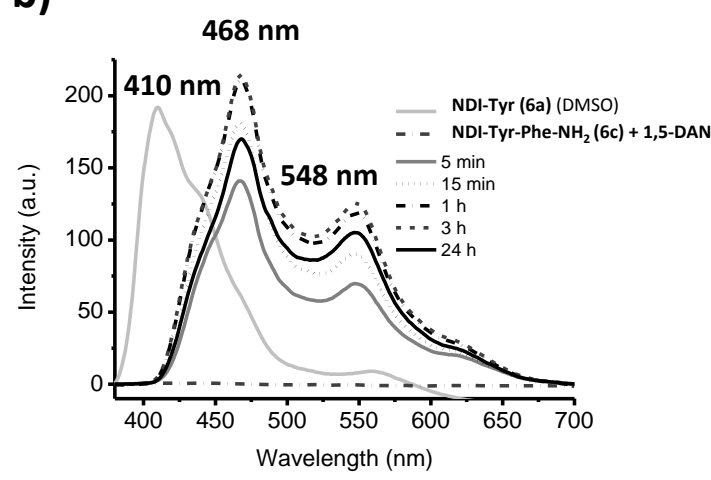

c)

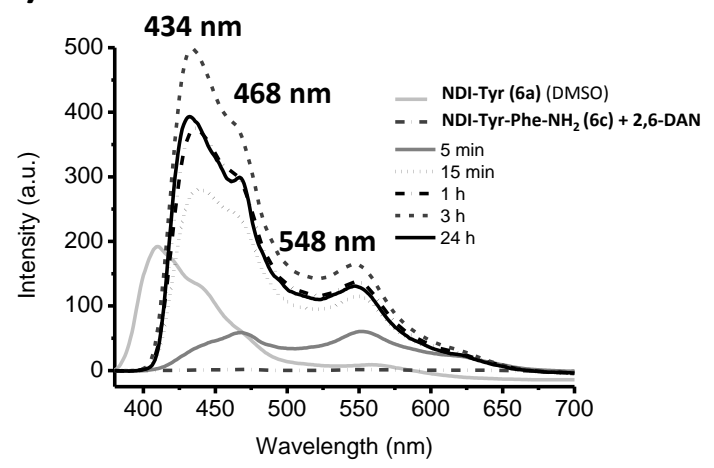

Figure SI_6.6.9. Time-dependent fluorescence emission spectra of NDI-Tyr (6a)/NDI-Tyr-Phe-NH $\mathbf{N}_{2}$ (6c) acceptor $(10 \mathrm{mM})$ at the acceptor excitation wavelength $\left(\lambda_{\mathrm{ex}}=360 \mathrm{~nm}\right)$. a) When no donor is present, b) In the presence of 1,5-DAN donor and c) In the presence of 2,6-DAN donor (10 mM).

a)

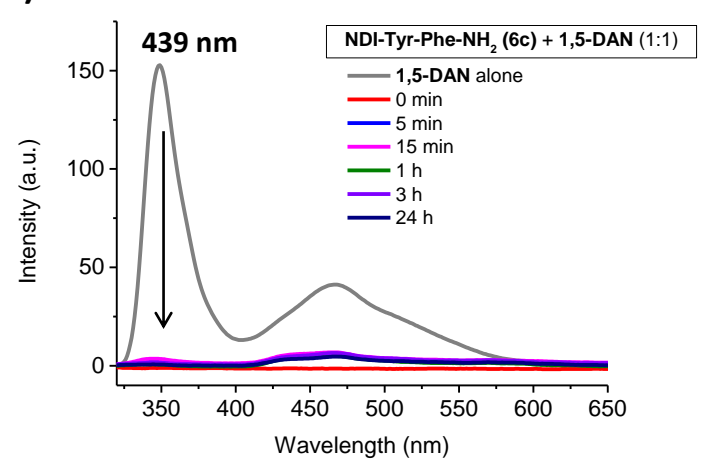

b)

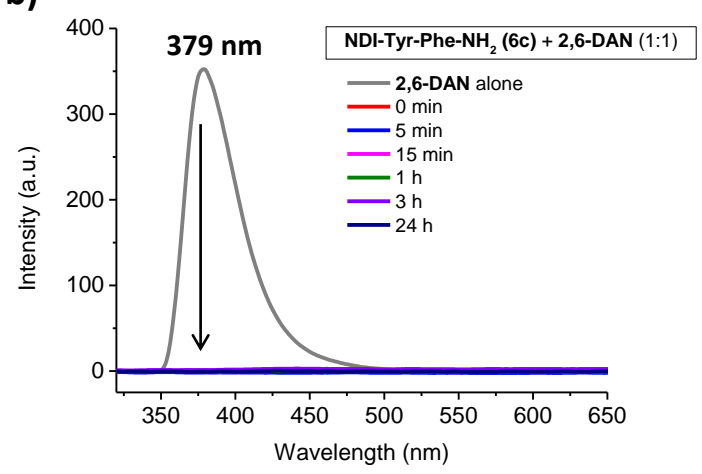

Figure SI_6.6.10. Fluorescence emission spectra showing the complete quenching upon charge-transfer complex formation between NDI derivative acceptor and either (a) 1,5-DAN donor or (b) 2,6-DAN donor at the donor excitation wavelength $\left(\lambda_{\mathrm{ex}}=296 \mathrm{~nm}\right)$. 


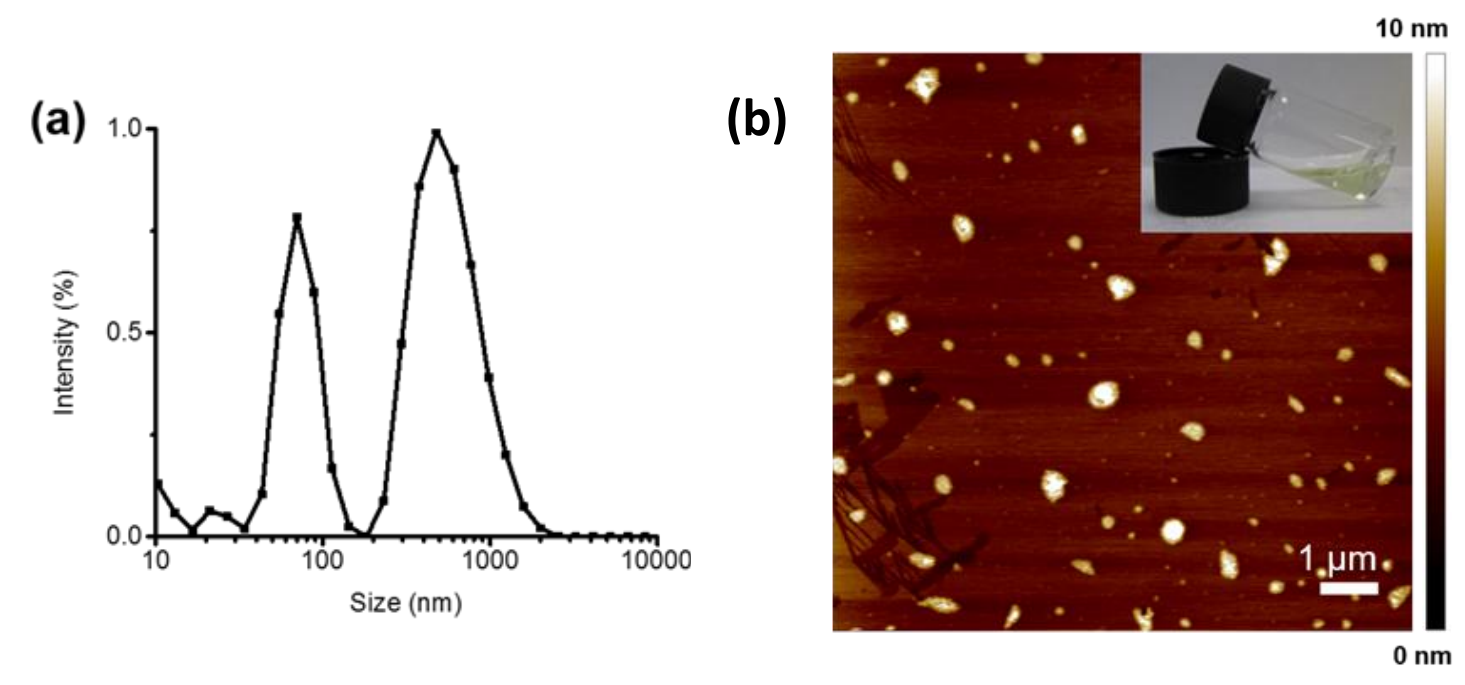

Figure SI_6.6.11. (a) Size distribution as determined by DLS for the charge-transfer plum coloured solution of NDI-Tyr (6a)/Phe- $\mathrm{NH}_{2}$ /1,5-DAN before exposing to thermolysin. Notably, the presence of spherical aggregates of two different populations of 72 and $490 \mathrm{~nm}$ as their average hydrodynamic radii. (b) AFM image of 1,5-DAN donor solution (10 mM) in $100 \mathrm{mM}$ phosphate buffer (pH 8). Inset: Digital photograph of the same sample.
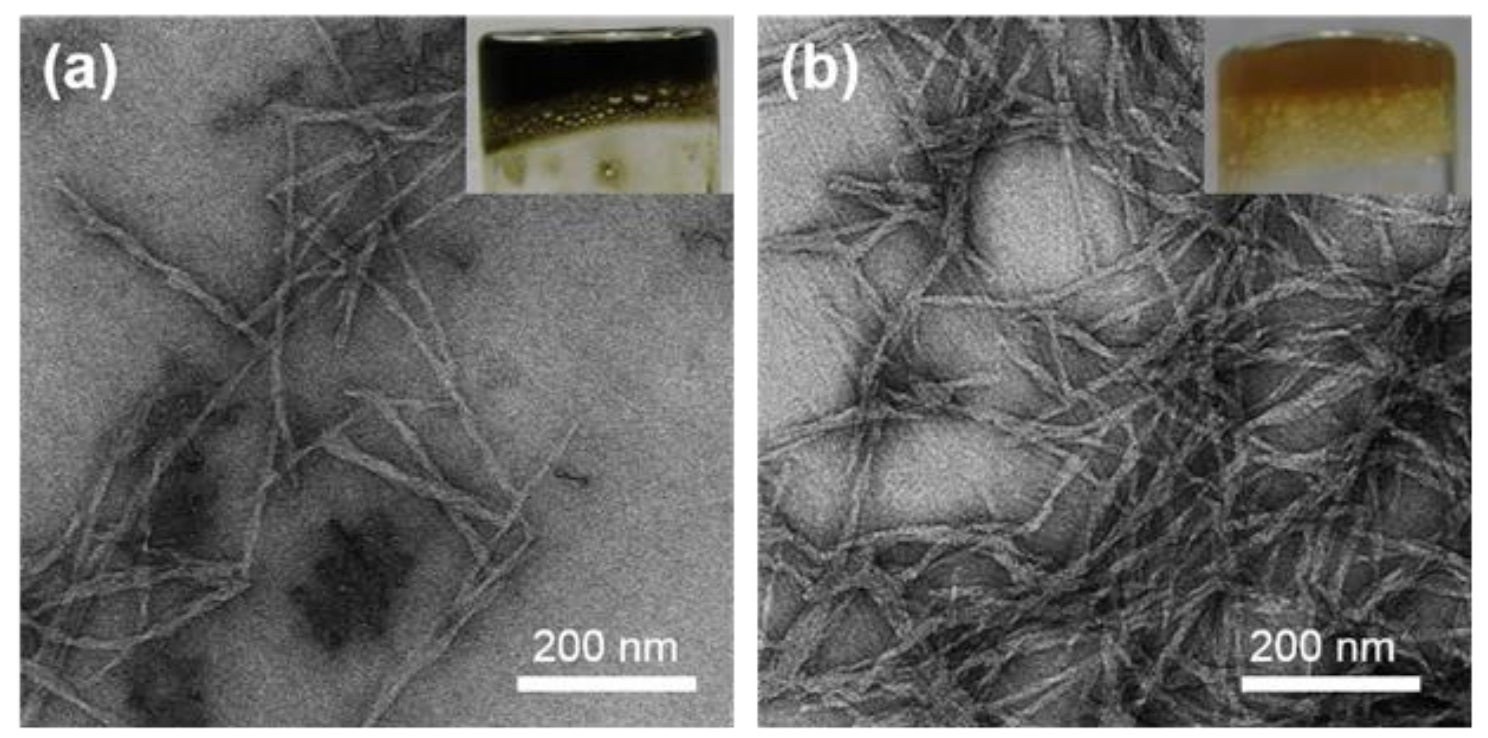

Figure SI_6.6.12. TEM images of the gel sample formed by NDI-Tyr-Phe- $\mathbf{N H}_{\mathbf{2}}(\mathbf{6 c})$ acceptor in the presence of either (a) 1,5-DHN donor or (b) 2,6-DHN donor. In each case, the formation of a dense network of micrometre-scale length helical nanofibres was observed. Inset: Digital photographs of the corresponding charge-transfer gel samples. 


\section{Product characterisation:}

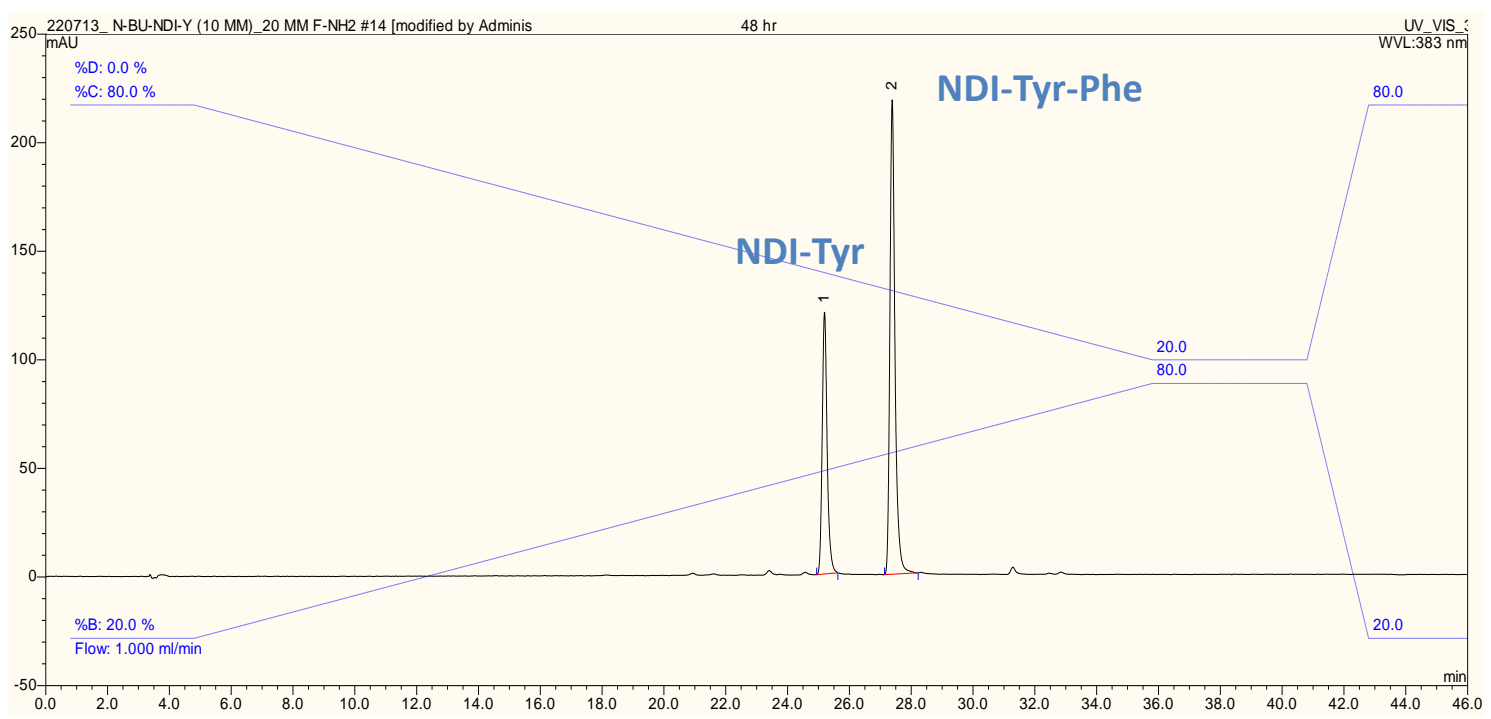

Figure SI_6.6.13. HPLC chromatogram showing the percentage conversion of the acceptor conjugate, NDITyr (6a) into NDI-Tyr-Phe- $\mathrm{NH}_{2}(\mathbf{6 c})$ upon the addition of thermolysin. The absorbance was monitored at 383 nm.

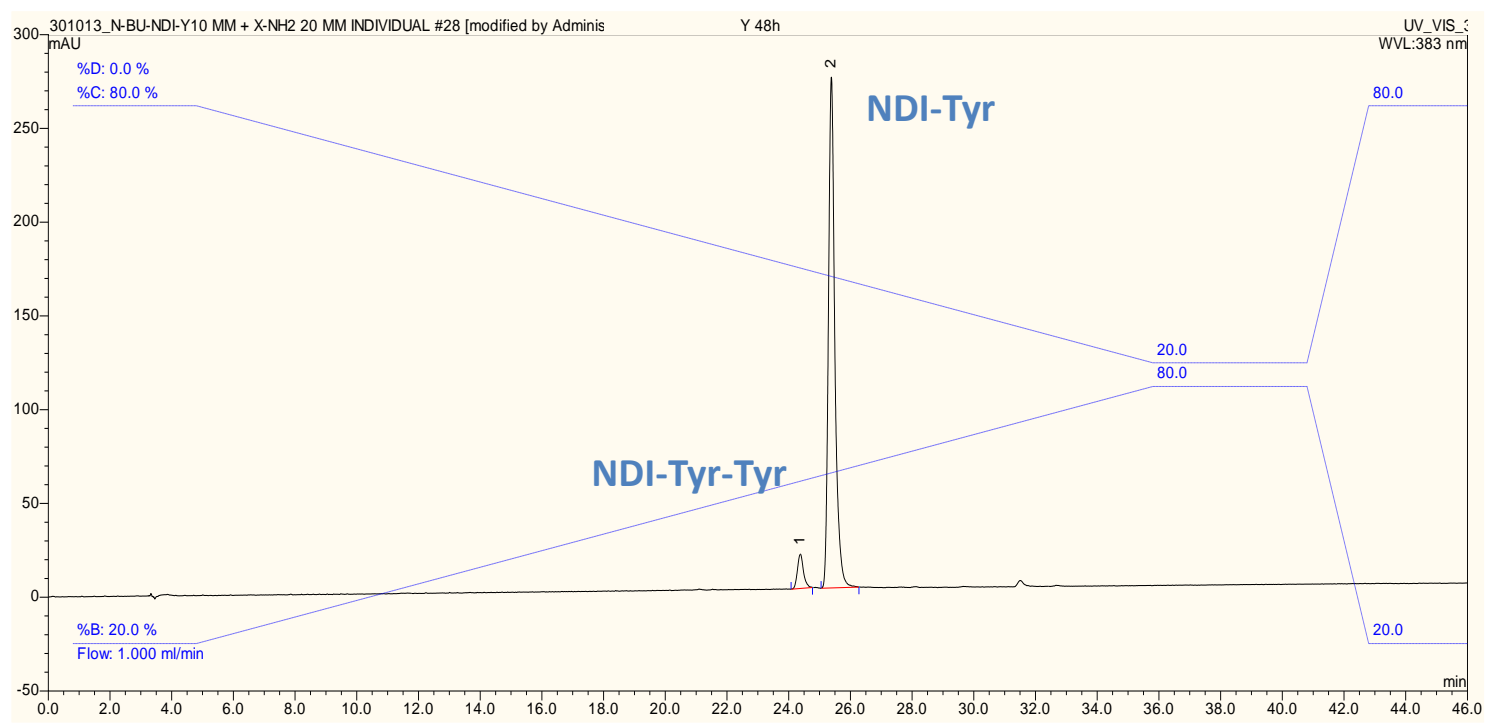

Figure SI_6.6.14. HPLC chromatogram showing the percentage conversion of the acceptor conjugate, NDITyr (6a) into NDI-Tyr-Tyr-NH 2 (6b) upon the addition of thermolysin. The absorbance was monitored at 383 $\mathrm{nm}$. 


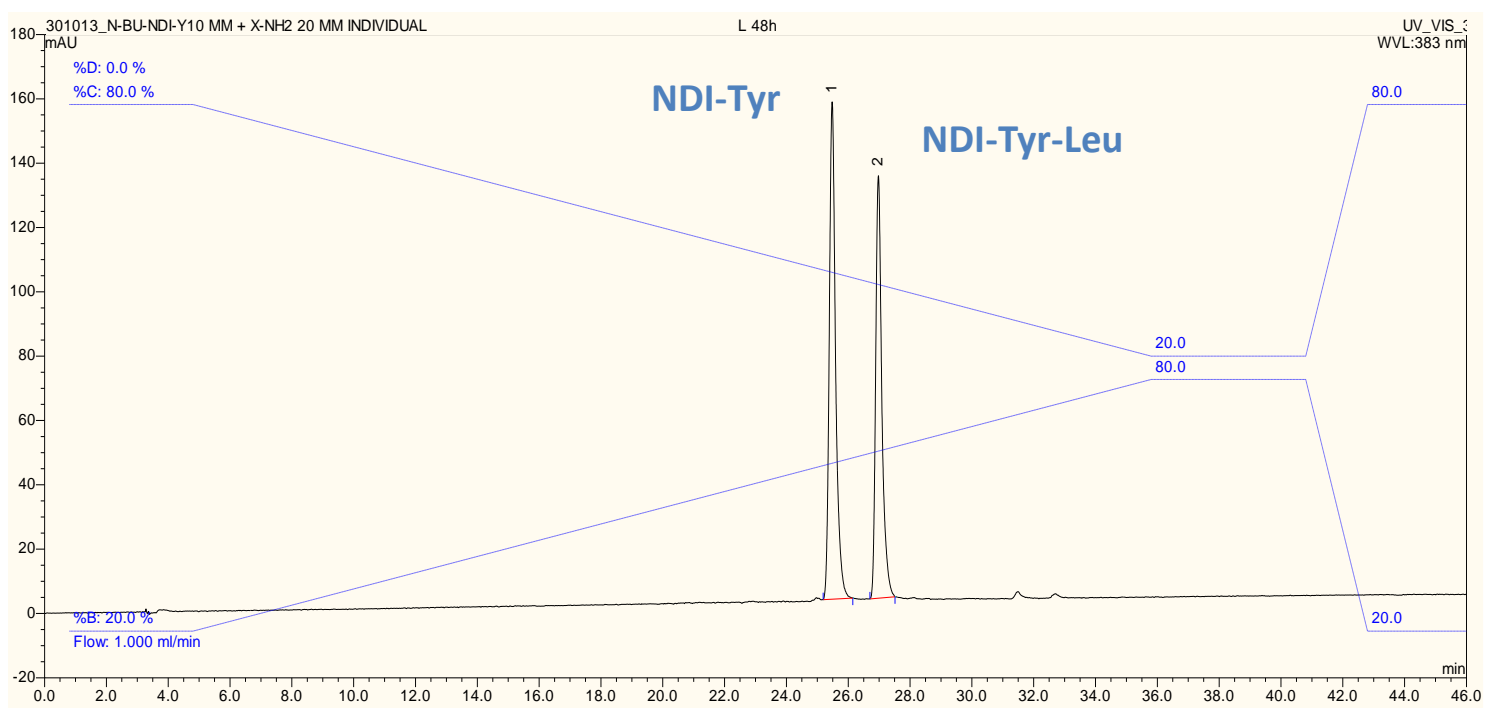

Figure SI_6.6.15. HPLC chromatogram showing the percentage conversion of the acceptor conjugate, NDITyr (6a) into NDI-Tyr-Leu- $\mathrm{NH}_{2}$ (6d) upon the addition of thermolysin. The absorbance was monitored at 383 $\mathrm{nm}$.

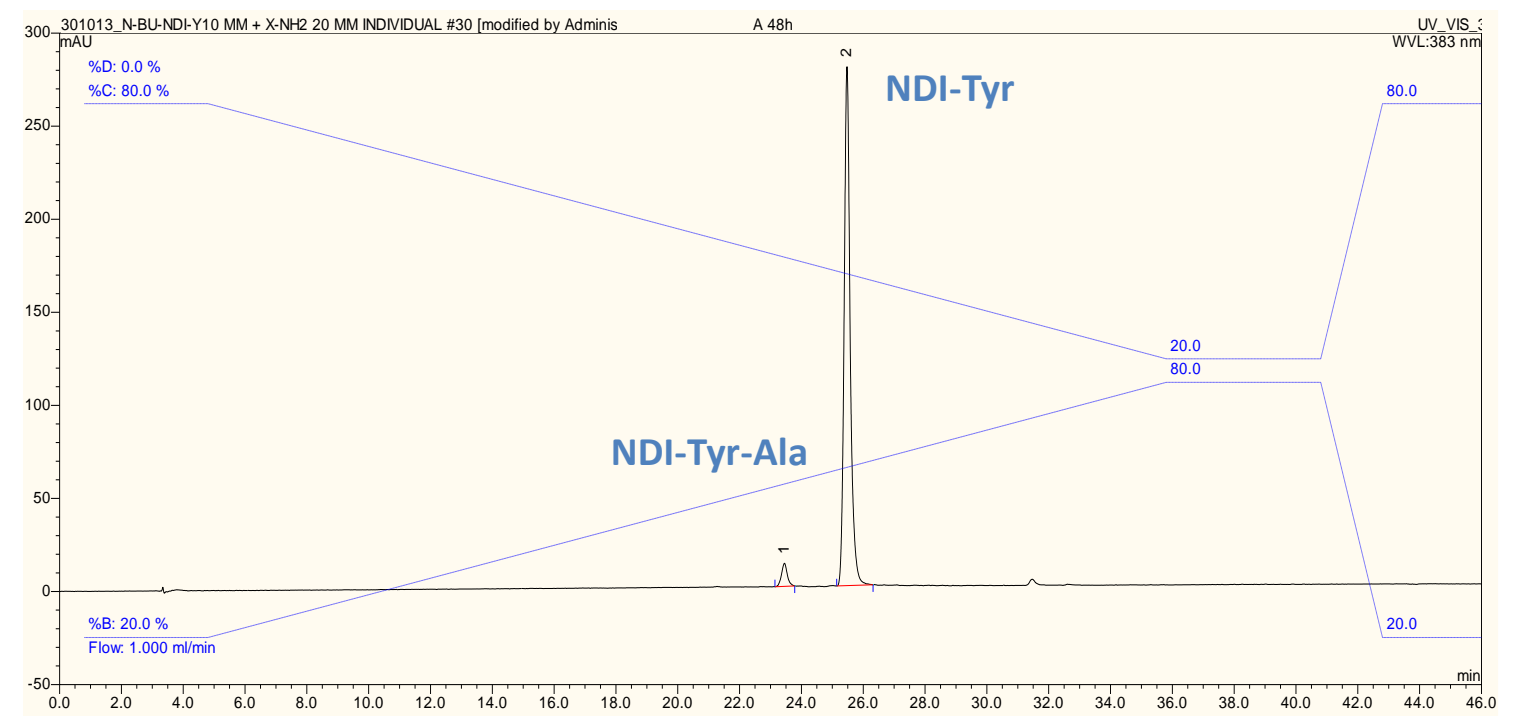

Figure SI_6.6.16. HPLC chromatogram showing the percentage conversion of the acceptor conjugate, NDITyr (6a) into NDI-Tyr-Ala- $\mathrm{NH}_{2}$ (6f) upon the addition of thermolysin. The absorbance was monitored at 383 $\mathrm{nm}$. 


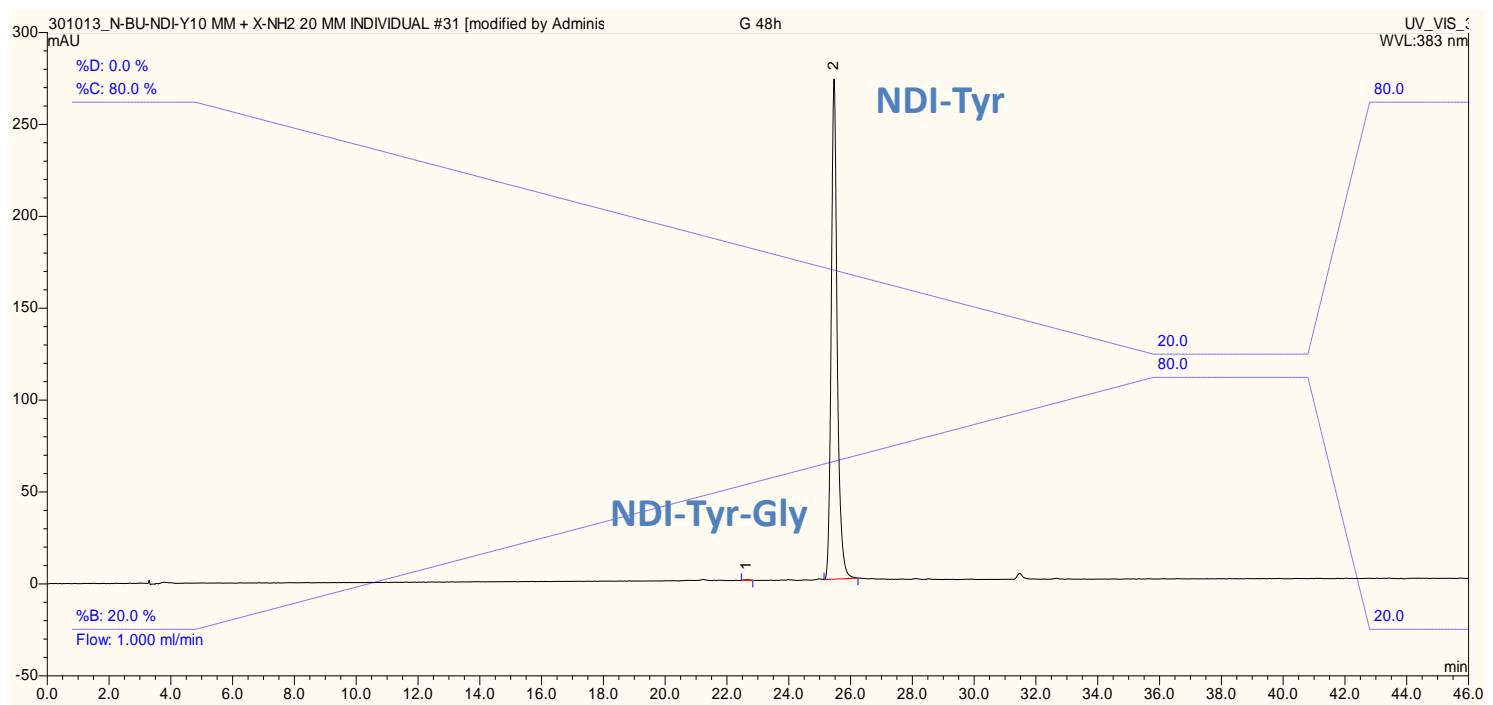

Figure SI_6.6.17. HPLC chromatogram showing the percentage conversion of the acceptor conjugate, NDITyr (6a) into NDI-Tyr-Gly- $\mathrm{NH}_{2}(\mathbf{6 g})$ upon the addition of thermolysin. The absorbance was monitored at 383 $\mathrm{nm}$. 


\subsection{References and Notes}

1. Facchetti, A. "Organic Semiconductors: Made to Order", Naural Mater., 2013, 12, 598600.

2. Forrest, S. R. "The Path to Ubiquitous and Low-Cost Organic Electronic Appliances on Plastic", Nature, 2004, 428, 911-918.

3. Meijer, E. W.; Schenning, A. P. H. J. "Chemistry: Material Marriage in Electronics", Nature, 2002, 419, 353-354.

4. Holdcroft, S. "Patterning $\pi$-Conjugated Polymers", Adv. Mater., 2001, 13, 1753-1765.

5. Cui, Y.; Wei, Q.; Park, H.; Lieber, C. M. "Nanowire Nanosensors for Highly Sensitive and Selective Detection of Biological and Chemical Species", Science, 2001, 293, 1289-1292.

6. Tans, S. J.; Verschueren, A. R. M.; Dekker, C. "Room-Temperature Transistor Based on a Single Carbon Nanotube", Nature, 1998, 393, 49-52.

7. Schenning, A. P.; Meijer, E. W. "Supramolecular Electronics; Nanowires from SelfAssembled pi-Conjugated Systems", Chem. Commun., 2005, 3245-3258.

8. Qin, Y.; Wang, X.; Wang, Z. L. "Microfibre-Nanowire Hybrid Structure for Energy Scavenging", Nature, 2008, 451, 809-813.

9. Merkoçi, A.; Pumera, M.; Llopis, X.; Pérez, B.; del Valle, M.; Alegret, S. "New Materials for Electrochemical Sensing VI: Carbon Nanotubes", Trends Anal. Chem., 2005, 24, 826-838.

10. Hoeben, F. J. M.; Jonkheijm, P.; Meijer, E. W.; Schenning, A. P. H. J. "About Supramolecular Assemblies of pi-Conjugated Systems", Chem. Rev., 2005, 105, 1491-1546.

11. (a) Diegelmann, S. R.; Gorham, J. M.; Tovar, J. D. "One-Dimensional Optoelectronic Nanostructures Derived from the Aqueous Self-Assembly of pi-Conjugated Oligopeptides", J. Am. Chem. Soc., 2008, 130, 13840-13841; (b) Xu, H. X.; Das, A. K.; Horie, M.; Shaik, M. S.; Smith, A. M.; Luo, Y.; Lu, X. F.; Collins, R.; Liem, S. Y.; Song, A. M.; Popelier, P. L. A.; Turner, M. L.; Xiao, P.; Kinloch, I. A.; Ulijn, R. V. "An Investigation of the Conductivity of Peptide Nanotube Networks Prepared by Enzyme-Triggered Self-Assembly", Nanoscale, 2010, 2, 960-966; (c) Torres, E.; Puigmarti-Luis, J.; Perez del Pino, A.; Ortuno, R. M.; Amabilino, D. B. "Use of Unnatural beta-Peptides as a Self-Assembling Component in Functional Organic Fibres", Org. Biomol. Chem., 2010, 8, 1661-1665.

12. Puigmarti-Luis, J.; Amabilino, D. B., "CHAPTER 7 Optic and Electronic Applications of Molecular Gels". In Functional Molecular Gels. RSC, Cambridge, 2014; pp 195-254.

13. (a) Puigmartí-Luís, J.; Laukhin, V.; Pérez del Pino, A.; Vidal-Gancedo, J.; Rovira, C.; Laukhina, E.; Amabilino, D. B. "Supramolecular Conducting Nanowires from Organogels", Angew. Chem. Int. Ed., 2007, 46, 238-241; (b) Hirst, A. R.; Escuder, B.; Miravet, J. F.; Smith, D. K. "High-Tech Applications of Self-Assembling Supramolecular Nanostructured GelPhase Materials: From Regenerative Medicine to Electronic Devices", Angew. Chem. Int. Ed., 2008, 47, 8002-8018; (c) Babu, S. S.; Prasanthkumar, S.; Ajayaghosh, A. "SelfAssembled Gelators for Organic Electronics", Angew. Chem. Int. Ed., 2012, 51, 1766-1776.

14. Kumar, M.; Rao, K. V.; George, S. J. "Supramolecular Charge Transfer Nanostructures", Phys. Chem. Chem. Phys., 2014, 16, 1300-1313. 
15. (a) Kistenmacher, T. J.; Phillips, T. E.; Cowan, D. O. "The Crystal Structure of the 1:1 Radical Cation-Radical Anion Salt of 2,2'-Bis-I,3-Dithiole (TTF) and 7,7,8,8Tetracyanoquinodimethane (TCNQ)", Acta Crystallogr., Sect. B, 1974, 30, 763-768; (b) Claessen, R.; Sing, M.; Schwingenschlögl, U.; Blaha, P.; Dressel, M.; Jacobsen, C. S. "Spectroscopic Signatures of Spin-Charge Separation in the Quasi-One-Dimensional Organic Conductor TTF-TCNQ", Phys. Rev. Lett., 2002, 88, 096402.

16. Das, A.; Ghosh, S. "Supramolecular Assemblies by Charge-Transfer Interactions between Donor and Acceptor Chromophores", Angew. Chem. Int. Ed., 2014, 53, 2038-2054.

17. Percec, V.; Glodde, M.; Bera, T. K.; Miura, Y.; Shiyanovskaya, I.; Singer, K. D.; Balagurusamy, V. S. K.; Heiney, P. A.; Schnell, I.; Rapp, A.; Spiess, H. W.; Hudson, S. D.; Duan, H. "Self-Organization of Supramolecular Helical Dendrimers into Complex Electronic Materials", Nature, 2002, 417, 384-387.

18. Alvey, P. M.; Reczek, J. J.; Lynch, V.; Iverson, B. L. "A Systematic Study of Thermochromic Aromatic Donor-Acceptor Materials", J. Org. Chem., 2010, 75, 7682-7690.

19. Au-Yeung, H. Y.; Pantos, G. D.; Sanders, J. K. M. "Dynamic Combinatorial Donor-Acceptor Catenanes in Water: Access to Unconventional and Unexpected Structures", J. Org. Chem., 2011, 76, 1257-1268.

20. Maitra, U.; Vijay Kumar, P.; Chandra, N.; J. D'Souza, L.; D. Prasanna, M.; R. Raju, A. "First Donor-Acceptor Interaction Promoted Gelation of Organic Fluids", Chem. Commun., 1999, 595-596.

21. Das, R. K.; Banerjee, S.; Raffy, G.; Del Guerzo, A.; Desvergne, J.-P.; Maitra, U. "Spectroscopic, Microscopic and First Rheological Investigations in Charge-Transfer Interaction Induced Organogels", J. Mater. Chem., 2010, 20, 7227-7235.

22. Bhosale, S. V.; Jani, C. H.; Langford, S. J. "Chemistry of Naphthalene Diimides", Chem. Soc. Rev., 2008, 37, 331-342.

23. Shao, H.; Seifert, J.; Romano, N. C.; Gao, M.; Helmus, J. J.; Jaroniec, C. P.; Modarelli, D. A.; Parquette, J. R. "Amphiphilic Self-Assembly of an n-Type Nanotube", Angew. Chem. Int. Ed., 2010, 49, 7688-7691.

24. Shao, H.; Nguyen, T.; Romano, N. C.; Modarelli, D. A.; Parquette, J. R. "Self-Assembly of 1D n-Type Nanostructures Based on Naphthalene Diimide-Appended Dipeptides", J. Am. Chem. Soc., 2009, 131, 16374-16376.

25. Shao, H.; Parquette, J. R. "A pi-Conjugated Hydrogel Based on an Fmoc-Dipeptide Naphthalene Diimide Semiconductor", Chem. Commun., 2010, 46, 4285-4287.

26. Basak, S.; Nanda, J.; Banerjee, A. "Assembly of Naphthalenediimide Conjugated Peptides: Aggregation Induced Changes in Fluorescence", Chem. Commun., 2013, 49, 6891-6893.

27. (a) Molla, M. R.; Ghosh, S. "Hydrogen-Bonding-Mediated Vesicular Assembly of Functionalized Naphthalene-Diimide-Based Bolaamphiphile and Guest-Induced Gelation in Water", Chem. Eur. J., 2012, 18, 9860-9869; (b) Das, A.; Ghosh, S. "Stimuli-Responsive Self-Assembly of a Naphthalene Diimide by Orthogonal Hydrogen Bonding and Its Coassembly with a Pyrene Derivative by a Pseudo-Intramolecular Charge-Transfer Interaction", Angew. Chem. Int. Ed., 2014, 53, 1092-1097. 
28. Mukhopadhyay, P.; Iwashita, Y.; Shirakawa, M.; Kawano, S.; Fujita, N.; Shinkai, S. "Spontaneous Colorimetric Sensing of the Positional Isomers of Dihydroxynaphthalene in a 1D Organogel Matrix", Angew. Chem. Int. Ed., 2006, 45, 1592-1595.

29. Li, J. W.; Nowak, P.; Otto, S. "Dynamic Combinatorial Libraries: From Exploring Molecular Recognition to Systems Chemistry", J. Am. Chem. Soc., 2013, 135, 9222-9239.

30. Otto, S.; Furlan, R. L. E.; Sanders, J. K. M. "Dynamic Combinatorial Chemistry", Drug Discov. Today, 2002, 7, 117-125.

31. (a) Williams, R. J.; Smith, A. M.; Collins, R.; Hodson, N.; Das, A. K.; Ulijn, R. V. "EnzymeAssisted Self-Assembly under Thermodynamic Control", Nat. Nanotechnol., 2009, 4, 1924; (b) Hughes, M.; Frederix, P. W. J. M.; Raeburn, J.; Birchall, L. S.; Sadownik, J.; Coomer, F. C.; Lin, I. H.; Cussen, E. J.; Hunt, N. T.; Tuttle, T.; Webb, S. J.; Adams, D. J.; Ulijn, R. V. "Sequence/Structure Relationships in Aromatic Dipeptide Hydrogels Formed under Thermodynamic Control by Enzyme-Assisted Self-Assembly", Soft Matter, 2012, 8, 55955602.

32. Moulin, E.; Cormos, G.; Giuseppone, N. "Dynamic Combinatorial Chemistry as a Tool for the Design of Functional Materials and Devices", Chem. Soc. Rev., 2012, 41, 1031-1049.

33. Sadownik, J. W.; Ulijn, R. V. "Dynamic Covalent Chemistry in Aid of Peptide SelfAssembly", Curr. Opin. Biotechnol., 2010, 21, 401-411.

34. Das, A. K.; Hirst, A. R.; Ulijn, R. V. "Evolving Nanomaterials Using Enzyme-Driven Dynamic Peptide Libraries (eDPL)", Faraday Discuss., 2009, 143, 293-303.

35. Lam, R. T.; Belenguer, A.; Roberts, S. L.; Naumann, C.; Jarrosson, T.; Otto, S.; Sanders, J. K. "Amplification of Acetylcholine-Binding Catenanes from Dynamic Combinatorial Libraries", Science, 2005, 308, 667-669.

36. Au-Yeung, H. Y.; Pengo, P.; Pantos, G. D.; Otto, S.; Sanders, J. K. M. "Templated Amplification of a Naphthalenediimide-Based Receptor from a Donor-Acceptor Dynamic Combinatorial Library in Water", Chem. Commun., 2009, 419-421.

37. Cooper, H. J.; Case, M. A.; McLendon, G. L.; Marshall, A. G. "Electrospray Ionization Fourier Transform Ion Cyclotron Resonance Mass Spectrometric Analysis of Metal-Ion Selected Dynamic Protein Libraries", J. Am. Chem. Soc., 2003, 125, 5331-5339.

38. Yang, Z.; Liang, G.; Xu, B. "Enzymatic Hydrogelation of Small Molecules", Acc. Chem. Res., 2008, 41, 315-326.

39. Yang, Z.; Liang, G.; Xu, B. "Enzymatic Control of the Self-Assembly of Small Molecules: a New Way to Generate Supramolecular Hydrogels", Soft Matter, 2007, 3, 515-520.

40. Debnath, S.; Ulijn, R. V., "CHAPTER 4 Enzyme-Responsive Molecular Gels". In Functional Molecular Gels. RSC, Cambridge, 2014; pp 95-116.

41. Ulijn, R. V. "Enzyme-Responsive Materials: a New Class of Smart Biomaterials", J. Mater. Chem., 2006, 16, 2217-2225.

42. Williams, R. J.; Mart, R. J.; Ulijn, R. V. "Exploiting Biocatalysis in Peptide Self-Assembly", Biopolymers, 2010, 94, 107-117. 
43. Roy, S.; Ulijn, R. V., "Exploiting Biocatalysis in the Synthesis of Supramolecular Polymers, Enzymatic Polymerization". In Advances in Polymer Science, 2010; Vol. 237.

44. Yang, Z.; Liang, G.; Ma, M.; Gao, Y.; Xu, B. "In Vitro and In Vivo Enzymatic Formation of Supramolecular Hydrogels Based on Self-Assembled Nanofibers of a $\beta$-Amino Acid Derivative", Small, 2007, 3, 558-562.

45. Klibanov, A. M. "Improving Enzymes by Using Them in Organic Solvents", Nature, 2001, 409, 241-246.

46. Ulijn, R. V.; De Martin, R.; Gardossi, L.; Halling, P. J. "Biocatalysis in Reaction Mixtures with Undissolved Solid Substrates and Products", Curr. Org. Chem., 2003, 7, 1333-1346.

47. Halling, P. J.; Ulijn, R. V.; Flitsch, S. L. "Understanding Enzyme Action on Immobilised Substrates", Curr. Opin. Biotechnol., 2005, 16, 385-392.

48. Horne, W. S.; Ashkenasy, N.; Ghadiri, M. R. "Modulating Charge Transfer through Cyclic D,L- $\alpha$-Peptide Self-Assembly", Chem. Eur. J., 2005, 11, 1137-1144.

49. Nalluri, S. K. M.; Ulijn, R. V. "Discovery of Energy Transfer Nanostructures Using GelationDriven Dynamic Combinatorial Libraries", Chem. Sci., 2013, 4, 3699-3705.

50. In the case of Valine derivative (6e), the retention times/peaks in the HPLC for the reactant (unreacted NDI-Tyr (6a)) and the product (NDI-Tyr-Val-NH $\mathbf{N}_{2}, 6 \mathbf{6 e}$ ) are superimposed using the HPLC established conditions. Thus, the conversion of such derivative could not be determined and hence we assumed that the formation of the NDITyr-Val- $\mathrm{NH}_{2}(6 \mathrm{e})$ in the library is clearly negligible

51. (a) Lokey, R. S.; Iverson, B. L. "Synthetic Molecules That Fold Into a Pleated Secondary Structure in Solution", Nature, 1995, 375, 303-305; (b) Das, A.; Molla, M. R.; Banerjee, A.; Paul, A.; Ghosh, S. "Hydrogen-Bonding Directed Assembly and Gelation of Donor-Acceptor Chromophores: Supramolecular Reorganization from a Charge-Transfer State to a SelfSorted State", Chem. Eur. J., 2011, 17, 6061-6066.

52. Higher concentrations could not be measured as the absorption of the charge-transfer band is saturated

53. (a) Andric, G.; Boas, J. F.; Bond, A. M.; Fallon, G. D.; Ghiggino, K. P.; Hogan, C. F.; Hutchison, J. A.; Lee, M. A. P.; Langford, S. J.; Pilbrow, J. R.; Troup, G. J.; Woodward, C. P. "Spectroscopy of Naphthalene Diimides and Their Anion Radicals", Aust. J. Chem., 2004, 57, 1011-1019; (b) Bell, T. D. M.; Bhosale, S. V.; Forsyth, C. M.; Hayne, D.; Ghiggino, K. P.; Hutchison, J. A.; Jani, C. H.; Langford, S. J.; Lee, M. A. P.; Woodward, C. P. "Melt-Induced Fluorescent Signature in a Simple Naphthalenediimide", Chem. Commun., 2010, 46, 48814883.

54. (a) Hong, Y.; Lam, J. W. Y.; Tang, B. Z. "Aggregation-Induced Emission: Phenomenon, Mechanism and Applications", Chem. Commun., 2009, 4332-4353; (b) Hong, Y.; Lam, J. W. Y.; Tang, B. Z. "Aggregation-Induced Emission", Chem. Soc. Rev., 2011, 40, 5361-5388.

55. The gel-sol transition was observed clearly only for the 1,5-DAN system, the systems with other donors formed a viscous liquid/gel phase

56. Gawroński, J.; Brzostowska, M.; Kacprzak, K.; Kołbon, H.; Skowronek, P. "Chirality of Aromatic Bis-Imides from their Circular Dichroism Spectra", Chirality, 2000, 12, 263-268. 
57. Shao, H.; Gao, M.; Kim, S. H.; Jaroniec, C. P.; Parquette, J. R. "Aqueous Self-Assembly of LLysine-Based Amphiphiles into 1D n-Type Nanotubes", Chem. Eur. J., 2011, 17, 1288212885.

58. Molla, M. R.; Das, A.; Ghosh, S. "Chiral Induction by Helical Neighbour: Spectroscopic Visualization of Macroscopic-Interaction Among Self-Sorted Donor and Acceptor piStacks", Chem. Commun., 2011, 47, 8934-8936. 


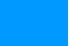




\subsection{Conclusions}

Several amphiphile and bolaamphiphlile peptide derivatives have been designed and synthesised following the model structure previously used as a gelator. In this way, a modular approach lets us introduce small modifications and functional groups to the structure in an effective way through a simple synthetic methodology. The synthetic routes based on peptide synthesis in solution allowed obtaining the desired products in good yields in grams scale without the need for complex purification methods.

Four proline derived dipeptidic compounds (3a-d) (differing on the central amino acid) have been prepared and their aggregation process studied in toluene. The results showed different molecular preferences on the disposition of the proline ring and conformational changes upon aggregation. This inspired a study of a gelator bolaamphiphile analogue (3e). An exhaustive study of its gelation behaviour in toluene and in water was carried out and its properties have been analysed from a structural and thermodynamic point of view. Additionally, its gelation ability in the presence of other gelator molecules (pyridine derivative (5e) in water) has been analysed.

With the formation of functional supramolecular gels in mind, several L-proline derivatives (3a-d) have been tested as efficient organocatalysts for the 1,4-conjugate addition of cyclohexanone to trans- $\beta$-nitrostyrene in toluene. In all the cases the activation of the catalytic activity was reached upon aggregation. The results highlight the importance of minor changes on the molecular structure of the catalyst, associated with differences in conformational arrangement of the aggregated molecules that lead to differences in catalytic activity. Finally, the catalytic activity was tested in gel-phase. The involvement of the fibrillar network on the catalytic activity was demonstrated and results showed quantitative conversion and moderate enantioselectivity.

On the other hand, the gelation behaviour of a proline amphiphile derivative (4a) has been studied in water. A polymorphic effect has been found and a full characterisation of the different structural packing arrangement upon very slight modifications was done with 4 polymorphic structures found. Additionally, the catalytic activity of this amphiphilic proline derivative (4a) was tested "on water" (the solubilisation of the reactants is not required). The different polymorphs found were 
tested with the benchmark direct aldol reaction of cyclohexanone with 4nitrobenzaldehyde. Small differences were found in the selectivity and in the reaction rates highlighting the importance of the structure/activity relationship of the supramolecular assemblies. A further study with this hydrogelator as a catalyst with several aliphatic ketones showed that its aggregation is driven by the hydrophobic effect causing the emergence of the catalytic behaviour, thus a selection of the most hydrophobic ketones to react in its active site has been demonstrated. Moreover, the enamine intermediate was found and analysed in order to gain a better understanding of the proline catalysed mechanism in gel-phase. Overall these results encourage the study of molecular gels as a new type of smart soft-materials to obtain novel highly selective catalysts that mimic the efficiency and the selectivity of natural catalysts, enzymes.

To continue with the design of functional smart materials, the introduction of stimuli-responsive groups in the molecular structure was investigated. In this case a family of compounds with ionisable groups were synthesised (5a-d), all of them bearing at least one succinic acid moiety susceptible to ionisation, and the acid-base equilibrium analysed. Some differences in the acid dissociation constant related with the aggregation ability have been found. Interestingly, the formation of self-assembled structures by charged species in solution has been suggested. Overall this study underlines the importance of a full characterisation of such materials with the aim to design smart $\mathrm{pH}$ responsive materials. The increase of the complexity in the system was then studied with the idea of finely tuning the properties of the gel system by the introduction of more than one gelator molecule with different $\mathrm{pH}$-responsive groups. A two-component hydrogelator has been designed by mixing the proline derivative (3e) with a pyridine derivative (5e) compound. Interestingly, the solubilisation of the pyridine derivative gel network by means of the presence of charged self-assembled micelles and its participation in possible mixed micelles has been hypothesised. As a result a system with differences in aggregating behaviour as a function of $\mathrm{pH}$ has been built and its use as a possible release system has been tested.

Finally, and following the same idea to construct functional materials, a new approach has been employed. Peptide-based functional dynamic combinatorial libraries (DCLs) have been successfully developed for selection and amplification of the most 
stable naphthalenediimide (NDI) self-assembled structure for its use as a material with electronic properties. Thus the formation of hydrogels in situ through the self-assembly of NDI-dipeptides (6b-g) via a fully reversible thermolysin-catalysed amide condensation has been demonstrated, with a full characterisation of the system carried out. Moreover, the effect of charge-transfer interactions has been analysed inside the DCL with the incorporation of suitable donors to the library. Results showed that the presence of additional charge-transfer interactions enhanced the amplification of the most stable functional peptide nanostructures, with the best donor being 1,5-DAN for the best acceptor structure NDI-Tyr-Phe- $\mathbf{N H}_{2}(6 \mathrm{c})$, where their co-assembly formed the most stable, highly ordered 1D functional aqueous charge-transfer peptide hydrogel nanostructure. It has been demonstrated that $\mathrm{DCL}$ is an effective tool to open up new possibilities for the discovery of functionalised nanomaterials.

To conclude, in this work a family of different compounds have been designed and synthesised using the rational modular approach, where a functional group has been introduced to the gelator molecule, as well as a combinatorial approach, by means of DCLs, in order to build functional gel-phase materials. Moreover, a better understanding of the aggregation process of these molecules has been gained to be used in future work. 



\section{CHAPTER 8}

\section{EXPERIMENTAL SECTION}





\subsection{General Remarks}

All solvents were used as HPLC quality. Otherwise stated, all the reactions were carried out under $\mathrm{N}_{2}$ atmosphere. All chemicals were commercially available products with purity higher than $98 \%$. All compounds synthesised were dried in a vacuum oven at $60 \stackrel{\circ}{C}$ to constant weight. NMR spectra were recorded at $500 \mathrm{MHz}, 300 \mathrm{MHz}\left({ }^{1} \mathrm{H} \mathrm{NMR}\right)$ and $125 \mathrm{MHz}, 75 \mathrm{MHz}\left({ }^{13} \mathrm{C} \mathrm{NMR}\right)$ in different solvents at $30 \stackrel{\circ}{ } \mathrm{C}$ with the solvent signals as internal reference. Mass spectra were run in the electrospray (ESI-MS) mode.

\subsection{Synthesis and Characterisation of New Compounds}

\subsubsection{Synthesis of Proline Derivatives}

a) Procedure for the synthesis of compounds 3a-d propyl derivatives

Compound ProValPr (3b)

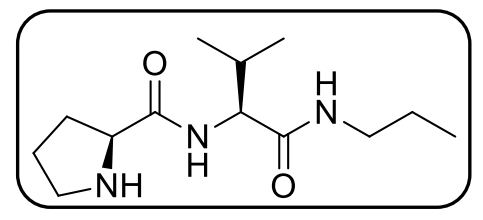

Compound ProValPr (3b) was synthesised as reported previously and both ${ }^{1} \mathrm{H}$ RNM and ${ }^{13} \mathrm{C}-\mathrm{NMR}$ were in good agreement with the literature. ${ }^{1}$

\section{Synthesis of compounds $\mathbf{3 . 1}$}

\section{Compound 3c.1}

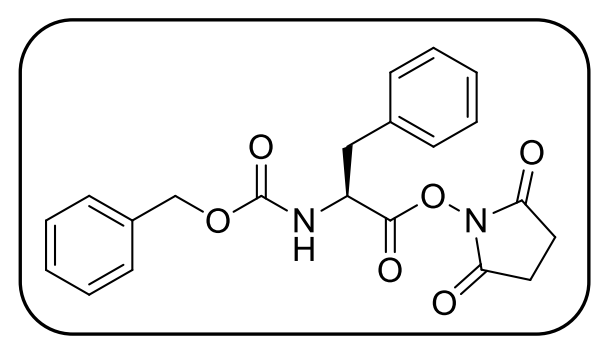

$\mathrm{N}$-Cbz-L-phenylalanine $(5.21 \mathrm{~g}, 16.4 \mathrm{mmol})$ and $\mathrm{N}$-hydroxysuccinimide $(1.97 \mathrm{~g}, 16.9$ $\mathrm{mmol}$ ) were dissolved in dry $\operatorname{THF}(50 \mathrm{~mL})$ at $0^{\circ} \mathrm{C}$. Once a clear solution had been obtained, $N, N^{\prime}$-dicyclohexylcarbodiimide (DCC) $(3.62 \mathrm{~g}, 17.3 \mathrm{mmol})$ in anhydrous THF (10 mL) was added in several aliquots and the resulting solution was stirred at $0-5^{\circ} \mathrm{C}$ for $3 \mathrm{~h}$. The 
dicyclohexylurea formed was filtered off and the filtrate was concentrated to dryness. The crude product was recrystallised from 2-propanol to furnish the pure product crystals (yield 93\%). Compound 3c.1 was previously described in literature and both ${ }^{1} \mathrm{H}$ and ${ }^{13} \mathrm{C}$ NMR spectra were in good agreement with the literature spectra. ${ }^{2}$

\section{Compound 3d.1}

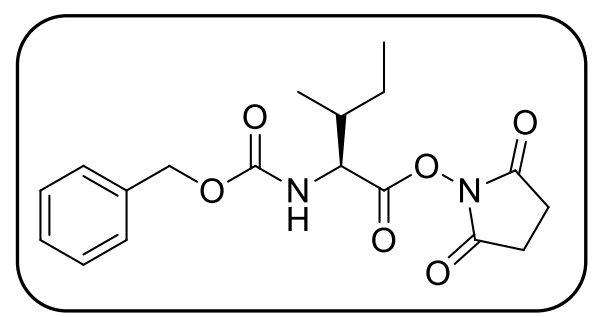

A similar procedure to that described for 3c.1 was used. Pure crystals were obtained (yield 89\%). ${ }^{1} \mathrm{H}$ NMR (300 MHz, $\mathrm{D}_{6}$-DMSO) $\delta$ (ppm): 8.03 (d, $J=8.1 \mathrm{~Hz}, 1 \mathrm{H}$ ), 7.35 $(\mathrm{m}, 5 \mathrm{H}), 5.07(\mathrm{~s}, 2 \mathrm{H}), 4.41-4.28(\mathrm{~m}, 1 \mathrm{H}), 2.80(\mathrm{~s}, 4 \mathrm{H}), 2.49(\mathrm{~m}, 4 \mathrm{H}), 1.90(\mathrm{~m}, 1 \mathrm{H}), 1.59-$ $1.45(\mathrm{~m}, 1 \mathrm{H}), 1.37-1.17(\mathrm{~m}, 1 \mathrm{H}), 0.97(\mathrm{~d}, J=6.8 \mathrm{~Hz}, 3 \mathrm{H}), 0.85(\mathrm{t}, J=7.3 \mathrm{~Hz}, 3 \mathrm{H}) ;{ }^{13} \mathrm{C} \mathrm{NMR}$ (75 MHz, $\mathrm{D}_{6}$-DMSO) $\delta$ (ppm): 170.3, 168.2, 156.5, 137.1, 128.8, 128.3, 128.2, 66.2, 57.5, 36.8, 25.9, 24.9, 15.3, 11.4; (ESI-TOF, positive mode) $\mathrm{m} / \mathrm{z}$ exp $[\mathrm{M}+\mathrm{Na}]^{+}$calcd for $\mathrm{C}_{18} \mathrm{H}_{22} \mathrm{~N}_{2} \mathrm{NaO}_{6}^{+} 385.1370$; found, 385.1381 .

\section{Synthesis of compounds $\mathbf{3 . 2}$}

\section{Compound 3a.2}

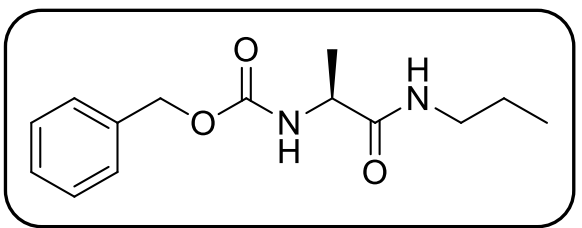

To an ice-cooled solution of the corresponding $\mathrm{N}$-Cbz-L-alanine $(5.03 \mathrm{~g}, 22.5 \mathrm{mmol})$ and triethylamine $(3.65 \mathrm{~mL}, 26.3 \mathrm{mmol})$ in THF $(40 \mathrm{~mL})$, a solution of ethyl chloroformate (2.00 mL, $25.7 \mathrm{mmol})$ in THF $(10 \mathrm{~mL})$ was added dropwise with vigorous stirring, a white precipitate was observed. After $30 \mathrm{~min}$ stirring in an ice-cold bath, a solution of propylamine $(2.15 \mathrm{~mL}, 26.1 \mathrm{mmol})$ in THF $(10 \mathrm{~mL})$ was added. The mixture was stirred at 0 o $\mathrm{C}$ for $1 \mathrm{~h}$ and was left overnight at room tempertature. The resulting white solid solution was filtered and the solvent was evaporated under vacuum. The resulting viscous solid 
was dissolved in dichloromethane $(20 \mathrm{~mL})$ and washed with $\mathrm{HCl} 0.1 \mathrm{M}(3 \times 20 \mathrm{~mL}), \mathrm{KOH}$ $0.1 \mathrm{M}(3 \times 20 \mathrm{~mL}), \mathrm{NaHCO}_{3}(1 \times 20 \mathrm{~mL})$ and water $(1 \times 20 \mathrm{~mL})$. The organic phase was dried with magnesium sulfate anhydrous and the solvent was evaporated under vacuum. The solid obtained was purified by column chromatography (silica gel, hexane/ ethyl acetate; 1:1). A white solid compound was obtained (yield 35\%). ${ }^{1} \mathrm{H}$ NMR (300 MHz, $\left.\mathrm{D}_{6}-\mathrm{DMSO}\right) \delta$ (ppm): $7.76(\mathrm{~s}, 1 \mathrm{H}), 7.45-7.15(\mathrm{~m}, 5 \mathrm{H}), 7.15(\mathrm{~m}, 1 \mathrm{H}), 4.99(\mathrm{~s}, 2 \mathrm{H}), 3.98(\mathrm{~m}, 1 \mathrm{H}), 3.11-$ $2.85(\mathrm{~m}, 2 \mathrm{H}), 1.50-1.30(\mathrm{~m}, 2 \mathrm{H}), 1.18(\mathrm{~d}, J=7.1 \mathrm{~Hz}, 3 \mathrm{H}), 0.81(\mathrm{t}, J=7.4 \mathrm{~Hz}, 3 \mathrm{H}) ;{ }^{13} \mathrm{C} \mathrm{NMR}$ (75 MHz, D 6 -DMSO) $\delta(p p m): 172.6,156.0,137.5,128.7,128.1,65.7,50.5,40.6,22.7,18.8$, 11.7; (ESI-TOF, positive mode) $\mathrm{m} / z$ exp $[\mathrm{M}+\mathrm{Na}]^{+}$calcd for $\mathrm{C}_{14} \mathrm{H}_{20} \mathrm{~N}_{2} \mathrm{O}_{3} \mathrm{Na}^{+} 287.1366$; found, 287.1372 .

\section{Compound 3c.2}

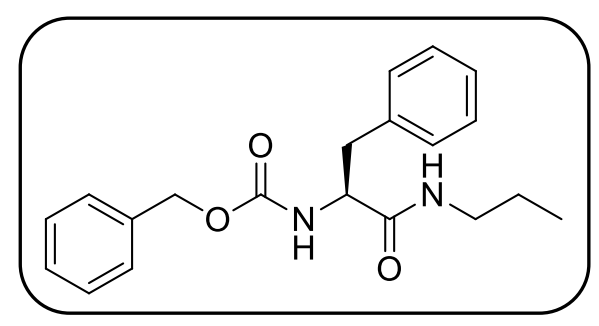

The $N$-hydroxysuccinimide ester 3c.1, (6.09 g, $15.3 \mathrm{mmol})$ was dissolved in DME $(100 \mathrm{~mL})$. Propylamine $(1.0 \mathrm{~g}, 16.9 \mathrm{mmol})$ dissolved in DME $(20 \mathrm{~mL})$ was added dropwise and the resulting solution was stirred at room temperature for 18 hours and then was warmed for 2 hours at 40-50 ㄷ. The solvent was evaporated under vacuum. The resulting solid was dissolved in dichloromethane $(25 \mathrm{~mL})$ and washed three times with $\mathrm{HCl} 0.1 \mathrm{M} \mathrm{(3}$ $\times 25 \mathrm{~mL}$ ) and water $(3 \times 25 \mathrm{~mL})$. The organic phase was dried with magnesium sulfate anhydrous and the solvent was evaporated under vacuum. A white solid was obtained

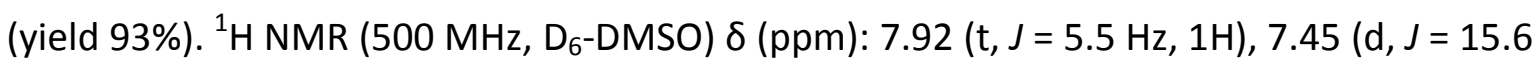
$\mathrm{Hz}, 1 \mathrm{H}), 7.38-7.10(\mathrm{~m}, 10 \mathrm{H}), 4.99-4.89(\mathrm{~s}, 2 \mathrm{H}), 4.21(\mathrm{~m}, 1 \mathrm{H}), 3.10-2.86(\mathrm{~m}, 3 \mathrm{H}), 2.77$ $(\mathrm{m}, 1 \mathrm{H}), 1.45-1.30(\mathrm{~m}, 2 \mathrm{H}), 0.80(\mathrm{t}, J=7.4 \mathrm{~Hz}, 3 \mathrm{H}) .{ }^{13} \mathrm{C}$ NMR (126 MHz, $\mathrm{D}_{6}$-DMSO) $\delta$ (ppm): 171.5, 156.2, 138.5, 137.5, 129.6, 128.7, 128.4, 128.0, 127.8, 126.6, 65.6, 56.7, 40.7, 38.2, 22.7, 11.7. (ESI-TOF, positive mode) $\mathrm{m} / \mathrm{z} \exp [\mathrm{M}+\mathrm{H}]^{+}$calcd for $\mathrm{C}_{20} \mathrm{H}_{25} \mathrm{~N}_{2} \mathrm{O}_{3}{ }^{+}$ 341.1865 ; found, 341.1860 . 


\section{Compound 3d.2}

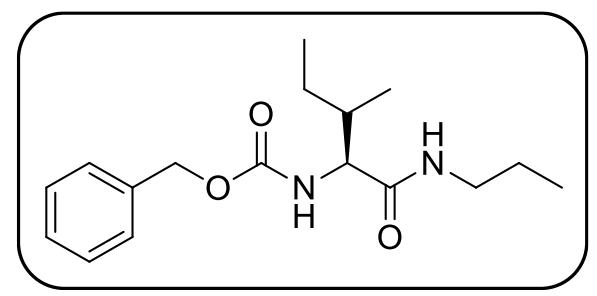

A similar procedure to that described for $\mathbf{3 c . 2}$ was used starting from the $\mathrm{N}$ hydroxysuccinimide ester of $\mathrm{N}$-Cbz-isoleucine (3d.1). A white solid was obtained (yield 84\%). ${ }^{1} \mathrm{H}$ NMR (300 MHz, D -DMSO) $\delta$ (ppm): 7.85 (s, 1H), $7.32(\mathrm{~m}, 5 \mathrm{H}), 7.18$ (d, J = $8.4 \mathrm{~Hz}$, $1 \mathrm{H}), 5.01(\mathrm{~s}, 2 \mathrm{H}), 3.80(\mathrm{t}, J=8.1 \mathrm{~Hz}, 1 \mathrm{H}), 3.11-2.86(\mathrm{~m}, 2 \mathrm{H}), 1.66(\mathrm{~s}, 1 \mathrm{H}), 1.38(\mathrm{~m}, 3 \mathrm{H}), 1.03$ (m, 1H), $0.95-0.66(\mathrm{~m}, 9 \mathrm{H}) ;{ }^{13} \mathrm{C}$ NMR (126 MHz, D $\left.-\mathrm{DMSO}\right) \delta$ (ppm): 171.4, 156.4, 137.5, 128.7, 128.1, 128.0, 65.7, 59.7, 40.6, 36.7, 24.8, 22.6, 15.8, 11.8, 11.3; (ESI-TOF, positive mode) $m / z \exp [\mathrm{M}+\mathrm{Na}]^{+}$calcd for $\mathrm{C}_{17} \mathrm{H}_{26} \mathrm{~N}_{2} \mathrm{NaO}_{3}{ }^{+} 329.1836$; found, 329.1840 .

\section{Synthesis of compounds $\mathbf{3 . 3}$}

\section{Compound 3a.3}

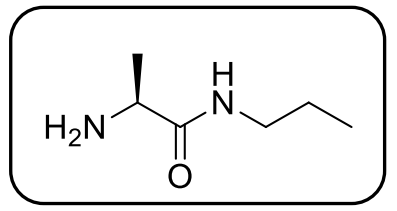

The corresponding $N$-benzyloxycarbonyl protected derivative $(0.48 \mathrm{~g}, 2.15 \mathrm{mmol})$ and a catalytic amount of Pd over activated carbon $(5-10 \% \mathrm{w} / \mathrm{w})$ were placed in a two necked round bottom flask and suspended in $\mathrm{MeOH}(50 \mathrm{~mL})$. The system was purged to remove the air with $\mathrm{N}_{2}$ and connected to $\mathrm{H}_{2}$ atmosphere. The grey suspension was stirred for several hours until it turned completely black (also checked with TLC, $\mathrm{MeOH}: \mathrm{CH}_{2} \mathrm{Cl}_{2}$ (1:4) and revealed with ninhydrin). The black suspension was filtered over Celite and the solvent was evaporated under reduced pressure. The resulting oil was dried in vacuum pump for 24 hours (yield 86\%). ${ }^{1} \mathrm{H}$ NMR (300 MHz, $\mathrm{D}_{6}$-DMSO) $\delta$ (ppm): $7.72(\mathrm{~s}, 1 \mathrm{H}), 3.20$ (dd, $J=13.7,6.9 \mathrm{~Hz}, 1 \mathrm{H}), 3.00(\mathrm{dd}, J=13.6,6.5 \mathrm{~Hz}, 2 \mathrm{H}), 1.52-1.27(\mathrm{~m}, 2 \mathrm{H}), 1.09$ (d, J = 6.9 $\mathrm{Hz}, 3 \mathrm{H}), 0.82$ (t, $J=7.4 \mathrm{~Hz}, 3 \mathrm{H}) ;{ }^{13} \mathrm{C}$ NMR (75 MHz, D $\left.\mathrm{D}_{6} \mathrm{DMSO}\right) \delta$ (ppm): 176.0, 50.7, 40.4, 22.8, 22.1, 11.7; (ESI-TOF, positive mode) $\mathrm{m} / \mathrm{z} \exp [\mathrm{M}+\mathrm{H}]^{+}$calcd for $\mathrm{C}_{6} \mathrm{H}_{15} \mathrm{~N}_{2} \mathrm{O}^{+} 131.1179$; found, 131.1181 . 


\section{Compound 3c.3}

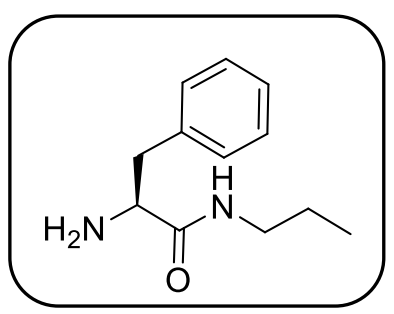

A similar procedure to that described for $\mathbf{3 a . 3}$ was used. A yellow oil was obtained (yield 96\%). ${ }^{1} \mathrm{H}$ NMR (300 MHz, $\mathrm{D}_{6}$-DMSO) $\delta$ (ppm): $7.74(\mathrm{~s}, 1 \mathrm{H}), 7.41-6.98(\mathrm{~m}, 5 \mathrm{H}), 3.39$ (m, 1H), $3.11-2.80(\mathrm{~m}, 3 \mathrm{H}), 2.60(\mathrm{dd}, J=13.3,8.0 \mathrm{~Hz}, 1 \mathrm{H}), 1.61(\mathrm{~s}, 1 \mathrm{H}), 1.34(\mathrm{~h}, J=7.2 \mathrm{~Hz}$, 2H), 0.77 (t, $J=7.4 \mathrm{~Hz}, 3 \mathrm{H}$ ); ${ }^{13} \mathrm{C}$ NMR (126 MHz, $\mathrm{D}_{6}$-DMSO) $\delta$ (ppm): 174.5, 139.2, 129.7, $128.4,126.4,56.7,41.7,40.5,22.7,11.7$; (ESI-TOF, positive mode) $\mathrm{m} / \mathrm{z} \exp \left[\mathrm{M}+\mathrm{H}^{+}\right.$calcd for $\mathrm{C}_{12} \mathrm{H}_{19} \mathrm{~N}_{2} \mathrm{O}^{+} 207.2915$; found, 207.1498.

\section{Compound 3d.3}

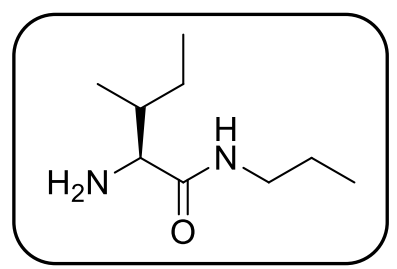

A similar procedure to that described for $\mathbf{3 a . 3}$ was used. An oil was obtained (yield 95\%). ${ }^{1} \mathrm{H}$ NMR (300 MHz, $\mathrm{D}_{6}$-DMSO) $\delta$ (ppm): $7.74(\mathrm{~s}, 1 \mathrm{H}), 3.14-2.87(\mathrm{~m}, 3 \mathrm{H}), 1.57$ (dd, $J=$ 8.9, $3.6 \mathrm{~Hz}, 1 \mathrm{H}), 1.45-1.28(\mathrm{~m}, 3 \mathrm{H}), 1.15-0.91(\mathrm{~m}, 1 \mathrm{H}), 0.88-0.69(\mathrm{~m}, 9 \mathrm{H}) ;{ }^{13} \mathrm{C} N M R(75$ $\mathrm{MHz}, \mathrm{D}_{6}$-DMSO) $\delta$ (ppm): 174.9, 59.8, 40.5, 38.9, 24.2, 22.8, 16.2, 11.9, 11.8; (ESI-TOF, positive mode) $m / z$ exp $[\mathrm{M}+\mathrm{H}]^{+}$calcd for $\mathrm{C}_{9} \mathrm{H}_{21} \mathrm{~N}_{2} \mathrm{O}^{+} 173.1648$; found, 176.1656 .

\section{Synthesis of compounds $\mathbf{3 . 4}$}

\section{Compound 3a.4}

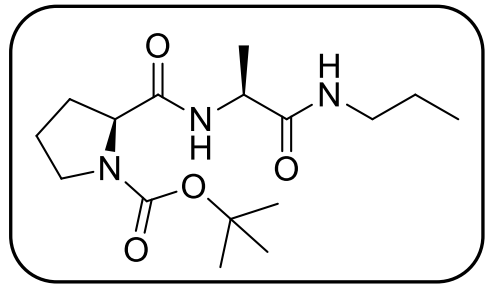


A solution of amino amide $3 a .3$ (0.64 g, 4.8 mmoles) in dry DME (10 mL) was added dropwise over a solution of Boc-L-Pro-OSu (1.87 g, $5.8 \mathrm{mmol})$ in dry DME $(100 \mathrm{~mL})$. The mixture was stirred at room temperature for $24 \mathrm{~h}$ and then at 40 o $C$ for $5 \mathrm{~h}$. The solvent was evaporated under vacuum and the resulting white solid was dissolved in dichloromethane $(50 \mathrm{~mL})$ and washed with $\mathrm{NaHCO}_{3}(3 \times 15 \mathrm{~mL})$. Afterwards, the organic layers were dried $\left(\mathrm{Na}_{2} \mathrm{SO}_{4}\right)$ and the solvent was evaporated under vacuum to yield a white

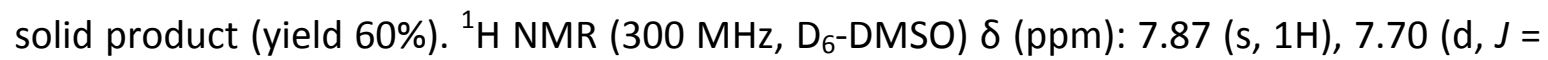
$50.9 \mathrm{~Hz}, 1 \mathrm{H}), 4.55(\mathrm{dd}, J=8.9,3.7 \mathrm{~Hz}, 1 \mathrm{H}), 4.08(\mathrm{dd}, J=8.2,3.4 \mathrm{~Hz}, 1 \mathrm{H}), 3.34(\mathrm{dd}, J=14.3$, $7.4 \mathrm{~Hz}, 2 \mathrm{H}$ ), 3.00 (dd, $J=12.8,6.8 \mathrm{~Hz}, 2 \mathrm{H}), 2.38(\mathrm{dd}, J=14.7,7.3 \mathrm{~Hz}, 1 \mathrm{H}), 2.15-1.94(\mathrm{~m}$, 1H), $1.93-1.59(\mathrm{~m}, 2 \mathrm{H}), 1.50-1.32(\mathrm{~m}, 9 \mathrm{H}), 1.30(\mathrm{~s}, 2 \mathrm{H}), 1.17(\mathrm{~d}, J=7.0 \mathrm{~Hz}, 3 \mathrm{H}), 0.80(\mathrm{t}, J$ $=7.4 \mathrm{~Hz}, 3 \mathrm{H}) ;{ }^{13} \mathrm{C}$ NMR $\left(75 \mathrm{MHz}, \mathrm{D}_{6}\right.$-DMSO) $\delta(\mathrm{ppm}): 172.3,153.7,78.8,59.7,48.4,46.9$, 40.6, 31.3, 28.4, 25.7, 22.7, 19.1, 11.68, 11.7; (ESI-TOF, positive mode) m/z exp [M + $\mathrm{H}]^{+}$calcd for $\mathrm{C}_{16} \mathrm{H}_{30} \mathrm{~N}_{3} \mathrm{O}_{4}{ }^{+} 328.2231$; found, 328.2237 .

\section{Compound 3c.4}

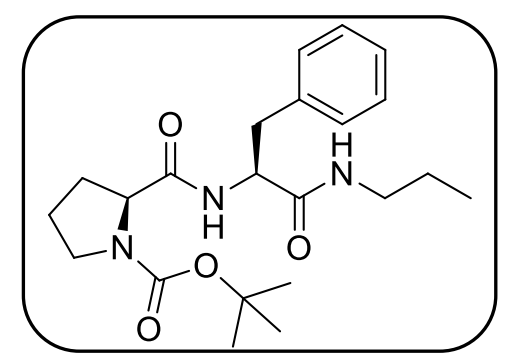

A similar procedure to that described for $3 a .4$ was used. A white solid product was obtained (yield 95\%). ${ }^{1} \mathrm{H}$ NMR (300 MHz, $\mathrm{D}_{6}$-DMSO) $\delta$ (ppm): 7.88 (s, 1H), 7.80 (d, J= 8.2 $\mathrm{Hz}, 1 \mathrm{H}), 7.22(\mathrm{~m}, 5 \mathrm{H}), 4.01(\mathrm{dd}, J=8.7,3.3 \mathrm{~Hz}, 1 \mathrm{H}), 3.41(\mathrm{~m}, 1 \mathrm{H}), 3.24-2.89(\mathrm{~m}, 6 \mathrm{H}), 2.00$ (s, 1H), 1.64 (d, J = $12.8 \mathrm{~Hz}, 3 \mathrm{H}), 1.36$ (dd, $J=14.1,5.9 \mathrm{~Hz}, 6 \mathrm{H}), 1.22(\mathrm{~d}, J=11.9 \mathrm{~Hz}, 5 \mathrm{H})$, $0.76(\mathrm{t}, J=7.4 \mathrm{~Hz}, 3 \mathrm{H}) ;{ }^{13} \mathrm{C}$ NMR (75 MHz, $\mathrm{D}_{6}$-DMSO) $\delta$ (ppm): 172.4, 171.1, 153.8, 138.2, 129.5, 128.4, 126.6, 78.8, 60.1, 54.3, 46.9, 38.4, 31.2, 28.3, 23.3, 22.6, 11.7; (ESI-TOF, positive mode) $\mathrm{m} / z \exp [\mathrm{M}+\mathrm{Na}]^{+}$calcd for $\mathrm{C}_{22} \mathrm{H}_{33} \mathrm{~N}_{3} \mathrm{O}_{4} \mathrm{Na}^{+}$426.2363; found, 426.2365. 


\section{Compound 3d.4}

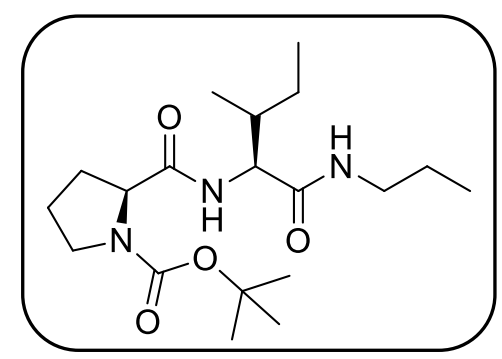

A similar procedure to that described for $\mathbf{3 a . 4}$ was used. A white solid product was obtained (yield 89\%). ${ }^{1} \mathrm{H}$ NMR (500 MHz, D6-DMSO) $\delta$ (ppm): $8.02-7.79$ (m, 1H), 7.61 (d, J $=20.3 \mathrm{~Hz}, 1 \mathrm{H}), 4.29-3.98(\mathrm{~m}, 1 \mathrm{H}), 3.51-3.30(\mathrm{~m}, 1 \mathrm{H}), 3.09-2.87(\mathrm{~m}, 2 \mathrm{H}), 2.76(\mathrm{~d}, J=$ $27.1 \mathrm{~Hz}, 2 \mathrm{H}), 2.16-1.95(\mathrm{~m}, 1 \mathrm{H}), 2.01-1.57(\mathrm{~m}, 4 \mathrm{H}), 1.48-1.20(\mathrm{~m}, 12 \mathrm{H}), 1.17-1.00(\mathrm{~m}$, 1H), $0.93-0.68(\mathrm{~m}, 9 \mathrm{H}) ;{ }^{13} \mathrm{C}$ NMR (126 MHz, $\mathrm{D}_{6}$-DMSO) $\delta$ (ppm): 172.4, 171.1, 153.8, 80.2, 59.9, 57.2, 46.9, 40.6, 37.1, 31.5, 28.1, 25.9, 24.9, 22.6, 15.8, 11.7, 11.2 ; (ESI-TOF, positive mode) $\mathrm{m} / z \exp [\mathrm{M}+\mathrm{Na}]^{+}$calcd for $\mathrm{C}_{19} \mathrm{H}_{35} \mathrm{~N}_{3} \mathrm{O}_{4} \mathrm{Na}^{+} 392.2520$; found, 392.2527.

\section{Synthesis of compounds 3a-d}

\section{Compound 3a}

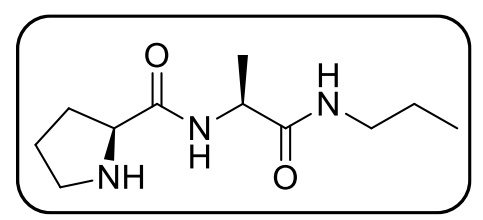

$\mathrm{N}$-protected compound $3 \mathrm{a} .4(2.00 \mathrm{~g}, 7.8 \mathrm{mmol})$ was dissolved in dichloromethane $(50 \mathrm{~mL})$ and after addition of TFA $(15 \mathrm{~mL})$ the mixture was stirred at room temperature for $2 \mathrm{~h}$. The solvent was evaporated under vacuum and then the resulting crude oil was dissolved in water $(50 \mathrm{~mL})$. The solution was treated with $\mathrm{NaOH}(\mathrm{pH}=12)$, and extracted with chloroform $(3 \times 15 \mathrm{~mL})$. The organic layers were washed with water and dried $\left(\mathrm{Na}_{2} \mathrm{SO}_{4}\right)$. The solvent was evaporated under vacuum to yield a white solid compound (yield 66\%). ${ }^{1} \mathrm{H}$ NMR (500 MHz, $\mathrm{D}_{6}$-DMSO) $\delta(\mathrm{ppm}): 8.09$ (d, J = $\left.7.7 \mathrm{~Hz}, 1 \mathrm{H}\right), 7.84(\mathrm{~d}, \mathrm{~J}=$ $97.2 \mathrm{~Hz}, 1 \mathrm{H}), 4.37-4.14(\mathrm{~m}, 1 \mathrm{H}), 3.60-3.38(\mathrm{~m}, 1 \mathrm{H}), 3.09-2.96(\mathrm{~m}, 2 \mathrm{H}), 2.90-2.80(\mathrm{~m}$, $1 \mathrm{H}), 2.77-2.67(\mathrm{~m}, 1 \mathrm{H}), 2.06-1.85(\mathrm{~m}, 1 \mathrm{H}), 1.70-1.60(\mathrm{~m}, 1 \mathrm{H}), 1.61-1.52(\mathrm{~m}, 2 \mathrm{H}), 1.46$ - $1.31(\mathrm{~m}, 2 \mathrm{H}), 1.15$ (dd, $J=6.9,2.1 \mathrm{~Hz}, 3 \mathrm{H}), 0.83(\mathrm{td}, J=7.4,2.1 \mathrm{~Hz}, 3 \mathrm{H}) ;{ }^{13} \mathrm{C}$ NMR $(75$ MHz, $\mathrm{D}_{6}$-DMSO) $\delta$ (ppm): 174.4, 172.5, 61.0, 48.3, 47.3, 41.3, 30.9, 26.2, 22.8, 19.8, 11.8; 
(ESI-TOF, positive mode) $\mathrm{m} / \mathrm{z}$ exp $[\mathrm{M}+\mathrm{H}]^{+}$calcd for $\mathrm{C}_{11} \mathrm{H}_{22} \mathrm{~N}_{3} \mathrm{O}_{2}^{+} 228.1707$; found, 228.1712 .

\section{Compound 3c}

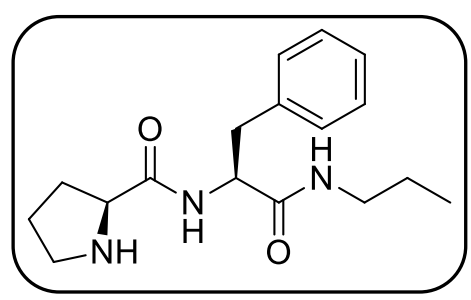

A similar procedure to that described for $3 a$ was used. A white solid product was obtained (yield 68\%). ${ }^{1} \mathrm{H}$ NMR (300 MHz, $\mathrm{D}_{6}$-DMSO) $\delta(\mathrm{ppm}): 8.00$ (dd, $J=14.5,7.4 \mathrm{~Hz}$, $2 \mathrm{H}), 7.30-7.02(\mathrm{~m}, 5 \mathrm{H}), 4.48(\mathrm{td}, J=8.6,5.5 \mathrm{~Hz}, 1 \mathrm{H}), 3.43(\mathrm{~m}, 1 \mathrm{H}), 3.12-2.86(\mathrm{~m}, 3 \mathrm{H})$, $2.85-2.69(\mathrm{~m}, 3 \mathrm{H}), 2.65-2.53(\mathrm{~m}, 1 \mathrm{H}), 1.81(\mathrm{~m}, 1 \mathrm{H}), 1.52-1.20(\mathrm{~m}, 5 \mathrm{H}), 0.79(\mathrm{t}, J=7.4$ $\mathrm{Hz}, 3 \mathrm{H}) ;{ }^{13} \mathrm{C}$ NMR (126 MHz, D - DMSO) $\delta$ (ppm): 174.1, 170.9, 137.7, 129.6, 128.3, 126.7, $60.4,53.2,46.9,40.7,38.9,30.6,26.0,22.6,11.7$; (ESI-TOF, positive mode) $\mathrm{m} / \mathrm{z} \exp [\mathrm{M}+$ $\mathrm{H}]^{+}$calcd for $\mathrm{C}_{17} \mathrm{H}_{26} \mathrm{~N}_{3} \mathrm{O}^{+}$304.2020; found, 304.2027.

\section{Compound 3d}

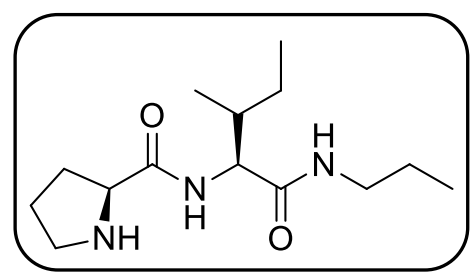

A similar procedure to that described for $3 a$ was used. A white solid product was obtain (yield 52\%). ${ }^{1} \mathrm{H}$ NMR (500 MHz, D -DMSO) $\delta$ (ppm): 8.13 - 7.89 (m, 2H), 4.11 (ddd, $J=25.7,13.2,7.8 \mathrm{~Hz}, 1 \mathrm{H}), 3.53(\mathrm{dd}, J=9.0,5.0 \mathrm{~Hz}, 1 \mathrm{H}), 3.14-2.91(\mathrm{~m}, 2 \mathrm{H}), 2.91-2.84$ (m, 1H), $2.72(d t, J=10.2,6.4 \mathrm{~Hz}, 1 \mathrm{H}), 1.94$ (ddd, $J=16.3,12.4,7.5 \mathrm{~Hz}, 1 \mathrm{H}$ ), $1.78-1.64$ $(\mathrm{m}, 2 \mathrm{H}), 1.63-1.48(\mathrm{~m}, 2 \mathrm{H}), 1.48-1.28(\mathrm{~m}, 3 \mathrm{H}), 1.08-0.91(\mathrm{~m}, 1 \mathrm{H}), 0.81(\mathrm{~m}, 9 \mathrm{H}) ;{ }^{13} \mathrm{C}$ NMR (300 MHz, D6-DMSO) $\delta$ (ppm): 174.2, 171.0, 60.6, 56.2, 47.1, 40.5, 37.8, 31.0, 26.3, 24.6, 22.6, 15.8, 11.8, 11.3;(ESI-TOF, positive mode) $\mathrm{m} / \mathrm{z} \exp [\mathrm{M}+\mathrm{H}]^{+}$calcd for $\mathrm{C}_{14} \mathrm{H}_{28} \mathrm{~N}_{3} \mathrm{O}^{+}$ 270.2176; found, 270.2186 . 
b) Procedure for the synthesis of compounds $3 e$

\section{Synthesis of compound 3e.2}

\section{Compound $3 e .2$}

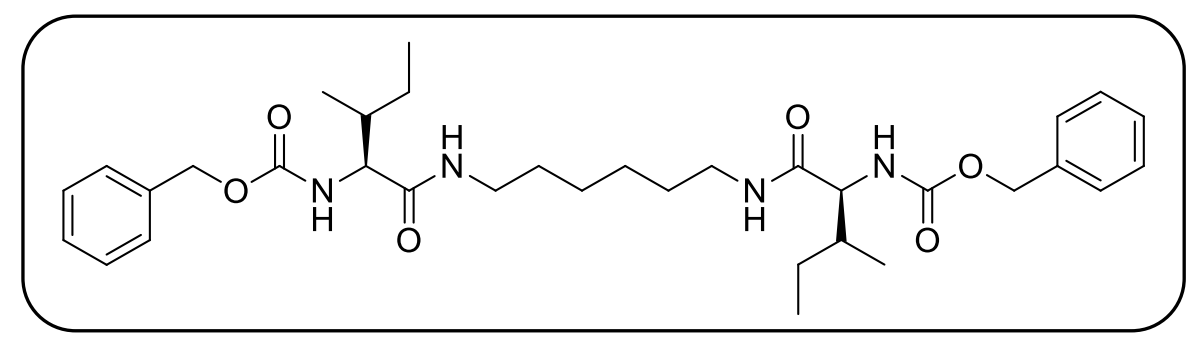

The $N$-hydroxysuccinimide ester of $N$-Cbz-L-Isoleucine (3d.1) (6.04 g, $16.68 \mathrm{mmol}$ ) was dissolved in anhydrous DME (100 mL) and cooled in an ice bath. 1,6-diaminohexane (0.99 $\mathrm{g}, 8.51 \mathrm{mmol})$ dissolved in dry DME $(10 \mathrm{~mL})$ was added in several portions. The reaction mixture was stirred at room temperature for $18 \mathrm{~h}$ and then was warmed for $6 \mathrm{~h}$ at $40-50{ }^{\circ} \mathrm{C}$. The white solid was filtered off and washed with cold water and cold methanol and was finally dried in a high-pressure oven (yield 85\%). ${ }^{1} \mathrm{H} N M R\left(500 \mathrm{MHz}, \mathrm{D}_{6}\right.$ DMSO) $\delta$ (ppm): 7.85 (s, 1H), $7.39-7.24(\mathrm{~m}, 5 \mathrm{H}), 7.18(\mathrm{~d}, J=8.1 \mathrm{~Hz}, 1 \mathrm{H}), 5.09-4.93(\mathrm{~m}$, 2H), $3.80(\mathrm{t}, J=8.1 \mathrm{~Hz}, 1 \mathrm{H}$ ), 3.02 (ddd, $J=18.5,12.7,6.2 \mathrm{~Hz}, 2 \mathrm{H}), 1.66(\mathrm{~s}, 1 \mathrm{H}), 1.35(\mathrm{~s}, 3 \mathrm{H})$, $1.22(\mathrm{~s}, 2 \mathrm{H}), 1.14-0.97(\mathrm{~m}, 1 \mathrm{H}), 0.79(\mathrm{~d}, J=3.3 \mathrm{~Hz}, 6 \mathrm{H}) .{ }^{13} \mathrm{C} N M R\left(126 \mathrm{MHz}, \mathrm{D}_{6}-\mathrm{DMSO}\right) \delta$ (ppm): 171.4, 156.4, 137.6, 128.7, 128.2, 128.0, 65.8, 59.7, 38.8, 36.8, 29.4, 26.5, 24.9, 15.8, 11.3; (ESI-TOF, positive mode) $\mathrm{m} / \mathrm{z} \exp [\mathrm{M}+\mathrm{Na}]^{+}$calcd for $\mathrm{C}_{34} \mathrm{H}_{50} \mathrm{~N}_{4} \mathrm{NaO}_{6}{ }^{+} 633.3623$; found, 633.3622 .

\section{Synthesis of compound 3e.3}

\section{Compound $3 e .3$}

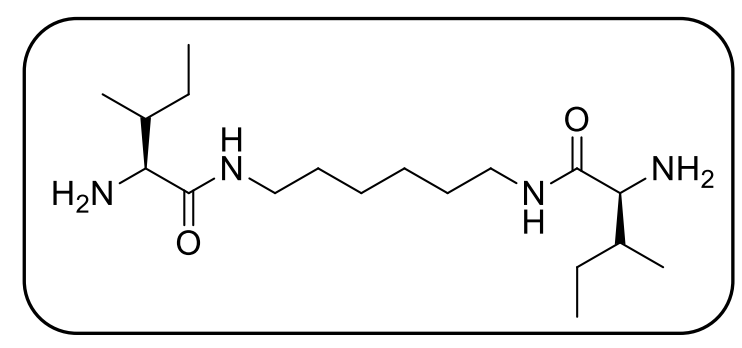

A similar procedure as that described for the deprotection of $\mathrm{N}$-Cbz group in 3a.3 was used (yield 95\%). ${ }^{1} \mathrm{H}$ NMR (500 MHz, $\mathrm{D}_{6}$-DMSO) $\delta(\mathrm{ppm}): 7.78$ (d, $\left.J=28.2 \mathrm{~Hz}, 1 \mathrm{H}\right), 3.11$ $-2.94(\mathrm{~m}, 2 \mathrm{H}), 2.92(\mathrm{~d}, J=5.4 \mathrm{~Hz}, 1 \mathrm{H}), 1.56(\mathrm{~d}, J=5.6 \mathrm{~Hz}, 1 \mathrm{H}), 1.48-1.32(\mathrm{~m}, 3 \mathrm{H}), 1.25(\mathrm{~s}$, 
2H), $1.05(\mathrm{tt}, J=15.6,7.6 \mathrm{~Hz}, 1 \mathrm{H}), 0.81(\mathrm{t}, J=7.6 \mathrm{~Hz}, 6 \mathrm{H}) ;{ }^{13} \mathrm{C} \mathrm{NMR}\left(126 \mathrm{MHz}, \mathrm{D}_{6}-\mathrm{DMSO}\right) \delta$ (ppm): 174.9, 59.8, 38.9, 38.6, 29.6, 26.5, 24.2, 16.3, 12.0; (ESI-TOF, positive mode) m/z $\exp [\mathrm{M}+\mathrm{H}]^{+}$calcd for $\mathrm{C}_{18} \mathrm{H}_{39} \mathrm{~N}_{4} \mathrm{O}_{2}{ }^{+} 343.5273$; found, 343.3076.

\section{Synthesis of compound $3 e .4$}

\section{Compound $3 e .4$}

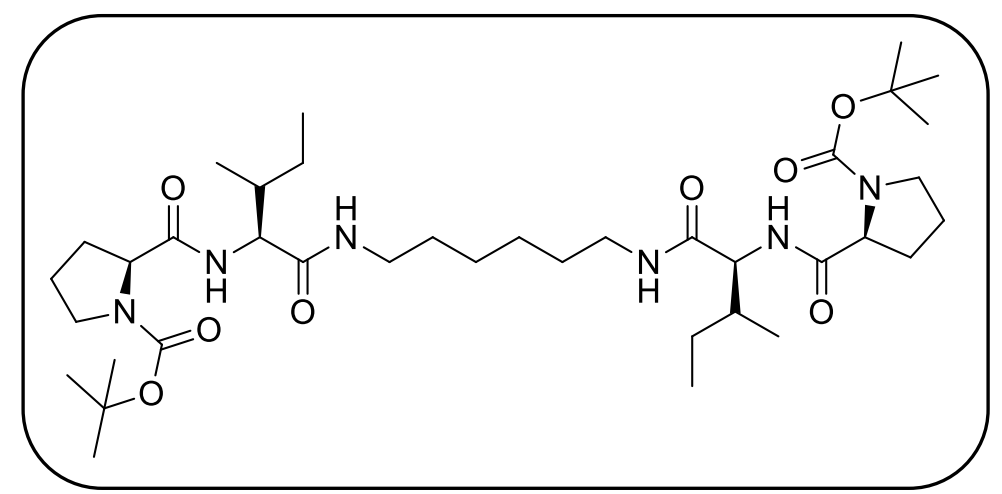

A similar procedure as described previously for the preparation of $N$-Boc-protected compounds (3a.4) previously was used, taking into account that must be used 2.1 equivalents of $N$-Boc-L-Pro-Osu for each equivalent of diamine (yield 95\%). ${ }^{1} \mathrm{H}$ NMR (500 $\mathrm{MHz}, \mathrm{D}_{6}$-DMSO) $\delta(\mathrm{ppm}): 7.89$ (d, $\left.J=48.0 \mathrm{~Hz}, 1 \mathrm{H}\right), 7.65(\mathrm{~d}, J=9.2 \mathrm{~Hz}, 21 \mathrm{H}), 4.17(\mathrm{~d}, J=9.0$ $\mathrm{Hz}, 1 \mathrm{H}), 4.10(\mathrm{t}, J=8.0 \mathrm{~Hz}, 1 \mathrm{H}), 3.39-3.32(\mathrm{~m}, 2 \mathrm{H}), 3.02$ (ddd, $J=49.2,13.1,6.4 \mathrm{~Hz}, 2 \mathrm{H})$, $2.81(\mathrm{~s}, 1 \mathrm{H}), 1.73(\mathrm{~s}, 4 \mathrm{H}), 1.45-1.28(\mathrm{~m}, 12 \mathrm{H}), 1.23(\mathrm{~s}, 2 \mathrm{H}), 1.12-0.99(\mathrm{~m}, 1 \mathrm{H}), 0.81(\mathrm{t}, J=$ $5.5 \mathrm{~Hz}, 9 \mathrm{H}) ;{ }^{13} \mathrm{C}$ NMR (126 MHz, D -DMSO) $\delta$ (ppm): 172.5, 171.1, 153.8, 78.8, 59.9, 57.1, 47.0, 38.7, 37.1, 31.6, 29.4, 28.4, 26.5, 24.8, 23.4, 15.8, 11.3; (ESI-TOF, positive mode) m/z $\exp [\mathrm{M}+\mathrm{Na}]^{+}$calcd for $\mathrm{C}_{38} \mathrm{H}_{68} \mathrm{~N}_{6} \mathrm{NaO}_{2}^{+} 759.4991 ;$ found, 759.5001 .

\section{Synthesis of compound $3 e$}

\section{Compound 3e}

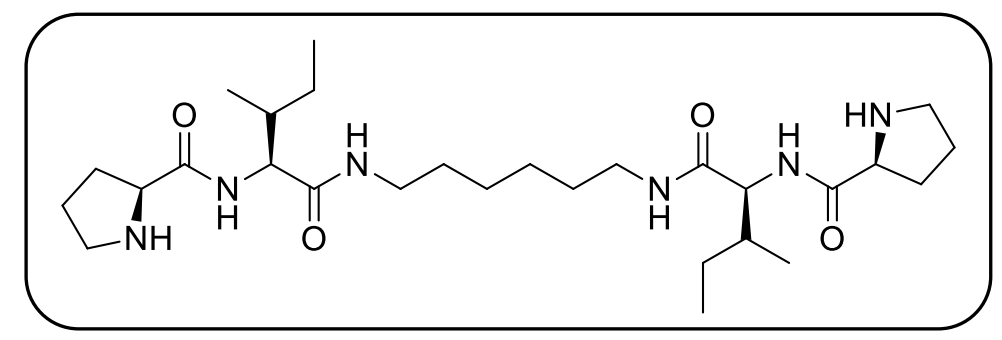


A similar procedure as described previously for the deprotection of $N$-Boc group for 3a previously was used (yield 68\%). ${ }^{1} \mathrm{H}$ NMR (500 MHz, D $\mathrm{D}_{6}$-DMSO) $\delta$ (ppm): 8.02 (dd, J $=10.5,5.9 \mathrm{~Hz}, 2 \mathrm{H}), 4.17-4.00(\mathrm{~m}, 1 \mathrm{H}), 3.52(\mathrm{dd}, \mathrm{J}=8.9,5.1 \mathrm{~Hz}, 1 \mathrm{H}), 3.13-2.91(\mathrm{~m}, 2 \mathrm{H})$, $2.88(d t, J=10.0,6.7 \mathrm{~Hz}, 1 \mathrm{H}), 2.71(\mathrm{dt}, \mathrm{J}=10.0,6.3 \mathrm{~Hz}, 1 \mathrm{H}), 1.92(\mathrm{ddd}, \mathrm{J}=16.3,12.4,7.6$ $\mathrm{Hz}, 1 \mathrm{H}), 1.74-1.59(\mathrm{~m}, 2 \mathrm{H}), 1.60-1.46(\mathrm{~m}, 2 \mathrm{H}), 1.39-1.29(\mathrm{~m}, 3 \mathrm{H}), 1.22(\mathrm{~s}, 2 \mathrm{H}), 0.95(\mathrm{dt}$, $J=15.6,7.0 \mathrm{~Hz}, 1 \mathrm{H}), 0.79(\mathrm{dd}, \mathrm{J}=13.6,7.0 \mathrm{~Hz}, 6 \mathrm{H}) ;{ }^{13} \mathrm{C}$ NMR $\left(75 \mathrm{MHz}, \mathrm{D}_{6}-\mathrm{DMSO}\right) \delta(\mathrm{ppm})$ : 174.3, 171.0, 60.6, 56.3 , 47.2, 38.7, 38.0, 31.0, 29.3, 26.4, 26.3, 24.7, 15.8, 11.5; (ESI-TOF, positive mode) $\mathrm{m} / \mathrm{z} \exp [\mathrm{M}+\mathrm{H}]^{+}$calcd for $\mathrm{C}_{28} \mathrm{H}_{53} \mathrm{~N}_{6} \mathrm{O}_{4}{ }^{+}$537.4123; found, 537.4133.

\section{c) Procedure for the synthesis of dodecyl amine derivative.}

\section{Compound ProValDoc (4a)}

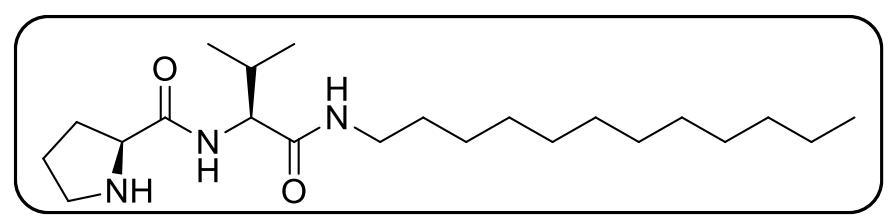

Compound ProValDoc (4a) was synthesised as reported previously and both ${ }^{1} \mathrm{H}$ RNM and ${ }^{13} \mathrm{C}-\mathrm{NMR}$ were in good agreement with the literature. ${ }^{3}$

\subsubsection{Synthesis of Succinic Acid Derivative}

\section{a) Procedure for the synthesis of compounds $5 a-b$}

\section{Compound 5a}

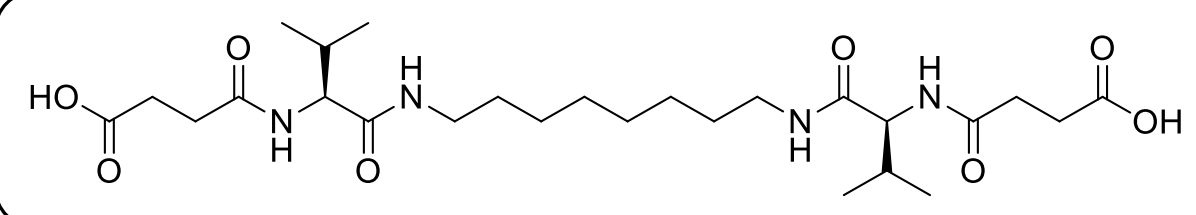

Compound SucVal8 (5a) was synthesised as reported previously and both ${ }^{1} \mathrm{H}-\mathrm{RNM}$ and ${ }^{13} \mathrm{C}-\mathrm{NMR}$ were in good agreement with the literature. ${ }^{4}$ 
Synthesis of compound 5 b.2 (see compound 3e.2)

Synthesis of compound $\mathbf{5 b . 3}$ (see compound 3e.3)

\section{Synthesis of compound $\mathbf{5 b}$}

\section{Compound 5b}

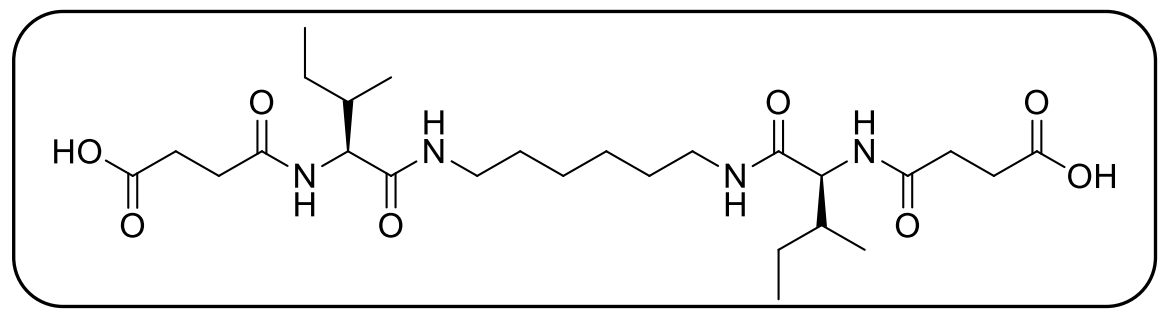

To a solution of compound $3 e .3(0.416 \mathrm{~g}, 1.26 \mathrm{mmol})$ in dry THF $(50 \mathrm{~mL})$, commercially available succinic anhydride $(0.307 \mathrm{~g}, 3.04 \mathrm{mmol})$ in dry THF $(20 \mathrm{~mL})$ was added dropwise under $\mathrm{N}_{2}$ at room temperature. The mixture was stirred under reflux for $16 \mathrm{~h}$. After this time, the solvent was evaporated under vacuum and the resulting white solid was washed (each wash was treated in ultrasound for 10-20 min and then filtered off) with cold $\mathrm{HCl} 0.1 \mathrm{M}(10 \mathrm{~mL})$ and cold water $(10 \mathrm{~mL})$. A white solid was obtained after drying in vacuum oven at $50 \stackrel{\circ}{\circ}$ for $24 \mathrm{~h}$ (yield 80\%). ${ }^{1} \mathrm{H} N M R\left(500 \mathrm{MHz}, \mathrm{D}_{6}-\mathrm{DMSO}\right) \delta$ (ppm): 12.05 (s, 1H), $7.93-7.76(\mathrm{~m}, 2 \mathrm{H}), 4.13$ (t, $J=8.0 \mathrm{~Hz}, 1 \mathrm{H}), 3.05$ (ddd, $J=60.3,12.8$, $6.1 \mathrm{~Hz}, 2 \mathrm{H}), 2.43(\mathrm{~m}, 4 \mathrm{H}), 1.72(\mathrm{~m}, 1 \mathrm{H}), 1.40(\mathrm{~m}, 3 \mathrm{H}), 1.26(\mathrm{~m}, 2 \mathrm{H}), 1.16-1.04(\mathrm{~m}, 1 \mathrm{H})$, $0.83(\mathrm{t}, J=7.2 \mathrm{~Hz}, 6 \mathrm{H}) ;{ }^{13} \mathrm{C}$ NMR $\left(75 \mathrm{MHz}, \mathrm{D}_{6}-\mathrm{DMSO}\right) \delta(\mathrm{ppm}): 174.3,171.3,171.2,57.3$, 38.7, 37.0, 30.3, 29.7, 29.4, 26.4, 24.8, 15.8, 11.5; (ESI-TOF, positive mode) $\mathrm{m} / \mathrm{z}$ exp [M + $\mathrm{H}]^{+}$calcd for $\mathrm{C}_{26} \mathrm{H}_{47} \mathrm{~N}_{4} \mathrm{O}_{8}{ }^{+} 543,3388$; found, 543.338 .

\section{b) Procedure for the synthesis of compounds 5c-d}

\section{Synthesis of compounds $5 c-d .2$}

\section{Compound 5c.2}<smiles>CCCCCCCCCCNC(=O)C(NC(=O)OCc1ccccc1)C(C)C</smiles> 
A solution of $N$-hydroxysuccinimide ester of Cbz-L-valine derivative (13.1 g, 37.6 $\mathrm{mmol})$ in THF $(150 \mathrm{~mL})$ was added dropwise to a solution of nonylamine $(7.6 \mathrm{~mL}, 41.4$ $\mathrm{mmol})$ in THF $(100 \mathrm{~mL})$ under $\mathrm{N}_{2}$ atmosphere at room temperature. The mixture was stirred for $5 \mathrm{~h}$ at $50 \stackrel{\circ}{ } \mathrm{C}$. After this time, the solvent was removed under vacuum and the resulting white solid was washed with aq. $\mathrm{HCl} 0.1 \mathrm{M}(50 \mathrm{~mL})$ and extracted several times with ethyl acetate. All the organic phases were collected and the solvent was removed under vacuum to yield a white solid after drying under vacuum in oven at 50 oc (yield 96\%). ${ }^{1} \mathrm{H}$ NMR (300 MHz, $\mathrm{D}_{6}$-DMSO) $\delta$ (ppm): $7.83(\mathrm{~m}, 1 \mathrm{H}), 7.34(\mathrm{~m}, 5 \mathrm{H}), 7.15$ (d, J = 8.9 $\mathrm{Hz}, 1 \mathrm{H}), 5.02(\mathrm{~s}, 2 \mathrm{H}), 3.84-3.68(\mathrm{~m}, 1 \mathrm{H}), 3.19-2.88(\mathrm{~m}, 2 \mathrm{H}), 1.92(\mathrm{dq}, \mathrm{J}=13.3,6.6 \mathrm{~Hz}$, $1 \mathrm{H}), 1.37(\mathrm{~m}, 2 \mathrm{H}), 1.23(\mathrm{~s}, 12 \mathrm{H}), 0.85(\mathrm{~m}, 9 \mathrm{H}) ;{ }^{13} \mathrm{C}$ NMR (75 MHz, D $\left.-\mathrm{DMSO}\right) \delta(\mathrm{ppm})$ : 170.8, 156.0, 137.1, 128.2(x2), 127.6, 127.5(x2), 65.3, 60.3, 38.3, 31.2, 30.2, 28.9, 28.7, 28.6, 26.3, 22.0, 19.1, 18.2, 13.9; (ESI-TOF, positive mode) $\mathrm{m} / \mathrm{z} \exp [\mathrm{M}+\mathrm{H}]^{+}$calcd for $\mathrm{C}_{22} \mathrm{H}_{37} \mathrm{~N}_{2} \mathrm{O}_{3}{ }^{+}$377.2799; found, 377.2802.

\section{Compound $5 \mathrm{~d} .2$}

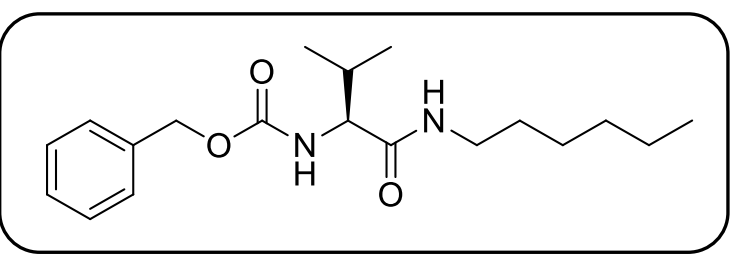

A similar procedure that described previously for $5 c .2$ was used (yield $96 \%$ ). ${ }^{1} \mathrm{H}$

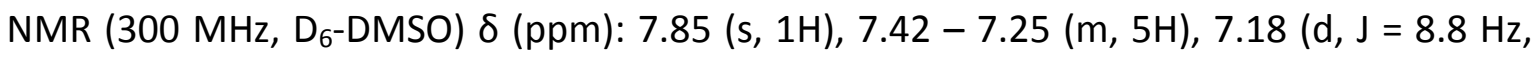
$1 \mathrm{H}), 5.02(\mathrm{~s}, 2 \mathrm{H}), 3.78(\mathrm{t}, \mathrm{J}=8.0 \mathrm{~Hz}, 1 \mathrm{H}), 3.17-2.88(\mathrm{~m}, 2 \mathrm{H}), 1.93(\mathrm{dt}, \mathrm{J}=13.5,6.8 \mathrm{~Hz}, 1 \mathrm{H})$, $1.46-1.14(\mathrm{~m}, 8 \mathrm{H}), 0.84(\mathrm{~m}, 9 \mathrm{H}) ;{ }^{13} \mathrm{C}$ NMR $\left(75 \mathrm{MHz}, \mathrm{D}_{6}-\mathrm{DMSO}\right) \delta$ (ppm): 170.8, 156.0, 128.3 (x3), 127.7, 127.5 (x2), 65.3, 60.3, 38.3, 30.9, 30.2, 28.9, 25.9, 22.0, 19.2, 18.2, 13.8; (ESI-TOF, positive mode) $\mathrm{m} / \mathrm{z}$ exp $[\mathrm{M}+\mathrm{H}]^{+}$calcd for $\mathrm{C}_{19} \mathrm{H}_{30} \mathrm{~N}_{2} \mathrm{O}_{3}{ }^{+} 335.2335$; found, : 335.2331 . 


\section{Synthesis of compounds $5 c-d .3$}

\section{Compound $5 c .3$}

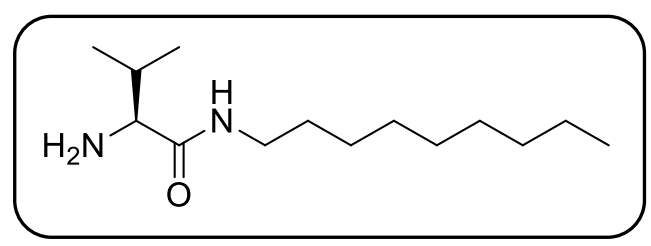

A similar procedure to that described for the deprotection of $\mathrm{N}$-Cbz group in 3a.3 was used (yield 95\%). ${ }^{1} \mathrm{H}$ NMR (500 MHz, D6-DMSO) $\delta(p p m): 7.75(\mathrm{~m}, 1 \mathrm{H}), 3.05(\mathrm{~m}, 2 \mathrm{H})$, $2.88(\mathrm{~d}, \mathrm{~J}=5.1 \mathrm{~Hz}, 1 \mathrm{H}), 1.83(\mathrm{dq}, \mathrm{J}=12.9,6.6 \mathrm{~Hz}, 1 \mathrm{H}), 1.38(\mathrm{~m}, 2 \mathrm{H}), 1.24(\mathrm{~s}, 12 \mathrm{H}), 0.85(\mathrm{~m}$, $6 \mathrm{H}), 0.77(\mathrm{~d}, \mathrm{~J}=6.8 \mathrm{~Hz}, 3 \mathrm{H}) ;{ }^{13} \mathrm{C}$ NMR (126 MHz, $\mathrm{D}_{6}$-DMSO) $\delta$ (ppm): 174.2, 60.0, 38.2, $31.6,31.2,29.2,28.9,28.70,28.6,26.3,22.0,19.5,17.1,13.9$.

\section{Compound $5 \mathrm{~d} .3$}

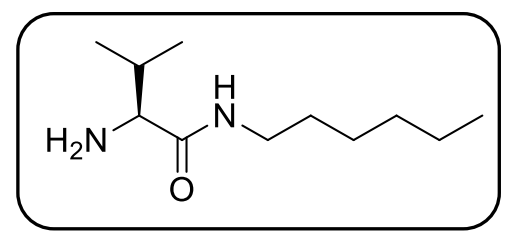

A similar procedure to that described for the deprotection of $\mathrm{N}$-Cbz groups in $\mathbf{5 c . 3}$ was used (yield 97\%). ${ }^{1} \mathrm{H}$ NMR (300 MHz, $\left.\mathrm{CDCl}_{3}\right) \delta(\mathrm{ppm}): 7.30(\mathrm{~s}, 1 \mathrm{H}), 3.12(\mathrm{~m}, 3 \mathrm{H}), 2.12$ $(\mathrm{m}, 1 \mathrm{H}), 1.96(\mathrm{~m}, 2 \mathrm{H}), 1.39(\mathrm{~m}, 2 \mathrm{H}), 1.17(\mathrm{~s}, 6 \mathrm{H}), 0.86(\mathrm{~d}, \mathrm{~J}=6.7 \mathrm{~Hz}, 3 \mathrm{H}), 0.79-0.66(\mathrm{~m}$, $6 \mathrm{H}) ;{ }^{13} \mathrm{C}-\mathrm{NMR}\left(75 \mathrm{MHz}, \mathrm{CDCl}_{3}\right) \delta$ (ppm): 174.0, 60.1, 38.9, 31.4, 30.9, 29.5, 26.5, 22.4, 19.5, 16.1, 13.9; (ESI-TOF, positive mode) $\mathrm{m} / \mathrm{z}$ exp $[\mathrm{M}+\mathrm{H}]^{+}$calcd for $\mathrm{C}_{11} \mathrm{H}_{25} \mathrm{~N}_{2} \mathrm{O}^{+}$201,1961; found, : 201.1967.

\section{Synthesis of compounds $\mathbf{5 c - d}$}

\section{Compound 5c}

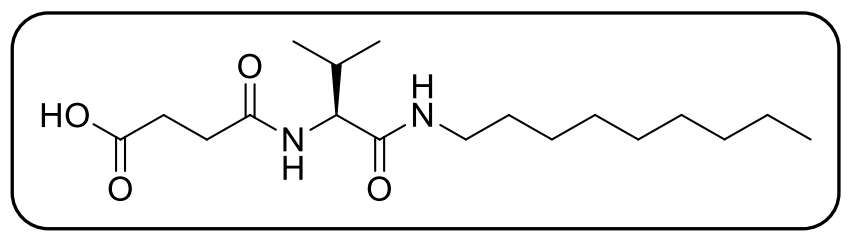


A solution of amine $5 \mathrm{c} .3(10.1 \mathrm{~g}, 41.6 \mathrm{mmol})$ in THF $(300 \mathrm{~mL})$ was treated at $0 \stackrel{\circ}{\circ}$ under $\mathrm{N}_{2}$ with solid $\mathrm{Na}_{2} \mathrm{CO}_{3}(14.3 \mathrm{~g}, 135 \mathrm{mmol})$. The mixture was stirred for 15 minutes at 0 으, after succinic anhydride $(7.1 \mathrm{~g}, 70.8 \mathrm{mmol})$ in THF $(100 \mathrm{~mL})$ was added dropwise. The mixture was further stirred vigorously for $16 \mathrm{~h}$ at room temperature. After this time, the solution was concentrated under reduced pressure and the crude residue was dissolved in water $(200 \mathrm{~mL})$, then $\mathrm{HCl}$ concentrate was added at $0 \stackrel{\circ}{\circ}$ until the formation of a white precipitate to $\mathrm{pH}=3$ was observed. The white solid obtained was filtered off under vacuum, and the residue was washed with water $(300 \mathrm{~mL})$. The compound was

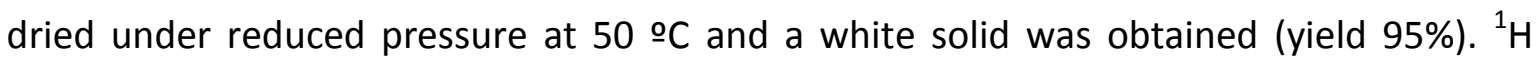
NMR (500 MHz, D D $\left.^{-D M S O}\right) \delta(p p m): 11.81(\mathrm{~s}, 1 \mathrm{H}), 7.82(\mathrm{~s}, 1 \mathrm{H}), 4.13-3.99(\mathrm{~m}, 1 \mathrm{H}), 3.02$ (ddd, J = 18.6, 12.8, 6.2 Hz, 2H), $2.40(\mathrm{~s}, 4 \mathrm{H}), 1.93(\mathrm{dd}, \mathrm{J}=13.3,6.6 \mathrm{~Hz}, 1 \mathrm{H}), 1.37(\mathrm{~s}, 2 \mathrm{H})$, $1.23(\mathrm{~s}, 12 \mathrm{H}), 0.93-0.76(\mathrm{~m}, 9 \mathrm{H}) ;{ }^{13} \mathrm{C}$ NMR (126 MHz, D $\left.-\mathrm{DMSO}\right) \delta(\mathrm{ppm}): 174.4,171.5$, $171.2,58.3,38.81,31.7,30.8,30.4,29.9,29.4,29.4,29.1,29.1,26.8,22.5,19.6,18.5$, 14.4; (ESI-TOF, positive mode) $\mathrm{m} / \mathrm{z} \exp [\mathrm{M}+\mathrm{H}]^{+}$calcd for $\mathrm{C}_{18} \mathrm{H}_{35} \mathrm{~N}_{2} \mathrm{O}_{4}{ }^{+} 343.2591$; found, 343.2591 .

\section{Compound 5d}

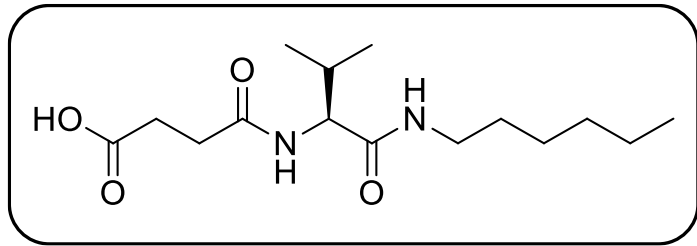

A similar procedure to that described for the compound $5 c$ was used (yield $93 \%$ ). ${ }^{1} \mathrm{H}-\mathrm{NMR}\left(\mathrm{D}_{6}\right.$-DMSO) $\delta(\mathrm{ppm}): 12.03(\mathrm{~s}, 1 \mathrm{H}), 7.84(\mathrm{~m}, 2 \mathrm{H}), 4.15-3.99(\mathrm{~m}, 1 \mathrm{H}), 3.17-2.87$ (m, 2H), $2.41(\mathrm{~s}, 4 \mathrm{H}), 1.93(\mathrm{td}, \mathrm{J}=13.4,6.7 \mathrm{~Hz}, 1 \mathrm{H}), 1.48-1.32(\mathrm{~m}, 2 \mathrm{H}), 1.24(\mathrm{~s}, 8 \mathrm{H}), 0.92-$ $0.66(\mathrm{~m}, 9 \mathrm{H}) ;{ }^{13} \mathrm{C}$ NMR (126 MHz, D D $\left.^{-D M S O}\right) \delta$ (ppm): 174.33, 171.47, 171.18, 58.31, $38.81,31.37,30.87,30.43,29.79,29.40,26.44,22.48,19.64,18.58,14.36 . ; \quad$ (ESI-TOF, positive mode) $\mathrm{m} / \mathrm{z} \exp [\mathrm{M}+\mathrm{Na}]^{+}$calcd for $\mathrm{C}_{15} \mathrm{H}_{28} \mathrm{~N}_{2} \mathrm{NaO}_{4}{ }^{+} 323,1947$; found, : 323, 1950 . 


\subsubsection{Synthesis of NDI Derivatives}

\section{Synthesis of compound 6 a}

\section{Compound 6a.1}

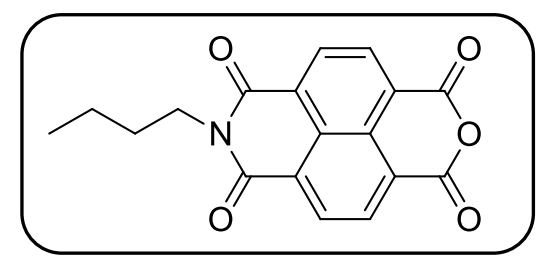

The 1,4,5,8-naphthalenetetracarboxylic acid dianhydride $(2.0 \mathrm{~g}, 7.46 \mathrm{mmol}$ ) was added in water $(350 \mathrm{~mL}$ ) followed by the addition of $1 \mathrm{M}$ aqueous $\mathrm{KOH}$ solution $(35 \mathrm{~mL})$. This mixture was vigorously stirred and heated until almost all the compound was dissolved. The $\mathrm{pH}$ of the resultant solution was acidified to 6.4 by adding $1 \mathrm{M} \mathrm{H}_{3} \mathrm{PO}_{4}$. To this solution, $n$-butyl amine $(0.74 \mathrm{ml}, 7.46 \mathrm{mmol})$ was added and the $\mathrm{pH}$ of the solution was readjusted to 6.4 with $1 \mathrm{M} \mathrm{H}_{3} \mathrm{PO}_{4}$. The mixture was heated to reflux overnight. It was then allowed to cool to room temperature and filtered. To the filtrate, acetic acid ( $5 \mathrm{~mL})$ was added, a solid precipitate was formed which was then filtered and dried under vacuum to give a pale yellow solid (yield 73\%). ${ }^{1} \mathrm{H}$ NMR (400 MHz, $\mathrm{D}_{6}$-DMSO) $\delta$ (ppm): 8.52 (d, $J=7.5 \mathrm{~Hz}, 2 \mathrm{H}), 8.14(\mathrm{~d}, J=7.5 \mathrm{~Hz}, 2 \mathrm{H}), 4.03(\mathrm{t}, J=7.4 \mathrm{~Hz}, 2 \mathrm{H}), 1.66-1.58(\mathrm{~m}, 2 \mathrm{H}), 1.35$ (h, $J=7.3 \mathrm{~Hz}, 2 \mathrm{H}), 0.92(\mathrm{t}, J=7.3 \mathrm{~Hz}, 3 \mathrm{H}) .{ }^{13} \mathrm{C}$ NMR (100 MHz, D 6 -DMSO) $\delta$ (ppm): 168.8, $162.8,137.9,130.0,128.8,128.5,125.4,124.1,40.1,29.5,19.8,13.7$. both in good agreement with the literature. ${ }^{5}$

\section{Compound 6a.2}

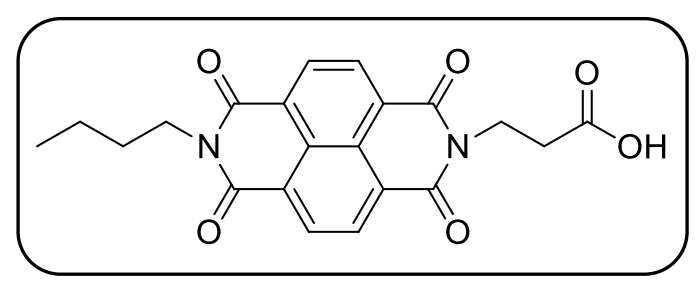

To a stirred solution of $\boldsymbol{n}$-Bu-NDI (6a.1) $(1.74 \mathrm{~g}, 5.38 \mathrm{mmol})$ in DMF (20 mL), $\beta$ alanine $(0.99 \mathrm{~g}, 11.11 \mathrm{mmol})$ and DIPEA $(1.94 \mathrm{ml}, 11.11 \mathrm{mmol})$ were sequentially added and the reaction mixture was stirred at $90^{\circ} \mathrm{C}$ overnight. The solvent was evaporated under vacuum and the crude residue was dissolved in 2:1 water/methanol (100 mL). The 
$\mathrm{pH}$ of the solution was adjusted to 3 by adding $\mathrm{HCl}(6 \mathrm{M})$. The mixture was concentrated under vacuum and the resulting precipitate was washed with methanol several times to afford a light orange solid (yield 78\%). ${ }^{1} \mathrm{H}$ NMR (400 MHz, D 6 -DMSO) $\delta$ (ppm): 12.44 (s, $1 \mathrm{H}), 8.45(\mathrm{~s}, 4 \mathrm{H}), 4.2\left(\mathrm{t}, J=7.5 \mathrm{~Hz}, 2 \mathrm{H}, \mathrm{NCH}_{2}\right), 3.97(\mathrm{t}, J=7.5 \mathrm{~Hz}, 2 \mathrm{H}), 2.62(\mathrm{t}, J=7.8 \mathrm{~Hz}$, 2H), $1.66-1.58(\mathrm{~m}, 2 \mathrm{H}), 1.38(\mathrm{~h}, J=7.4 \mathrm{~Hz}, 2 \mathrm{H}), 0.94(\mathrm{t}, J=7.3 \mathrm{~Hz}, 3 \mathrm{H}) .{ }^{13} \mathrm{C} N M R(100$ $\mathrm{MHz}, \mathrm{D}_{6}$-DMSO) $\delta$ (ppm): 172.4, 162.2, 162.1, 130.3, 130.3, 125.9, 125.8, 125.6, 40.1, 36.0, 31.9, 29.5, 19.8, 13.7; ESI-HRMS (m/z) exp [M - H] calcd for $\mathrm{C}_{21} \mathrm{H}_{17} \mathrm{~N}_{2} \mathrm{O}_{6}{ }^{-}$393.1092; found, 393.1096 .

\section{Compound 6a.3}

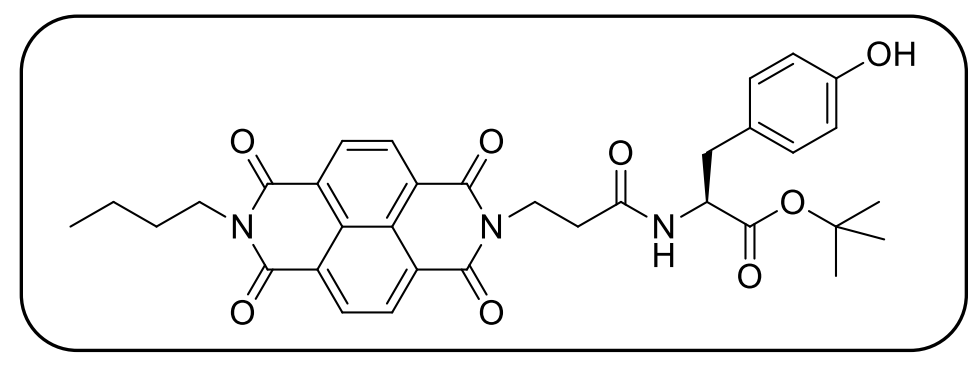

To a stirred solution of the mixture of $\boldsymbol{n}$-Bu-NDI- $\boldsymbol{\beta}$-alanine (6a.2) (1.6 g, 4.06 mmol), L-tyrosine-tert-butyl ester (1.06 g, $4.47 \mathrm{mmol})$ and HBTU (1.69 g, $4.47 \mathrm{mmol})$ in dry DMF (30 mL), DIPEA $(1.78 \mathrm{~mL}, 10.15 \mathrm{mmol})$ was added and the reaction mixture was left to stir at room temperature overnight. The resulting solution dissolved in ethyl acetate $(70 \mathrm{~mL})$ and sequentially washed with equal volumes $(50 \mathrm{ml})$ of $1 \mathrm{M}$ saturated $\mathrm{NaHCO}_{3}$, brine, $1 \mathrm{M} \mathrm{HCl}$ and brine. The organic layer was then collected, dried over magnesium sulfate and the solvent was removed by evaporation in vacuum. The crude residue was finally purified by silica gel column chromatography (eluent, $100 \%$ chloroform followed by $2 \%$ methanol in chloroform) to afford NDI-Tyr-OtBu (6a.3) as a light red solid (yield 56\%). ${ }^{1} \mathrm{H}$ NMR (400 MHz, $\left.\mathrm{CDCl}_{3}\right) \delta$ (ppm): $8.63(\mathrm{~s}, 4 \mathrm{H}), 6.92-6.85$ (m, $2 \mathrm{H}), 6.57-6.49(\mathrm{~m}, 2 \mathrm{H}), 6.39(\mathrm{~d}, J=8.0 \mathrm{~Hz}, 1 \mathrm{H}), 4.73-4.68(\mathrm{~m}, 1 \mathrm{H}), 4.53-4.36(\mathrm{~m}, 2 \mathrm{H})$, $4.20-4.11(\mathrm{~m}, 2 \mathrm{H}), 3.00-2.88(\mathrm{~m}, 2 \mathrm{H}), 2.70-2.65(\mathrm{~m}, 2 \mathrm{H}), 1.76-1.64(\mathrm{~m}, 2 \mathrm{H}), 1.50-$ $1.41(\mathrm{~m}, 2 \mathrm{H}), 1.39(\mathrm{~s}, 9 \mathrm{H}), 0.97(\mathrm{t}, J=7.3 \mathrm{~Hz}, 3 \mathrm{H}) .{ }^{13} \mathrm{C} \mathrm{NMR}\left(100 \mathrm{MHz} \mathrm{CDCl}_{3}\right) \delta(\mathrm{ppm})$ : 171.0, 170.1, 162.8, 155.3, 131.1, 130.9, 130.4, 127.3, 126.7, 126.6, 126.4, 115.3, 82.7, 53.9, 40.9, 37.2, 34.6, 30.2, 28.1, 20.4, 13.9; ESI-HRMS (m/z) $\exp [\mathrm{M}+\mathrm{H}]^{+}$calcd for $\mathrm{C}_{34} \mathrm{H}_{36} \mathrm{~N}_{3} \mathrm{O}_{8}^{+} 614.2497$; found, 614.2498; HPLC analysis: > 99\% pure. 


\section{Compound 6a}

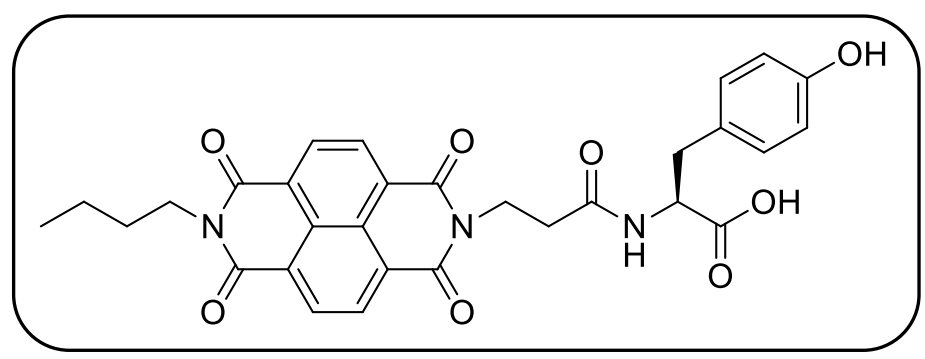

NDI-Tyr-OtBu (6a.3) (1.3 g, $3.32 \mathrm{mmol}$ ) was dissolved in trifluoroacetic acid (15 $\mathrm{mL}$ ) and left to stir at room temperature overnight. The solvent was then removed by evaporation in vacuum and the residual trifluoroacetic acid was removed by azeotropic distillation with toluene. The crude product was then taken up in cold diethyl ether (100 $\mathrm{ml}$ ) followed by sonication for few minutes resulted in an emulsion. The pure product was then collected by decantation and this procedure was repeated several times to afford pure NDI-Tyr (6a) as a light orange powder (yield 85\%). ${ }^{1} \mathrm{H}$ NMR (400 MHz, $\mathrm{D}_{6}$-DMSO) $\delta$ (ppm): $12.65(\mathrm{~s}, 1 \mathrm{H}), 9.16(\mathrm{~s}, 1 \mathrm{H}), 8.61(\mathrm{~s}, 4 \mathrm{H}), 8.28(\mathrm{~d}, J=8.0 \mathrm{~Hz}, 1 \mathrm{H}), 7.01-6.94(\mathrm{~m}, 2 \mathrm{H})$, $6.62-6.54(m, 2 H), 4.38-4.27(m, 1 H), 4.26-4.12(m, 2 H), 4.10-3.97(m, 2 H), 2.94-$ $2.82(\mathrm{~m} 1 \mathrm{H}), 2.78-2.64(\mathrm{~m}, 1 \mathrm{H}), 2.59-2.52(\mathrm{~m}), 1.68-1.60(\mathrm{~m}, 2 \mathrm{H}), 1.38(\mathrm{~h}, J=7.4 \mathrm{~Hz}$, $2 \mathrm{H}), 0.94$ (t, $J=7.3 \mathrm{~Hz}, 3 \mathrm{H}) .{ }^{13} \mathrm{C}$ NMR (100 MHz, D 6 -DMSO) $\delta$ (ppm): 173.1, 169.6, 162.5, $162.4,155.8,130.3,130.3,129.9,127.6,126.2,126.2,126.0,114.9,53.8,36.7,36.0,33.0$, 29.5, 19.8, 13.7; ESI-HRMS (m/z) exp $[\mathrm{M}+\mathrm{H}]^{+}$calcd for $\mathrm{C}_{30} \mathrm{H}_{28} \mathrm{~N}_{3} \mathrm{O}_{8}{ }^{+}$558.1871; found, 558.1874; HPLC analysis: > 98\% pure.

\section{Synthesis of compounds $\mathbf{6 b}$}

\section{Compound 6b}

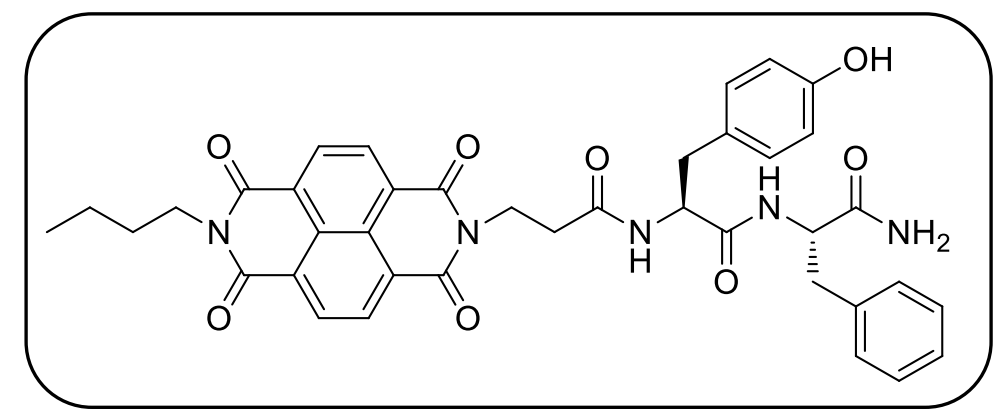

To a stirred solution of the mixture of NDI-Tyr (6a) $(0.2 \mathrm{~g}, 0.36 \mathrm{mmol}), \mathrm{L}-$ phenylalanine amide $(0.07 \mathrm{~g}, 0.43 \mathrm{mmol})$ and HBTU $(0.16 \mathrm{~g}, 0.43 \mathrm{mmol})$ in dry DMF (15 
$\mathrm{mL})$, DIPEA $(0.16 \mathrm{~mL}, 0.9 \mathrm{mmol})$ was added and the reaction mixture was left to stir at room temperature for $24 \mathrm{~h}$. The resulting solution was taken up in ethyl acetate $(70 \mathrm{~mL})$ and sequentially washed with equal volumes $(50 \mathrm{ml})$ of $1 \mathrm{M}$ saturated $\mathrm{NaHCO}_{3}$, brine, $1 \mathrm{M}$ $\mathrm{HCl}$ and brine. The organic layer was then collected and the solvent was removed by evaporation in vacuum. The product could not be further purified because of its extreme poor solubility in most of the common solvents tested. ${ }^{1} \mathrm{H} N M R\left(400 \mathrm{MHz}, \mathrm{D}_{6}-\mathrm{DMSO}\right) \delta$ (ppm): 9.10 (s, 1H), 8.65 (s, 4H), 8.13 (d, J = 9.0 Hz, 1H), 7.95 (d, J=8.1 Hz, 1H), $7.35-7.23$ $(\mathrm{m}, 6 \mathrm{H}), 7.06(\mathrm{~s}, 1 \mathrm{H}), 6.95(\mathrm{~d}, J=7.9 \mathrm{~Hz}, 2 \mathrm{H}), 6.56(\mathrm{~d}, J=7.9 \mathrm{~Hz}, 2 \mathrm{H}), 4.39$ (overlapped with solvent peaks, $1 \mathrm{H}$ ), 4.21 (overlapped with solvent peaks, $1 \mathrm{H}$ ), $4.07(\mathrm{~d}, J=8.1 \mathrm{~Hz}, 2 \mathrm{H}), 3.18$ $-2.91(\mathrm{~m}, 4 \mathrm{H}), 2.79(\mathrm{~d}, \mathrm{~m}, J=15.1 \mathrm{~Hz}, 2 \mathrm{H}), 1.72-1.55(\mathrm{~m}, 2 \mathrm{H}), 1.38(\mathrm{q}, J=7.6 \mathrm{~Hz}, 2 \mathrm{H})$, $0.94(t, J=7.2 \mathrm{~Hz}, 3 \mathrm{H}) ;{ }^{13} \mathrm{C}$ NMR $\left(100 \mathrm{MHz}, \mathrm{D}_{6}\right.$-DMSO) The spectra could not be measured because of its extreme poor solubility in most of the common solvents tested. HPLC analysis: $>98 \%$ pure.

\subsubsection{Synthesis of DAN Derivatives}

\section{Compound 1,5-DAN}

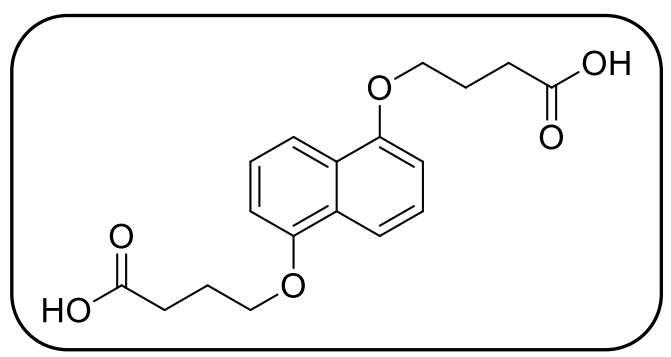

Compound 1,5-DAN was synthesised as reported previously and both ${ }^{1} \mathrm{H}-\mathrm{RNM}$ and ${ }^{13} \mathrm{C}$-NMR were in good agreement with the literature. ${ }^{6}$

\section{Compound 2,6-DAN}

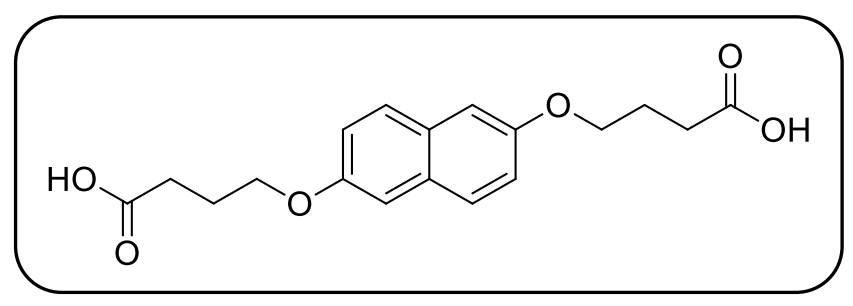


Compound 2,6-DAN was synthesised as reported previously and both ${ }^{1} \mathrm{H}-\mathrm{RNM}$ and ${ }^{13} \mathrm{C}-\mathrm{NMR}$ were in good agreement with the literature. ${ }^{7}$

\subsection{Methodology of Characterisation of Gels}

\subsubsection{Gelation Procedures}

Gelation studies were carried out in cylindrical screw capped vials and tube inversion approach was used to determine the formation of the gel. In all cases the weight of the different compounds were measured in an analytical balance and the volume of solvent used was measured with analytical precision pipettes.

Different methods have been employed to obtain the gels described in this work. Otherwise stated, the standard method was used.

\section{Standard Gelation}

The required amount of gelator was introduced in a screw capped vial with a volume of solvent necessary to reach the desired concentration. The complete dissolution of the gelator was achieved by heating (using a heating gun) and gentle mechanical stirring of the vials. The sample was left to cool at room temperature during $24 \mathrm{~h}$. The formation of a gel was evidenced by a solid-like soft material that did not flow upon inversion of the flask.

\section{Hydrogelation of Prolle6 (3e)}

The required amount of gelator was introduced in a screw capped vial and was firstly dissolved in $40 \mu \mathrm{L}$ of DMSO (4\%). After this, $960 \mu \mathrm{L}$ of water was added and the complete dissolution of the gelator was achieved by heating (using a heating gun) and gentle mechanical stirring of the vials. The sample was sonicated and left to cool at room temperature during $24 \mathrm{~h}$. The formation of a white gel was evidenced minutes after by a solid-like soft material that did not flow upon inversion of the flask.

\section{Hydrogelation of Two-Component Mixture}

The required amount of Prolle6 (3e) was introduced in a screw capped vial and was firstly dissolved in $20 \mu \mathrm{L}$ of DMSO (4\%). After this, $480 \mu \mathrm{L}$ of water was added and the 
complete dissolution of the gelator was achieved by heating (using a heating gun) and gentle mechanical stirring of the vial. The sample was sonicated and left to cool at room temperature for $1 \mathrm{~min}$ with the formation of a white gel. Then the PyrVal3 (5e) was added on the top of the gel and the mixture was heated again until complete dissolution and sonicated for a few seconds. After the sample was left to cool down at room temperature over $24 \mathrm{~h}$. The formation of a gel was evidenced within $1 \mathrm{~h}$ by the formation of a solid-like soft material that did not flow upon inversion of the flask.

\subsubsection{Thermal Stability}

Gel-to-solution transition temperature $\left(T_{\text {gel }}\right)$ was determined by the typical 'inversion-tube-method'. In a glass cylindrical screw capped vial the gel was prepared as described above. The vial was immersed in a silicone bath up-side-down and slowly heated in $5^{\circ} \mathrm{C}$ steps stabilizing for 5 minutes at each temperature. The temperature where the gel melted and flowed to the bottom of the vial was considered as $\mathrm{T}_{\mathrm{gel}}$.

\subsubsection{Electron Microscopy}

\section{Field-Emission Scanning Electron Microscopy (FESEM)}

Field-emission scanning electron micrographs were taken on a JEOL 7001F microscope equipped with a digital camera. The corresponding freeze-dried gels were placed on top of an aluminium specimen mount stub and sputtered with Pt.

\section{Transmission Electron Microscopy (TEM)}

Samples were applied directly onto a 200 mesh carbon coated copper grids. Excess solvent was carefully removed by capillary action using filter paper. TEM images were recorded in a JEOL 2100 Transmission Electron Microscope.

Organogels: the samples where sputtered with Pt

Hydrogel: For ProValDoc (4a) samples the grids were stained with one drop of phosphotungstic acid $1 \%$ solution immediately after the sample was placed on the grid and left it to act for $1 \mathrm{~min}$. Excess of stain was removed by capillarity. Otherwise stated, for the Prolle6 (3a) or its mixture with PyrVal3 (5e) samples, no dye was required. 
Atomic Force Microscopy (AFM) (University of Strathclyde Glasgow)

For AFM experiments, $20 \mu \mathrm{L}$ of sample solution (as a gel) was diluted to $1000 \mu \mathrm{L}$ of deionized water and then deposited onto a freshly cleaved mica surface (G250-2 Mica sheets 1" x 1" x 0.006"; Agar Scientific Ltd, Essex, UK). Each sample was air dried overnight in a dust-free environment prior to AFM imaging. The images were obtained by scanning the mica surface in air under ambient conditions using a Veeco dilNNOVA Scanning Probe Microscope (VEECO/BRUKER, Santa Barbara, CA, USA) operated in tapping mode. The AFM measurements were obtained using sharp silicon probes (RTESPA; Veeco Instruments SAS, Dourdan, France). AFM scans were taken at $512 \times 512$ pixels resolution and produced topographic images of the samples in which the brightness of features increases as a function of height. Typical scanning parameters were as follows: tapping frequency $326 \mathrm{kHz}$, integral and proportional gains 0.1 and 0.3 , respectively, set point $0.5-0.7 \mathrm{~V}$ and scanning speed $1.0 \mathrm{~Hz}$. AFM images $(10 \mu \mathrm{m} \times 10$ $\mu \mathrm{m}$ ) were collected from two different samples and at random spot surface sampling (at least five areas). The images were analysed using NanoScope Analysis software Version 1.40 .

Transmission Electron Microscopy (TEM) (University of Strathclyde Glasgow)

Transmission electron microscopy (TEM) images were captured using a LEO 912 energy filtering transmission electron microscope operating at $120 \mathrm{kV}$ fitted with $14 \mathrm{bit} / 2$ K Proscan CCD camera. Carbon-coated copper grids (200 mesh) were glow discharged in air for 30 seconds. The support film was touched onto the gel surface for 3 seconds and blotted down using filter paper. Each sample was allowed to dry afterwards for few minutes in a dust-free environment prior to TEM imaging. Negative stain (20 $\mu \mathrm{L}, 1 \%$ aqueous methylamine vanadate) was applied and the mixture blotted again using filter paper to remove excess. The dried specimens were then imaged using the microscope.

\subsubsection{NMR Studies}

Spectra were recorded at $500 \mathrm{MHz}, 300 \mathrm{MHz}\left({ }^{1} \mathrm{H}\right.$ NMR) and $125 \mathrm{MHz}, 75 \mathrm{MHz}\left({ }^{13} \mathrm{C}\right.$ NMR) at 30 ㄷ. NOESY-1D experiments were recorded using a mixing time of $800 \mathrm{~ms}$ at 30 으. 
University of Strathclyde, Glasgow: ${ }^{1} \mathrm{H}$ and ${ }^{13} \mathrm{C}$ nuclear magnetic resonance (NMR) spectra were recorded on Bruker AV400 spectrometer at 30 ㅇ․

\section{DOSY}

Diffusion coefficients were calculated using the values of the intensity of the observed signal for 15 different gradient strengths in the Stejskal-Tanner equation $\left(\ln (I / / 0)=-\gamma g^{2} \delta^{2}(\Delta-\delta / 3) D\right){ }^{8}$ where $I$ and $/ 0$ are the signal intensities in the presence and absence of the pulsed-field gradients respectively, $\nu$ is the gyromagnetic ratio $\left(\operatorname{rad~s~G}^{-1}\right), g$ is the strength of the diffusion gradients $\left(\mathrm{G} \mathrm{m}^{-1}\right), D$ is the diffusion coefficient of the observed spins $\left(\mathrm{m}^{2} \mathrm{~s}^{-1}\right), \delta$ is the length of the diffusion gradients (s) and $\Delta$ is the time separation between the leading edges of the two diffusion pulsed gradients (s).

\section{Solubility}

Gel Sample Preparation. Gels were prepared in deuterated solvent. The hot solution of the gelator was transferred into a NMR tube and aged for $24 \mathrm{~h}$ at room temperature before all the measurements were performed. For measurements in $\mathrm{H}_{2} \mathrm{O}$ a capillary tube with hydroquinone was used as reference for chemical shifts and integrals. For solubility measurements in toluene, 1,1,2,2-tetrachloroethane was used as an internal standard.

Solubility Analysis. ${ }^{1}$ H NMR spectra of the gels were used to estimate the solubility constants at different temperatures. The measurements were recorded at the desired temperature after stabilization for 2 minutes. The relative integral value of proton signals to the internal standard was measured. To obtain samples with all the gelator in solution, the gel was disassembled in the NMR tube by the addition of: $10-30 \mu \mathrm{L}$ of DMSO for organogels and $10-20 \mu \mathrm{L}$ of trifluoracetic acid for proline and pyridine hydrogels. The results were reproduced successfully at least twice. Enthalpy and entropy of solubilisation values were obtained from the VT NMR measurements using the van't Hoff equation where solubility $\left(K_{s}\right)$ at a given temperature can be expressed by:

$$
L n K_{s}=-\left(\frac{\Delta H}{R T}\right)+\left(\frac{\Delta S}{R}\right)
$$


where $\Delta \mathrm{H}$ and $\Delta \mathrm{S}$ denotes the molar enthalpy and the molar entropy for the dissolution process (i.e. gel to solution transformation), $T$ is the temperature $(K)$ and $R$ is the gas constant $\left(8.3145 \mathrm{~J} \cdot \mathrm{K}^{-1} \cdot \mathrm{mol}^{-1}\right)$. Plots of $L n K_{s}$ vs $(1 / T)$ were used for the extraction of $\Delta \mathrm{H}$ and $\Delta \mathrm{S}$ values for the process of gel dissolution.

\subsubsection{UV-Vis Measurements}

UV-Vis spectra were recorded in a Jasco V-630 spectrometer. In all samples quartz cuvettes with $1 \mathrm{~cm}$ path length were employed. The spectra were acquired at $25^{\circ} \mathrm{C}$ and where treated with Spectra Manager Software.

University of Strathclyde Glasgow: UV-vis absorption spectra were recorded on a Jasco V-660 spectrophotometer. Samples were prepared in PMMA cuvettes (from Fisher Scientific). The experimental data was acquired in triplicate and the average data was shown.

\subsubsection{Fluorescence Emission Spectroscopy (University of Strathclyde Glasgow)}

Fluorescence emission spectra were measured on a Jasco FP-6500 spectrofluorometer with light measured orthogonally to the excitation light, at a scanning speed of $200 \mathrm{~nm} \mathrm{~min}{ }^{-1}$. Samples were prepared in PMMA cuvettes. The freshly prepared samples were directly taken in the cuvette and the time-dependent spectra were recorded immediately. The experiments were carried out directly under the described concentrations and the gels were used as such for characterization. The experimental data were acquired in triplicate and the average values shown

\subsubsection{Oscillatory Rheology (University of Strathclyde Glasgow)}

The mechanical properties of the hydrogels were investigated by dynamic frequency sweep experiments which were carried out by strain controlled rheometer (Kinexus Pro Rheometer) by employing parallel plates of $20 \mathrm{~mm}$ diameter with $0.5 \mathrm{~mm}$ gap. To ensure the measurements were made in the linear viscoelastic regime, an amplitude sweep was performed and the results showed no variation in elastic modulus $\left(G^{\prime}\right)$ and viscous modulus $\left(G^{\prime \prime}\right)$ up to a strain of $1 \%$. The dynamic modulus of the hydrogels 
(after $24 \mathrm{~h}$ ) was measured as a frequency function, where the frequency sweeps were carried out between 0.1 and $10 \mathrm{~Hz}$. The experiments were performed at $25^{\circ} \mathrm{C}$ and were repeated three times to ensure reproducibility, with the average data shown.

\subsubsection{Fourier Transform Infrared (FTIR) Spectroscopy (University of Strathclyde Glasgow)}

FTIR spectra were recorded on a Bruker optics Vertex 70 spectrophotometer. The hydrogels were loaded between two $\mathrm{CaF}_{2}$ windows using a $5 \mu \mathrm{m}$ PTFE spacer. The spectra were taken in the region between 800 and $4000 \mathrm{~cm}^{-1}$ with a resolution of $1 \mathrm{~cm}^{-1}$ and averaged over 20 scans. Spectra were background-subtracted to correct for atmospheric interference. For all measurements, $100 \mathrm{mM}$ phosphate buffer in $\mathrm{D}_{2} \mathrm{O}$ was used as the solvent. The spectra were recorded at $24 \mathrm{~h}$ after the equilibration of each sample under ambient conditions. The spectra shown in the figures are cumulatively offset by $0.15 \mathrm{OD}$ (absorbance).

\subsubsection{Circular Dichroism (CD) (University of Strathclyde Glasgow)}

Circular dichroism (CD) spectra were measured on a Jasco J600 spectropolarimeter in a $0.1 \mathrm{~mm}$ path-length cylindrical cell, with $1 \mathrm{~s}$ integration, step resolution of $1 \mathrm{~mm}$, response of $0.5 \mathrm{~s}$ with a bandwidth of $1 \mathrm{~nm}$ and slit width of $1 \mathrm{~mm}$. The freshly prepared samples were directly added to the cell using a pipette and the spectra were recorded after equilibration for $24 \mathrm{~h}$. Due to the high opacity of the gels under these conditions, the High Tension (HT) voltage value reaches maximum below the wavelengths of $235 \mathrm{~nm}$ and the CD could not be measured.

\subsubsection{Molecular Modelling}

The models reported were obtained by molecular mechanics calculations performed with MacroModel using AMBER* as force field in vacuum. Exhaustive Monte Carlo conformational search (1000 steps of torsional angles variation) was carried out for the isolated molecules. The structures described for the folded conformations correspond to energy minimized structures near the global minimum. The models for the aggregates were built manually from unfolded energy minimized conformers. Then these aggregated structures were energy minimized to the nearest local energy minimum. 


\subsubsection{Determination the Hydrodynamic Diameter}

Dynamic Light Scattering (DLS) measurements were performed at $25^{\circ} \mathrm{C}$ using a Malvern Zetasizer NanozS instrument, equipped with a $532 \mathrm{~nm}$ laser at a fixed scattering angle of $90^{\circ}$. Samples were prepared dissolving the solid in water with the addition of $\mathrm{NaOH}$ pearls. The solution was sonicated for $10 \mathrm{~min}$ and filtered off through a $0.22 \mu \mathrm{m}$ cellulose membrane filter before analysis. Micelle size distribution by volume was measured (diameter, $\mathrm{nm}$ ) for each sample $(\mathrm{n} \geq 3)$.

University of Strathclyde Glasgow: The dynamic light scattering (DLS) was carried out by using a ALV (ALV, GMBH, Germany) using vertically polarized He-Ne laser light (25 $\mathrm{mW}$ with wavelength of $632.8 \mathrm{~nm}$ ) with an avalanche photodiode detector. The DLS measurements were carried out at angle of $90^{\circ}$ and SLS measurements were performed at angles between $15^{\circ}$ and $100^{\circ}$ at $25^{\circ} \mathrm{C}$.

\subsection{Catalysis Study}

\subsubsection{Michael Reaction in Toluene}

\section{Catalytic System in Solution}

Catalyst $(0.033 \mathrm{mmol}$ ) was dissolved in a vial in $1 \mathrm{~mL}$ of toluene (for diluted systems, $1 \mathrm{mM}$ and $2.5 \mathrm{mM}, 0.006 \mathrm{mmol}$ of catalyst were added). The sample was left to cool at room temperature and afterwards was stabilised for $24 \mathrm{~h}$.

\section{Catalytic System in Gel Phase}

$0.016 \mathrm{mmol}$ of the gelator in toluene $(1 \mathrm{~mL})$ were heated in a screw-capped vial until it was completely dissolved. The sample was left to cool at room temperature and afterwards it was stabilized during $24 \mathrm{~h}$. The formation of an organogel was evidenced by the formation of a solid-like soft material that did not flow upon inversion of the flask.

\section{General Procedure for the 1,4-Conjugated Michael Addition Reaction ${ }^{9}$}

A solution of trans- $\beta$-nitrostyrene $(0.16 \mathrm{mmol})$ and cyclohexanone $(3.29 \mathrm{mmol})$ was added to the catalytic system in a volume to reach the desired concentration. The 
reaction was left at room temperature the required time. The reaction was quenched by addition of a $0.25 \mathrm{M}$ aqueous acetic acid solution $(2 \mathrm{~mL})$ and toluene $(1 \mathrm{~mL})$. The aqueous layer was extracted with toluene $(2 \times 2 \mathrm{~mL})$. Then the solvent of the combined organic extracts were removed until dryness. The reaction crude was analysed by ${ }^{1} \mathrm{H}-\mathrm{NMR}$ in $\mathrm{CDCl}_{3}$ in order to determine the yield and the diastereoselectivity (syn=3.76 ppm; anti= $4.01 \mathrm{ppm})^{10}$ and was further purified by column chromatography (silica gel, mixture of hexane /ethyl acetate, $3: 1$ ) to isolate the pure product used to determine enantioselectivity.

\section{Determination of the Enantiomeric Excess ${ }^{9}$}

The enantiomeric excess was determined by HPLC using a Chiral Pack IA column, hexane/IPA ( $/ \mathrm{v}: 85 / 15)$, flow rate $=1 \mathrm{~mL} / \mathrm{min}, \lambda=210 \mathrm{~nm}, \mathrm{t}_{1}=7.76 \mathrm{~min}\left(2 \mathrm{~S}, 1^{\prime} \mathrm{R}\right), \mathrm{t}_{2}=8.76$ $\min (2 \mathrm{R}, 1 \mathrm{~S})$.

\section{Kinetic Studies}

Kinetic constants were estimated from the reaction yield at a given final time. The concentration of cyclohexanone was approximated to be constant and the system analysed as having a pseudo first order kinetics.

\subsubsection{Aldol Reaction in Water}

\section{Direct Aldol Reaction between 4-Nitrobenzaldehyde and Cyclohexanone}

$6.04 \mathrm{mg}(0.04 \mathrm{mmol})$ of 4-nitrobenzaldehyde were dissolved in $41 \mu \mathrm{L}(0.4 \mathrm{mmol})$ of cyclohexanone, added on top of the hydrogel $(0.008 \mathrm{mmol})$ and left to diffuse and react at $25 \stackrel{\circ}{ } \mathrm{C}$. The reaction was quenched by addition of $2 \mathrm{~mL}$ of $0.1 \mathrm{M} \mathrm{HCl}$, diluted with $10 \mathrm{~mL}$ of water and extracted twice with $5 \mathrm{~mL}$ of dichloromethane. The combined organic extracts were dried with anhydrous sodium sulfate and the solvent evaporated under vacuum. The resulting yellow solid was analysed by ${ }^{1} \mathrm{H}-\mathrm{NMR}$ in $\mathrm{CDCl}_{3}$ in order to determine the yield and diastereoselectivity, and by chiral HPLC to determine the enantioselectivity. ${ }^{3}$ 


\section{Direct Aldol Reaction between 4-Nitrobenzaldehyde and Linear Ketones}

$3 \mathrm{mg}(0.008 \mathrm{mmol})$ of gelator were suspended in $4 \mathrm{~mL}$ of water in a screw-capped vial which was heated during $3 \mathrm{~min}$ in a bath at $100 \stackrel{\circ}{ } \mathrm{C}$ until complete dissolution. Then the vial was immersed in an ultrasounds bath for $1 \mathrm{~min}$ and then left at room temperature. After 1 hour, the formation of a hydrogel can be observed. Samples were aged for $24 \mathrm{~h}$ at room temperature. Afterwards $0.04 \mathrm{mmol}$ of 4-nitrobenzaldehyde and $0.4 \mathrm{mmol}$ of ketone were mixed and added simultaneously on top of the hydrogel and left to diffuse and react at room temperature. The reaction was quenched by addition of $2 \mathrm{~mL}$ of $0.1 \mathrm{M} \mathrm{HCl}$ and extracted twice with $2 \mathrm{~mL}$ of dichloromethane. The combined organic extracts were dried with anhydrous sodium sulfate and the solvent evaporated. The resulting yellow solid was analysed by ${ }^{1} \mathrm{H}-\mathrm{NMR}$ in $\mathrm{CDCl}_{3}$ in order to determine the yield. Finally, the crude product was purified by column chromatography on silica gel (hexane: ethyl acetate, 3:1) to give the aldol product. Then the enantioselectivity was determined by chiral phase HPLC: HPLC conditions Chiralpak IA ( $n$-hexane/THF: 80/20, $1.0 \mathrm{~mL} / \mathrm{min}$, $\lambda=250 \mathrm{~nm})$

\subsection{Polymorphism Study}

Hydrogels and dispersions were prepared by placing ProValDoc (4a) ( $3 \mathrm{mg}, 0.008$ $\mathrm{mmol}$ ) and solvent $(4 \mathrm{~mL})$ in $8 \mathrm{~mL}$ screw-capped vials under the experimental conditions described in Table 4.2.4.1. Samples for $\mathrm{pH}$-controlled aggregation were prepared by addition of the required volumes of phosphate buffer solution over a sample of ProValDoc (4a) dissolved in $\cdot \mathrm{HCl} 10 \mathrm{mM}$. Xerogels were obtained after lyophilisation of the samples by immersion into liquid nitrogen and connected to a Telstar Lyoquest freezedrying system operating at $-85^{\circ} \mathrm{C}$ and $0.005 \mathrm{mBar}$. All the samples were freeze-dried for at least $72 \mathrm{~h}$.

\section{Wide-Angle X-Ray Diffraction}

Data collection was performed at room temperature with a Bruker D4 Endeavor Xray powder diffractometer by using $\mathrm{Cu}-\alpha$ radiation. A sample of the respective freezedried powder was placed on a sample holder and data were collected for $2 \theta$ values between $2^{\circ}$ and $40^{\circ}$ with a step size of $0.03^{\circ}$ and a time step of $10 \mathrm{~s}$. 


\section{Differential Scanning Calorimetry}

A freeze-dried gel was placed in a sealed aluminium pan. DSC thermographs were recorded on a METTLER TOLEDO DSC 821

\section{Single Crystal X-Ray Diffraction}

Single crystals of $\mathrm{C}_{22} \mathrm{H}_{48} \mathrm{~N}_{3} \mathrm{O}_{4} \mathrm{Cl}$ (ProValDov (4a) $\cdot \mathbf{H C l} \cdot 2 \mathrm{H}_{2} \mathrm{O}$ ) were obtained from an acidic aqueous solution. A suitable crystal was selected and mounted on a SuperNova, Dual, $\mathrm{Cu}$ at zero, Atlas diffractometer. The crystal was kept at 200.00(10) K during data collection. Using Olex $2{ }^{11}$ the structure was solved with the ShelXS ${ }^{12}$ structure solution program using Direct Methods and refined with the ShelXL ${ }^{12}$ refinement package using Least Squares minimisation.

Crystal structure determination of $\mathrm{C}_{22} \mathrm{H}_{48} \mathrm{~N}_{3} \mathrm{O}_{4} \mathrm{Cl}$ (ProValDoc (4a) $\cdot \mathbf{H C l} \cdot 2 \mathrm{H}_{2} \mathrm{O}$ )

Crystal Data. $\mathrm{C}_{22} \mathrm{H}_{48} \mathrm{~N}_{3} \mathrm{O}_{4} \mathrm{Cl}, \quad M=454.08$, monoclinic, $a=4.83104(13) \AA$ $7.1526(2) \AA, c=38.7650(12) \AA, B=90.420(3)^{\circ}, V=1339.47(7) \AA^{3}, T=200.00(10)$, space group $\mathrm{P} 2_{1}$ (no. 4), $Z=2, \mu(\mathrm{Cu} \mathrm{K \alpha})=1.491,7253$ reflections measured, 4335 unique $\left(R_{\text {int }}=\right.$ 0.0434 ) which were used in all calculations. The final $w R_{2}$ was 0.1916 (all data) and $R_{1}$ was 0.0495 (>2sigma(I)).

Deposition number: CCDC 951964.

\section{Crystal data collection and refinement for $4.1 \cdot \mathrm{HCl} \cdot 2 \mathrm{H}_{2} \mathrm{O}$}

\begin{tabular}{|c|c|}
\hline Identification code & CCDC 951964 \\
\hline Empirical formula & $\mathrm{C}_{22} \mathrm{H}_{48} \mathrm{~N}_{3} \mathrm{O}_{4} \mathrm{Cl}$ \\
\hline Formula weight & 454.08 \\
\hline Temperature/K & $200.00(10)$ \\
\hline Crystal system & monoclinic \\
\hline Space group & $\mathrm{P} 2_{1}$ \\
\hline$a / \AA ̊$ & $4.83104(13)$ \\
\hline$b / A ̊$ & $7.1526(2)$ \\
\hline
\end{tabular}




\begin{tabular}{|c|c|}
\hline$c / \AA ̊$ & $38.7650(12)$ \\
\hline$\alpha /^{\circ}$ & 90.00 \\
\hline$\beta /^{\circ}$ & $90.420(3)$ \\
\hline$\gamma /{ }^{\circ}$ & 90.00 \\
\hline Volume $/ \AA^{3}$ & 1339.47(7) \\
\hline Z & 2 \\
\hline$\rho_{\text {calc }} \mathrm{mg} / \mathrm{mm}^{3}$ & 1.126 \\
\hline $\mathrm{m} / \mathrm{mm}^{-1}$ & 1.491 \\
\hline$F(000)$ & 500.0 \\
\hline Crystal size $/ \mathrm{mm}^{3}$ & $0.533 \times 0.044 \times 0.038$ \\
\hline $2 \Theta$ range for data & 9.12 to $130.98^{\circ}$ \\
\hline Index ranges & $-5 \leq h \leq 5,-8 \leq k \leq 8,-45$ \\
\hline Reflections collected & 7253 \\
\hline Independent & $4335[R$ (int) $=0.0434]$ \\
\hline Data/restraints/para & $4335 / 1 / 288$ \\
\hline Goodness-of-fit on $F^{2}$ & 1.085 \\
\hline Final $\mathrm{R}$ indexes & $\mathrm{R}_{1}=0.0495, \quad w R_{2}=$ \\
\hline Final $R$ indexes [all] & $\mathrm{R}_{1}=0.0596, \quad \mathrm{wR}_{2}=$ \\
\hline $\begin{array}{ll}\text { Largest } & \text { diff. }\end{array}$ & $0.31 /-0.35$ \\
\hline Flack parameter & $-0.02(2)$ \\
\hline
\end{tabular}

Table 8.5.2. Fractional Atomic Coordinates $\left(\times 10^{4}\right)$ and Equivalent Isotropic Displacement Parameters $\left(\AA^{2} \times 10^{3}\right)$ for $4 \mathbf{a} \cdot \mathbf{H C l} \cdot 2 \mathrm{H}_{2} \mathbf{O}$. $U_{\text {eq }}$ is defined as $1 / 3$ of of the trace of the orthogonalised $U_{I J}$ tensor.

\begin{tabular}{|l|r|r|r|r|}
\hline Atom & \multicolumn{1}{|c|}{$\boldsymbol{x}$} & $\boldsymbol{y}$ & $\mathbf{U}$ \\
\hline C4 & $-7745(11)$ & $3444(7)$ & $-4329.2(10)$ & $45.6(11)$ \\
\hline C22 & $-7281(15)$ & $-1099(8)$ & $-2477.1(13)$ & $66.5(16)$ \\
\hline C20 & $-6240(10)$ & $-172(7)$ & $-2805.7(10)$ & $43.1(9)$ \\
\hline C6 & $-7142(7)$ & $1876(5)$ & $-2816.4(8)$ & $23.9(7)$ \\
\hline C21 & $-7235(16)$ & $-1214(7)$ & $-3123.6(13)$ & $70.1(18)$ \\
\hline C11 & $-8121(7)$ & $3615(5)$ & $-991.2(8)$ & $24.2(7)$ \\
\hline C15 & $-8095(7)$ & $3617(5)$ & $326.2(8)$ & $22.5(7)$ \\
\hline C3 & $-7194(10)$ & $5462(6)$ & $-4255.3(10)$ & $40.2(10)$ \\
\hline C13 & $-8124(7)$ & $3622(5)$ & $-333.1(8)$ & $22.0(7)$ \\
\hline
\end{tabular}




\begin{tabular}{|l|r|r|r|r|}
\hline C14 & $-6909(7)$ & $4437(5)$ & $-5.1(8)$ & $22.0(7)$ \\
\hline C9 & $-8006(7)$ & $3566(5)$ & $-1649.7(9)$ & $25.7(7)$ \\
\hline C17 & $-8077(7)$ & $3661(5)$ & $984.8(8)$ & $22.5(7)$ \\
\hline C16 & $-6862(7)$ & $4454(5)$ & $653.2(8)$ & $22.4(7)$ \\
\hline C7 & $-5963(7)$ & $2909(5)$ & $-2506.1(8)$ & $24.6(7)$ \\
\hline C2 & $-7777(9)$ & $5690(6)$ & $-3871.3(10)$ & $35.3(9)$ \\
\hline C18 & $-6852(7)$ & $4538(6)$ & $1310.1(8)$ & $28.2(8)$ \\
\hline C5 & $-7989(7)$ & $3271(5)$ & $-3382.9(8)$ & $21.1(7)$ \\
\hline C1 & $-6682(7)$ & $3872(5)$ & $-3722.0(9)$ & $24.5(7)$ \\
\hline C12 & $-6941(7)$ & $4457(5)$ & $-662.5(8)$ & $22.6(7)$ \\
\hline C8 & $-6983(8)$ & $4552(6)$ & $-1970.3(9)$ & $29.2(8)$ \\
\hline C10 & $-6974(7)$ & $4480(5)$ & $-1319.7(8)$ & $23.9(7)$ \\
\hline C19 & $-8116(9)$ & $3759(7)$ & $1640.2(9)$ & $39.5(10)$ \\
\hline N3 & $-7799(6)$ & $3634(5)$ & $-2287.4(7)$ & $26.3(6)$ \\
\hline N1 & $-7428(6)$ & $2434(4)$ & $-3991.1(7)$ & $24.7(7)$ \\
\hline N2 & $-6216(6)$ & $2775(4)$ & $-3132.9(7)$ & $22.0(6)$ \\
\hline O1 & $-10514(5)$ & $3225(5)$ & $-3354.8(6)$ & $35.8(6)$ \\
\hline O2 & $-3441(5)$ & $3041(6)$ & $-2463.1(7)$ & $49.3(9)$ \\
\hline C11 & $-2502.0(17)$ & $-251.0(14)$ & $-3981.6(2)$ & $36.5(3)$ \\
\hline O4 & $-12510(6)$ & $4973(5)$ & $-4939.3(8)$ & $-5223.1(8)$ \\
\hline O3 & $-7545(6)$ & $3780(5)$ & & $4(7)$ \\
\hline
\end{tabular}

Table 8.5.3. Anisotropic Displacement Parameters $\left(\AA^{2} \times 10^{3}\right)$ for $\mathbf{4 a} \cdot \mathbf{H C l} \cdot 2 \mathrm{H}_{2} \mathrm{O}$. The Anisotropic displacement factor exponent takes the form: $-2 \pi^{2}\left[h^{2} a^{2} U_{11}+\ldots+2 h k a \times b \times U_{12}\right]$

\begin{tabular}{|l|r|r|r|r|r|r|}
\hline \multicolumn{1}{|c|}{ Atom } & \multicolumn{1}{c|}{$\mathbf{U}_{11}$} & \multicolumn{1}{c|}{$\mathbf{U}_{22}$} & $\mathbf{U}_{33}$ & \multicolumn{1}{c|}{$\mathbf{U}_{23}$} & \multicolumn{1}{c|}{$\mathbf{U}_{13}$} & \multicolumn{1}{c|}{$\mathbf{U}_{12}$} \\
\hline C4 & $70(3)$ & $48(2)$ & $19.6(17)$ & $2.6(18)$ & $-4.9(19)$ & $-7(2)$ \\
\hline C22 & $116(5)$ & $49(3)$ & $35(2)$ & $17(2)$ & $6(3)$ & $-1(3)$ \\
\hline C20 & $60(3)$ & $43(2)$ & $26.4(18)$ & $5.2(19)$ & $6.1(17)$ & $2(2)$ \\
\hline C6 & $16.3(16)$ & $41.7(18)$ & $13.8(14)$ & $0.4(14)$ & $6.2(12)$ & $-4.6(14)$ \\
\hline C21 & $135(6)$ & $39(2)$ & $37(2)$ & $-7(2)$ & $4(3)$ & $-6(3)$ \\
\hline C11 & $23.8(17)$ & $27.6(17)$ & $21.1(16)$ & $1.4(13)$ & $1.9(13)$ & $-1.6(14)$ \\
\hline C15 & $18.6(16)$ & $29.2(18)$ & $19.6(15)$ & $2.6(14)$ & $2.4(13)$ & $1.0(13)$ \\
\hline C3 & $56(3)$ & $39(2)$ & $25.9(18)$ & $4.5(16)$ & $2.5(18)$ & $-1.3(19)$ \\
\hline C13 & $22.8(16)$ & $27.1(17)$ & $16.1(15)$ & $-0.4(13)$ & $3.9(13)$ & $1.0(13)$ \\
\hline C14 & $19.2(16)$ & $26.0(17)$ & $20.7(16)$ & $3.9(13)$ & $3.5(13)$ & $-1.2(13)$ \\
\hline
\end{tabular}




\begin{tabular}{|l|r|r|r|r|r|r|}
\hline C9 & $26.9(18)$ & $28.5(17)$ & $21.8(16)$ & $0.4(14)$ & $1.4(13)$ & $-5.2(14)$ \\
\hline C17 & $20.4(16)$ & $27.4(17)$ & $19.7(16)$ & $0.0(13)$ & $2.6(13)$ & $-0.5(14)$ \\
\hline C16 & $21.1(17)$ & $25.3(17)$ & $20.8(16)$ & $1.8(13)$ & $2.0(13)$ & $-0.2(13)$ \\
\hline C7 & $13.8(16)$ & $42.9(19)$ & $17.0(15)$ & $0.3(13)$ & $4.8(12)$ & $-1.3(14)$ \\
\hline C2 & $48(2)$ & $30.3(19)$ & $28.1(18)$ & $0.0(16)$ & $9.3(17)$ & $1.2(17)$ \\
\hline C5 & $25.4(17)$ & $38(2)$ & $21.3(16)$ & $-1.8(15)$ & $0.6(13)$ & $1.0(15)$ \\
\hline C1 & $16.1(15)$ & $31.5(17)$ & $15.8(14)$ & $-3.5(12)$ & $7.0(12)$ & $-1.9(13)$ \\
\hline C12 & $17.8(16)$ & $34.6(18)$ & $21.1(16)$ & $-1.1(14)$ & $5.1(13)$ & $-4.3(14)$ \\
\hline C8 & $23.1(17)$ & $26.8(18)$ & $18.0(15)$ & $2.7(13)$ & $2.9(12)$ & $0.0(13)$ \\
\hline C10 & $28.8(18)$ & $38(2)$ & $20.8(15)$ & $-2.6(15)$ & $6.0(13)$ & $-10.9(17)$ \\
\hline C19 & $25.1(17)$ & $26.7(17)$ & $19.9(16)$ & $2.5(13)$ & $3.2(12)$ & $-2.4(14)$ \\
\hline N3 & $43(2)$ & $55(3)$ & $21.0(17)$ & $3.2(17)$ & $1.0(16)$ & $5(2)$ \\
\hline N1 & $14.1(13)$ & $46.3(18)$ & $18.4(13)$ & $-2.1(13)$ & $2.6(11)$ & $-2.7(12)$ \\
\hline N2 & $24.0(16)$ & $29.6(16)$ & $20.5(14)$ & $0.4(11)$ & $8.0(12)$ & $0.3(12)$ \\
\hline O1 & $12.1(13)$ & $38.4(15)$ & $15.6(12)$ & $3.3(11)$ & $4.9(10)$ & $-1.2(11)$ \\
\hline O2 & $14.9(12)$ & $66.7(18)$ & $25.8(12)$ & $7.8(12)$ & $4.7(10)$ & $-1.2(12)$ \\
\hline C11 & $13.0(13)$ & $97(3)$ & $37.7(15)$ & $-17.5(16)$ & $0.1(11)$ & $-2.4(15)$ \\
\hline O4 & $25.4(4)$ & $34.4(4)$ & $49.9(5)$ & $-4.7(4)$ & $6.3(4)$ & $0.8(4)$ \\
\hline O3 & $37.6(15)$ & $47.9(17)$ & $49.7(16)$ & $-4.4(14)$ & $0.1(13)$ & $-1.0(14)$ \\
\hline & $33.5(16)$ & $50.0(16)$ & $47.0(17)$ & $0.7(15)$ & $4.1(13)$ & $3.9(13)$ \\
\hline
\end{tabular}

\begin{tabular}{|c|c|c|c|c|c|}
\hline \multicolumn{6}{|c|}{ Table 8.5.4. Bond Lengths for $4 \mathrm{a} \cdot \mathrm{HCl} \cdot 2 \mathrm{H}_{2} \mathrm{O}$. } \\
\hline Atom & Atom & Length/Å & Atom & Atom & Length/Å \\
\hline C4 & C3 & $1.495(7)$ & C9 & C8 & $1.515(5)$ \\
\hline $\mathrm{C4}$ & N1 & $1.503(5)$ & C9 & C10 & $1.518(5)$ \\
\hline $\mathrm{C} 22$ & $\mathrm{C} 20$ & $1.525(6)$ & C17 & C16 & $1.526(4)$ \\
\hline $\mathrm{C} 20$ & C6 & $1.529(6)$ & C17 & C18 & $1.524(4)$ \\
\hline $\mathrm{C} 20$ & C21 & $1.515(7)$ & C7 & N3 & $1.336(5)$ \\
\hline C6 & C7 & $1.519(5)$ & C7 & $\mathrm{O} 2$ & $1.232(4)$ \\
\hline C6 & N2 & $1.458(4)$ & $\mathrm{C} 2$ & C1 & $1.517(5)$ \\
\hline C11 & C12 & $1.517(4)$ & C18 & C19 & $1.527(5)$ \\
\hline C11 & C10 & $1.524(5)$ & C5 & C1 & $1.524(4)$ \\
\hline C15 & C14 & $1.527(4)$ & C5 & N2 & $1.336(4)$ \\
\hline C15 & C16 & $1.520(4)$ & C5 & 01 & $1.226(4)$ \\
\hline $\mathrm{C3}$ & $\mathrm{C} 2$ & $1.526(5)$ & C1 & N1 & $1.507(4)$ \\
\hline C13 & C14 & $1.514(4)$ & C8 & N3 & $1.446(5)$ \\
\hline C13 & C12 & $1.524(4)$ & & & \\
\hline
\end{tabular}




\begin{tabular}{|l|l|l|l||l|l|l|r|}
\hline \multicolumn{2}{|l|}{ Table 8.5.5. Bond Angles for $\mathbf{4 a} \cdot \mathbf{H C l} \cdot \mathbf{2} \mathbf{H}_{\mathbf{2}} \mathbf{O}}$. \\
\hline Atom & Atom & Atom & \multicolumn{1}{|l|}{ Angle/ $^{\circ}$} & Atom & Atom Atom & Angle/ $^{\circ}$ \\
\hline C3 & C4 & N1 & $106.3(3)$ & O2 & C7 & C6 & $120.6(3)$ \\
\hline C22 & C20 & C6 & $110.1(4)$ & O2 & C7 & N3 & $123.0(3)$ \\
\hline C21 & C20 & C22 & $111.1(5)$ & C1 & C2 & C3 & $102.4(3)$ \\
\hline C21 & C20 & C6 & $111.2(4)$ & C17 & C18 & C19 & $112.8(3)$ \\
\hline C7 & C6 & C20 & $109.9(3)$ & N2 & C5 & C1 & $115.6(3)$ \\
\hline N2 & C6 & C20 & $110.9(3)$ & O1 & C5 & C1 & $120.2(3)$ \\
\hline N2 & C6 & C7 & $109.7(3)$ & O1 & C5 & N2 & $124.1(3)$ \\
\hline C12 & C11 & C10 & $113.8(3)$ & C2 & C1 & C5 & $115.2(3)$ \\
\hline C16 & C15 & C14 & $113.8(3)$ & N1 & C1 & C2 & $103.9(3)$ \\
\hline C4 & C3 & C2 & $104.8(3)$ & N1 & C1 & C5 & $107.8(3)$ \\
\hline C14 & C13 & C12 & $114.0(3)$ & C11 & C12 & C13 & $114.0(3)$ \\
\hline C13 & C14 & C15 & $114.4(3)$ & N3 & C8 & C9 & $113.4(3)$ \\
\hline C8 & C9 & C10 & $112.6(3)$ & C9 & C10 & C11 & $114.2(3)$ \\
\hline C18 & C17 & C16 & $113.3(3)$ & C7 & N3 & C8 & $122.5(3)$ \\
\hline C15 & C16 & C17 & $113.9(3)$ & C4 & N1 & C1 & $107.3(3)$ \\
\hline N3 & C7 & C6 & $116.4(3)$ & C5 & N2 & C6 & $121.9(3)$ \\
\hline
\end{tabular}

\begin{tabular}{|c|c|c|c|c|}
\hline Atom & $x$ & $y$ & $z$ & $\mathrm{U}(\mathrm{eq})$ \\
\hline $\mathrm{H} 1 \mathrm{~A}$ & $-5770(100)$ & $1450(70)$ & $-3996(13)$ & $43(13)$ \\
\hline $\mathrm{H} 1 \mathrm{~B}$ & $-9110(90)$ & $1720(60)$ & $-3943(12)$ & $31(11)$ \\
\hline $\mathrm{H} 4 \mathrm{~A}$ & -6434 & 2969 & -4496 & 55 \\
\hline H4B & -9603 & 3281 & -4421 & 55 \\
\hline $\mathrm{H} 22 \mathrm{~A}$ & -6677 & -389 & -2280 & 100 \\
\hline $\mathrm{H} 22 \mathrm{~B}$ & -6555 & -2346 & -2462 & 100 \\
\hline $\mathrm{H} 22 \mathrm{C}$ & -9267 & -1147 & -2482 & 100 \\
\hline $\mathrm{H} 2 \mathrm{O}$ & -4212 & -209 & -2802 & 52 \\
\hline H6 & -9167 & 1932 & -2808 & 29 \\
\hline $\mathrm{H} 21 \mathrm{~A}$ & -6855 & -2524 & -3097 & 105 \\
\hline $\mathrm{H} 21 \mathrm{~B}$ & -6289 & -748 & -3323 & 105 \\
\hline $\mathrm{H} 21 \mathrm{C}$ & -9192 & -1031 & -3152 & 105 \\
\hline $\mathrm{H} 11 \mathrm{~A}$ & -10116 & 3767 & -991 & 29 \\
\hline $\mathrm{H} 11 \mathrm{~B}$ & -7731 & 2284 & -993 & 29 \\
\hline $\mathrm{H} 15 \mathrm{~A}$ & -10081 & 3816 & 327 & 27 \\
\hline H15B & -7772 & 2279 & 328 & 27 \\
\hline
\end{tabular}




\begin{tabular}{|c|c|c|c|c|}
\hline $\mathrm{H} 3 \mathrm{~A}$ & -8406 & 6256 & -4392 & 48 \\
\hline H3B & -5287 & 5779 & -4306 & 48 \\
\hline $\mathrm{H} 13 \mathrm{~A}$ & -10111 & 3817 & -333 & 26 \\
\hline $\mathrm{H} 13 \mathrm{~B}$ & -7797 & 2284 & -334 & 26 \\
\hline $\mathrm{H} 14 \mathrm{~A}$ & -7224 & 5777 & -5 & 26 \\
\hline $\mathrm{H} 14 \mathrm{~B}$ & -4923 & 4236 & -5 & 26 \\
\hline H9A & -7394 & 2275 & -1654 & 31 \\
\hline H9B & -10014 & 3568 & -1651 & 31 \\
\hline H17A & -10063 & 3861 & 983 & 27 \\
\hline $\mathrm{H} 17 \mathrm{~B}$ & -7753 & 2323 & 991 & 27 \\
\hline $\mathrm{H} 16 \mathrm{~A}$ & -7157 & 5796 & 650 & 27 \\
\hline H16B & -4880 & 4237 & 654 & 27 \\
\hline $\mathrm{H} 2 \mathrm{~A}$ & -6801 & 6760 & -3776 & 42 \\
\hline $\mathrm{H} 2 \mathrm{~B}$ & -9743 & 5830 & -3829 & 42 \\
\hline $\mathrm{H} 18 \mathrm{~A}$ & -7149 & 5879 & 1303 & 34 \\
\hline $\mathrm{H} 18 \mathrm{~B}$ & -4871 & 4319 & 1315 & 34 \\
\hline $\mathrm{H} 1$ & -4666 & 3942 & -3695 & 29 \\
\hline $\mathrm{H} 12 \mathrm{~A}$ & -7299 & 5792 & -664 & 27 \\
\hline $\mathrm{H} 12 \mathrm{~B}$ & -4950 & 4283 & -661 & 27 \\
\hline $\mathrm{H} 8 \mathrm{~A}$ & -7694 & 5820 & -1972 & 35 \\
\hline $\mathrm{H} 8 \mathrm{~B}$ & -4979 & 4623 & -1960 & 35 \\
\hline $\mathrm{H} 10 \mathrm{~A}$ & -7467 & 5795 & -1325 & 29 \\
\hline $\mathrm{H} 10 \mathrm{~B}$ & -4970 & 4400 & -1312 & 29 \\
\hline $\mathrm{H} 19 \mathrm{~A}$ & -10094 & 3883 & 1629 & 59 \\
\hline $\mathrm{H} 19 \mathrm{~B}$ & -7415 & 4442 & 1835 & 59 \\
\hline $\mathrm{H} 19 \mathrm{C}$ & -7635 & 2463 & 1664 & 59 \\
\hline $\mathrm{H} 3$ & -9532 & 3551 & -2337 & 32 \\
\hline $\mathrm{H} 2$ & -4480 & 2992 & -3160 & 26 \\
\hline $\mathrm{H} 4 \mathrm{C}$ & -12531 & 6153 & -4915 & 68 \\
\hline H4D & -13879 & 4639 & -5064 & 68 \\
\hline $\mathrm{H} 3 \mathrm{C}$ & -9038 & 4104 & -5125 & 65 \\
\hline$H 3 \mathrm{D}$ & -7678 & 3990 & -5438 & 65 \\
\hline
\end{tabular}

\section{FTIR and Circular Dichroism.}

Sample discs were prepared by mixing the freeze-dried solids with $200-300 \mathrm{mg}$ of $\mathrm{KBr}$ in an agate mortar and pressing the mixture at 10 ton for ca. $10 \mathrm{~min}$. Transparent discs of less than $0.5 \mathrm{~mm}$ were obtained. FT-IR spectra were collected in a JASCO FTIR- 
6200 spectrometer. CD spectra were recorded in a JASCO J-810 spectrometer. CD spectra were randomly taken in at least six different positions of the disc and averaged.

\subsection{Determination of Acid-Base Thermodynamics}

Potentiometric titrations to determine acid-base constants were carried out at 298 K. In a typical experiment an aqueous solution of the corresponding dissolved compound were titrated with a normalized solution of aqueous hydrogen chloride (for succinic acid derivatives) or sodium hydroxide (for proline and pyridine derivatives) with vigorous stirring. The $\mathrm{pH}$ was monitored every 10 seconds (in a S220 Seven Compact pH-meter Mettler Toledo). Thermodynamic constants for the species in solution could be calculated for all the compounds analysing the titration data with HYPERQUAD-2008. Then the acidbase constants were calculated iteratively with HYSS2009, adjusting its value to fit the calculated and experimental $\mathrm{pH}$.

\subsection{Study of Dynamic Combinatorial Libraries (University of Strathclyde Glasgow)}

\section{Hydrogelation of NDY-Tyr (6a)}

The precursor NDI-Tyr (6a) (500 mM) was prepared as a stock solution in DMSO. The required volume of NDI-Tyr (6a) stock solution (e.g. $20 \mu \mathrm{L}$ for $10 \mathrm{mM}$ ) was added to 1 $\mathrm{mL}$ of $100 \mathrm{mM}$ phosphate buffer $(\mathrm{pH} 8)$. The solution was vortexed for few seconds and sonicated for about $1 \mathrm{~min}$ to ensure dissolution. This solution formed a self-supporting hydrogel within $2 \mathrm{~min}$. Gelation was considered to have occurred when a homogeneous solid-like material was obtained that exhibited no gravitational flow in each case.

\section{Enzyme-triggered hydrogelation}

Similarly, the precursors NDI-Tyr (6a) (10 mM) and each $\mathbf{X}-\mathbf{N H}_{\mathbf{2}}(20 \mathrm{mM}$, where "X" refers tyrosine, Tyr; phenylalanine, Phe; leucine, Leu; valine, Val; alanine, Ala and glycine, Gly; were mixed (at 1:2 ratio) in a glass vial. The mixture was suspended in $1.0 \mathrm{~mL}$ of 100 $\mathrm{mM}$ phosphate buffer $(\mathrm{pH} 8)$ with the addition of $1 \mathrm{mg} \mathrm{mL}^{-1}$ lyophilised thermolysin powder (bacillus Thermoproteolyticus rokko, mol wt $34.6 \mathrm{kDa}$ by amino acid sequence). The mixture was vortexed for few seconds and sonicated for about $1 \mathrm{~min}$ to ensure 
dissolution which later on developed into a self-supporting hydrogel. Samples were incubated at room temperature for $24 \mathrm{~h}$ before analysis, unless otherwise stated.

\section{Library procedure}

In the case of dynamic combinatorial library (DCL), NDI-Tyr (6a) (10 mM) and various $\mathbf{X}-\mathbf{N H}_{2}$ (20 $\mathrm{mM}$ each) derivatives were mixed and the above procedure was followed to form a self-supporting hydrogel.

In the case of mixed charge transfer donor-acceptor system, donor was first dissolved in buffer at the required concentration $(10 \mathrm{mM}$, at 1:1 donor/acceptor ratio, 1 $\mathrm{mM}$ at 10:1 ratio, $5 \mathrm{mM}$ at 1:2 ratio and $20 \mathrm{mM}$ at 2:1 ratio respectively) followed by the sequential additions of each $\mathbf{X}-\mathbf{N H}_{\mathbf{2}}, \mathbf{N D I}-\mathbf{T y r}(\mathbf{6 a})$ and thermolysin, mixed as described above, to form self-supporting charge transfer hydrogels.

\section{High-performance liquid chromatography (HPLC)}

A Dionex P680 HPLC system was used to quantify the percentage conversion of the enzymatic reaction. A $50 \mu \mathrm{L}$ sample was injected onto a Macherey-Nagel C18 column of $250 \mathrm{~mm}$ length with an internal diameter of $4.6 \mathrm{~mm}$ and $5 \mu \mathrm{m}$ fused silica particles at a flow rate of $1 \mathrm{~mL}$ per minute (eluting solvent system: linear gradient of $20 \%(\mathrm{v} / \mathrm{v})$ acetonitrile in water for 4 min, gradually rising to $80 \%(v / v)$ acetonitrile in water at 35 min. This concentration was kept constant until 40 min when the gradient was decreased to $20 \%(\mathrm{v} / \mathrm{v})$ acetonitrile in water at $42 \mathrm{~min}$ ). Sample preparation involved mixing $20 \mu \mathrm{L}$ of the sample with $1 \mathrm{~mL}$ of acetonitrile-water (50:50 mixture) containing $0.1 \%$ trifluoroacetic acid. The intensity of each identified peak was determined by UV detection at $383 \mathrm{~nm}$ for

(NDI derivatives) and $296 \mathrm{~nm}$ (for donors). The experimental data were acquired in triplicate and the average values are shown. The samples were vortexed before collecting the aliquots for HPLC and the percentage yields are calculated from HPLC integrated peak areas. 


\subsection{References and Notes}

1. (a) Escuder, B.; Martí, S.; Miravet, J. F. "Organogel Formation by Coaggregation of Adaptable Amidocarbamates and their Tetraamide Analogues", Langmuir, 2005, 21, 67766787; (b) Rodríguez-Llansola, F.; Escuder, B.; Miravet, J. F. "Switchable Perfomance of an L-Proline-Derived Basic Catalyst Controlled by Supramolecular Gelation", J. Am. Chem. Soc., 2009, 131, 11478-11484.

2. Becerril, J.; Bolte, M.; Burguete, M. I.; Galindo, F.; García-España, E.; Luis, S. V.; Miravet, J. F. "Efficient Macrocyclization of U-Turn Preorganized Peptidomimetics: The Role of Intramolecular H-Bond and Solvophobic Effects", J. Am. Chem. Soc., 2003, 125, 6677-6686.

3. Rodríguez-Llansola, F.; Miravet, J. F.; Escuder, B. "A Supramolecular Hydrogel as a Reusable Heterogeneous Catalyst for the Direct Aldol Reaction", Chem. Commun., 2009, 7303-7305.

4. Fontanillo, M.; Angulo-Pachón, C. A.; Escuder, B.; Miravet, J. F. "In Situ Synthesis-Gelation at Room Temperature vs. Heating-Cooling Procedure. Fine Tuning of Molecular Gels Derived from Succinic Acid and L-Valine", J. Colloid Interface Sci., 2013, 412, 65-71.

5. Horne, W. S.; Ashkenasy, N.; Ghadiri, M. R. "Modulating Charge Transfer through Cyclic D,L- $\alpha$-Peptide Self-Assembly", Chem. Eur. J., 2005, 11, 1137-1144.

6. Au-Yeung, H. Y.; Pantos, G. D.; Sanders, J. K. M. "Dynamic Combinatorial Synthesis of a Catenane Based on Donor-Acceptor Interactions in Water", Proc. Natl. Acad. Sci. U. S. A., 2009, 106, 10466-10470.

7. Au-Yeung, H. Y.; Pantos, G. D.; Sanders, J. K. M. "A Water Soluble Donor-Acceptor [2]Catenane that Can Switch between a Coplanar and a Gemini-Sign Conformation", Angew. Chem. Int. Ed., 2010, 49, 5331-5334.

8. Stejskal, E. O.; Tanner, J. E. "Spin Diffusion Measurements: Spin Echoes in the Presence of a Time-Dependent Field Gradient", J. Chem. Phys., 1965, 42, 288-292.

9. Rodríguez-Llansola, F.; Miravet, J. F.; Escuder, B. "Supramolecular Catalysis with Extended Aggregates and Gels: Inversion of Stereoselectivity Caused by Self-Assembly", Chem. Eur. J., 2010, 16, 8480-8486.

10. Cobb, A. J.; Shaw, D. M.; Longbottom, D. A.; Gold, J. B.; Ley, S. V. "Organocatalysis with Proline Derivatives: Improved Catalysts for the Asymmetric Mannich, Nitro-Michael and Aldol Reactions", Org. Biomol. Chem., 2005, 3, 84-96.

11. Dolomanov, O. V.; Bourhis, L. J.; Gildea, R. J.; Howard, J. A. K.; Puschmann, H. "OLEX2: a Complete Structure Solution, Refinement and Analysis Program", J. Appl. Crystallogr., 2009, 42, 339-341.

12. Sheldrick, G. M. "A Short History of SHELX", Acta Crystallogr., Sect. A, 2008, 64, 112-122. 

ANNEXES 



\section{Annex I. Summary in Spanish}

\section{Tema y objetivos}

Los geles moleculares constituyen un ejemplo de materiales auto-ensamblados. Estos materiales blandos están constituidos por moléculas de bajo peso molecular que son capaces de auto-ensamblarse anisotrópicamente mediante interacciones de naturaleza no covalente (enlaces de hidrogeno, van de Waals, etc) dando lugar a agregados que crecen preferentemente en una dirección. Estos agregados posteriormente pueden seguir asociándose para dar lugar a fibras que a su vez contribuyen a la formación de redes tridimensionales (Figura 9.1). Estas redes de agregados tienen la capacidad de retener el disolvente en el que tiene lugar el proceso confiriendo a estos materiales de propiedades visco-elásticas y de un aspecto característico de "gel" en forma de sólido blando.

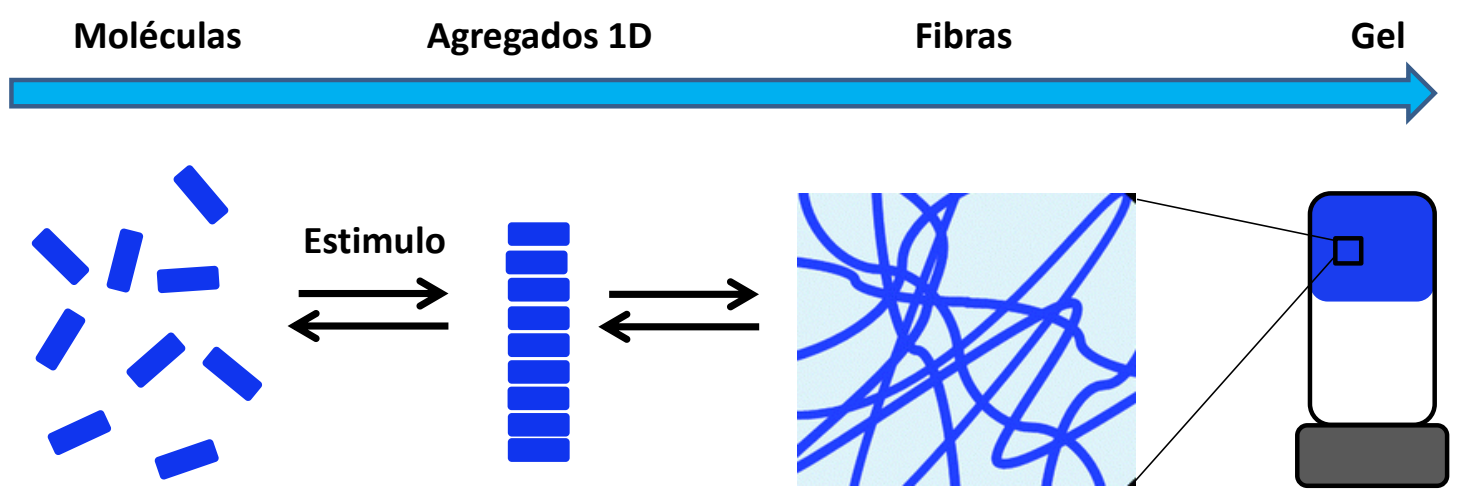

Figura 9.1. Representación del proceso de agregación y de la formación de geles moleculares.

El auto-ensamblaje jerárquico de moléculas es una herramienta que ofrece una amplia variedad de posibilidades para conseguir sistemas que presenten una organización precisa de grupos funcionales, imitando los centros de reconocimiento y catálisis por excelencia, las enzimas. Este tipo de proceso denominado también "construcción de abajo a arriba" es ventajoso desde el punto de vista de la economía atómica y energética. Además, la participación de enlaces de naturaleza no covalente dota a estos sistemas de reversibilidad y les confiere propiedades dinámicas que permiten la autocorrección de 
errores en el proceso de asociación y la obtención de estructuras altamente ordenadas libres de defectos.

Un gel molecular funcional puede considerarse como un material que presenta una amplia superficie activa y que presenta una organización precisa de grupos funcionales (por ejemplo, catalíticos, capaces del reconocimiento molecular, que respondan a estímulos, etc.). Los reactivos pueden difundir a través del gel e interaccionar, de forma covalente o no covalente, con los grupos funcionales que este contenga. En el caso de grupos funcionales catalíticos, se espera que la agregación de los mismos dé lugar a cambios en la eficiencia y la selectividad del catalizador. Estos cambios estarían relacionados, por ejemplo, con cambios en la movilidad conformacional, orientación en la aproximación de los sustratos o cooperación entre grupos catalíticos próximos. Por otro lado, la presencia de grupos funcionales capaces de interaccionar con especies moleculares (mediante enlaces de $\mathrm{H}$, interacciones solvofóbicas, interacciones covalentes reversibles, etc.) puede dar lugar a procesos de reconocimiento molecular. El reconocimiento sería el resultado de las características intrínsecas de los grupos funcionales o de la cooperación de los mismos en la interacción con sustratos. Esta interacción modificaría las propiedades del gel molecular, dando lugar a respuestas asociadas a los cambios producidos como, por ejemplo, liberación de especies activas o cambio de volumen (respuesta mecánica) de interés en una variedad de aplicaciones (Figura 9.2).

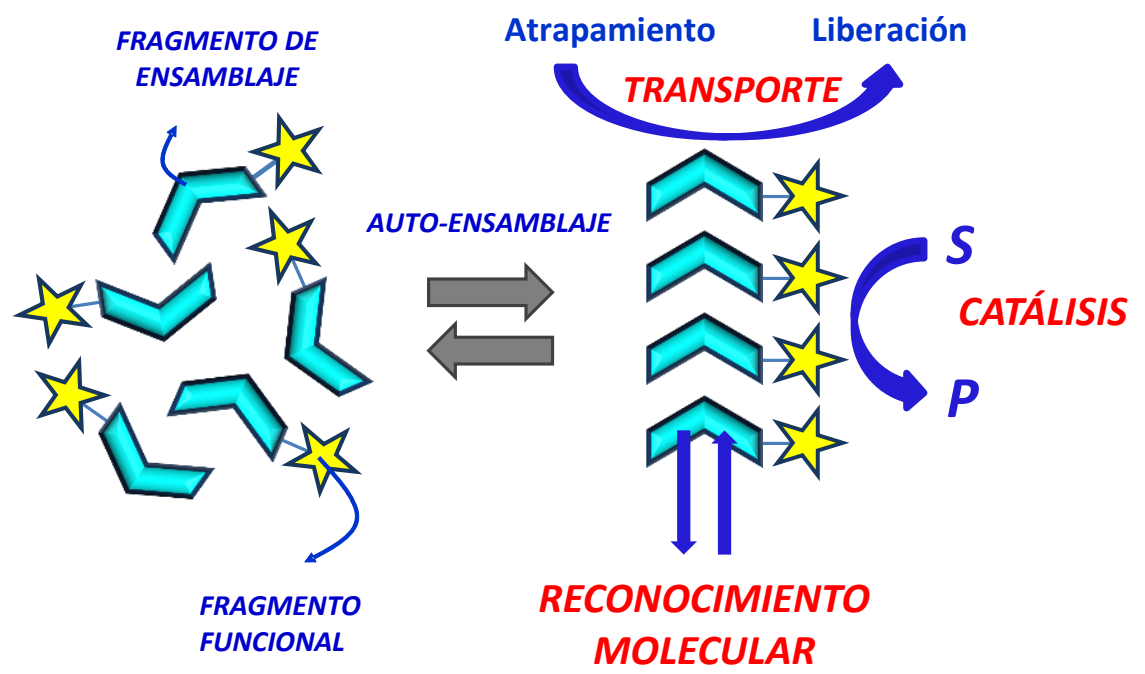

Figura 9.2. Esquema del proceso de auto-ensamblaje para la obtención de materiales funcionalizados. 
El objetivo de esta tesis es por lo tanto el diseño de nuevos gelantes de bajo peso molecular derivados de péptidos que incorporen fragmentos funcionales para la obtención de materiales funcionalizados con aplicaciones en campos como la catálisis o los materiales que respondan a estímulos. Se espera que la ordenación de los grupos dentro de las fibras del gel de lugar a propiedades que difieran de las observadas en disolución.

\section{Planteamiento y metodología}

La idea principal en esta tesis es por lo tanto el diseño de estructuras gelantes que generarán materiales blandos funcionalizados. Basado en esto, el diseño de los gelantes ha sido pensado siguiendo un enfoque modular. Partiendo de una estructura general con un esqueleto peptídico, la modificación de la misma da lugar a las estructuras finales que combinan fragmentos de auto-ensamblaje con fragmentos funcionales (Figura 9.3), responsables de otorgar las propiedades finales al material. De este modo se ha diseñado una familia de anfífilos y bolaanfífilos con el objetivo de formar geles tanto en disolventes orgánicos (organogeles) como en medio acuoso (hidrogeles).

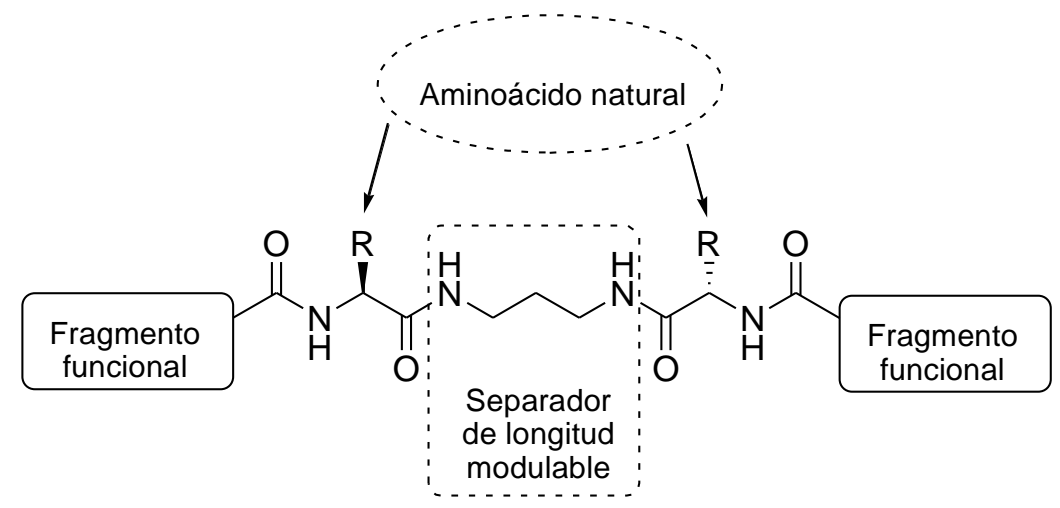

Figura 9.3. Representación del enfoque modular usado en el diseño de los gelantes.

Por lo tanto, la metodología general empleada para llevar a cabo esta tesis comporta diferentes etapas que van desde el diseño y síntesis del gelante, con la selección del grupo funcional adecuado para cada tipo de aplicación, el estudio de gelación en los diferentes disolventes de interés para cada caso, la caracterización del gel y por último, su aplicación funcional. A continuación se describe el plan de trabajo y la metodología a utilizar en cada uno de los apartados propuestos: 
1) Diseño y síntesis de gelantes moleculares

Se ha diseñado una familia de gelantes derivados de péptidos partiendo de estructuras generales previamente estudiadas en nuestro grupo. Debido al carácter modular, diferentes variaciones estructurales así como varios grupos funcionales se han introducidos. De este modo las moléculas gelantes se han preparado siguiendo un procedimiento sintético convencional de síntesis en disolución.

2) Estudio del proceso de agregación y caracterización de geles moleculares

La habilidad gelante de los compuestos sintetizados se ha evaluado en disolventes orgánicos así como en medio acuoso. Se ha analizado el proceso de agregación para poder tener una mejor comprensión de las reglas que lo controlan. Además, se han estudiado las propiedades gelantes de los compuestos desde un punto de vista macroscópico y microscópico y se han determinado parámetros como la concentración mínima de gelación o la morfología del gelante.

3) Geles catalíticos

Gracias a la creciente popularidad del uso de derivados de prolina como catalizadores eficientes $\mathrm{y}$ apoyados por los buenos resultados previamente obtenidos al incorporar este aminoácido en la estructura del gelante como grupo funcional, se ha evaluado a una familia de compuestos derivados de L-Prolina como organocatalizadores para la reacción de Michael en disolventes orgánicos. Por otra parte, se ha testado un derivado anfífilo como catalizador en medio acuoso para la reacción aldólica con diferentes sustratos prestando atención al efecto hidrofóbico.

4) Geles como materiales que responden a estímulos

Con el objetivo de obtener materiales funcionalizados inteligentes, se ha estudiado la introducción de grupos funcionales susceptibles a estímulos. Estos sistemas se definen como materiales capaces de cambiar sus propiedades ante la presencia de estímulos externos como un cambio en el $\mathrm{pH}$, luz, calor, etc. Basado en esto, se ha estudiado una familia de hidrogelantes ionizables mediante cambios de $\mathrm{pH}$. Adicionalmente, se ha analizado un sistema multi-componente complejo, basado en la diferente basicidad de cada uno de los componentes.

5) Bibliotecas combinatorias dinámicas para el descubrimiento de geles como materiales con propiedades electrónicas

Finalmente se ha evaluado el uso de la química combinatoria dinámica como herramienta para el descubrimiento de nuevas estructuras funcionalizadas. Una familia de derivados peptídicos conteniendo naftalendiimidas (NDI), grupo usado 
en materiales orgánicos conductores, se ha combinado con una serie de derivados de aminoácidos para descubrir la mejor estructura. Además, se ha evaluado la incorporación de dadores de electrones al sistema, que aportan interacciones adicionales de transferencia de carga. Para terminar, se ha llevado a cabo una completa caracterización del mejor sistema.

\section{Aportaciones originales}

- Una amplia familia de compuestos, todos ellos derivados de péptidos, han sido diseñados y sintetizados (ver moléculas en Annex II) siguiendo el enfoque modular donde pequeñas modificaciones han sido introducidas para generar los diferentes gelantes. Así mismo, este enfoque nos ha permitido funcionalizar las moléculas permitiendo introducir el grupo funcional deseado dentro de la estructura molecular de una forma efectiva a través de una metodología sintética simple en disolución. Esta metodología ha permitido obtener los diferentes compuestos en escala de gramos y con buenos rendimientos sin la necesidad de métodos complejos de purificación.

- Se ha estudiado el proceso de agregación de varios compuestos en tolueno. Para ello, han sido utilizados cuatro diferentes dipéptidos (3a-d), todos ellos derivados de Lprolina a los cuales se les ha modificado el aminoácido central. Se ha llevada a cabo una caracterización completa del proceso de agregación así como un estudio de la disposición molecular, tanto en disolución como en estado agregado. Los resultados muestran una diferente preferencia conformacional respecto al anillo de prolina, dependiendo del aminoácido central utilizado, antes y después del proceso de agregación (ver Esquema 3.2.2.2).

- Han sido evaluados los diferentes dipéptidos derivados de L-Prolina (3a-d) como posibles organocatalizadores en la reacción de Michael usada como modelo entre la ciclohexanona y el trans- $\beta$-nitroestireno en tolueno. Los resultados han mostrado actividad catalítica después de la agregación para todos ellos. Adicionalmente, se ha encontrado que las diferencias en las preferencias conformacionales obtenidas previamente, influyen en la selectividad de dicha reacción, siendo una conformación (la "anti" respecto al anillo de prolina, ver Figura 3.2.2.3) ligeramente más selectiva hacia la formación del enantiómero mayoritario. 
- El estudio del proceso de agregación de los análogos solubles así como de su actividad como organocatalizadores, ha servido para escoger el mejor aminoácido y de esta forma diseñar la estructura gelante bolaanfífilica derivada (3e). La habilidad gelante de este compuesto ha sido completamente estudiada y sus propiedades y características han sido analizadas desde un punto de vista tanto macroscópico como microscópico en tolueno.

- Adicionalmente ha sido evaluado el gelante derivado de L-Prolina (3e) como organocatalizador supramolecular en fase gel para la reacción de Michael entre la ciclohexanona y el trans- $\beta$-nitroestireno en tolueno. Se ha demostrado la participación de la red fibrilar en la actividad catalítica y se han obtenido resultados de conversiones cuantitativas así como una moderada selectividad.

- Se ha estudiado la habilidad gelante del anfífilo derivado de L-Prolina (4a) en medio acuoso. Se han encontrado y caracterizado cuatro diferentes polimorfos (A-D) dependiendo del método de gelación empleado. Los resultados muestran pequeñas diferencias en el empaquetamiento de las estructuras moleculares después de la agregación. Adicionalmente, se ha evaluado la actividad catalítica de este derivado para la reacción aldólica entre la ciclohexanona y el 4-nitrobenzaldehído en medio acuoso "sobre agua" (donde la disolución de los reactivos no es necesaria). Los resultados han mostrado diferencias en la selectividad y en la velocidad de reacción para los diferentes polimorfos utilizados, destacando la importancia de la caracterización de estas estructuras supramoleculares para su uso como materiales funcionalizados.

- Se ha realizado un estudio adicional usando el anfífilo derivado de L-prolina (4a) en agua como organocatalizador en fase gel para la reacción aldólica entre el 4nitrobenzaldehído y diferentes cetonas alifáticas de longitud de cadena variable. Los resultados han mostrado que el efecto hidrofóbico juega un papel importante tanto en la agregación como en el comportamiento catalítico. Se ha observado la selección de los sustratos más hidrofóbicos para llevar a cabo la reacción en las fibras catalíticas. Adicionalmente, se ha estudiado el mecanismo de la reacción aldólica en este sistema, detectándose intermedios de tipo enamina. 
- Se ha estudiado la introducción de grupos que responden a estímulos con el objetivo de generar materiales inteligentes. Para ello se ha diseñado una familia de moléculas a las cuales se les ha introducido un grupo ionizable (derivado del ácido succínico) (5a-d), y se han evaluado para su uso como materiales que respondan a cambios en el $\mathrm{pH}$. Se ha analizado su capacidad gelante así como el equilibrio acido-base, tanto en disolución como después de la agregación, y se han obtenido las correspondientes constantes de ionización. Los resultados han mostrado algunas diferencias relacionadas con las propiedades agregantes, además de la posible presencia de agregados para las especies solubles cargadas involucradas en el equilibrio de ionización.

- Se ha llevado a cabo un estudio de sistemas complejos multicomponente en medio acuoso. En este caso, se ha preparado y analizado un sistema en fase gel formado por el gelante derivado de L-prolina·(3e) en presencia de un derivado de piridina (5e). El estudio de la influencia del $\mathrm{pH}$ en la habilidad gelante de este sistema ha mostrado la sorprendentemente solubilización de la red fibrilar del derivado de piridina a valores de $\mathrm{pH}$ en los cuales este compuesto no es soluble. Se ha propuesto explicar este comportamiento por la formación de agregados micelares mixtos entre el derivado de prolina cargado y el derivado de piridina neutro.

- Se ha estudiado el sistema multicomponente (3e+5e) como material para su uso en la liberación controlada de sustancias. Los resultados han demostrado diferentes perfiles de liberación en función del pH del sistema y se ha corroborado por lo tanto, su uso como sistema inteligente que responde a estímulos.

- Se ha probado un nuevo enfoque en el descubrimiento de moléculas gelantes. En él, la química combinatoria dinámica se ha empleado para elegir al mejor candidato entre una mezcla de moléculas. En este estudio, se ha usado un derivado de naftalendiimida NDI (6a), al cual se le ha unido un fragmento de tipo aminoácido, como precursor para la generación de una familia de compuestos gelantes basada en péptidos. En esta familia la agregación tiene lugar a partir de la formación de un enlace peptídico por medio de la acción de una enzima. Se ha demostrado la formación de varios gelantes (6b-g) y se ha visto la amplificación del mejor gelante en 
una mezcla de derivados de aminoácido. Además, se ha visto que la presencia de diferentes dadores de electrones amplifica la selección del mejor gelante gracias a interacciones adicionales de transferencia de carga entre los dadores de electrones y los derivados de NDI que actúan como aceptores de electrones.

- Finalmente se ha llevado a cabo una completa caracterización de las propiedades del mejor sistema obtenido en la librería (6c) en presencia y en ausencia de diferentes dadores de electrones. Con este estudio se ha conseguido una mejor compresión del proceso de agregación.

Estos resultados han conducido a la redacción de los artículos de investigación que se citan a continuación:

Substrate Selective Catalytic Molecular Hydrogels: the Role of the Hydrophobic Effect. C. Berdugo; J. F. Miravet; B. Escuder, Chem. Commun., 2013, 49, 10608-10610.

Biocatalytic Self-Assembly of Supramolecular Charge-Transfer Nanostructures Based on $\mathbf{n}$-Type Semiconductor-Appended Peptides. S. K. M. Nalluri; C. Berdugo; N. Javid; P. W. J. M. Frederix; R. V. Ulijn, Angew. Chem. Int. Ed., 2014, 53, $5882-5887$.

Structural Insight into the Aggregation of L-Prolyl Dipeptides and its Effect on Organocatalytic Performance. C. Berdugo; J. F. Miravet; B. Escuder, Org. Biomol. Chem., 2015, 13, 592-600.

Study of the Effect of Polymorphism on the Self-Assembly and Catalytic Performance of an LProline Based Molecular Hydrogelator. S. Díaz-Oltra; C. Berdugo; J. F. Miravet; B. Escuder, New J. Chem., 2015, DOI: 10.1039/C5NJ00072F.

A Biocatalytically Driven Dynamic Combinatorial Library Approach for the Discovery of Aqueous Gel Phase Charge Transfer Nanostructures. C. Berdugo; S. K. M. Nalluri; N. Javid; B. Escuder; J. F. Miravet; R. V. Ulijn (manuscrito en preparación).

\section{Conclusiones y futuras líneas de investigación}

En conclusión en este trabajo, se han diseñado y sintetizado diferentes compuestos derivados de péptidos, tanto anfífilos como bolaanfífilos, para ser usados como gelantes mediante un enfoque modular, en donde diferentes grupos funcionales pueden ser introducidos en la estructura de la molécula para así poder generar compuestos funcionalizados. De este modo, han sido introducidos varios grupos funcionales y los gelantes así construidos han sido probados en diferentes campos de estudio como la catálisis o los materiales con propiedades electrónicas. Además se ha 
demostrado que la naturaleza no covalente de estos materiales supramoleculares provoca que estos puedan responder a diferentes estímulos (como el $\mathrm{pH}$ ), y que tengan aplicaciones en la construcción de materiales inteligentes.

Adicionalmente se ha comprobado el uso de la química combinatoria dinámica como una herramienta potente para el descubrimiento de materiales funcionalizados, y la gelación por medio de enzimas como una metodología eficiente para la generación de sistemas en fase gel.

Los resultados descritos abren el camino para futuras líneas de investigación como por ejemplo:

- El desarrollo de redes fibrilares catalíticas en medios acuosos con elevada selectividad y eficiencia. Estos materiales presentarían regulación de la actividad catalítica en presencia de estímulos, dando lugar a propiedades biomiméticas.

- La preparación de nuevos materiales orgánicos con propiedades optoelectrónicas mediante la metodología basada en química combinatoria dinámica y el uso de enzimas descrito en el trabajo.

- El desarrollo de geles biocompatibles para la liberación controlada de fármacos con propiedades regulables en función de estímulos. 
Annex II. List of Molecules

\section{List of Compounds:}

\section{- Amphiphiles}

\section{Proline derivatives}

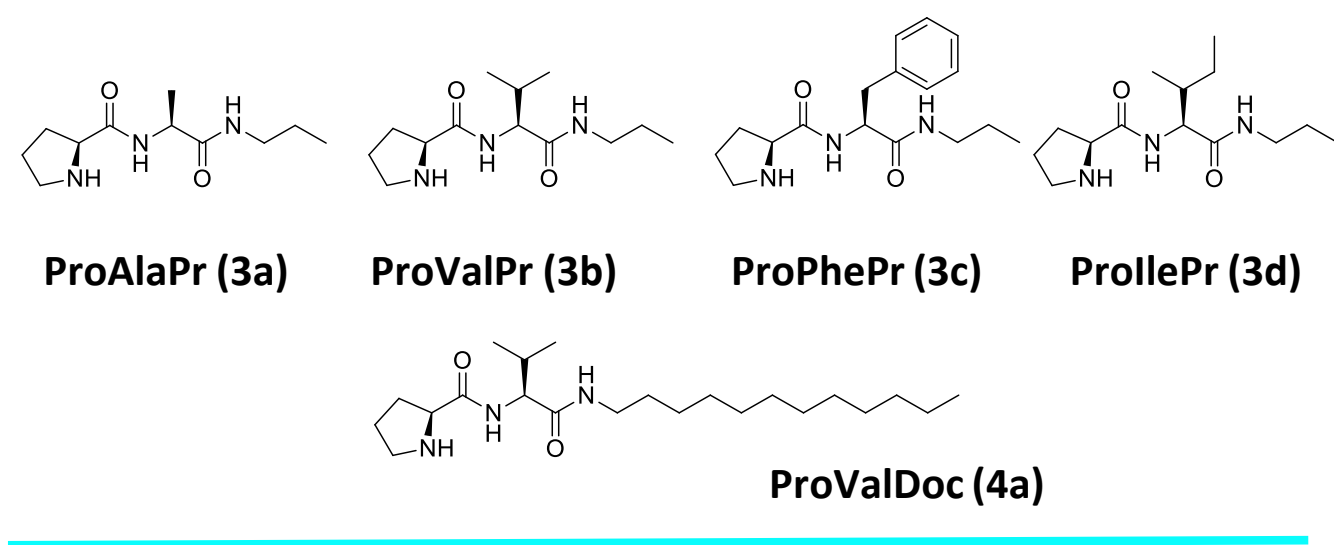

Succinic acid derivatives
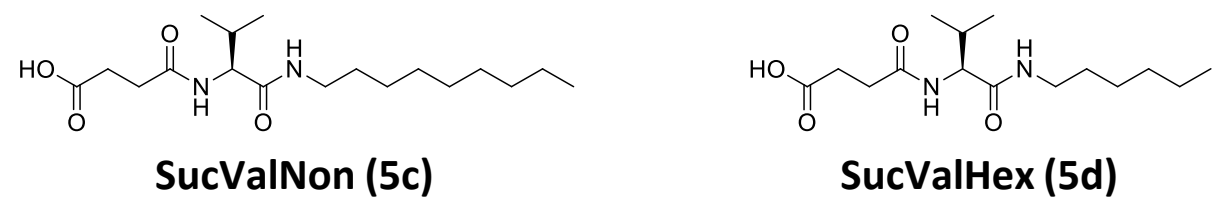

\section{- Bolaamphiphiles}

Proline derivatives

$$
\text { Prolle6(3e) }
$$

Succinic acid derivatives
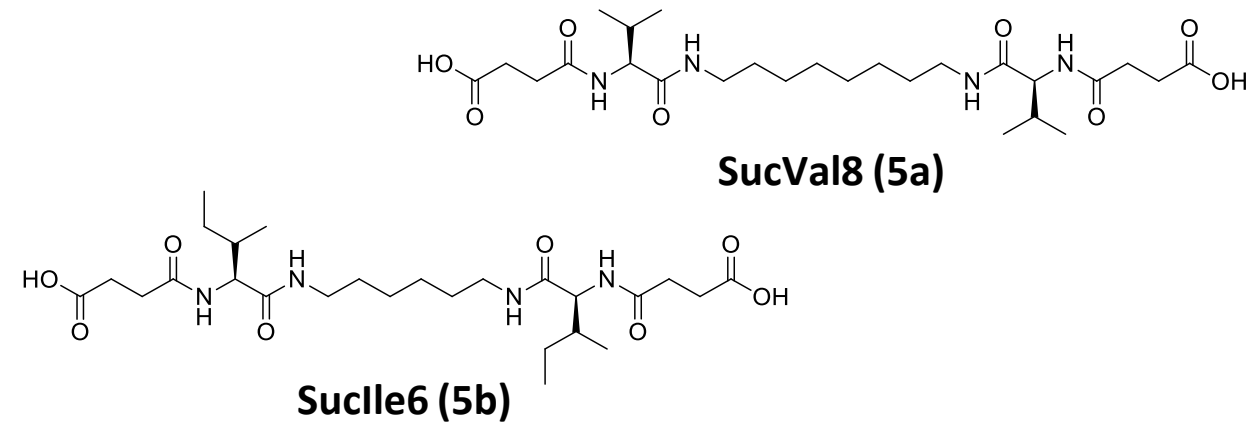


\section{$\underline{\text { NDI derivatives }}$}
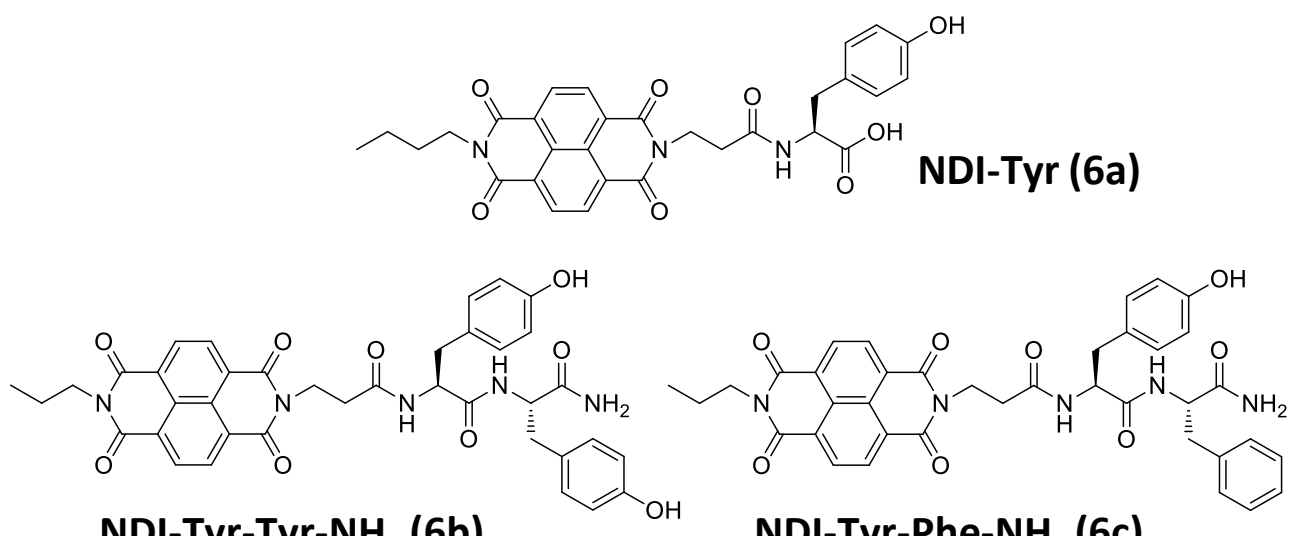

NDI-Tyr-Tyr- $\mathrm{NH}_{2}$ (6b)

NDI-Tyr-Phe- $\mathrm{NH}_{2}(6 \mathrm{c})$

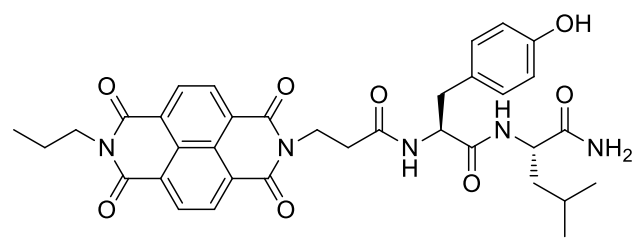

NDI-Tyr-Leu-NH ${ }_{2}$ (6d)

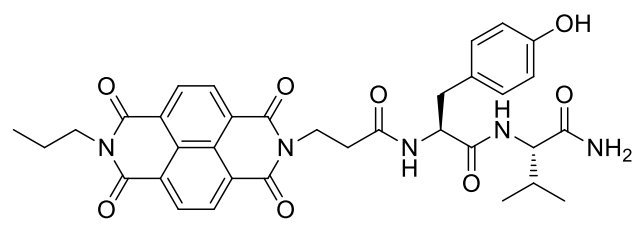

NDI-Tyr-Val- $\mathrm{NH}_{2}$ (6e) (n)

NDI-Tyr-Ala-NH $\mathrm{N}_{2}$ (6f)

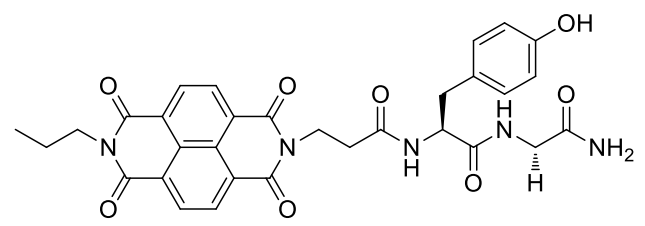

NDI-Tyr-Gly-NH 2 (6g)

\section{- Rest of compounds}

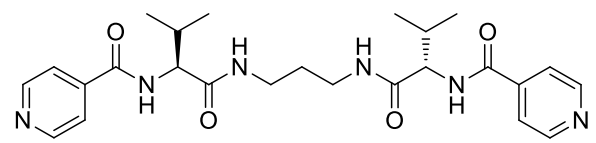

\section{PyrVal3 (5e)}<smiles>N#C[AsH3]</smiles><smiles>Oc1ccc2cc(O)ccc2c1</smiles>

2,6-DHN

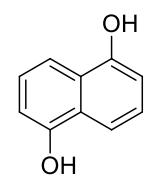

1,5-DHN<smiles></smiles>

2,6-DAN<smiles>Nc1cccc2c(N)cccc12</smiles>

1,5-DAmN

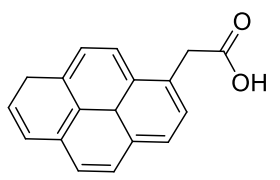

1-PAA 